\title{
Total Synthesis of Various Hormaomycin Analogues with Modified Amino Acid Residues
}

\author{
Dissertation \\ zur Erlangung des Doktorgrades \\ der Mathematisch-Naturwissenschaftlichen Fakultäten \\ der Georg-August-Universität zu Göttingen
}

vorgelegt von

Vitaly Raev

aus Murmansk / UdSSR

Göttingen 2008 
D 7

Referent: Prof. Dr. A. de Meijere

Korreferent: Prof. Dr. A. Zeeck

Tag der mündlichen Prüfung: 02.07.2008 
Meinem Lehrer, Herrn Prof. Dr. A. de Meijere, danke ich herzlich für die interessante Themenstellung, die während dieser Arbeit erwiesene Unterstützung und die stete Diskussionsbereitschaft zu Fragen dieser Arbeit. 

Dedicated to my family - with gratitude for understanding and patience 


\section{TABLE OF CONTENTS}

INTRODUCTION .1

MAIN PART 11

1. (Mono-, (Di- and (Trifluoromethyl)substituted cyclopropylalanines.................................11

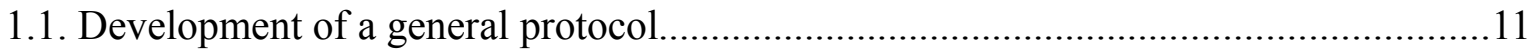

1.2. Attempted syntheses according to the proposed synthetic route..................................14

2. New and improved syntheses of some other non-proteinogenic amino acids....................22

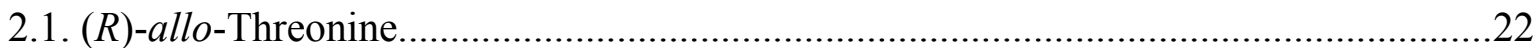

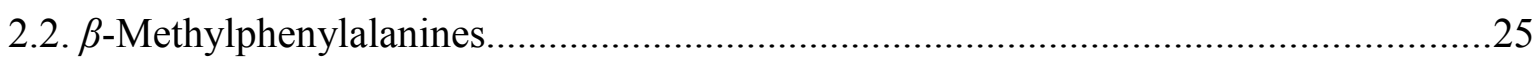

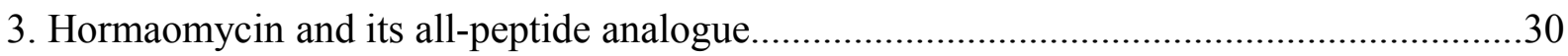

4. Hormaomycin analogues with fluoromethyl-substituted cyclopropylalanine residuess.......38

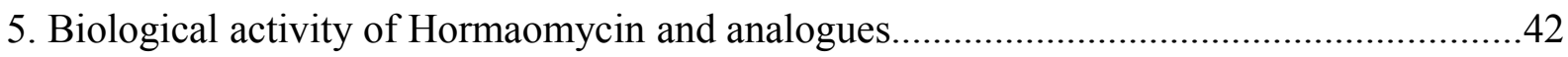

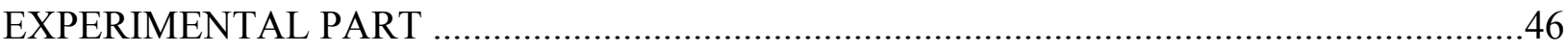

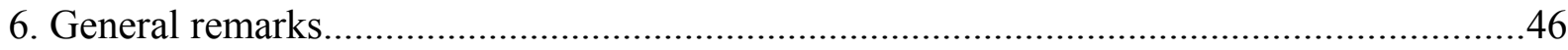

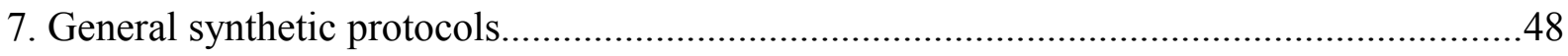

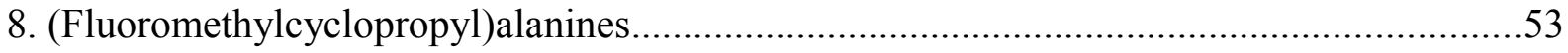

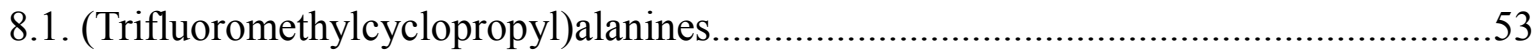

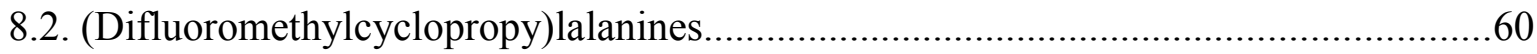

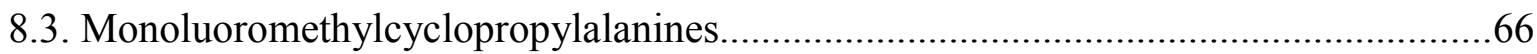

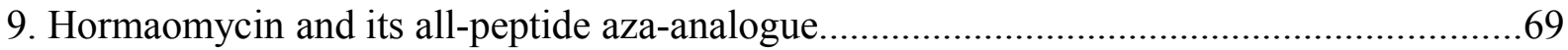

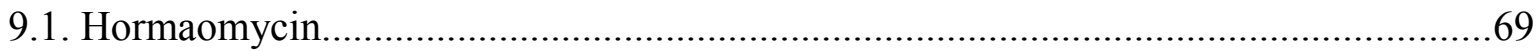

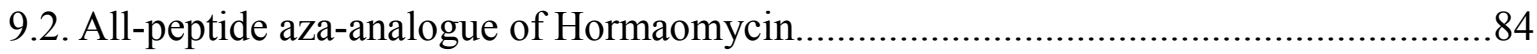

10. Hormaomycin analogues containing (fluoromethylcyclopropyl)alanine moieties............94

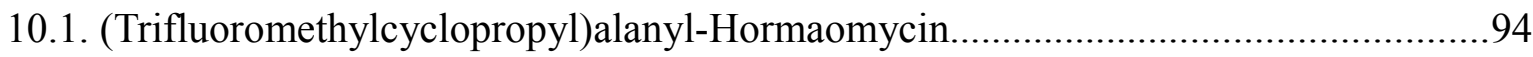

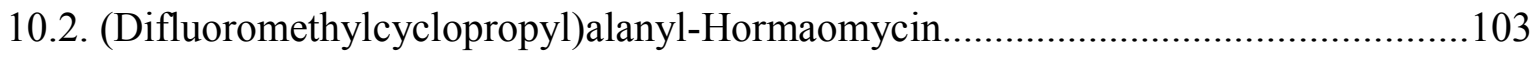

10.3. (Monofluoromethylcyclopropyl)alanyl-Hormaomycin.........................................115

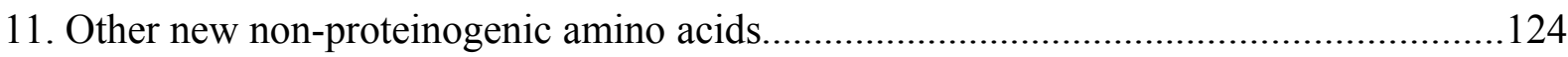




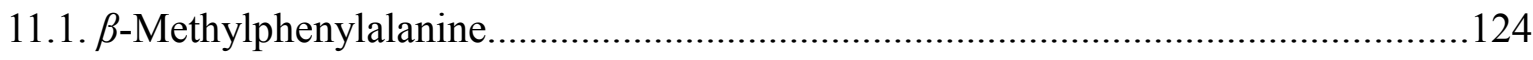

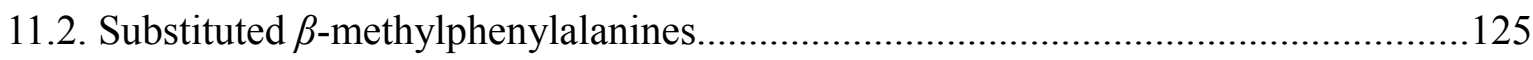

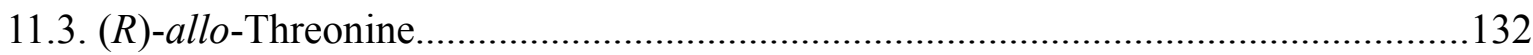

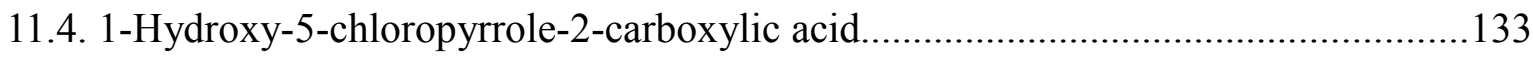

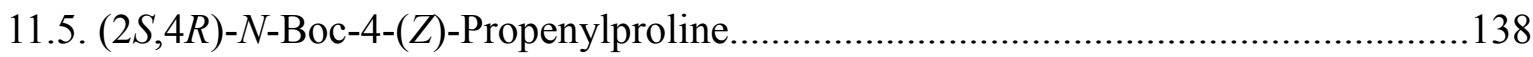

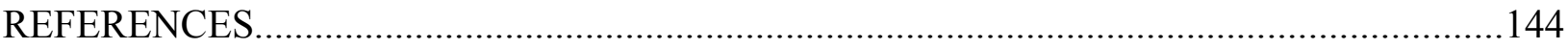

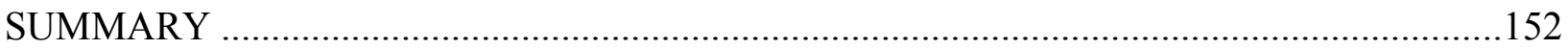

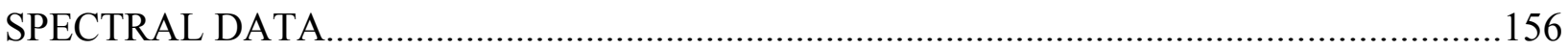

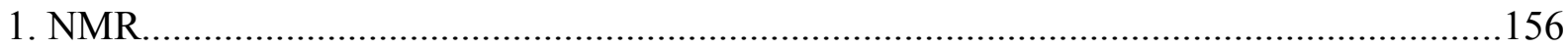

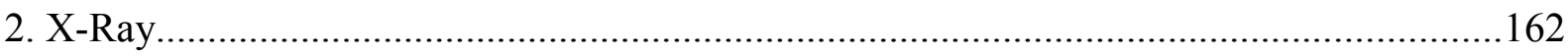

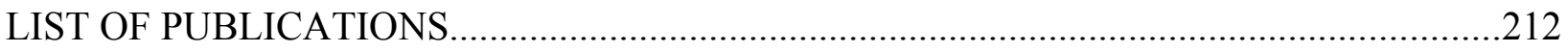

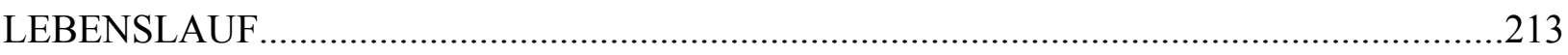

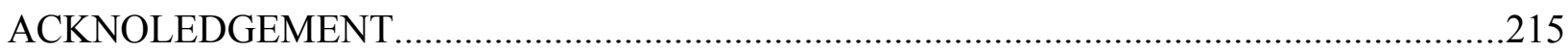




\section{ABBREVIATIONS}

DCPM dicyclopropylmethyl;

DIEA $\quad N, N$-diisopropylethylamine;

DMAP 4-dimethylaminopyridine;

EDC $\quad N^{\prime}$-(3-dimethylaminopropyl)- $N$-ethylcarbodiimide hydrochloride;

Fmoc 9-fluorenylmethyloxycarbonyl;

FmocOSu $O$-(9-fluorenylmethyloxycarbonyl)-1-hydroxypyrrolidine-2,5-dione;

HATU O-(7-azabenzotriazole-1-yl)- $N, N, N^{\prime}, N^{\prime}$-tetramethyluronium hexafluorophosphate;

HOAt 7-aza-1-hydroxybenzotriazole;

MeZ 4-methylbenzyloxycarbonyl;

MeZOSu O-(4-methylbenzyloxycarbonyl)-1-hydroxypyrrolidine-2,5-dione;

MOM methoxymethyl;

Teoc (2-trimethylsilylethyl)-oxycarbonyl;

TeocOSu $O$-[(2-trimethylsilylethyl)-oxycarbonyl]-1-hydroxypyrrolidine-2,5-dione;

TMP 2,4,6-collidine;

Z benzyloxycarbonyl;

$\mathrm{ZOSu} \quad O$-benzyloxycarbonyl-1-hydroxypyrrolidine-2,5-dione. 


\section{INTRODUCTION}

The exploration of microorganisms as sources of medicinally relevant compounds has a much shorter and less well-known history than the use of substances of plant or animal origin. Nevertheless, from the time of the discovery of the antibacterial effect of penicillin by A. Flemming in $1928^{[1]}$ and the beginning of its widespread application and manufacturing during the Second World War, such substances, which are produced by bacteria and fungi, have been attracting an ever increasing attention of scientists. Among all the chemical entities, which are "manufactured" by these miniature "pharmaceutical factories", the so-called secondary metabolites occupy a special role. Secondary metabolites are those naturally produced substances, which do not play an apparent role in the internal economy of an organism that produces them. In microorganisms the ability to produce such compounds may have evolved because of certain selection advantages conferred upon them as a result of the interactions of the compounds with specific receptors in other organisms. Although almost 20000 microbial metabolites and approximately 100000 plant products have been described so far, secondary metabolites still appear to be an inexhaustible source of lead structures for new antimicrobials, antiviral, antitumor and immunosuppressive drugs as well as plant protecting agents. In addition, numerous secondary metabolites, such as Benzylpenicillin ${ }^{[2]}$, Cephalosporin, Erythromycin, Strobilurin, etc. were lead structures that later became the basis for synthetic and semi-synthetic derivatives with improved pharmacological properties. ${ }^{[3]}$ Some of these compounds play a key role not only in defense mechanisms of microorganisms, but may be used as signal substances for intercellular communication with a function similar to those of hormones and pheromones in higher organisms. ${ }^{[4]}$ There are a lot of processes during the life cycle of a bacterium, which are regulated by such substances. For example, they regulate the metabolic capability and the quorum sensing ${ }^{[5]}$ in Gram-negative pathogenic bacteria, the competence ${ }^{[6]}$ and sporulation in Bacillus, the sporulation, multicellular differentiation and motility in Myxococcus, the antibiotic production, morphological differentiation and sporulation in Streptomyces and Erwinia, and gene transfer mechanisms in Enterococcus. ${ }^{[7,8,9]}$ It appears to be very attractive to employ the knowledge about such compounds either in terms of controlling cellular proliferation or conversely to increase the production of a particular secondary metabolite. The latter possibility was first realized in the 1960s, when several metabolites of Actinomycetes were shown to control the production of antibiotics and the morphological differentiation (aerial mycelium formation) even in nanomolar concentrations. All these compounds were structurally very similar 2,3-disubstituted $\gamma$-butyrolactones, which nevertheless showed remarkably different spectra of 
action: a so-called A-factor (Khokhlov factor) stimulated the production of Streptomycin, socalled IM-type regulators stimulated the production of Staphylomycin and so-called VB-type regulators stimulated Virginiamycin production in different Streptomyces species. ${ }^{[10,11]}$ There are also modified homoserine lactones, i.e. $N$-( $\beta$-ketocaproyl)-(S)-homoserine lactone (KHL) of $V$. fischeri, which can stimulate the Carbapenem antibiotic biosynthesis in E. carovora, and the B-factor of A. mediterranei, an adenosine derivative, which induces Rifamycin B synthesis in Nocardia species. ${ }^{[12]}$

The peptolide Hormaomycin 1 was isolated from Streptomyces griseoflavus, strain W-384, during the screening of intermolecular signal substances by Zäner et al. in $1989 \cdot{ }^{[13,14]}$ It was the first ever discovered such substance with a peptide structure, which induced the antibiotics production and aerial mycelium formation not only in the producing strain itself, but also in other Streptomyces species. Its e. g. the production of Hydroxystreptomycin in S. flaveolus, of Streptolin in S. fridae, of Tirandamycin in S. griseoflavus, strain 1306, and of Bafilomycin in S. griseus. A significant increase in the antibiotics production was observed already at a $0.05 \mu \mathrm{g} /$ $\mathrm{L}$ concentration of Hormaomycin. This compound also showed strong antibiotic activity against coryneform ${ }^{[15]}$ bacteria (MIC $=0.0005 \mu \mathrm{g} / \mathrm{mL}$ for Arthrobacter oxydans) ${ }^{[16]}$

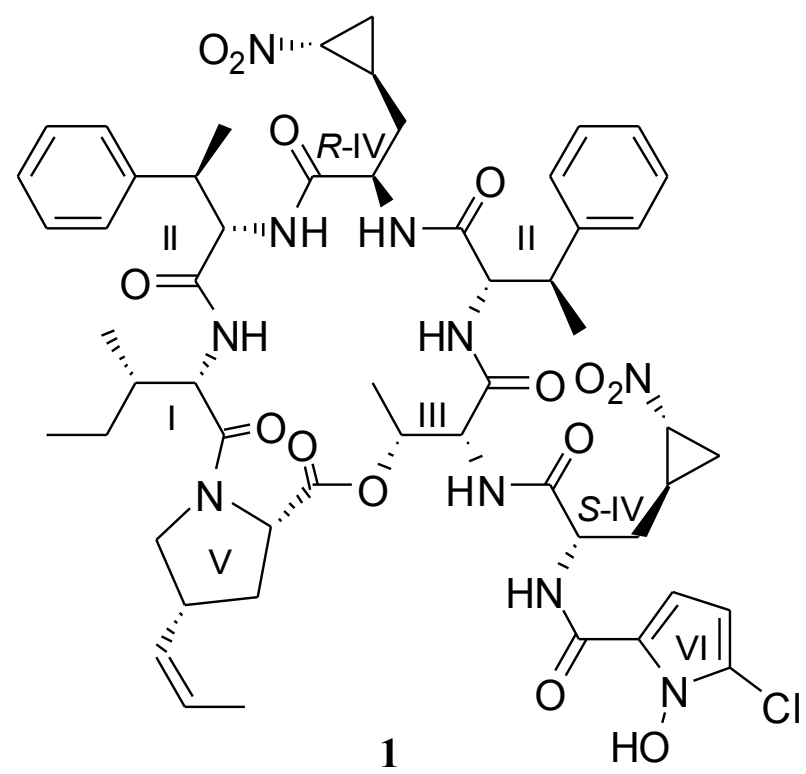

Figure 1. Structure and absolute configuration of Hormaomycin. I ( $S$ )-Ile; II $(2 S, 3 R)-(\beta \mathrm{Me}) \mathrm{Phe} ; \mathrm{III}(R)-\mathrm{a}-\mathrm{Thr}$; IV $\left(1^{\prime} R, 2^{\prime} R\right)-(3-\mathrm{Ncp}) \mathrm{Ala}$; V $(2 S, 4 R)-4-(Z)-(4-P e)$ Pro; VI Chpca.

The constitution of this cyclic depsipeptide showed features unusual even for this structurally flexible class of compounds. Initial structural investigations performed by Zeeck et al. ${ }^{[14,16,17]}$ 
disclosed that along with one residue of the proteinogenic $(S)$-isoleucine [Ile], Hormaomycin contains two units of 3-(2S,3R)-methylphenylalanine [( $\beta \mathrm{Me}) \mathrm{Phe}, \mathrm{MeF}]$, one of $(2 R)$-allo-threonine [a-Thr] as well as two moieties of 3-(trans-2'-nitrocyclopropyl)alanine [(3-Ncp)Ala] and one of 4-(Z)-propenylproline [(4-Pe)Pro]. The side chain of $\mathbf{1}$ is terminated with a residue of 5-chloro-1-hydroxypyrrole-2-carboxylic acid [Chpca] (Figure 1). The latter three elements had never been found in any natural product before. A partial assignment of the absolute configuration of the (3-Ncp)Ala residues in $\mathbf{1}$ was later made by Zindel and de Meijere. ${ }^{[18,19]}$ The retention times of the derivatized synthetically prepared enantiomerically pure mixtures of the diastereomers of 3-(trans-2'-nitrocyclopropyl)alanine were compared with the derivatized components in the total hydrolysate of natural Hormaomycin. These experiments unambiguously proved that both (3-Ncp)Ala residues in the cyclic depsipeptide 1 have the same $\left(1^{\prime} R, 2^{\prime} R\right)$ configuration in the 2-nitrosubstituted cyclopropyl moiety and the opposite configurations at the $\alpha$-carbons. However, the assignment as to which diastereomer of the (3-Ncp)Ala residue is incorporated in the ring of $\mathbf{1}$ and which is attached in the side-chain, remained unsolved. To clarify the situation, feeding experiments with enantiomerically pure deuterium-labelled 3-(trans-2'-nitrocyclopropyl)alanine were carried out. (2S,1'RS,2'RS)-3,3-Dideuterio-3-(trans-2'nitrocyclopropyl)alanine was first synthesized by Loscha ${ }^{[20]}$ and the correspondingly deuteriumlabelled Hormaomycin was indeed obtained after the appropriate feeding experiments, which were carried out by Alvermann. ${ }^{[2]}{ }^{1} \mathrm{H}-,{ }^{2} \mathrm{H}-\mathrm{NMR}$ and MS-ESI spectra of these labelled compounds unequivocally showed that the labelled amino acid had been incorporated twice. The possible explanation for this fact is that the (2S)-epimer initially administered, can later in the cell be epimerized by a specific enzyme, an epimerase, before the assembly of the peptide chain of Hormaomycin starts, or during this process, after the amino acid has been bound to the multienzyme complex. The relative and absolute configuration of the 4-(Z)-propenylproline moiety remained unclear, and no attempts to elucidate it had been made before Zlatopolski et al. [22] provided this amino acid in deuterium-labelled form for feeding experiments. This investigation disclosed the absolute configuration for the 4-(Z)-propenylproline moiety as well as for the $(2 R)$ - and the $(2 S)-3-\left(1^{\prime} R, 2^{\prime} R\right)-\left(2^{\prime}-\right.$-nitrocyclopropyl)alanine residues.

While several synthetic accesses to D-allo-threonine and (2S,3R)-3-methylphenylalanine have been reported in the literature, the enantio- and diastereoselective synthesis of the previously unknown 3-(trans-2'-nitrocyclopropyl)alanine has mainly been investigated in our group. At

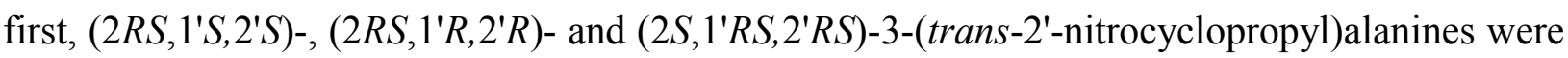
successfully prepared ${ }^{[18,23]}$ Unfortunately, a great number of steps and a relatively low overall yield strongly decreased the preparative value of this synthetic route. In fact, this procedure even 
did not enable one to obtain any enantiomerically pure diastereomer of 3-(trans-2'-nitrocyclopropyl)alanine. The improvement of the originally reported procedure was connected with the progress in the preparation of the enantiomerically pure $\left(1^{\prime} R, 2^{\prime} R\right)-\left(2^{\prime}\right.$-nitrocyclopropyl)methanol which served as the key intermediate in this synthesis developed by Brandl and de Meijere et al. ${ }^{[24]}$ Finally, all four possible diastereomers of 3-(trans-2'-nitrocyclopropyl)alanine were synthesized by Larionov and de Meijere, et al. ${ }^{[25]}$ in enantiomerically pure form and in good to excellent yields. Significant progress was also achieved in the preparation of 4-(Z)-propenylproline. This compound was first prepared from 5-(2-dimethylaminopropyl)piperidone-2 in 1958, but only as a mixture of all four possible stereoisomers along with all possible stereoisomers of 4 -allylproline. ${ }^{[26]}$ The procedure more recently proposed by Melotto ${ }^{[27]}$ allowed one to prepare 4-(Z)-propenylproline as an individual compound starting from $\mathrm{N}, \mathrm{O}$-diprotected pyroglutamic acid. The protocol, eventually developed by Zlatopolskiy, starts from natural $(2 S, 4 R)$-4-hydroxyproline and leads to (2S,4R)-4-(Z)-propenylproline of good purity and, after 8 steps, with an overall yield of more than $10 \%{ }^{[28]}$ Initially, $N$-Boc-protected ${ }^{[29]}(2 S, 4 R)$-4-hydroxyproline 2 was converted to the corresponding prolinol 3 by sodium borohydride reduction of the mixed anhydride prepared with ethyl chloroformate. The primary hydroxy group of the resulting diol was selectively protected with $t \mathrm{BuMe}_{2} \mathrm{SiCl}_{,}^{[30]}$ and the secondary hydroxy group was converted to a methanesulfonyloxy group to be $\mathrm{S}_{\mathrm{N}} 2$-substituted with cyanide with inversion of the configuration. The resulting nitrile 6 was reduced to the corresponding aldehyde 7 with di- $n$-butylaluminum hydride and the (Z)-configured double bond was installed by a Wittig alkenation with triphenylethylphosphonium bromide. The hydroxy group in the aminoalcohol $\mathbf{8}$ was deprotected with tetrabutylammonium fluoride and the hydroxymethyl group in $\mathbf{9}$ was oxidized to the carboxylic acid functionality with Jones reagent to give the $N$-Boc-protected (2S,4R)-4-(Z)-propenylproline $\mathbf{1 0}$. 
$\mathrm{HO}$,<smiles>O=C(O)C1CCCN1C(=O)O</smiles>

$\mathrm{HO}$,<smiles></smiles>

NC<smiles></smiles>
6

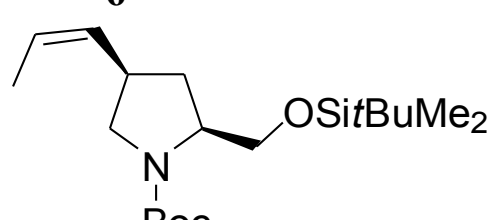

Boc

8

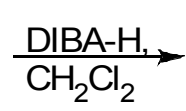

98\%

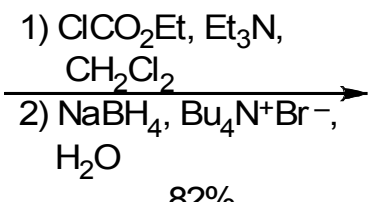

1) $\mathrm{ClCO}_{2} \mathrm{Et}, \mathrm{Et}_{3} \mathrm{~N}$,

$\mathrm{H}_{2} \mathrm{O}$

$82 \%$

$\mathrm{MsO}$,

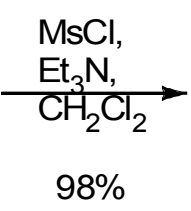

$\mathrm{HO}$,

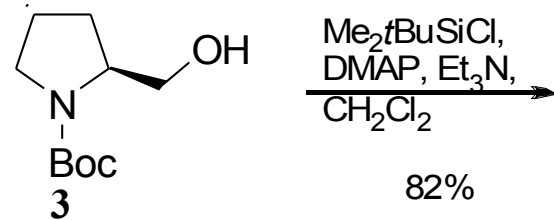

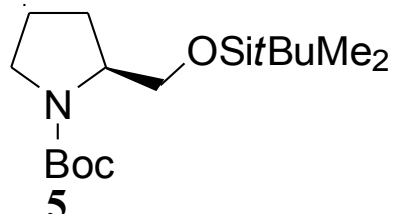

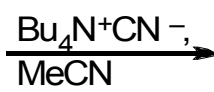

$60 \%$

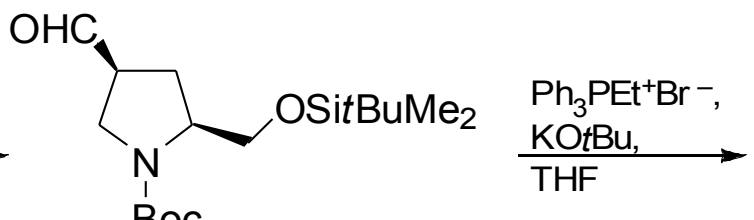

Boc

7
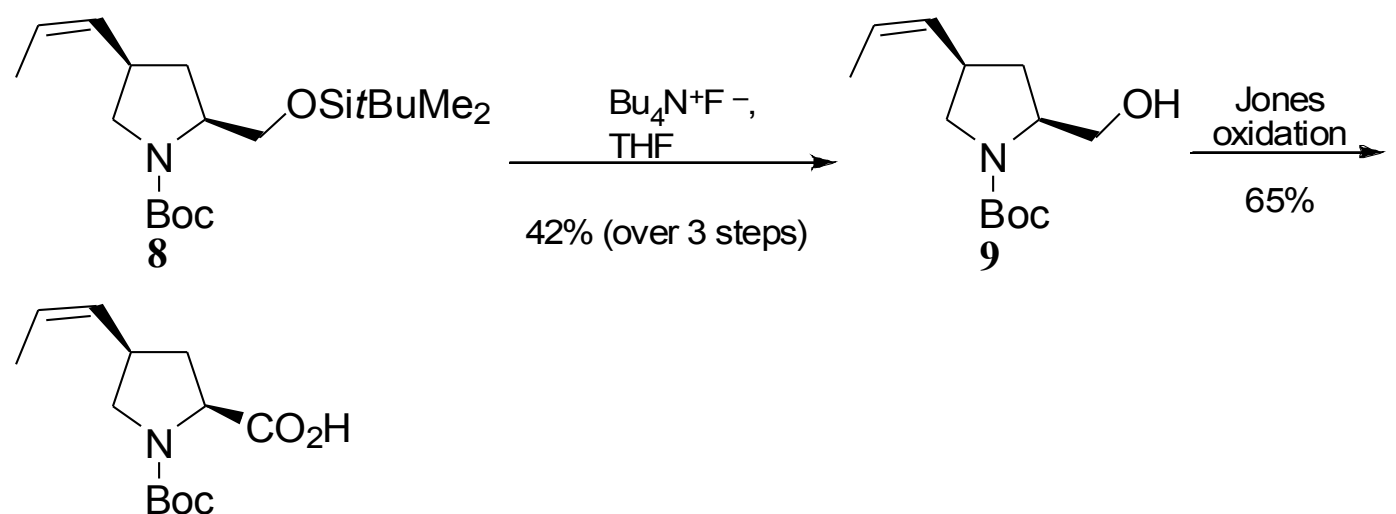

10

Scheme 1. Synthetic route to $(2 S, 4 R)-4-(Z)$-propenylproline moiety.

Prior to the work of Zlatopolskiy, ${ }^{[28]}$ no procedure for the synthesis of $N$-hydroxypyrrolecarboxylic acids or $N$-hydroxypyrrolecarboxamides had been reported in the literature. An attempted synthesis of 5-chloro-1-hydroxypyrrole-2-carboxylic acid by Ritzau turned out unsuccessful. ${ }^{[3]]}$

Structure-activity relationships for Hormaomycin were investigated to some extent using analogues obtained by modification of the natural compound and also by precursor-induced biosynthesis employing certain synthetic amino acids. The cleavage of the lactone ring of Hormaomycin with potassium carbonate in methanol, which was carried out by Rössner, ${ }^{[16]}$ produced only biologically inactive material. The same author performed a hydrogenation of the natural depsipeptide over a palladium on charcoal catalyst in methanol, which not only led to reduction of the double bond in the 4-(Z)-propenylproline moiety, but also an elimination of 
water and the reductive dehalogenation of the Chpca fragment as well as partial reduction of the nitro groups in both (3-Ncp)Ala residues. The resulting mixture of Hormaomycin-like substances did not show any antibiotic activity. Later, a fine tuning of the hydrogenation conditions of the native depsipeptide allowed Ritzau ${ }^{[31]}$ to successfully prepare a Hormaomycin analogue containing a 4-propylproline instead of the original 4-(Z)-propenylproline moiety. This analogue did initiate the aerial mycelium formation in Streptomyces species even more pronouncedly than native Hormaomycin. It also showed antibiotic activity against coryneform bacteria, although its activity was noticeably lower than that of the unmodified depsipeptide. The same author prepared an analogue of Hormaomycin, which contained a bromine instead of a chlorine substituent in the Chpca fragment. This substitution caused only a little loss of the capability to induce the formation of the aerial mycelium, but a drastic decrease of the antibiotic activity. Feeding experiments with synthetic 2-(trans-2'-nitrocyclopropyl)glycine ${ }^{[32]}$ and 3-(trans-2'-methoxycarbonylcyclopropyl)alanine ${ }^{[33]}$ enabled Alvermann ${ }^{[21]}$ to obtain both possible Hormaomycin analogues containing a 2-(trans-2'-nitrocyclopropyl)glycine residue instead of one (3-Ncp)Ala moiety and depsipeptides with both (3-Ncp)Ala fragments being substituted either by 2-(trans-2'-nitrocyclopropyl)glycine or by 3-(trans-2'-methoxycarbonylcyclopropyl)alanine moieties. All these analogues did not display any Hormaomycin-like activity.

As was already mentioned, Hormaomycin contains two moieties of 3-(trans-2'-nitrocyclopropyl)alanine. Aliphatic nitro compounds are very rare in nature, in fact, less than thirty such compounds have been isolated till now, and among them is the dipeptide nitropeptine 11 isolated from S. xanthochromogenus, which displayed noticeable antifungal activity. ${ }^{[34]}$ One might therefore be tempted to suppose that the unique biological activity of Hormaomycin would be connected with its nitro group containing fragments. 3-(trans-2'-Nitrocyclopropyl)alanine itself (at least as a mixture of all possible isomers) was already tested and turned out to be inactive, but this inactivity might be due to the low capability of many amino acids to permeate across cell walls in the absence of a special transport mechanism because of their low lipophilicity. It was also known, that one of the new potent inhibitors of influenza neuraminidase, compound A-315675 12, contains a 4-(Z)-propenylproline fragment. ${ }^{[35]}$ Therefore a more detailed study of the role of this fragment for the biological activity of Hormaomycin would be necessary. 
<smiles>CC(C)CC(N)C(=O)NC(C(=O)O)C(CC(=O)O)[N+](=O)[O-]</smiles>

11

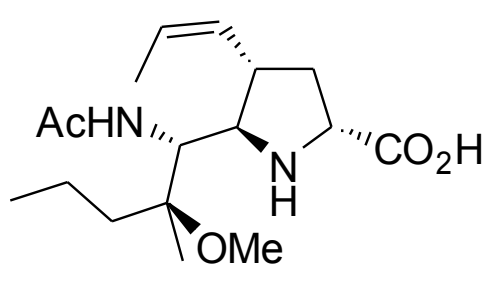

12

Figure 2. Nitropeptine 11 and the natural product A-315675 12.

There is significant interest in the preparation of modified proteins containing unnatural amino acids, in particular, fluorinated amino acid analogues, and this is due, on the one hand, to the possibility of solving a number of fundamental problems related to the studies of protein structures as well as structure-property relationships, and, on the other hand, to the probable practical application of these proteins. ${ }^{[36,37,38]}$ The replacement of amino acid residues in proteins by their analogues may give rise to proteins with new properties and, in particular, may favorably change the properties of well-known proteins toward their practical use. In particular, their lipophilicity, their substrate specificity, their stability, their $\mathrm{pK}_{\mathrm{a}}$ values, their in vivo availability and improved permeation capability through certain body barriers, as well as their temperature optimum of action and folding kinetics can be modified. ${ }^{[39,40,41,42,43]}$ Transport rates of peptides through membranes in vivo are known to be enhanced by increasing the lipophilicity. The site specific incorporation of highly lipophilic amino acids and amino acid analogues into biologically active peptides appears to be a major aim in modern peptide chemistry.

Fluorinated amino acids and derived peptides - both analogues of naturally occurring compounds and synthetic substances - claim an extraordinary interest in chemistry and biochemistry as well as in medicinal research because of their enormous variety of biological activities. ${ }^{[44,45]}$ Thus the replacement of the phenylalanine residues in PvuII-endonuclease by 3-fluorophenylalanine leads to a twice as high specific activity compared to that of the native enzyme, while the introduction of 4-fluorophenylalanine reduces it fourfold. ${ }^{[46]}$ An X-ray diffraction structure analysis of glutathione transferase M-1, in which Tyr residues were replaced by 3-fluorotyrosine has revealed multiple conformational changes in the structure of the modified enzyme, which changed its spectral and kinetic characteristics. ${ }^{[47]}$

Because of the high electron density, the trifluoromethyl group is capable to participate in hydrogen bonding ${ }^{[48]}$ and may act also as a coordination site in metal complexes. Furthermore, 
the fluorine atoms can serve as powerful NMR labels for spectroscopic studies of metabolism and conformation.

The replacement of substantial amino acids in microbial proteins by synthetic analogues is a route to the preparation of compounds with potentially increased biological activity based on previously known microbial products.

The previously achieved progress in the synthesis of the Hormaomycin and its analogues as well as the investigation of the structure-activity relationships for these compounds which is briefly described above has lead to a list of desirable goals for the presented research:

- Synthesis of Hormaomycin and its all-peptide analogue to obtain enough material for in vivo biological tests.

- Synthesis of new Hormaomycin analogues, containing $(2 R)$ - and $(2 S)-3-\left(1^{\prime} S, 2^{\prime} R\right)-\left(2^{\prime}-\right.$ fluoromethylcyclopropyl)alanine moieties instead of $(2 R)$ - and $(2 S)-3-\left(1^{\prime} R, 2^{\prime} R\right)-\left(2^{\prime}\right.$-nitrocyclopropyl)alanine.

- Synthesis of $(2 R)$ - and (2S)-3-(1'S,2'R)-(2'-fluoromethylcyclopropyl)alanines (mono-, diand trifluoromethyl derivatives).

- Development of new improved protocols for the synthesis of $(R)$-allo-threonine and $\beta$-methylphenylalanine moieties. 


\section{MAIN PART}

Once the absolute configuration of the native Hormaomycin had been established and the strategy of the synthesis and the route to Hormaomycin were developed by Zlatopolskiy, ${ }^{[49]}$ the main aim of the present work was to synthesize 2'-fluoromethyl-substituted cyclopropylalanines and build the corresponding Hormaomycin analogues to test their biological activities to contribute to a wider knowledge of the structure-activity relations.

\section{1. (Mono-, (Di- and (Trifluoromethyl)-substituted cyclopropylalanines}

\subsection{Development of a general protocol}

Fluoromethyl-substituted cyclopropylalanines have never been described before. Like for the approach to 2'-nitrocyclopropylalanines, ${ }^{[25]}$ the Belokon' method was chosen as a viable access route to all of the fluoromethyl-substituted cyclopropylalanines, employing the $\mathrm{Ni}(\mathrm{II})$-complex of the Schiff base derived from glycine and $(S)$ - or $(R)-2-[(N$-benzylprolyl)amino $]$ benzophenone 13 as a reusable chiral auxiliary (Figure 3). In general, the configuration of the stereocenter, formed upon alkylation of C-2 of the glycine moiety, is the same as the configuration of the C-2 atom of the proline moiety; other stereocenters are neither generated nor involved in this transformation.
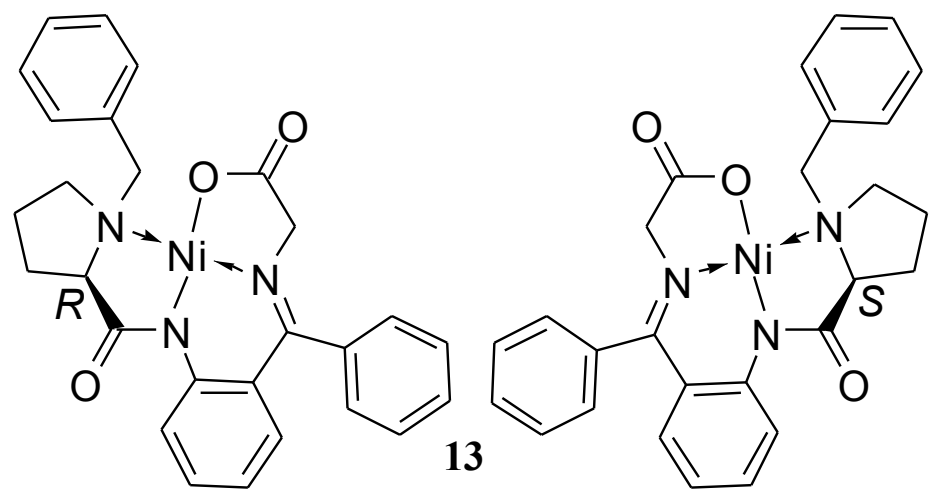

Figure 3. The Ni(II)-complexes of the Schiff base derived from glycine and $(R)$ - or $(S)$-2-[(N-Benzylprolyl)amino]benzophenone $\quad[(R)-\quad$ and $\quad(S)$-Belokon' glycine complexes, $(R)$ - and $(S)$-BGC].

2'-Fluoromethyl-substituted cyclopropylmethyl iodides were intended to be obtained by transformation of the corresponding alcohols, obtained by reduction of the corresponding carboxylates (Scheme 2). The fluoro-derivatives could be obtained from corresponding oxygen- 
functionalised derivatives by treatment with different fluorinating reagents, e. g. with the pyridine-HF complex ${ }^{[50]}$ for tertiary alcohols, with N,N-diethyl- $\alpha, \alpha$-difluoro( $m$-methylbenzyl)amine ${ }^{[51]}$ for sugars and with Xenon difluoride for aryl perfluoroalkyl sulfides. [52]

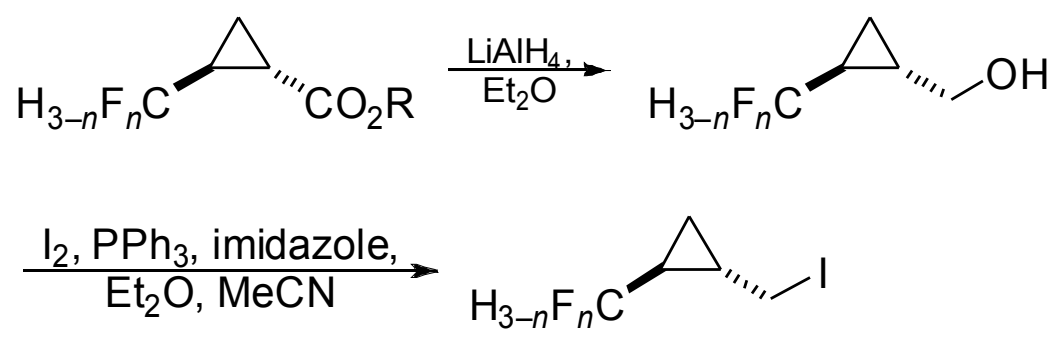

Scheme 2. Synthetic route to trans-fluoromethyl-substituted cyclopropylmethyl iodides.

Without taking into account the most exotic reagents (like $\mathrm{XeF}_{2}$ or $\mathrm{MoF}_{6}{ }^{[53]}$ ), almost all other fluorinating agents should be suitable to achieve the target. The most universal one is $\mathrm{SF}_{4}$, as it successfully converts carboxylic acids to trifluoromethyl derivatives, ${ }^{[54]}$ aldehydes and ketones to the corresponding difluorides ${ }^{[5]}$ and alcohols to monofluorides (Scheme 3). However, $\mathrm{SF}_{4}$ is problematic in handling because of its low boiling point $\left(-40^{\circ} \mathrm{C}\right)^{[56]}$ and its extreme corrosireness to glass. With $\mathrm{SF}_{4}$ it is necessary to use steel autoclaves (the usual reaction temperature is $+130{ }^{\circ} \mathrm{C}$, but $+200{ }^{\circ} \mathrm{C}$ and even $+270{ }^{\circ} \mathrm{C}^{[54]}$ can be required for some compounds) for such transformations.
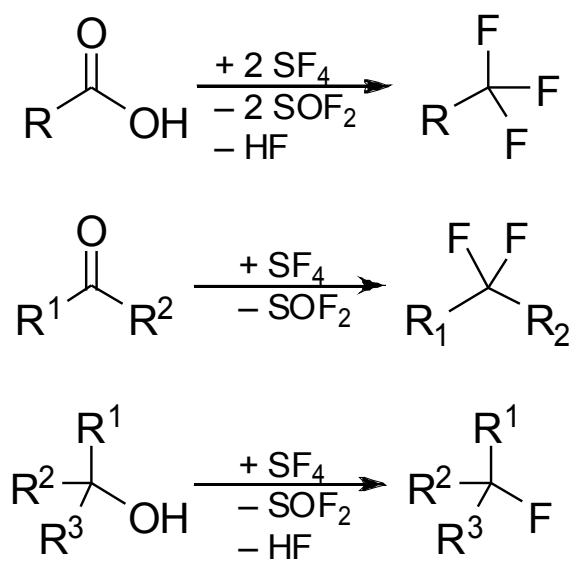

Scheme 3. Fluorinations with $\mathrm{SF}_{4}$.

Other fluoro derivatives of sulfur (IV) which have been widely used in recent years for fluorinatuons of organic compounds are the dialkylaminosulfur trifluorides. They were first prepared in $1964^{[57]}$ and first used as nucleophilic fluorination agents in 1973. ${ }^{[58]}$ These 
substances are liquid under normal conditions and, as a rule, are able to bring about such transformations under much milder conditions. More recent publications brought forward bis(2-methoxyethyl)aminosulfur trifluoride (Deoxo-Fluor $\left.{ }^{\circledR}\right) \mathbf{1 4}$ as a thermally stable and soft fluorinating agent, which ought to be applicable for virtually all of the above mentioned transformations. ${ }^{[59]}$ Unfortunately, the neat reagent is not available in Germany, so a solution of 14 in THF $(50 \% \mathrm{w} / \mathrm{w})$ was used initially.

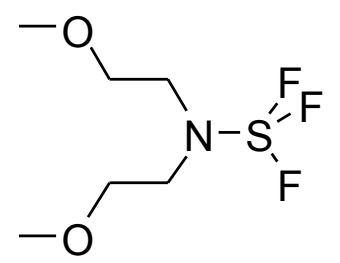

14

Deoxo-Fluor ${ }^{\circledR}$.

The synthetic route outlined in Scheme $4^{[60]}$ was initially designed to access all three desired 2-fluoromethyl-substituted cyclopropanecarboxylates.

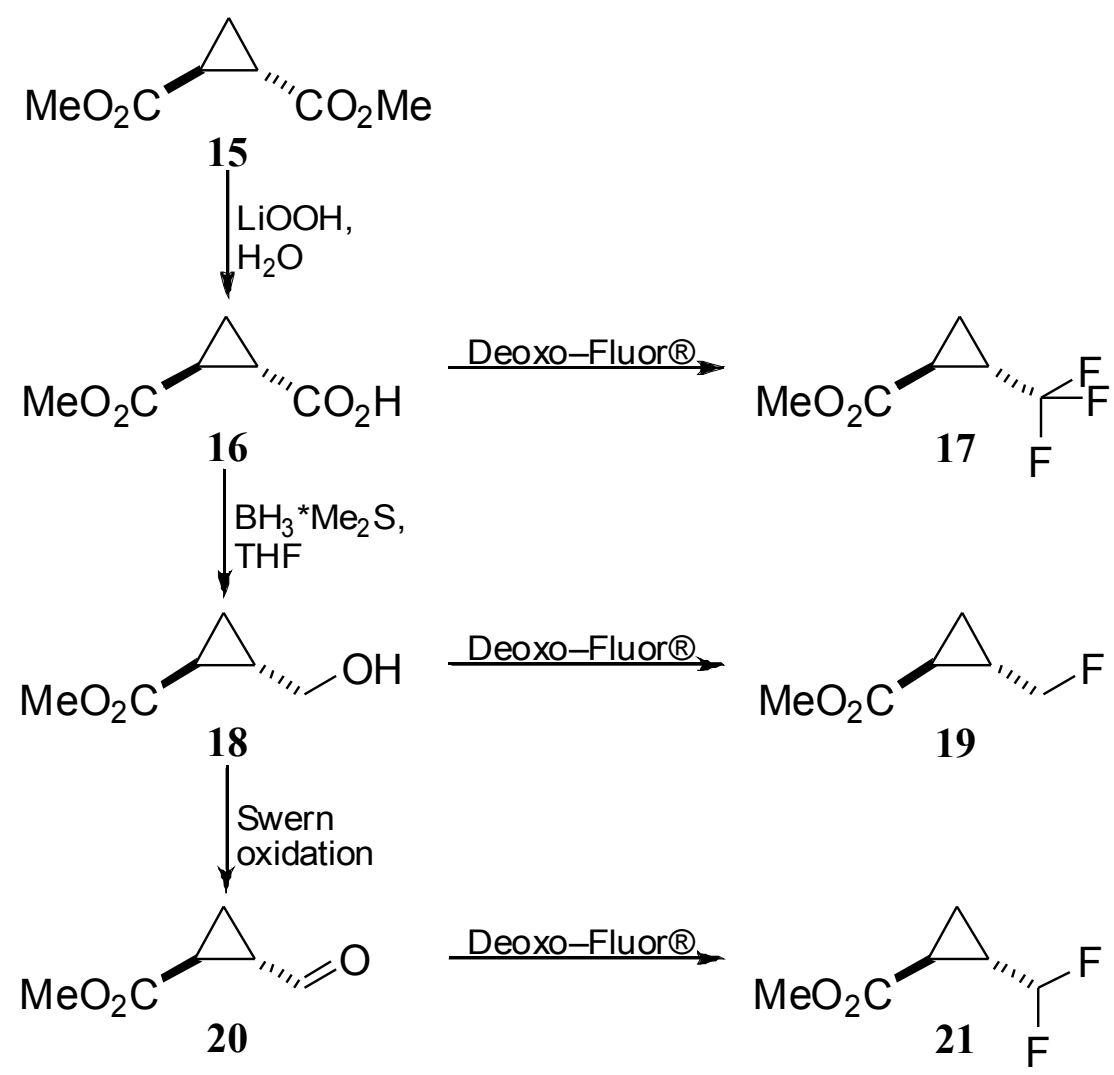

Scheme 4. Synthetic route to 2-fluoromethylcyclopropanecarboxylates. 


\subsection{Attempted syntheses according to the proposed synthetic route}

The very first attempt to access methyl trans-2-monofluoromethylcyclopropanecarboxylate 19 from the corresponding alcohol $\mathbf{1 8}$ gave unexpected results: the target monofluoromethylsubstituted ester was obtained in very low yield (only 14\%), and a mixture of derivatives 24 was obtained ( $\sim 31 \%$ using peak intensities in ESI-MS, main component with $n=5)$ as well as compounds $22(15 \%)$ and $23(12 \%)$.

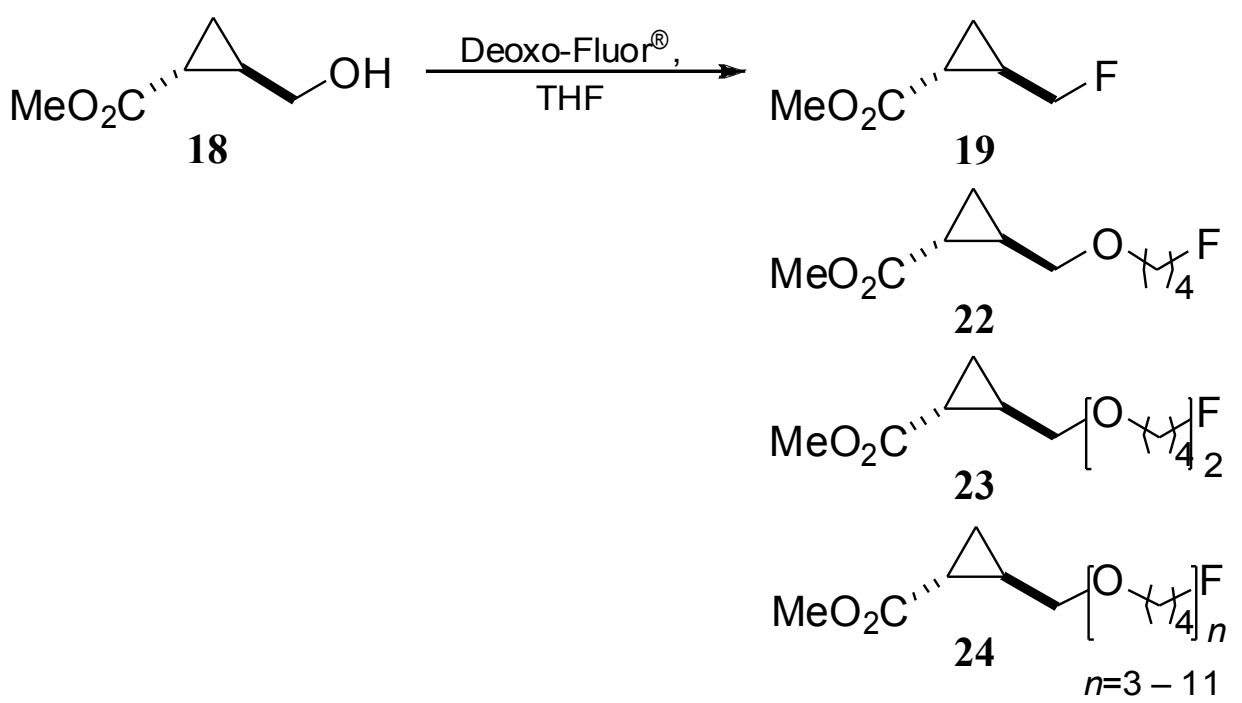

Scheme 5. The reaction of Deoxo-Fluor ${ }^{\circledR}$ in THF solution with methyl 2-hydroxymethylcyclopropanecarboxylate at ambient temperature.

The latter products are apparently formed by THF ring cleavage and a formal insertion of a 1,4-butanediol moiety into the $\mathrm{C}-\mathrm{OH}$ bond before transformation to a $\mathrm{C}-\mathrm{F}$ bond. The closest previously observed analogues of the observed reaction are the incorporation of THF into cycloadducts of tetracyanoethylene to dispiro[2.0.2.4]deca-7,9-diene, ${ }^{[61]}$ and the reaction of alkyl chlorosulfinates with THF. ${ }^{[62]}$

The formation of products $\mathbf{2 2}-\mathbf{2 4}$ can be rationalized assuming primary attack of the reagent $\mathbf{1 4}$ by the alcohol 18 molecule to form the HF molecule and the amidoester $\mathbf{2 6}$ which, in turn, produces target fluoride 19 and di-(2-methoxyethyl)-fluorosulfinamide $\mathbf{2 7}$. The latter attaches on the oxygen of a tetrahydrofurane molecule to yield an oxonium ylide $\mathbf{2 8}$ which would first be attached either by a molecule of the hydroxymethylcyclopropanecarboxylate to yield the new ylide 29 or by another molecule of tetrahydrofurane to furnish $\mathbf{3 0}$. Nucleofilic transfer of fluorine from sulfur to the vicinal carbon in $\mathbf{2 9}$ would yield $\mathbf{2 2}$, and analogously $\mathbf{2 3}$ would come about from 30 after reaction with 18. Further consecutive reactions of 30 with tetrahydrofuran and 
eventually with $\mathbf{1 8}$ and fluorine transfer would lead to the higher oligomeric products of type $\mathbf{2 4}$ (Scheme 6).

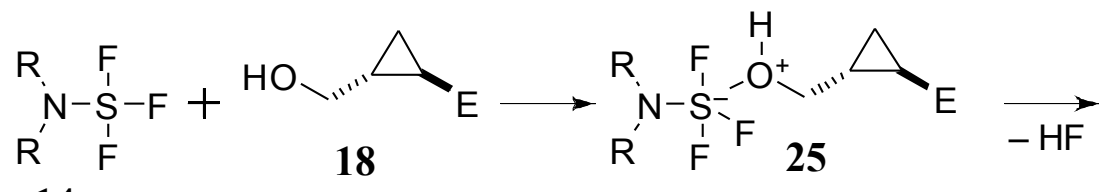

14
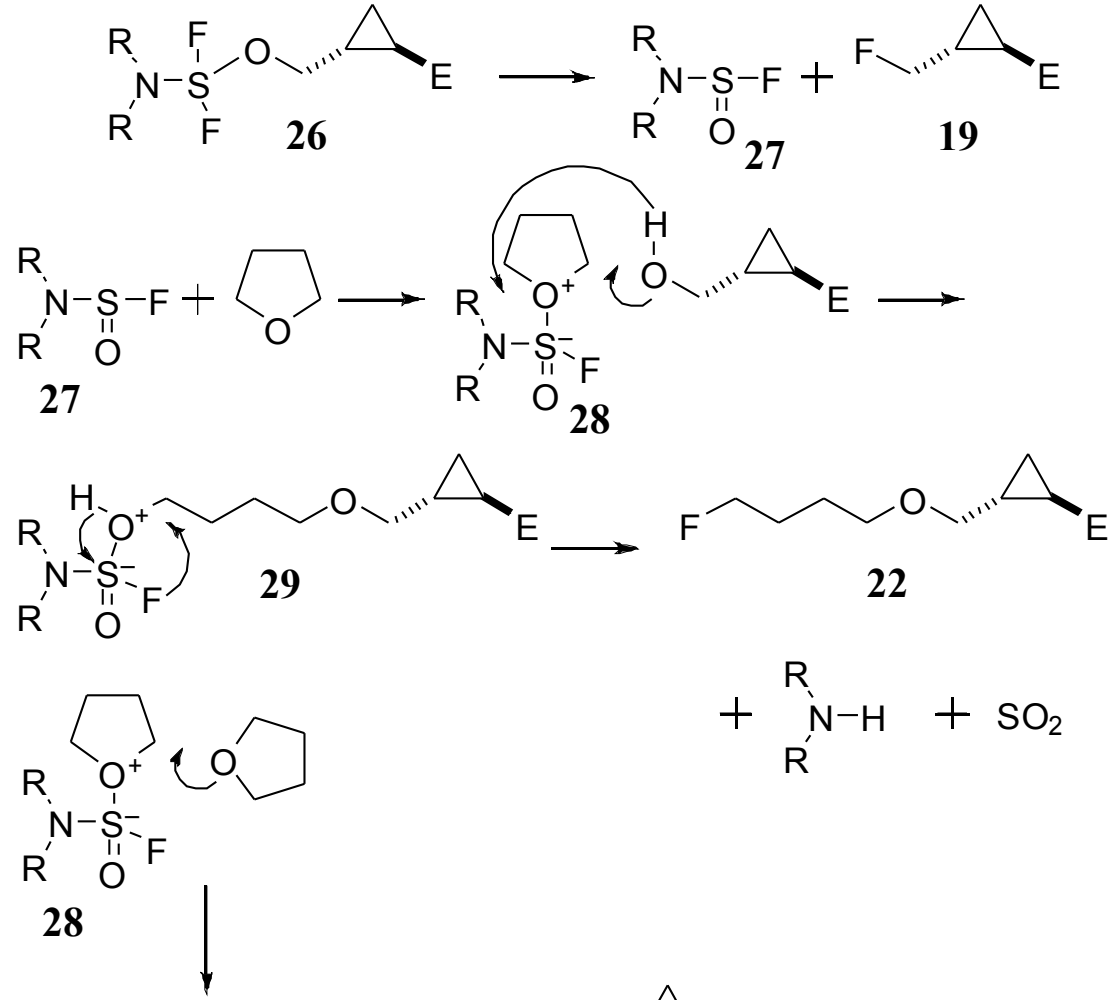

$$
+\underset{\mathrm{R}^{\prime}}{\mathrm{R}-\mathrm{H}}+\mathrm{SO}_{2}
$$

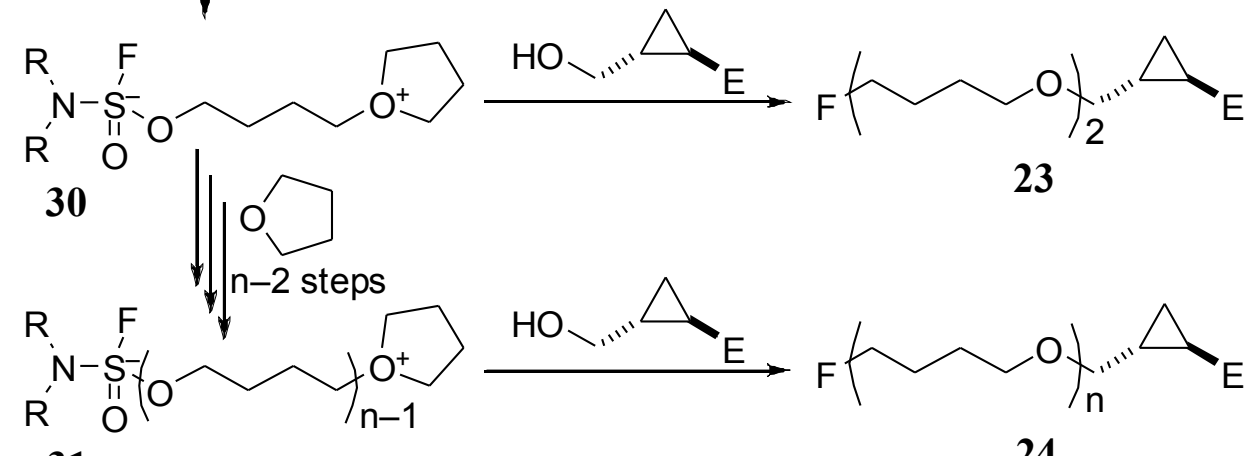

31

24

$\mathrm{R}=2$-methoxyethyl

$\mathrm{E}=\mathrm{CO}_{2} \mathrm{Me}$

Scheme 6. Mechanistic rationalization of the oligoether formation upon reaction of 2-(hydroxymethyl)cyclopropanecarboxylate with Deoxo-Fluor ${ }^{\circledR}$ in tetrahydrofuran.

When the Deoxo-Fluor ${ }^{\circledR}$ reagent was employed as a solution in toluene $(50 \% \mathrm{w} / \mathrm{w})$, transformation of the alcohol 18 to the fluoride 19 occured smoothly (47\% yield), and the 
carbaldehyde under the same conditions furnished the 2-(difluoromethyl)cyclopropanecarboxylate in $51 \%$ yield.

The attempted transformation of the carboxylic acid $\mathbf{1 6}$ to the trifluoromethyl derivative with this Deoxo-Fluor $^{\circledR}$ solution was not successful. The acid was easily converted to the acyl fluoride, the latter, however, did not react any further with the fluorination reagent, not even at reflux.

An alternative route to trans-2-(trifluoromethyl)cyclopropylmethanol is by way of the Claisen condensation product of diethyl succinate with ethyl trifluoroacetate ${ }^{[63]}$ according to known procedure. However, the previously used conditions were modified for two of the four steps in order to achieve a better overall yield ${ }^{[64]}$ (Scheme 7).<smiles>CCOC(=O)CCOC(=O)C(=O)C(CC(=O)OCC)C(=O)OCC</smiles><smiles>CCOC(=O)CCC(O)C(F)(F)CCC(=O)OCC</smiles>

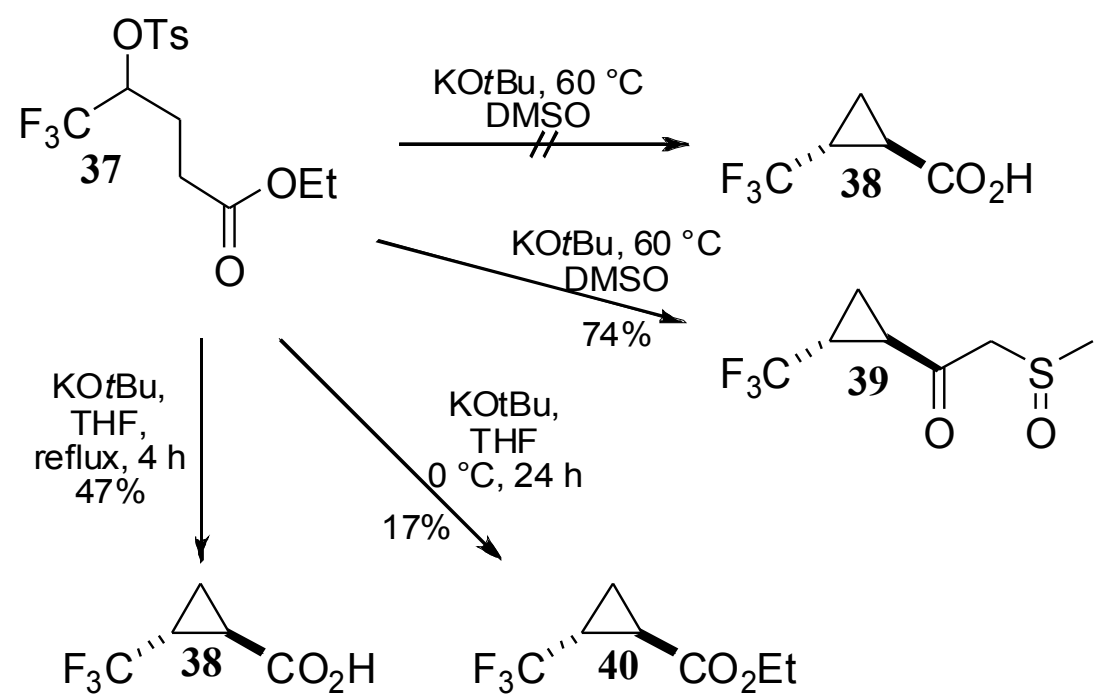

Scheme 7. Synthesis of trans-2-trifluoromethylcyclopropane carboxylic acid (yields are given after work-up). 
Surprisingly, the reduction of the ketoester $\mathbf{3 5}$ to the hydroxyester $\mathbf{3 6}$ proceeded very slowly under the previously described conditions $\left(\mathrm{H}_{2} / \mathrm{PtO}_{2}\right)$ - in several attempts $(2 \mathrm{~h}, 24 \mathrm{~h}$ and $72 \mathrm{~h})$ the yield of 36 was never better than $60 \%$, and about $25 \%$ of the ketoester 35 was recovered. Yet, the reduction of $\mathbf{3 5}$ with crushed sodium borohydride in diethyl ether gave quantitative conversion and an excellent yield of $\mathbf{3 6}$.

The final step, the attempted intramolecular 1,3-dehydrotosylation with potassium tert-butoxide in dimethylsulfoxide, also gave an unexpected result. The intermolecular condensation product $\mathbf{3 9}$ of the expected cyclopropanecarboxylate $\mathbf{4 0}$ with dimethylsulfoxide rather than $\mathbf{4 0}$ or the free acid 38, was obtained in $74 \%$ yield. Among several other base/solvent combinations tested $\mathrm{NaOEt} / \mathrm{EtOH}, \mathrm{NaOMe} / \mathrm{MeOH}, \mathrm{KO} t \mathrm{Bu} / \mathrm{BuOH}, \mathrm{NaH} / \mathrm{THF}$ and $\mathrm{KO} t \mathrm{Bu} / \mathrm{THF}$ the last one gave the best yield (up to $45 \%$ at reflux) of the target acid 38, as well as of the corresponding ethyl ester $40\left(\sim 17 \%\right.$ at $\left.0{ }^{\circ} \mathrm{C}\right)$; the latter was not obtained using any other solvent/base combinations.

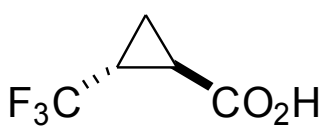

38

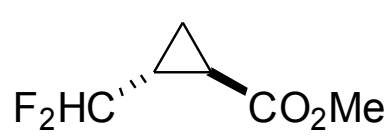

21

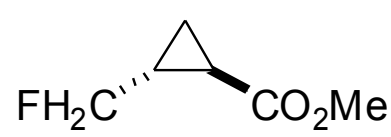

19

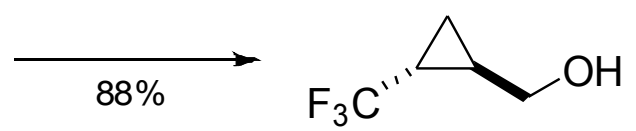

41

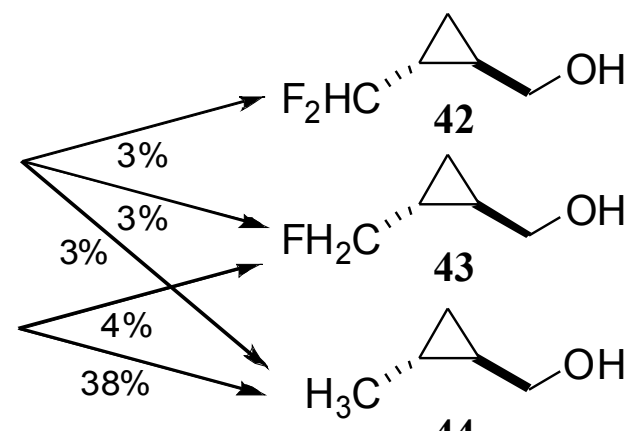

44

Scheme 8. Reductions of trans-2-fluoromethylcyclopropane carboxylic acid and esters with an excess of $\mathrm{LiAlH}_{4}$ (2 equiv. $\mathrm{LiAlH}_{4}$ in $\mathrm{Et}_{2} \mathrm{O}$, reflux).

The conversion of the carboxylic acid $\mathbf{3 8}$ and esters 21, $\mathbf{1 9}$ to the corresponding cyclopropylmethyl alcohols was attempted according to the standard protocol by adding the substrate to a twofold excess of $\mathrm{LiAlH}_{4}$ in diethyl ether under reflux. (2-Trifluoromethylcyclopropyl)methanol 41 thus was obtained in excellent yield (88\%), but the difluoromethyl- 42 and especially monofluoromethylcyclopropylmethanol 43, respectively, were obtained from the corresponding methyl cyclopropanecarboxylates 21 and 19, respectively, in much poorer yield (3\% and $4 \%$, respectively). In the case of monofluoride the main product was trans-2-methyl- 
cyclopropylmethanol $44(38 \%)$. In the case of the difluoride 21 , a mixture of the mono- 43 and difluoromethylcyclopropylmethanol 42 along with the non-fluorinated alcohol 44 was obtained in a ratio of approximately 1:1:1 (Scheme 8).

To avoid this overreduction, inverce addition of 1.1 equivalent of $\mathrm{LiAlH}_{4}$ in diethyl ether solution (ca. $1 \mathrm{M}$ ) to the solution of the ester or the acid (in the case of the trifluoride) in diethyl ether (ca. $1 \mathrm{M}$ ) was practiced. This way, the desired alcohols were obtained in good yields ( $76 \%$, $82 \%$ and $88 \%$ for $\mathbf{4 3}, \mathbf{4 2}$ and $\mathbf{4 1}$ respectively).

The racemic trans-2-fluoromethylcyclopropylmethanols upon treatment with iodine/triphenylphosphine in the presence of imidasole were smoothly converted to corresponding iodides in very good yields (Scheme 9).
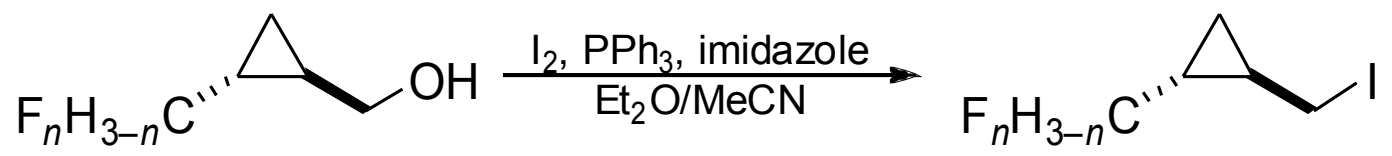

n $\quad 1 \quad 2 \quad 3$

n $\quad 1 \quad 2 \quad 3$

$\begin{array}{lll}43 & 42 & 41\end{array}$

$45 \quad 46 \quad 47$

Yield (\%): $91 \quad 88 \quad 90$

Scheme 9. Synthesis of trans-2-(fluoromethyl)cyclopropylmethyl iodides from the corresponding cyclopropylmethanols.

Alkylation of the glycine equivalents derived from $(R)$ - and $(S)-2-[(N-$ benzylprolyl)amino]benzophenone $[(R)$ - and $(S)$-BGC 13] as reusable chiral auxiliaries with the racemic iodides $45-\mathbf{4 7}$, employing the protocol of Larionov and de Meijere et al, ${ }^{[25]}$ in each case led to a mixture of diastereomeric products, which could be separated by column chromatography. Unfortunately, the diastereomers could not be separated by fractional crystallization as was previously reported for the corresponding 3-(trans-2-nitrocyclopropyl)alanine derivatives. ${ }^{[25]}$ Absolute configuration of the Belokon' (2S,1'R,2'S)-3-(2'trifluoromethylcyclopropy)lalanine complex was determined by a single crystal X-ray analysis. 


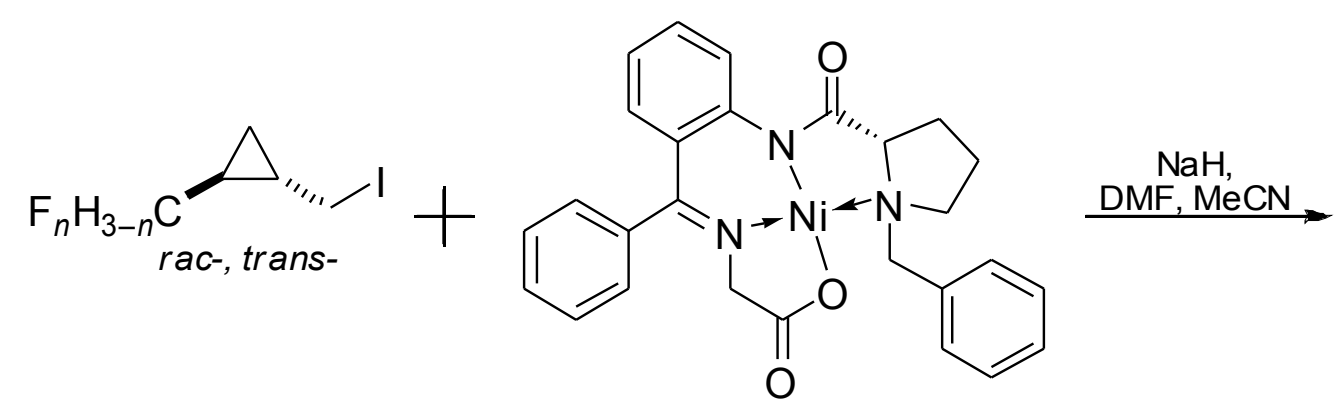<smiles>O=C(CCC1C[C@@H]1C(F)(F)F)O[N+]1(OCc2ccccc2)N(Cc2ccccc2)C(=O)[C@@H]2CCCN21</smiles>

$(S)-13$

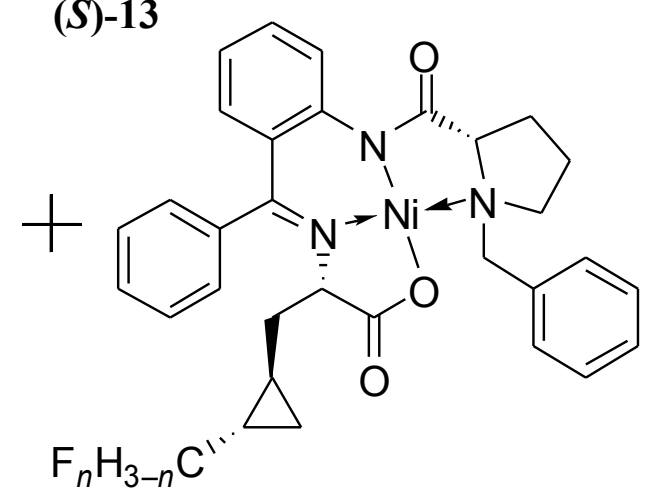

1) $\mathrm{HCl}, \mathrm{H}_{2} \mathrm{O}, \mathrm{MeOH}$, reflux 2) ion-exchange chromatography<smiles>N[C@@H](C[C@@H]1C[C@H]1CCP)C(=O)O</smiles>

(2S, 1'S, 2'R) -3-(2'- fluoromethylcyclopropyl)alanines<smiles>N[C@@H](C[C@@H]1C[C@H]1CCP)C(=O)O</smiles>

(2S, 1'R, 2'S) -3-(2' - fluoromethylcyclopropyl)alanines

Scheme 10. Synthesis of (2S)-3-(trans-2'-fluoromethylcyclopropyl)alanines by alkylation of the (S)-configured Belokon' glycine complex [(S)-BGC 13] with the racemic trans-2-fluoromethylcyclopropylmethyl iodides $45-47$. For details see Table 1.

Table 1. Yields of products of reaction of corresponding racemic iodides with $(S)$ - and $(R)$ BGC 13 (\% BGC).

\begin{tabular}{ccccc}
\hline \multirow{2}{*}{ Iodide } & \multicolumn{4}{c}{ Yield (\% on BGC) } \\
\cline { 2 - 5 } & $2 S, 1^{\prime} S, 2^{\prime} R$ & $2 S, 1^{\prime} R, 2^{\prime} S$ & $2 R, 1^{\prime} S, 2^{\prime} R$ & $2 R, 1^{\prime} R, 2^{\prime} S$ \\
\cline { 2 - 5 } & 43.7 & 44.8 & 46.7 & 42.3 \\
\hline $\mathbf{4 5}$ & 44.7 & 48.3 & 47.3 & 45.4 \\
\hline $\mathbf{4 6}$ & 45.5 & 49.1 & 43.7 & 42.4 \\
\hline $\mathbf{4 7}$ & &
\end{tabular}


The separated target $\mathrm{Ni}$ complexes were decomposed by treatment with refluxing aqeousmethanolic $\mathrm{HCl}$ to give, after ion-exchange chromatography, the corresponding $\left(2 S, 1^{\prime} S, 2^{\prime} R\right)$ - [see Scheme 10, derived from (S)-BGC] and $\left(2 R, 1^{\prime} S, 2^{\prime} R\right)$-3-(2'-fluoromethylcyclopropyl)alanines [derived from $(R)-\mathrm{BGC}$ ] in good to excellent yields. The chiral auxiliary was recovered as the hydrochloride of 2-[(N-benzylprolyl)amino]benzophenone ( $\sim 95 \%)$.

\section{New and improved syntheses of some other non-proteinogenic amino acids}

\section{1. (R)-allo-Threonine}

$(R)$-allo-threonine is commercially available, but extremely expensive (from $77.80 €$ for $250 \mathrm{mg}$ from Alpha Aesar to $60.80 €$ for $25 \mathrm{mg}$ from Fluka). Therefore a simple and inexpensive access to $(R)$-allo-threonine was desirable.

There are at least three principally different ways to approach this target amino acid:

1) Separation of the mixture of all four stereoisomers to provide individual substances or at least pairs of enantiomers, which should be resolved.

2) Preparation of mixture of two diastereomers and subsequent separation.

3) Enantioselective synthesis of the target stereoisomer from an achiral or from a chiral precursor.

One of the best ways to obtain the target amino acid in a diastereomerically pure state is by in vitro synthesis under enzyme catalysis. ${ }^{[65,66,67,68]}$

An enantioselective synthesis of $(R)$-allo-threonine from an achiral precursor employing the Sharpless asymmetric epoxidation ${ }^{[69]}$ or an asymmetric aldol reaction under catalysis with a chiral gold complex ${ }^{[70]}$ also should be possible.

The synthesis of $(R)$-allo-threonine from $(R)$-threonine as a chiral precursor was used by Zlatopolskiy. ${ }^{[7]]}$ Although $(R)$-threonine is less expensive $(21-37 €$ for $5 \mathrm{~g})$ than the target amino acid, the conversion requires five steps, and the overall yield is not better than $72 \%$.

The separation of the mixture of all four stereoisomers, produced by a non-stereoselective synthesis, is well known, but tedious. ${ }^{[72,73,74,75]}$

The separation of diastereomers is much easier and does not require chiral phases for chromatography, one just has to determine an appropriate derivative and the proper conditions 
for satisfactory separation. In fact, the synthesis of threonine diastereomers by aldol-type condensation of acetaldehyde with the enolates of glycine equivalents is well described in the literature. ${ }^{[76,77]}$ The question is just to choose the route with the best enantiomeric/diastereomeric excess. The yield is not so important, because the starting materials are inexpensive and the chiral auxiliary or catalyst should be recycled. The Belokon' protocols are among the best to access enantiomerically pure non-proteogenic amino acids. Nickel(II) or copper(II) complexes of the Schiff bases derived from glycine and $(S)$ - or $(R)-2-N-\left(N^{\prime}\right.$-benzylprolyl)aminobenzophenone $(\mathrm{BPB}),{ }^{[78,79]}$ aminoacetophenone $(\mathrm{BPA})^{[80]}$ or aminobenzaldehyd $(\mathrm{BPH})^{[81]}$ can be used as chiral nucleophilic glycine equivalents in reactions with alkyl halides or carbonyl compounds. The most versatile one is the nickel (II) aminobenzophenone derivative.

It is interesting that nickel(II) complexes of Schiff bases derived from 2-bromoglycine and $(S)$-BPB can be used as electrophilic glycine equivalents. ${ }^{[82]}$

Alkylations of the nickel(II) complexes of Schiff bases derived from glycine and $(S)$ - or $(R)$-BPB 13 with alkyl halides virtually yields a single stereoisomer, in which the configuration of the newly formed stereogenic centerat C-2 of the amino acid moiety is the same as that in the proline moiety of the chiral auxiliary in the starting material.

In the reaction of enolate of this chiral glycine equivalent with aldehydes the situation is more complicated. The reaction of $(S)$-BGC with acetaldehyde under strongly basic conditions lead to $(R)$-threonine (inverse configuration relative to that of the proline moiety of $(S)$-BGC due to epimerization on C-2), but when a weaker base like triethylamine was employed, a mixture of $(R)$-threonine and $(S)$-allo-threonine ${ }^{[83]}$ was obtained.

The hypothesis that the reaction of BGC with aldehydes under strongly basic conditions proceeds in two steps and is thermodimacally controlled was corroborated by experimental tests. $^{\left[{ }^{[8]}\right]}$ The initially formed main product in the aldol reaction of acetaldehyde with BGC had the same configuration at $\mathrm{C}-2$ as the proline unit in $\mathrm{BGC}$, but the product ratio changed in time from 95:5 after $30 \mathrm{~s}$ through 70:18 after $10 \mathrm{~min}$ to 5:95 after $24 \mathrm{~h}$ at ambient temperature. This epimerization comes along with possible rearrangement in the Ni complex. The newly formed hydroxide group of the product can coordinate the Ni atom liberating the carboxylate moiety and thus making the proton at $\mathrm{C}-2$ accessible to base attack (Scheme 11). In order to obtain $(R)$-allo-threonine, it is necessary to carry out the aldol reaction of $(R)$-BGC with an excess of acetaldehyde under strongly basic conditions at low temperature and quench the reaction after a short time to avoid epimerisation. 
This modified protocol indeed gave the $(R)$-allo-threonine in relatively poor yield $(7.5 \%$ for $\mathrm{Ni}$ complex, $6 \%$ for amino acid), but with high enantiomeric purity in two steps. Bearing in mind that the starting materials are inexpensive and the chiral auxiliary is reusable ( $\geq 95 \%$ recovery), this protocol represents one of the best route to the extremely expensive $(R)$-allo-threonine.

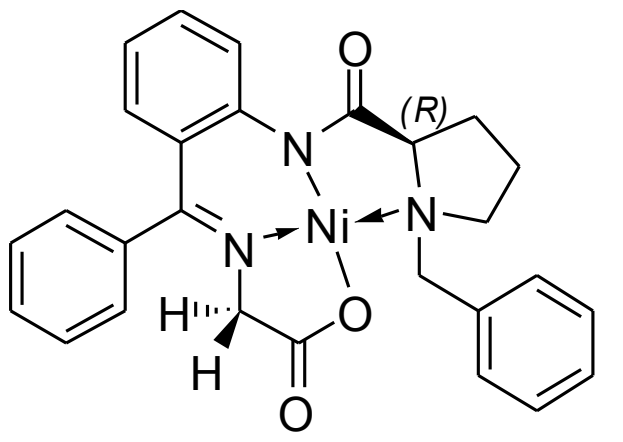<smiles>CC(O)CO[Na]</smiles>

$(R)-13$

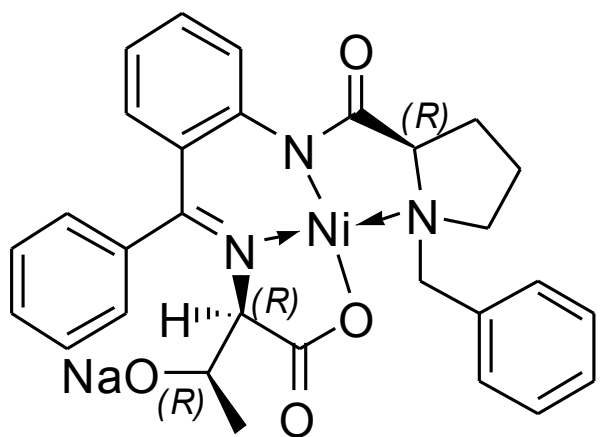<smiles>[N-]=[13NH2+]</smiles>

$\mathrm{ONa}$<smiles>CC(C)OC1CCCCC1</smiles>

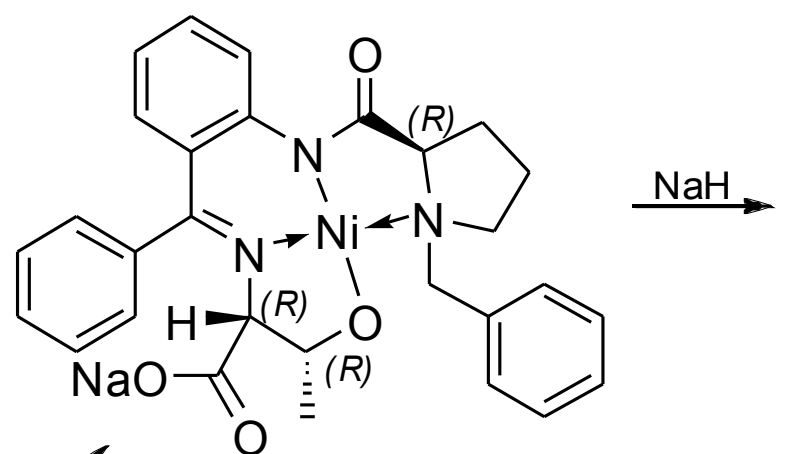<smiles>CC1CCCN1Cc1ccccc1</smiles>

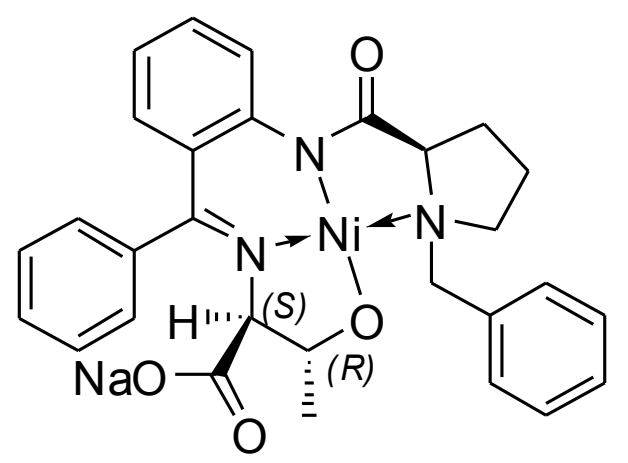

Thermodynamically more stable

Scheme 11. Mechanism of epimerisation of the threonine Belokon' complex.

It is also possible to obtain the $(R)$-allo-threonine starting from $(R)$-BGC and acetaldehyde under thermodynamic control $\left(\mathrm{Et}_{3} \mathrm{~N}\right.$ as base, $(S)$-threonine: $(R)$-allo-threonine $\left.=1: 7\right)$, but it is necessary to leave the reaction mixture for two months for the reaction to go to completion. ${ }^{[85]}$ 


\section{2. $\quad \beta$-Methylphenylalanines}

(2S,3R)-3-Methylphenylalanine ( $L-\beta$-methylphenylalanine, $(\beta \mathrm{Me}) \mathrm{Phe}, \mathrm{MeF})$ is a constituent of the peptidolactone Hormaomycin and is contained in the molecule twice. Thus it is required for the synthesis of Hormaomycin and the analogues envisaged here. In addition, a versatile protocol for the preparation of other $\beta$-alkylarylalanines would be desirable for incorporation in Hormaomycin analogues as well as in other peptides, as the incorporation of conformationally constrained $\alpha$-amino acids into peptides is frequently used to study structure-activity relationships. ${ }^{[86,87,88]}$ In this context, special attention should be paid to constrained analogues of phenylalanine such as these $\beta$-methylphenylalanines, since the naturally occurring phenylalanine unit is directly involved in a large number of molecular recognition processes. ${ }^{[89,90]}$

In all cases, the three-dimensional arrangement of the phenylalanine residue is crucial in eliciting the desired response. The residue can be conformationally constrained by introducing an alkyl group at the $\beta$-position of an phenylalanine residue without significantly perturbing the backbone conformation. In particular, aromatic $\beta$-methyl- $\alpha$-amino acids have been incorporated into peptides $^{[89,91,92,93]}$ and confer on these systems a conformational side-chain rigidity that is very valuable for the study of both the specific topochemical arrays of the side chains and topochemical nature of the binding site.

The preparation of analogues of $\beta$-methylphenylalanine in enantiopure form is a challenging area in synthetic organic chemistry. Several strategies have been developed, and these include classical resolution, ${ }^{[94]}$ enzymatic resolution in conjunction with HPLC,${ }^{[91]}$ or HPLC separation of derived peptides, ${ }^{[92]}$ chiral preparative HPLC separation, ${ }^{[95]}$ asymmetric synthesis from chiral precursors $^{[96,97]}$ including the stereoselective alkylation of aromatic compounds with triflates of threonine stereoisomers, ${ }^{[98]}$ the chiral auxiliary $\operatorname{approach}^{[99,100,101,102]}$ and enantioselective hydrogenation over a chiral catalyst. ${ }^{[103,104]}$

All these approaches ought to be applicable to prepare unsubstituted $\beta$-methylphenylalanine. Separation protocols are suitable to approach any substituted amino acid, which can be synthesized. A stereoselective synthesis requires an optically active precursor, which, in turn, should be prepared or purchased; in many cases, these precursors are quite expensive or difficult to prepare. Chiral auxiliary approaches are better, and routes employing a chiral catalyst even better, although requires optically active auxiliaries or catalysts may have to be prepared. 
To the best of our knowledge, synthetic way to $\beta$-methylphenylalanine using so-called "Evans amide" as chiral auxiliary is the most common approach to $\beta$-branched arylalanines and could be shown by Scheme 12:<smiles>COC(N)C(=O)O</smiles>

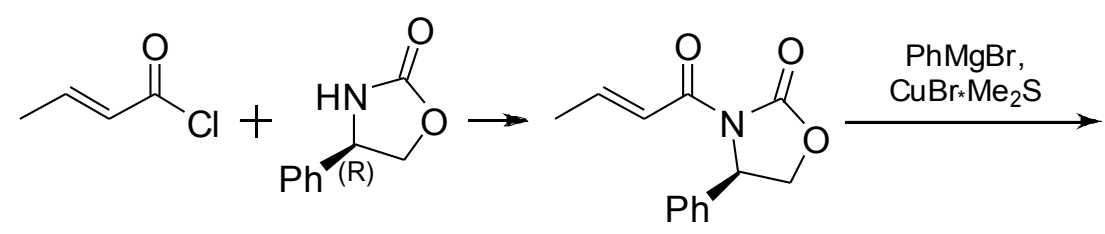<smiles>C[C@H](CC(=O)N1C(=O)OC[C@H]1c1ccccc1)c1ccccc1</smiles>

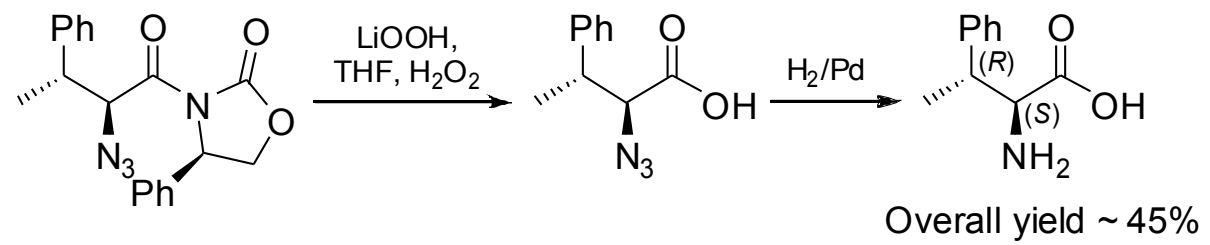

Scheme 12. The classic "Evans" approach to $(2 S, 3 R)-\beta$-methylphenylalanine.

The crucial step in the "Evans" sequence is the Michael addition of the organometallic (usually arylcuprate) reagent to the crotonoyl moiety attached to the chiral auxiliary. The respective arylcuprate can be produced from the corresponding arylmagnesium halide and $\mathrm{CuBr} \times \mathrm{Me}_{2} \mathrm{~S}$ complex. The organomagnesium reagent can be easily obtained from the corresponding aryl halide and metallic magnesium, or, in difficult situations, by the Knochel protocol with $\mathrm{iPrMgCl} \times \mathrm{LiCl}$ with subsequent transmetallation with $\mathrm{CuCN} \times 2 \mathrm{LiCl}^{[105,106]}$ but in the case of oligohalogen-substituted arenes it could lead to mixtures of organometallic compounds and, in turn, mixtures of products.

The "Evans" route, with the employment of phenylmagnesium bromide has led to $\beta$-methylphenylalanine in eight steps (including the transmetallation) with an overall yield of approx. $45 \%$ (based on the crotonated chiral auxiliary) ${ }^{[107,108]}$.

In view of the good performance of the Belokon' protocol for various eletrophilic reagents, it was straightforward to apply this approach to $\beta$-methylphenylalanines as well (Scheme 13). 
Towards this, the $(S)$-configured Belokon' glycine complex $(S)$-BGC, $(\boldsymbol{S})$-13 was alkylated with 1-phenylethyl iodide and various analogues with substituents in the aryl moiety, all in racemic form.

The diastereomeric Ni(II) complex products obtained in each case, could be separated by column chromatography, and the pure diastereomeres were decomposed with aqueous-methanolic $\mathrm{HCl}$ solution to furnish the target amino acids which were purified by ion-exchange chromatography. The obtained yields were very good (Table 2).

Table 2. Substituted $\beta$-methylphenylalanines by alkylation of the Belokon' glycine complex $(S)$-BGC with 1-arylethyl iodides (yields based on used $(S)$-BGC, d.e. $\geq 98 \%$ ). See Scheme 13 .

\begin{tabular}{ccccc}
\hline $\mathbf{X}$ & Product & Yield (\%) & Product & Yield (\%) \\
\hline $\mathrm{H}$ & $(2 S, 3 S)-\mathbf{4 8}$ & 35 & $(2 S, 3 R)-\mathbf{4 8}$ & 38 \\
$o-\mathrm{Cl}$ & $(2 S, 3 S)-\mathbf{4 9}$ & 30 & $(2 S, 3 R)-\mathbf{4 9}$ & 33 \\
$m-\mathrm{Cl}$ & $(2 S, 3 S)-\mathbf{5 0}$ & 34 & $(2 S, 3 R)-\mathbf{5 0}$ & 35 \\
$p-\mathrm{Cl}$ & $(2 S, 3 S)-\mathbf{5 1}$ & 33 & $(2 S, 3 R)-\mathbf{5 1}$ & 33 \\
$p-\mathrm{F}$ & $(2 S, 3 S)-\mathbf{5 2}$ & 37 & $(2 S, 3 R)-\mathbf{5 2}$ & 36 \\
\hline
\end{tabular}


$\overbrace{\oplus}^{\infty}+\overbrace{\mathrm{O}}^{\mathrm{CH}_{2}}$

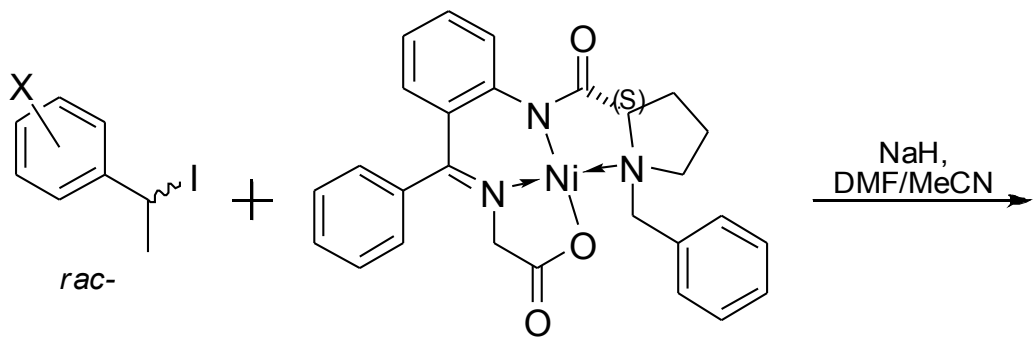

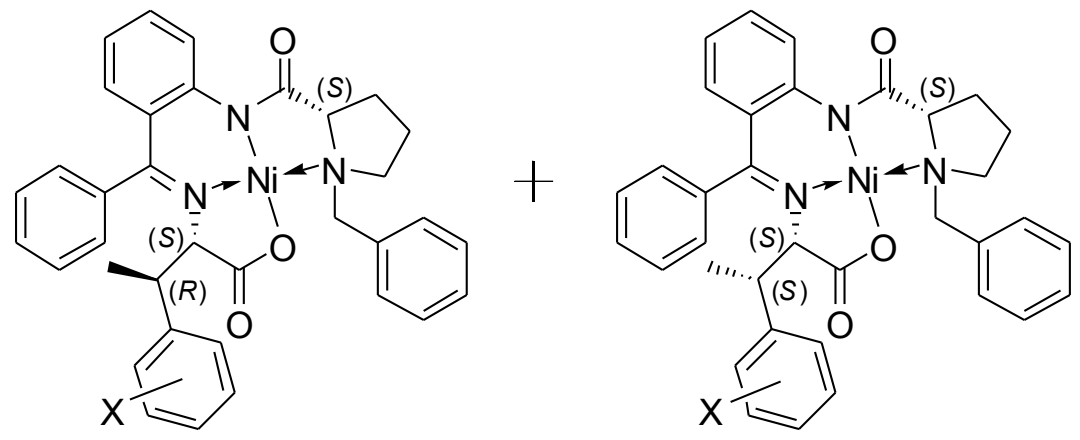

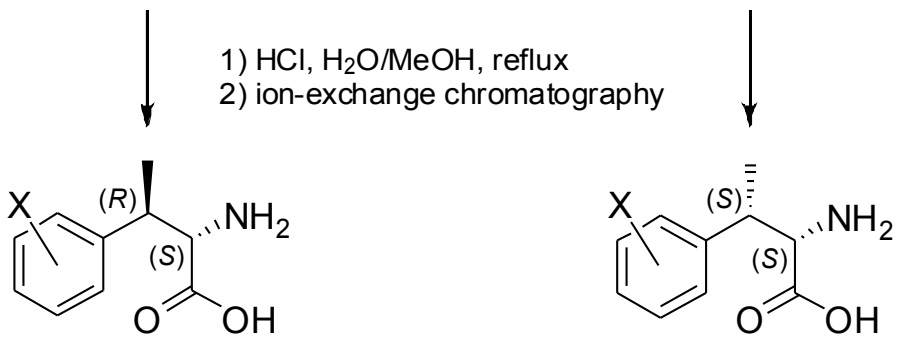

Scheme 13. A new general route to $(2 S, 3 R)-\beta$-methylarylalanines by alkylation of the Belokon' glycine complex $(S)$-BGC with 1-arylethyl iodides. For details see Table 2. 


\section{Hormaomycin and its all-peptide analogue}

The total syntheses of Hormaomycin 1 itself and its all-peptide aza-analogue 53, developed by B. Zlatopolskiy, ${ }^{[109,110]}$ were reproduced in order to provide large enough quantities (39 and $25 \mathrm{mg}$, respectively) for biological tests of their antimalarial activities ${ }^{[111]}$.

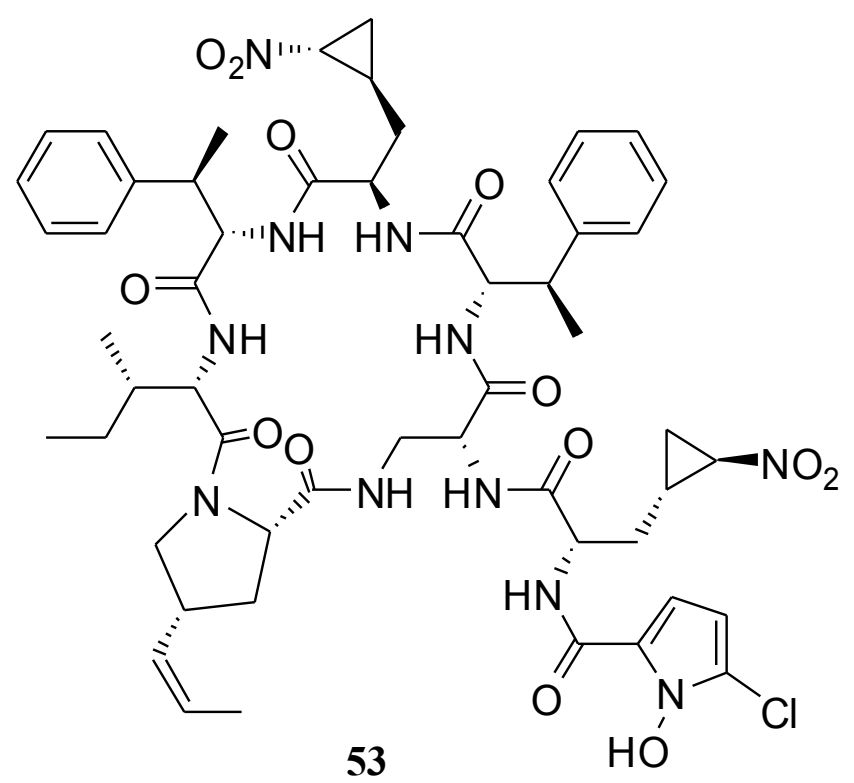

Figure 4. The all-peptide analogue of Hormaomycin, which showed the best antiparasitic activity in vitro. ${ }^{[111]}$

At first sight, the oligopeptide assembly leading to Hormaomycin does no appear to be a very complicated problem. "State of the art" peptide coupling methodology ${ }^{[112]}$ allows one to prepare almost any peptides, that do not contain extremely sterically congested fragments such as $\alpha, \alpha$-dialkyl amino acids, $N$-alkyl amino acids or even more challenging $N$-aryl amino acids. With a proper choice of the coupling reagent, solvent and other experimental conditions, the oligopeptides are obtained in high yields and in high optical purities. As almost all amino acids, which comprise Hormaomycin itself and its anticipated analogues, are $\beta$-branched with the exception of 3-(2'-nitrocyclopropyl)alanine and the 3-(2'-fluoromethylcyclopropyl)alanines, HATU, as well as the combination of EDC and 7-aza-1-hydroxybenzotriazole (HOAt) ${ }^{[113]}$ were used for each condensation step to ensure high yields. The most unusual fragment in Hormaomycin is the ester bond between the secondary (4-Pe)Pro moiety and the hydroxy group of $a$-Thr. Among several methods described in the literature for the creation of such bonds, the dialkylaminopyridine-promoted carbodiimide-mediated esterification was chosen. ${ }^{[114]}$ 
On the other hand, the reactivities of the double bond in the 4-(Z)-propenylproline residue and the nitrogroups in the nitrocyclopropylalanine moieties as well as, what is not so obvious, the ester bond between the propenylproline and $(R)$-allo-threonine residues make a proper choice of the protecting groups and also the conditions for their deprotection a real challenge. Thus, the presence of the double bond hampers the application of catalytic hydrogenolysis and $\mathrm{HBr} / \mathrm{AcOH}$ reagent for the deprotection of peptides containing the propenylproline residue. The aliphatic nitrogroup in (3-Ncp)Ala is not compatible with reductive cleavage conditions, and the threonine ester bond is sensitive to alkaline and basic conditions. ${ }^{[15]}$ Because of this base sensitivity the Fmoc strategy is unsuitable for the depsipeptide fragment, and other protecting groups had to be chosen for the ester moiety as well as for manipulations of intermediates that contain it.

The key step in the synthesis of Hormaomycin is the formation of the macrocycle. The greater facility, with which amide bonds can be formed, a consequence of the superior nucleophilicity of the amine over the hydroxy group, makes macrolactamization the preferred mode for ring closure. The amide bond between the $(\beta \mathrm{Me}) \mathrm{Phe}$ and Ile residues appears to be least suitable for this cyclization because of the possibility of a cyclo-[Ile-(4-Pe)Pro] diketopiperazine formation ( $\beta$-position to the ester bond) as well as significant epimerization and expected low yield, which are connected with the bulk of the side chains of these amino acids. To form the bond between $\mathrm{a}-\mathrm{Thr}$ and $(\beta \mathrm{Me}) \mathrm{Phe}$ as the last one is more preferable, because racemization would be suppressed by the urethane protection, and between the $(\beta \mathrm{Me}) \mathrm{Phe}$ and $(R)-(3-\mathrm{Ncp})$ Ala residues cyclization would proceed faster, since these residues have opposite configurations at their $\alpha$-centers. ${ }^{[116]}$ A ring closure forming the amide bond between Ile and (4-Pe)Pro should go along with a larger degree of epimerization, because proline is more basic than any primary amino acid, and that between $(R)-(3-\mathrm{Ncp})$ Ala and $(\beta \mathrm{Me}) \mathrm{Phe}$ is also less preferable, since the bulky side chain of the latter shields its amino group. 


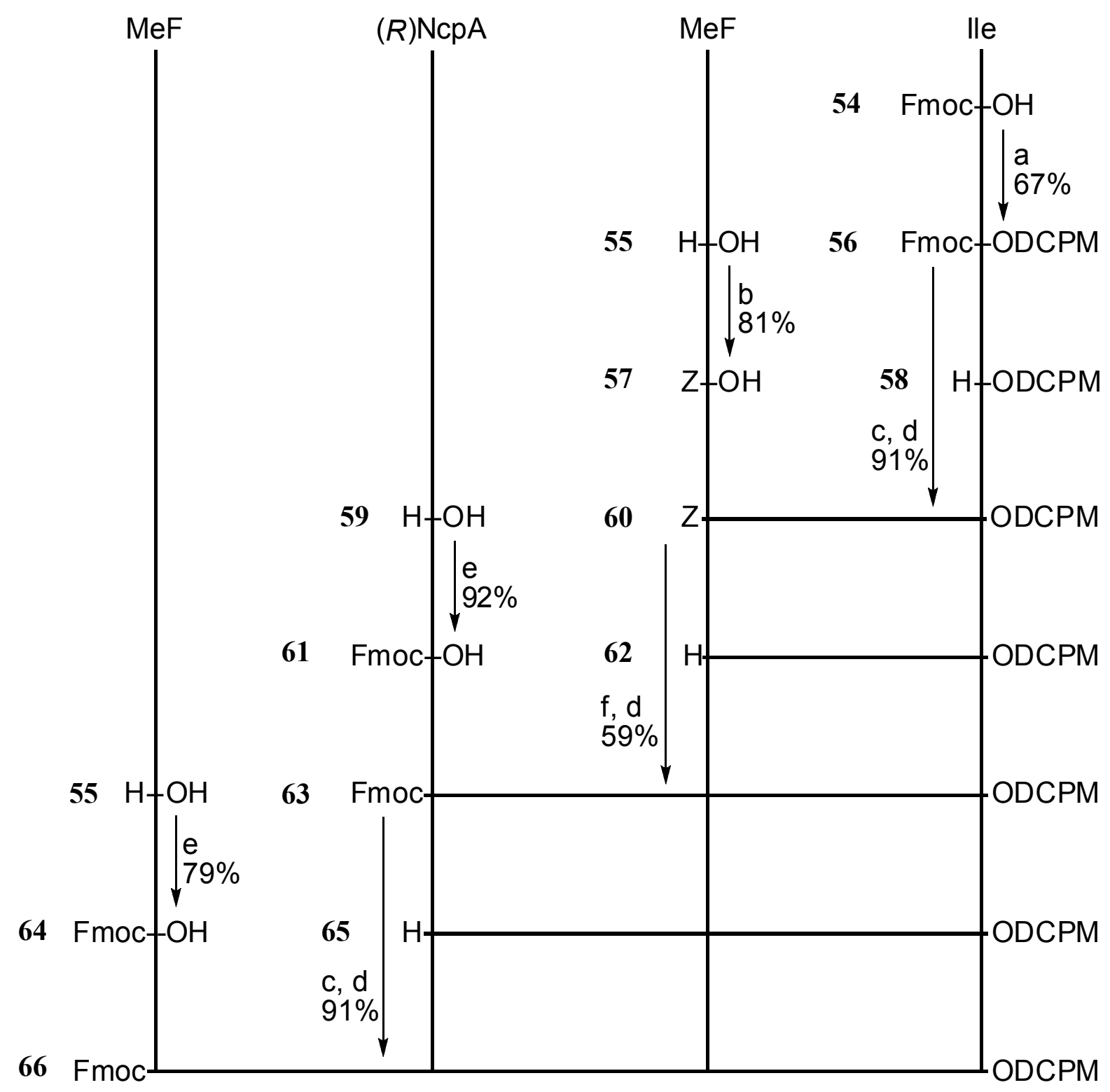

Scheme 14. Synthesis of tetrapeptide precursor $\mathbf{6 6}$ of Hormaomycin and its all-peptide analogue.

a) oxalyl chloride, pyridine/dicyclopropylmethanol, DMAP, $\mathrm{CH}_{2} \mathrm{Cl}_{2}, 0 \rightarrow 20{ }^{\circ} \mathrm{C}, 20 \mathrm{~h}$;

b) $\mathrm{ZOSu}, \mathrm{NaHCO}_{3}$, acetone/water, $2 \mathrm{~h}$; c) $50 \% \mathrm{Et}_{2} \mathrm{NH} / \mathrm{THF}, 2{ }^{\circ} \mathrm{C}, 1 \mathrm{~h}$; d) EDC, HOAt, DIEA, 2,4,6-collidine, $\mathrm{CH}_{2} \mathrm{Cl}_{2}, 0 \rightarrow 20^{\circ} \mathrm{C}, 14 \mathrm{~h}$; e) FmocOSu, $\mathrm{NaHCO}_{3}$, acetone/water, $4 \mathrm{~h}$; f) $\mathrm{H}_{2}, \mathrm{Pd} /$ C, EtOAc, $20^{\circ} \mathrm{C}, 40 \mathrm{~min}$. 


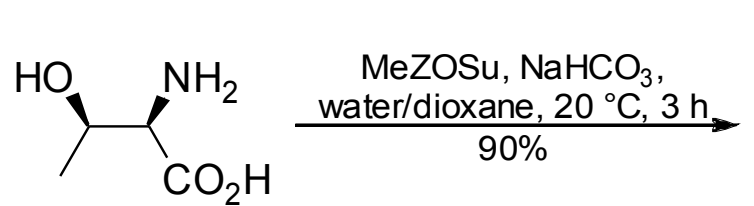

67
MeZ

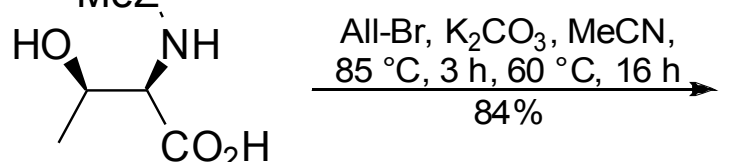

68<smiles>C/C=C\[C@H]1C[C@@H](C(=O)O)N([R6](=O)[O-])C1</smiles><smiles>C/C=C\[C@H]1C[C@@H](C(=O)O[C@H](C)[C@H](NC(C)=O)C(=O)O)N(C(=O)OCc2ccccc2)C1</smiles><smiles>C/C=C\[C@H]1C[C@@H](C(=O)O[C@H](C)[C@H](NC(C)=O)C(=O)O)N(C(=O)OC(C)(C)C)C1</smiles>

Scheme 15. Synthesis of diprotected ester acid $\mathbf{7 1 .}$ 


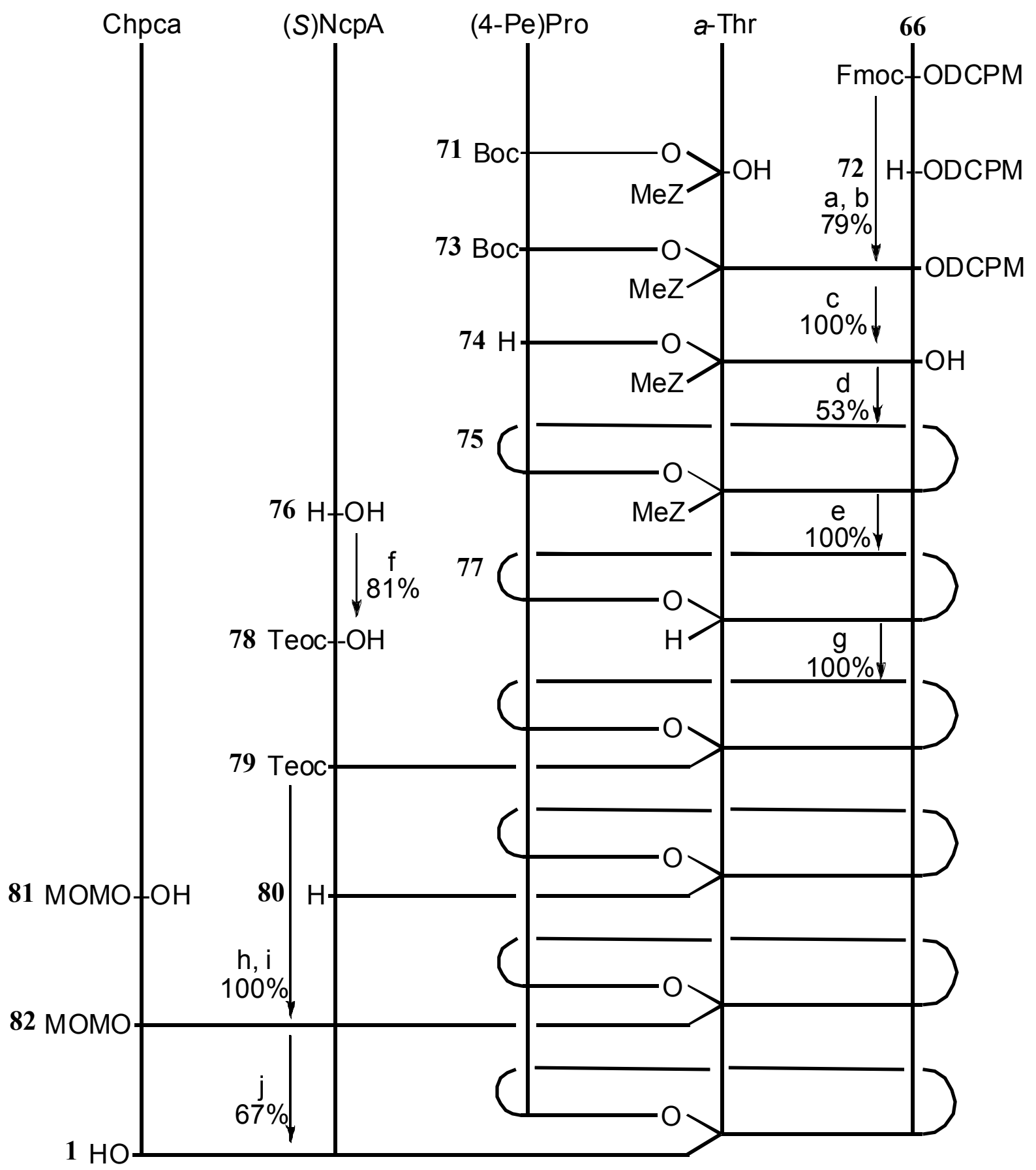

Scheme 16. Synthesis of Hormaomycin 1.

a) $50 \% \mathrm{Et}_{2} \mathrm{NH} / \mathrm{THF}, 20^{\circ} \mathrm{C}, 1 \mathrm{~h}$; b) HATU, HOAt, DIEA, TMP, $\mathrm{CH}_{2} \mathrm{Cl}_{2}, 0 \rightarrow 20^{\circ} \mathrm{C}, 24 \mathrm{~h}$;

c) $2 \mathrm{M} \mathrm{HCl}$ in EtOAc, $20^{\circ} \mathrm{C}, 45 \mathrm{~min}$; d) HATU, DIEA,TMP, $\mathrm{CH}_{2} \mathrm{Cl}_{2}, 0 \rightarrow 20{ }^{\circ} \mathrm{C}, 16 \mathrm{~h}$; e) anisole, TFA, $20{ }^{\circ} \mathrm{C}, 2 \mathrm{~h}$; f) TeocOSu, $\mathrm{NaHCO}_{3}, \mathrm{~N}, \mathrm{~N}-$ Dimethylaminopropylamine, water/acetone, $20{ }^{\circ} \mathrm{C}$, $2 \mathrm{~h}$; g) HATU, HOAt, DIEA, TMP, $\mathrm{CH}_{2} \mathrm{Cl}_{2}, 20{ }^{\circ} \mathrm{C}, 6 \mathrm{~h}$; h) TFA, $20{ }^{\circ} \mathrm{C}, 1 \mathrm{~h}$; i) HATU, HOAt, DIEA, TMP, $\mathrm{CH}_{2} \mathrm{Cl}_{2}, 20{ }^{\circ} \mathrm{C}, 4 \mathrm{~h}$; j) $\mathrm{MgBr}_{2} \cdot \mathrm{Et}_{2} \mathrm{O}, \mathrm{EtSH}, \mathrm{CH}_{2} \mathrm{Cl}_{2}, 20{ }^{\circ} \mathrm{C}, 3.5 \mathrm{~h}$. 
Having in mind to ring-close an acyclic precursor already containing the ester bond between the (4-Pe)Pro and the $a$-Thr residues, by forming the peptide bond between the Ile and (4-Pe)Pro moieties, the dicyclopropylmethyl ester of Ile $\mathbf{5 6}$ was condensed with $\mathrm{N}$-Z-protected $(\beta \mathrm{Me}) \mathrm{Phe}-\mathrm{OH}$ 57. After removal of the $\mathrm{Z}$ group from the $N$-terminus of the resulting dipeptide 60 by catalytic hydrogenation, the latter was coupled with $N$-Fmoc-protected-(2R,1'R,2'R)-(3-Ncp)Ala-OH 61 to yield the tripeptide $\mathbf{6 3}$, which, in turn, after deprotection with $\mathrm{Et}_{2} \mathrm{NH} / \mathrm{THF}$, was coupled with $N$-Fmoc-protected $(\beta \mathrm{Me}) \mathrm{Phe}-\mathrm{OH} \mathbf{6 4}$ to give the $N, C$-protected tetrapeptide $\mathbf{6 6}$.

In the case of Hormaomycin 1 itself, the 4-pyrrolidinopyridine-catalyzed condensation of the $N$-Boc-protected (4-Pe)Pro-OH 69 and N,C-protected a-Thr 118 gave the ester 70, which, after palladium-promoted removal of the allyl group, was coupled with the tetrapeptide $\mathbf{6 6}$ using the HATU reagent in the presence of HOAt to give the hexadepsipeptide $\mathbf{7 3}$.
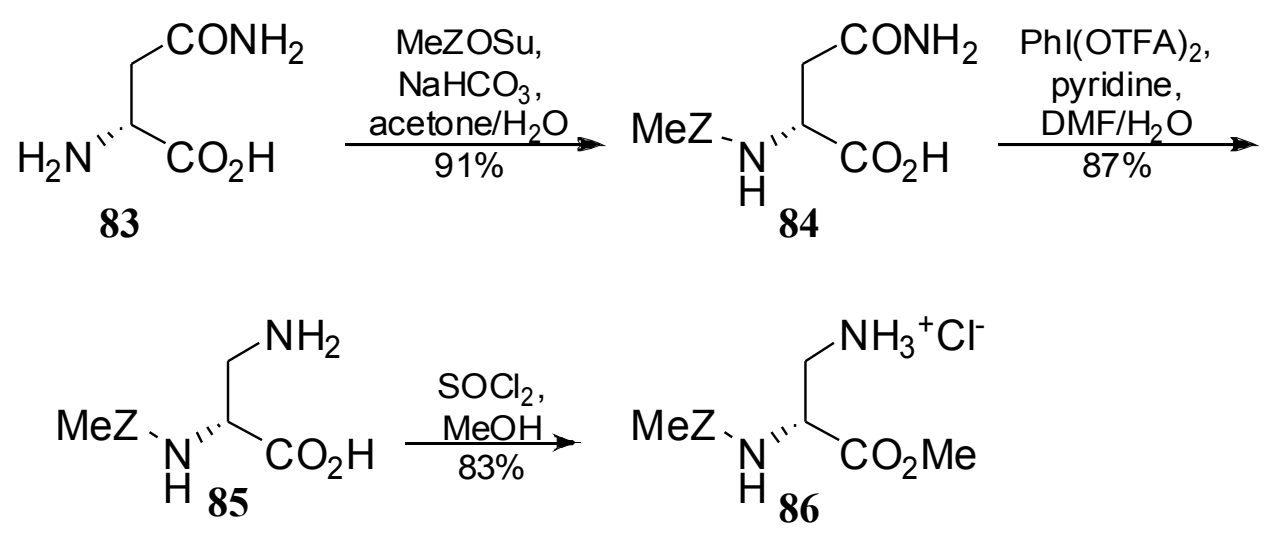

Scheme 17. Synthesis of $N$-(4-methylbenzyloxycarbonyl-protected) methyl (2R)-2,3diaminopropionate.

In the case of the all-peptide analogue 53, the $N_{\alpha}$-MeZ-protected 2,3-diaminopropionic acid ester 86 was obtained (Scheme 17) as the hydrochloride by esterification with methanol of the intermediate 85, which in turn was prepared in 79\% yield over two steps starting from $(R)$-asparagine $\mathbf{8 3}$ by initial acylation with MeZOSu and subsequent oxidation of the amide $\mathbf{8 4}$ with iodobenzene bis(trifluoroacetate) in close analogy to the published procedure. ${ }^{[117]}$ 
<smiles>C/C=C\C1CC(C(=O)O)C(C(=O)OC(C)(C)C)C1</smiles>

10

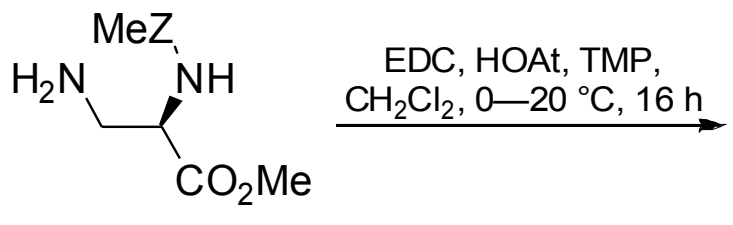

86

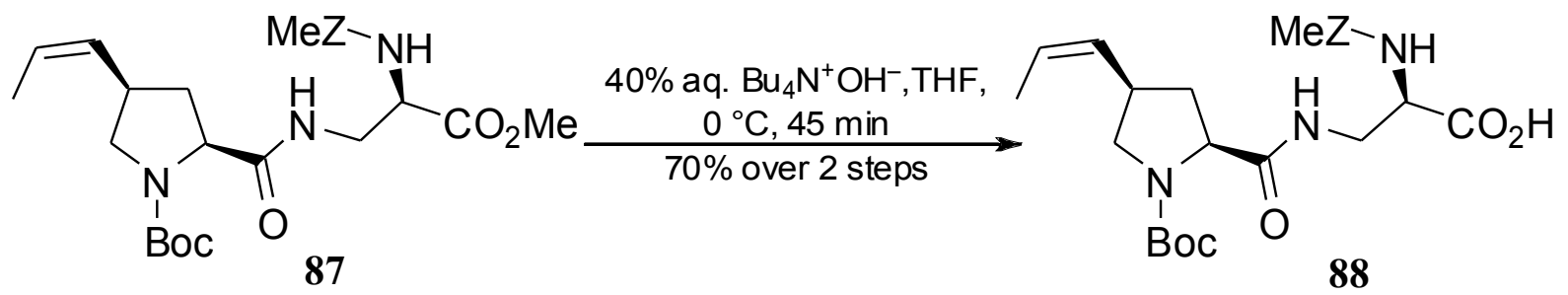

Scheme 18. Synthesis of diprotected dipeptide acid $\mathbf{8 8}$.

The diamino ester 86 was coupled with the $N$-Boc-protected (2S,3R)-4-(Z)-propenylproline 10 using EDC and HOAt to give the intermediate dipeptide methyl ester 97. Treatment of the latter with tetrabutylammonium hydroxide ${ }^{[118]}$ gave the dipeptide acid $\mathbf{9 8}$ (70\% yield over two steps), which was coupled with the O-dicyclopropylmethyl (DCPM) protected tetrapeptide $\mathbf{6 6}$ after deprotection of its terminal amino groups, to yield the branched hexapeptide 89 (59\%).

These intermediates, the hexadepsipeptide $\mathbf{7 3}$ and the hexapeptide $\mathbf{8 9}$, should not to be purified by column chromatography, because the DCPM protective group is labile towards silica gel.

The DCPM and Boc groups were removed from the termini of the hexadepsipeptide/ hexapeptide (the ESI-MS spectrum showed that the MeZ group stayed intact), and the cyclizing peptide condensation succeeded under high dilution conditions, using the HATU reagent. The cyclodepsipeptide 75 and cyclopeptide 91 were obtained in 53\% and 34\%, respectively, yield after HPLC purification. 


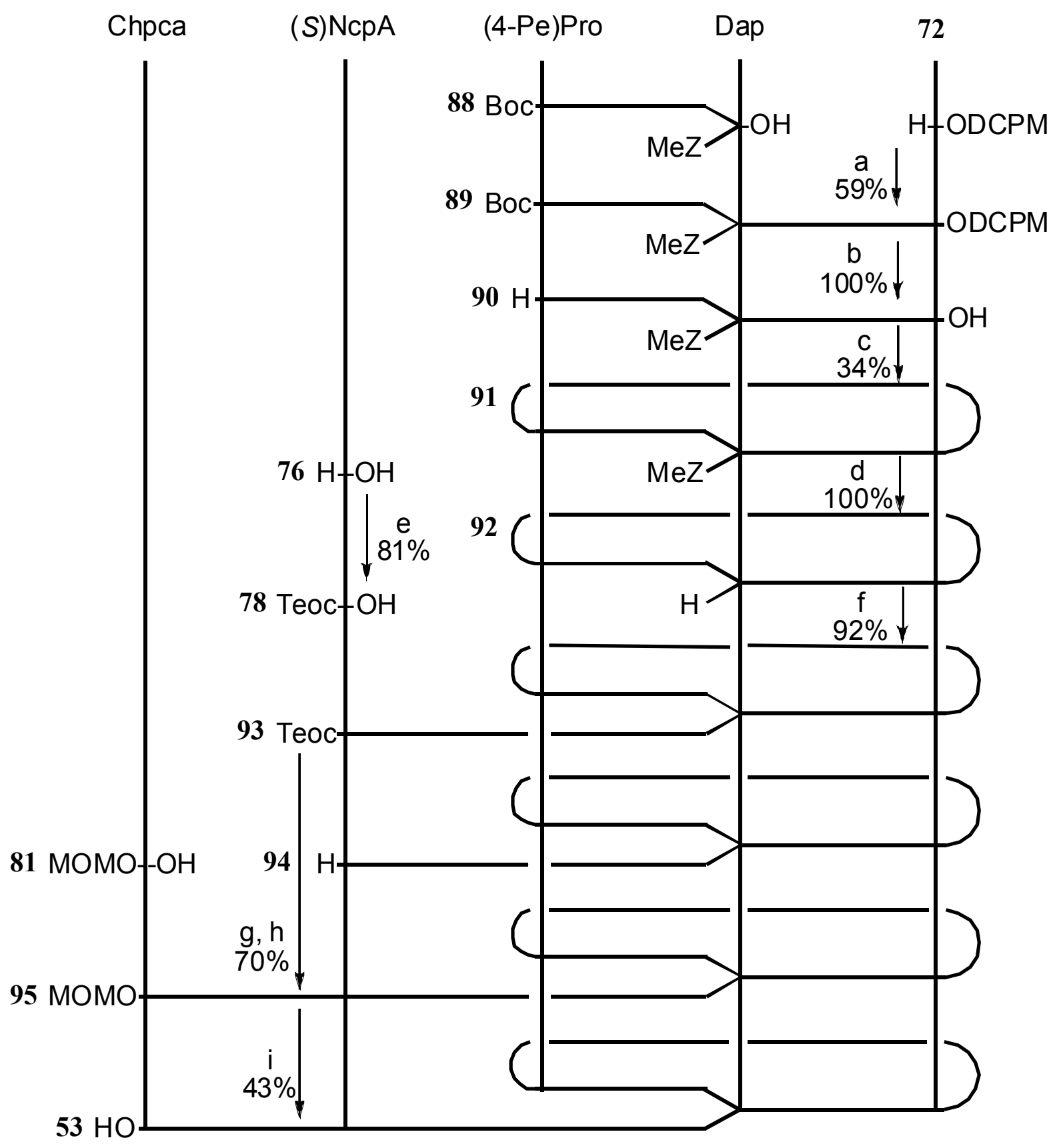

Scheme 19. Synthesis of Hormaomycin all-peptide analogue 53.

a) HATU, HOAt, DIEA, TMP, $\mathrm{CH}_{2} \mathrm{Cl}_{2}, 0 \rightarrow 20{ }^{\circ} \mathrm{C}, 24 \mathrm{~h}$; b) $2 \mathrm{M} \mathrm{HCl}$ in EtOAc, $20^{\circ} \mathrm{C}, 45 \mathrm{~min}$; c) HATU, DIEA, TMP, $\mathrm{CH}_{2} \mathrm{Cl}_{2}, 0 \rightarrow 20{ }^{\circ} \mathrm{C}, 16 \mathrm{~h}$, d) anisole, TFA, $20^{\circ} \mathrm{C}, 2 \mathrm{~h}$; e) TeocOSu, $\mathrm{NaHCO}_{3}, \mathrm{~N}, \mathrm{~N}-$ Dimethylaminopropylamine, water/acetone, $20{ }^{\circ} \mathrm{C}, 2 \mathrm{~h}$; f) HATU, HOAt, DIEA, TMP, $\mathrm{CH}_{2} \mathrm{Cl}_{2}, 20{ }^{\circ} \mathrm{C}, 6 \mathrm{~h}$; g) TFA, $20{ }^{\circ} \mathrm{C}, 1 \mathrm{~h}$; h) HATU, HOAt, DIEA, TMP, $\mathrm{CH}_{2} \mathrm{Cl}_{2}, 20{ }^{\circ} \mathrm{C}$, $4 \mathrm{~h}$; i) $\mathrm{MgBr}_{2} \cdot \mathrm{Et}_{2} \mathrm{O}, \mathrm{EtSH}, \mathrm{CH}_{2} \mathrm{Cl}_{2}, 20^{\circ} \mathrm{C}, 3.5 \mathrm{~h}$.

To complete the assembly of the target compounds, the $N$-MeZ-protected cyclic intermediates $\mathbf{7 5}$ and 91 were deprotected and first coupled with $N$-Teoc-protected 
$\left(2 S, 1^{\prime} R, 2^{\prime} R\right)-(3-\mathrm{Ncp}) \mathrm{Ala}-\mathrm{OH}$ 78. After removal of the Teoc-group, the intermediates 80 and 94 in turn were coupled with the 1-OMOM-protected 5-chloro-1-hydroxypyrrole-2-carboxylic acid $\mathbf{8 1}$. Finally, the MOM group was removed by treatment with $\mathrm{MgBr}_{2} \cdot \mathrm{Et}_{2} \mathrm{O}$ and $\mathrm{EtSH}$ in dichloromethane to give the target compounds $\mathbf{1}$ (Scheme 16) and $\mathbf{5 3}$ (Scheme 19) in 28\% and in $13 \%$, respectively, yield over 8 steps.

\section{Hormaomycin analogues with fluoromethyl-substituted cyclopropylalanine residues}

Once sufficient quantities of $\left(2 S, 1^{\prime} S, 2^{\prime} R\right)$ - and ( $\left.2 R, 1^{\prime} S, 2^{\prime} R\right)-3-\left(2^{\prime}\right.$-fluoromethylcyclopropyl)alanine, $N$-Boc-protected $(2 S, 4 R)-4-(Z)$-propenylproline, as well as the $O$-MOM protected 5-chloro-1-hydroxypyrrole-2-carboxylic acid, $(R)$-allo-threonine and $(2 S, 3 R)$ - $\beta$ methylphenylalanine had been prepared, the assembly of the Hormaomycin analogues with 3-(2'-fluoromethylcyclopropyl)alanine residues could be initiated.

The same sequence that was developed by Zlatopolskiy for the synthesis of Hormaomycin and its aza-analogue, was successfully employed toward the synthesis of these new Hormaomycin analogues as well.

To prepare the Hormaomycin with fluoromethyl-substituted cyclopropylalanine moieties, the dicyclopropylmethyl ester of Ile 54, was condensed with $N$-Z-protected ( $\beta \mathrm{Me}) \mathrm{Phe}-\mathrm{OH} \mathbf{5 5}$. After removal of the $\mathrm{Z}$ group from the $\mathrm{N}$-terminus of the resulting dipeptide $\mathbf{6 0}$ by catalytic hydrogenation, the latter was coupled with $N$-Fmoc-protected $\left(2 R, 1^{\prime} R, 2^{\prime} R\right)$-(3-(mono-, di- or tri-)fluoromethylcyclopropyl)alanines 97 a-c to yield tripeptides 98 a-c, which, in turn, after deprotection with $\mathrm{Et}_{2} \mathrm{NH} / \mathrm{THF}$, were coupled with $N$-Fmoc-protected ( $\left.\beta \mathrm{Me}\right) \mathrm{Phe}-\mathrm{OH} \mathbf{6 4}$ to give $N, C$-protected tetrapeptides $\mathbf{1 0 0}$ a-c.

The 4-pyrrolidinopyridine-catalyzed condensation of the $N$-Boc-protected (4-Pe)Pro-OH 10 and $N, C$-protected $a$-Thr 69 gave the ester 70, which, after palladium-promoted removal of the allyl group, was coupled with the tetrapeptides using the HATU reagent in the presence of HOAt to give the corresponding hexadepsipeptides 101 a-c.

The DCPM and Boc groups were removed from the termini of the hexadepsipeptides (the ESI-MS spectrum showed that the MeZ group stayed intact), and the cyclizing peptide condensation succeeded under high dilution conditions, using the HATU reagent. The cyclodepsipeptides 103 a-c were obtained in 53, 60 and 54\%, respectively, yield over 8 steps, after HPLC purification. 
To complete the assembly of the corresponding Hormaomycin analogues, the $N$-MeZ-protected cyclic intermediates $\mathbf{1 0 3}$ a-c were deprotected and first coupled with the corresponding $N$-Teoc-protected $\left(2 S, 1^{\prime} R, 2^{\prime} R\right)$-(3-(mono-, di- or tri-)fluoromethylcyclopropyl)alanines 105 a-c. After removal of the Teoc group, the intermediates 107 a-c in turn were coupled with the 1-OMOM-protected 5-chloro-1-hydroxypyrrole-2-carboxylic acid 81. Finally, the MOM group was removed by treatment with $\mathrm{MgBr}_{2} \cdot \mathrm{Et}_{2} \mathrm{O}$ and $\mathrm{EtSH}$ in dichloromethane to give after HPLC purification the target compounds 109 a-c in 84, 82 and 72\%, respectively (Scheme 20).

Because it was found, that MeZ-protected cyclohexadepsipeptide core of the native Hormaomycin has a significant antiparasitic activity, $N$-acetylated $\mathbf{1 1 0}$ c and $N$-trifluoroacetylated 111 c derivatives were prepared by coupling the deprotected cyclic intermediate $104 \mathbf{c}$ with acetic and trifluoroacetic acid.

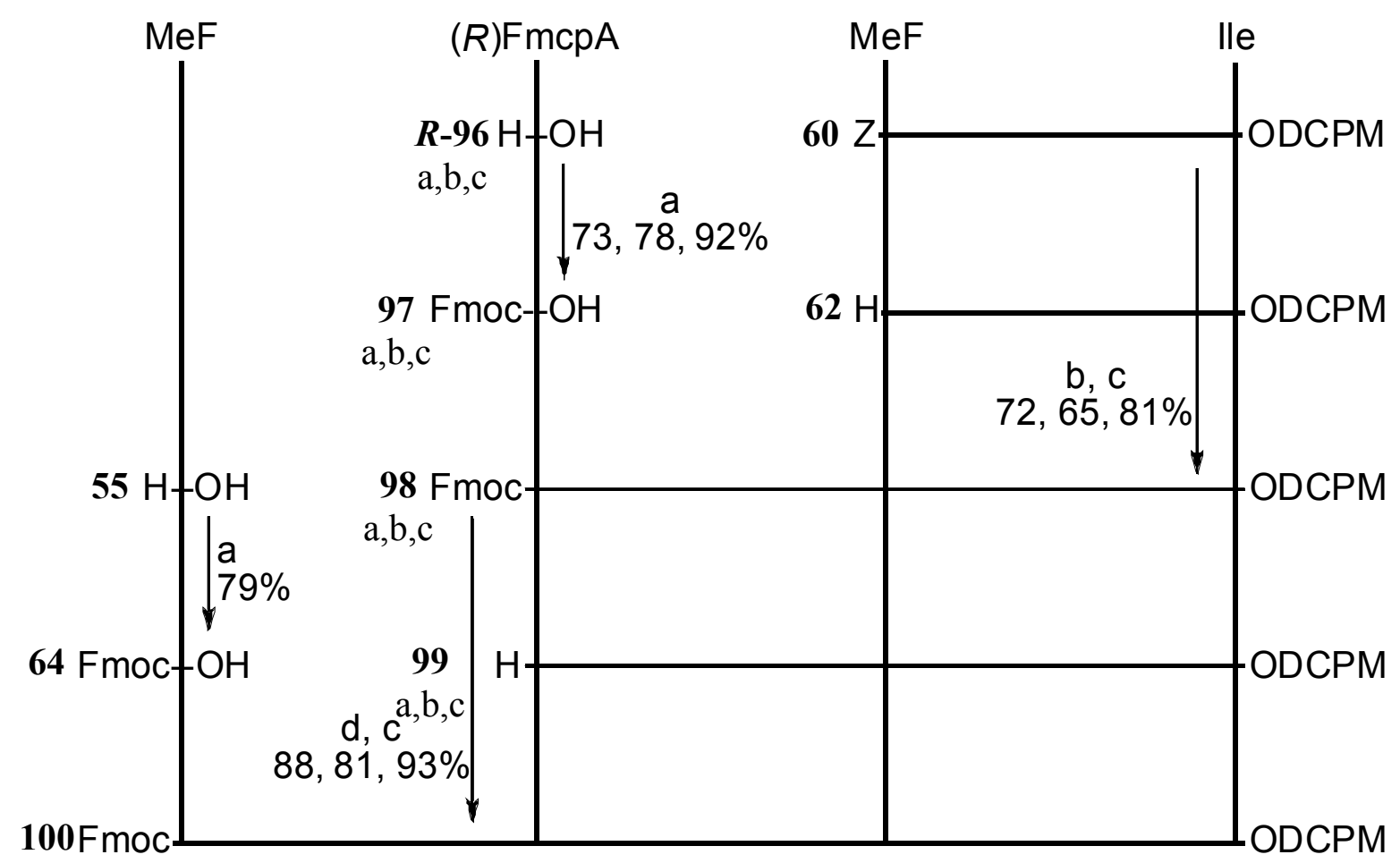

$\mathrm{a}, \mathrm{b}, \mathrm{c}$

Scheme 20. Synthesis of the tetrapeptide precursors of Hormaomycin analogues with a: monofluoromethyl-, b: difluoromethyl-, c: trifluoromethylcyclopropylalanine residues.

a) FmocOSu, $\mathrm{NaHCO}_{3}$, acetone/water, $4 \mathrm{~h}$; b) $\mathrm{H}_{2}$, $\mathrm{Pd} / \mathrm{C}$, EtOAc, $20{ }^{\circ} \mathrm{C}, 40 \mathrm{~min}$; c) EDC, HOAt, DIEA, 2,4,6-collidine, $\mathrm{CH}_{2} \mathrm{Cl}_{2}, 0 \rightarrow 20{ }^{\circ} \mathrm{C}, 14 \mathrm{~h}$; d) $50 \% \mathrm{Et}_{2} \mathrm{NH} / \mathrm{THF}, 20{ }^{\circ} \mathrm{C}, 1 \mathrm{~h}$. 


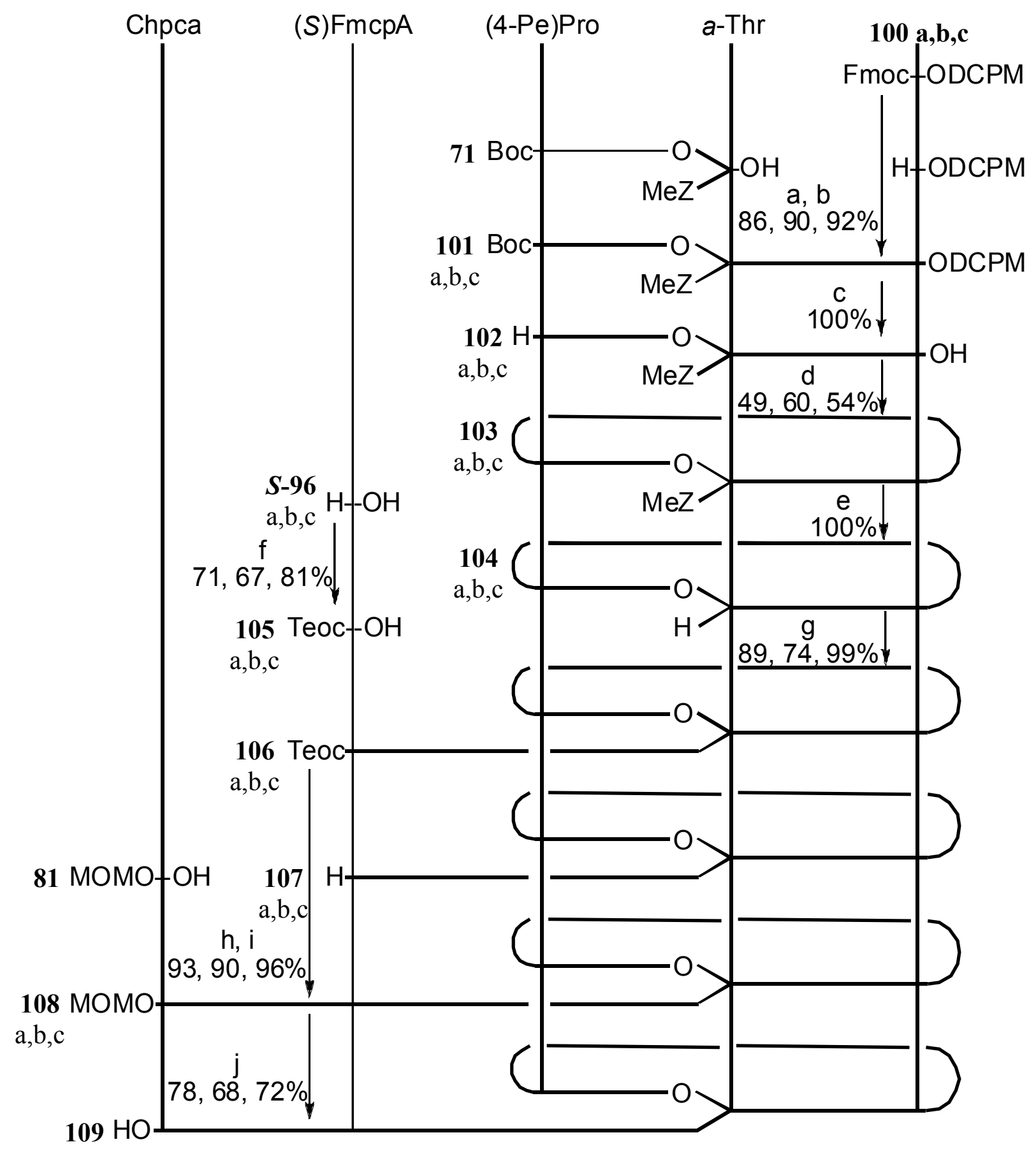

$\mathrm{a}, \mathrm{b}, \mathrm{c}$

Scheme 21. Synthesis of of Hormaomycin analogues with a: monofluoromethyl-, b: difluoromethyl-, c: trifluoromethylcyclopropylalanine residues.

a) $50 \% \mathrm{Et}_{2} \mathrm{NH} / \mathrm{THF}, 2{ }^{\circ} \mathrm{C}, 1 \mathrm{~h}$; b) HATU, HOAt, DIEA, TMP, $\mathrm{CH}_{2} \mathrm{Cl}_{2}, 0 \rightarrow 20{ }^{\circ} \mathrm{C}, 24 \mathrm{~h}$; c) $2 \mathrm{M}$ $\mathrm{HCl}$ in EtOAc, $20^{\circ} \mathrm{C}, 45 \mathrm{~min}$; d) HATU, DIEA,TMP, $\mathrm{CH}_{2} \mathrm{Cl}_{2}, 0 \rightarrow 20^{\circ} \mathrm{C}, 16 \mathrm{~h}$; e) anisole, TFA, $20{ }^{\circ} \mathrm{C}, 2 \mathrm{~h}$; f) TeocOSu, $\mathrm{NaHCO}_{3}, N, N$-Dimethylaminopropylamine, water/acetone, $20{ }^{\circ} \mathrm{C}, 2 \mathrm{~h}$; g) HATU, HOAt, DIEA, TMP, $\mathrm{CH}_{2} \mathrm{Cl}_{2}, 20{ }^{\circ} \mathrm{C}, 6 \mathrm{~h}$; h) TFA, $20{ }^{\circ} \mathrm{C}, 1 \mathrm{~h}$; i) HATU, HOAt, DIEA, TMP, $\mathrm{CH}_{2} \mathrm{Cl}_{2}, 2{ }^{\circ} \mathrm{C}, 4 \mathrm{~h}$; j) $\mathrm{MgBr}_{2} \cdot \mathrm{Et}_{2} \mathrm{O}, \mathrm{EtSH}, \mathrm{CH}_{2} \mathrm{Cl}_{2}, 2{ }^{\circ} \mathrm{C}, 3.5 \mathrm{~h}$. 


\section{Biological activity of Hormaomycin and analogues}

Malaria (lat.: mala aria = bad air) is an infection caused by human-pathogenic Protozoen. The pathogens are transferred by the female Anopheles gnat, leading to a primary infection of the liver. In the following 'blood phase' of the illness erythrocytes are injured, in which the parasites are developing. During the release of the Protozoen from the infected erythrocytes, cell destruction is occurred, that lead to the characteristic fever. The Plasmodium falciparum causes the heaviest of the four observed disease pictures, called Malaria tropica. This pathogen causes the storage of specific proteins in the erythrocytes membranes, which lead to an adhering of infected blood cells at pre-venous capillaries. It causes thrombosis at the blood flow leading finally to death. Currently approx. 2.2 billion humans live in Plasmodium-endemic regions, approximately 500 million of them get sick with malaria annually. The estimated number of deaths caused by malaria is 1.5-3.0 million annually. Despite these, only five medicines of altogether 1300 developed since 1975 are used in malaria treating.

Anti-malaria active substances from plants:

- Quinine was the first chemically pure substance in the malaria therapy. The natural substance was first isolated in 1820 from the crust of the Cinchona tree resident in the Andes. Indians used the crust for the fever lowering, giving the first example of the often successful ethnomedical approach to the active substance search. On the basis of the structure of the Quinine synthetic analogues were developed.

- In the traditional Chinese medicine the Wormwood (Artemisia annua) has been used for more than 1500 years for the treatment of bleeding and against fever. The isolation of the active component, Artemisinin, was succeeded in 1972, but the substance, like many natural substances from plants, can be isolated only in very small yields, which causes high costs.

Anti-malaria active substances from microorganisms:

- Tetracyclines (like e.g. Doxycyclin) are antibacterial substances from microorganisms, that show a high activity against Gram-positive and negative organisms as well as against Plasmodium.

- Another antibacterially effective secondary metabolite with an activity against Plasmodium is Borrelidin. 
- Among all secondary metabolites active against Plasmodium falciparum Gramicidin D holds an outstanding position with a subnanomolar activity and small toxicity. From the chemical point of view, it is the linear peptide, that is able to form ion channels in cell membranes.

There are some cyclic peptides, antiplasmoidale activities of which have been proved. Examples for this are Enniatine and Hormaomycin.

Resistance of Plasmodium falciparum against medicines is developing, like that observed for bacteria. In Africa most strains are Chloroquin-resistant, at the same time the effectiveness of Artemisinin in Asia slowly decreases.

So, it is necessary to provide new medicines for malaria treating and Hormaomycin is one of the best drug candidates.

Biological activity of Hormaomicyn and analogues was tested at the group of Dr. Marcel Kaiser (Parasite Chemotherapy group, Swiss Tropical Institute, Basel).

Activity table (IC-50 ${ }^{[119]}$ for substances and parasites, concentration in $\mu \mathrm{g} / \mathrm{ml}$ ):

\begin{tabular}{|c|c|c|}
\hline Compound & $\begin{array}{c}\text { Leishmania donovani axen } \\
\text { strain MHOM-ET-67/L82 }\end{array}$ & $\begin{array}{c}\text { Plasmodium falciparum } \\
\text { strain K1 }\end{array}$ \\
\hline Miltefosine & 0.143 & - \\
\hline Chloroquine & - & 0.089 \\
\hline 103 c & 2.125 & 0.042 \\
\hline 110 c & 1.730 & 0.151 \\
\hline 109 c & 0.205 & 0.183 \\
\hline 111 c & 2.370 & 0.265 \\
\hline Chloroquine/Artemisinin & - & 0.045 \\
\hline 53 & 4.8 & 0.023 \\
\hline 91 & -- & 0.061 \\
\hline
\end{tabular}

Positions suggested for in vivo studies are marked yellow. Reference drugs are marked cyan. 
Compounds identification:

110 c: $\quad$ Acetylated cyclohexadepsipeptide with (2R,1'R,2'R)-3-(2'-Trifluoromethylcyclopropyl)alanine ( $R$-tFmcpA).

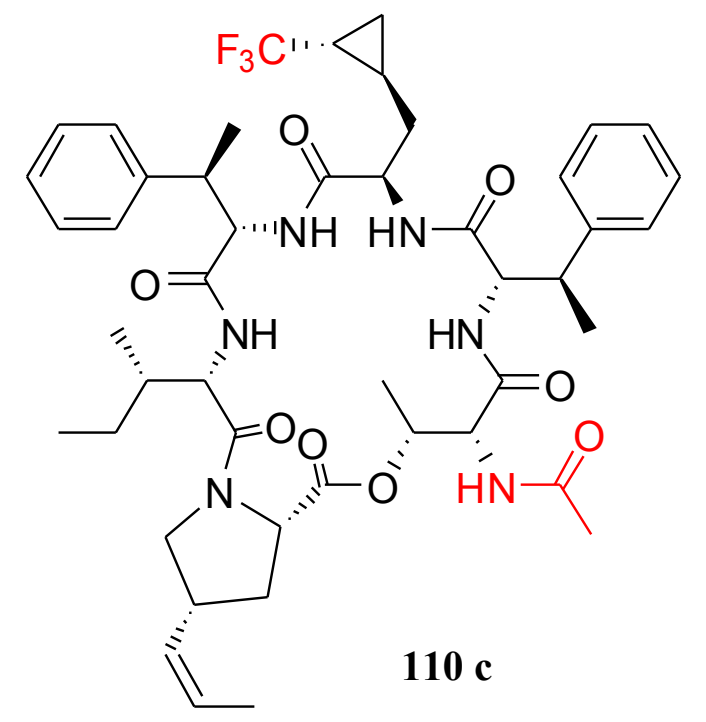

111 c: $\quad$ Trifluoroacetylated cyclohexadepsipeptide with (2R,1'R,2'R)-3-(2'-Trifluoromethylcyclopropyl)alanine ( $R$-tFmcpA).

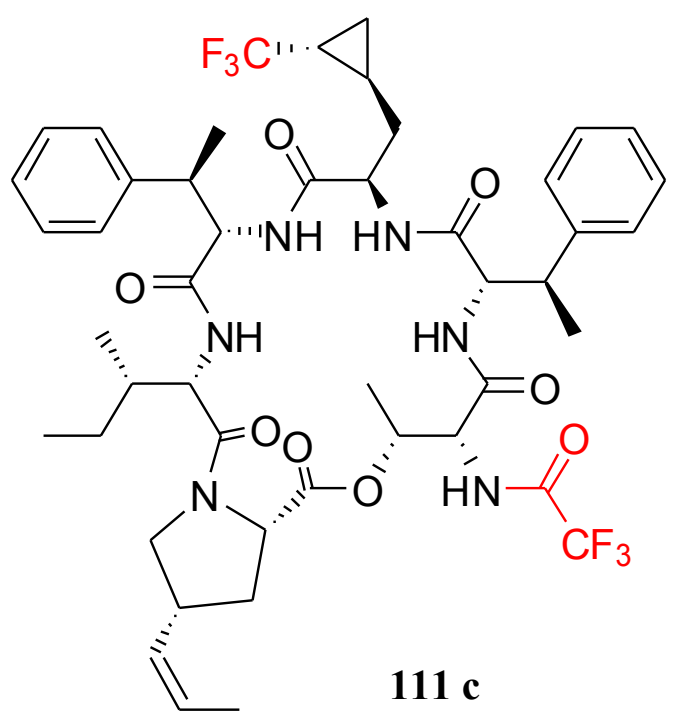




\section{EXPERIMENTAL PART}

\section{General remarks}

'H NMR: Bruker AM $250(250 \mathrm{MHz})$ ， Varian Unity 300 (300 MHz)， Inova $500(500 \mathrm{MHz})$, Inova $600(600 \mathrm{MHz}) .{ }^{1} \mathrm{H}$ chemical shifts are reported in ppm relative to residual peaks of deuterated solvents: $\delta(\mathrm{ppm})=2.49$ for $\left[\mathrm{D}_{5}\right] \mathrm{DMSO}, \quad 4.65$ for $\mathrm{HOD}$ in $\mathrm{D}_{2} \mathrm{O}, \quad 7.26$ for $\mathrm{CHCl}_{3}$, 1.73 and 3.55 for $\left[\mathrm{D}_{7}\right] \mathrm{THF}, 3.35$ for $\mathrm{CHD}_{2} \mathrm{OD}$. Higher-order NMR spectra were approximately interpreted as first-order spectra, if possible. For the characterization of the observed signal multiplicities the following abbreviations have been applied: $\mathrm{s}=$ singlet, $\mathrm{d}=$ doublet, $\mathrm{t}=$ triplet, $\mathrm{q}=$ quartet, quin $=$ quintet, $\mathrm{m}=$ multiplet, as well $\mathrm{as} \mathrm{b}=$ broad.

${ }^{13} \mathrm{C}$ NMR [additional DEPT (Distortionless Enhancement by Polarization Transfer) or APT (Attached Proton Test)]: Bruker AM $250(62.9 \mathrm{MHz}), \quad$ AMX $300(75.5 \mathrm{MHz}) \quad$ or Varian Unity $300(75.5 \mathrm{MHz})$, Inova $500(125.7 \mathrm{MHz})$, Inova $600(125.7 \mathrm{MHz})$ instruments. ${ }^{13} \mathrm{C}$ chemical shifts are reported relative to peaks of deuterated solvents: $\delta(\mathrm{ppm})=39.5$ for $\left[\mathrm{D}_{6}\right] \mathrm{DMSO}, 77.0$ for $\mathrm{CDCl}_{3}, 25.5$ and 3.55 for $\left[\mathrm{D}_{8}\right] \mathrm{THF}, 3.35$ for $\mathrm{CD}_{3} \mathrm{OD}$ or to methanol in $\mathrm{D}_{2} \mathrm{O}(\delta=49.5 \mathrm{ppm})$. The following abbreviations were applied: DEPT: $+=$ primary or tertiary (positive signal in DEPT), $-=$ secondary (negative signal in DEPT), Cquat $=$ quaternary $($ no signal in DEPT); APT: $+=$ primary or tertiary (positive signal in DEPT),$-=$ secondary or quaternary (negative signal in APT).

IR measured as $\mathrm{KBr}$ pellets or thin films between $\mathrm{KBr}$ plates on a Bruker IFS 66 (FT-IR) spectrometer.

MS: EI-MS: Finnigan MAT 95, $70 \mathrm{eV}$, high resolution EI-MS spectra with perfluorkerosene as reference substance; DCI-MS: Finnigan MAT 95, $200 \mathrm{eV}$, reactant gas $\mathrm{NH}_{3}$; ESI-MS: Finnigan

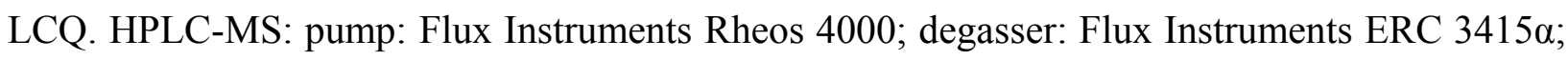
detector: Linear UVIS-205; data system: Flux Instruments Janeiro; ESI: Finnigan LCQ, positive and negative ion mode; data system: Finnigan LCQ Xcalibur; column: Crom Superspher 100 RP-18 endcapped ( $4 \mu \mathrm{m}, 2 \times 100 \mathrm{~mm})$; HPLC conditions: eluent A: $\mathrm{H}_{2} \mathrm{O}(0.1 \%$ TFA), eluent B: $\mathrm{MeCN}(0.1 \%$ TFA). Analytical HPLC: instrument Instrumentelle Analytik Goebel GmbH, autosampler SA 360, pump 420, detector Celeno DAD UV, software Geminyx Version 1.91, column Nucleodur ${ }^{\circledR} \mathrm{C} 18(250 \mathrm{~mm} \times 3 \mathrm{~mm}, 5 \mu \mathrm{m}, 100 \AA)$, flow rate $0.5 \mathrm{ml} / \mathrm{min}$. Preparative HPLC: instrument Jasco, pump Jasco PU-1587, detector Jasco UV-1575, Software Jasco- 
BORWIN HSS-2000, column Nucleodur ${ }^{\circledR} \mathrm{C} 18(250 \mathrm{~mm} \times 20 \mathrm{~mm}, 5 \mu \mathrm{m}, 100 \AA)$, flow rate $18.0 \mathrm{ml} / \mathrm{min}$.

Optical rotations: Perkin-Elmer 241 digital polarimeter, 1-dm cell; optical rotation values are given in $10^{-1} \mathrm{deg} \mathrm{cm}^{2} \mathrm{~g}^{-1}$; concentrations (c) are given in $\mathrm{g} / 100 \mathrm{~mL}$.

M.p.: Büchi 510 capillary melting point apparatus, uncorrected values.

TLC: Macherey-Nagel pre-coated sheets, $0.25 \mathrm{~mm}$ Sil G/UV254. The chromatograms were viewed under UV light and/or by treatment with phosphomolybdic acid (10\% in ethanol), or ninhydrine $\left(0.2 \%\right.$ in ethanol), or $\mathrm{I}_{2}$ vapor.

Column chromatography: Merck silica gel, grade 60, 230-400 mesh and Baker silica gel, 40-140 mesh.

Elemental analyses: Mikroanalytisches Laboratorium des Instituts für Organische und Biomolekulare Chemie der Universität Göttingen.

Starting materials: Anhydrous solvents were prepared according to standard methods by distillation over drying agents and were stored under nitrogen. All other solvents were distilled before use.

All reactions were carried out with magnetic stirring and, when employing air- or moisturesensitive materials, in flame-dried glassware under argon or nitrogen.

\section{General synthetic protocols}

\subsection{Deprotection of N-Fmoc-protected peptides (GP 1)}

The respective protected peptide $(1 \mathrm{mmol})$ was taken up with acetonitrile or THF $(2 \mathrm{~mL})$, diethylamine $(2 \mathrm{~mL})$ was added, and the resulting mixture left at ambient temperature for $40 \mathrm{~min}$. All volatiles were evaporated under reduced pressure, the residue was taken up with toluene $(2 \times 5 \mathrm{~mL})$, which was evaporated under reduced pressure to remove the last traces of diethylamine. The obtained crude $N$-deprotected peptide was directly used in the next condensation step.

\subsection{Peptide condensation step for the preparation of peptides using EDC/HOAt - mediated coupling (GP 2)}

EDC $(1.03 \mathrm{mmol})$ and HOAt $(1.05 \mathrm{mmol})$ were added to a cooled $\left(4{ }^{\circ} \mathrm{C}\right)$ solution of the respective $N$-protected amino acid $(1 \mathrm{mmol})$ in anhydrous $\mathrm{CH}_{2} \mathrm{Cl}_{2}(3 \mathrm{~mL})$. After $20 \mathrm{~min}$, the solution of the appropriate crude $N$-deprotected peptide $(0.97 \mathrm{mmol})$ and TMP $(3 \mathrm{mmol})$ in 
anhydrous $\mathrm{CH}_{2} \mathrm{Cl}_{2}(1 \mathrm{~mL})$ was added at the same temperature. The temperature was allowed to reach $20^{\circ} \mathrm{C}$ and stirring was continued for $15 \mathrm{~h}$. Then the reaction mixture was diluted with diethyl ether or ethyl acetate $(30 \mathrm{~mL})$ and washed with water $(2 \times 5 \mathrm{~mL}), 1 \mathrm{M} \mathrm{KHSO}_{4}$ $(3 \times 5 \mathrm{~mL})$, water $(2 \times 5 \mathrm{~mL}), 5 \%$ aqueous $\mathrm{NaHCO}_{3}$ solution $(3 \times 5 \mathrm{~mL})$, water $(3 \times 5 \mathrm{~mL})$, brine $(2 \times 5 \mathrm{~mL})$, dried over $\mathrm{MgSO}_{4}$, filtered and concentrated under reduced pressure. The residue was purified by column chromatography or recrystallization.

\subsection{Preparation of hexadepsipeptides and hexapeptides using HATU/HOAt mediated coupling (GP 3)}

Deprotected according to GP 1 tetrapeptide $(0.100 \mathrm{mmol})$ was dissolved in anhydrous $\mathrm{CH}_{2} \mathrm{Cl}_{2}$ $(3 \mathrm{~mL})$, ester acid / dipeptide acid $(0.110 \mathrm{mmol})$, HATU $(0.107 \mathrm{mmol})$ and HOAt $(0.110 \mathrm{mmol})$ were added and the reaction mixture was cooled to $4{ }^{\circ} \mathrm{C}$. DIEA $(0.110 \mathrm{mmol})$ and TMP $(0.300 \mathrm{mmol})$ were then added, the mixture was allowed to warm to $20^{\circ} \mathrm{C}$ and stirring continued for an additional $15 \mathrm{~h}$. The mixture was then taken up with $\mathrm{Et}_{2} \mathrm{O}(40 \mathrm{~mL})$ and after usual aqueous work-up (GP 2) the organic layer was concentrated to leave crude hexadepsipeptide/hexapeptide, which was purified by recrystallization and/or column chromatography.

\subsection{Preparation of cyclohexadepsipeptides (GP 4)}

The respective acyclic hexadepsipeptide $(105 \mu \mathrm{mol})$ was deprotected by stirring with $2 \mathrm{M} \mathrm{HCl}$ solution in ethyl acetate $(2 \mathrm{~mL})$ at $20^{\circ} \mathrm{C}$ for $1 \mathrm{~h}$ in dark place and followed concentration under reduced pressure to solid residue. The deprotected material was then dissolved in $\mathrm{CH}_{2} \mathrm{Cl}_{2}(1.0 \mathrm{~L})$. The solution was cooled to $4{ }^{\circ} \mathrm{C}$ (internal temperature), HATU $(122 \mu \mathrm{mol})$ and HOAt (104 $\mu \mathrm{mol})$ were added, the mixture was stirred for $30 \mathrm{~min}$, and then the solution of DIEA (305 $\mu \mathrm{mol})$ in $\mathrm{CH}_{2} \mathrm{Cl}_{2}(50 \mathrm{~mL}$, over a period of $30 \mathrm{~min}$ ). The cooling bath was removed, and stirring was continued for an additional $2 \mathrm{~h}$ at ambient temperature. Then the reaction mixture was cooled again to $4{ }^{\circ} \mathrm{C}$ and second portions of HATU $(122 \mu \mathrm{mol})$ and HOAt $(104 \mu \mathrm{mol})$ were added, followed by a solution of DIEA ( $305 \mu \mathrm{mol})$ in $\mathrm{CH}_{2} \mathrm{Cl}_{2}(50 \mathrm{~mL}$, over a period of $30 \mathrm{~min})$. The temperature was allowed to reach $20^{\circ} \mathrm{C}$, and stirring was continued for $15 \mathrm{~h}$. After this, the solvent was removed under reduced pressure, the residue was taken up with diethyl ether $(50 \mathrm{~mL})$, subjected to the usual aqueous work-up (see GP 2) and concentrated under reduced pressure, to give the crude product, which was finally purified by preparative HPLC. 


\subsection{Deprotection of $\mathrm{N}-\mathrm{MeZ}$ protected cyclohexadepsipeptides (GP 5)}

The respective $N$-MeZ protected cyclodepsipeptide $(10 \mu \mathrm{mol})$ was treated with $10 \%$ anisole in TFA $(1 \mathrm{~mL})$ in the dark for $2 \mathrm{~h}$. All volatiles were then removed under reduced pressure at 20 ${ }^{\circ} \mathrm{C}$. The solid residue was taken up with toluene $(2 \times 10 \mathrm{~mL})$, which was distilled off under reduced pressure to remove the last traces of anisole and TFA. The resulting crude deprotected depsipeptide was directly used for the appropriate coupling reaction.

7.6. Preparation of heptadepsipeptides and Hormaomycines using HATU/HOAt mediated coupling (GP 6)

Deprotected according to GP 5 depsidipeptide $(0.100 \mathrm{mmol})$ was dissolved in anhydrous $\mathrm{CH}_{2} \mathrm{Cl}_{2}(4 \mathrm{~mL}), \quad N$-protected amino acid $(0.320 \mathrm{mmol})$, HATU $(0.300 \mathrm{mmol})$ and HOAt $(0.300 \mathrm{mmol})$ were added and the reaction mixture was cooled to $4{ }^{\circ} \mathrm{C}$. DIEA $(0.102 \mathrm{mmol})$ and TMP $(0.900 \mathrm{mmol})$ were then added as a solution in anhydrous $\mathrm{CH}_{2} \mathrm{Cl}_{2}(2 \mathrm{~mL})$, the mixture was allowed to warm to $20^{\circ} \mathrm{C}$ and stirring continued for an additional $15 \mathrm{~h}$. The mixture was then taken up with EtOAc $(100 \mathrm{~mL})$ and after usual aqueous work-up (GP 2) the organic layer was concentrated to leave crude depsipeptide, which was purified by recrystallization and/or chromatography.

\subsection{Removal of the MOM ether group using $\mathrm{MgBr}_{2} \bullet \mathrm{Et}_{2} \mathrm{O}$ and $\mathrm{EtSH}(\mathrm{GP} 7)$}

$\mathrm{MgBr}_{2} \bullet \mathrm{Et}_{2} \mathrm{O}(1 \mathrm{mmol})$ and $\mathrm{EtSH}(0.5 \mathrm{mmol})$ were added to a vigorously stirred solution of the respective $O$-MOM protected derivative $(0.1 \mathrm{mmol})$ in $\mathrm{CH}_{2} \mathrm{Cl}_{2}(15 \mathrm{~mL})$, and stirring was continued for an additional $3.5 \mathrm{~h}$ (TLC control was impossible as the starting material and the product in all cases showed exactly the same $R_{\mathrm{f}}$ in all tested solvent systems). The mixture was then taken up with EtOAc $(40 \mathrm{~mL})$ and washed with $1 \mathrm{M} \mathrm{KHSO}_{4}(3 \times 10 \mathrm{~mL})$, water $(5 \times 10 \mathrm{~mL})$, brine $(2 \times 5 \mathrm{~mL})$, dried over $\mathrm{MgSO}_{4}$, filtered and concentrated under reduced pressure. The residue was purified first by crystallization and the crude product was finally purified with HPLC.

\subsection{Reduction with $\mathrm{LiAlH}_{4}$ (reverse addition) (GP 8)}

A solution of $\mathrm{LiAlH}_{4}$ in diethyl ether $(1 \mathrm{M}, 6 \mathrm{~mL}, 6 \mathrm{mmol})$ was added dropwise to the cooled (dry ice/acetone bath) solution of the respective carbonyl compound (20 mmol for ketones, $10 \mathrm{mmol}$ for esters, 7,5 mmol for carboxylic acid) in diethyl ether $(20 \mathrm{ml})$ and the mixture was stirred for an additional $30 \mathrm{~min}$ at $-78{ }^{\circ} \mathrm{C}$. The flask was immersed to ice/water bath, the mixture was stirred for an additional 2 hour and the saturated aqueous $\mathrm{NH}_{4} \mathrm{Cl}$ solution was added dropwise under vigorous stirring (carefully - foam!) till $\mathrm{H}_{2}$ gas evolution ceased. The mixture 
was stirred for an additional $15 \mathrm{~min}$, filtered with suction through Celite ${ }^{\circledR}$ pad, filter cake was washed with diethyl ether $(3 \times 50 \mathrm{ml})$, combined filtrates concentrated under reduced pressure, giving the target alcohol.

\subsection{Conversion alcohols to iodides (GP 9)}

The respective racemic alcohol $(12 \mathrm{mmol})$ was added to the solution of triphenylphosphine (5.5 g, $21 \mathrm{mmol})$ and imidazole $(1.5 \mathrm{~g}, 22 \mathrm{mmol})$ in corresponding solvent mixture and the solution was cooled down to $-5^{\circ} \mathrm{C}$ (internal temperature, ice/salt bath). The solid iodine $(6.0 \mathrm{~g}$, $24 \mathrm{mmol}$ ) was added as one portion and the mixture was stirred for an additional $30 \mathrm{~min}$ at this temperature, bath was removed and the mixture was stirred at ambient temperature for 3 hour. The mixture was poured to pentane $(200 \mathrm{ml})$ under vigorous stirring and the resulting mixture was washed with $20 \%\left(\right.$ w/w) aqueous $\mathrm{Na}_{2} \mathrm{~S}_{2} \mathrm{O}_{3} \times 5 \mathrm{H}_{2} \mathrm{O}(100 \mathrm{~mL})$, upper pentane layer was separated, water layer was extracted with pentane $(2 \times 50 \mathrm{~mL})$ and discarded. Combined pentane solution (washing and extracts) was washed with $20 \%$ (w/w) aqueous $\mathrm{Na}_{2} \mathrm{~S}_{2} \mathrm{O}_{3} \times 5 \mathrm{H}_{2} \mathrm{O}(100 \mathrm{~mL})$, brine $(100 \mathrm{~mL})$, dried over $\mathrm{MgSO}_{4}$, filtered and concentrated under reduced pressure to give crude product as colorless liquid containing solid. This was purified with the column chromatography (silica gel, eluting with pentane), giving the pure corresponding target iodide.

7.10. Alkylation of the Ni(II)-complexes of Schiff bases, derived from glycine and $(S)$ - or (R)-2-[(N-Benzylprolyl)amino]benzophenone (Belokon' glycine complexes; (S)- or (R)-BGC) (GP 10)

The respective Belokon' glycine complex (BGC) $(2.00 \mathrm{~g}, 4.02 \mathrm{mmol})$ was suspended in $\mathrm{DMF} / \mathrm{MeCN}$ mixture $(2+4 \mathrm{~mL})$ and degassed in two freeze-pump-thaw cycles (dry ice/acetone bath) under stirring, then $\mathrm{NaH}(60 \%$ in oil, $193 \mathrm{mg}, 4.8 \mathrm{mmol})$ was added to the cold mixture and the system was thawed to $0^{\circ} \mathrm{C}$ under stirring till the color of the reaction mixture changed from orange to dark-brown. The mixture was frozen, the respective racemic iodide $(4.22 \mathrm{mmol})$ was added with stirring, the bath was removed and the mixture was left to warm to $0{ }^{\circ} \mathrm{C}$ with stirring. When ice cover on flask started to thaw, the flask was immersed in an ice/water bath, and stirring was continued until all starting BGC had been consumed (TLC monitoring, chloroform/acetone 7:1, $R_{f}=0.12$ ). After ca. $1 \mathrm{~h}, 60 \%$ aqueous acetic acid $(2 \mathrm{~mL})$ was added dropwise. After an additional 10 min of stirring, the mixture was poured into vigorously stirred $\mathrm{H}_{2} \mathrm{O}(100 \mathrm{~mL})$. The resulting suspension was stirred for ca. $1 \mathrm{~h}$, and the crude product (diastereomeric mixture) was filtered off, the filter cake was washed with $\mathrm{H}_{2} \mathrm{O}(3 \times 10 \mathrm{~mL})$ and dried overnight over $\mathrm{P}_{2} \mathrm{O}_{5}$ under reduced pressure. The diastereomers were separated by column chromatography (silica gel, eluting with ethyl acetate). 


\subsection{Decomposition of Belokon' amino acid complexes to obtain enantiomerically pure amino acids (GP 11)}

$6 \mathrm{M} \mathrm{HCl}(50 \mathrm{~mL})$ was added to a refluxing solution of the respective Belokon' amino acid complex $(1 \mathrm{mmol})$ in methanol $(25 \mathrm{~mL})$, the mixture was heated at reflux for an additional $10 \mathrm{~min}$ and concentrated under reduced pressure to leave behind a wet hydrochloride salt. The residue was treated with $\mathrm{H}_{2} \mathrm{O}(100 \mathrm{~mL})$, precipitated ligand (2-[(N-Benzylprolyl)amino]benzophenone) as a hydrochloride salt was filtered off, washed with $\mathrm{H}_{2} \mathrm{O}(3 \times 30 \mathrm{~mL})$, dried and collected for recycling. The filtrate was combined with the washings, neutralized to $\mathrm{pH}=6.0$ with $5 \%$ aqueous ammonia and extracted with $\mathrm{CHCl}_{3}(3 \times 30 \mathrm{~mL})$. The aqueous fraction was concentrated to ca. $10 \mathrm{~mL}$ and neutralized with $5 \%$ aqueous ammonia to $\mathrm{pH}=6.5$. The amino acid was separated from the nickel salts by elution of the neutralized concentrate through an $\mathrm{H}^{+}$-form DOWEX ionexchange resin column (ca. $150 \mathrm{~g}$ of resin) with 5-7\% aqueous ammonia. The fraction of the eluate that showed red pigmentation on developing with ninhydrin, was collected. This was concentrated under reduced pressure at $40-45^{\circ} \mathrm{C}$. The crude amino acid was dissolved in minimal volume of hot water, the hot turbid solution was filtered and diluted with an equal volume of ethanol. The precipitate, formed after storing at $-20{ }^{\circ} \mathrm{C}$ for $1 \mathrm{~h}$, was filtered off, washed with cold ethanol $(10 \mathrm{~mL})$, and dried in vacuo at $40{ }^{\circ} \mathrm{C}$ to give the target amino acid.

\section{8. (Fluoromethylcyclopropyl)alanines}

\section{1. (Trifluoromethylcyclopropyl)alanines}

Racemic diethyl 2-trifluoroacetyl succinate (34): ${ }^{[120]}$ Ethyl trifluoroacetate 32 (31.0 g, 220 mmol)<smiles>CCOC(=O)CC(C(=O)OCC)C(=O)C(F)(F)F</smiles>
and diethyl succinate $33(76.0 \mathrm{~g}, 440 \mathrm{mmol})$ were mixed in a $250-\mathrm{mL}$ round-bottomed flask and sodium metal (thin plates, $5.0 \mathrm{~g}, 220 \mathrm{mmol}$ ) was

added in one portion. The mixture was heated at reflux with vigorous stirring for $12 \mathrm{~h}$, the reflux condenser was replaced by distillation head, and all volatiles were distilled out (ethanol at ambient pressure and excess of diethyl succinate at reduced pressure). After the mixture had cooled down to ambient temperature, the black tar residue was treated with $5 \mathrm{M}$ aqueous $\mathrm{H}_{2} \mathrm{SO}_{4}(150 \mathrm{~mL})$, the organic layer was separated, the water layer was extracted with diethyl ether $(3 \times 50 \mathrm{~mL})$, the combined organic phases were washed with water $(3 \times 50 \mathrm{~mL})$, dried over $\mathrm{MgSO}_{4}$, filtered and concentrated under reduced pressure. The viscous black residue was fractioned through a $15-\mathrm{cm}$ Vigreux column in vacuo, giving $8.1 \mathrm{~g}$ of a predistillate (the product and starting diethyl succinate), $35.6 \mathrm{~g}$ main fraction (the target product) and $3.4 \mathrm{~g}$ of tail distillate (the target product 
and the side product, diethyl 2,5-dioxo-cyclohexane-1,4-dicarboxylate). Yield $35.6 \mathrm{~g}$, $131.7 \mathrm{mmol}$, 59.8\%. 1H NMR (250 MHz, $\left.\mathrm{CDCl}_{3}\right): \delta=1.20$ (t, $\left.J=7.1 \mathrm{~Hz}, 3 \mathrm{H}\right), 1.22$ (t, $J=7.1 \mathrm{~Hz}, 3 \mathrm{H}), 2.85-3.15(\mathrm{~m}, 2 \mathrm{H}), 4.11$ (q, $J=7.1 \mathrm{~Hz}, 2 \mathrm{H}), 4.19$ (q, $J=7.1 \mathrm{~Hz}, 2 \mathrm{H}), 4.27$ $4.38(\mathrm{~m}, 1 \mathrm{H}) ;{ }^{13} \mathrm{C} \mathrm{NMR}\left(62.9 \mathrm{MHz}, \mathrm{CDCl}_{3}\right): \delta=13.67,13.89,32.42,48.45,61.54,62.66$, $115.09(\mathrm{q}, J=291.2 \mathrm{~Hz}), 166.03,169.97,186.81$ (q, $J=36.9 \mathrm{~Hz})$.

Diethyl 2,5-dihydroxycyclohexa-1.4-diene-1.4-dicarboxylate (side product of 34): ${ }^{1} \mathrm{H}$ NMR

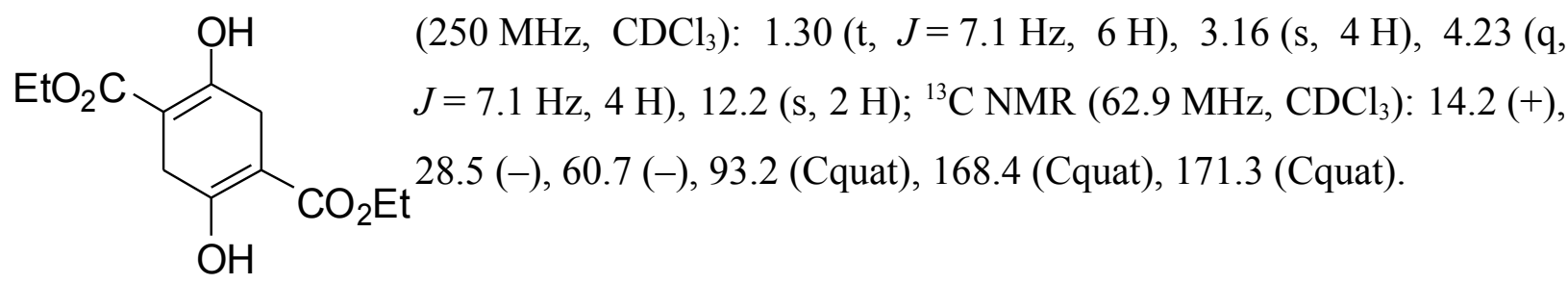

Ethyl 5,5,5-Trifluoro-4-oxovalerate (35 ${ }^{[64]}$ : Racemic diethyl 2-trifluoroacetosuccinate 34<smiles>CCOC(=O)CCC(=O)C(F)(F)F</smiles>
$(35.1 \mathrm{~g}, 130 \mathrm{mmol})$ was mixed with boric acid $(8.1 \mathrm{~g}, 130 \mathrm{mmol})$ in a round-bottomed flask, equipped with a distillation head, and the mixture was stirred at $170{ }^{\circ} \mathrm{C}$ (bath temperature) overnight. The distilled ethanol was discarded, the residue was fractioned over a 15-cm Vigreux column in vacuo, giving the target ester as a colorless liquid (13,7 g, $69 \mathrm{mmol}, 53 \%)$. B. p. : 50-55 ${ }^{\circ} \mathrm{C} / 10-11 \mathrm{mbar} ;{ }^{1} \mathrm{H} \mathrm{NMR}\left(250 \mathrm{MHz}, \mathrm{CDCl}_{3}\right): \delta=1,23(\mathrm{t}, J=7.2 \mathrm{~Hz}, 3 \mathrm{H}), 2.68$ (t, $J=6.3 \mathrm{~Hz}, 2 \mathrm{H}), 3.01(\mathrm{t}, J=6.3 \mathrm{~Hz}, 2 \mathrm{H}), 4.13(\mathrm{q}, J=7.2 \mathrm{~Hz}, 2 \mathrm{H}) ;{ }^{13} \mathrm{C} \mathrm{NMR}(250 \mathrm{MHz}$, $\left.\mathrm{CDCl}_{3}\right): \delta=14.00, \quad 26.90, \quad 31.40, \quad 61.10, \quad 115.50(\mathrm{q}, J=291.0 \mathrm{~Hz}), \quad 171.20,190.10(\mathrm{q}, J=$ $36.0 \mathrm{~Hz})$.

Racemic ethyl 5,5,5-Trifluoro-4-oxivalerate (36) ${ }^{[64]}$ : To a solution of racemic ethyl 5,5,5-<smiles>CCOC(=O)CCC(O)C(F)(F)F</smilestrifluoro-4-oxovalerate $\mathbf{3 5}$ (15,8 g, 79,7 mmol) in anhydrous diethyl ether $(160 \mathrm{~mL})$, cooled in an ice/salt bath, was added crushed sodium borohydride $(1.51 \mathrm{~g}, 40.0 \mathrm{mmol})$ in one portion. The cold mixture was stirred for $10 \mathrm{~min}$, the bath was removed, and the mixture was stirred for 4 h. $1 \mathrm{M}$ aq. $\mathrm{KHSO}_{4}(50 \mathrm{~mL})$ was added slowly (carefully - foam!), the organic layer was separated, and the water layer was extracted with diethyl ether $(3 \times 30 \mathrm{~mL})$. The combined organic layers were dried over $\mathrm{MgSO}_{4}$, filtered and concentrated under reduced pressure, giving the target hydroxyester $(15.6 \mathrm{~g}, 77.9 \mathrm{mmol}, 97.8 \%)$ as yellowish liquid. ${ }^{1} \mathrm{H}$ NMR $\left(250 \mathrm{MHz}, \mathrm{CDCl}_{3}\right): \quad \delta=1,25(\mathrm{t}, J=7.1 \mathrm{~Hz}, 3 \mathrm{H}), 1.80-2.12 \quad(\mathrm{~m}, 2 \mathrm{H}), 2.54(\mathrm{t}$, $J=7.1 \mathrm{~Hz}, 2 \mathrm{H}), \quad 3.38-3.76$ (bs, $1 \mathrm{H}), 3.91-4.07$ (m, $1 \mathrm{H}), 4.14$ (q, $J=7.1 \mathrm{~Hz}, 2 \mathrm{H})$; 
${ }^{13} \mathrm{C}$ NMR: $\delta=9.03, \quad 19.58, \quad 24.57, \quad 56.08, \quad 64.59(\mathrm{q}, J=31.2 \mathrm{~Hz}), \quad 124.90(\mathrm{q}, J=281.9 \mathrm{~Hz})$, 173.83.

Racemic ethyl 5,5,5-Trifluoro-4-tosyloxivalerate (37): To a solution of ethyl 5,5,5-trifluoro-4-<smiles>CCOC(=O)CCC([O-])C(F)(F)F</smileshydroxyvalerate $\mathbf{3 6}(5.87 \mathrm{~g}, 29.3 \mathrm{mmol})$ in anhydrous pyridine $(30 \mathrm{~mL})$, cooled in ice/salt bath was added tosyl chloride $(11.2 \mathrm{~g}, 58.6 \mathrm{mmol})$ as one portion, followed by DMAP $(0.72 \mathrm{~g}, 5.9 \mathrm{mmol})$. The cold mixture was stirred for $10 \mathrm{~min}$, the bath was removed, and the mixture was stirred at ambient temperature for $20 \mathrm{~h}$. Water $(5 \mathrm{~mL})$ was added, the mixture was stirred for $30 \mathrm{~min}$, poured into a vigorously stirred mixture of water $(50 \mathrm{~mL})$ and diethyl ether $(100 \mathrm{~mL})$ and stirred for an additional $10 \mathrm{~min}$. The organic phase was separated, washed with aq. $6 \mathrm{M} \mathrm{HCl}(50 \mathrm{~mL})$, water $(50 \mathrm{~mL})$, dried over $\mathrm{MgSO}_{4}$, filtered and concentrated under reduced pressure to give target ester (9.3 g, $26.4 \mathrm{mmol}, 90 \%)$ as light yellow viscous liquid. ${ }^{1} \mathrm{H}$ NMR $\left(250 \mathrm{MHz}, \mathrm{CDCl}_{3}\right)$ : $\delta=1,27(\mathrm{t}, J=7.14 \mathrm{~Hz}, 3 \mathrm{H}), 1.93-2.08(\mathrm{~m}, 1 \mathrm{H}), 2.11-2.25(\mathrm{~m}, 1 \mathrm{H}), 2.44(\mathrm{~s}, 3 \mathrm{H}), 2.50(\mathrm{t}$, $J=7.20 \mathrm{~Hz}, 2 \mathrm{H}), 4.15$ (q, $J=7.14 \mathrm{~Hz}, 2 \mathrm{H}), 4.93-5.08(\mathrm{~m}, 1 \mathrm{H}), 7.34(\mathrm{~d}, J=8.25 \mathrm{~Hz}, 2 \mathrm{H})$, $7.78(\mathrm{~d}, J=8.32 \mathrm{~Hz}, 2 \mathrm{H}) ;{ }^{13} \mathrm{C} \mathrm{NMR}\left(62.9 \mathrm{MHz}, \mathrm{CDCl}_{3}\right): \delta=14.07,21.63,23.65 \quad(\mathrm{q}$, $J=1.7 \mathrm{~Hz}), 28.23,60.82,75.40(\mathrm{q}, J=33.0 \mathrm{~Hz}), 127.90,129.86,130.78$ (q, $J=243.5 \mathrm{~Hz})$, $145.57,156.52,171.82$.

Racemic trans-2-trifluoromethylcyclopropanecarboxylic acid (38): The solution of the ethyl<smiles>O=C(O)[C@@H]1C[C@H]1C(F)(F)F</smiles>
5,5,5-trifluoro-4-tosyloxivalerate $\mathbf{3 7}(6.4 \mathrm{~g}, 18 \mathrm{mmol})$ in anhydrous THF $(10 \mathrm{~mL})$ was added via syringe during 3 hour to vigorously stirred refluxing solution of potassium tert-butoxide $(9.0 \mathrm{~g}, 80 \mathrm{mmol})$ in anhydrous THF $(50 \mathrm{~mL})$ under $\mathrm{N}_{2}$-flow and the solution was refluxed for $4 \mathrm{~h}$. The resulting mixture after cooling was diluted with water $(100 \mathrm{~mL})$ and organics were distilled out under reduced pressure. Alkaline water phase was washed with diethyl ether $(3 \times 30 \mathrm{~mL})$, acidified with aqueous $6 \mathrm{M} \mathrm{HCl}$ to $\mathrm{pH} \sim 1$ and extracted with diethyl ether $(5 \times 50 \mathrm{~mL})$. Combined extracts were dried over $\mathrm{MgSO}_{4}$, filtered and concentrated under reduced pressure, giving crude product as black tar. Molecular distillation of this crude product gives target acid as colorless liquid (1.3 g, $8.4 \mathrm{mmol}, 47 \%) .{ }^{1} \mathrm{H} \mathrm{NMR}\left(250 \mathrm{MHz} \mathrm{CDCl}_{3}\right): \delta=1.25-1.48(\mathrm{~m}, 2 \mathrm{H}), 1.97-2.11$ (m, $1 \mathrm{H}), 2.12-2.29(\mathrm{~m}, 1 \mathrm{H}), 10.36(\mathrm{bs}, 1 \mathrm{H}) ;{ }^{13} \mathrm{C} \mathrm{NMR}\left(62.9 \mathrm{MHz}, \mathrm{CDCl}_{3}\right): \delta=10.83(-, \mathrm{q}$, $J=3.2 \mathrm{~Hz}), 16.56(+, \mathrm{q}, J=2.6 \mathrm{~Hz}), 22.56(+, \mathrm{q}, J=38.3 \mathrm{~Hz}), 124.50(-, \mathrm{q}, J=271.2 \mathrm{~Hz})$, 177.70 . 
Racemic 2-Methanesulfinyl-1-(trans-2-trifluoromethyl-cyclopropyl)-ethanone (39): Potassium<smiles>CS(=O)CC(=O)[C@@H]1C[C@H]1C(F)(F)F</smiles>
tert-butoxide $(4.9 \mathrm{~g}, 44 \mathrm{mmol})$ was dissolved in anhydrous DMSO $(30 \mathrm{~mL})$ under $\mathrm{N}_{2}$-flow at ambient temperature and the solution was stirred for 1 hour. The solution of the ethyl 5,5,5-trifluoro-4tosyloxyvalerate $37(3.4 \mathrm{~g}, 10.0 \mathrm{mmol})$ in $5 \mathrm{ml}$ of anhydrous DMSO was added dropwise and the resulting mixture was stirred for 24 hours at $50^{\circ} \mathrm{C}$ and 12 hours at $60^{\circ} \mathrm{C}$ (bath temperature). The mixture was diluted with water $(50 \mathrm{~mL})$ and washed with diethyl ether $(5 \times 20 \mathrm{~mL})$, organic phases were discarded. The resulting alkaline solution was acidified with aqueous $\mathrm{HCl}(6 \mathrm{M})$ to $\mathrm{pH} \sim 1$ and extracted with diethyl ether $(10 \times 20 \mathrm{~mL})$. Combined organic phases were washed with water $(3 \times 50 \mathrm{~mL})$, dried over $\mathrm{MgSO}_{4}$, filtered and concentrated under reduced pressure, giving crude product as viscous dark oil containing solid. The molecular distillation of the crude product gives the yellow liquid, solidifying when stored at ambient temperature to waxy solid $(6.6 \mathrm{~g}, 31 \mathrm{mmol}, 70 \%)$. ${ }^{1} \mathrm{H} \mathrm{NMR}\left(250 \mathrm{MHz}, \mathrm{CDCl}_{3}\right)$ : $\delta=1.30-1.42(\mathrm{~m}, 2 \mathrm{H}), 2.07(\mathrm{~s}, 2 \mathrm{H}), 2.08(\mathrm{~s}, 3 \mathrm{H}), 2.11-2.22(\mathrm{~m}, 1 \mathrm{H}), 2.65-2.75(\mathrm{~m}, 1 \mathrm{H})$; ${ }^{13} \mathrm{C}$ NMR (62.9 MHz, $\mathrm{CDCl}_{3}$ ): $\delta=12.85,21.20,23.17,61.82,126.09$ (q, $J=270.3 \mathrm{~Hz}$ ), 155.62; MS-EI $\left.(77 \mathrm{eV}): \quad \mathrm{m} / \mathrm{z} \quad(\%) \quad 214 \quad(10, \mathrm{M}\rceil+), 137 \quad\left(14, \quad \mathrm{M}-\mathrm{CH}_{2} \mathrm{SOCH}_{3}\right]^{+}\right), \quad 109$ (8, M$\left.\left.\left.\left.\mathrm{COCH}_{2} \mathrm{SOCH}_{3}\right\rceil^{+}\right), 89\left(10, \mathrm{C}_{4} \mathrm{H}_{3} \mathrm{~F}_{2}\right\rceil^{+}\right), 77\left(100, \mathrm{CH}_{3} \mathrm{SOCH}_{2}\right\rceil^{+}\right)$.

Racemic ethyl trans-2-trifluoromethylcyclopropanecarboxylate (40): The solution of the ethyl<smiles>CCOC(=O)[C@@H]1C[C@H]1C(F)(F)F</smiles>
5,5,5-trifluoro-4-tosyloxivalerate $(9.4 \mathrm{~g}, 26 \mathrm{mmol})$ in anhydrous THF (20 mL) was added via syringe during 1 hour to vigorously stirred solution of potassium tert-butoxide $(14.9 \mathrm{~g}, 133 \mathrm{mmol})$ in anhydrous THF (100 mL) under $\mathrm{N}_{2}$-flow and the solution was stirred for 24 hours. The resulting mixture was concentrated under reduced pressure at ambient temperature and diluted with water $(100 \mathrm{~mL})$. Alkaline water solution was extracted with diethyl ether $(5 \times 50 \mathrm{~mL})$, combined extracts were dried over $\mathrm{MgSO}_{4}$, filtered and concentrated under reduced pressure, giving crude product as black tar. Molecular distillation of this crude product gives target ester as colorless liquid $(0.81 \mathrm{~g}, 4.4 \mathrm{mmol}, 17 \%)$. Water phase work-up gives no other products, but tar. ${ }^{1} \mathrm{H} \mathrm{NMR}\left(250 \mathrm{MHz}, \mathrm{CDCl}_{3}\right): \delta=1.18-1.41(\mathrm{~m}, 2 \mathrm{H}), 1.27(\mathrm{t}, J=7.1 \mathrm{~Hz}, 3 \mathrm{H}), 1.93-2.30$ (m, $2 \mathrm{H}), 4.08-4.29(\mathrm{q}, J=7.1 \mathrm{~Hz}, 2 \mathrm{H}) ;{ }^{13} \mathrm{C} \mathrm{NMR}\left(62.9 \mathrm{MHz}, \mathrm{CDCl}_{3}\right): \delta=10.18(-, \mathrm{q}, J=2.5 \mathrm{~Hz})$, $13.93(+), \quad 16.71(+, \quad \mathrm{q}, \quad J=2.9 \mathrm{~Hz}), \quad 21.85(+, \quad \mathrm{q}, \quad J=37.8 \mathrm{~Hz}), \quad 61.27(-), 124.78(-, \quad \mathrm{q}$, $J=266.7 \mathrm{~Hz}), 171.24(-)$.

Racemic (2-Trifluoromethyl-cyclopropyl) methanol(41): Racemic trans-2-trifluoromethyl$\mathrm{F}_{3} \mathrm{C}^{\prime \prime} \triangle \mathrm{OH}$ cyclopropanecarboxylic acid $(3.4 \mathrm{~g}, 18.6 \mathrm{mmol})$ was reduced with the 
lithium aluminum hydride in diethyl ether $(1.13 \mathrm{M}, 8.3 \mathrm{~mL}, 9.4 \mathrm{mmol})$ according to GP 8 , giving target alcohol as colorless liquid $(2.3 \mathrm{~g}, 16.4 \mathrm{mmol}, 88 \%)$. ${ }^{1} \mathrm{H} \mathrm{NMR}\left(250 \mathrm{MHz}, \mathrm{CDCl}_{3}\right): 0.70$ $0.83(\mathrm{~m}, 1 \mathrm{H}), 0.94-1.06(\mathrm{~m}, 1 \mathrm{H}), 1.39-1.58(\mathrm{~m}, 2 \mathrm{H}), 1.95(\mathrm{bd}, J=19.7 \mathrm{~Hz}, 1 \mathrm{H}), 3.40-$ $3.70(\mathrm{~m}, \quad 2 \mathrm{H}) ; \quad{ }^{13} \mathrm{C} \mathrm{NMR}\left(62.9 \mathrm{MHz}, \mathrm{CDCl}_{3}\right): \quad 6.28, \quad 17.44, \quad 17.86(\mathrm{q}, J=37.0 \mathrm{~Hz}), \quad 63.68$, 126.09 (q, $J=270.3 \mathrm{~Hz})$.

Racemic trans-(2-Trifluoromethylcyclopropyl)methyl iodide (47): Racemic trans-(2$\mathrm{F}_{3} \mathrm{C}^{\prime \prime}$ trifluoromethylcyclopropyl $)$ methanol $(1.7 \mathrm{~g}, 12 \mathrm{mmol})$ was iodinated $(1.5 \mathrm{~g}, 22 \mathrm{mmol})$ and solid iodine $(6.0 \mathrm{~g}, 24 \mathrm{mmol})$ in diethyl ether/acetonitrile mixture $(36+24 \mathrm{ml})$, giving the target iodide as slightly yellowish liquid $(2.7 \mathrm{~g}, 11 \mathrm{mmol}, 90 \%)$. TLC: $R_{\mathrm{f}}=0.36$, pentane; ${ }^{1} \mathrm{H}$ NMR $\left(250 \mathrm{MHz}, \mathrm{CDCl}_{3}\right): 0.75-0.87(\mathrm{~m}$, $1 \mathrm{H}), 1.21-1.33(\mathrm{~m}, 1 \mathrm{H}), 1.41-1.57(\mathrm{~m}, 1 \mathrm{H}), 1.63-1.79(\mathrm{~m}, 1 \mathrm{H}), 3.03-3.21(\mathrm{~m}, 2 \mathrm{H}) ;{ }^{13} \mathrm{C} \mathrm{NMR}$ (62.9 MHz, $\left.\mathrm{CDCl}_{3}\right): \quad 6.76, \quad 13.51(\mathrm{q}, \quad J=3.0 \mathrm{~Hz}), \quad 19.42(\mathrm{q}, J=2.7 \mathrm{~Hz}), \quad 25.06$, $130.00(\mathrm{q}, J=280.5 \mathrm{~Hz})$

(R)-Belokon' 3-(2-trifluoromethylcyclopropyl)alanine complex [(R)-B(tFmcpA)C, $\left.\left(2 R, 1^{\prime} S, 2^{\prime} R\right)-112\right]:(R)-\mathrm{BGC}(3.17 \mathrm{~g}, 6.4 \mathrm{mmol})$ was alkylated
thyliodide $47(1.67 \mathrm{~g}, 6.7 \mathrm{mmol})$ according to GP 10 using separation (silica gel, eluted with EtOAc), $\left(2 R, 1^{\prime} S, 2^{\prime} R\right)$ component $(1.73 \mathrm{~g}, 2.79 \mathrm{mmol}, 43.7 \%$ on (R)-BGC, d.e. $\geq 98 \%), \quad\left(2 R, 1^{\prime} R, 2^{\prime} S\right)$ component $(1.68 \mathrm{~g}, 2.71 \mathrm{mmol}, 42.4 \%$ on $(R)$-BGC, d.e. $\geq 98 \%)$ and mixed fractions $(0.17 \mathrm{~g}, 0.27 \mathrm{mmol}, 4.3 \%$ on $(R)$-BGC) as well as products of the anion oxidation $(0.12 \mathrm{~g})$. For $\left(2 R, 1^{\prime} S, 2^{\prime} R\right)$ component ${ }^{1} \mathrm{H}$ NMR $\left(250 \mathrm{MHz}, \mathrm{CDCl}_{3}\right): \delta=-0.15--$ 0.09 (m, $1 \mathrm{H}), 0.80-0.86$ (m, $1 \mathrm{H}), 0.90-0.98$ (m, $1 \mathrm{H}), 1.10-1.16(\mathrm{~m}, 1 \mathrm{H}), 1.76-1.84$ (m, $1 \mathrm{H})$, 2.04-2.13 (m, $1 \mathrm{H}), 2.13-2.21(\mathrm{~m}, 1 \mathrm{H}), 2.43-2.52(\mathrm{~m}, 1 \mathrm{H}), 2.57-2.71(\mathrm{~m}, 2 \mathrm{H}), 3.46(\mathrm{dd}$, $J=5.6 \mathrm{~Hz}, 11.1 \mathrm{~Hz}, 1 \mathrm{H}), 3.49-3.60(\mathrm{~m}, 3 \mathrm{H}), 3.92(\mathrm{dd}, J=3.5 \mathrm{~Hz}, 9.1 \mathrm{~Hz}, 1 \mathrm{H}), 4.42(\mathrm{~d}$, $J=12.7 \mathrm{~Hz}, 1 \mathrm{H}), 6.56-6.65(\mathrm{~m}, 2 \mathrm{H}), 6.86(\mathrm{~d}, J=7.6 \mathrm{~Hz}, 1 \mathrm{H}), 7.11(\mathrm{ddd}, J=1.8 \mathrm{~Hz}, 6.8 \mathrm{~Hz}$, $8.6 \mathrm{~Hz}, 1 \mathrm{H}), 7.17(\mathrm{t}, J=7.5 \mathrm{~Hz}, 1 \mathrm{H}), 7.25-7.29(\mathrm{~m}, 1 \mathrm{H}), 7.33(\mathrm{t}, J=7.7 \mathrm{~Hz}, 2 \mathrm{H}), 7.41-$ 
$7.46(\mathrm{~m}, 1 \mathrm{H}), 7.47-7.54(\mathrm{~m}, 2 \mathrm{H}), 8.05(\mathrm{~d}, J=7.1 \mathrm{~Hz}, 2 \mathrm{H}), 8.09(\mathrm{~d}, J=8.7 \mathrm{~Hz}, 1 \mathrm{H}) ;{ }^{13} \mathrm{C} \mathrm{NMR}$ $\left(125.7 \mathrm{MHz}, \mathrm{CDCl}_{3}\right): \delta=8.41,11.74,20.09$ (q, $J=36.9 \mathrm{~Hz}$ ), 23.93, 30.70, 38.41, 57.24, 63.17, $69.52,70.11,120.70,123.74,125.93$ (q, $J=270.3 \mathrm{~Hz}$ ), 126.23, 127.32, 127.35, 128.84, 128.89, $128.90,129.05,129.84,131.48,132.28,133.12,133.24,133.69,142.35,170.75,178.65,180.45$ MS-ESI: (positive) m/z (\%) $\left.\left.1882(100,3 \mathrm{M}+\mathrm{Na}\rceil^{+}\right), 1263(66,2 \mathrm{M}+\mathrm{Na}\rceil^{+}\right), 810(25,2 \mathrm{M}+\mathrm{Na}-$ $\left.\left.\mathrm{C}_{13} \mathrm{H}_{12} 7^{+}\right), 642\left(15, \mathrm{M}+\mathrm{Na}^{+}\right), 620(3, \mathrm{M}+\mathrm{H}\rceil^{+}\right),\left(\right.$negative) $\mathrm{m} / \mathrm{z}(\%) 618\left(100, \mathrm{M}-\mathrm{H}^{-}\right), 528(40$, $\left.\mathrm{M}-\mathrm{C}_{7} \mathrm{H}_{7}\right\rceil^{-}$).

(2R, 1'S,2'R)-3-(2-trifluoromethylcyclopropyl)alanine $\quad[(R) t F m c p A, \quad \boldsymbol{R}-96 \mathbf{c}]: \quad$ Compound

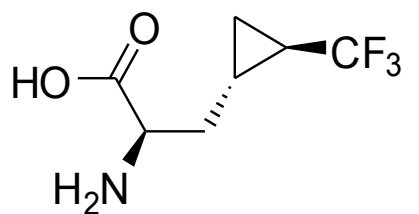

$\left(2 R, 1^{\prime} S, 2^{\prime} R\right)-112$ (980 $\left.\mathrm{mg}, 1.58 \mathrm{mmol}\right)$ was decomposed and the amino acid was separated and purified according to GP 11 to give pure target amino acid R-96 c (275 mg, $1.39 \mathrm{mmol}, 88 \%)$. $[\alpha]_{\mathrm{D}}{ }^{20}-20.0(\mathrm{c}=0.2 \mathrm{in}$

$\mathrm{MeOH}) ;{ }^{1} \mathrm{H}$ NMR $\left(250 \mathrm{MHz}, \mathrm{CD}_{3} \mathrm{OD}\right): \delta=0.75-0.87(\mathrm{~m}, 1 \mathrm{H}), 0.94-1.07(\mathrm{~m}, 1 \mathrm{H}), 1.31-$ $1.44(\mathrm{~m}, 1 \mathrm{H}), 1.47-1.67(\mathrm{~m}, 2 \mathrm{H}), 2.06-2.22(\mathrm{~m}, 1 \mathrm{H}), 3.54-3.63(\mathrm{~m}, 1 \mathrm{H}), 4.94(\mathrm{bs}, 3 \mathrm{H})$; ${ }^{13} \mathrm{C}$ NMR (62.9 MHz, $\left.\mathrm{CD}_{3} \mathrm{OD}\right): \delta=9.64,13.10,20.12(\mathrm{q}, J=36.9 \mathrm{~Hz}), 34.88,55.99,127.80(\mathrm{q}$, $J=269.6 \mathrm{~Hz}), 173.72$; MS-EI $\left.\left.\left.(70 \mathrm{eV}): \mathrm{m} / \mathrm{z}(\%) 152\left(100, \mathrm{M}-\mathrm{CO}_{2} \mathrm{H}\right\rceil^{+}\right), 74\left(75, \mathrm{C}_{2} \mathrm{H}_{4} \mathrm{NO}_{2}\right\rceil^{+}\right)\right)$; MS-ESI: (positive) m/z (\%) $\left.198(100, \mathrm{M}+\mathrm{H}\rceil^{+}\right)$, (negative) m/z (\%) $196\left(60, \mathrm{M}-\mathrm{H}^{-}\right)$.

(S)-Belokon' 3-(2-trifluoromethylcyclopropyl)alanine complex [(S)-B(tFmcpA)C,

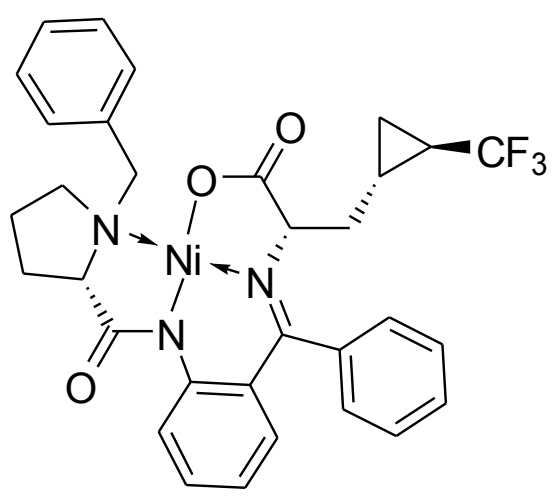
(2S,1'S,2'R)-113]: (S)-BGC $\quad(645 \mathrm{mg}, \quad 1.29 \mathrm{mmol}) \quad$ was alkylated with racemic (2-trifluoromethylcyclopropyl)methyl iodide 47 (340 mg, $1.36 \mathrm{mmol})$ according to GP 10 using $\mathrm{NaH}(60 \%$ in oil, $62 \mathrm{mg}, 1.55 \mathrm{mmol})$ in $\mathrm{DMF} / \mathrm{MeCN}$ mixture $(1+2 \mathrm{~mL})$ during $4 \mathrm{~h}$, giving after chromatographycal separation (silica gel, eluted with EtOAc), (2S,1'R,2'S) component $(393 \mathrm{mg}, \quad 634 \mu \mathrm{mol}, 49.1 \%$ on $(S)$-BGC, d.e. $\geq 98 \%), \quad\left(2 S, 1^{\prime} S, 2^{\prime} R\right) \quad$ component $\quad(364 \mathrm{mg}, \quad 587 \mu \mathrm{mol}$, $45.5 \%$ on (S)-BGC, d.e. $\geq 98 \%$ ) and mixed fractions (33 mg, $53 \mu \mathrm{mol}, 4.1 \%$ on $(S)$-BGC). For $\left(2 S, 1^{\prime} S, 2^{\prime} R\right)$ component ${ }^{1} \mathrm{H} \mathrm{NMR}\left(250 \mathrm{MHz}, \mathrm{CDCl}_{3}\right): \delta=0.43-0.61(\mathrm{~m}, 2 \mathrm{H}), 1.00-1.13(\mathrm{~m}$, $1 \mathrm{H}), 1.40-1.70$ (m, 2 H), 1.99-2.26 (m, 3 H), 2.40-2.78 (m, 2 H), 3.33-3.65 (m, 4 H), 3.99 (dd, $J=8.9 \mathrm{~Hz}, 3.3 \mathrm{~Hz}, 1 \mathrm{H}), 4.46(\mathrm{~d}, J=12.6 \mathrm{~Hz}, 1 \mathrm{H}), 6.58-6.71(\mathrm{~m}, 2 \mathrm{H}), 6.84(\mathrm{~d}, J=7.5 \mathrm{~Hz}, 1 \mathrm{H})$, 7.09-7.23 (m, $2 \mathrm{H}), 7.29-7.40(\mathrm{~m}, 3 \mathrm{H}), 7.41-7.63(\mathrm{~m}, 3 \mathrm{H}), 8.04-8.12(\mathrm{~m}, 3 \mathrm{H}) ;{ }^{13} \mathrm{C} \mathrm{NMR}$ $\left(62.9 \mathrm{MHz}, \mathrm{CDCl}_{3}\right): \delta=23.89$ (q, $\left.J=1.3 \mathrm{~Hz}\right), 30.67,38.77,57.24,60.33,63.21,69.68,70.14$, 
$77.29,120.74,125.58$ (q, $J=237.9 \mathrm{~Hz}), 126.24,127.28,128.35,128.54,128.86,128.88,128.89$, $129.25,129.90,131.45,131.93$, 132.09, 132.24, 133.14, 133.34, 133.62, 142.27, 156.25, 156.27, $156.32,170.47,178.68,180.43$.

(2S, 1'S,2'R)-3-(2-trifluoromethylcyclopropyl)alanine $\quad[(S) t F m c p A, \quad S-96$ c $]$ : $\quad$ Compound<smiles>N[C@@H](C[C@@H]1C[C@H]1C(F)(F)F)C(=O)O</smiles>

$\left(2 S, 1^{\prime} S, 2^{\prime} R\right)-113(210 \mathrm{mg}, 339 \mu \mathrm{mol})$ was decomposed and the amino acid was separated and purified according to GP 11 to give pure target amino acid c (61 mg, $311 \mu \mathrm{mol}, 92 \%)$.

\section{2. (Difluoromethylcyclopropy)lalanines}

Racemic monomethyl cyclopropane-trans-1,2-dicarboxylate (16) ${ }^{[121]}$ : The $\mathrm{LiOH} \times \mathrm{H}_{2} \mathrm{O}(4.2 \mathrm{~g}$,<smiles>COC(=O)[C@H]1CC1C(=O)O</smiles>
$100 \mathrm{mmol})$ solution in methanol $(100 \mathrm{~mL})$ was added dropwise for 1 hour to vigorously stirred solution of racemic dimethyl cyclopropanetrans-1,2-dicarboxylate 15 (15.8 g, $100 \mathrm{mmol})$ in THF (400 mL) under $\mathrm{N}_{2}$-flow and the resulting mixture was stirred for an additional $1 \mathrm{~h}$. Solvents were evaporated under reduced pressure at ambient temperature, the residue was diluted with water $(80 \mathrm{~mL})$ and washed with diethyl ether. Organic phases were discarded, water phase was acidified with concentrated aqueous $\mathrm{HCl}(37 \%, 10 \mathrm{~mL})$, saturated with solid $\mathrm{NaCl}$ and extracted with diethyl ether $(3 \times 50 \mathrm{~mL})$. Combined organic phases were dried over $\mathrm{MgSO}_{4}$, filtered and concentrated under reduced pressure, giving clear oil, solidifying when dried in vacuo overnight (12.1 g, $84 \mathrm{mmol}, 84 \%) .{ }^{1} \mathrm{H} \mathrm{NMR}\left(250 \mathrm{MHz}, \mathrm{CDCl}_{3}\right): \delta=1.42-1.57(\mathrm{~m}, 2 \mathrm{H}), 2.11-2.27(\mathrm{~m}, 2 \mathrm{H})$, $3.71(\mathrm{~s}, 3 \mathrm{H}), 9.76(\mathrm{bs}, 1 \mathrm{H}) ;{ }^{13} \mathrm{C} \mathrm{NMR}\left(62.9 \mathrm{MHz}, \mathrm{CDCl}_{3}\right): \delta=15.87,22.07,22.75,52.31$, $171.86,178.13$.

Racemic methyl trans-2-hydroxymethylcyclopropanecarboxylate $(\mathbf{1 8})^{[122]}$ : The borane - dimethyl

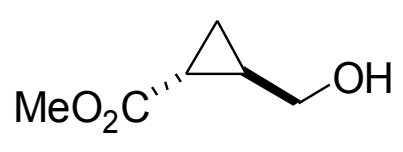
sulfide complex (10 M in $\left.\mathrm{Me}_{2} \mathrm{~S}, 10.8 \mathrm{~mL}, 108 \mathrm{mmol}\right)$ was added dropwise for $30 \mathrm{~min}$ to cold (ice/water bath) solution of the racemic monomethyl cyclopropane-trans-1,2-dicarboxylate $\mathbf{1 6}(12.9 \mathrm{~g}$,

$90 \mathrm{mmol})$ in THF $(40 \mathrm{~mL})$ and the resulting mixture was left to stir in melting bath overnight. The mixture was re-cooled (ice/water bath) and methanol $(5 \mathrm{~mL})$ was added dropwise under stirring. After $\mathrm{H}_{2}$ gas evolution ceased, the mixture was diluted with methanol $(100 \mathrm{~mL})$ and concentrated under reduced pressure, this dilution-concentration procedure was repeated 3 times, giving crude product as clear oil (11.9 g), which was purified with chromatography (silica gel, eluted with $\left.\mathrm{Et}_{2} \mathrm{O}\right)$ to give pure hydroxyester 18 as colorless clear oil (11.1 g, $\left.85 \mathrm{mmol}, 95 \%\right)$. TLC: $R_{\mathrm{f}}=0.26\left(\mathrm{Et}_{2} \mathrm{O}\right) ;{ }^{1} \mathrm{H} \mathrm{NMR}\left(250 \mathrm{MHz}, \mathrm{CDCl}_{3}\right): 1.06-1.29(\mathrm{~m}, 2 \mathrm{H}), 1.36-1.50(\mathrm{~m}, 2 \mathrm{H})$, 
1.99-2.25 (m, $2 \mathrm{H}), 3.65$ (s, $3 \mathrm{H}), 4.58$ (bs, $1 \mathrm{H}) ;{ }^{13} \mathrm{C} \mathrm{NMR}\left(62.9 \mathrm{MHz}, \mathrm{CDCl}_{3}\right)$ : 12.7, 18.1, 24.2, $51.8,64.3,174.5$.

Racemic methyl trans-2-formylcyclopropanecarboxylate $(\mathbf{2 0})^{[121]}$ : To a vigorously stirred solution

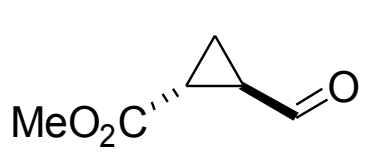
of oxalyl chloride $(5.23 \mathrm{~g}, 3.5 \mathrm{~mL}, 41.2 \mathrm{mmol})$ in anhydrous $\mathrm{CH}_{2} \mathrm{Cl}_{2}$

$(70 \mathrm{~mL})$ cooled to $-78^{\circ} \mathrm{C}$ (dry ice/acetone bath) under nitrogen flow, was added a solution of anhydrous DMSO (6.89 $\mathrm{g}, 6.3 \mathrm{~mL}, 88.2 \mathrm{mmol})$ in anhydrous $\mathrm{CH}_{2} \mathrm{Cl}_{2}(3 \mathrm{~mL})$ at such a rate that the temperature of the reaction mixture did not exceed $-70{ }^{\circ} \mathrm{C}$ (about $40 \mathrm{~min}$ ). After the mixture was stirred at $-70{ }^{\circ} \mathrm{C}$ for an additional $30 \mathrm{~min}$, a solution of the racemic methyl trans-2-hydroxymethylcyclopropanecarboxylate 18 (4.44 g, $34.1 \mathrm{mmol}$ ) was added dropwise under vigorous stirring keeping the temperature of the reaction mixture under $-70{ }^{\circ} \mathrm{C}$. The mixture was stirred at this temperature for an additional $1 \mathrm{~h}$ and anhydrous triethylamine $(17.2 \mathrm{~g}, 24 \mathrm{~mL}, 170 \mathrm{mmol})$ was gradually added at $-78^{\circ} \mathrm{C}$. After the addition was complete, the cooling bath was removed and the stirred mixture was allowed to reach room temperature. Then water $(20 \mathrm{~mL})$ was added and the mixture was acidified with aq. $12 \mathrm{M} \mathrm{HCl}(15 \mathrm{~mL})$ at $0{ }^{\circ} \mathrm{C}$ (ice/salt bath). The organic layer was separated and the aqueous phase was extracted with diethyl ether $(3 \times 20 \mathrm{~mL})$. Combined organic layers were washed with water $(20 \mathrm{~mL})$, brine $(2 \times 20 \mathrm{~mL})$, dried over $\mathrm{MgSO}_{4}$, filtered and concentrated under reduced pressure, giving the target aldehyde as colorless clear oil (4.19 g, $32.7 \mathrm{mmol}, 96 \%)$. ${ }^{1} \mathrm{H}$ NMR $\left(250 \mathrm{MHz}, \quad \mathrm{CDCl}_{3}\right): \quad 1.38-1.66(\mathrm{~m}, 2 \mathrm{H}), \quad 2.08-2.53(\mathrm{~m}, 2 \mathrm{H}), \quad 3.68(\mathrm{~s}, 3 \mathrm{H}), \quad 9.27(\mathrm{~d}, 4.2 \mathrm{~Hz})$; ${ }^{13} \mathrm{C}$ NMR (62.9 MHz, $\left.\mathrm{CDCl}_{3}\right): 14.8,21.9,30.5,52.2,171.5,198.1$.

Racemic methyl trans-2-difluoromethylcyclopropanecarboxylate (21): The reaction was provided $\mathrm{MeO}_{2} \mathrm{C}^{\prime \prime}{ }_{\mathrm{CHF}_{2}}$ in PTFE flask. Deoxo-Fluor ${ }^{\circledR} 14$ solution in toluene $(50 \% \mathrm{w} / \mathrm{w}, 26.4 \mathrm{~g}$, $59.7 \mathrm{mmol}$ )was added under $\mathrm{N}_{2}$-flow with stirring to the solution of racemic methyl trans-2-formyl-cyclopropanecarboxylate 20 (4.5 g, $35.1 \mathrm{mmol})$ in anhydrous $\mathrm{CH}_{2} \mathrm{Cl}_{2}(6 \mathrm{ml})$. Ethanol $(0.1 \mathrm{~mL})$ was added and the mixture was stirred for 48 hours at ambient temperature. Resulting solution was poured to vigorously stirred sat. aq. $\mathrm{NaHCO}_{3}(150 \mathrm{~mL})$, stirred till $\mathrm{CO}_{2}$ gas evolution ceased, organic phase was separated, water phase was extracted with $\mathrm{CH}_{2} \mathrm{Cl}_{2}(3 \times 50 \mathrm{~mL})$, combined organic phases were dried over $\mathrm{MgSO}_{4}$, filtered and concentrated under reduced pressure. The crude product was purified with the column chromatography (silica gel, eluted with pentane/diethyl ether 4:1) to give pure target difluoroester 21 as colorless liquid $(2.7 \mathrm{~g}, 18.0 \mathrm{mmol}, 51 \%)$. ${ }^{1} \mathrm{H} \mathrm{NMR}\left(250 \mathrm{MHz}, \mathrm{CDCl}_{3}\right)$ : $\delta=1.09-1.21(\mathrm{~m}, 1 \mathrm{H}), 1.21-1.33(\mathrm{~m}, 1 \mathrm{H}), 1.82-2.00(\mathrm{~m}, 2 \mathrm{H}), 3.69(\mathrm{~s}, 3 \mathrm{H}), 5.76(\mathrm{td}$, $J=57.3 \mathrm{~Hz}, 3.4 \mathrm{~Hz}, 1 \mathrm{H}) ;{ }^{13} \mathrm{C} \mathrm{NMR}\left(62.9 \mathrm{MHz}, \mathrm{CDCl}_{3}\right): \delta=9.7(-, \mathrm{t}, J=4.3 \mathrm{~Hz}), 15.5(+, \mathrm{t}$, 
$J=4.4 \mathrm{~Hz}), \quad 22.8(+, \quad \mathrm{t}, \quad J=27.0 \mathrm{~Hz}), \quad 51.9(+), \quad 114.6(+, \quad \mathrm{t}, \quad J=239.0 \mathrm{~Hz}), \quad 172.5(-) ; \quad$ MS$\left.\left.\left.\mathrm{EI}(70 \mathrm{eV}): \mathrm{m} / \mathrm{z} \quad(\%) \quad 150.1(10 \%, \mathrm{M}\rceil^{+}\right), \quad 149.1(9 \%, \mathrm{M}-\mathrm{H}\rceil^{+}\right), \quad 119.1(100 \%, \mathrm{M}-\mathrm{MeO}\rceil^{+}\right)$, $\left.\left.99.1\left(29 \%, \mathrm{C}_{5} \mathrm{H}_{4} \mathrm{FO}\right\rceil^{+}\right), 91.1\left(30 \%, \mathrm{M}-\mathrm{CO}_{2} \mathrm{Me}^{+}\right), 59.0\left(28 \%, \mathrm{C}_{3} \mathrm{H}_{4} \mathrm{~F}\right\rceil^{+}\right)$, .

Racemic trans-(2-difluoromethylcyclopropyl) methanol (42): Racemic methyl

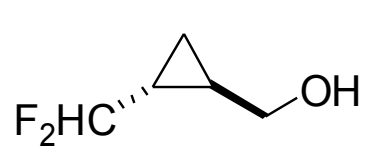
trans-2-difluoromethylcyclopropanecarboxylate 21 (2.52 g, $16.8 \mathrm{mmol})$ was reduced with the lithium aluminum hydride in diethyl ether $(1.13 \mathrm{M}$, $7.4 \mathrm{~mL}, 8.4 \mathrm{mmol}$ ) according to GP 9. The crude product was purified with the column chromatography (silica gel, eluted with pentane/diethyl ether 1:1) to give the target difluoroalcohol 42 as colorless liquid (1.68 g, $13.8 \mathrm{mmol}, 82 \%)$. TLC: $R_{\mathrm{f}}=0.17$ (pentane/ $\left.\mathrm{Et}_{2} \mathrm{O}=4: 1\right)$; ${ }^{1} \mathrm{H}$ NMR $\left(600 \mathrm{MHz}, \mathrm{CDCl}_{3}\right): \delta=0.58-0.63(\mathrm{~m}, 1 \mathrm{H}), 0.76-0.80(\mathrm{~m}, 1 \mathrm{H}), 1.15-$ $1.24(\mathrm{~m}, 1 \mathrm{H}), 1.26-1.32(\mathrm{~m}, 1 \mathrm{H}), 2.57(\mathrm{bs}, 1 \mathrm{H}), 3.40-3.53(\mathrm{~m}, 2 \mathrm{H}), 5.56(\mathrm{td}, J=57.4 \mathrm{~Hz}$, $4.5 \mathrm{~Hz}, 1 \mathrm{H}) ;{ }^{13} \mathrm{C} \mathrm{NMR}\left(125.7 \mathrm{MHz}, \mathrm{CDCl}_{3}\right): \delta=5.48(-, \mathrm{t}, J=4.5 \mathrm{~Hz}), 16.58(+, \mathrm{t}, J=4.0 \mathrm{~Hz})$, $18.59(+, \mathrm{t}, J=27.1 \mathrm{~Hz}), 64.42(-), 116.78(+, \mathrm{t}, J=237.5 \mathrm{~Hz})$.

Racemic trans-(2-difluoromethylcyclopropyl)methyl iodide (46): Racemic trans-(2-

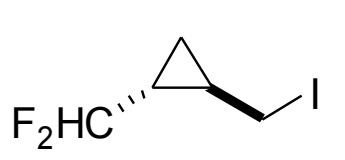

difluoromethylcyclopropyl) methanol 42 (1.68 g, $13.8 \mathrm{mmol})$ was iodinated according to GP 9 with triphenylphosphine $(6.26 \mathrm{~g}, 23.9 \mathrm{mmol})$, imidazole $(1.71 \mathrm{~g}, 25.1 \mathrm{mmol})$ and solid iodine $(6.75 \mathrm{~g}, 26.5 \mathrm{mmol})$ in diethyl ether/acetonitrile mixture $(41+27 \mathrm{ml})$, giving the target iodide as slightly yellowish liquid $(2.81 \mathrm{~g}, 12.1 \mathrm{mmol}, 88 \%)$. TLC: $R_{\mathrm{f}}=0.17$, pentane; ${ }^{1} \mathrm{H} \mathrm{NMR}\left(300 \mathrm{MHz}, \mathrm{CDCl}_{3}\right): \delta=0.63-$ $0.74(\mathrm{~m}, 1 \mathrm{H}), 1.06-1.16$ (m, $1 \mathrm{H}), 1.16-1.33$ (m, $1 \mathrm{H}), 1.48-1.61$ (m, $1 \mathrm{H}), 3.04-3.17$ (m, $2 \mathrm{H})$, $5.63(\mathrm{td}, J=57.4 \mathrm{~Hz}, 3.9 \mathrm{~Hz}, 1 \mathrm{H}) ;{ }^{13} \mathrm{C} \mathrm{NMR}\left(125.7 \mathrm{MHz}, \mathrm{CDCl}_{3}\right): \delta=8.60(-), 12.95(-, \mathrm{t}$, $J=4.5 \mathrm{~Hz}), \quad 18.85(+, \mathrm{t}, J=4.6 \mathrm{~Hz}), 25.73(+, \mathrm{t}, J=26.7 \mathrm{~Hz}), 115.70(+, \mathrm{t}, J=238.4 \mathrm{~Hz})$; MS-EI $\left.(70 \mathrm{eV}): \mathrm{m} / \mathrm{z}(\%) \quad 105,1(100 \%, \mathrm{M}-\mathrm{I}\rceil^{+}\right), \quad 85.1\left(20 \%, \mathrm{C}_{5} \mathrm{H}_{6} \mathrm{~F}^{+}\right), \quad 77.0\left(30 \%, \mathrm{C}_{4} \mathrm{H}_{10} \mathrm{~F}^{+}\right)$, $\left.\left.59.1\left(95 \%, \mathrm{C}_{3} \mathrm{H}_{4} \mathrm{~F}\right\rceil^{+}\right), 41.2\left(31 \%, \mathrm{C}_{3} \mathrm{H}_{5}\right\rceil^{+}\right)$.

(R)-Belokon' 3-(2-difluoromethylcyclopropyl)alanine complex $\quad[(R)$-B(dFmcpA)C,

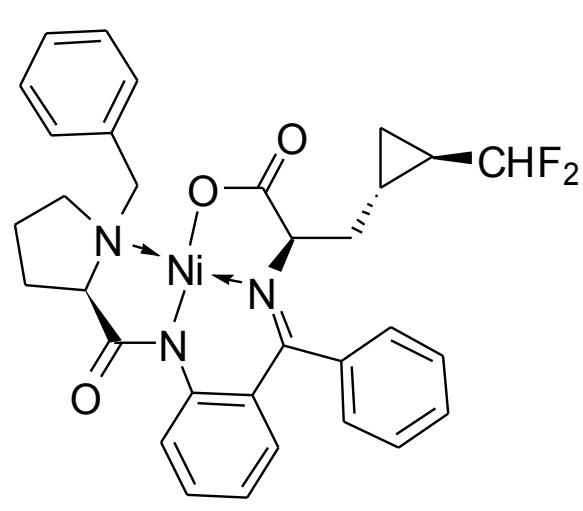
(2R,1'S,2'R)-114]: $(R)$-BGC $\quad(2.00 \mathrm{~g}, \quad 4.02 \mathrm{mmol}) \quad$ was alkylated with racemic trans-(2-difluoromethylcyclopropyl)methyl iodide 46 (980 $\mathrm{mg}, 4.22 \mathrm{mmol})$ according to GP 10 using $\mathrm{NaH}$ (60\% in oil, $193 \mathrm{mg}, 4.8 \mathrm{mmol})$ in $\mathrm{DMF} /$ $\mathrm{MeCN}$ mixture $(2+4 \mathrm{~mL})$ during $1 \mathrm{~h}$, giving $\left(2 R, 1^{\prime} S, 2^{\prime} R\right)$ component $(1.14 \mathrm{~g}, 1.90 \mathrm{mmol}, 47.3 \%$ on $(R)$-BGC, d.e. $\geq 98 \%), \quad\left(2 R, 1^{\prime} R, 2^{\prime} S\right)$ component $(1.10 \mathrm{~g}, 1.82 \mathrm{mmol}$, $45.4 \%$ on $(R)$-BGC, d.e. $\geq 98 \%$ ) and mixed fractions 
$\left(0.107 \mathrm{~g}, \quad 0.18 \mathrm{mmol}, 4.4 \%\right.$ on $(R)$-BGC). For $\left(2 R, 1^{\prime} S, 2^{\prime} R\right)$ component $[\alpha]_{\mathrm{D}}{ }^{20}=-2830.0^{\circ}$ $\left(\mathrm{c}=0.2, \mathrm{CHCl}_{3}\right) ;{ }^{1} \mathrm{H}$ NMR $\left(600 \mathrm{MHz}, \mathrm{CDCl}_{3}\right): \delta=-0.3--0.2(\mathrm{~m}, 1 \mathrm{H}), 0.6-0.7(\mathrm{~m}, 1 \mathrm{H}), 0.9-1.0$ (m, $1 \mathrm{H}), 1.0-1.1(\mathrm{~m}, 1 \mathrm{H}), 1.4-1.5(\mathrm{~m}, 1 \mathrm{H}), 2.0-2.1(\mathrm{~m}, 1 \mathrm{H}), 2.1-2.2(\mathrm{~m}, 1 \mathrm{H}), 2.4-2.5(\mathrm{~m}$, $1 \mathrm{H}), 2.55-2.7$ (m, $2 \mathrm{H}), 3.4-3.5(\mathrm{~m}, 1 \mathrm{H}), 3.5-3.6(\mathrm{~m}, 2 \mathrm{H}), 3.55$ (d, $J=12.7 \mathrm{~Hz}, 1 \mathrm{H}), 3.90$ (dd, $J=3.5 \mathrm{~Hz}, 9.6 \mathrm{~Hz}, 1 \mathrm{H}), 4.43(\mathrm{~d}, J=12.7 \mathrm{~Hz}, 1 \mathrm{H}), 5.51(\mathrm{td}, J=57.4 \mathrm{~Hz}, 4.4 \mathrm{~Hz}, 1 \mathrm{H}), 6.55-$ $6.65(\mathrm{~m}, 2 \mathrm{H}), 6.87(\mathrm{~d}, J=7.2 \mathrm{~Hz}, 1 \mathrm{H}), 7.07-7.12(\mathrm{~m}, 1 \mathrm{H}), 7.12-7.20$ (m, $1 \mathrm{H}), 7.22-7.28$ (m, $1 \mathrm{H}), 7.28-7.36(\mathrm{~m}, 2 \mathrm{H}), 7.38-7.44(\mathrm{~m}, 1 \mathrm{H}), 7.46-7.54(\mathrm{~m}, 2 \mathrm{H}), 8.00-8.50(\mathrm{~m}, 3 \mathrm{H}) ;{ }^{13} \mathrm{C} \mathrm{NMR}$ $\left(125.7 \mathrm{MHz}, \mathrm{CDCl}_{3}\right): \delta=7.23(-), 11.01(+, \mathrm{t}, J=4.5 \mathrm{~Hz}), 20.94(+, \mathrm{t}, J=26.9 \mathrm{~Hz}), 23.95(-)$, $30.66(-), 39.07(-), 57.21(-), 63.10(-), 69.84(+), 70.08(+), 116.73(+, \mathrm{t}, J=238.0 \mathrm{~Hz})$, $120.66(+), 123.70(+), 126.23(-), 127.31(+), 127.35(+), 128.11(+), 128.80(+), 128.85(+)$, $128.92(+) 128.97(+), 129.77(+), 129.81(+), 131.46(+), 132.17(+), 133.07(+), 133.23(-)$, $133.61(-), \quad 142.24(-), \quad 170.45(-), 178.79(-), \quad 180.39(-)$; MS-ESI: (positive) $\mathrm{m} / \mathrm{z}$ (\%) $\left.\left.\left.624.2(100 \%, \quad \mathrm{M}+\mathrm{Na}\rceil^{+}\right), \quad 1225.0(90 \%, \quad 2 \mathrm{M}+\mathrm{Na}\rceil^{+}\right), \quad 1827.4(55 \%, \quad 3 \mathrm{M}+\mathrm{Na}\rceil^{+}\right), \quad 602.2(16 \%$, $\left.\mathrm{M}+\mathrm{H}\rceil^{+}\right)$.

$\left(2 R, 1^{\prime} S, 2^{\prime} R\right)-3-(2-d i f l u o r o m e t h y l c y c l o p r o p y l) a l a n i n e \quad(\boldsymbol{R}-96 \mathbf{b}): \quad\left(2 R, 1^{\prime} S, 2^{\prime} R\right)-\mathrm{B}(\mathrm{dFmcpA}) \mathrm{C}$

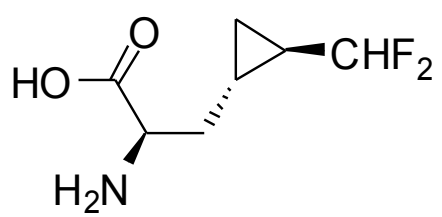
(730 $\mathrm{mg}, 1.21 \mathrm{mmol}$ ) was decomposed and the amino acid was separated and purified according to GP 11 to give pure target amino acid $(210 \mathrm{mg}, 1.17 \mathrm{mmol}, 97 \%) .[\alpha]_{\mathrm{D}}^{20}=+30.4^{\circ} \quad\left(\mathrm{c}=0.5, \mathrm{H}_{2} \mathrm{O}\right)$; ${ }^{1} \mathrm{H}$ NMR $\left(600 \mathrm{MHz}, \mathrm{D}_{2} \mathrm{O}\right): \delta=1.21-1.30(\mathrm{~m}, 1 \mathrm{H}), 1.63-1.75(\mathrm{~m}, 1 \mathrm{H}), 1.98-2.08(\mathrm{~m}, 1 \mathrm{H}), 3.81$ (t, $J=6.0 \mathrm{~Hz}, 1 \mathrm{H}), 5.67$ (td, $J=57.1 \mathrm{~Hz}, 4.8 \mathrm{~Hz}, 1 \mathrm{H})$; MS-ESI: (positive) m/z (\%) 180 (100, $\left.\mathrm{M}+\mathrm{H}^{+}\right)$, (negative) $\left.\left.\mathrm{m} / \mathrm{z}(\%) 357(100,2 \mathrm{M}-\mathrm{H}\rceil^{-}\right), 178(55, \mathrm{M}-\mathrm{H}\rceil^{-}\right)$.

(S)-Belokon' 3-(2-difluoromethylcyclopropyl)alanine complex [(S)-B(dFmcpA)C,

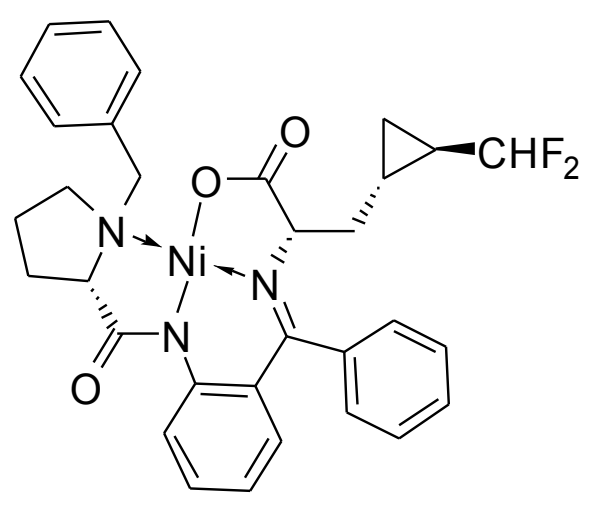
(2S,1'S,2'R)-115]: (S)-BGC $\quad(756 \mathrm{mg}, \quad 1.52 \mathrm{mmol}) \quad$ was alkylated with racemic trans-(2-difluoromethylcyclopropyl)methyl iodide 46 (370 $\mathrm{mg}, 1.60 \mathrm{mmol})$ according to GP 10 using $\mathrm{NaH}(60 \%$ in oil, $73 \mathrm{mg}, 1.82 \mathrm{mmol})$ in $\mathrm{DMF} /$ $\mathrm{MeCN}$ mixture $(1+2 \mathrm{~mL})$ during 1 hour, giving $\left(2 S, 1^{\prime} R, 2 ' S\right)$ component (442 mg, $734 \mu \mathrm{mol}, 48,3 \%$ on $(S)$-BGC, d.e. $\geq 98 \%), \quad\left(2 S, 1^{\prime} S, 2^{\prime} R\right) \quad$ component $\quad(409 \mathrm{mg}$, $679 \mu \mathrm{mol}, 44.7 \%$ on $(S)$-BGC, d.e. $\geq 98 \%)$ and mixed fractions $\left(40 \mathrm{mg}, 67 \mu \mathrm{mol}, 4.4 \%\right.$ on $(S)$-BGC). For $\left(2 S, 1^{\prime} S, 2^{\prime} R\right)$ component $[\alpha]_{\mathrm{D}}{ }^{20}=+2200^{\circ}$ $\left(\mathrm{c}=0.2, \mathrm{CHCl}_{3}\right) ;{ }^{1} \mathrm{H} \mathrm{NMR}\left(600 \mathrm{MHz}, \mathrm{CDCl}_{3}\right): \delta=0.25-0.37(\mathrm{~m}, 1 \mathrm{H}), 0.37-0.41(\mathrm{~m}, 1 \mathrm{H}), 0.85-$ 0.95 (m, 1 H), 1.30-1.45 (m, 2 H), 2.00-2.10 (m, 1 H), 2.10-2.19 (m, 1 H), 2.19-2.23 (m, 1 H), 
2.43-2.53 (m, $1 \mathrm{H}), \quad 2.60-2.70(\mathrm{~m}, 1-\mathrm{H}), 3.40-3.47(\mathrm{~m}, 1-\mathrm{H}), 3.47-3.6(\mathrm{~m}, 2 \mathrm{H}), 3.53(\mathrm{~d}$, $J=12.7 \mathrm{~Hz}, 1 \mathrm{H}), 3.90-3.97(\mathrm{~m}, 1 \mathrm{H}), 4.41(\mathrm{~d}, J=12.7 \mathrm{~Hz}, 1 \mathrm{H}), 5.41$ (td, $J=57.2 \mathrm{~Hz}, 4.1 \mathrm{~Hz}$, $1 \mathrm{H}), 6.55-6.60(\mathrm{~m}, 1 \mathrm{H}), 6.60-6.65(\mathrm{~m}, 1 \mathrm{H}), 6.78-6.83(\mathrm{~m}, 1 \mathrm{H}), 7.08-7.12(\mathrm{~m}, 1 \mathrm{H}), 7.12-$ 7.17 (m, $1 \mathrm{H}), 7.25-7.38$ (m, $3 \mathrm{H}), 7.39-7.46$ (m, $1 \mathrm{H})$, 7.46-7.57 (m, $2 \mathrm{H}), 8.00-8.10$ (m, $3 \mathrm{H})$; ${ }^{13} \mathrm{C}$ NMR (125.7 MHz, $\left.\mathrm{CDCl}_{3}\right): \delta=8.38(-, \mathrm{t}, J=4.2 \mathrm{~Hz}), 11.38(+, \mathrm{dd}, J=4 \mathrm{~Hz}, 5 \mathrm{~Hz}), 20.10(+$, dd, $26.1 \mathrm{~Hz}, 28.0 \mathrm{~Hz}), 23.85(-), 30.67(-), 39.18(-), 57.19(-), 63.16(-), 69.96(+), 70.15(+)$, $116.44(+, \mathrm{t}, J=32.0 \mathrm{~Hz}), 120.66(+), 123.66(+), 126.25(-), 127.33(+), 127.44(+), 128.13(+)$, $128.81(+), 128.85(+), 129.04(+), 129.12(+), 129.83(+), 131.29(+), 131.45(+), 132.15(+)$, 133,11(+), $133.28(-), 133.62(-), 142.29(-), 170.33(-), 178.76(-), 180.36(-)$; MS-ESI: (positive) $\left.\left.\left.\mathrm{m} / \mathrm{z} \quad(\%) \quad 1828(55 \%, 3 \mathrm{M}+\mathrm{Na}\rceil^{+}\right), \quad 1225(100 \%, 2 \mathrm{M}+\mathrm{Na}\rceil^{+}\right), \quad 624(70 \%, \mathrm{M}+\mathrm{Na}\rceil^{+}\right)$, $\left.602(14 \%, \mathrm{M}+\mathrm{H}\rceil^{+}\right),\left(\right.$negative) $\left.\mathrm{m} / \mathrm{z}(\%) 600(100 \%, \mathrm{M}-\mathrm{H}\rceil^{-}\right)$.

(2S, 1'S, 2'R)-3-(2-difluoromethylcyclopropyl)alanine

(S-96 b): $\quad\left(2 S, 1^{\prime} S, 2^{\prime} R\right)-\mathrm{B}(\mathrm{dFmcpA}) \mathrm{C}$ (180 mg, $299 \mu \mathrm{mol})$ was decomposed and the amino acid was

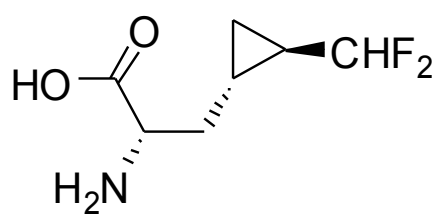
separated and purified according to GP 11 to give pure target amino acid $(50 \mathrm{mg}, 278 \mu \mathrm{mol}, 93 \%) .[\alpha]_{\mathrm{D}}^{20}=-16.0^{\circ}\left(\mathrm{c}=0.3\right.$ in $\left.\mathrm{H}_{2} \mathrm{O}\right)$;

${ }^{1} \mathrm{H}$ NMR $\left(600 \mathrm{MHz}, \mathrm{D}_{2} \mathrm{O}\right): \delta=0.65-0.75(\mathrm{~m}, 1 \mathrm{H}), 0.90-1.00(\mathrm{~m}$, $1 \mathrm{H}), \quad 1.10-1.20(\mathrm{~m}, 1 \mathrm{H}), \quad 1.30-1.41(\mathrm{~m}, 1 \mathrm{H}), 1.80-1.88(\mathrm{~m}, 1 \mathrm{H}), 2.01-2.08(\mathrm{~m}, 1 \mathrm{H})$, $3.87(\mathrm{dd}, J=6.6 \mathrm{~Hz}, 5.4 \mathrm{~Hz}, 1 \mathrm{H}), 4.72(\mathrm{bs}, 3 \mathrm{H}), 5.76$ (td, $J=57.2 \mathrm{~Hz}, 4.8 \mathrm{~Hz}, 1 \mathrm{H}) ;{ }^{13} \mathrm{C} \mathrm{NMR}$ $\left(125.7 \mathrm{MHz}, \mathrm{D}_{2} \mathrm{O}\right): \delta=6.93(-, \mathrm{dd}, J=5.6 \mathrm{~Hz}, 3.3 \mathrm{~Hz}), 10.10(+, \mathrm{dd}, J=5.9 \mathrm{~Hz}, 3.5 \mathrm{~Hz})$, $19.92(+, \mathrm{t}, J=27.1 \mathrm{~Hz}), 117.99(+, \mathrm{t}, J=235.4 \mathrm{~Hz}), 174.20(-)$; MS-ESI: (positive) m/z (\%) $\left.180.0(100 \%, \mathrm{M}+\mathrm{H}\rceil^{+}\right)$.

\subsection{Monoluoromethylcyclopropylalanines}

Racemic methyl trans-2-monofluoromethylcyclopropanecarboxylate (19): The reaction was $\mathrm{MeO}_{2} \mathrm{C}^{\prime \prime}{ }_{\mathrm{CH}_{2} \mathrm{~F}}$ provided in PTFE flask. Deoxo-Fluor ${ }^{\circledR}$ solution in toluene $(50 \% \mathrm{w} / \mathrm{w}$, $24.3 \mathrm{~g}, 55.0 \mathrm{mmol}$ ) was added under $\mathrm{N}_{2}$-flow with stirring to the solution of racemic methyl trans-2-hydroximethyl-cyclopropanecarboxylate $18(6.5 \mathrm{~g}, 50.0 \mathrm{mmol})$ in anhydrous $\mathrm{CH}_{2} \mathrm{Cl}_{2}(5 \mathrm{ml})$ and the mixture was stirred overnight at ambient temperature. Resulting solution was poured to vigorously stirred sat. aq. $\mathrm{NaHCO}_{3}$ $(150 \mathrm{~mL})$, stirred till $\mathrm{CO}_{2}$ gas evolution ceased, organic phase was separated, water phase was extracted with $\mathrm{CH}_{2} \mathrm{Cl}_{2}(3 \times 50 \mathrm{~mL})$, combined organic phases were dried over $\mathrm{MgSO}_{4}$, filtered and concentrated under reduced pressure. The crude product was purified with the column chromatography (silica gel, eluted with pentane/diethyl ether 4:1) to give pure target 
monofluoroester as colorless liquid $(3.1 \mathrm{~g}, 23.6 \mathrm{mmol}, 47 \%)$. ${ }^{1} \mathrm{H} \mathrm{NMR}\left(250 \mathrm{MHz}, \mathrm{CDCl}_{3}\right)$ : $\delta=0.80-0.98(\mathrm{~m}, 1 \mathrm{H}), 1.15-1.37(\mathrm{~m}, 1 \mathrm{H}), 1.60-1.72(\mathrm{~m}, 1 \mathrm{H}), 1.73-1.92(\mathrm{~m}, 1 \mathrm{H}), 3.67(\mathrm{~s}$, $3 \mathrm{H}), 4.18$ (ddd, $J=7.2 \mathrm{~Hz}, 9.8 \mathrm{~Hz}, 48.2 \mathrm{~Hz}, 1 \mathrm{H}), 4.40$ (ddd, $J=6.0 \mathrm{~Hz}, 9.8 \mathrm{~Hz}, 48.2 \mathrm{~Hz}, 1 \mathrm{H}$ ); ${ }^{13} \mathrm{C}$ NMR $\left(62.9 \mathrm{MHz}, \mathrm{CDCl}_{3}\right): \delta=12.1(-, \mathrm{d}, J=7.0 \mathrm{~Hz}), 18.0(+, \mathrm{d}, J=5.8 \mathrm{~Hz}), 21.3(+, \mathrm{d}$, $J=24.7 \mathrm{~Hz}), 51.8(+), 84.7(-, \mathrm{d}, J=168.7 \mathrm{~Hz}), 173.46(-, \mathrm{d}, J=1.7 \mathrm{~Hz}) ; \mathrm{MS}-\mathrm{EI}(70 \mathrm{eV}): \mathrm{m} / \mathrm{z}$ (\%) $\left.\left.\left.132.1\left(25 \%, \mathrm{M}^{+}\right), 131.1(20 \%, \mathrm{M}-\mathrm{H}\rceil^{+}\right), 101.0(100 \%, \mathrm{M}-\mathrm{MeO}\rceil^{+}\right), 71.0\left(30 \%, \mathrm{C}_{4} \mathrm{H}_{4} \mathrm{~F}\right\rceil^{+}\right)$, $\left.47.1\left(80 \%, \mathrm{C}_{2} \mathrm{H}_{4} \mathrm{~F}\right\rceil^{+}\right)$.

Racemic trans-(2-monofluoromethylcyclopropyl) methanol (43): Racemic methyl $\mathrm{FH}_{2} \mathrm{C}^{\prime \prime} \begin{aligned} & \text { trans-2-monofluoromethylcyclopropanecarboxylate } \\ & 16.7 \mathrm{mmol}) \text { was reduced with the lithium aluminum hydride in diethyl } \\ & \text { ether }(1.13 \mathrm{M}, 7.4 \mathrm{~mL}, 8.4 \mathrm{mmol}) \text { according to GP } 8 \text {. The crude product }\end{aligned}$ was purified with the column chromatography (silica gel, eluted with pentane/diethyl ether 1:1) to give the target monofluoroalcohol as colorless liquid $(1.33 \mathrm{~g}, 12,8 \mathrm{mmol}, 76 \%)$. ${ }^{1} \mathrm{H} \mathrm{NMR}$ (600 MHz, $\left.\mathrm{CDCl}_{3}\right)$ : 0.47-0.55 (m, $\left.2 \mathrm{H}\right), 1.00-1.11$ (m, $\left.2 \mathrm{H}\right), 2.64-2.74$ (bs, $\left.1 \mathrm{H}\right), 3.33-3.49$ (m, $2 \mathrm{H}), 4.07-4.31(\mathrm{~m}, 2 \mathrm{H}) ;{ }^{13} \mathrm{C} \mathrm{NMR}\left(125.7 \mathrm{MHz}, \mathrm{CDCl}_{3}\right): 7.59$ (-, d, $\left.J=7.3 \mathrm{~Hz}\right), 16.49(+, \mathrm{d}$, $J=25.1 \mathrm{~Hz}), 19.21(+, \mathrm{d}, J=5.7 \mathrm{~Hz}), 65.31(-, \mathrm{d}, J=1.3 \mathrm{~Hz}), 86.90(-, \mathrm{d}, J=165.8 \mathrm{~Hz})$.

Racemic trans-(2-monofluoromethylcyclopropyl)methyl iodide (45): Racemic trans-(2$\mathrm{FH}_{2} \mathrm{C}^{\prime \prime} \triangle \mathrm{l}$ monofluoromethylcyclopropyl) methanol 43 (1.33 g, $\left.12.8 \mathrm{mmol}\right)$ was imidazole $(1.58 \mathrm{~g}, 23.3 \mathrm{mmol})$ and solid iodine $(6.25 \mathrm{~g}, 24.6 \mathrm{mmol})$ in diethyl ether/acetonitrile mixture $(38+25 \mathrm{ml})$, giving the target iodide as light yellow liquid $(2.50 \mathrm{~g}, 17.7 \mathrm{mmol}, 91 \%)$. TLC: $R_{\mathrm{f}}=0.11$, pentane; ${ }^{1} \mathrm{H}$ NMR $\left(300 \mathrm{MHz}, \mathrm{CDCl}_{3}\right)$ : 0.59-0.68 (m, $\left.1 \mathrm{H}\right), 0.82-0.91(\mathrm{~m}, 1 \mathrm{H})$, 1.11-1.25 (m, $1 \mathrm{H}), 1.26-1.38$ (m, $1 \mathrm{H}), 3.13$ (d, $J=7.6 \mathrm{~Hz}, 2 \mathrm{H}), 4.22(\mathrm{dd}, J=7.0 \mathrm{~Hz}, 48.4 \mathrm{~Hz}$, $2 \mathrm{H}) ;{ }^{13} \mathrm{C}$ NMR $\left(125.7 \mathrm{MHz}, \mathrm{CDCl}_{3}\right): 10.54(-, \mathrm{d}, J=0.9 \mathrm{~Hz}) 15.40(-, \mathrm{d}, J=7.0 \mathrm{~Hz}), 21.38(+$, d, $J=6.6 \mathrm{~Hz}), 24.06(+, \mathrm{d}, J=25.2 \mathrm{~Hz}), 85.96(-, \mathrm{d}, J=167.6 \mathrm{~Hz}) ;$ MS-EI $(70 \mathrm{eV}): \mathrm{m} / \mathrm{z}(\%)$ $\left.\left.41.2\left(100 \%, \mathrm{C}_{3} \mathrm{H}_{5}\right\rceil^{+}\right), 87.1(38 \%, \mathrm{M}-\mathrm{I}\rceil^{+}\right), 67.1\left(36 \%, \mathrm{C}_{5} \mathrm{H}_{7} 7^{+}\right)$.

(R)-Belokon' 3-(2-monofluoromethylcyclopropyl)alanine complex $\quad[(R)-B(m F m c p A) C$,

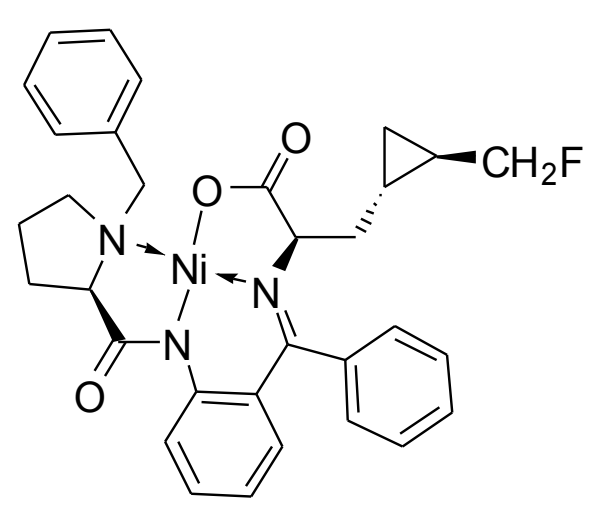
(2R,1'S,2'R)-116]: (R)-BGC $\boldsymbol{R}-\mathbf{1 3}(2.40 \mathrm{~g}, 4.8 \mathrm{mmol})$ was alkylated with racemic trans-(2-monofluoromethylcyclopropyl)methyl iodide 45 (1.07 g, $5.0 \mathrm{mmol})$ according to GP 10, using $\mathrm{NaH}$ (60\% in oil, $230 \mathrm{mg}, 5.7 \mathrm{mmol})$ in DMF/ $\mathrm{MeCN}$ mixture $(2.5+5 \mathrm{~mL})$ during $3 \mathrm{~h}$, giving after chromatographycal separation (silica gel, eluted with 
EtOAc) $\left(2 R, 1^{\prime} S, 2^{\prime} R\right)$ component $(1.31 \mathrm{~g}, 2.24 \mathrm{mmol}, 46.7 \%$ on $(R)$-BGC, d.e. $\geq 98 \%),\left(2 R, 1^{\prime} R, 2^{\prime} S\right)$ component $(1.19 \mathrm{~g}, 2.03 \mathrm{mmol}, 42.3 \%$ on $(R)$-BGC, d.e. $\geq 98 \%)$ and mixed fractions $(0.102 \mathrm{~g}$, $0.17 \mathrm{mmol}, 3.6 \%$ on $(R)$ - BGC).

(2R, 1'S, 2'R)-3-(2-monofluoromethylcyclopropyl)alanine $\quad\left(\boldsymbol{R}-96\right.$ a): $\quad\left(2 R, 1^{\prime} S, 2^{\prime} R\right)-\mathrm{B}(\mathrm{mFmcpA}) \mathrm{C}$<smiles>N[C@@H](C[C@@H]1C[C@H]1CF)C(=O)O</smiles>
$(1.11 \mathrm{~g}, 1.90 \mathrm{mmol})$ was decomposed and the amino acid was separated and purified according to GP 11 to give pure target amino acid (172 mg, $1.06 \mathrm{mmol}, 96 \%)$.

(S)-Belokon' 3-(2'-monofluoromethylcyclopropyl)alanine complex [(S)-B(mFmcpA)C,

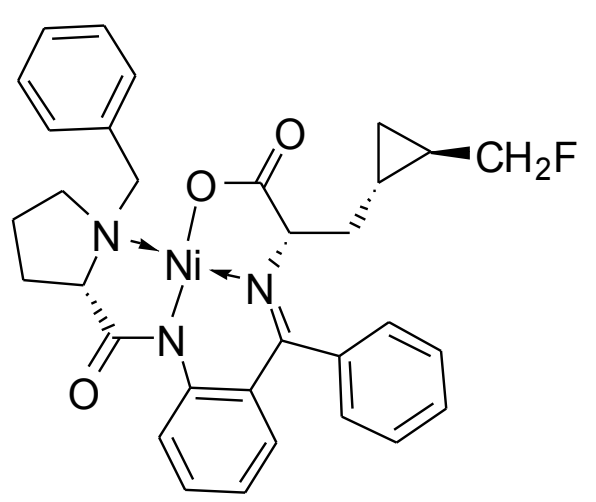
(2S,1'S,2'R)-117]: (S)-BGC $\boldsymbol{S}$-13 (2.40 g, $4.8 \mathrm{mmol})$ was alkylated with racemic trans-(2-monofluoromethylcyclopropyl)methyl iodide $\mathbf{4 5}(1.07 \mathrm{mg}, \quad 5.0 \mathrm{mmol})$ according to GP 10, using $\mathrm{NaH}(60 \%$ in oil, $230 \mathrm{mg}$, $5.7 \mathrm{mmol})$ in $\mathrm{DMF} / \mathrm{MeCN}$ mixture $(2.5+5 \mathrm{~mL})$ during $3 \mathrm{~h}$, giving after chromatographycal separation (silica gel, eluted with EtOAc) $\left(2 S, 1^{\prime} R, 2^{\prime} S\right)$ component $(1.25 \mathrm{~g}, 2.15 \mathrm{mmol}$, $44.8 \%$ on $(S)$-BGC, d.e. $\geq 98 \%), \quad\left(2 S, 1^{\prime} S, 2^{\prime} R\right)$ component $(1.23 \mathrm{~g}, 2.10 \mathrm{mmol}, 43.7 \%$ on $(S)-B G C$, d.e. $\geq 98 \%)$ and mixed fractions (143 mg, $244 \mu \mathrm{mol}$, $5.1 \%$ on $(S)-B G C)$.

(2S, 1'S, 2'R)-3-(2-monofluoromethylcyclopropyl)alanine $\quad(\boldsymbol{S}-96 \mathbf{a}): \quad\left(2 S, 1^{\prime} S, 2^{\prime} R\right)-\mathrm{B}(\mathrm{mFmcpA}) \mathrm{C}$<smiles>N[C@@H](C[C@@H]1C[C@@H]1F)C(=O)O</smiles>
$(1.20 \mathrm{~g}, 2.05 \mathrm{mmol})$ was decomposed and the amino acid was separated and purified according to GP 11 to give pure target amino acid (311 mg, $1.93 \mathrm{mmol}, 94 \%)$. 


\section{Hormaomycin and its all-peptide aza-analogue}

\subsection{Hormaomycin}

N-Fmoc Isoleucine dicyclopropylmethyl ester (Fmoc-Ile-ODCPM, 56): ${ }^{[109]}$ To a stirred ice-cold

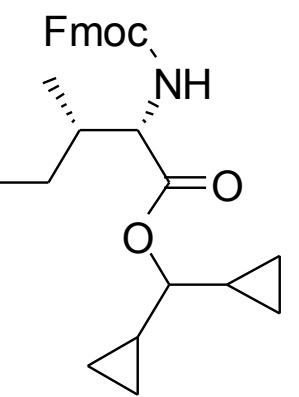
solution of $\mathrm{N}$-Fmoc protected isoleucine $54(3.53 \mathrm{~g}, 10.0 \mathrm{mmol})$ in anhydrous $\mathrm{CH}_{2} \mathrm{Cl}_{2}(35 \mathrm{~mL})$ oxalyl chloride $(3.17 \mathrm{~g}, 25.0 \mathrm{mmol})$ and then DMF (15 drops) were added and stirring continued at the same temperature for $2 \mathrm{~h}$. The mixture was then allowed to warm to $20{ }^{\circ} \mathrm{C}$ and stirred for an additional $1 \mathrm{~h}$. Solvents were removed under reduced pressure at ambient temperature and the crude acylchloride was dried at 0.01 Torr for $2 \mathrm{~h}$ and used further without purification. The acylchloride was dissolved in anhydrous $\mathrm{CH}_{2} \mathrm{Cl}_{2}(35 \mathrm{~mL})$ and the mixture of pyridine/dicyclopropylmethanol $(1: 1 \mathrm{v} / \mathrm{v}, 5.2 \mathrm{~mL})$ was then added. After 40 min DMAP (0.02 g) was added to the mixture and stirring continued overnight under $\mathrm{N}_{2}$-flow. The reaction mixture was then diluted with diethyl ether $(150 \mathrm{~mL})$, washed with aq. $1 \mathrm{M} \mathrm{KHSO}_{4}$ $(3 \times 20 \mathrm{~mL})$, water $(2 \times 20 \mathrm{~mL})$, aq. $5 \% \mathrm{NaHCO}_{3}(3 \times 20 \mathrm{~mL})$, water $(3 \times 20 \mathrm{~mL})$, brine $(2 \times 20$ $\mathrm{mL}$ ), dried over $\mathrm{MgSO}_{4}$, filtered and concentrated under reduced pressure. The residue was

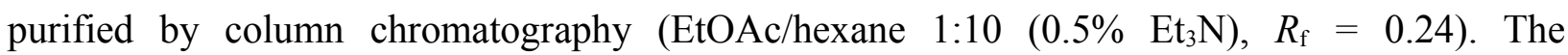
appropriate fractions were pooled, concentrated under reduced pressure, taken up with $\mathrm{Et}_{2} \mathrm{O} /$ hexane 1:1 $(100 \mathrm{~mL})$, washed with water $(3 \times 20 \mathrm{~mL}), 3 \%$ aqueous $\mathrm{NaHCO}_{3}(3 \times 20 \mathrm{~mL})$, water $(3 \times 20 \mathrm{~mL})$, brine $(2 \times 10 \mathrm{~mL})$, dried, filtered and concentrated under reduced pressure to give di-protected amino acid $(3.0 \mathrm{~g}, 6.7 \mathrm{mmol}, 67 \%)$ as a turbid oil. $[\alpha]_{\mathrm{D}}{ }^{20}-3.8(\mathrm{c}=0.26$, $\left.\mathrm{CHCl}_{3}\right) ;{ }^{1} \mathrm{H}$ NMR $\left(250 \mathrm{MHz}, \mathrm{CDCl}_{3}\right): \delta=0.16-0.38(\mathrm{~m}, 4 \mathrm{H}), 0.38-0.51(\mathrm{~m}, 2 \mathrm{H}), 0.51-0.64(\mathrm{~m}$, $2 \mathrm{H}), 0.94$ (t, $J=7.5 \mathrm{~Hz}, 3 \mathrm{H}), 0.96$ (d, $J=7,5 \mathrm{~Hz}, 3 \mathrm{H}), 1.02-1.16(\mathrm{~m}, 2 \mathrm{H}), 1.17-1.34$ (m, $1 \mathrm{H})$, 1.39-1.47 (m, 1 H), 1.86-2.09 (m, $1 \mathrm{H}), 3.90$ (t, $J=8,8 \mathrm{~Hz}, 1 \mathrm{H}), 4.20-4.27$ (m, $1 \mathrm{H}), 4.34-$ $4.44(\mathrm{~m}, 3 \mathrm{H}), 5,36(\mathrm{~d}, J=9,8 \mathrm{~Hz}, 1 \mathrm{H}), 5,16(\mathrm{~d}, J=6,0 \mathrm{~Hz}, 1 \mathrm{H}), 7,23-7,46(\mathrm{~m}, 4 \mathrm{H})$, 7,6 (d, $J=7,5 \mathrm{~Hz}, 2 \mathrm{H}), 7,76(\mathrm{~d}, J=8,3 \mathrm{~Hz}, 2 \mathrm{H}) ;{ }^{13} \mathrm{C} \mathrm{NMR}\left(62,9 \mathrm{MHz}, \mathrm{CDCl}_{3}\right): \delta=2.4,2.6,2.9(-)$, $11.6(+), 14.5,15.3(+), 14.6(+), 24.9(-), 38.1(+), 47.1(+), 58.3(+), 66.8(-), 83.4(+), 119.8$, $125.0,126.9,127.5(+), 141.1\left(\mathrm{C}_{\text {quat }}\right), 143.7,143.8\left(\mathrm{C}_{\text {quat }}\right), 156.0\left(\mathrm{C}_{\text {quat }}\right), 171.5,\left(\mathrm{C}_{\text {quat }}\right)$.

$N-Z-(2 S, 3 R)-\beta-M e t h y l p h e n y l a l a n i n e(Z-M e F, 57):{ }^{[109]}$ A solution of ZOSu (489 mg, $\left.1.96 \mathrm{mmol}\right)$

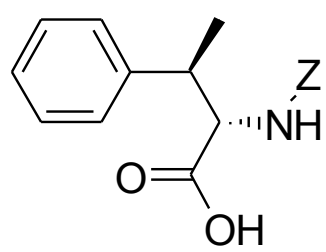

in acetone $(6 \mathrm{~mL})$ was added to a vigorously stirred solution of 3-(2S,3R)-

Z methylphenylalanine 55 (359 mg, $2.00 \mathrm{mmol})$ and $\mathrm{NaHCO}_{3}(505 \mathrm{mg}$, $6.00 \mathrm{mmol})$ in water $(6 \mathrm{~mL})$; stirring was continued for $2 \mathrm{~h}$ (if an emulsion formed, acetone and/or water were added to obtain a homogeneous 
solution).Acetone was then removed under reduced pressure, the residual fraction was diluted with water $(25 \mathrm{~mL})$ and washed with diethyl ether $(3 \times 10 \mathrm{~mL})$. The organic fraction was backextracted with aq. $5 \% \mathrm{NaHCO}_{3}(3 \times 10 \mathrm{~mL})$, the $\mathrm{pH}$ of the combined water fractions was adjusted to $\sim 1$ with aq. $1 \mathrm{M} \mathrm{HCl}$ and the resulting emulsion was extracted with diethyl ether $(2 \times 50 \mathrm{~mL})$. The organic layer was washed with aq. $1 \mathrm{M} \mathrm{KHSO}_{4}(2 \times 10 \mathrm{~mL})$, water $(5 \times 10 \mathrm{~mL})$, brine $(2 \times 10 \mathrm{~mL})$, dried over $\mathrm{MgSO}_{4}$, filtered and concentrated under reduced pressure. The residual oil was dissolved in diethyl ether $(3 \mathrm{~mL})$ and dicyclohexylamine $(342 \mathrm{mg}$, $1.88 \mathrm{mmol})$ was added followed by hexane $(20 \mathrm{~mL})$ and the resulting precipitate was filtered and crystallized twice from EtOAc/hexane to give the dicyclohexylammonium salt of the target Nprotected amino acid (800 mg, $1.62 \mathrm{mmol}, 81 \%$ ) as a white solid. To obtain an analytical sample, a small quantity of the dicyclohexylammonium salt dissolved in EtOAc and washed twice with aq. $1 \mathrm{M} \mathrm{KHSO}_{4}$, three times with water, twice with brine to give, after prolonged drying at 0.02 Torr and $60{ }^{\circ} \mathrm{C}$, the target N-protected amino acid. M.p. $77-79{ }^{\circ} \mathrm{C} ;[\alpha]_{\mathrm{D}}{ }^{20} 17.3(\mathrm{c}=0.76$, $\left.\mathrm{CHCl}_{3}\right) ;{ }^{1} \mathrm{H} \mathrm{NMR}\left(250 \mathrm{MHz}, \mathrm{CDCl}_{3}\right): \delta=1.31,1.36(2 \times \mathrm{d}, J=7.0 \mathrm{~Hz}, 3 \mathrm{H}), 3.27-4.01(\mathrm{~m}$, $1 \mathrm{H}), 4.43-4.68(\mathrm{~m}, 1 \mathrm{H}), 4.74-5.20(\mathrm{~m}, 2 \mathrm{H}), 5.30(\mathrm{~d}, J=9.0 \mathrm{~Hz}, 0.75 \mathrm{H}), 6.25$ (d, $J=8.8 \mathrm{~Hz}$, $0.25 \mathrm{H}), 7.03-7.35(\mathrm{~m}, 10 \mathrm{H}), 7.30-7.90(\mathrm{bs}, 1 \mathrm{H}) ;{ }^{13} \mathrm{C} \mathrm{NMR}\left(62.9 \mathrm{MHz}, \mathrm{CDCl}_{3}\right): \delta=14.3$, $15.8(+), 41.5,41.8(+), 59.1,59.8(+), 67.0,67.4(-), 127.0(+), 127.5(+), 127.6(+), 127.9(+)$, $128.0(+), 128.3,128.3(+), 135.2,135.9\left(\mathrm{C}_{\text {quat }}\right), 140.9,141.4\left(\mathrm{C}_{\text {quat }}\right), 156.0,157.1\left(\mathrm{C}_{\text {quat }}\right), 175.1$, $175.4\left(\mathrm{C}_{\text {quat }}\right)$.

Z-MeF-Ile-ODCPM (60):: ${ }^{[109]}$ The di-protected isoleucine $56(334 \mathrm{mg}, 750 \mu \mathrm{mol})$ was $N$ -

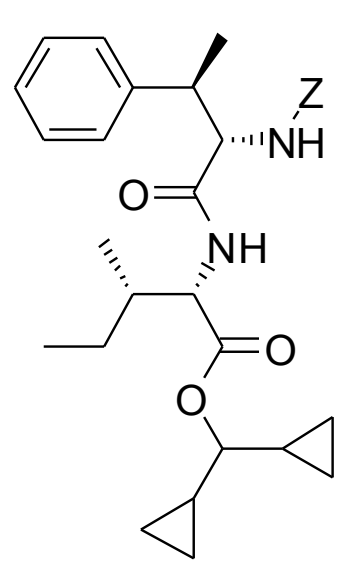
deprotected according to GP 1 and the resulting amino ester was coupled Z with $N$-Z-protected $\beta$-methylphenylalanine $57 \quad(223 \mathrm{mg}, \quad 710 \mu \mathrm{mol})$ employing EDC (140 mg, $730 \mu \mathrm{mol})$, HOAt $(100 \mathrm{mg}, 730 \mu \mathrm{mol})$ and TMP (260 $\mathrm{mg}, 2,13 \mathrm{mmol})$ in $\mathrm{CH}_{2} \mathrm{Cl}_{2}(5 \mathrm{~mL})$ according to GP 2. After $6 \mathrm{~h}$, the reaction mixture was subjected usual aqueous work-up and the resulting crude product was triturated with pentane and then purified by crystallization from hexanes to give target dipeptide $(336 \mathrm{mg}, 640 \mu \mathrm{mol}$, 91\%) as a white solid. $R_{\mathrm{f}}=0.17, \mathrm{EtOAc} /$ hexanes 1:6 $\left(0.5 \% \mathrm{Et}_{3} \mathrm{~N}\right) ;$ m.p. $105-106{ }^{\circ} \mathrm{C} ;[\alpha]_{\mathrm{D}}{ }^{20} 9,0\left(\mathrm{c}=0.31, \mathrm{CHCl}_{3}\right) ;{ }^{1} \mathrm{H} \mathrm{NMR}\left(250 \mathrm{MHz}, \mathrm{CDCl}_{3}\right): \delta=0.23-0.41(\mathrm{~m}, 4 \mathrm{H})$, $0.41-0.53(\mathrm{~m}, 2 \mathrm{H}), 0.53-0.64(\mathrm{~m}, 2 \mathrm{H}), 0.81(\mathrm{~d}, J=6.8 \mathrm{~Hz}, 3 \mathrm{H}), 0.89(\mathrm{t}, J=7.3 \mathrm{~Hz}, 3 \mathrm{H})$, 0.95-1.21 (m, $3 \mathrm{H}), 1.35(\mathrm{~d}, J=7.3 \mathrm{~Hz}, 3 \mathrm{H}), 1.37-1.48(\mathrm{~m}, 1 \mathrm{H}), 1.71-1.90(\mathrm{~m}, 1 \mathrm{H}), 3.15-$ $3.22(\mathrm{~m}, 1 \mathrm{H}), 3.90$ (t, $J=8.5 \mathrm{~Hz}, 1 \mathrm{H}), 4.26-4.42(\mathrm{~m}, 2 \mathrm{H}), 5.09$ (s, $2 \mathrm{H}), 5.42$ (d, $J=9.0 \mathrm{~Hz}$, $1 \mathrm{H}), 6.06(\mathrm{~d}, J=7.5 \mathrm{~Hz}, 1 \mathrm{H}), 7.15-7.30(\mathrm{~m}, 6 \mathrm{H}), 7.30-7.40(\mathrm{~m}, 4 \mathrm{H}) ;{ }^{13} \mathrm{C} \mathrm{NMR}(62.9 \mathrm{MHz}$, $\left.\mathrm{CDCl}_{3}\right): \delta=2.4,2.7(-), 11.4(+), 14.3,14.5(+), 14.8(+), 16.7(+), 24.9(-), 37.9(+), 42.2(+)$, 
$56.1(+), 60.1(+), 66.5(-), 82.9(+), 126.5,127.4,127.5,127.6,128.0(+), 128.1(+), 136.2$, $141.9\left(\mathrm{C}_{\text {quat }}\right), 156.1\left(\mathrm{C}_{\text {quat }}\right), 170.2,170.5\left(\mathrm{C}_{\text {quat }}\right)$.

N-Fmoc-(2R, 1'R, 2'R)-3-(2'-Nitrocyclopropyl)alanine $[(R) N c p A, 61]::^{[109]}$ A solution of FmocOSu

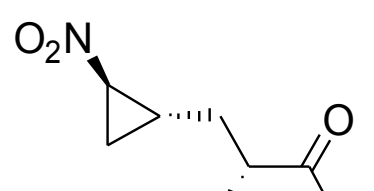
$(0.416 \mathrm{~g}, 1.36 \mathrm{mmol})$ in acetone $(7 \mathrm{~mL})$ was added to a vigorously stirred

Fmoc $-\mathrm{NH} \quad \mathrm{OH}$ water $(5 \mathrm{~mL}$ ) (if precipitate formed acetone and/or water were added to obtain homogeneous solution) and stirring continued for an additional $3 \mathrm{~h}$. Acetone was then removed under reduced pressure and $\mathrm{pH}$ of the residual water solution was adjusted to $\sim 1$ with aq. $1 \mathrm{M} \mathrm{KHSO}_{4}$. The resulting emulsion was extracted with $\mathrm{Et}_{2} \mathrm{O}(30 \mathrm{~mL})$ and the ethereal layer was then back extracted with aq. $5 \% \mathrm{NaHCO}_{3}(5 \times 10 \mathrm{~mL})$. Combined aqueous fractions were washed with $\mathrm{Et}_{2} \mathrm{O}(2 \times 10 \mathrm{~mL})$, acidified to $\mathrm{pH} 2$ with aq. $1 \mathrm{M} \mathrm{KHSO}_{4}$ and the resulting emulsion was extracted with $\mathrm{Et}_{2} \mathrm{O}(4 \times 10 \mathrm{~mL})$. The organic phase was washed with aq. $1 \mathrm{M} \mathrm{KHSO}_{4}(2 \times 10 \mathrm{~mL})$, water $(3 \times 10 \mathrm{~mL})$, brine $(2 \times 5 \mathrm{~mL})$, dried over $\mathrm{MgSO}_{4}$, filtered and concentrated under reduced pressure. The residue was triturated with cold pentane and filtered. The resulting semisolid was dried at 0.02 Torr for prolonged time to give $61(0.423 \mathrm{~g}$, $93 \%$ ) as a white foam. $R_{\mathrm{f}}=0.08 \mathrm{EtOAc} /$ hexanes $1: 1$; m.p. (softening) $50-57^{\circ} \mathrm{C} ;[\alpha]_{\mathrm{D}}{ }^{20} 56.7$ (c $=$ $\left.0.36, \mathrm{CHCl}_{3}\right) ;{ }^{1} \mathrm{H} \mathrm{NMR}\left(250 \mathrm{MHz}, \mathrm{CDCl}_{3}\right): \delta=0.71-0.82(\mathrm{~m}, 0.4 \mathrm{H}), 1.11(\mathrm{~m}, 0.6 \mathrm{H}), 1.17-$ $1.51(\mathrm{~m}, 1 \mathrm{H}), 1.75-2.13(\mathrm{~m}, 2 \mathrm{H}), 3.61-3.76(\mathrm{~m}, 1 \mathrm{H}), 3.76-3.89(\mathrm{~m}, 1 \mathrm{H}), 3.99-4.27$ (m, $2 \mathrm{H})$, $4.27-4.56(\mathrm{~m}, 2 \mathrm{H}), 4.56-4.69(\mathrm{~m}, 1 \mathrm{H}), 4.71-4.87(\mathrm{~m}, 1 \mathrm{H}), 5.48(\mathrm{~d}, J=7.0 \mathrm{~Hz}, 0.6 \mathrm{H}), 7.01-$ $7.13(\mathrm{~m}, \quad 0.4 \mathrm{H}), \quad 7.23-7.42(\mathrm{~m}, \quad 5 \mathrm{H}), \quad 7.42-7.61(\mathrm{~m}, \quad 2 \mathrm{H}), \quad 7.75(\mathrm{~d}, \quad J=7.5 \mathrm{~Hz}, \quad 2 \mathrm{H})$; ${ }^{13} \mathrm{C}$ NMR (62.9 MHz, CDCl3): $\delta=17.3,17.6,21.4,22.0(+), 32.8,33.2,46.8(+), 53.0(+), 59.0$ $(+), 66.7,67.0,119.8(+), 124.2,124.4(+), 124.8(+), 126.9,127.6(+), 141.1\left(\mathrm{C}_{\text {quat }}\right), 143.0$, $143.3\left(\mathrm{C}_{\text {quat }}\right), 143.3,143.5\left(\mathrm{C}_{\text {quat }}\right), 155.9,156.8\left(\mathrm{C}_{\text {quat }}\right), 173.8,174.6\left(\mathrm{C}_{\text {quat }}\right)$.

Fmoc-(R)NcpA-MeF-Ile-ODCPM (63): ${ }^{[109]}$ Dipeptide 60 (0.35 g, $\left.0.67 \mathrm{mmol}\right)$ was taken up with

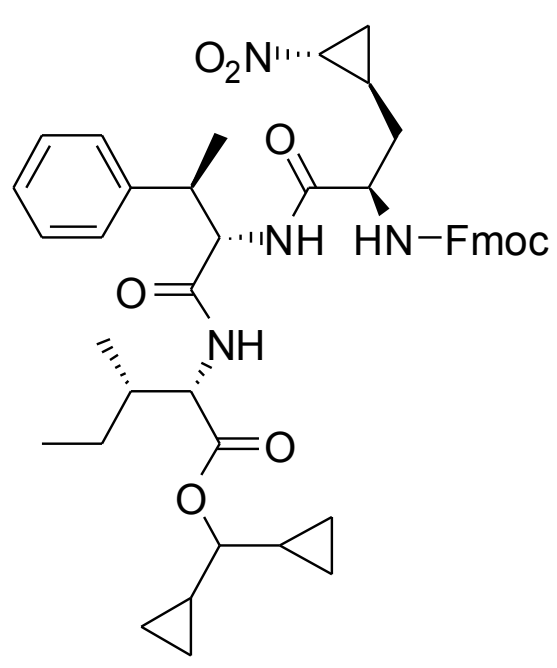
EtOAc $(10 \mathrm{~mL})$ and hydrogenated over $10 \% \mathrm{Pd} / \mathrm{C}(0.15 \mathrm{~g})$ under ambient pressure of hydrogen for $2 \mathrm{~h}$. The reaction mixture was filtered through a pad of Celite and concentrated under reduced pressure to give deprotected dipeptide 62 , which was directly used for the coupling with 61 (274 mg, $0.69 \mathrm{mmol}$ ), using EDC (137 mg, $0.72 \mathrm{mmol}$ ), HOAt (96 mg, $0.71 \mathrm{mmol})$ and TMP (0.25 $\mathrm{mL}, 2.02 \mathrm{mmol})$ according to GP 2 to give tripeptide 63 (405 mg, 79\%) as a colorless solid 
after 2 recrystallizations from THF/hexanes $1: 1 . R_{\mathrm{f}}=0.52$, EtOAc/hexanes 2:3; m.p 151-155 ${ }^{\circ} \mathrm{C}$, $[\alpha]_{\mathrm{D}}{ }^{20} 3.8\left(\mathrm{c}=0.26, \mathrm{CHCl}_{3}\right) ;{ }^{1} \mathrm{H} \mathrm{NMR}\left(250 \mathrm{MHz}, \mathrm{CDCl}_{3}\right): \delta=0.20-0.38(\mathrm{~m}, 4 \mathrm{H}), 0.37-0.49(\mathrm{~m}$, $2 \mathrm{H}), 0.49-0.62(\mathrm{~m}, 2 \mathrm{H}), 0.80(\mathrm{~d}, J=7.0 \mathrm{~Hz}, 3 \mathrm{H}), 0.86$ (t, $J=7.5 \mathrm{~Hz}, 3 \mathrm{H}), 0.94-1.20$ (m, $4 \mathrm{H})$, $1.33-1.49(\mathrm{~m}, 1 \mathrm{H}), 1.34(\mathrm{~d}, J=7.0 \mathrm{~Hz}, 3 \mathrm{H}), 1.50-1.68(\mathrm{~m}, 1 \mathrm{H}), 1.71-1.85(\mathrm{~m}, 2 \mathrm{H}), 1.85-$ $2.05(\mathrm{~m}, 2 \mathrm{H}), 3.15-3.29(\mathrm{~m}, 1 \mathrm{H}), 3.79(\mathrm{t}, J=8.6 \mathrm{~Hz}, 1 \mathrm{H}), 4.01-4.1(\mathrm{~m}, 1 \mathrm{H}), 4.16-4.29$ (m, $1 \mathrm{H}), 4.26-4.43(\mathrm{~m}, 3 \mathrm{H}), 4.49(\mathrm{dd}, J=10.3 \mathrm{~Hz}, 7.0 \mathrm{~Hz}, 1 \mathrm{H}), 4.57-4.65(\mathrm{~m}, 1 \mathrm{H}), 5.58(\mathrm{~d}$, $J=8.3 \mathrm{~Hz}, 1 \mathrm{H}), 6.14(\mathrm{~d}, J=7.8 \mathrm{~Hz}, 1 \mathrm{H}), 6.84(\mathrm{~d}, J=8.0 \mathrm{~Hz}, 1 \mathrm{H}), 7.12-7.31(\mathrm{~m}, 5 \mathrm{H}), 7.33$ (d, $J=7.8 \mathrm{~Hz}, 2 \mathrm{H}), 7.36-7.45(\mathrm{~m}, 2 \mathrm{H}), 7.58(\mathrm{~d}, J=7.0 \mathrm{~Hz}, 2 \mathrm{H}), 7.77(\mathrm{~d}, J=7.3 \mathrm{~Hz}, 2 \mathrm{H})$; ${ }^{13} \mathrm{C}$ NMR (62.9 MHz, $\left.\mathrm{CDCl}_{3}\right): \delta=2.4,2.7(-), 11.4(+), 14.1,14.4(+), 14.8(+), 16.7(+), 17.7$ $(-), 21.97(+), 24.9(-), 33.9(-), 38.1(+), 41.7(+), 46.8(+), 53.8(+), 56.2(+), 58.5(+), 58.8$ $(+), 67.0(-), 83.1(+), 119.7,124.8,126.6,127.3,127.4,128.2,128.3,140.96,141.49\left(\mathrm{C}_{\text {quat }}\right)$, $141.5\left(\mathrm{C}_{\text {quat }}\right), 143.5,143.6\left(\mathrm{C}_{\text {quat }}\right), 155.9\left(\mathrm{C}_{\text {quat }}\right), 169.8,170.5,171.0\left(\mathrm{C}_{\text {quat }}\right)$.

N-Fmoc-(2S,3R)- $\beta$-Methylphenylalanine (Fmoc-MeF, 64): A solution of FmocOSu (810 mg,

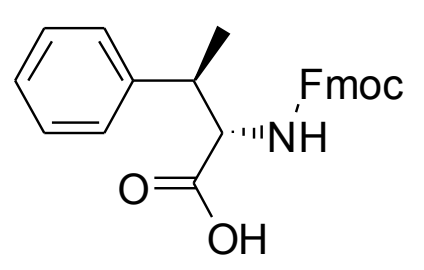
$2.40 \mathrm{mmol})$ in acetone $(12 \mathrm{~mL})$ was added to a vigorously stirred added to obtain a homogeneous solution). Acetone was then removed under reduced pressure, the residual fraction was diluted with water $(25 \mathrm{~mL})$ and washed with diethyl ether $(3 \times 10 \mathrm{~mL})$. The organic fraction was back-extracted with $5 \%$ aqueous $\mathrm{NaHCO}_{3}(3 \times 10 \mathrm{~mL})$, the $\mathrm{pH}$ of the combined water fractions was adjusted to 1 with aq. $1 \mathrm{M} \mathrm{KHSO}_{4}$ and the resulting emulsion was extracted with diethyl ether $(3 \times 30 \mathrm{~mL})$. Combined organic layers were washed with aq. $1 \mathrm{M} \mathrm{KHSO}_{4}(2 \times 10 \mathrm{~mL})$, water $(5 \times 10 \mathrm{~mL})$, brine $(2 \times 10 \mathrm{~mL})$, dried over $\mathrm{MgSO}_{4}$, filtered and concentrated under reduced pressure to give, after prolonged drying at 0.02 Torr and $60{ }^{\circ} \mathrm{C}$, the target $N$-protected amino acid 64 as colorless foam $(636 \mathrm{mg}, 1.58 \mathrm{mmol}, 79 \%)$. M.p. 77$79{ }^{\circ} \mathrm{C} ;[\alpha]_{\mathrm{D}}{ }^{20} 17.3\left(\mathrm{c}=0.76, \mathrm{CHCl}_{3}\right) ;{ }^{1} \mathrm{H} \mathrm{NMR}\left(250 \mathrm{MHz}, \mathrm{CDCl}_{3}\right): \delta=1.40(\mathrm{~d}, J=7.2 \mathrm{~Hz}, 3 \mathrm{H})$, 3.24-3.39 (m, $1 \mathrm{H}), 4.13-4.24(\mathrm{~m}, 1 \mathrm{H}), 4.32(\mathrm{dd}, J=10.1 \mathrm{~Hz}, 6.8 \mathrm{~Hz}, 2 \mathrm{H}), 4.38-4.49$ (m, $1 \mathrm{H})$, $4.64(\mathrm{dd}, J=9.2 \mathrm{~Hz}, 5.6 \mathrm{~Hz}, 1 \mathrm{H}), 5.30(\mathrm{~d}, J=9.2 \mathrm{~Hz}, 1 \mathrm{H}), 7.10-7.36(\mathrm{~m}, 6 \mathrm{H}), 7.41$ (t, $J=7.2 \mathrm{~Hz}, 2 \mathrm{H}), 7.57$ (d, $J=7.4 \mathrm{~Hz}, 1 \mathrm{H}), 7.77(\mathrm{~d}, J=7.3 \mathrm{~Hz}, 2 \mathrm{H}) ;{ }^{13} \mathrm{C} \mathrm{NMR}(62.9 \mathrm{MHz}$, $\left.\mathrm{CDCl}_{3}\right): \delta=14.3,15.8(+), 41.5,41.8(+), 59.1,59.8(+), 67.0,67.4(-), 127.0(+), 127.5(+)$, $127.6(+), 127.9(+), 128.0(+), 128.3,128.3(+), 135.2,135.9\left(\mathrm{C}_{\text {quat }}\right), 140.9,141.4\left(\mathrm{C}_{\text {quat }}\right), 156.0$, $157.1\left(\mathrm{C}_{\text {quat }}\right), 175.1,175.4\left(\mathrm{C}_{\text {quat }}\right)$. 
Fmoc-MeF-(S)NcpA-MeF-Ile-ODCPM (66): ${ }^{[109]}$ The tripeptide 63 (0.420 g, 0.549 mmol) was

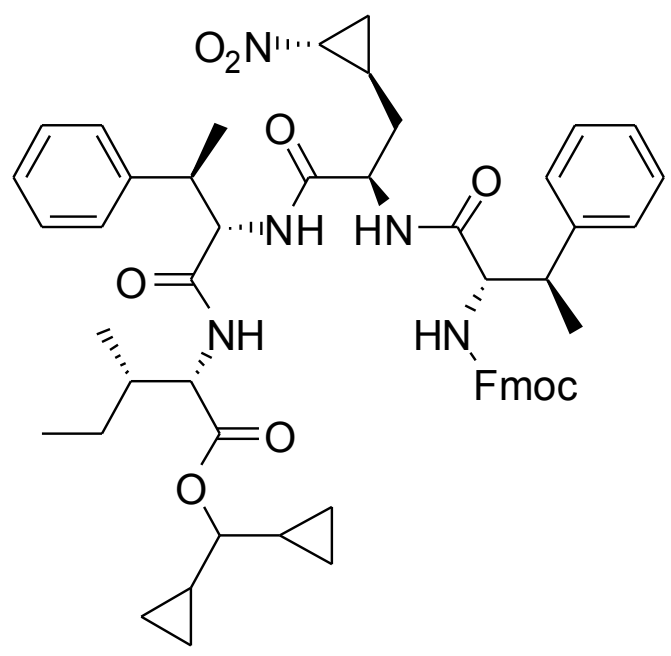
deprotected according to GP 1 and the resulting product 65 was then directly coupled with $N$-Fmocprotected $\beta$-methylphenylalanine $64 \quad(0.242 \mathrm{~g}$, $0.603 \mathrm{mmol})$ according to GP 2 using $\operatorname{EDC}(0.114 \mathrm{~g}$, $0.595 \mathrm{mmol})$, HOAt (0.080 g, $0.592 \mathrm{mmol})$ and TMP (0.200 g, $1.650 \mathrm{mmol})$ in $\mathrm{CH}_{2} \mathrm{Cl}_{2}(3 \mathrm{~mL})$. After $1 \mathrm{~h}$, a precipitate appeared in the reaction mixture and anhydrous DMF $(2 \mathrm{~mL})$ was added to obtain homogeneous solution. After $15 \mathrm{~h}$ the reaction mixture was concentrated under reduced pressure. The resulting solid was washed with water $(100 \mathrm{~mL}), 5 \%$ aqueous $\mathrm{NaHCO}_{3}(100 \mathrm{~mL})$, water $(100 \mathrm{~mL}), \mathrm{Et}_{2} \mathrm{O}(100 \mathrm{~mL})$, pentane $(50 \mathrm{~mL})$, dried at 0.5 Torr and then crystallized twice from $\mathrm{THF} /$ hexanes to give $66(0.430 \mathrm{~g}, 85 \%)$ as an off-white solid. $R_{\mathrm{f}}=0.29, \mathrm{CHCl}_{3} / \mathrm{MeOH} 70: 1 ; \mathrm{m} . \mathrm{p}$ 210-215 ${ }^{\circ} \mathrm{C}$ (decomp.), $[\alpha]_{\mathrm{D}}{ }^{20}-26.3$ (c $=0.32$, THF); ${ }^{1} \mathrm{H}$ NMR $(250 \mathrm{MHz},[\mathrm{D} 8] \mathrm{THF}): \delta=0.22-$ $0.57(\mathrm{~m}, 7 \mathrm{H}), 0.67-0.90(\mathrm{~m}, 2 \mathrm{H}), 0.78-0.86(\mathrm{~m}, 1 \mathrm{H}), 0.81(\mathrm{~d}, J=6.5 \mathrm{~Hz}, 3 \mathrm{H}), 0.83$ (t, $J=7.3$ $\mathrm{Hz}, 3 \mathrm{H}), 0.92-1.19$ (m, $4 \mathrm{H}), 1.20$ (d, J=7.3 Hz, $3 \mathrm{H}), 1.32$ (d, J=7.0 Hz, $3 \mathrm{H}), 1.30-1.50$ (m, $4 \mathrm{H}), 3.07-3.16(\mathrm{~m}, 1 \mathrm{H}), 3.18-3.31(\mathrm{~m}, 1 \mathrm{H}), 3.81(\mathrm{t}, J=8.5 \mathrm{~Hz}, 1 \mathrm{H}), 3.89-4.01(\mathrm{~m}, 1 \mathrm{H})$, 4.13-4.41 (m, $5 \mathrm{H}), 4.41-4.51(\mathrm{~m}, 1 \mathrm{H}), 4.55-4.68(\mathrm{~m}, 1 \mathrm{H}), 6.97-7.40(\mathrm{~m}, 16 \mathrm{H}), 7.48(\mathrm{~d}$, $J=8.5 \mathrm{~Hz}, 1 \mathrm{H}), 7.58(\mathrm{~d}, J=8.3 \mathrm{~Hz}, 1 \mathrm{H}), 7.63-7.70(\mathrm{~m}, 2 \mathrm{H}), 7.77(\mathrm{~d}, J=7.8 \mathrm{~Hz}, 2 \mathrm{H})$; ${ }^{13} \mathrm{C}$ NMR (62.9 MHz, [D8]THF): $\delta=3.3,3.5,3.6,3.7(-),, 12.2(+), 15.6,15.9(+), 16.1(+), 17.7$ $(+), 18.7(+), 18.9(-), 23.2(+), 26.2(-), 35.0(-), 39.1\left(^{+}\right), 42.8(+), 44.1(+), 48.5(+), 52.8(+)$, $57.4(+), 59.3(+), 60.2(+), 62.1(+), 67.8(-),, 83.4(+), 126.3,126.4,127.5,127.7,128.1,128.6$, $129.1,129.1,129.3,129.4(+), 142.5\left(\mathrm{C}_{\text {quat }}\right), 144.1\left(\mathrm{C}_{\text {quat }}\right), 144.4\left(\mathrm{C}_{\text {quat }}\right), 145.4,145.6\left(\mathrm{C}_{\text {quat }}\right), 157.8$ $\left(\mathrm{C}_{\text {quat }}\right), 171.1,171.5,171.9,172.1$ ( $\left.\mathrm{C}_{\text {quat }}\right)$.

MeZ-a-Thr-OH (68): ${ }^{[109]} \mathrm{NaHCO}_{3}(0.180 \mathrm{~g}, 2.14 \mathrm{mmol})$ and then a solution of MeZOSu $(0.608 \mathrm{~g}$, $2.31 \mathrm{mmol})$ in dioxane $(7 \mathrm{~mL})$ were added to a vigorously stirred solution of $\mathrm{HOH}(R)$-allo-threonine $67(0.25 \mathrm{~g}, 2.10 \mathrm{mmol})$ in water $(7 \mathrm{~mL})$ and stirring
$\mathrm{HO}-\mathrm{ZMe}$ continued for $3 \mathrm{~h}$ (if precipitate formed dioxane and/or water were added to reduced pressure, diluted with water $(40 \mathrm{~mL})$ and washed with $\mathrm{CH}_{2} \mathrm{Cl}_{2}(4 \times 10 \mathrm{~mL})$. $\mathrm{pH}$ of the water fraction was adjusted to $\sim 2$ with solid $\mathrm{KHSO}_{4}$ and the resulting emulsion was extracted with EtOAc $(2 \times 40 \mathrm{~mL})$. The organic layer was washed with water $(4 \times 20 \mathrm{~mL})$, brine $(2 \times 10$ $\mathrm{mL}$ ), dried and concentrated under reduced pressure. The residue was recrystallized from 
$\mathrm{Et}_{2} \mathrm{O} /$ hexanes and then from $\mathrm{CH}_{2} \mathrm{Cl}_{2} /$ hexanes to give $\mathbf{6 8}(0.175 \mathrm{~g}, 39 \%)$ as a white solid. The mother liquor from the second crystallization was concentrated and recrystallized again from $\mathrm{Et}_{2} \mathrm{O} /$ hexanes to give a second crop of $\mathbf{6 8}(0.23 \mathrm{~g}, 90 \%$ overall yield $) . R_{\mathrm{f}}=0.13 \mathrm{EtOAc} /$ hexanes $(2 \% \mathrm{AcOH}), 3$ runs; m.p. $78-80{ }^{\circ} \mathrm{C} ;[\alpha]_{\mathrm{D}}{ }^{20} 24.6\left(\mathrm{c}=0.32, \mathrm{CHCl}_{3}\right) ;{ }^{1} \mathrm{H} \mathrm{NMR}\left(250 \mathrm{MHz}, \mathrm{CDCl}_{3}\right)$ : $\delta=1.27(\mathrm{~d}, J=6.5 \mathrm{~Hz}, 3 \mathrm{H}), 2.34(\mathrm{~s}, 3 \mathrm{H}), 3.70-4.40(\mathrm{bs}, 1 \mathrm{H}), 4.05-4.27(\mathrm{~m}, 1 \mathrm{H}), 4.33-$ $4.44(\mathrm{~m}, 1 \mathrm{H}), 5.07(\mathrm{~s}, 2 \mathrm{H}), 5.77(\mathrm{~d}, J=7.5 \mathrm{~Hz}, 1 \mathrm{H}), 7.15(\mathrm{~d}, J=8.0 \mathrm{~Hz}, 2 \mathrm{H}), 7.24$ (d, $J=8.0 \mathrm{~Hz}, 2 \mathrm{H}) ;{ }^{13} \mathrm{C} \mathrm{NMR}(62.9 \mathrm{MHz}, \mathrm{CDCl} 3): \delta=18.7(+), 21.1(+), 59.3(+), 67.4(-), 69.0$ $(+), 128.3,129.2(+), 132.7,138.1\left(\mathrm{C}_{\text {quat }}\right), 156.9\left(\mathrm{C}_{\text {quat }}\right), 173.3\left(\mathrm{C}_{\text {quat }}\right)$.

MeZ-a-Thr-OAll (69): ${ }^{[109]}$ A suspension of dried $\mathrm{K}_{2} \mathrm{CO}_{3}(0.034 \mathrm{~g}, 0.247 \mathrm{mmol})$ in a solution of the $N$-protected acid $68(0.12 \mathrm{~g}, 0.449 \mathrm{mmol})$ and allyl bromide $(0.08 \mathrm{~mL}$, $\bigcup_{\mathrm{HO}}^{\mathrm{O}}$ OAII $0.946 \mathrm{mmol})$ in anhydrous $\mathrm{MeCN}(4 \mathrm{~mL})$ was vigorously stirred in a sealed tube at $85^{\circ} \mathrm{C}$ for $2 \mathrm{~h}$. The mixture was then allowed to cool to $60{ }^{\circ} \mathrm{C}$ and stirring continued for an additional $16 \mathrm{~h}$. The reaction mixture was cooled to $20{ }^{\circ} \mathrm{C}$, and $\mathrm{Et}_{2} \mathrm{O}(50 \mathrm{~mL})$ and water $(20 \mathrm{~mL})$ were then added. The organic layer was washed with water $(4 \times 10 \mathrm{~mL})$, sat. aq. $\mathrm{NaHCO}_{3}(2 \times 10 \mathrm{~mL})$, brine $(2 \times 5 \mathrm{~mL})$, dried over $\mathrm{MgSO}_{4}$, filtered and concentrated under reduced pressure. The residual oil was triturated with $\mathrm{Et}_{2} \mathrm{O}$ /pentane 1:2 to give a white solid. Then more pentane was added to complete precipitation and the precipitate was filtered off and dried under reduced pressure $(0.116 \mathrm{~g}, 84 \%) . R_{\mathrm{f}}=0.16$ EtOAc/hexanes 1:3; m.p. $47-48{ }^{\circ} \mathrm{C} ;[\alpha]_{\mathrm{D}}{ }^{20}-20.4\left(\mathrm{c}=0.30, \mathrm{CHCl}_{3}\right) ;{ }^{1} \mathrm{H} \mathrm{NMR}(250 \mathrm{MHz}$, CDCl3): $\delta=1.20(\mathrm{~d}, J=6.5 \mathrm{~Hz}, 3 \mathrm{H}), 2.35(\mathrm{~s}, 3 \mathrm{H}), 2.74(\mathrm{~d}, J=6.3 \mathrm{~Hz}, 1 \mathrm{H}), 4.09-4.26(\mathrm{~m}$, $1 \mathrm{H}), 4.47$ (dd, $J=7.5 \mathrm{~Hz}, 3.8 \mathrm{~Hz}, 1 \mathrm{H}), 4.67$ (d, $J=5.3 \mathrm{~Hz}, 2 \mathrm{H}), 5.08$ (s, 2 H), 5.20-5.41 (m, $2 \mathrm{H}), 5.66(\mathrm{~d}, J=7.3 \mathrm{~Hz}, 1 \mathrm{H}), 5.83-5.97(\mathrm{~m}, 1 \mathrm{H}), 7.17(\mathrm{~d}, J=8.0 \mathrm{~Hz}, 2 \mathrm{H}), 7.26(\mathrm{~d}, J=8.0 \mathrm{~Hz}$, $2 \mathrm{H}) ;{ }^{13} \mathrm{C} \mathrm{NMR}\left(62.9 \mathrm{MHz}, \mathrm{CDCl}_{3}\right): \delta=18.7(+), 20.9(+), 59.3(+), 65.9(-), 67.4(-), 68.4(+)$, $118.7(-), 128.0,128.9(+), 132.8(+), 132.8,137.7\left(\mathrm{C}_{\text {quat }}\right), 156.4\left(\mathrm{C}_{\text {quat }}\right), 169.9\left(\mathrm{C}_{\text {quat }}\right)$.

Boc-(4-Pe)Pro-[MeZ-a-Thr]-OAll (70): ${ }^{[109]}$ EDC (0.324 g, $\left.1.69 \mathrm{mmol}\right)$ was added to a cooled

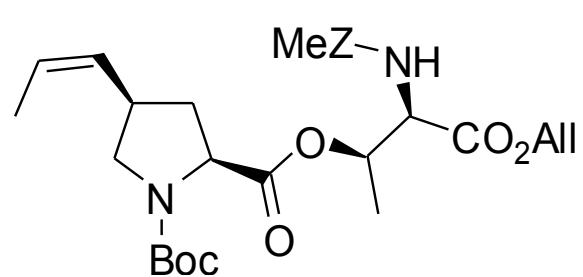
$\left(4{ }^{\circ} \mathrm{C}\right)$ solution of the $N$-Boc-protected $(2 S, 4 R)-4-(Z)-$ propenylproline $118(0.340 \mathrm{~g}, 1.33 \mathrm{mmol})$, the $N, C$ protected amino acid $69(0.400 \mathrm{~g}, 1.30 \mathrm{mmol})$ and 4pyrrolidinopyridine $(0.250 \mathrm{~g}, 1.69 \mathrm{mmol})$ in anhydrous $\mathrm{CH}_{2} \mathrm{Cl}_{2}(3 \mathrm{~mL})$. The temperature was allowed to reach $20^{\circ} \mathrm{C}$, and stirring was continued for $15 \mathrm{~h}$. Then the reaction mixture was diluted with $\mathrm{Et}_{2} \mathrm{O}(30 \mathrm{~mL})$ and washed with aq. $1 \mathrm{M} \mathrm{KHSO}_{4}$ $(3 \times 5 \mathrm{~mL})$, water $(2 \times 5 \mathrm{~mL})$, aq. $3 \% \mathrm{NaHCO}_{3}(3 \times 5 \mathrm{~mL})$, water $(3 \times 5 \mathrm{~mL})$, brine $(2 \times 5 \mathrm{~mL})$, dried over $\mathrm{MgSO}_{4}$, filtered and concentrated under reduced pressure. The residue was purified by 
column chromatography (silica gel, eluted with EtOAc/hexane 1:6) to give $\mathbf{7 0}(0.588 \mathrm{~g}, 83 \%)$ as a turbid oil. $R_{\mathrm{f}}=0.43($ EtOAc/hexane $1: 3) ;[\alpha]_{\mathrm{D}}{ }^{20}-35.4\left(\mathrm{c}=0.28, \mathrm{CHCl}_{3}\right) ;{ }^{1} \mathrm{H} \mathrm{NMR}(250 \mathrm{MHz}$, $\left.\mathrm{CDCl}_{3}\right): \delta=1.34(\mathrm{~d}, J=6.5 \mathrm{~Hz}, 3 \mathrm{H}), 1.36+1.39(2 \times \mathrm{s}, 9 \mathrm{H}), 1.64(\mathrm{dd}, J=1.5 \mathrm{~Hz}, 7.0 \mathrm{~Hz}, 3 \mathrm{H})$, $1.63-1.81(\mathrm{~m}, 1 \mathrm{H}), 2.21-2.46(\mathrm{~m}, 1 \mathrm{H}), 2.33+2.35(2 \times \mathrm{s}, 3 \mathrm{H}), 2.93-3.19(\mathrm{~m}, 2 \mathrm{H}), 3.51-$ $3.67+3.69-3.83(2 \times \mathrm{m}, 1 \mathrm{H}), 4.10-4.27(\mathrm{~m}, 1 \mathrm{H}), 4.48-4.65(\mathrm{~m}, 1 \mathrm{H}), 4.68(\mathrm{~d}, J=5.2 \mathrm{~Hz}, 2 \mathrm{H})$, 4.97-5.13 (m, 2 H), 5.17-5.43 (m, 4 H), 5.54 (dq, $J=10.2$ Hz, 7.0 Hz, 1 H), 5.80-6.01 (m, 1 H), $6.42(\mathrm{~d}, J=9.2 \mathrm{~Hz}, 1 \mathrm{H}), 7.13+7.17(2 \times \mathrm{d}, J=7.9 \mathrm{~Hz}, 2 \mathrm{H}), 7.23+7.25(\mathrm{~d}, J=7.9 \mathrm{~Hz}, 2 \mathrm{H})$; ${ }^{13} \mathrm{C}$ NMR (62.9 MHz, $\left.\mathrm{CDCl}_{3}\right): \delta=12.8(+), 15.8,16.3(+), 20.8(+), 27.9(+), 35.3,36.1(+)$, 35.8, $36.9(-), 51.1,51.3(-), 57.0,57.4(+), 59.0,59.1(+), 65.5,66.0(-), 66.4,67.0(-), 70.8$, $70.8(+), 79.6,79.7$ (Cquat) 118.2, $118.9(-), 126.3,126.3(+), 127.9(+), 128.7,128.8(+), 129.0$, $129.1(+), 130.9,131.2(+), 132.7,133.1$ (Cquat), 137.2, 137.6 (Cquat), 153.0, 153.5 (Cquat), 155.4, 155.9 (Cquat), 168.4, 168.4 (Cquat), 171.6, 171.9 (Cquat).

Boc-(4-Pe)Pro-[MeZ-a-Thr]-OH (71): ${ }^{[109]} \mathrm{Pd}\left[\mathrm{P}(\mathrm{Ph})_{3}\right]_{4}(0.034 \mathrm{~g}, 2.94 \mu \mathrm{mol})$ was added to a

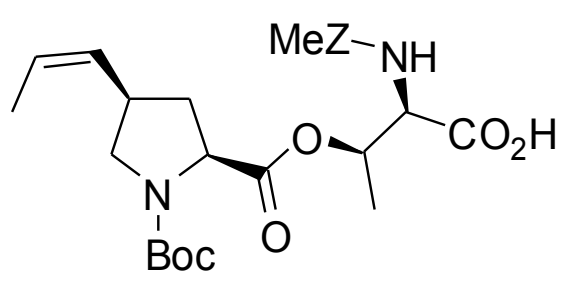
vigorously stirred solution of ester $70(0.108 \mathrm{~g}, 0.198 \mathrm{mmol})$ and $N$-methyl aniline $(0.08 \mathrm{~mL}, 0.738 \mathrm{mmol})$ in DME $(3.5$ $\mathrm{mL}$ ) and the resulting suspension was stirred at ambient temperature for $2 \mathrm{~h}$. The reaction mixture was then diluted with $\mathrm{Et}_{2} \mathrm{O}(40 \mathrm{~mL})$, washed with aq. $1 \mathrm{M} \mathrm{KHSO}_{4}(3 \times 10$ $\mathrm{mL})$, water $(10 \times 10 \mathrm{~mL})$, brine $(2 \times 10 \mathrm{~mL})$, dried over $\mathrm{MgSO}_{4}$, filtered and concentrated under reduced pressure. The residue was taken up with $\mathrm{Et}_{2} \mathrm{O} /$ hexanes 1:2, filtered, concentrated and purified by column chromatography (silica gel, eluted with EtOAc/hexanes 1:2 $+1.5 \% \mathrm{AcOH}$, $\left.R_{f}=0.34\right) \quad$ to give $71(0.121 \quad \mathrm{~g}, \quad 90 \%)$ as an yellow oil. $[\alpha]_{\mathrm{D}}{ }^{20}-71.7\left(\mathrm{c}=0.32, \mathrm{CHCl}_{3}\right) ;{ }^{1} \mathrm{H} \mathrm{NMR}\left(250 \mathrm{MHz}, \mathrm{CDCl}_{3}\right): \delta=1.35+1.40(2 \times \mathrm{s}, 9 \mathrm{H})$, $1.41+1.43(2 \times \mathrm{d}, J=5.7 \mathrm{~Hz}, 3 \mathrm{H}), 1.63+1.65(2 \times \mathrm{dd}, J=1.5 \mathrm{~Hz}, 6.7 \mathrm{~Hz}, 3 \mathrm{H}), 1.67-1.86(\mathrm{~m}$, $1 \mathrm{H}), 2.24-2.49(\mathrm{~m}, 1 \mathrm{H}), 2.33+2.34(2 \times \mathrm{s}, 3 \mathrm{H}), 2.91-3.20(\mathrm{~m}, 2 \mathrm{H}), 3.40-4.30(\mathrm{bs}, 1 \mathrm{H})$, 3.61-3.73 (m, 1 H), 4.11-4.29 (m, $1 \mathrm{H}), 4.51(\mathrm{dd}, J=8.6 \mathrm{~Hz}, 3.5 \mathrm{~Hz}, 1 \mathrm{H}), 5.05(\mathrm{~s}, 2 \mathrm{H}), 5.15-$ $5.31(\mathrm{~m}, 1 \mathrm{H}), 5.31-5.44(\mathrm{~m}, 1 \mathrm{H}), 5.45-5.60(\mathrm{~m}, 1 \mathrm{H}), 5.63+6.46(2 \times \mathrm{d}, J=9.2 \mathrm{~Hz}, 1 \mathrm{H})$, $7.13+7.17(2 \times \mathrm{d}, J=7.9 \mathrm{~Hz}, 2 \mathrm{H}), 7.21+7.22(2 \times \mathrm{d}, J=7.9 \mathrm{~Hz}, 2 \mathrm{H}) ;{ }^{13} \mathrm{C} \mathrm{NMR}(62.9 \mathrm{MHz}$, $\left.\mathrm{CDCl}_{3}\right): \delta=13.0(+), 15.8,16.3(+), 21.0(+), 28.1(+), 35.5,36.2(+), 36.0,37.0(-), 51.3$, $51.6(-), 57.0,57.5(+), 59.1,59.3(+), 66.8,67.0(-), 71.2,71.4(+), 80.5,80.6\left(\mathrm{C}_{\text {quat }}\right), 126.5$, $126.5(+), 128.1(+), 128.1(+), 128.9,129.0(+), 132.8,133.0\left(\mathrm{C}_{\text {quat }}\right), 137.6,137.9\left(\mathrm{C}_{\text {quat }}\right), 153.7$, 154.2 $\left(\mathrm{C}_{\text {quat }}\right), 155.8,156.4\left(\mathrm{C}_{\text {quat }}\right), 171.2,171.7\left(\mathrm{C}_{\text {quat }}\right), 172.0,172.2\left(\mathrm{C}_{\text {quat }}\right)$. 
Boc-(4-Pe)Pro-[MeZ-a-Thr]-MeF-(S)NcpA-MeF-Ile-ODCPM (73): Tetrapeptide 66 (180 mg,

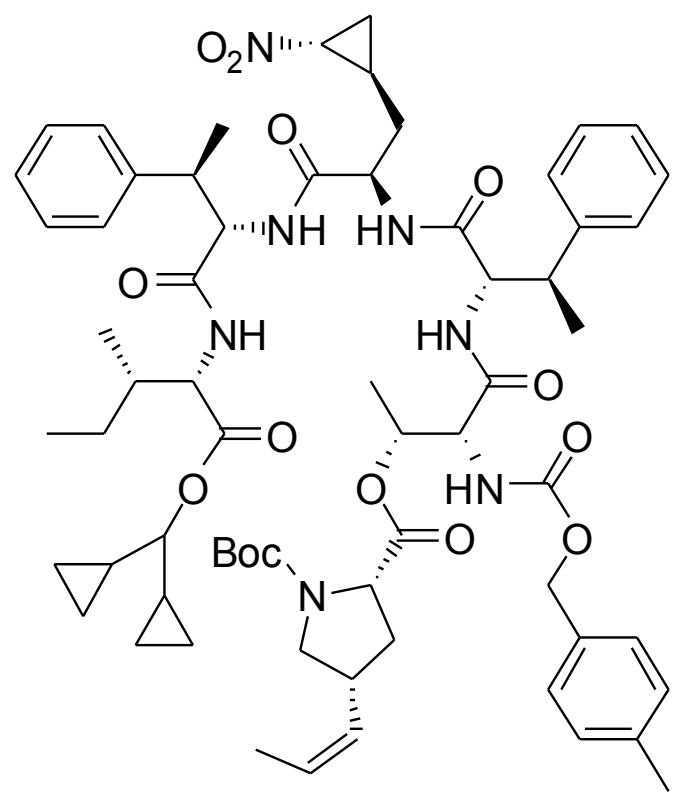
$0.21 \mathrm{mmol}$ ) was deprotected according to GP 1 in THF $(2 \mathrm{~mL})$, taken up with anhydrous $\mathrm{CH}_{2} \mathrm{Cl}_{2}(5 \mathrm{~mL})$, ester acid 71 (0.114 g, $0.23 \mathrm{mmol})$, HATU (96 mg, 0.25 mmol) and HOAt (31 mg, $0.23 \mathrm{mmol}$ ) were added and the reaction mixture was cooled to $4{ }^{\circ} \mathrm{C}$. After this, a solution of DIEA (29 $\mathrm{mg}, 0,22 \mathrm{mmol})$ and TMP (75 mg, $0.62 \mathrm{mmol})$ in $\mathrm{CH}_{2} \mathrm{Cl}_{2}(2 \mathrm{~mL})$ were added at the same temperature within $5 \mathrm{~min}$. The temperature was allowed to reach $20^{\circ} \mathrm{C}$, and stirring continued for an additional $15 \mathrm{~h}$. After aqueous work-up according to GP 2 and two recrystallizations from EtOAc/hexanes 1:2, depsipeptide 73 (185 $\mathrm{mg}, 79 \%$ ) was obtained as a colorless powder. $R_{\mathrm{f}}=0.46($ EtOAc/hexanes $1: 1)$; m.p. $125-127^{\circ} \mathrm{C},[\alpha]_{\mathrm{D}}{ }^{20}-29.0(\mathrm{c}=0.2$, THF); ${ }^{1} \mathrm{H}$ NMR (600 MHz, $\left.\mathrm{CDCl}_{3}\right): \delta=0.27-0.33(\mathrm{~m}, 1 \mathrm{H}), 0.33-0.44(\mathrm{~m}, 3 \mathrm{H}), 0.44-0.49(\mathrm{~m}, 1 \mathrm{H})$, 0.49-0.57 (m, 2 H), 0.59-0.65 (m, $1 \mathrm{H}), 0.75(\mathrm{~d}, J=6.8 \mathrm{~Hz}, 3 \mathrm{H}), 0.89(\mathrm{t}, J=7.3 \mathrm{~Hz}, 3 \mathrm{H})$, 0.95-1.11 (m, $4 \mathrm{H}), 1.11-1.60(\mathrm{~m}, 5 \mathrm{H}), 1.24(\mathrm{~d}, J=7.5 \mathrm{~Hz}, 3 \mathrm{H}), 1.27(\mathrm{~d}, J=7.5 \mathrm{~Hz}, 3 \mathrm{H})$, $1.36(\mathrm{~s}, 9 \mathrm{H}), 1.43(\mathrm{~d}, J=6.5 \mathrm{~Hz}, 3 \mathrm{H}), 1.66(\mathrm{dd}, J=6.9 \mathrm{~Hz}, 1.7 \mathrm{~Hz}, 3 \mathrm{H}), 1.68-1.76(\mathrm{~m}, 1 \mathrm{H})$, 1.80-1.90 (m, $1 \mathrm{H}), 2.30$ (s, $3 \mathrm{H}), 2.35-2.42(\mathrm{~m}, 1 \mathrm{H}), 3.06-3.13(\mathrm{~m}, 1 \mathrm{H}), 3.13$ (t, J=10.5 Hz, $1 \mathrm{H}), 3.18-3.31(\mathrm{~m}, 2 \mathrm{H}), 3.67$ (dd, $J=10.2 \mathrm{~Hz}, 7.8 \mathrm{~Hz}, 1 \mathrm{H}), 3.80-3.84$ (m, $1 \mathrm{H}), 4.02-4.07$ (m, $1 \mathrm{H}), 4.14(\mathrm{dd}, J=10.7 \mathrm{~Hz}, 6.2 \mathrm{~Hz}, 1 \mathrm{H}), 4.33(\mathrm{dd}, J=8.9 \mathrm{~Hz}, 4.6 \mathrm{~Hz}, 1 \mathrm{H}), 4.37$ (dd, $J=9.5 \mathrm{~Hz}, 2.2 \mathrm{~Hz}, 1 \mathrm{H}), 4.45(\mathrm{dd}, J=9.9 \mathrm{~Hz}, 6.9 \mathrm{~Hz} 1 \mathrm{H}), 4.57$ (dt, $J=9.6 \mathrm{~Hz}, 5.1 \mathrm{~Hz}, 1 \mathrm{H})$, $4.62(\mathrm{t}, J=10.3 \mathrm{~Hz}, 1 \mathrm{H}), 4.96-5.06(\mathrm{~m}, 2 \mathrm{H}), 5.22-5.28(\mathrm{~m}, 1 \mathrm{H}), 5.46-5.51(\mathrm{~m}, 1 \mathrm{H}), 5.52-$ $5.58(\mathrm{~m}, 1 \mathrm{H}), 6.54(\mathrm{~d}, J=8.2 \mathrm{~Hz}, 1 \mathrm{H}), 6.89(\mathrm{~d}, J=8.9 \mathrm{~Hz}, 1 \mathrm{H}), 6.99(\mathrm{~d}, J=9.6 \mathrm{~Hz}, 1 \mathrm{H})$, 7.06-7.12 (m, 2 H), 7.14-7.25 (m, $12 \mathrm{H}) 7.49$ (d, $J=9.8 \mathrm{~Hz}, 1 \mathrm{H}), 7.86(\mathrm{~d}, J=5.9 \mathrm{~Hz}, 1 \mathrm{H})$; ${ }^{13} \mathrm{C}$ NMR $\left(62.9 \mathrm{MHz}, \mathrm{CDCl}_{3}\right): \delta=2.48(-), 2.82(-), 2.90(-), 3.01(-), 11.59(+), 13.20(+)$, $14.16(+), 14.64(+), 15.57(+), 17.77(+), 18.41(-), 18.86(+), 19.43(+), 21.11(+), 21.77(+)$, $25.23(-), 26.85(-), 28.23(+), 31.46(-), 31.53(-), 36.28(-), 36.32(+), 37.30(+), 40.45(+)$, $42.00(+), 50.62(+), 52.08(+), 56.43(+), 59.30\left(_{+}\right), 59.49(+), 61.01(+), 61.62(+), 61.99\left(^{+}\right)$, $66.89(-), 70.53(+), 80.93\left(\mathrm{C}_{\text {quat }}\right), 83.24(+), 127.00(+), 127.06(+), 127.10(+), 127.60(+)$, $127.69(+), 128.47(+), 128.63(+), 128.68(+), 128.82(+), 128.93(+), 133.21\left(\mathrm{C}_{\text {quat }}\right)$, $137.83\left(\mathrm{C}_{\text {quat }}\right), \quad 141.73\left(\mathrm{C}_{\text {quat }}\right), \quad 141.90\left(\mathrm{C}_{\text {quat }}\right), \quad 154.76\left(\mathrm{C}_{\text {quat }}\right), \quad 155.75\left(\mathrm{C}_{\text {quat }}\right), \quad 170.38\left(\mathrm{C}_{\text {quat }}\right)$, $170.43\left(\mathrm{C}_{\text {quat }}\right), 170.79\left(\mathrm{C}_{\text {quat }}\right), 171.37\left(\mathrm{C}_{\text {quat }}\right), 173.41\left(\mathrm{C}_{\text {quat }}\right), 174.06$ (Cquat); MS-ESI: (positive), $\left.\mathrm{m} / \mathrm{z}(\%) 1163(100, \mathrm{M}+\mathrm{Na}\rceil^{+}\right)$; (negative), m/z (\%) $\left.\left.1138(50, \mathrm{M}-\mathrm{H}\rceil^{-}\right), 1175(50, \mathrm{M}+\mathrm{Cl}\rceil^{-}\right)$. 
$N-M e Z$ protected cyclohexadepsipeptide (75): To the hexadepsipeptide 74 (0.188 g, 0.165 mmol)

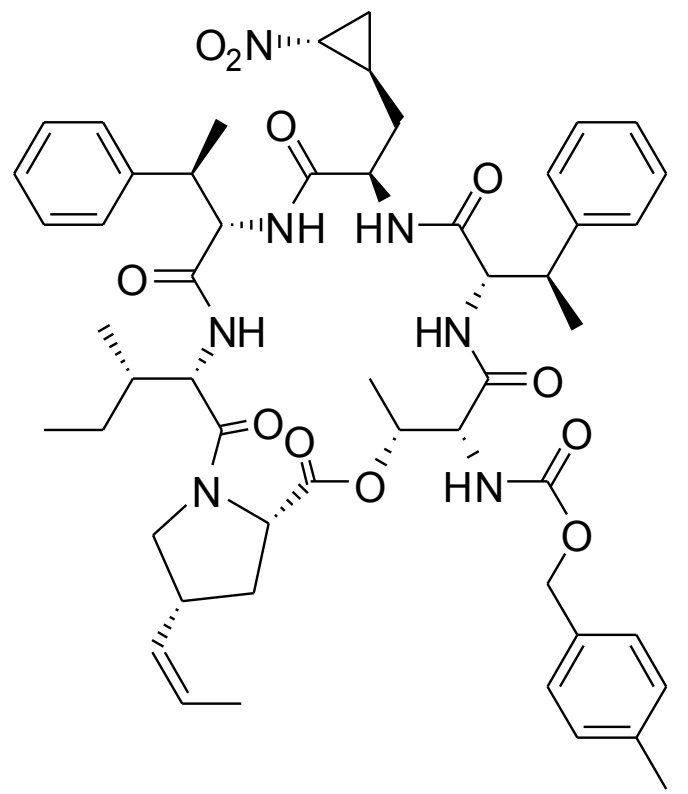
$2 \mathrm{M} \mathrm{HCl}$ in EtOAc $(2 \mathrm{~mL})$ was added, the reaction mixture was stirred for $45 \mathrm{~min}$ at ambient temperature in the dark place and then was concentrated under reduced pressure without any heating. The residue was triturated with dry $\mathrm{Et}_{2} \mathrm{O}$ to give deprotected material $\mathbf{7 4}$ as a white solid [0.160 g; MS-ESI: positive mode, $\mathrm{m} /$ $\left.\left.\mathrm{z}(\%)=997(100, \mathrm{M}+\mathrm{H}\rceil^{+}\right), 1019(5, \mathrm{M}+\mathrm{Na}\rceil^{+}\right)$; negative mode, $\left.\mathrm{m} / \mathrm{z}(\%)=995\left(50, \mathrm{M}-\mathrm{H}^{-}\right)\right]$, which was taken up with anhydrous $\mathrm{CH}_{2} \mathrm{Cl}_{2}(1.5 \mathrm{~L})$ and cyclized employing HATU $(2 \times 0.073 \mathrm{~g}, 2 \times 0.192 \mathrm{mmol})$ and HOAt $(2 \times 0.022 \mathrm{~g}, 2 \times 0.163 \mathrm{mmol})$ and solution of DIEA $(2 \times 0.062 \mathrm{~g}, 2 \times 0.480 \mathrm{mmol})$ in $\mathrm{CH}_{2} \mathrm{Cl}_{2}(2 \times 50 \mathrm{~mL})$ according to $\mathrm{GP} 4$ for $16 \mathrm{~h}$. After this, the solvent was removed under reduced pressure, the residue was taken up with $\mathrm{Et}_{2} \mathrm{O}(50$ $\mathrm{mL}$ ), and after usual aqueous work-up (GP 2), drying and filtration, the organic layer was concentrated under reduced pressure. The residue was purified first by column chromatography (acetone/hexanes 1:3, $\left.R_{f}=0.17\right)$ and then by recrystallization $\left(\mathrm{Et}_{2} \mathrm{O} /\right.$ pentane) to give crude product $(0.12 \mathrm{~g})$, which was finally purified by preparative HPLC to give cyclodepsipeptide $\mathbf{7 5}$ (86 mg, 53\% on 2 steps) as a white solid. Preparative HPLC: isocratic, 70\% B, $\mathrm{t}_{\mathrm{R}}=19.3 \mathrm{~min}$, purity $>98 \%$; analytical HPLC: gradient $20 \% \rightarrow 100 \% \mathrm{~B}$ for $20 \mathrm{~min}$, then isocratic $100 \% \mathrm{~B}$ for $5 \min \mathrm{t}_{\mathrm{R}}=16.7 \mathrm{~min}$, purity $>98 \% ;[\alpha]_{\mathrm{D}}{ }^{20}-15.5\left(\mathrm{c}=0.20, \mathrm{CHCl}_{3}\right) ;{ }^{1} \mathrm{H} \mathrm{NMR}\left(600 \mathrm{MHz}, \mathrm{CDCl}_{3}\right)$ : $\delta=0.61-0.67(\mathrm{~m}, 1 \mathrm{H}), 0.72(\mathrm{~d}, J=6.6 \mathrm{~Hz}, 3 \mathrm{H}), 0.71-0.77(\mathrm{~m}, 1 \mathrm{H}), 0.79(\mathrm{t}, J=7.2 \mathrm{~Hz}, 3 \mathrm{H})$, $1.04-1.12(\mathrm{~m}, 1 \mathrm{H}), 1.23(\mathrm{~d}, J=6.6 \mathrm{~Hz}, 3 \mathrm{H}), 1.27-1.34(\mathrm{~m}, 1 \mathrm{H}), 1.37(\mathrm{~d}, J=6.6 \mathrm{~Hz}, 3 \mathrm{H})$, 1.37-1.43 (m, $1 \mathrm{H}), 1.45-1.54(\mathrm{~m}, 1 \mathrm{H}), 1.54-1.57$ (m, $1 \mathrm{H}), 1.57$ (d, $J=6.6 \mathrm{~Hz}, 3 \mathrm{H}), 1.65$ (dd, $J=6.6 \mathrm{~Hz}, 1.5 \mathrm{~Hz}, 3 \mathrm{H}), 1.66-1.76(\mathrm{~m}, 2 \mathrm{H}), 2.20-2.25(\mathrm{~m}, 1 \mathrm{H}), 2.35$ (s, 3 H), 3.01-3.07 (m, $1 \mathrm{H}), 3.15-3.28(\mathrm{~m}, 2 \mathrm{H}), 3.54(\mathrm{dq}, J=7.2 \mathrm{~Hz}, 6.6 \mathrm{~Hz}, 1 \mathrm{H}), 3.71(\mathrm{dd}, J=6.0 \mathrm{~Hz}, 5.4 \mathrm{~Hz}, 1 \mathrm{H})$, 3.74-3.77 (m, $1 \mathrm{H}), 3.98$ (dd, $J=10.5 \mathrm{~Hz}, 6.3 \mathrm{~Hz}, 1 \mathrm{H}), 4.01-4.08$ (m, $1 \mathrm{H}), 4.46-4.54$ (m, $2 \mathrm{H})$, $4.52-4.55(\mathrm{~m}, 1 \mathrm{H}), 4.67-4.70(\mathrm{~m}, 1 \mathrm{H}), 5.03(\mathrm{~d}, J=12.0 \mathrm{~Hz}, 1 \mathrm{H}), 5.15(\mathrm{~d}, J=12.0 \mathrm{~Hz}, 1 \mathrm{H})$, $5.19-5.25(\mathrm{~m}, 1 \mathrm{H}), 5.39(\mathrm{qd}, J=6.6 \mathrm{~Hz}, 1.8 \mathrm{~Hz}, 1 \mathrm{H}), 5.56(\mathrm{dq}, J=10.8 \mathrm{~Hz}, 6.6 \mathrm{~Hz}, 1 \mathrm{H})$, $5.96(\mathrm{~d}, J=5.3 \mathrm{~Hz}, 1 \mathrm{H}), 6.20(\mathrm{~d}, J=7.4 \mathrm{~Hz}, 1 \mathrm{H}), 6.28(\mathrm{~d}, J=9.7 \mathrm{~Hz}, 1 \mathrm{H}), 6.49$ (d, $J=9.4 \mathrm{~Hz}$, $1 \mathrm{H}), \quad 7.11-7.37(\mathrm{~m}, 14 \mathrm{H}) \quad 7.32(\mathrm{~d}, J=7.4 \mathrm{~Hz}, 1 \mathrm{H}), 7.45(\mathrm{~d}, J=8.7 \mathrm{~Hz}, 1 \mathrm{H}) ;{ }^{13} \mathrm{C} \mathrm{NMR}$ $\left(150.8 \mathrm{MHz}, \mathrm{CDCl}_{3}\right): \delta=10.3(+), 13.3(+), 14.6(+), 17.3(-), 17.7(+), 18.4(+), 21.2(+)$, $21.3(+), 24.7(-), 32.0(-), 35.4(-), 36.6(+), 39.4(+), 44.5(+), 52.5(-), 53.3(+), 54.6(+)$, $58.6(+), 59.0(+), 59.4(+), 60.1(+), 60.7(+), 67.2(-), 72.6(+), 127.1(+), 127.2(+), 127.5(+)$, 
$127.6(+), 128.3(+), 128.6(+), 128.8(+), 129.2(+), 127.8(+), 128.0(+), 133.2\left(\mathrm{C}_{\text {quat }}\right)$, $137.9\left(\mathrm{C}_{\text {quat }}\right), 140.9\left(\mathrm{C}_{\text {quat }}\right), 142.6\left(\mathrm{C}_{\text {quat }}\right), 156.3\left(\mathrm{C}_{\text {quat }}\right), 169.0\left(\mathrm{C}_{\text {quat }}\right), 170.3\left(\mathrm{C}_{\text {quat }}\right), 170.6\left(\mathrm{C}_{\text {quat }}\right)$, $171.1\left(\mathrm{C}_{\text {quat }}\right), 171.4\left(\mathrm{C}_{\text {quat }}\right), 173.1\left(\mathrm{C}_{\text {quat }}\right)$.

N-Teoc-(2S, 1'R, 2'R)-3-(2'-Nitrocyclopropyl)alanine (78): ${ }^{[109]}$ A solution of TeocOSu (0.358 g, $1.38 \mathrm{mmol})$ in acetone $(5 \mathrm{~mL})$ was added to a vigorously stirred solution
of $\left(2 S, 1^{\prime} R, 2^{\prime} R\right)-3-\left(2^{\prime}\right.$-nitrocyclopropyl)alanine $76(0.200 \mathrm{~g}, 1.15 \mathrm{mmol})$ $(0.055 \mathrm{~mL}, 0.44 \mathrm{mmol})$ was then added. After an additional $10 \mathrm{~min}$ acetone was removed under reduced pressure and $\mathrm{pH}$ of the residual water solution was adjusted to 2-3 with aq. $1 \mathrm{M} \mathrm{KHSO}_{4}$. The resulting emulsion was extracted with $\mathrm{Et}_{2} \mathrm{O}(50 \mathrm{~mL})$ and the ethereal layer was washed with aq. $1 \mathrm{M} \mathrm{KHSO}_{4}(2 \times 10 \mathrm{~mL})$, water $(10 \times 10 \mathrm{~mL})$, brine $(2 \times 5 \mathrm{~mL})$, dried, filtered and concentrated under reduced pressure. The residual oil $(0.300 \mathrm{~g})$ was dissolved in $\mathrm{Et}_{2} \mathrm{O}(5 \mathrm{~mL})$ and cyclohexylamine $(0.094 \mathrm{~g}, 0.95 \mathrm{mmol})$ was added. The mixture was concentrated under reduced pressure and treated with boiling hexanes. The resulting precipitate was filtered off and washed with $\mathrm{Et}_{2} \mathrm{O} /$ pentane 1:4 to give cyclohexylammonium salt of $78(0.386 \mathrm{~g}, 81 \%)$ as a white solid. $R_{\mathrm{f}}=0.24$ EtOAc/hexanes $1: 3(2 \% \mathrm{AcOH}) ;[\alpha]_{\mathrm{D}}^{20} 22.80 \quad\left(\mathrm{c}=0.46, \mathrm{CHCl}_{3}\right)$ for cyclohexylammonium salt; ${ }^{1} \mathrm{H}$ NMR $\left(250 \mathrm{MHz} \mathrm{CDCl}_{3}\right): \delta=0.04(\mathrm{~s}, 9 \mathrm{H}), 1.00$ (dd, $J=9.5 \mathrm{~Hz}$, $7.3 \mathrm{~Hz}, 2 \mathrm{H}), 1.09-1.18$ (m, $1 \mathrm{H}), 1.20-1.43$ (m, $5 \mathrm{H}), 1.50-1.70$ (m, $2 \mathrm{H}), 1.70-1.90$ (m, $3 \mathrm{H})$, 1.90-2.19 (m, 4 H), 2.80-3.05 (m, $1 \mathrm{H}) 3.95-4.23(\mathrm{~m}, 4 \mathrm{H}), 5.88(\mathrm{~d}, J=8.1 \mathrm{~Hz}, 1 \mathrm{H}), 7.25-$ $8.10(\mathrm{bs}, 3 \mathrm{H}) ;{ }^{13} \mathrm{C} \mathrm{NMR}\left(62.9 \mathrm{MHz}, \mathrm{CDCl}_{3}\right): \delta=-1.9(+), 17.3(-), 17.3(-), 22.0(+), 33.1,33.3$ $(-), 52.7,53.2(+), 59.0(+), 63.7,64.8(-), 156.4,157.4\left(\mathrm{C}_{\text {quat }}\right), 174.5,174.8\left(\mathrm{C}_{\text {quat }}\right)$.

$N$-Teoc protected heptadepsipeptide (79): ${ }^{[109]}$ An ethereal solution $(50 \mathrm{~mL})$ of the

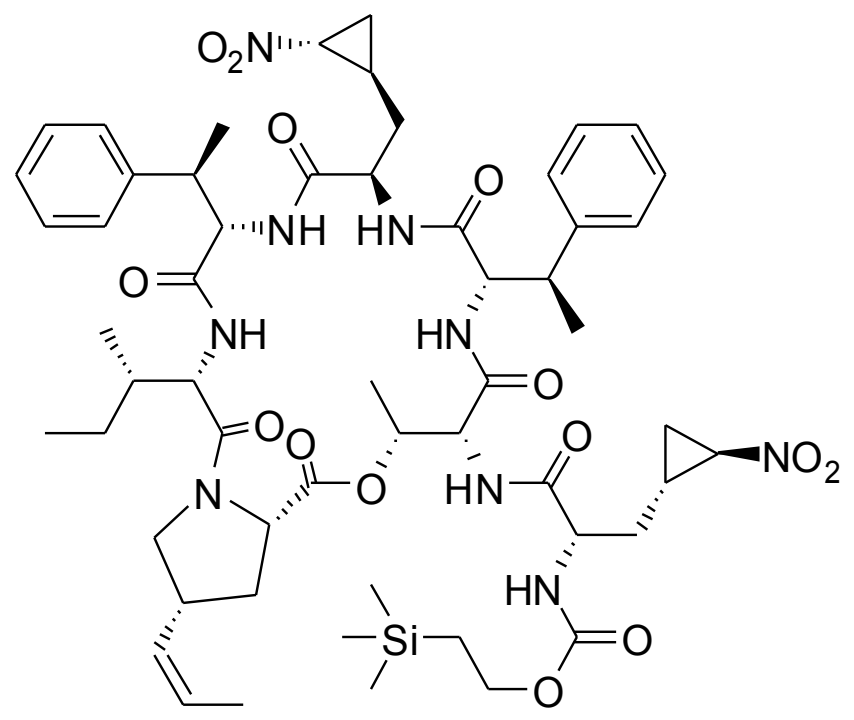
cyclohexylammonium salt of $N$-Teoc protected $\quad\left(2 S, 1^{\prime} R, 2^{\prime} R\right)-3-\left(2^{\prime}-\right.$ nitrocyclopropyl)alanine 78 (8.1 mg, $19.41 \mu \mathrm{mol})$ was washed with aq. $1 \mathrm{M} \mathrm{H}_{2} \mathrm{SO}_{4}(3 \times 5 \mathrm{~mL})$, aq. $1 \mathrm{M} \mathrm{KHSO}_{4}(2 \times 5 \mathrm{~mL})$, water $(3 \times 5 \mathrm{~mL})$, brine $(2 \times 5 \mathrm{~mL})$, dried, filtered and concentrated under reduced pressure. The resulting $N$-protected amino acid $\mathbf{7 8}$ was dried at 0.02 Torr for $2 \mathrm{~h}$ and then coupled 
with the depsipeptide 77 [obtained after deprotection of $N$-MeZ protected cyclodepsipeptide $\mathbf{7 5}$ (9.5 mg, $9.71 \mu \mathrm{mol})$ with $10 \%$ anisole in TFA $(1.1 \mathrm{~mL})$ for $2 \mathrm{~h}$ according to GP 5] using HATU (7.4 mg, $19.46 \mu \mathrm{mol})$, HOAt $(2.6 \mathrm{mg}, 19.24 \mu \mathrm{mol})$, DIEA $(1.25 \mathrm{mg}, 9.67 \mu \mathrm{mol})$ and TMP (7.04 $\mathrm{mg}, 58.10 \mu \mathrm{mol})$ in $\mathrm{CH}_{2} \mathrm{Cl}_{2}(0.7 \mathrm{~mL})$ according to $\mathrm{GP} 6$ for $15 \mathrm{~h}$. The mixture was then diluted with $\mathrm{EtOAc} / \mathrm{Et}_{2} \mathrm{O}$ 1:1 (20 mL) to give after usual aqueous work-up (GP 2) the crude product 79 $\left(8.0 \mathrm{mg}, 73 \%, R_{\mathrm{f}}=0.43\right.$ acetone/hexanes 1:2) as a colorless glass which was used for the next step without any characterization.

MOM-O-protected Hormaomycin (82): ${ }^{[109]}$ Teoc group was cleaved from the compound 79 (8.0

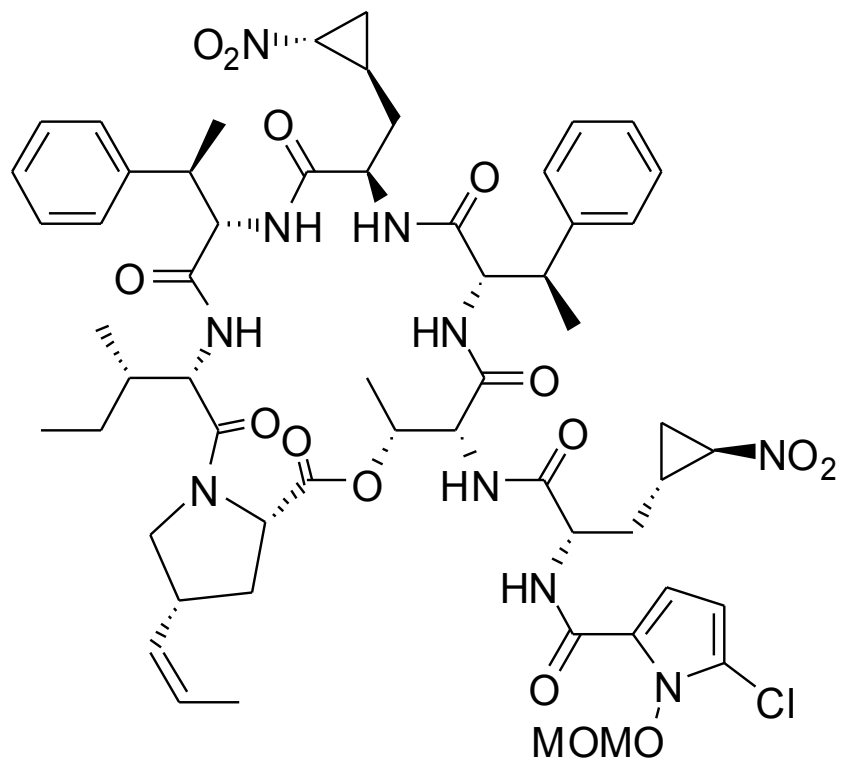
mg, $7.08 \mu \mathrm{mol})$ with TFA $(0.6 \mathrm{~mL})$ for $1 \mathrm{~h}$. The mixture was concentrated under reduced pressure at $20^{\circ} \mathrm{C}$ and then taken up with toluene $(3 \times 15 \mathrm{~mL})$ which was distilled off to remove the last traces of TFA. The resulting deprotected depsipeptide $\mathbf{8 0}$ was coupled with $O$-MOM protected acid $\mathbf{8 1}$ (2.9 mg, $14.10 \mu \mathrm{mol})$ using HATU $(5.4 \mathrm{mg}$, $14.20 \mu \mathrm{mol})$, DIEA $(0.92 \mathrm{mg}, 7.12 \mu \mathrm{mol})$ and TMP $(5.14 \mathrm{mg}, 42.42 \mu \mathrm{mol})$ in $\mathrm{CH}_{2} \mathrm{Cl}_{2}$ $(1 \mathrm{~mL})$ according to GP 6 for $2.5 \mathrm{~h}$. The mixture was then taken up with $\mathrm{Et}_{2} \mathrm{O}(20 \mathrm{~mL})$ and the crude product obtained after usual aqueous work-up (GP 2) was crystallized from $\mathrm{CH}_{2} \mathrm{Cl}_{2} /$ pentane to give $O$-MOM protected Hormaomycin $82\left(8.0 \mathrm{mg}, 96 \%, R_{\mathrm{f}}=0.36\right.$ acetone/hexanes 1:2) as a colorless glass which was used for the next step without any characterization.

Hormaomycin (1): $O$-MOM protected Hormaomycin $82(8.0 \mathrm{mg}, 6.82 \mu \mathrm{mol})$ was deprotected

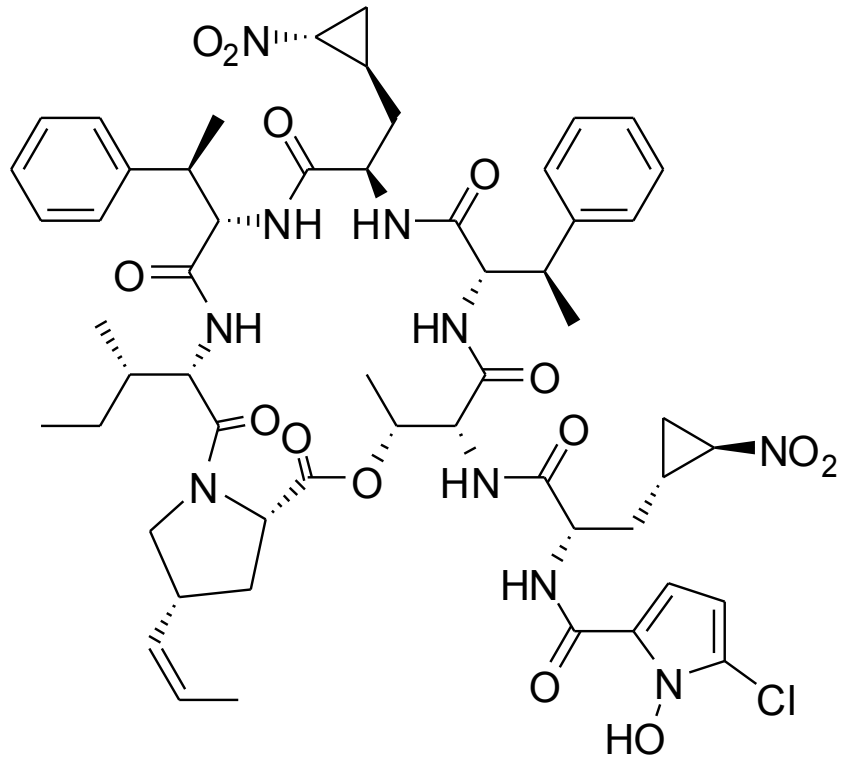
using $\mathrm{MgBr}_{2} \times \mathrm{Et}_{2} \mathrm{O}(52 \mathrm{mg}, 201.36 \mu \mathrm{mol})$ and $\mathrm{EtSH}(0.10 \mathrm{~mL}, 1.9 \mathrm{mmol})$ in $\mathrm{CH}_{2} \mathrm{Cl}_{2}$ $(10 \mathrm{~mL})$ according to $\mathrm{GP} 7$ for $3 \mathrm{~h}$. The mixture was taken up with EtOAc and the crude product obtained after usual aqueous work-up was crystallized from $\mathrm{CH}_{2} \mathrm{Cl}_{2} /$ pentane to give 1 (5.5 $\mathrm{mg}, 72 \%$, $50 \%$ on 5 steps from 75 ) as a white solid, which was finally purified with preparative 
HPLC. $R_{\mathrm{f}}=0.24$ acetone/hexanes 3:7; Preparative HPLC: isocratic, $62 \% \mathrm{~B}$ for $15 \mathrm{~min}$, then gradient $62 \% \rightarrow 100 \% \mathrm{~B}$ for $1 \mathrm{~min}$, then isocratic $100 \% \mathrm{~B}$ for $4 \mathrm{~min}$, then gradient $100 \% \rightarrow 62 \% \mathrm{~B}$ for $1 \mathrm{~min}$, then isocratic $62 \% \mathrm{~B}$ for $10 \mathrm{~min}, \mathrm{t}_{\mathrm{R}}=5.7 \mathrm{~min}$, purity $>98 \%$; analytical HPLC: gradient $20 \% \rightarrow 100 \% \mathrm{~B}$ for $20 \mathrm{~min}$, then isocratic $100 \% \mathrm{~B}$ for $5 \mathrm{~min} \mathrm{t}_{\mathrm{R}}=15.3 \mathrm{~min}$, purity $>$ $98 \%$; $[\alpha]_{\mathrm{D}}{ }^{20} 20.0(\mathrm{c}=0.1, \mathrm{MeOH}) ;{ }^{1} \mathrm{H}$ NMR $(600 \mathrm{MHz}, \mathrm{CDCl} 3): \delta=-0.71--0.63(\mathrm{~m}, 1 \mathrm{H}),-$ $0.20-0.10(\mathrm{~m}, 1 \mathrm{H}), 0.23-0.32(\mathrm{~m}, 1 \mathrm{H}), 0.49-0.56(\mathrm{~m}, 1 \mathrm{H}), 0.88(\mathrm{t}, J=7.4 \mathrm{~Hz}, 3 \mathrm{H}), 0.95-$ $1.01(\mathrm{~m}, 1 \mathrm{H}), 1.02(\mathrm{~d}, J=6.9 \mathrm{~Hz}, 3 \mathrm{H}), 1.04-1.14(\mathrm{~m}, 1 \mathrm{H}), 1.17-1.35(\mathrm{~m}, 1 \mathrm{H}), 1.30(\mathrm{~d}$, $J=7.0 \mathrm{~Hz}, 3 \mathrm{H}), 1.39$ (d, $J=7.3 \mathrm{~Hz}, 3 \mathrm{H}), 1.47-1.54(\mathrm{~m}, 1 \mathrm{H}), 1.53$ (d, $J=6.9 \mathrm{~Hz}, 3 \mathrm{H}), 1.55-$ $1.62(\mathrm{~m}, 1 \mathrm{H}), 1.67$ (dd, $J=6.9 \mathrm{~Hz}, 1.6 \mathrm{~Hz}, 3 \mathrm{H}), 1.75-1.84$ (m, $2 \mathrm{H}), 1.85-1.94$ (m, $3 \mathrm{H}), 2.30$ 2.40 (m, $1 \mathrm{H}), 2.88-2.91(\mathrm{~m}, 1 \mathrm{H}), 2.96-3.02(\mathrm{~m}, 1 \mathrm{H}), 3.22-3.31(\mathrm{~m}, 2 \mathrm{H}), 3.43-3.50$ (m, $1 \mathrm{H})$, 3.62-3.70 (m, $1 \mathrm{H}), 3.93-4.00(\mathrm{~m}, 1 \mathrm{H}), 4.01-4.08(\mathrm{~m}, 1 \mathrm{H}), 4.26(\mathrm{dd}, J=11.5 \mathrm{~Hz}, 6.0 \mathrm{~Hz}, 1 \mathrm{H})$, $4.38(\mathrm{dd}, J=10.6 \mathrm{~Hz}, 10.6 \mathrm{~Hz}, 1 \mathrm{H}), 4.46(\mathrm{dd}, J=9.4 \mathrm{~Hz}, 4.5 \mathrm{~Hz}, 1 \mathrm{H}), 4.57$ (dd, $J=9.3 \mathrm{~Hz}$, $2.3 \mathrm{~Hz}, 1 \mathrm{H}), 4.66(\mathrm{dd}, J=9.0 \mathrm{~Hz}, 9.0 \mathrm{~Hz}, 1 \mathrm{H}), 5.08-5.14(\mathrm{~m}, 1 \mathrm{H}), 5.22-5.28(\mathrm{~m}, 1 \mathrm{H})$, $5.40(\mathrm{qd}, J=6.9 \mathrm{~Hz}, 2.4 \mathrm{~Hz}, 1 \mathrm{H}), 5.58-5.65(\mathrm{~m}, 1 \mathrm{H}), 6.13(\mathrm{~d}, J=4.7 \mathrm{~Hz}, 1 \mathrm{H}), 6.56(\mathrm{~d}$, $J=6.5 \mathrm{~Hz}, 1 \mathrm{H}), 6.80(\mathrm{~d}, J=9.3 \mathrm{~Hz}, 1 \mathrm{H}), 6.82(\mathrm{~d}, J=4.7 \mathrm{~Hz}, 1 \mathrm{H}), 6.98-7.03$ (m, $1 \mathrm{H}), 7.09$ $7.18(\mathrm{~m}, 5 \mathrm{H}), 7.20-7.27(\mathrm{~m}, 7 \mathrm{H}), \quad 8.05(\mathrm{~d}, J=9.1 \mathrm{~Hz}, 1 \mathrm{H}), 9.06(\mathrm{~d}, J=9.3 \mathrm{~Hz}, 1 \mathrm{H})$; ${ }^{13} \mathrm{C}$ NMR $\left(150.8 \mathrm{MHz}, \mathrm{CDCl}_{3}\right): \delta=10.50(+), 13.24(+), 13.33(+), 14.94(+), 16.99(+), 17.41$ $(-), 17.74(+), 20.00(+), 21.66(+), 24.90(-), 26.88(-), 33.02(-), 35.03(-), 35.51(-), 36.66$ $(-), 37.97(+), 39.24(+), 41.75(+), 50.99(+), 51.79\left(_{(+)}, 52.78(-), 54.61(+), 54.93(+), 58.11\right.$ $(+), 59.12(+), 59.86(+), 60.04(+), 61.37(+), 69.07(+), 103.59(+), 109.85(+), 119.86\left(\mathrm{C}_{\text {quat }}\right)$, $121.55\left(\mathrm{C}_{\text {quat }}\right), 126.98(+), 127.17(+), 127.44(+), 127.47(+), 127.67(+), 128.33(+), 128.49(+)$, $128.64(+), 141.55\left(\mathrm{C}_{\text {quat }}\right), 142.11\left(\mathrm{C}_{\text {quat }}\right), 159.27\left(\mathrm{C}_{\text {quat }}\right), 168.54\left(\mathrm{C}_{\text {quat }}\right), 168.73\left(\mathrm{C}_{\text {quat }}\right), 169.75$ $\left(\mathrm{C}_{\text {quat }}\right), 170.74\left(\mathrm{C}_{\text {quat }}\right), 171.26\left(\mathrm{C}_{\text {quat }}\right), 171.55\left(\mathrm{C}_{\text {quat }}\right), 172.86\left(\mathrm{C}_{\text {quat }}\right)$; MS-ESI: positive, $\mathrm{m} / \mathrm{z}=292$ (100), $\left.1151(80, \mathrm{M}+\mathrm{Na}\rceil^{+}\right)$; negative, $\left.\mathrm{m} / \mathrm{z}=1127(100, \mathrm{M}-\mathrm{H}\rceil^{-}\right)$.

\subsection{All-peptide aza-analogue of Hormaomycin}

MeZ-(R)-Asn-OH (84): ${ }^{[110]} \mathrm{NaHCO}_{3}(0.520 \mathrm{~g}, 6.18 \mathrm{mmol})$ and then a solution of MeZOSu

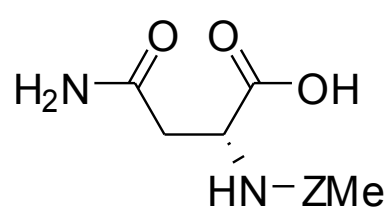

$(0.775 \mathrm{~g}, 2.97 \mathrm{mmol})$ in acetone $(7 \mathrm{~mL})$ were added to a vigorously stirred solution of $(R)$-aspargine $(0.442 \mathrm{~g}, 2.94 \mathrm{mmol})$ in water $(10 \mathrm{~mL})$, and stirring was continued for $3 \mathrm{~h}$ (if a precipitate formed, acetone and/or water was added to obtain a homogeneous solution). The mixture was then concentrated under reduced pressure, diluted with water $(40 \mathrm{~mL})$ and washed with $\mathrm{CH}_{2} \mathrm{Cl}_{2}(3 \times 10 \mathrm{~mL})$. The $\mathrm{pH}$ of the water fraction was adjusted to $\sim 1$ with solid $\mathrm{KHSO}_{4}$, the 
resulting precipitate was filtered off, washed with $\mathrm{H}_{2} \mathrm{O}(5 \times 20 \mathrm{~mL}), \mathrm{Et}_{2} \mathrm{O}(5 \times 20 \mathrm{~mL})$ and dried to give $84(0.75 \mathrm{~g}, 2.67 \mathrm{mmol}, 91 \%)$ as a colorless solid. M.p. $181-183{ }^{\circ} \mathrm{C} ;[\alpha]_{\mathrm{D}}{ }^{20}=6.5(\mathrm{c}=1.00$, DMF); ${ }^{1} \mathrm{H}$ NMR (500 MHz, [D6]acetone): $\delta=2.30$ (s, $\left.3 \mathrm{H}\right), 2.50-3.55$ (bs, $\left.3 \mathrm{H}\right), 2.65-2.85$ (m, $2 \mathrm{H}), 4.39-4.53(\mathrm{~m}, 1 \mathrm{H}), 5.03(\mathrm{~s}, 2 \mathrm{H}), 6.39-6.61(\mathrm{bs}, 1 \mathrm{H}), 7.15(\mathrm{~d}, J=8.0 \mathrm{~Hz}, 2 \mathrm{H}), 7.26(\mathrm{~d}$, $J=8.0 \mathrm{~Hz}, 2 \mathrm{H}) ;{ }^{13} \mathrm{C}$ NMR (125.7 MHz, [D6]DMSO): $\delta=20.7(+), 36.7(-), 50.5(+), 65.3(-)$, $127.8(+), 128.8(+), 133.8\left(\mathrm{C}_{\text {quat }}\right), 137.0\left(\mathrm{C}_{\text {quat }}\right), 155.7\left(\mathrm{C}_{\text {quat }}\right), 170.7\left(\mathrm{C}_{\text {quat }}\right), 173.0\left(\mathrm{C}_{\text {quat }}\right)$.

$N_{\alpha}$-MeZ-2,3-diaminopropionic acid (MeZ-Dap-OH, 85): ${ }^{[110]}$ Iodobenzene bis(trifluoroacetate)<smiles>C[14CH2]NCC(=O)O</smiles>
(1.46 g, $3.40 \mathrm{mmol})$ and 84 were suspended by stirring in $50 \%(\mathrm{v} / \mathrm{v})$ aqueous DMF (20 mL). After $15 \mathrm{~min}$, pyridine (0.367 g, $4.64 \mathrm{mmol})$ was added, and the mixture was stirred for an additional $5 \mathrm{~h}$. The emulsion formed was evaporated at $40-45^{\circ} \mathrm{C}$ under reduced pressure. The residue was taken up with water $(2 \times 15 \mathrm{~mL})$, which was evaporated under reduced pressure. The residual oil was taken up in water $(50 \mathrm{~mL})$ and washed with chloroform $(3 \times 10 \mathrm{~mL})$. The aqueous layer was once more concentrated in vacuo, and the residue was dissolved in ethanol $(20 \mathrm{~mL})$. The $\mathrm{pH}$ value was adjusted to about 7 with pyridine, and the formed suspension was left at $4{ }^{\circ} \mathrm{C}$ for $12 \mathrm{~h}$. The precipitate was filtered off and washed with ether $(5 \times 20 \mathrm{~mL})$ to give, after drying, $N_{\alpha}$-protected diamino acid $85(0.51 \mathrm{~g}, 87 \%)$ as a colorless powder. $R_{\mathrm{f}}=0.32$ (MeCN/AcOH/ $\mathrm{H}_{2} \mathrm{O}$ 10:1:1); m.p. $210-216{ }^{\circ} \mathrm{C}$ (decomp.); $[\alpha]_{\mathrm{D}}{ }^{20} 38.1$ (c=0.31, $\left.0.1 \mathrm{~N} \mathrm{HCl}\right) ;{ }^{1} \mathrm{H}$ NMR (300 MHz, DCl in $\left.\mathrm{D}_{2} \mathrm{O}\right): \delta=2.28(\mathrm{~s}, 3 \mathrm{H}), 3.28(\mathrm{dd}, J=12.6 \mathrm{~Hz}, 9.6 \mathrm{~Hz}, 1 \mathrm{H}), 3.49(\mathrm{dd}$, $J=12.6 \mathrm{~Hz}, 4.5 \mathrm{~Hz}, 1 \mathrm{H}), 4.44-4.55(\mathrm{~m}, 1 \mathrm{H}), 5.07$ (s, $2 \mathrm{H}), 7.22(\mathrm{~d}, J=7.5 \mathrm{~Hz}, 2 \mathrm{H}), 7.28$ (d, $J=7.5 \mathrm{~Hz}, 2 \mathrm{H})$.

MeZ-Dap-OMe hydrochloride (86): ${ }^{[110]}$ To a solution of thionyl chloride (0.52 $\left.\mathrm{mL}, 7.26 \mathrm{mmol}\right)$ in ZMe OMe anhydrous $\mathrm{MeOH}(10 \mathrm{~mL})$ at $-20^{\circ} \mathrm{C}$ was added with stirring after $10 \mathrm{~min}$ the amino acid $85(0.50 \mathrm{~g}, 1.98 \mathrm{mmol})$. The resulting thick suspension was stirred at $20^{\circ} \mathrm{C}$ for $24 \mathrm{~h}$ to give a clear solution, which was then left at $20{ }^{\circ} \mathrm{C}$ for $16 \mathrm{~h} . \mathrm{Et}_{2} \mathrm{O}(40 \mathrm{~mL})$ was added to complete the precipitation, and the solid was filtered off to give $86(0.47 \mathrm{~g}, 78 \%)$ as long colorless needles. The mother liquor was concentrated, and the residue was recrystallized from $\mathrm{MeOH} / \mathrm{Et}_{2} \mathrm{O}$ to give a second crop of 86 (26 mg, 83\% overall yield). M.p. $159-161{ }^{\circ} \mathrm{C} ;[\alpha]_{\mathrm{D}}{ }^{20}=32.3\left(\mathrm{c}=0.86\right.$, DMSO); ${ }^{1} \mathrm{H}$ NMR (250 MHz, [D6]DMSO): $\delta=2.28$ (s, $3 \mathrm{H}), 2.98-3.29$ (m, $2 \mathrm{H}), 3.66$ (s, $3 \mathrm{H}), 4.37-4.49$ (m, $1 \mathrm{H})$, 5.08 (s, 2 H), 7.17 (d, J=7.9 Hz, 2 H), 7.25 (d, J=7.9 Hz, 2 H), 7.52 (d, J=8.3 Hz, $1 \mathrm{H}), 8.15-$ $8.55(\mathrm{bs}, 3 \mathrm{H}) ;{ }^{13} \mathrm{C} \mathrm{NMR}(62.9 \mathrm{MHz},[\mathrm{D} 6] \mathrm{DMSO}): \delta=21.0(+), 39.2(-), 52.0(+), 52.8(+), 66.0$ $(-), 128.2(+), 129.1(+), 133.8\left(\mathrm{C}_{\text {quat }}\right), 137.4\left(\mathrm{C}_{\text {quat }}\right), 156.3\left(\mathrm{C}_{\text {quat }}\right), 173.6\left(\mathrm{C}_{\text {quat }}\right)$. 
Boc-(4-Pe)Pro-[MeZ-Dap]-OMe (87): ${ }^{[110]}$ Compound 86 (0.127 g, $\left.0.42 \mathrm{mmol}\right)$ was coupled with the $N$-Boc protected (4-propenyl)proline $\mathbf{1 0}(0.11 \mathrm{~g}$,<smiles>[Z10]N[C@@H](CNC(=O)[C@@H]1C[C@H](/C=C\C)CN1C(=O)OC(C)(C)C)C(C)=O</smiles>
$0.431 \mathrm{mmol})$ by treatment with EDC $(85 \mathrm{mg}, 0.44 \mathrm{mmol})$, HOAt (60 mg, $0.44 \mathrm{mmol})$ and TMP (0.314 g, $2.59 \mathrm{mmol})$ in $\mathrm{CH}_{2} \mathrm{Cl}_{2}(5 \mathrm{~mL})$ according to GP 2 for $16 \mathrm{~h}$. The crude product obtained after the usual aqueous workup (GP 2) was further purified by column chromatography (silica gel, eluted with acetone/hexane 2:5, $R_{\mathrm{f}}=0.13$ ) to give an oily residue which was triturated with pentane to furnish the dipeptide ester $87(0.14 \mathrm{~g}, 66 \%)$ as a colorless solid. The mother liquor was cooled to $4{ }^{\circ} \mathrm{C}$, and the precipitate was filtered off to give a second crop of the 87 (10 mg, $71 \%$ overall yield). M.p. $160-162{ }^{\circ} \mathrm{C}$; $[\alpha]_{\mathrm{D}}^{20}-41.4\left(\mathrm{c}=0.35, \mathrm{CHCl}_{3}\right) ;{ }^{1} \mathrm{H} \mathrm{NMR}\left(300 \mathrm{MHz}, \mathrm{CDCl}_{3}\right): \delta=1.40(\mathrm{~s}, 9 \mathrm{H}), 1.64(\mathrm{dd}$, $J=6.9 \mathrm{~Hz}, 1.8 \mathrm{~Hz}, 3 \mathrm{H}), 1.78-2.04(\mathrm{~m}, 1 \mathrm{H}), 2.11-2.57(\mathrm{~m}, 1 \mathrm{H}), 2.34(\mathrm{~s}, 3 \mathrm{H}), 2.97-3.15(\mathrm{~m}$, $1 \mathrm{H}), 2.99$ (dd, $J=9.3 \mathrm{~Hz}, 9.1 \mathrm{~Hz}, 1 \mathrm{H}), 3.51-3.92$ (m, $3 \mathrm{H}), 3.75$ (s, $3 \mathrm{H}), 4.12$ (dd, $J=8.2 \mathrm{~Hz}$, $8.1 \mathrm{~Hz}, 1 \mathrm{H}), 4.34-4.51(\mathrm{~m}, 1 \mathrm{H}), 5.02(\mathrm{~d}, J=12.3 \mathrm{~Hz}, 1 \mathrm{H}), 5.08(\mathrm{~d}, J=12.3 \mathrm{~Hz}, 1 \mathrm{H}), 5.17-$ $5.30(\mathrm{~m}, 1 \mathrm{H}), 5.52(\mathrm{dq}, J=10.5 \mathrm{~Hz}, 6.9 \mathrm{~Hz}, 1 \mathrm{H}), 5.74-6.17(\mathrm{bs}, 1 \mathrm{H}), 6.43-6.85(\mathrm{bs}, 1 \mathrm{H})$, $7.14(\mathrm{~d}, J=8.1 \mathrm{~Hz}, 2 \mathrm{H}), 7.33(\mathrm{~d}, J=8.1 \mathrm{~Hz}, 2 \mathrm{H}) ;{ }^{13} \mathrm{C} \mathrm{NMR}\left(75.5 \mathrm{MHz}, \mathrm{CDCl}_{3}\right): \delta=13.2(+)$, $21.2(+), 28.3(+), 36.0(+), 38.1(-), 40.8(-), 41.5(-), 52.4(-), 52.7(+), 54.3(+), 60.8(+), 61.4$ $(+), 67.0(-), 80.7\left(\mathrm{C}_{\text {quat }}\right), 126.5(+), 128.3(+), 129.1(+), 129.4(+), 133.2\left(\mathrm{C}_{\text {quat }}\right), 137.9\left(\mathrm{C}_{\text {quat }}\right)$, 154.4, $155.1\left(\mathrm{C}_{\text {quat }}\right), 156.3\left(\mathrm{C}_{\text {quat }}\right), 170.2,171.0\left(\mathrm{C}_{\text {quat }}\right), 170.9,173.0\left(\mathrm{C}_{\text {quat }}\right)$.

Boc-(4-Pe)Pro-[MeZ-Dap]-OH (88)::[110] A 40\% aqueous solution of tetra- $n$-butylammonium<smiles>[Z10]N[C@@H](CNC(=O)[C@@H]1C[C@H](/C=C\C)CN1C(=O)OC(C)(C)C)C(C)=O</smiles>
hydroxide $(0.20 \mathrm{~g}, 0.31 \mathrm{mmol})$ was added dropwise to an ice-cold solution of the dipeptide ester $87(0.13 \mathrm{~g}$, $0.26 \mathrm{mmol})$ in THF $(2.0 \mathrm{~mL})$ within $3 \mathrm{~min}$, and stirring was continued at the same temperature for an additional $45 \mathrm{~min}$ (TLC monitoring to detect complete consuming of the starting material). A aq. $1 \mathrm{M} \mathrm{H}_{2} \mathrm{SO}_{4}$ $(1 \mathrm{~mL})$ was then added, and the mixture was diluted with $\mathrm{Et}_{2} \mathrm{O}(50 \mathrm{~mL})$. The organic layer was separated and washed with aq. $1 \mathrm{M} \mathrm{KHSO}_{4}(2 \times 10 \mathrm{~mL})$, water $(5 \times 10 \mathrm{~mL})$, brine $(2 \times 5 \mathrm{~mL})$, dried over $\mathrm{MgSO}_{4}$ and filtered. The filtrate was concentrated under reduced pressure to give the crude product which was finally purified by column chromatography (silica gel, eluted with acetone/hexane $\left.4: 7+2 \% \mathrm{AcOH}, R_{\mathrm{f}}=0.36\right)$ to give dipeptide acid $\mathbf{8 8}(0.126 \mathrm{~g}, 99 \%)$ as an extremely viscous turbid oil. ${ }^{1} \mathrm{H}$ NMR $\left(250 \mathrm{MHz}, \mathrm{CDCl}_{3}\right): \delta=1.31+1.41(2 \times \mathrm{s}, 9 \mathrm{H}), 1.65(\mathrm{~d}$, $J=6.0 \mathrm{~Hz}, 3 \mathrm{H}), 1.75-1.98(\mathrm{~m}, 1 \mathrm{H}), 2.33(\mathrm{~s}, 3 \mathrm{H}), 2.21-2.53(\mathrm{~m}, 1 \mathrm{H}), 2.93-3.21(\mathrm{~m}, 2 \mathrm{H})$, $3.44-3.60(\mathrm{~m}, 2 \mathrm{H}), 3.60-4.03(\mathrm{~m}, 1 \mathrm{H}), 4.03-4.24(\mathrm{~m}, 2 \mathrm{H}), 4.30-4.39+4.41-4.54(2 \times \mathrm{bs}$, 
$1 \mathrm{H}), 5.04(\mathrm{~s}, 2 \mathrm{H}), 5.15-5.32(\mathrm{~m}, 1 \mathrm{H}), 5.55(\mathrm{dq}, J=10.8 \mathrm{~Hz}, 7.0 \mathrm{~Hz}, 1 \mathrm{H}), 6.25(\mathrm{~d}, J=6.5 \mathrm{~Hz}$, $1 \mathrm{H}), 7.12(\mathrm{~d}, J=7.5 \mathrm{~Hz}, 2 \mathrm{H}), 7.22(\mathrm{~d}, J=7.5 \mathrm{~Hz}, 2 \mathrm{H}), 7.42-7.65$ (bs, $1 \mathrm{H})$.

Boc-(4-Pe)Pro-[MeZ-Dap]-MeF-(S)NcpA-MeF-Ile-ODCPM (89): :[110] Tetrapeptide 66 (180 mg,

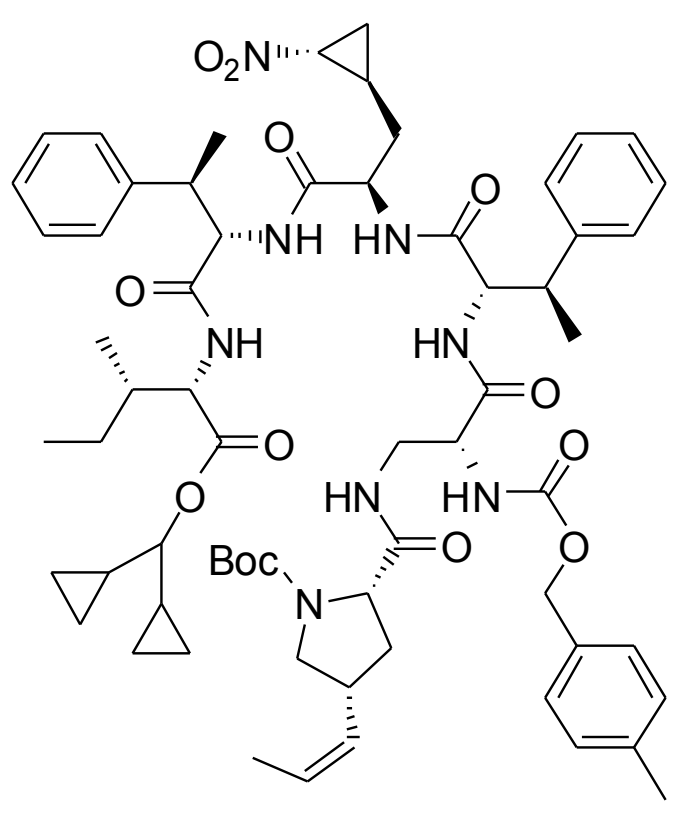
$0.21 \mathrm{mmol}$ ) was deprotected according to GP 1 in THF $(2 \mathrm{~mL})$, taken up with anhydrous $\mathrm{CH}_{2} \mathrm{Cl}_{2}(5 \mathrm{~mL})$, dipeptide acid $\mathbf{8 8}(0.114 \mathrm{~g}, 0.23 \mathrm{mmol})$, HATU (96 mg, $0.25 \mathrm{mmol}$ ) and HOAt (31 $\mathrm{mg}, 0.23 \mathrm{mmol})$ were added and the reaction mixture was cooled to $4{ }^{\circ} \mathrm{C}$. After this, a solution of DIEA (29 mg, $0.22 \mathrm{mmol})$ and TMP (75 mg, $0.62 \mathrm{mmol})$ in $\mathrm{CH}_{2} \mathrm{Cl}_{2}(2 \mathrm{~mL})$ were added at the same temperature within $5 \mathrm{~min}$. The temperature was allowed to reach $20{ }^{\circ} \mathrm{C}$, and stirring continued for an additional $15 \mathrm{~h}$. After aqueous work-up according to GP 2 and two recrystallizations from EtOAc/hexanes 1:2, triprotected peptide 89 (185 mg, 79\%) was obtained as a colorless powder. $R_{\mathrm{f}}=0.46$ (EtOAc/hexanes 1:1); m.p. $125-127^{\circ} \mathrm{C},[\alpha]_{\mathrm{D}}{ }^{20}-29.0(\mathrm{c}=0.2, \mathrm{THF}) ;{ }^{1} \mathrm{H}$ NMR $\left(600 \mathrm{MHz}, \mathrm{CDCl}_{3}\right): \delta=0.27-0.33(\mathrm{~m}$, $1 \mathrm{H}), 0.33-0.44(\mathrm{~m}, 3 \mathrm{H}), 0.44-0.49(\mathrm{~m}, 1 \mathrm{H}), 0.49-0.57$ (m, $2 \mathrm{H}), 0.59-0.65(\mathrm{~m}, 1 \mathrm{H}), 0.75$ (d, $J=6.8 \mathrm{~Hz}, 3 \mathrm{H}), 0.89(\mathrm{t}, J=7.3 \mathrm{~Hz}, 3 \mathrm{H}), 0.95-1.11(\mathrm{~m}, 4 \mathrm{H}), 1.11-1.60(\mathrm{~m}, 5 \mathrm{H}), 1.24(\mathrm{~d}$, $J=7.5 \mathrm{~Hz}, 3 \mathrm{H}), 1.27(\mathrm{~d}, J=7.5 \mathrm{~Hz}, 3 \mathrm{H}), 1.36(\mathrm{~s}, 9 \mathrm{H}), 1.43$ (d, $J=6.5 \mathrm{~Hz}, 3 \mathrm{H}), 1.66$ (dd, $J=6.9 \mathrm{~Hz}, 1.7 \mathrm{~Hz}, 3 \mathrm{H}), 1.68-1.76(\mathrm{~m}, 1 \mathrm{H}), 1.80-1.90(\mathrm{~m}, 1 \mathrm{H}), 2.30$ (s, 3 H), 2.35-2.42 (m, $1 \mathrm{H}), 3.06-3.13(\mathrm{~m}, 1 \mathrm{H}), 3.13(\mathrm{t}, J=10.5 \mathrm{~Hz}, 1 \mathrm{H}), 3.18-3.31(\mathrm{~m}, 2 \mathrm{H}), 3.67(\mathrm{dd}, J=10.2 \mathrm{~Hz}$, $7.8 \mathrm{~Hz}, 1 \mathrm{H}), 3.80-3.84(\mathrm{~m}, 1 \mathrm{H}), 4.02-4.07(\mathrm{~m}, 1 \mathrm{H}), 4.14(\mathrm{dd}, J=10.7 \mathrm{~Hz}, 6.2 \mathrm{~Hz}, 1 \mathrm{H})$, $4.33(\mathrm{dd}, J=8.9 \mathrm{~Hz}, 4.6 \mathrm{~Hz}, 1 \mathrm{H}), 4.37(\mathrm{dd}, J=9.5 \mathrm{~Hz}, 2.2 \mathrm{~Hz}, 1 \mathrm{H}), 4.45(\mathrm{dd}, J=9.9 \mathrm{~Hz}$, $6.9 \mathrm{~Hz} 1 \mathrm{H}), 4.57(\mathrm{dt}, J=9.6 \mathrm{~Hz}, 5.1 \mathrm{~Hz}, 1 \mathrm{H}), 4.62$ (t, $J=10.3 \mathrm{~Hz}, 1 \mathrm{H}), 4.96-5.06(\mathrm{~m}, 2 \mathrm{H})$, $5.22-5.28(\mathrm{~m}, 1 \mathrm{H}), 5.46-5.51(\mathrm{~m}, 1 \mathrm{H}), 5.52-5.58(\mathrm{~m}, 1 \mathrm{H}), 6.54(\mathrm{~d}, J=8.2 \mathrm{~Hz}, 1 \mathrm{H}), 6.89(\mathrm{~d}$, $J=8.9 \mathrm{~Hz}, 1 \mathrm{H}), 6.99(\mathrm{~d}, J=9.6 \mathrm{~Hz}, 1 \mathrm{H}), 7.06-7.12(\mathrm{~m}, 2 \mathrm{H}), 7.14-7.25(\mathrm{~m}, 12 \mathrm{H}) 7.49$ (d, $J=9.8 \mathrm{~Hz}, 1 \mathrm{H}), 7.86(\mathrm{~d}, J=5.9 \mathrm{~Hz}, 1 \mathrm{H}) ;{ }^{13} \mathrm{C} \mathrm{NMR}\left(62.9 \mathrm{MHz}, \mathrm{CDCl}_{3}\right): \delta=2.48(-), 2.82(-)$, $2.90(-), 3.01(-), 11.59(+), 13.20(+), 14.16(+), 14.64(+), 15.57(+), 17.77(+), 18.41(-)$, $18.86(+), 19.43(+), 21.11(+), 21.77(+), 25.23(-), 26.85(-), 28.23(+), 31.46(-), 31.53(-)$, $36.28(-), 36.32(+), 37.30(+), 40.45(+), 42.00(+), 50.62(+), 52.08(+), 56.43(+), 59.30(+)$, $59.49(+), 61.01(+), 61.62(+), 61.99(+), 66.89(-), 70.53(+), 80.93\left(\mathrm{C}_{\text {quat }}\right), 83.24(+), 127.00$ $(+), 127.06(+), 127.10(+), 127.60(+), 127.69(+), 128.47(+), 128.63(+), 128.68(+), 128.82$ $(+), 128.93(+), 133.21\left(\mathrm{C}_{\text {quat }}\right), 137.83\left(\mathrm{C}_{\text {quat }}\right), 141.73\left(\mathrm{C}_{\text {quat }}\right), 141.90\left(\mathrm{C}_{\text {quat }}\right), 154.76\left(\mathrm{C}_{\text {quat }}\right)$, 
$155.75\left(\mathrm{C}_{\text {quat }}\right), 170.38\left(\mathrm{C}_{\text {quat }}\right), 170.43\left(\mathrm{C}_{\text {quat }}\right), 170.79\left(\mathrm{C}_{\text {quat }}\right), 171.37\left(\mathrm{C}_{\text {quat }}\right), 173.41\left(\mathrm{C}_{\text {quat }}\right), 174.06$ $\left(\mathrm{C}_{\text {quat }}\right)$.

$N-M e Z$ protected cyclohexapeptide (91):[110] To the hexapeptide 89 (0.188 g, 0.165 mmol)

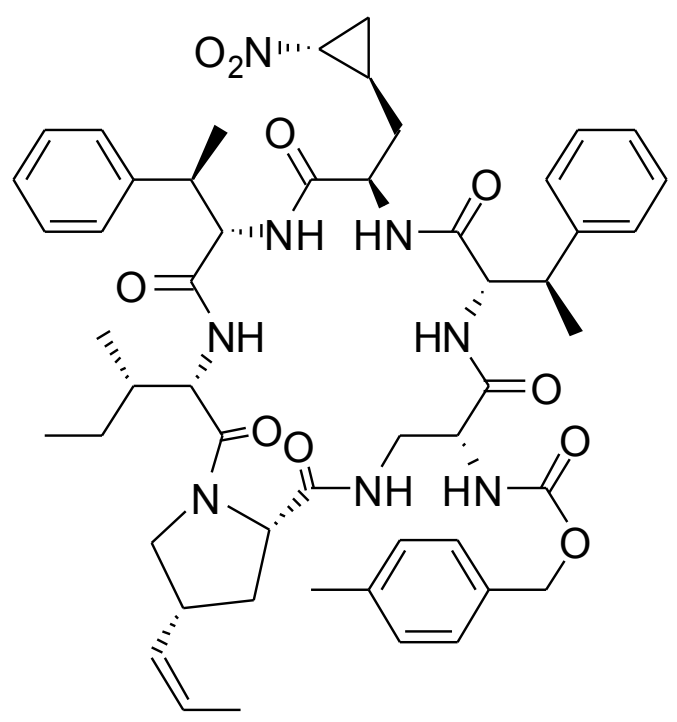
$2 \mathrm{MHCl}$ in EtOAc $(2 \mathrm{~mL})$ was added, the reaction mixture was stirred for 45 min at ambient temperature in the dark place and then was concentrated under reduced pressure without any heating. The residue was triturated with dry $\mathrm{Et}_{2} \mathrm{O}$ to give deprotected material $\mathbf{9 0}$ as a white solid, which was taken up with anhydrous $\mathrm{CH}_{2} \mathrm{Cl}_{2} \quad(1.5 \mathrm{~L})$ and cyclized employing HATU $(2 \times 0.073 \mathrm{~g}, 2 \times 0.192 \mathrm{mmol})$ and HOAt $(2 \times 0.022 \mathrm{~g}$, $2 \times 0.163 \mathrm{mmol})$ and solution of DIEA $(2 \times 0.062 \mathrm{~g}$, $2 \times 0.480 \mathrm{mmol})$ in $\mathrm{CH}_{2} \mathrm{Cl}_{2}(2 \times 50 \mathrm{~mL})$ according to GP 4 for $16 \mathrm{~h}$. After this, the solvent was removed under reduced pressure, the residue was taken up with $\mathrm{Et}_{2} \mathrm{O}(50 \mathrm{~mL})$, and after usual aqueous work-up (GP 2), drying and filtration, the organic layer was concentrated under reduced pressure. The residue was purified first by column chromatography (silica gel, eluted with acetone/hexanes $2: 3, R_{\mathrm{f}}=0.31$ ) and then by recrystallization $\left(\mathrm{Et}_{2} \mathrm{O} /\right.$ pentane $)$ to give crude product $(0.12 \mathrm{~g})$, which was finally purified by preparative HPLC to give cyclohexapeptide 91 (86 mg, 53\% on 2 steps) as a white solid. Preparative HPLC: isocratic, 70\% B for $30 \mathrm{~min}, \mathrm{t}_{\mathrm{R}}=21.5 \mathrm{~min}$, purity $>98 \%$; analytical HPLC: isocratic, $62 \% \mathrm{~B}$ for, $\mathrm{t}_{\mathrm{R}}=22.9 \mathrm{~min}$, purity $>98 \%[\alpha]_{\mathrm{D}}{ }^{20}-15.5\left(\mathrm{c}=0.20, \mathrm{CHCl}_{3}\right) ;{ }^{1} \mathrm{H} \mathrm{NMR}$ $\left(600 \mathrm{MHz}, \mathrm{CDCl}_{3}\right): \delta=0.61-0.67(\mathrm{~m}, 1 \mathrm{H}), 0.72(\mathrm{~d}, J=6.6 \mathrm{~Hz}, 3 \mathrm{H}), 0.71-0.77(\mathrm{~m}, 1 \mathrm{H})$, $0.79(\mathrm{t}, J=7.2 \mathrm{~Hz}, 3 \mathrm{H}), 1.04-1.12(\mathrm{~m}, 1 \mathrm{H}), 1.23(\mathrm{~d}, J=6.6 \mathrm{~Hz}, 3 \mathrm{H}), 1.27-1.34(\mathrm{~m}, 1 \mathrm{H})$, $1.37(\mathrm{~d}, J=6.6 \mathrm{~Hz}, 3 \mathrm{H}), 1.37-1.43(\mathrm{~m}, 1 \mathrm{H}), 1.45-1.54(\mathrm{~m}, 1 \mathrm{H}), 1.54-1.57(\mathrm{~m}, 1 \mathrm{H}), 1.57(\mathrm{~d}$, $J=6.6 \mathrm{~Hz}, 3 \mathrm{H}), 1.65(\mathrm{dd}, J=6.6 \mathrm{~Hz}, 1.5 \mathrm{~Hz}, 3 \mathrm{H}), 1.66-1.76(\mathrm{~m}, 2 \mathrm{H}), 2.20-2.25(\mathrm{~m}, 1 \mathrm{H})$, $2.35(\mathrm{~s}, 3 \mathrm{H}), 3.01-3.07(\mathrm{~m}, 1 \mathrm{H}), 3.15-3.28(\mathrm{~m}, 2 \mathrm{H}), 3.54(\mathrm{dq}, J=7.2 \mathrm{~Hz}, 6.6 \mathrm{~Hz}, 1 \mathrm{H})$, $3.71(\mathrm{dd}, J=6.0 \mathrm{~Hz}, 5.4 \mathrm{~Hz}, 1 \mathrm{H}), 3.74-3.77$ (m, $1 \mathrm{H}), 3.98$ (dd, $J=10.5 \mathrm{~Hz}, 6.3 \mathrm{~Hz}, 1 \mathrm{H}), 4.01-$ 4.08 (m, $1 \mathrm{H}), 4.46-4.54(\mathrm{~m}, 2 \mathrm{H}), 4.52-4.55$ (m, $1 \mathrm{H}), 4.67-4.70(\mathrm{~m}, 1 \mathrm{H}), 5.03$ (d, J=12.0 Hz, $1 \mathrm{H}), 5.15(\mathrm{~d}, J=12.0 \mathrm{~Hz}, 1 \mathrm{H}), 5.19-5.25(\mathrm{~m}, 1 \mathrm{H}), 5.39(\mathrm{qd}, J=6.6 \mathrm{~Hz}, 1.8 \mathrm{~Hz}, 1 \mathrm{H}), 5.56(\mathrm{dq}$, $J=10.8 \mathrm{~Hz}, 6.6 \mathrm{~Hz}, 1 \mathrm{H}), 5.96(\mathrm{~d}, J=5.3 \mathrm{~Hz}, 1 \mathrm{H}), 6.20(\mathrm{~d}, J=7.4 \mathrm{~Hz}, 1 \mathrm{H}), 6.28(\mathrm{~d}, J=$ $9.7 \mathrm{~Hz}, 1 \mathrm{H}), 6.49$ (d, $J=9.4 \mathrm{~Hz}, 1 \mathrm{H}), 7.11-7.37$ (m, $14 \mathrm{H}) 7.32$ (d, $J=7.4 \mathrm{~Hz}, 1 \mathrm{H}), 7.45$ (d, $J=8.7 \mathrm{~Hz}, 1 \mathrm{H}) ;{ }^{13} \mathrm{C} \mathrm{NMR}\left(150.8 \mathrm{MHz}, \mathrm{CDCl}_{3}\right): \delta=10.3(+), 13.3(+), 14.6(+), 17.3(-), 17.7$ $(+), 18.4(+), 21.2(+), 21.3(+), 24.7(-), 32.0(-), 35.4(-), 36.6(+), 39.4(+), 44.5(+), 52.5(-)$, 
$53.3(+), 54.6(+), 58.6(+), 59.0(+), 59.4(+), 60.1(+), 60.7(+), 67.2(-), 72.6(+), 127.1(+)$, $127.2(+), 127.5(+), 127.6(+), 128.3(+), 128.6(+), 128.8(+), 129.2(+), 127.8(+), 128.0(+)$, $133.2\left(\mathrm{C}_{\text {quat }}\right), 137.9\left(\mathrm{C}_{\text {quat }}\right), 140.9\left(\mathrm{C}_{\text {quat }}\right), 142.6\left(\mathrm{C}_{\text {quat }}\right), 156.3\left(\mathrm{C}_{\text {quat }}\right), 169.0\left(\mathrm{C}_{\text {quat }}\right), 170.3\left(\mathrm{C}_{\text {quat }}\right)$, $170.6\left(\mathrm{C}_{\text {quat }}\right), 171.1\left(\mathrm{C}_{\text {quat }}\right), 171.4\left(\mathrm{C}_{\text {quat }}\right), 173.1\left(\mathrm{C}_{\text {quat }}\right)$; MS-ESI: positive mode, $\mathrm{m} / \mathrm{z}(\%)=1001$ $\left.(100, \mathrm{M}+\mathrm{Na}\rceil^{+}\right)$; negative mode, $\left.\mathrm{m} / \mathrm{z}(\%)=977(100, \mathrm{M}-\mathrm{H}\rceil^{-}\right)$.

$\mathrm{N}$-Teoc protected heptapeptide (93): ${ }^{[110]}$ An ethereal solution $(50 \mathrm{~mL})$ of the

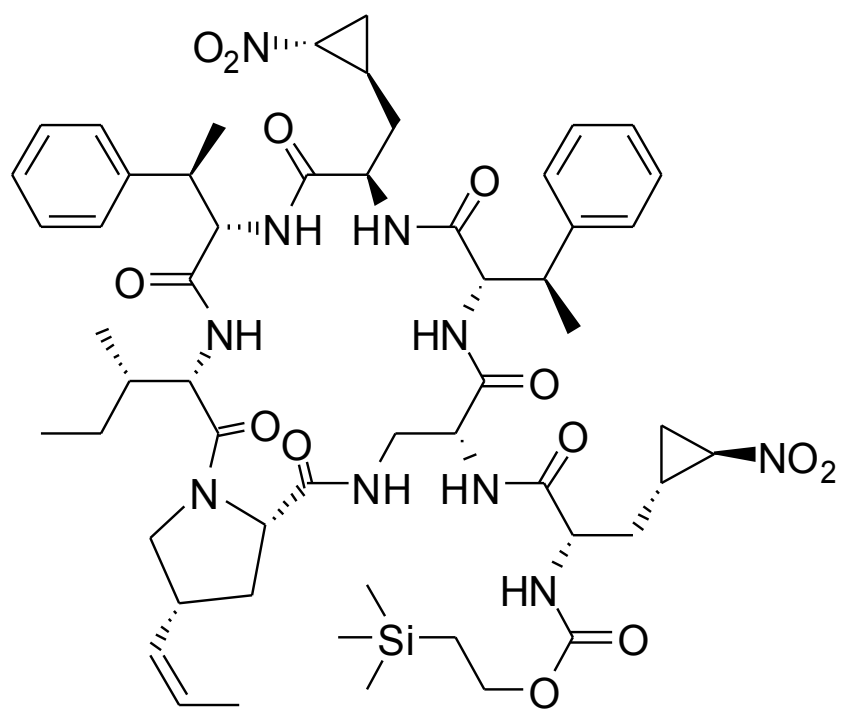
cyclohexylammonium salt of $\mathrm{N}$-Teoc protected $\quad\left(2 S, 1^{\prime} R, 2^{\prime} R\right)-3-\left(2^{\prime}-\right.$ nitrocyclopropyl)alanine 78 (8.1 mg, $19.41 \mu \mathrm{mol})$ was washed with aq. $1 \mathrm{M} \mathrm{H}_{2} \mathrm{SO}_{4}(3 \times 5 \mathrm{~mL})$, aq. $1 \mathrm{M} \mathrm{KHSO}_{4}(2 \times 5 \mathrm{~mL})$, water $(3 \times 5 \mathrm{~mL})$, brine $(2 \times 5 \mathrm{~mL})$, dried, filtered and concentrated under reduced pressure. The resulting $N$-protected amino acid $\mathbf{7 8}$ was dried at 0.02 Torr for $2 \mathrm{~h}$ and then coupled with the cyclohexapeptide 92 [obtained after deprotection of $N$-MeZ protected cyclohexapeptide $91(9.5 \mathrm{mg}, 9.71 \mu \mathrm{mol})$ with $10 \%$ anisole in TFA $(1.1 \mathrm{~mL})$ for $2 \mathrm{~h}$ according to GP 5] using HATU (7.4 mg, $19.46 \mu \mathrm{mol})$, HOAt (2.6 mg, $19.24 \mu \mathrm{mol})$, DIEA (1.25 mg, 9.67 $\mu \mathrm{mol})$ and TMP $(7.04 \mathrm{mg}, 58.10 \mu \mathrm{mol})$ in $\mathrm{CH}_{2} \mathrm{Cl}_{2}(0.7 \mathrm{~mL})$ according to $\mathrm{GP} 6$ for $15 \mathrm{~h}$. The mixture was then diluted with $\mathrm{EtOAc} / \mathrm{Et}_{2} \mathrm{O} 1: 1(20 \mathrm{~mL})$ to give after usual aqueous work-up (GP 2) the crude product $79\left(8.0 \mathrm{mg}, 73 \%, R_{\mathrm{f}}=0.43\right.$ acetone/hexanes $\left.1: 2\right)$ as a colorless glass which was used for the next step without any characterization.

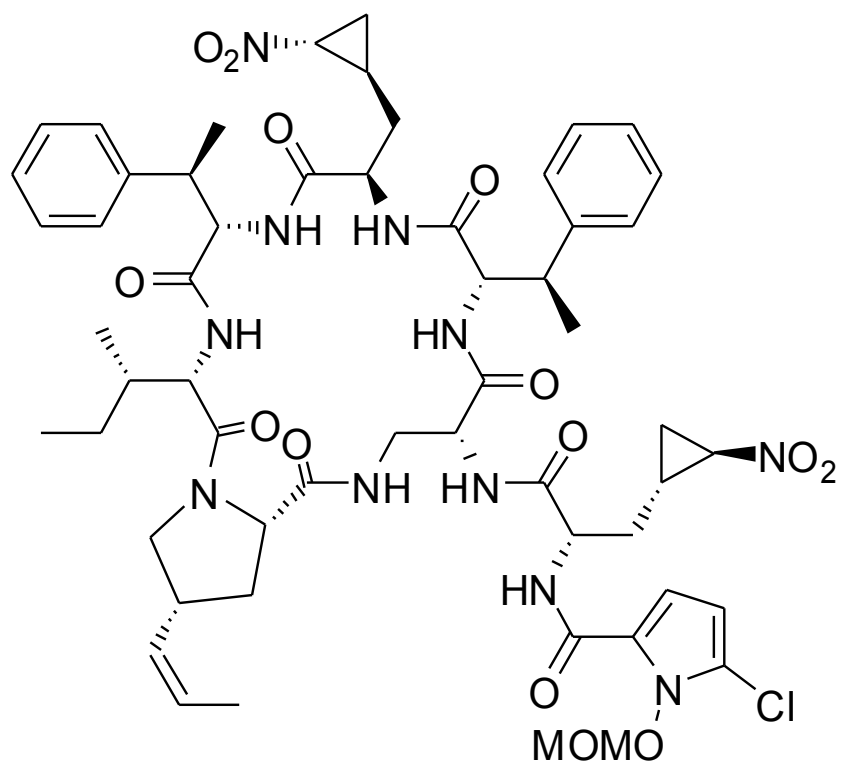

MOM-O-protected Hormaomycin allpeptide analogue (95): ${ }^{[110]}$ Teoc group was cleaved from the compound $\mathbf{9 3}(8.0 \mathrm{mg}, 7.08$ $\mu \mathrm{mol})$ with TFA $(0.6 \mathrm{~mL})$ for $1 \mathrm{~h}$. The mixture was concentrated under reduced pressure at $20^{\circ} \mathrm{C}$ and then taken up with toluene $(3 \times 15 \mathrm{~mL})$ which was distilled off to remove the last traces of TFA. The resulting deprotected peptide $\mathbf{9 4}$ was coupled with $O$-MOM protected acid 81 (2.9 
mg, $14.10 \mu \mathrm{mol})$ using HATU $(5.4 \mathrm{mg}, 14.20 \mu \mathrm{mol})$, DIEA (0.92 mg, $7.12 \mu \mathrm{mol})$ and TMP (5.14 mg, $42.42 \mu \mathrm{mol})$ in $\mathrm{CH}_{2} \mathrm{Cl}_{2}(1 \mathrm{~mL})$ according to GP 6 for $2.5 \mathrm{~h}$. The mixture was then taken up with $\mathrm{Et}_{2} \mathrm{O}(20 \mathrm{~mL})$ and the crude product obtained after usual aqueous work-up (GP 2) was crystallized from $\mathrm{CH}_{2} \mathrm{Cl}_{2} /$ pentane to give $O$-MOM protected all-peptide Hormaomycin analogue 95 (8.0 mg, 96\%, $R_{\mathrm{f}}=0.36$ acetone/hexanes 1:2) as a colorless glass which was used for the next step without any characterization.

Hormaomycin all-peptide analogue (53): $:^{[110]}$ O-MOM protected all-peptide Hormaomycin

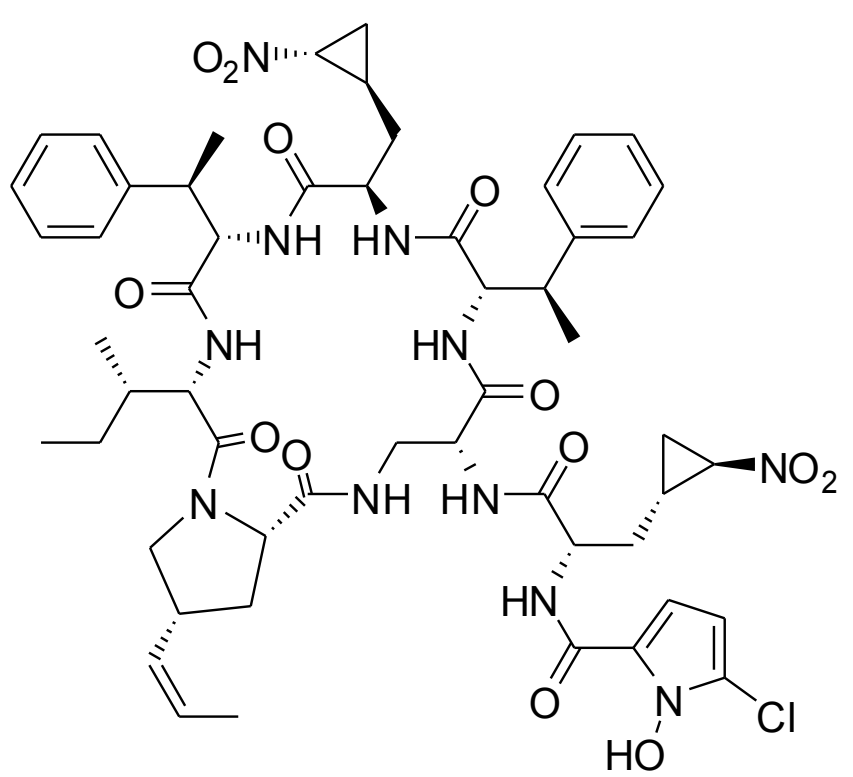
analogue $95(8.0 \mathrm{mg}, 6.82 \mu \mathrm{mol})$ was deprotected using $\mathrm{MgBr}_{2} \times \mathrm{Et}_{2} \mathrm{O}$ (52 mg, $201 \mu \mathrm{mol})$ and $\operatorname{EtSH}(0.10 \mathrm{~mL}, 1.9 \mathrm{mmol})$ in $\mathrm{CH}_{2} \mathrm{Cl}_{2}(10 \mathrm{~mL})$ according to GP 7 for $3 \mathrm{~h}$. The mixture was taken up with EtOAc and the crude product obtained after usual aqueous work-up (GP 2) was crystallized from $\mathrm{CH}_{2} \mathrm{Cl}_{2} /$ pentane to give $\mathbf{5 3}(5.5 \mathrm{mg}$, $72 \%, 50 \%$ on 5 steps from 91) as a white solid, which was finally purified with preparative HPLC. $\quad R_{\mathrm{f}}=0.24$

acetone/hexanes 3:7; preparative HPLC: isocratic, 75\% B for $12 \mathrm{~min}$, then gradient $62 \% \rightarrow 90 \%$ $\mathrm{B}$ for $1 \mathrm{~min}$, then isocratic $90 \% \mathrm{~B}$ for $2 \mathrm{~min}$, then gradient $90 \% \rightarrow 75 \% \mathrm{~B}$ for $1 \mathrm{~min}$, then isocratic $75 \% \mathrm{~B}$ for $14 \mathrm{~min}, \mathrm{t}_{\mathrm{R}}=10.4 \mathrm{~min}$, purity $>98 \%$; analytical HPLC: isocratic, $75 \% \mathrm{~B} \mathrm{t}_{\mathrm{R}}=7.3 \mathrm{~min}$ $[\alpha]_{\mathrm{D}}^{20} 61.0(\mathrm{c}=0.1, \mathrm{MeOH}) ;{ }^{1} \mathrm{H} \mathrm{NMR}\left(600 \mathrm{MHz}, \mathrm{CDCl}_{3}\right): \delta=-0.60--0.54(\mathrm{~m}, 1 \mathrm{H}),-0.20-$ $0.02(\mathrm{~m}, 1 \mathrm{H}), 0.25-0.31(\mathrm{~m}, 1 \mathrm{H}), 0.52(\mathrm{ddd}, J=13.8 \mathrm{~Hz}, 4.8 \mathrm{~Hz}, 4.8 \mathrm{~Hz}, 1 \mathrm{H}), 0.89(\mathrm{t}$, $J=7.2 \mathrm{~Hz}, 3 \mathrm{H}), \quad 0.98-1.05(\mathrm{~m}, 2 \mathrm{H}), 1.07(\mathrm{~d}, J=7.2 \mathrm{~Hz}), 1.26-1.32(\mathrm{~m}, 1 \mathrm{H}) 1.30(\mathrm{~d}$, $J=7.2 \mathrm{~Hz}, 3 \mathrm{H}), 1.40$ (d, $J=7.2 \mathrm{~Hz}, 3 \mathrm{H}), 1.54-1.60(\mathrm{~m}, 1 \mathrm{H}), 1.67(\mathrm{~d}, J=6.6 \mathrm{~Hz}, 3 \mathrm{H}), 1.67-$ $1.75(\mathrm{~m}, 2 \mathrm{H}), 1.84-1.93$ (m, $3 \mathrm{H}), 1.95-2.01$ (m, $1 \mathrm{H}), 2.20-2.27$ (m, $1 \mathrm{H}), 2.85-2.88$ (m, $1 \mathrm{H})$, $3.04(\mathrm{dq}, J=10.5 \mathrm{~Hz}, 7.2 \mathrm{~Hz}, 1 \mathrm{H}), 3.18-3.30(\mathrm{~m}, 2 \mathrm{H}), 3.33(\mathrm{~d}, J=13.8 \mathrm{~Hz}, 1 \mathrm{H}), 3.47-$ $3.51(\mathrm{~m}, 1 \mathrm{H}), 3.68(\mathrm{dq}, J=4.8 \mathrm{~Hz}, 7.2 \mathrm{~Hz}, 1 \mathrm{H}), 3.93(\mathrm{dd}, J=12.0 \mathrm{~Hz}, 5.4 \mathrm{~Hz}, 2 \mathrm{H}), 3.94-$ $3.98(\mathrm{~m}, 1 \mathrm{H}), 4.02-4.05(\mathrm{~m}, 1 \mathrm{H}), 4.11-4.23(\mathrm{~m}, 1 \mathrm{H}), 4.33(\mathrm{dd}, J=10.5 \mathrm{~Hz}, 10.5 \mathrm{~Hz}, 1 \mathrm{H})$, $4.47(\mathrm{dd}, J=9.6 \mathrm{~Hz}, 4.8 \mathrm{~Hz}, 1 \mathrm{H}), 4.50$ (dd, $J=9.0 \mathrm{~Hz}, 3.0 \mathrm{~Hz}, 1 \mathrm{H}), 4.60-4.68$ (m, $2 \mathrm{H}), 5.14$ $5.20(\mathrm{~m}, 1 \mathrm{H}), 5.24-5.30(\mathrm{~m}, 1 \mathrm{H}), 5.61(\mathrm{dq}, J=10.8 \mathrm{~Hz}, 6.6 \mathrm{~Hz}, 1 \mathrm{H}), 6.15(\mathrm{~d}, J=4.8 \mathrm{~Hz}, 1 \mathrm{H})$, $6.46(\mathrm{~d}, J=6.6 \mathrm{~Hz}, 1 \mathrm{H}), 6.78-6.83(\mathrm{bs}, 1 \mathrm{H}), 6.83(\mathrm{~d}, J=4.8 \mathrm{~Hz}, 1 \mathrm{H}), 7.02-7.06(\mathrm{~m}, 2 \mathrm{H})$, $7.10-7.19(\mathrm{~m}, 6 \mathrm{H}), 7.20-7.24(\mathrm{~m}, 5 \mathrm{H}), 7.32(\mathrm{~d}, J=9.0 \mathrm{~Hz}, 1 \mathrm{H}), 8.17(\mathrm{~d}, J=7.8 \mathrm{~Hz}, 1 \mathrm{H})$, 
$8.75(\mathrm{~d}, J=8.4 \mathrm{~Hz}, 1 \mathrm{H}), 10.75-11.15$ (bs, $1 \mathrm{H}) ;{ }^{13} \mathrm{C} \mathrm{NMR}\left(150.8 \mathrm{MHz}, \mathrm{CDCl}_{3}\right): \delta=10.3(+)$, $13.2(+), 13.3(+), 14.8(+), 16.9(-), 17.1(-), 17.5(+), 20.0(+), 21.6(+), 25.1(-), 32.9(-), 35.0$ $(-), 35.7(-), 36.3(+), 37.8(+), 38.0(-), 39.1(+), 41.6(+), 50.9(+), 51.8(+), 52.0(+), 53.0(-)$, $54.5(+), 58.0(+), 59.2(+), 60.0(+), 60.3(+), 63.5(+), 103.6(+), 109.8(+), 119.9\left(C_{\text {quat }}\right), 121.6$ $\left(\mathrm{C}_{\text {quat }}\right), 126.9(+), 127.3(+), 127.4(+), 127.6(+), 127.8(+), 127.9(+), 128.5(+), 128.6(+), 141.3$ $\left(\mathrm{C}_{\text {quat }}\right), 142.1\left(\mathrm{C}_{\text {quat }}\right), 159.2\left(\mathrm{C}_{\text {quat }}\right), 168.4\left(\mathrm{C}_{\text {quat }}\right), 169.5\left(\mathrm{C}_{\text {quat }}\right), 170.3\left(\mathrm{C}_{\text {quat }}\right), 170.8\left(\mathrm{C}_{\text {quat }}\right), 171.7$ $\left(\mathrm{C}_{\text {quat }}\right), 172.4\left(\mathrm{C}_{\text {quat }}\right), 172.5\left(\mathrm{C}_{\text {quat }}\right) ; \mathrm{MS}(\mathrm{ESI})$ : (positive) $\left.\mathrm{m} / \mathrm{z}(\%): 1137(100, \mathrm{M}+\mathrm{Na}\rceil^{+}\right), 1115(32$, $\left.\mathrm{M}+\mathrm{H}\rceil^{+}\right)$, (negative) $\left.\mathrm{m} / \mathrm{z}(\%): 1113(72, \mathrm{M}-\mathrm{H}\rceil\right)$. 


\section{Hormaomycin analogues containing (fluoromethylcyclopropyl)alanine moieties}

\section{1. (Trifluoromethylcyclopropyl)alanyl-Hormaomycin}

N-Fmoc-(2R, 1'R, 2'R)-3-(2'-Trifluoromethylcyclopropyl)alanine (Fmoc-(R)tFmcpA-OH, 97 c): A

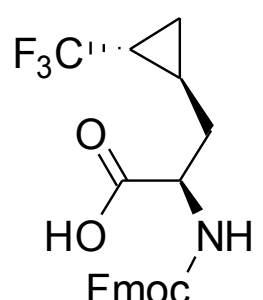

Fmoc solution of Fmoc-OSu (459 mg, $1.36 \mathrm{mmol})$ in acetone $(7 \mathrm{~mL})$ was added to a vigorously stirred solution of $\left(2 R, 1^{\prime} R, 2^{\prime} R\right)-3-\left(2^{\prime}\right.$-trifluoromethylcyclopropyl)alanine $\boldsymbol{R}-96$ c (225 mg, $1.14 \mathrm{mmol})$ and $\mathrm{NaHCO}_{3}(0.202 \mathrm{~g}, 2.40 \mathrm{mmol})$ in water $(5 \mathrm{~mL})$ (if a precipitate formed, acetone and/or water was added to obtain a homogeneous solution) and stirring continued for an additional $3 \mathrm{~h}$. Acetone was then removed under reduced pressure, and the $\mathrm{pH}$ of the residual water solution was adjusted to 1 with aq. $1 \mathrm{M} \mathrm{KHSO}_{4}$. The resulting emulsion was extracted with diethyl ether $(30 \mathrm{~mL})$ and the ethereal layer was back-extracted with aq. $3 \% \mathrm{NaHCO}_{3}(5 \times 10 \mathrm{~mL})$. The combined aqueous fractions were washed with diethyl ether $(2 \times 10 \mathrm{~mL})$, acidified to $\mathrm{pH} 2$ with aq. $1 \mathrm{M} \mathrm{KHSO}_{4}$, and the resulting emulsion was extracted with diethyl ether $(4 \times 10 \mathrm{~mL})$. The organic phase was washed with aq. $1 \mathrm{MKHSO}_{4}(2 \times 10 \mathrm{~mL})$, water $(3 \times 10 \mathrm{~mL})$, brine $(2 \times 5 \mathrm{~mL})$, dried, filtered and concentrated under reduced pressure. The residue was triturated with cold pentane and filtered. The resulting extremely viscous oil was dried at 0.02 Torr for prolonged time to give the target protected amino acid $97 \mathbf{c}(390 \mathrm{mg}, 0.930 \mathrm{mmol}, 82 \%)$ as a colorless foam. $R_{\mathrm{f}}=0.08$ (EtOAc/hexane 1:1); m.p. (softening) $50-57^{\circ} \mathrm{C} ;[\alpha]_{\mathrm{D}}{ }^{20}-56.7(\mathrm{c}=0.36$, $\left.\mathrm{CHCl}_{3}\right) ;{ }^{1} \mathrm{H} \mathrm{NMR}\left(600 \mathrm{MHz}, \mathrm{CDCl}_{3}\right): 0.38-0.44+0.57-0.64+0.79-0.86(3 \times \mathrm{m}, 1 \mathrm{H}), 0.91-$ $0.97+1.00-1.09(2 \times \mathrm{m}, \quad 1 \mathrm{H}), 1.14-1.22+1.26-1.34(2 \times \mathrm{m}, \quad 1 \mathrm{H}), \quad 1.35-1.53+1.85-1.88$ $(2 \times \mathrm{m}, 1 \mathrm{H}), 1.80-1.85(\mathrm{~m}, 1 \mathrm{H}), 3.75-3.79+4.53-4.67(2 \times \mathrm{m}, 1 \mathrm{H}), 3.95-4.01+4.47-4.52$ $(2 \times \mathrm{m}, 1 \mathrm{H}), 4.16-4.23(\mathrm{~m}, 1 \mathrm{H}), 4.36-4.47(\mathrm{~m}, 1 \mathrm{H}), 5.52(\mathrm{~d}, J=7.9 \mathrm{~Hz}, 0.7 \mathrm{H}), 6.76(\mathrm{~d}$, $J=5.9 \mathrm{~Hz}, 0.3 \mathrm{H}), 7.27-7.31(\mathrm{~m}, 2 \mathrm{H}), 7.35-7.41(\mathrm{~m}, 2 \mathrm{H}), 7.51(\mathrm{t}, \mathrm{J}=8.1 \mathrm{~Hz}, 0.6 \mathrm{H}), 7.58(\mathrm{t}$, $\mathrm{J}=8.4 \mathrm{~Hz}, 1.4 \mathrm{H}), 7.74(\mathrm{~d}, J=7.4 \mathrm{~Hz}, 2 \mathrm{H}), 7.85-8.65(\mathrm{bs}, 1 \mathrm{H}) ;{ }^{13} \mathrm{C}$ NMR $(125.7 \mathrm{MHz}$, $\left.\mathrm{CDCl}_{3}\right): \delta=7.94(-), 11.46(+), 19.73(+, \mathrm{q}, J=37.0 \mathrm{~Hz}), 34.65(-), 47.10(+), 53.48+53.82$ $(+), 67.14+67.87(-), 120.00(+), 124.97(+), 125.96(-$, q, $J=272.4 \mathrm{~Hz}), 127.07(+), 127.76$ $(+), 141.32\left(\mathrm{C}_{\text {quat }}\right), 143.51\left(\mathrm{C}_{\text {quat }}\right), 143.76\left(\mathrm{C}_{\text {quat }}\right), 155.79+156.71\left(\mathrm{C}_{\text {quat }}\right), 174.96+175.79\left(\mathrm{C}_{\text {quat }}\right)$; MS-ESI: (positive) m/z (\%) $\left.\left.1302\left(35,3 \mathrm{M}-\mathrm{H}+2 \mathrm{Na}^{+}\right), 861(100,2 \mathrm{M}+\mathrm{Na}\rceil^{+}\right), 442(\mathrm{M}+\mathrm{Na}\rceil^{+}\right)$, (negative) m/z (\%) $\left.\left.\left.837(100,2 \mathrm{M}-\mathrm{H}\rceil^{-}\right), 418(16, \mathrm{M}-\mathrm{H}\rceil^{-}\right), 222(14, \mathrm{M}-\mathrm{FmOH}-\mathrm{H}\rceil^{-}\right), 196(15$, $\left.\mathrm{FmOH}^{-}\right)$. 
Fmoc-(R)tFmcpA-MeF-Ile-ODCPM (98 c): Dipeptide 60 (434 mg, $834 \mu \mathrm{mol})$ was taken up with

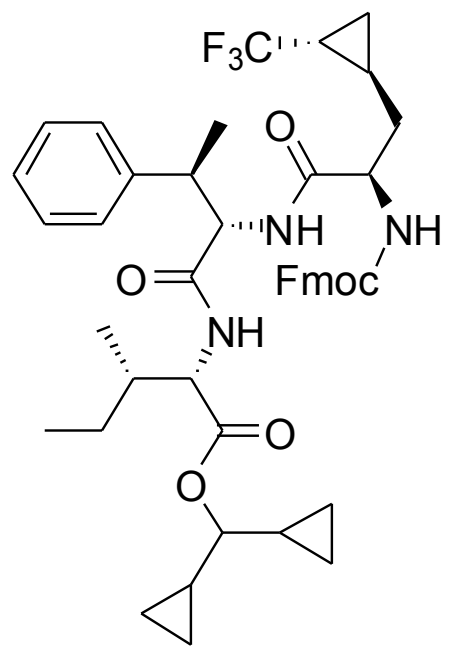

EtOAc $(20 \mathrm{~mL})$ and hydrogenated over $10 \% \mathrm{Pd} / \mathrm{C}(250 \mathrm{mg})$ under ambient pressure of hydrogen for $2 \mathrm{~h}$. The reaction mixture was filtered through a pad of Celite ${ }^{\circledR}$ and concentrated under reduced pressure to give deprotected dipeptide 62, which was directly used for the coupling with Fmoc- $(R)$ tFmcpA-OH 97 c (360 mg, $860 \mu \mathrm{mol})$, using $\operatorname{EDC}(172 \mathrm{mg}, 896 \mu \mathrm{mol})$, HOAt (120 mg, $883 \mu \mathrm{mol})$ and TMP $(310 \mu \mathrm{L}, 2.5 \mathrm{mmol})$ according to GP 2. During reaction the white precipitate appeared. The mixture was diluted with diethyl ether $(50 \mathrm{~mL})$, stirred for $30 \mathrm{~min}$ and filtered, giving the crude product ( $1^{\text {st }}$ crop, $473 \mathrm{mg}$ after drying in vacuo). The filtrate was concentrated under reduced pressure at ambient temperature and diluted with diethyl ether $(20 \mathrm{~mL})$ giving the crude product ( $2^{\text {nd }}$ crop, $130 \mathrm{mg}$ after drying in vacuo). The residing filtrate was subjected usual aqueous work-up according to GP 2 to give the last portion of crude product ( $3^{\text {rd }}$ crop, $100 \mathrm{mg}$ after drying in vacuo). Combined crude product was re-crystallized from $\mathrm{THF} /$ hexane and the resulting off-white solid was dissolved in chloroform $(50 \mathrm{~mL})$ and subjected usual aqueous work-up according to GP 2 to give the pure tripeptide as white solid (535 $\mathrm{mg}, 679 \mu \mathrm{mol}, 81 \%$ ). $R_{\mathrm{f}}=0.52$; EtOAc/hexane 2:3; m.p. $151-155^{\circ} \mathrm{C} ; \quad[\alpha]_{\mathrm{D}}{ }^{20}-3,8 \quad\left(c=0.26, \mathrm{CHCl}_{3}\right)$; ${ }^{1} \mathrm{H}$ NMR $\left(500 \mathrm{MHz}, \mathrm{CDCl}_{3}\right): \delta=0.28-0.40(\mathrm{~m}, 4 \mathrm{H}), 0.43-0.53(\mathrm{~m}, 2 \mathrm{H}), 0.57-0.66(\mathrm{~m}, 3 \mathrm{H})$, $0.88(\mathrm{~d}, J=6.7 \mathrm{~Hz}, 3 \mathrm{H}), 0.90(\mathrm{t}, J=7.4 \mathrm{~Hz}, 3 \mathrm{H}), 0.94-1.00(\mathrm{~m}, 1 \mathrm{H}), 1.03-1.13(\mathrm{~m}, 2 \mathrm{H})$, 1.13-1.22 (m, 2 H), 1.30-1.50 (m, 2 H), 1.41 (d, $J=6.8$ Hz, 3 H), 1.58-1.70 (m, 1 H), 1.81-1.97 (m, $2 \mathrm{H}), 3.28-3.38(\mathrm{~m}, 1 \mathrm{H}), 3.86(\mathrm{t}, J=8.4 \mathrm{~Hz}, 1 \mathrm{H}), 4.28(\mathrm{t}, J=6.9 \mathrm{~Hz}, 1 \mathrm{H}), 4.39-4.48(\mathrm{~m}$, $3 \mathrm{H}), 4.48-4.57(\mathrm{~m}, 1 \mathrm{H}), 4.76(\mathrm{t}, J=8.0 \mathrm{~Hz}, 1 \mathrm{H}), 5.76(\mathrm{~d}, J=7.7 \mathrm{~Hz}, 1 \mathrm{H}), 6.40(\mathrm{~d}, J=7.5 \mathrm{~Hz}$, $1 \mathrm{H}), 7.11(\mathrm{~d}, J=8.1 \mathrm{~Hz}, 1 \mathrm{H}), 7.20-7.34(\mathrm{~m}, 5 \mathrm{H}), 7.35$ (t, $J=7.4 \mathrm{~Hz}, 2 \mathrm{H}), 7.46$ (t, $J=7.4 \mathrm{~Hz}$, $2 \mathrm{H}), 7.60(\mathrm{t}, J=8.0 \mathrm{~Hz}, 2 \mathrm{H}), 7.79(\mathrm{~d}, J=7.5 \mathrm{~Hz}, 2 \mathrm{H}) ;{ }^{13} \mathrm{C} \mathrm{NMR}\left(125.7 \mathrm{MHz}, \mathrm{CDCl}_{3}\right) \delta=2.57$; 2.90, 8.18, 11.62,14.39, 14.66, 15.07, 15.07, 16.86, 19.70 (q, $J=37.1 \mathrm{~Hz}), 25.14,35.08,38.13$, 42.05, 47.07, 54.69,56.46, 58.79, 67.18, 83.44, 119.94, 124.99, 127.06, 127.55, 127.71, 128.54, $141.23,141.28,141.70,143.57,143.78,155.95,169.64,170.55,170.94$. 
Fmoc-MeF-(R)tFmcpA-MeF-Ile-ODCPM (100 c): The tripeptide 98 c (394 mg, $500 \mu$ mol) was

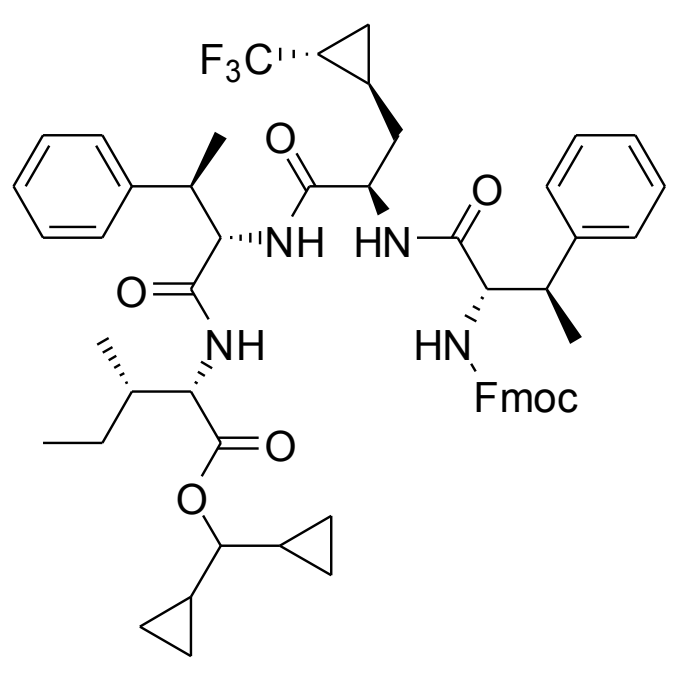
deprotected according to GP 1 and the resulting $C$ protected tripeptide $99 \mathbf{c}$ was then directly coupled with Fmoc-MeF-OH 64 (211 mg, $525 \mu \mathrm{mol})$ according to GP 2 using EDC (99 mg, $518 \mu \mathrm{mol})$, HOAt (70 mg, $512 \mu \mathrm{mol})$ and TMP (175 mg, $1440 \mu \mathrm{mol})$ in $\mathrm{CH}_{2} \mathrm{Cl}_{2}$ $(3 \mathrm{~mL})$. After $16 \mathrm{~h}$ the reaction mixture was diluted with chloroform $(50 \mathrm{~mL})$ and subjected usual aqueous work-up according to GP 2 to give the crude tetrapeptide, which was twice re-crystallized from $\mathrm{THF} /$ hexane, giving the pure target tetrapeptide as offwhite solid (440 mg, $463 \mu \mathrm{mol}, 93 \%) . \quad R_{\mathrm{f}}=0.29 ; \mathrm{CHCl}_{3} / \mathrm{MeOH}$ 70:1; m.p. $210-215^{\circ} \mathrm{C}$ (decomp.); $[\alpha]_{\mathrm{D}}{ }^{20}-26,3\left(c=0.32\right.$, THF); ${ }^{1} \mathrm{H}$ NMR $\left(250 \mathrm{MHz}, \mathrm{CDCl}_{3}\right): \delta=0.22-0.36(\mathrm{~m}, 4 \mathrm{H})$, $0.41(\mathrm{t}, J=8.9 \mathrm{~Hz}, 3 \mathrm{H}), 0.48-0.61(\mathrm{~m}, 2 \mathrm{H}), 0.62-0.71(\mathrm{~m}, 1 \mathrm{H}), 0.76(\mathrm{~d}, J=6.9 \mathrm{~Hz}, 3 \mathrm{H})$, $0.82(\mathrm{t}, J=7.3 \mathrm{~Hz}, 3 \mathrm{H}), 0.92-1.09$ (m, $4 \mathrm{H}), 1.09-1.18(\mathrm{~m}, 1 \mathrm{H}), 1.22-1.46(\mathrm{~m}, 2 \mathrm{H}), 1.26(\mathrm{~d}$, $J=6.9 \mathrm{~Hz}, 3 \mathrm{H}), 1.39(\mathrm{~d}, J=6.6 \mathrm{~Hz}, 3 \mathrm{H}), 1.71-1.89(\mathrm{~m}, 1 \mathrm{H}), 3.09-3.36(\mathrm{~m}, 3 \mathrm{H}), 3.81$ (t, $J=8.4 \mathrm{~Hz}, 1 \mathrm{H}), 4.15-4.33(\mathrm{~m}, 3 \mathrm{H}), 4.35-4.64(\mathrm{~m}, 3 \mathrm{H}), 4.62(\mathrm{t}, J=8.3 \mathrm{~Hz}, 1 \mathrm{H}), 5.95(\mathrm{~d}$, $J=7.1 \mathrm{~Hz}, 1 \mathrm{H}), 6.40(\mathrm{~d}, J=7.4 \mathrm{~Hz}, 1 \mathrm{H}), 7.08-7.45(\mathrm{~m}, 16 \mathrm{H}), 7.57(\mathrm{t}, J=8.9 \mathrm{~Hz}, 2 \mathrm{H})$, $7.76(\mathrm{~d}, J=7.3 \mathrm{~Hz}, 2 \mathrm{H}) ;{ }^{13} \mathrm{C} \mathrm{NMR}\left(62.9 \mathrm{MHz}, \mathrm{CDCl}_{3}\right): \delta=3.3(-), 3.5(-), 3.6(-), 3.7(-), 12.2$ $(+), 15.6(+), 15.9(+), 16.1(+), 17.7(+), 18.7(+), 18.9(-), 23.2(+), 26.2(-), 35.0(-), 39.1\left(^{+}\right)$, $42.8(+), 44.1(+), 48.5(+), 52.8(+), 57.4(+), 59.3(+), 60.2(+), 62.1(+), 67.8(-), 83.4(+)$, $115,1(+, \mathrm{q}, J=291.4 \mathrm{~Hz}), 126.4(+), 127.5(+), 127.7(+), 128.1(+), 128.6(+), 129.1(+), 129.1$ $(+), 129.3(+), 129.4(+), 142.5\left(\mathrm{C}_{\text {quat }}\right), 144.1\left(\mathrm{C}_{\text {quat }}\right), 144.4\left(\mathrm{C}_{\text {quat }}\right), 145.4\left(\mathrm{C}_{\text {quat }}\right), 145.6\left(\mathrm{C}_{\text {quat }}\right)$, $157.8\left(\mathrm{C}_{\text {quat }}\right), 171.1\left(\mathrm{C}_{\text {quat }}\right), 171.5\left(\mathrm{C}_{\text {quat }}\right), 171.9\left(\mathrm{C}_{\text {quat }}\right), 172.1\left(\mathrm{C}_{\text {quat }}\right)$. 
Boc-(4-Pe)Pro-[MeZ-a-Thr]-MeF-(S)tFmcpA-MeF-Ile-ODCPM (101 c): The tetrapeptide 100 c

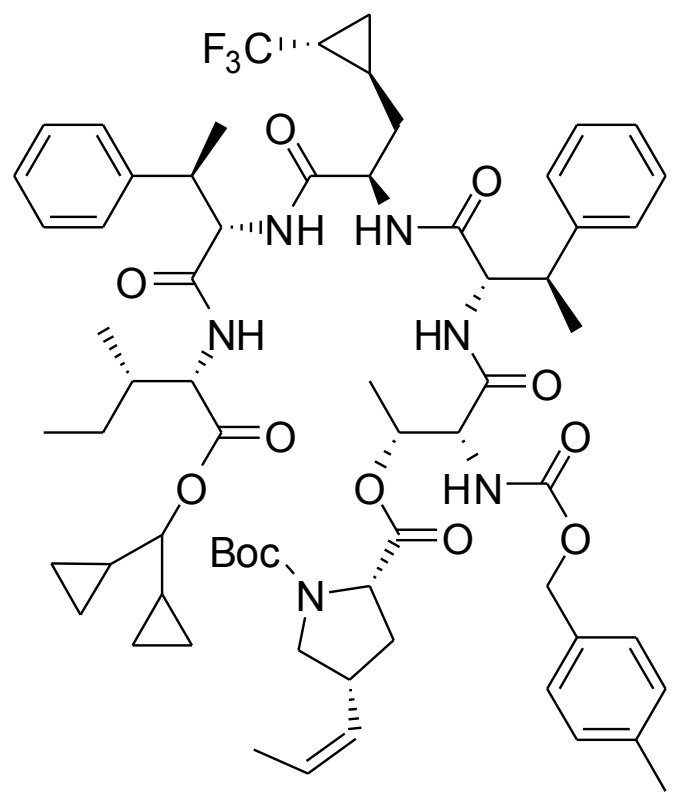
(332 mg, $350 \mu \mathrm{mol}$ ) was $N$-deprotected according to GP 1 with diethylamine $(5 \mathrm{~mL})$ and THF $(5 \mathrm{~mL})$, taken up with anhydrous $\mathrm{CH}_{2} \mathrm{Cl}_{2}(5 \mathrm{~mL})$, the solution of the ester acid 71 (194 mg, $385 \mu \mathrm{mol})$, HATU (160 mg, $420 \mu \mathrm{mol})$ and $\mathrm{HOAt}(53 \mathrm{mg}, 385 \mu \mathrm{mol})$ in $\mathrm{CH}_{2} \mathrm{Cl}_{2}$ $(3 \mathrm{~mL})$ was added, and the reaction mixture was cooled to $4{ }^{\circ} \mathrm{C}$. After this, a solution of DIEA $(65 \mu \mathrm{L}, 48 \mathrm{mg}$, $368 \mu \mathrm{mol})$ and TMP $(140 \mu \mathrm{L}, 127 \mathrm{mg}, 1050 \mu \mathrm{mol})$ in $\mathrm{CH}_{2} \mathrm{Cl}_{2}(2 \mathrm{~mL})$ was added at the same temperature within $5 \mathrm{~min}$. The temperature was allowed to reach $20{ }^{\circ} \mathrm{C}$, and stirring was continued for an additional 15 hours. After aqueous work-up according to GP 2 and two recrystallizations from EtOAc/hexane (1:2), the target hexadepsipeptide 101 c (390 mg, $321 \mu \mathrm{mol}, 92 \%)$ was obtained as a colorless solid. $R_{\mathrm{f}}=0.46\left(\right.$ EtOAc/hexane 1:1); m.p. $125-127^{\circ} \mathrm{C} ;[\alpha]_{\mathrm{D}}{ }^{20}-29.0(\mathrm{c}=0.2$, THF); ${ }^{1} \mathrm{H}$ NMR $\left(250 \mathrm{MHz}, \mathrm{CDCl}_{3}\right): \delta=0.24-0.68(\mathrm{~m}, 12 \mathrm{H}), 0.75(\mathrm{~d}, J=6.9 \mathrm{~Hz}, 3 \mathrm{H}), 0.88(\mathrm{t}$, $J=7.1 \mathrm{~Hz}, 3 \mathrm{H}), 0.98-1.17(\mathrm{~m}, 5 \mathrm{H}), 1.18-1.46(\mathrm{~m}, 1 \mathrm{H}), 1.24(\mathrm{~d}, J=6.6 \mathrm{~Hz}, 3 \mathrm{H}), 1.27$ (d, $J=6.7 \mathrm{~Hz}, 3 \mathrm{H}), 1.36(\mathrm{~s}, 9 \mathrm{H}), 1.40(\mathrm{~d}, J=6.7 \mathrm{~Hz}, 3 \mathrm{H}), 1.68(\mathrm{~d}, J=7.0 \mathrm{~Hz}, 3 \mathrm{H}), 1.75-1.94(\mathrm{~m}$, $2 \mathrm{H}), 2.29-2.46(\mathrm{~m}, 1 \mathrm{H}), 2.32(\mathrm{~s}, 3 \mathrm{H}), 3.07-3.33(\mathrm{~m}, 4 \mathrm{H}), 3.68(\mathrm{t}, J=8.6 \mathrm{~Hz}, 1 \mathrm{H}), 3.93(\mathrm{t}$, $J=8.3 \mathrm{~Hz}, 1 \mathrm{H}), 4.18-4.27(\mathrm{~m}, 1 \mathrm{H}), 4.32-4.54(\mathrm{~m}, 4 \mathrm{H}), 4.64(\mathrm{t}, J=9.6 \mathrm{~Hz}, 1 \mathrm{H}), 4.95-$ $5.13(\mathrm{~m}, 2 \mathrm{H}), 5.20-5.34(\mathrm{~m}, 1 \mathrm{H}), 5.44-5.63(\mathrm{~m}, 2 \mathrm{H}), 6.60(\mathrm{~d}, J=7.7 \mathrm{~Hz}, 1 \mathrm{H}), 6.70(\mathrm{~d}$, $J=8.8 \mathrm{~Hz}, 1 \mathrm{H}), 6.82(\mathrm{~d}, J=8.4 \mathrm{~Hz}, 1 \mathrm{H}), 7.05-7.37(\mathrm{~m}, 14 \mathrm{H}), 7.45(\mathrm{~d}, J=10.1 \mathrm{~Hz}, 1 \mathrm{H})$, $7.76(\mathrm{~d}, J=8.1 \mathrm{~Hz}, 1 \mathrm{H}) ;{ }^{13} \mathrm{C} \mathrm{NMR}\left(62.9 \mathrm{MHz}, \mathrm{CDCl}_{3}\right): \delta=2.48(-), 2.82(-), 2.90(-), 3.01(-)$, $11.59(+), 13.20(+), 14.16(+), 14.64(+), 15.57(+), 17.77(+), 18.41(-), 18.86(+), 19.43(+)$, $21.11(+), 21.77(+), 25.23(-), 26.85(-), 28.23(+), 31.46(-), 31.53(-), 36.28(-), 36.32(+)$, $37.30(+), 40.45(+), 42.00(+), 50.62(+), 52.08(+), 56.43(+), 59.30(+), 59.49(+), 61.01(+)$, $61.62(+), 61.99(+), 66.89(-), 70.53(+), 80.93$ (Cquat), $83.24(+), 116,2(+, \mathrm{q}, J=287.3 \mathrm{~Hz})$, $127.00(+), 127.06(+), 127.10(+), 127.60(+), 127.69(+), 128.47(+), 128.63(+), 128.68(+)$, $128.82(+), 128.93(+), 133.21$ (Cquat), 137.83 (Cquat), 141.73 (Cquat), 141.90 (Cquat), 154.76 (Cquat), 155.75 (Cquat), 170.38 (Cquat), 170.43 (Cquat), 170.79 (Cquat), 171.37 (Cquat), 173.41 (Cquat), 174.06 (Cquat). 
$N$-MeZ-protected cyclohexadepsipeptide 103 c (Cyclo- $\left.F_{3} 6-M e Z\right)$ : The hexadepsipeptide

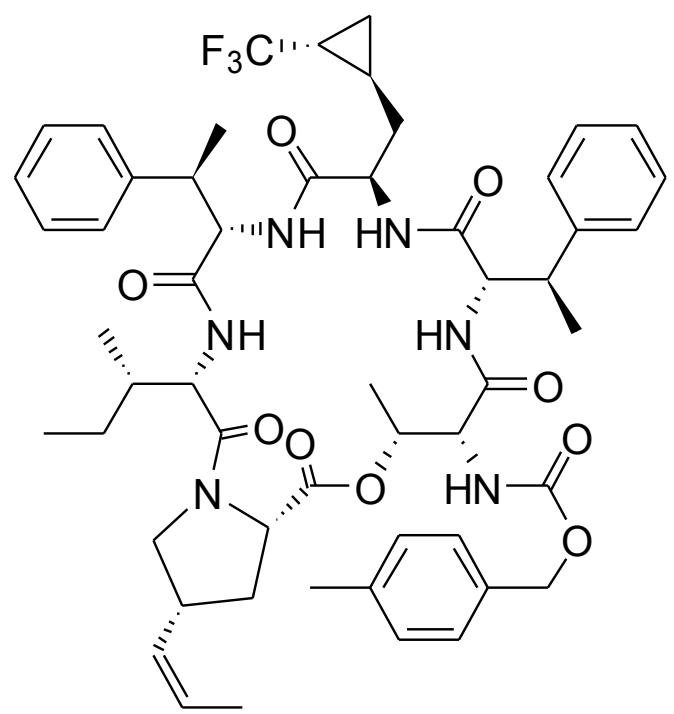

Boc-(4-Pe)Pro-[MeZ-a-Thr]-MeF-(S)tFmcpA-MeFIle-ODCPM (300 mg, $247 \mu \mathrm{mol})$ was ends-deprotected by treating with $2 \mathrm{M} \mathrm{HCl}$ solution in ethyl acetate $(5 \mathrm{~mL})$. The reaction mixture was stirred for $20 \mathrm{~min}$ in dark place (Al foil jacket) at ambient temperature and all volatiles were removed under reduced pressure in vacuo without any heating. The residue was triturated with anhydrous diethyl ether to give the hydrochloride of the deprotected material as a colorless solid (232 mg, $220 \mu \mathrm{mol}, 89 \%)$. The ends-deprotected hexadepsipeptide, HATU (110 mg, $288 \mu \mathrm{mol})$ and HOAt $(33 \mathrm{mg}, 244 \mu \mathrm{mol})$ were dissolved in cold $\left(4^{\circ} \mathrm{C}\right.$, internal temperature) anhydrous $\mathrm{CH}_{2} \mathrm{Cl}_{2}(2,5 \mathrm{~L})$, and the solution of DIEA $(120 \mu \mathrm{L}$, $93 \mathrm{mg}, 720 \mu \mathrm{mol})$ in $\mathrm{CH}_{2} \mathrm{Cl}_{2}(100 \mathrm{~mL})$ was added dropwise within 1 hour, the cooling bath was removed and the mixture was stirred for 2 hours at ambient temperature. Then the mixture was cooled again to $4^{\circ} \mathrm{C}$ (internal temperature), the second portions of HATU (110 mg, $\left.288 \mu \mathrm{mol}\right)$ and HOAt (33 mg, $244 \mu \mathrm{mol}$ ) were added, followed with dropwise addition of the solution of DIEA $(120 \mu \mathrm{L}, 93 \mathrm{mg}, 720 \mu \mathrm{mol})$ in $\mathrm{CH}_{2} \mathrm{Cl}_{2}(100 \mathrm{~mL})$ within 1 hour. The cooling bath was removed and the mixture was stirred for 18 hours at ambient temperature. The mixture was concentrated under reduced pressure, subjected to aqueous work-up according to GP 2 to give the crude protected cyclohexadepsipeptide $(180 \mathrm{mg}, 180 \mu \mathrm{mol}, 73 \%)$ which was finally purified with the HPLC to give pure product (132 mg, $132 \mu \mathrm{mol}, 54 \%)$. Preparative HPLC: isocratic, $82 \%$ B for $25 \mathrm{~min}, \mathrm{t}_{\mathrm{R}}=15.4 \mathrm{~min}$, purity $>98 \%$; analytical HPLC: gradient $20 \% \rightarrow 100 \%$ B for $20 \mathrm{~min}$, then isocratic $100 \% \mathrm{~B}$ for $5 \mathrm{~min} \mathrm{t}_{\mathrm{R}}=11.9 \mathrm{~min}$, purity $>98 \%$; $[\alpha]_{\mathrm{D}}{ }^{20}-15.5(\mathrm{c}=0.20$, $\left.\mathrm{CHCl}_{3}\right) ;{ }^{1} \mathrm{H}$ NMR $\left(600 \mathrm{MHz}, \mathrm{CDCl}_{3}\right): \delta=0.61-0.67(\mathrm{~m}, 1 \mathrm{H}), 0.72(\mathrm{~d}, J=6.6 \mathrm{~Hz}, 3 \mathrm{H}), 0.71-$ $0.77(\mathrm{~m}, 1 \mathrm{H}), 0.79(\mathrm{t}, J=7.2 \mathrm{~Hz}, 3 \mathrm{H}), 1.04-1.12(\mathrm{~m}, 1 \mathrm{H}), 1.23(\mathrm{~d}, J=6.6 \mathrm{~Hz}, 3 \mathrm{H}), 1.27-$ 1.34 (m, $1 \mathrm{H}), 1.37$ (d, $J=6.6 \mathrm{~Hz}, 3 \mathrm{H}), 1.37-1.43$ (m, $1 \mathrm{H}), 1.45-1.54$ (m, $1 \mathrm{H}), 1.54-1.57$ (m, $1 \mathrm{H}), 1.57(\mathrm{~d}, J=6.6 \mathrm{~Hz}, 3 \mathrm{H}), 1.65(\mathrm{dd}, J=6.6 \mathrm{~Hz}, 1.5 \mathrm{~Hz}, 3 \mathrm{H}), 1.66-1.76(\mathrm{~m}, 2 \mathrm{H}), 2.20$ $2.25(\mathrm{~m}, 1 \mathrm{H}), 2.35(\mathrm{~s}, 3 \mathrm{H}), 3.01-3.07(\mathrm{~m}, 1 \mathrm{H}), 3.15-3.28(\mathrm{~m}, 2 \mathrm{H}), 3.54(\mathrm{dq}, J=7.2 \mathrm{~Hz}$, $6.6 \mathrm{~Hz}, 1 \mathrm{H}), 3.71(\mathrm{dd}, J=6.0 \mathrm{~Hz}, 5.4 \mathrm{~Hz}, 1 \mathrm{H}), 3.74-3.77(\mathrm{~m}, 1 \mathrm{H}), 3.98(\mathrm{dd}, J=10.5 \mathrm{~Hz}$, $6.3 \mathrm{~Hz}, 1 \mathrm{H}), 4.01-4.08(\mathrm{~m}, 1 \mathrm{H}), 4.46-4.54(\mathrm{~m}, 2 \mathrm{H}), 4.52-4.55(\mathrm{~m}, 1 \mathrm{H}), 4.67-4.70(\mathrm{~m}, 1 \mathrm{H})$, $5.03(\mathrm{~d}, J=12.0 \mathrm{~Hz}, 1 \mathrm{H}), 5.15(\mathrm{~d}, J=12.0 \mathrm{~Hz}, 1 \mathrm{H}), 5.19-5.25(\mathrm{~m}, 1 \mathrm{H}), 5.39$ (qd, $J=6.6 \mathrm{~Hz}$, $1.8 \mathrm{~Hz}, 1 \mathrm{H}), 5.56(\mathrm{dq}, J=10.8 \mathrm{~Hz}, 6.6 \mathrm{~Hz}, 1 \mathrm{H}), 5.96(\mathrm{~d}, J=5.3 \mathrm{~Hz}, 1 \mathrm{H}), 6.20(\mathrm{~d}, J=7.4 \mathrm{~Hz}$, $1 \mathrm{H}), \quad 6.28(\mathrm{~d}, J=9.7 \mathrm{~Hz}, 1 \mathrm{H}), \quad 6.49(\mathrm{~d}, J=9.4 \mathrm{~Hz}, 1 \mathrm{H}), \quad 7.11-7.37(\mathrm{~m}, 14 \mathrm{H}) 7.32(\mathrm{~d}$, 
$J=7.4 \mathrm{~Hz}, 1 \mathrm{H}), 7.45(\mathrm{~d}, J=8.7 \mathrm{~Hz}, 1 \mathrm{H}) ;{ }^{13} \mathrm{C} \mathrm{NMR}\left(150.8 \mathrm{MHz}, \mathrm{CDCl}_{3}\right): \delta=10.3(+), 13.3(+)$, $14.6(+), 17.3(-), 17.7(+), 18.4(+), 21.2(+), 21.3(+), 24.7(-), 32.0(-), 35.4(-), 36.6(+), 39.4$ $(+), 44.5(+), 52.5(-), 53.3(+), 54.6(+), 58.6(+), 59.0(+), 59.4(+), 60.1(+), 60.7(+), 67.2(-)$, $72.6(+), 127.1(+), 127.2(+), 127.5(+), 127.6(+), 128.3(+), 128.6(+), 128.8(+), 129.2\left(_{+}\right)$, $127.8(+), 128.0(+), 133.2\left(\mathrm{C}_{\text {quat }}\right), 137.9\left(\mathrm{C}_{\text {quat }}\right), 140.9\left(\mathrm{C}_{\text {quat }}\right), 142.6\left(\mathrm{C}_{\text {quat }}\right), 156.3\left(\mathrm{C}_{\text {quat }}\right), 169.0$ $\left(\mathrm{C}_{\text {quat }}\right), 170.3\left(\mathrm{C}_{\text {quat }}\right), 170.6\left(\mathrm{C}_{\text {quat }}\right), 171.1\left(\mathrm{C}_{\text {quat }}\right), 171.4\left(\mathrm{C}_{\text {quat }}\right), 173.1\left(\mathrm{C}_{\text {quat }}\right)$.

$\mathrm{N}$-Teoc-(2S,1'S,2'R)-3-(2'-Trifluoromethylcyclopropyl)alanine (Teoc-(S)tFmcA-OH, 105 c): A<smiles>C[Si](C)(C)CCOC(=O)NCCC[C@H]1C[C@H]1C(F)(F)F</smiles>
solution of TeocOSu (43 mg, $164 \mu \mathrm{mol})$ in acetone $(1 \mathrm{~mL})$ was added to a vigorously stirred solution of $\left(2 S, 1^{\prime} S, 2^{\prime} R\right)-3-\left(2^{\prime}-\right.$ trifluoromethylcyclopropyl)alanine $\mathbf{S - 9 6}$ c $(27 \mathrm{mg}, 137 \mu \mathrm{mol})$ and $\mathrm{NaHCO}_{3}(24 \mathrm{mg}, 286 \mu \mathrm{mol})$ in water $(1 \mathrm{~mL})$ (if an emulsion formed, acetone and/or water was added to obtain a homogeneous solution), and stirring was continued for another $2 \mathrm{~h}$. $N, N$-dimethylaminopropylamine $(8 \mu \mathrm{L}, 6,4 \mathrm{mg}$, $52 \mu \mathrm{mol})$ was then added. After an additional $10 \mathrm{~min}$ acetone was removed under reduced pressure and the $\mathrm{pH}$ of the residual water solution was adjusted to 2-3 with aq. $1 \mathrm{M} \mathrm{KHSO}_{4}$. The resulting emulsion was extracted with diethyl ether $(50 \mathrm{~mL})$, and the ethereal layer was washed with aq. $1 \mathrm{M} \mathrm{KHSO}_{4}(2 \times 10 \mathrm{~mL})$, water $(3 \times 10 \mathrm{~mL})$, brine $(2 \times 5 \mathrm{~mL})$, dried over $\mathrm{MgSO}_{4}$, filtered and concentrated under reduced pressure. The residual oil was dried overnight in vacuo to give glass-like product (38 mg, $111 \mu \mathrm{mol}, 81 \%) . R_{\mathrm{f}}=0.24$ [EtOAc/hexane 1:3 (2\% AcOH)]; $[\alpha]_{\mathrm{D}}{ }^{20} 22.80\left(\mathrm{c}=0.46, \mathrm{CHCl}_{3}\right) ;{ }^{1} \mathrm{H} \mathrm{NMR}\left(250 \mathrm{MHz}, \mathrm{CDCl}_{3}\right): \delta=0.04(\mathrm{~s}, 9 \mathrm{H}), 1.00(\mathrm{dd}, J=$ $9.5 \mathrm{~Hz}, 7.3 \mathrm{~Hz}, 2 \mathrm{H}), 1.11-1.18$ (m, $1 \mathrm{H}), 1.60-1.95$ (m, $2 \mathrm{H}), 1.98-2.19$ (m, $2 \mathrm{H}), 4.14-4.23$ (m, $3 \mathrm{H}), \quad 4.33-4.59 \quad(\mathrm{~m}, \quad 1 \mathrm{H}), \quad 5.33-5.46 \quad(\mathrm{~m}, \quad 1 \mathrm{H}), \quad 7.08-7.25 \quad$ (bs, $1 \mathrm{H})$; ${ }^{13} \mathrm{C} \mathrm{NMR}\left(62.9 \mathrm{MHz}, \mathrm{CDCl}_{3}\right): \delta=-1.9(+), 10.2(-), 17.3(-), 22.0(+), 33.1+33.3(-), 52.7(+)$, $53.2(+), 59.0(+), 63.7(+), 64.8(-), 115.6(\mathrm{q}, J=271.4 \mathrm{~Hz}), 157.4$ (Cquat), $174.5+174.8$ (Cquat). 
Teoc-(S)tFmcpA-Cyclo- $F_{3} 6$ (106 c): $N$-MeZ-protected cyclohexadepsipeptide $\mathbf{1 0 3}$ c (25 mg,

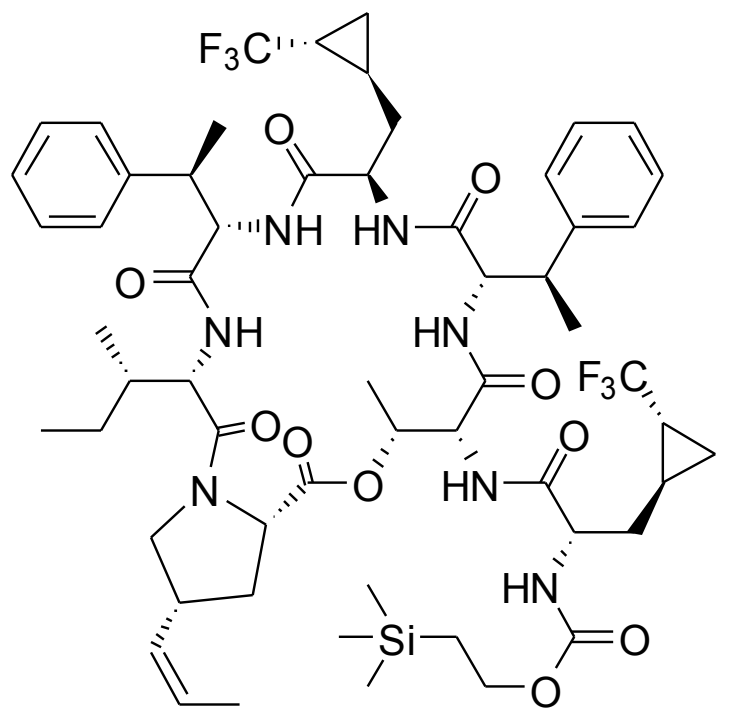

$25 \mu \mathrm{mol}$ ) was deprotected with $10 \%$ anisole in TFA $(1.1 \mathrm{~mL})$ in the dark at ambient temperature for $2 \mathrm{~h}$, the residue was treated with toluene $(5 \mathrm{~mL})$, concentrated under reduced pressure and the residue was dried in vacuo at ambient temperature for 2 hours. The solution of Teoc-(S)tFmcpA-OH 105 c, HATU $(29 \mathrm{mg}, 75 \mu \mathrm{mol})$ and HOAt $(10 \mathrm{mg}$, $75 \mu \mathrm{mol})$ in $\mathrm{CH}_{2} \mathrm{Cl}_{2}(1.5 \mathrm{~mL})$ was added at $4{ }^{\circ} \mathrm{C}$, followed with DIEA (3 $5 \mathrm{mg}, 27 \mu \mathrm{mol})$ and TMP (27 mg, $225 \mu \mathrm{mol})$ solution in $\mathrm{CH}_{2} \mathrm{Cl}_{2}(1.5 \mathrm{~mL})$ and the mixture was stirred at ambient temperature for

$15 \mathrm{~h}$. The reaction mixture was then diluted with diethyl ether $(50 \mathrm{~mL})$ and the crude product obtained after the usual aqueous work-up (GP 2) was purified by crystallization from $\mathrm{CH}_{2} \mathrm{Cl}_{2} /$ pentane to give Teoc-(S)tFmcpA-Cyclo- $\mathrm{F}_{3} 6(\mathbf{1 0 6} \mathbf{c})(29 \mathrm{mg}, 24,7 \mu \mathrm{mol}, \mathbf{9 9 \%})$ as a colorless solid $\left(R_{\mathrm{f}}=0.43\right.$, acetone/hexane $\left.1: 2\right)$ which was used for the next step without any characterization.

MOM-O-protected Trifluoromethylcyclopropylalanyl Hormaomycin (MOM-O-F ${ }_{3} H o r m, 108$ c):

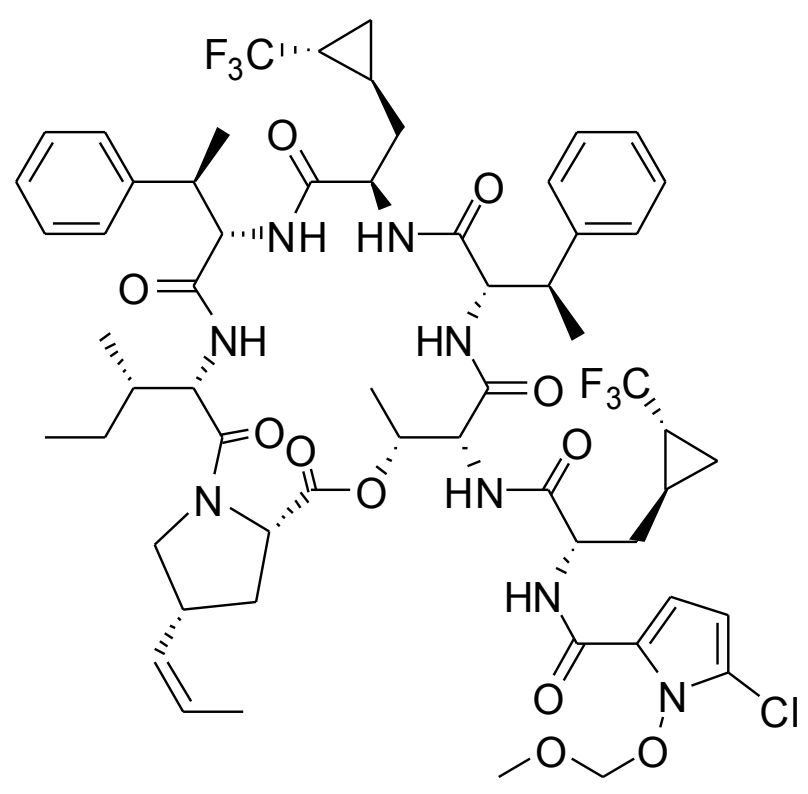

Teoc group was cleaved from the compound 106 c $(8.0 \mathrm{mg}, 7.08 \mu \mathrm{mol})$ with TFA $(0.6 \mathrm{~mL})$ for $1 \mathrm{~h}$. The mixture was concentrated under reduced pressure at $20{ }^{\circ} \mathrm{C}$ and then taken up with toluene $(3 \times 15 \mathrm{~mL})$ which was distilled off to remove the last traces of TFA. The resulting deprotected depsipeptide 107 c was coupled with $O$-MOM protected acid 81 (2.9 mg, $14.10 \mu \mathrm{mol})$ using HATU (5.4 mg, $14.20 \mu \mathrm{mol})$, DIEA $(0.92 \mathrm{mg}, 7.12 \mu \mathrm{mol})$ and TMP (5.14 mg, $42.42 \mu \mathrm{mol})$ in $\mathrm{CH}_{2} \mathrm{Cl}_{2}(1 \mathrm{~mL})$ according to GP 6 for $2.5 \mathrm{~h}$. The mixture was then taken up with $\mathrm{Et}_{2} \mathrm{O}(20 \mathrm{~mL})$ and the crude product obtained after usual aqueous work-up (GP 2) was crystallized from $\mathrm{CH}_{2} \mathrm{Cl}_{2} /$ pentane to give $O$-MOM protected 
trifluoromethylcyclopropylalanyl Hormaomycin 108 c $\left(8.0 \mathrm{mg}, 96 \%, R_{\mathrm{f}}=0.36\right.$ acetone/hexanes $1: 2)$ as a colorless glass which was used for the next step without any characterization.

Trifluoromethylcyclopropylalanyl Hormaomycin $\left(F_{3} H o r m, 109\right.$ c): O-MOM protected trifluoro-

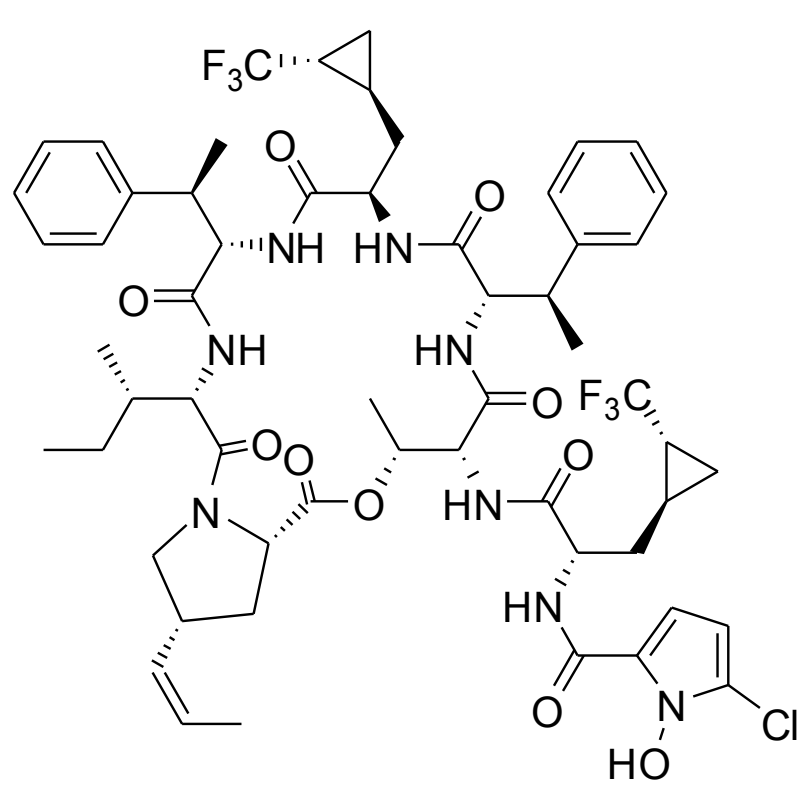
methylcyclopropylalanyl Hormaomycin 108 c $(8.0 \mathrm{mg}, 6.82 \mu \mathrm{mol})$ was deprotected using $\mathrm{MgBr}_{2} \times \mathrm{Et}_{2} \mathrm{O}(52 \mathrm{mg}, 201 \mu \mathrm{mol})$ and $\mathrm{EtSH}$ $(0.10 \mathrm{~mL}, 1.9 \mathrm{mmol})$ in $\mathrm{CH}_{2} \mathrm{Cl}_{2}(10 \mathrm{~mL})$ according to GP 7 for $3 \mathrm{~h}$. The mixture was taken up with EtOAc and the crude product obtained after usual aqueous work-up was crystallized from $\mathrm{CH}_{2} \mathrm{Cl}_{2} /$ pentane to give $109 \mathrm{c}$ as a white solid, which was finally purified with preparative HPLC. Yield $5.5 \mathrm{mg}$ $(72 \%, 55 \%$ over 5 steps from $103 \mathrm{c}) \cdot R_{\mathrm{f}}=0.24$ acetone/hexanes 3:7; preparative HPLC: isocratic, $82 \% \mathrm{~B}$ for $30 \mathrm{~min}, \mathrm{t}_{\mathrm{R}}=16.8 \mathrm{~min}$, purity > 98\%; analytical HPLC: gradient $20 \% \rightarrow 100 \%$ B for $20 \mathrm{~min}$, then isocratic $100 \% \mathrm{~B}$ for $5 \mathrm{~min}$ $\mathrm{t}_{\mathrm{R}}=15.3$ min, purity $>98 \% ;[\alpha]_{\mathrm{D}}^{20} 20.0(\mathrm{c}=0.1, \mathrm{MeOH}) ;{ }^{1} \mathrm{H}$ NMR $(600 \mathrm{MHz}$, $\left.\mathrm{CDCl}_{3}\right): \delta=-0.95--0.89(\mathrm{~m}, 1 \mathrm{H}),-0.41--0.33(\mathrm{~m}, 1 \mathrm{H}),-0.11-0.01(\mathrm{~m}, 2 \mathrm{H}), 0.05-$ 0.09 (m, $1 \mathrm{H}), 0.47-0.56(\mathrm{~m}, 2 \mathrm{H}), 0.86(\mathrm{t}, J=7.1 \mathrm{~Hz}, 1 \mathrm{H}), 0.89$ (t, $J=7.4 \mathrm{~Hz}, 3 \mathrm{H}), 1.01$ (d, $J=6.8 \mathrm{~Hz}, 3 \mathrm{H}), 1.03-1.09(\mathrm{~m}, 2 \mathrm{H}), 1.25-1.34(\mathrm{~m}, 1 \mathrm{H}), 1.30(\mathrm{~d}, J=7.1 \mathrm{~Hz}, 3 \mathrm{H}), 1.52(\mathrm{~d}$, $J=6.9 \mathrm{~Hz}, 3 \mathrm{H}), 1.66$ (dd, $J=7.1 \mathrm{~Hz}, 1.7 \mathrm{~Hz}, 3 \mathrm{H}), 1.79$ (q, $J=11.7 \mathrm{~Hz}, 1 \mathrm{H}), 1.84$ (bs, $1 \mathrm{H}$ ), 1.87-1.95 (m, $1 \mathrm{H}), 1.97-2.04(\mathrm{~m}, 1 \mathrm{H}), 2.31-2.37(\mathrm{~m}, 1 \mathrm{H}), 2.96-3.05(\mathrm{~m}, 1 \mathrm{H}), 3.03(\mathrm{dq}$, $J=11.2 \mathrm{~Hz}, 6.8 \mathrm{~Hz}, 1 \mathrm{H}), 3.06-3.20$ (m, $2 \mathrm{H}), 3.21-3.30$ (m, $2 \mathrm{H}), 3.45-3.53(\mathrm{~m}, 1 \mathrm{H}), 3.66(\mathrm{dq}$, $J=7.1 \mathrm{~Hz}, 5.0 \mathrm{~Hz}, 1 \mathrm{H}), 3.72-3.83(\mathrm{~m}, 1 \mathrm{H}), 3.89-4.02(\mathrm{~m}, 2 \mathrm{H}), 4.27(\mathrm{dd}, J=10.6 \mathrm{~Hz}, 5.9 \mathrm{~Hz}$, $1 \mathrm{H}), 4.35$ (t, $J=10.4 \mathrm{~Hz}, 1 \mathrm{H}), 4.50$ (dd, $J=9.3 \mathrm{~Hz}, 4.7 \mathrm{~Hz}, 1 \mathrm{H}), 4.54$ (dd, $J=9.2 \mathrm{~Hz}, 2.4 \mathrm{~Hz}$, $1 \mathrm{H}), 4.63(\mathrm{t}, J=9.3 \mathrm{~Hz}, 1 \mathrm{H}), 5.15(\mathrm{td}, J=9.1 \mathrm{~Hz}, 6.2 \mathrm{~Hz}, 1 \mathrm{H}), 5.25(\mathrm{tt}, J=8.8 \mathrm{~Hz}, 1.8 \mathrm{~Hz}$, $1 \mathrm{H}), 5.35(\mathrm{qd}, J=6.9 \mathrm{~Hz}, 2.3 \mathrm{~Hz}, 1 \mathrm{H}), 5.61(\mathrm{dq}, J=10.8 \mathrm{~Hz}, 6.9 \mathrm{~Hz}, 1 \mathrm{H}), 5.95,6.09(2 \times \mathrm{d}$, $J=4.5 \mathrm{~Hz}, J=4.7 \mathrm{~Hz}, 1 \mathrm{H}), 6.37,6.79(2 \times \mathrm{d}, J=4.9 \mathrm{~Hz}, J=4.7 \mathrm{~Hz}, 1 \mathrm{H}), 6.43(\mathrm{~d}, J=7.7 \mathrm{~Hz}$, $1 \mathrm{H}), 6.88(\mathrm{~d}, J=9.8 \mathrm{~Hz}, 1 \mathrm{H}), 7.07-7.32(\mathrm{~m}, 12 \mathrm{H}), 7.41(\mathrm{~d}, J=9.6 \mathrm{~Hz}, 1 \mathrm{H}), 9.17(\mathrm{~d}$, $J=9.2 \mathrm{~Hz}, 1 \mathrm{H}) ;{ }^{13} \mathrm{C} \mathrm{NMR}\left(150.8 \mathrm{MHz}, \mathrm{CDCl}_{3}\right): \delta=8.69(-, \quad \mathrm{q}, J=6.2 \mathrm{~Hz}), 9.84(-, \mathrm{q}$, $J=4.7 \mathrm{~Hz}), 10.41(+), 11.07(+), 11.84(+), 13.31,13.60,14.10,14.80,17.13(-), 17.93(+)$, $18.57(+, \mathrm{q}, J=38.5 \mathrm{~Hz}), 19.47(+, \mathrm{q}, J=36.9 \mathrm{~Hz}), 22.68(+), 25.05(+), 29.64(-), 29.69(-)$, $31.91(-), 34.56(-), 35.51(-), 36.67(-), 36.71(+), 37.86(+) 39.42(+), 41.76,42.11,43.45$, 44.57, $51.44(+), 52.68(+), 52.75(-), 54.65(+), 54.93(+), 59.86(+), 60.17(+), 61.36(+)$, 
64.28, 66.56, $69.07(+), 103.29(+), 109.25(+), 119.28,121.63,126.93(+), 127.31(+), 127.44$ $(+), 127.55(+), 127.63(+), 128.19(+), 128.45(+), 128.67(+), 141.68$ (Cquat), 141.97 (Cquat), 159.14 (Cquat), 168.72 (Cquat), 168.92 (Cquat), 170.07 (Cquat), 170.68 (Cquat), 171.21 (Cquat), 171.69 (Cquat), 172.65 (Cquat); HRMS: $\mathrm{M}+\mathrm{H}\rceil^{+}$calculated 1175.48021 measured 1175.47956; $\mathrm{M}+\mathrm{NH}_{4}{ }^{+}$calculated 1192.50676 measured $\left.1192.50686 ; \mathrm{M}+\mathrm{Na}\right\rceil^{+}$calculated 1197.46216 measured 1197.46120.

\section{2. (Difluoromethylcyclopropyl)alanyl-Hormaomycin}

N-Fmoc-(2R, 1'R, 2'R)-3-(2'-Difluoromethylcyclopropyl)alanine (Fmoc-(R)dFmcpA-OH, 97 b): A

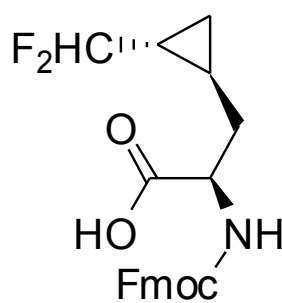

Fmoc

solution of Fmoc-OSu (459 mg, $1.36 \mathrm{mmol})$ in acetone $(7 \mathrm{~mL})$ was added to a vigorously stirred solution of $\left(2 R, 1^{\prime} R, 2^{\prime} R\right)-3-\left(2^{\prime}\right.$-difluoromethyl cyclopropyl) alanine $\boldsymbol{R}-96 \mathbf{b}$ (225 mg, $1.14 \mathrm{mmol})$ and $\mathrm{NaHCO}_{3}(0.202 \mathrm{~g}, 2.40 \mathrm{mmol})$ in water $(5 \mathrm{~mL}$ ) (if a precipitate formed, acetone and/or water was added to obtain a homogeneous solution) and stirring continued for an additional $3 \mathrm{~h}$.

Acetone was then removed under reduced pressure, and the $\mathrm{pH}$ of the residual water solution was adjusted to 1 with aq. $1 \mathrm{M} \mathrm{KHSO}_{4}$. The resulting emulsion was extracted with diethyl ether $(30 \mathrm{~mL})$ and the ethereal layer was back-extracted with $3 \%$ aq. $\mathrm{NaHCO}_{3}(5 \times 10 \mathrm{~mL}$, TLC control for the completeness of extraction was necessary). The combined aqueous fractions were washed with diethyl ether $(2 \times 10 \mathrm{~mL})$, acidified to $\mathrm{pH} 2$ with aq. $1 \mathrm{M} \mathrm{KHSO}_{4}$, and the resulting emulsion was extracted with diethyl ether $(4 \times 10 \mathrm{~mL})$. The organic phase was washed with aq. $1 \mathrm{M} \mathrm{KHSO}_{4}(2 \times 10 \mathrm{~mL})$, water $(3 \times 10 \mathrm{~mL})$, brine $(2 \times 5 \mathrm{~mL})$, dried, filtered and concentrated under reduced pressure. The residue was triturated with cold pentane and filtered. The resulting extremely viscous oil was dried at 0.02 Torr for prolonged time to give the target protected amino acid $97 \mathrm{~b}(390 \mathrm{mg}, 0.930 \mathrm{mmol}, 78 \%)$ as a colorless foam. $R_{\mathrm{f}}=0.08$ (EtOAc/hexane 1:1); m.p. (softening) $50-57{ }^{\circ} \mathrm{C} ;[\alpha]_{\mathrm{D}}{ }^{20}-56.7 \quad\left(\mathrm{c}=0.36, \mathrm{CHCl}_{3}\right) ;{ }^{1} \mathrm{H} \mathrm{NMR}$ $\left(600 \mathrm{MHz}, \quad \mathrm{CDCl}_{3}\right): \quad 0.38-0.44+0.57-0.64+0.79-0.86(3 \times \mathrm{m}, \quad 1 \mathrm{H}), \quad 0.91-0.97+1.00-$ $1.09(2 \times \mathrm{m}, 1 \mathrm{H}), 1.14-1.22+1.26-1.34(2 \times \mathrm{m}, 1 \mathrm{H}), 1.35-1.53+1.85-1.88(2 \times \mathrm{m}, 1 \mathrm{H})$, 1.80-1.85 (m, 1 H), 3.75-3.79+4.53-4.67 (2× m, 1 H), 3.95-4.01+4.47-4.52 (2× m, 1 H), 4.16-4.23 (m, $1 \mathrm{H}), 4.36-4.47(\mathrm{~m}, 1 \mathrm{H}), 5.52(\mathrm{~d}, J=7.9 \mathrm{~Hz}, 0.7 \mathrm{H}), 6.76(\mathrm{~d}, J=5.9 \mathrm{~Hz}, 0.3 \mathrm{H})$, $7.27-7.31(\mathrm{~m}, 2 \mathrm{H}), 7.35-7.41(\mathrm{~m}, 2 \mathrm{H}), 7.51(\mathrm{t}, J=8.1 \mathrm{~Hz}, 0.6 \mathrm{H}), 7.58(\mathrm{t}, J=8.4 \mathrm{~Hz}, 1.4 \mathrm{H})$, $7.74(\mathrm{~d}, J=7.4 \mathrm{~Hz}, 2 \mathrm{H}), 7.85-8.65(\mathrm{bs}, 1 \mathrm{H}) ;{ }^{13} \mathrm{C} \mathrm{NMR}\left(125.7 \mathrm{MHz}, \mathrm{CDCl}_{3}\right): \delta=7.94(-)$, $11.46(+), 19.73(+, \mathrm{q}, J=37.0 \mathrm{~Hz}), 34.65(-), 47.10(+), 53.48+53.82(+), 67.14+67.87(-)$, $120.00(+), 124.97(+), 125.96(-$, q, $J=272.4 \mathrm{~Hz}), 127.07(+), 127.76(+), 141.32\left(\mathrm{C}_{\text {quat }}\right)$, $143.51\left(\mathrm{C}_{\text {quat }}\right), 143.76\left(\mathrm{C}_{\text {quat }}\right), 155.79+156.71\left(\mathrm{C}_{\text {quat }}\right), 174.96+175.79\left(\mathrm{C}_{\text {quat }}\right)$; MS-ESI: (positive $)$ 
$\left.\left.\mathrm{m} / \mathrm{z}(\%): 1302(35,3 \mathrm{M}-\mathrm{H}+2 \mathrm{Na}\rceil^{+}\right), 861\left(100,2 \mathrm{M}+\mathrm{Na}^{+}\right), 442(\mathrm{M}+\mathrm{Na}\rceil^{+}\right)$, (negative) $\mathrm{m} / \mathrm{z}(\%): 837$ $\left.\left(100,2 \mathrm{M}-\mathrm{H}^{\top}{ }^{-}\right), 418(16, \mathrm{M}-\mathrm{H}\rceil^{-}\right), 222\left(14, \mathrm{M}-\mathrm{FmOH}-\mathrm{H}^{-}\right), 196\left(15, \mathrm{FmOH}^{-}\right)$.

Fmoc-(R)dFmcpA-MeF-Ile-ODCPM (98 b): Dipeptide 60 (434 mg, $834 \mu \mathrm{mol})$ was taken up

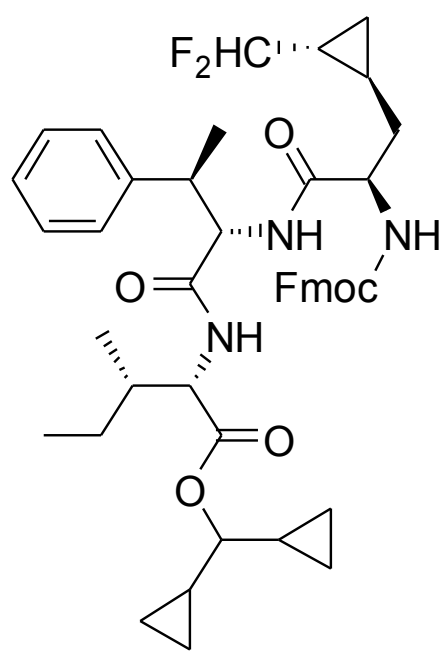
with EtOAc $(20 \mathrm{~mL})$ and hydrogenated over $10 \% \mathrm{Pd} / \mathrm{C}(250 \mathrm{mg})$ under ambient pressure of hydrogen for $2 \mathrm{~h}$. The reaction mixture was filtered through a pad of Celite ${ }^{\circledR}$ and concentrated under reduced pressure to give deprotected dipeptide 62, which was directly used for the coupling with Fmoc- $(R)$ dFmcpA-OH $97 \mathbf{b}$ $(360 \mathrm{mg}, 860 \mu \mathrm{mol})$, using EDC $(172 \mathrm{mg}, 896 \mu \mathrm{mol})$, HOAt (120 mg, $883 \mu \mathrm{mol})$ and TMP ( $310 \mu \mathrm{L}, 2.5 \mathrm{mmol})$ according to GP 2. During reaction the white precipitate appeared. The mixture was diluted with diethyl ether $(50 \mathrm{~mL})$, stirred for $30 \mathrm{~min}$ and filtered, giving the crude product $\left(1^{\text {st }}\right.$ crop, $173 \mathrm{mg}$ after drying in

vacuo). The filtrate was concentrated under reduced pressure at ambient temperature and diluted with diethyl ether $(20 \mathrm{~mL})$ and subjected usual aqueous work-up according to GP 2 to give the last portion of crude product ( $2^{\text {nd }} \mathrm{crop}, 112 \mathrm{mg}$ after drying in vacuo). Combined crude product was re-crystallized from THF/hexane and the resulting off-white solid was dissolved in chloroform $(50 \mathrm{~mL})$ and subjected usual aqueous work-up according to GP 2 to give the pure tripeptide $98 \mathrm{~b}$ as white solid ( $253 \mathrm{mg}, 320 \mu \mathrm{mol}, 65 \%) . R_{\mathrm{f}}=0.52$; EtOAc/hexane 2:3; m.p. 151$155^{\circ} \mathrm{C} ;[\alpha]_{\mathrm{D}}^{20}-3,8\left(\mathrm{c}=0.26, \mathrm{CHCl}_{3}\right) ;{ }^{1} \mathrm{H} \mathrm{NMR}\left(500 \mathrm{MHz}, \mathrm{CDCl}_{3}\right): \delta=0.28-0.40(\mathrm{~m}, 4 \mathrm{H})$, $0.43-0.53(\mathrm{~m}, 2 \mathrm{H}), 0.57-0.66(\mathrm{~m}, 3 \mathrm{H}), 0.88(\mathrm{~d}, J=6.7 \mathrm{~Hz}, 3 \mathrm{H}), 0.90(\mathrm{t}, J=7.4 \mathrm{~Hz}, 3 \mathrm{H})$, 0.94-1.00 (m, $1 \mathrm{H}), 1.03-1.13(\mathrm{~m}, 2 \mathrm{H}), 1.13-1.22(\mathrm{~m}, 2 \mathrm{H}), 1.30-1.50(\mathrm{~m}, 2 \mathrm{H}), 1.41(\mathrm{~d}$, $J=6.8 \mathrm{~Hz}, \quad 3 \mathrm{H}), \quad 1.58-1.70(\mathrm{~m}, 1 \mathrm{H}), \quad 1.81-1.97(\mathrm{~m}, 2 \mathrm{H}), \quad 3.28-3.38(\mathrm{~m}, 1 \mathrm{H}), \quad 3.86(\mathrm{t}$, $J=8.4 \mathrm{~Hz}, 1 \mathrm{H}), 4.28(\mathrm{t}, J=6.9 \mathrm{~Hz}, 1 \mathrm{H}), 4.39-4.48(\mathrm{~m}, 3 \mathrm{H}), 4.48-4.57(\mathrm{~m}, 1 \mathrm{H}), 4.76(\mathrm{t}$, $J=8.0 \mathrm{~Hz}, 1 \mathrm{H}), 5.76(\mathrm{~d}, J=7.7 \mathrm{~Hz}, 1 \mathrm{H}), 6.40(\mathrm{~d}, J=7.5 \mathrm{~Hz}, 1 \mathrm{H}), 7.11(\mathrm{~d}, J=8.1 \mathrm{~Hz}, 1 \mathrm{H})$, $7.20-7.34$ (m, 5 H), 7.35 (t, $J=7.4 \mathrm{~Hz}, 2 \mathrm{H}), 7.46$ (t, $J=7.4 \mathrm{~Hz}, 2 \mathrm{H}), 7.60$ (t, $J=8.0 \mathrm{~Hz}, 2 \mathrm{H}$ ), $7.79(\mathrm{~d}, J=7.5 \mathrm{~Hz}, 2 \mathrm{H}) ;{ }^{13} \mathrm{C}$ NMR $\left(125.7 \mathrm{MHz}, \mathrm{CDCl}_{3}\right): \delta=2.57,2.90,8.18,11.62,14.39$, 14.66, 15.07, 15.07, 16.86, $19.70(\mathrm{t}, J=37.1 \mathrm{~Hz}), 25.14,35.08,38.13,42.05,47.07,54.69,56.46$, $58.79,67.18,83.44,119.94,124.99,127.06,127.55,127.71,128.54,141.23,141.28,141.70$, $143.57,143.78,155.95,169.64,170.55,170.94$; HRMS: for $\mathrm{M}+\mathrm{H}\rceil^{+}$calculated 770.33752 , found 770.39756 . 
Fmoc-MeF-(R)dFmcpA-MeF-Ile-ODCPM (100 b): The tripeptide 98 b (394 mg, $500 \mu$ mol) was

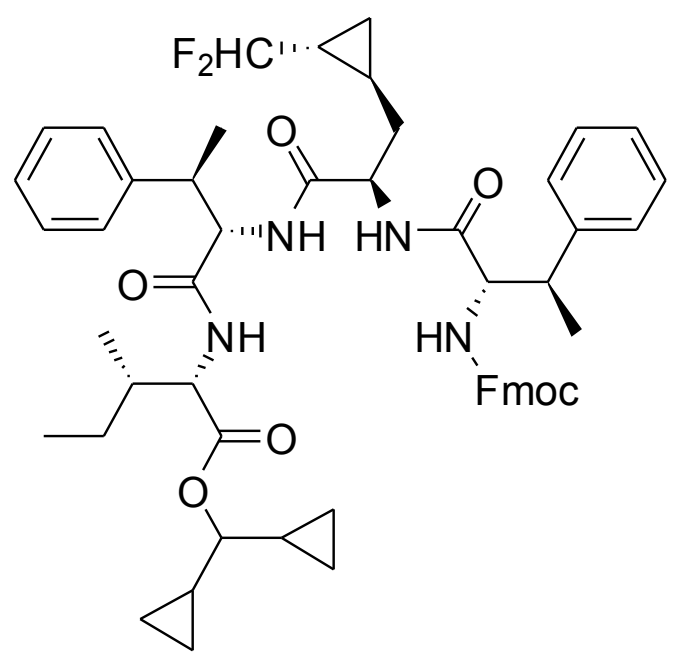
deprotected according to GP 1 and the resulting $C$-protected tripeptide $99 \mathrm{~b}$ was then directly coupled with Fmoc-MeF-OH 64 (211 mg, 525 mol) according to GP 2 using EDC (99 mg, $518 \mu \mathrm{mol})$, HOAt (70 mg, $512 \mu \mathrm{mol})$ and TMP (175 mg, $1440 \mu \mathrm{mol})$ in $\mathrm{CH}_{2} \mathrm{Cl}_{2}$ $(3 \mathrm{~mL})$. After $16 \mathrm{~h}$ the reaction mixture was diluted with chloroform $(50 \mathrm{~mL})$ and subjected usual aqueous work-up according to GP 2 to give the crude tetrapeptide, which was twice re-crystallized from $\mathrm{THF} /$ hexane, giving the pure target tetrapeptide $\mathbf{1 0 0} \mathbf{b}$ as off-white solid (440 mg, $463 \mu \mathrm{mol}, 81 \%$ ). $R_{\mathrm{f}}=0.29 ; \mathrm{CHCl}_{3} / \mathrm{MeOH} 70: 1 ;$ m.p. $210-215^{\circ} \mathrm{C}$ (decomp.); $[\alpha]_{\mathrm{D}}{ }^{20}-16.0$ (c=0.5 in THF); ${ }^{1} \mathrm{H}$ NMR (500 MHz, [D8]THF): $\delta=0.23-0.32$ (m, $4 \mathrm{H}$ ), $0.32-0.39(\mathrm{~m}, 2 \mathrm{H}), 0.40-0.55(\mathrm{~m}, 4 \mathrm{H}), 0.82(\mathrm{~d}, J=7.0 \mathrm{~Hz}, 3 \mathrm{H}), 0.83(\mathrm{~d}, J=7.3 \mathrm{~Hz}, 3 \mathrm{H})$, 0.82-0.91 (m, $1 \mathrm{H}), 0.94-1.05(\mathrm{~m}, 3 \mathrm{H}), 1.08-1.19(\mathrm{~m}, 2 \mathrm{H}), 1.22(\mathrm{~d}, J=7.1 \mathrm{~Hz}, 3 \mathrm{H}), 1.32(\mathrm{~d}$, $J=7.0 \mathrm{~Hz}, 3 \mathrm{H}), 1.36-1.47$ (m, $2 \mathrm{H}), 2.63$ (bs, $1 \mathrm{H}), 3.17$ (dq, $J=9.2 \mathrm{~Hz}, 7.2 \mathrm{~Hz}, 1 \mathrm{H}), 3.29$ (dq, $J=7.2 \mathrm{~Hz}, 7.1 \mathrm{~Hz}, 1 \mathrm{H}), 3.82(\mathrm{t}, J=8.28,1 \mathrm{H}), 4.18-4.25(\mathrm{~m}, 2 \mathrm{H}), 4.25-4.31(\mathrm{~m}, 1 \mathrm{H}), 4.33-$ 4.40 (m, 2 H), 4.41-4.48 (m, 1 H), 4.65-4.71 (m, $1 \mathrm{H}), 5.40$ (td, $J=57.5 \mathrm{~Hz}, 4.8 \mathrm{~Hz}, 1 \mathrm{H}), 7.02-$ $7.08(\mathrm{~m}, 1 \mathrm{H}), 7.09-7.29(\mathrm{~m}, 12 \mathrm{H}), 7.30-7.36(\mathrm{~m}, 3 \mathrm{H}), 7.49(\mathrm{t}, J=9.3 \mathrm{~Hz}, 2 \mathrm{H}), 7.63(\mathrm{~d}$, $J=7.5 \mathrm{~Hz}, 1 \mathrm{H}), 7.67(\mathrm{~d}, J=7.7 \mathrm{~Hz}, 1 \mathrm{H}), 7.76(\mathrm{~d}, J=7.6 \mathrm{~Hz}, 2 \mathrm{H}) ;{ }^{13} \mathrm{C} \mathrm{NMR}(125.7 \mathrm{MHz}$, [D8]THF): $\delta=2.76(-), 2.98(-), 3.04(-), 3.21(-), 7.67(-), 11.68(+), 11.99(+, \mathrm{t}, J=4.2 \mathrm{~Hz})$, $15.11(+), \quad 15.36(+), 15.60(+), 17.04(+), 18.02(+), 21.13(+, \mathrm{t}, J=27.1 \mathrm{~Hz}), 25.80(-)$, $36.03(-), 38.53(+), 42.03(+), 43.33(+), 48.06(+), 53.44(+), 56.91(+), 58.78(+), 61.62(+)$, $67.28(-), 82.82(+), 118.34(+, \mathrm{t}, J=236.9 \mathrm{~Hz}), 120.34(+), 120.35(+), 125.92(+), 125.96(+)$, $126.83(+), 127.17(+), 127.57(+), 127.60(+), 128.08(+), 128.49(+), 128.59(+), 128.69(+)$, $128.84(+), 142.02(-), 142.05(-), 143.87(-), 144.12(-), 145.01(-), 145.10(-), 157.27(-)$, $170.64(-), 171.46(-), 171.50(-)$; HRMS: for $\mathrm{C}_{55} \mathrm{H}_{65} \mathrm{O}_{7} \mathrm{~N}_{4} \mathrm{~F}_{2}\left([\mathrm{M}+\mathrm{H}]^{+}\right)$calculated: 931.48158 ; measured: 931.48094 . 
Boc-(4-Pe)Pro-[MeZ-a-Thr]-MeF-(S)dFmcpA-MeF-Ile-ODCPM (101 b): The tetrapeptide 100 b

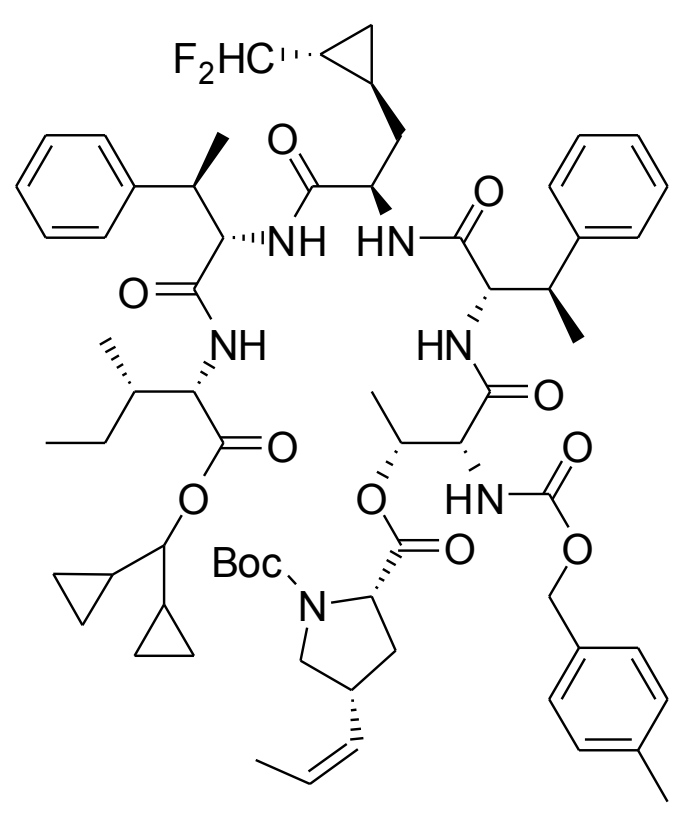
(200 mg, $215 \mu \mathrm{mol})$ was $N$-deprotected according to GP 1 with diethylamine $(5 \mathrm{~mL})$ and THF $(5 \mathrm{~mL})$, taken up with anhydrous $\mathrm{CH}_{2} \mathrm{Cl}_{2}(5 \mathrm{~mL})$, the solution of the ester acid 71 (119 mg, $235 \mu \mathrm{mol})$, HATU (98 mg, $256 \mu \mathrm{mol})$ and $\mathrm{HOAt}(32 \mathrm{mg}, 235 \mu \mathrm{mol})$ in $\mathrm{CH}_{2} \mathrm{Cl}_{2}$ $(3 \mathrm{~mL})$ was added, and the reaction mixture was cooled to $4{ }^{\circ} \mathrm{C}$. After this, a solution of DIEA $(30 \mathrm{mg}$, $225 \mu \mathrm{mol})$ and TMP (77 mg, $635 \mu \mathrm{mol})$ in $\mathrm{CH}_{2} \mathrm{Cl}_{2}$ $(2 \mathrm{~mL})$ was added at the same temperature within $5 \mathrm{~min}$. The temperature was allowed to reach $20^{\circ} \mathrm{C}$, and stirring was continued for an additional $15 \mathrm{~h}$. After aqueous work-up according to GP 2 and two recrystallizations from EtOAc/hexane (1:2), the crude hexadepsipeptide was finally purified with column chromatography (silica gel). All impurities were eluted out with EtOAc/hexane (1:1) and the substance was eluted out with methanol. After solvent evaporation under reduced pressure $C$ deprotected hexadepsipeptide $\mathbf{1 0 2}$ b (236 mg, $198 \mu \mathrm{mol}, 90 \%)$ was obtained as a colorless solid. $R_{\mathrm{f}}=0.46(\mathrm{THF}) ;[\alpha]_{\mathrm{D}}{ }^{20}-18.5$ (c=0.2, THF); ${ }^{1} \mathrm{H}$ NMR $(600 \mathrm{MHz},[\mathrm{D} 8] \mathrm{THF}): \delta=0.23-0.30(\mathrm{~m}$, $1 \mathrm{H}), \quad 0.30-0.37(\mathrm{~m}, 0.5 \mathrm{H}), \quad 0.37-0.43(\mathrm{~m}, 0.5 \mathrm{H}), 0.44-0.53(\mathrm{~m}, 1 \mathrm{H}), 0.79-1.02(\mathrm{~m}, 2 \mathrm{H})$ $0.84(\mathrm{~d}, J=7.3 \mathrm{~Hz}, 3 \mathrm{H}), 0.86(d, J=7.1 \mathrm{~Hz}, 3 \mathrm{H}), 1.13(\mathrm{~d}, J=6.1 \mathrm{~Hz}, 2 \mathrm{H}), 1.16(\mathrm{~d}, J=5.9 \mathrm{~Hz}$, $2 \mathrm{H}), 1.20(\mathrm{t}, J=5.4 \mathrm{~Hz}, 2 \mathrm{H}), 1.28(\mathrm{t}, J=6.0 \mathrm{~Hz}, 2 \mathrm{H}), 1.37+1.39(2 \mathrm{~s}, 9 \mathrm{H}), 1.42-1.53$ (m, $2 \mathrm{H}), 1.64(\mathrm{t}, J=6.7 \mathrm{~Hz}, 2 \mathrm{H}), 1.78-1.87(\mathrm{~m}, 1 \mathrm{H}), 2.26-2.33(\mathrm{~m}, 1 \mathrm{H}), 2.27(\mathrm{~s}, 3 \mathrm{H}), 2.91-$ $3.60(\mathrm{bs}, 1 \mathrm{H}), 2.93(\mathrm{t}, J=10.3 \mathrm{~Hz}, 1 \mathrm{H}), 2.99$ (t, $J=10.2 \mathrm{~Hz}, 1 \mathrm{H}), 3.05(\mathrm{q}, J=8.6 \mathrm{~Hz}, 1 \mathrm{H})$, 3.16 (q, $J=8.7 \mathrm{~Hz}, 1 \mathrm{H}), 3.17-3.28$ (m, $2 \mathrm{H}), 3.35-3.46$ (m, $2 \mathrm{H}), 3.64$ (dd, $J=10.0 \mathrm{~Hz}, 7.5 \mathrm{~Hz}$, $1 \mathrm{H}), 4.07-4.35(\mathrm{~m}, 3 \mathrm{H}), 4.55-4.72(\mathrm{~m}, 3 \mathrm{H}), 4.91(\mathrm{~d}, J=12.3 \mathrm{~Hz}, 1 \mathrm{H}), 5.04$ (dd, $J=24.1 \mathrm{~Hz}$, $12.2 \mathrm{~Hz}, 1 \mathrm{H}), 5.19-5.36(\mathrm{~m}, 3 \mathrm{H}), 5.45-5.53(\mathrm{~m}, 1 \mathrm{H}), 6.90(\mathrm{~d}, J=8.1 \mathrm{~Hz}, 0.6 \mathrm{H}), 6.97(\mathrm{~d}$, $J=8.7 \mathrm{~Hz}, 0.4 \mathrm{H}), 7.03-7.29(\mathrm{~m}, 14 \mathrm{H}), 7.40(\mathrm{~d}, J=7.0 \mathrm{~Hz}, 0.5 \mathrm{H}), 7.45(\mathrm{~d}, J=6.4 \mathrm{~Hz}, 0.5 \mathrm{H})$, $7.53-7.64(\mathrm{~m}, \quad 2 \mathrm{H}), \quad 7.93(\mathrm{~d}, \quad J=5.4 \mathrm{~Hz}, \quad 0.6 \mathrm{H}), \quad 7.97(\mathrm{~d}, \quad J=6.1 \mathrm{~Hz}, \quad 0.4 \mathrm{H}) ; \quad{ }^{13} \mathrm{C} \mathrm{NMR}$ (125.7 MHz, [D8]THF): $\delta=7.79,11.71,11.96,12.02,12.99,13.06,15.78,21.00,21.01,25.60$, 25.89, 28.43, 28.49, 35.08, 35.38, 36.39, 36.64, 37.12, 37.67, 38.08, 41.30, 41.55, 42.38, 52.01, $52.45,53.77,53.88,60.18,67.74,79.44,80.04,116.61,118.49,120.37,126.39,126.54,126.76$, $126.78,127.09,127.15,128.37,128.40,128.57,128.59,128.77,128.85,129.43,129.47,130.69$, $130.94,134.93$, 137.85, 143.87, 144.00, 153.38, 154.52, 156.96, 157.22, 169.91, 169.94, 170.87, 
170.92, 171.35, 171.45, 171.93, 172.15, 172.41; MS-ESI: (positive) $\mathrm{m} / \mathrm{z}$ (\%) 1101.57 $\left.(100, \mathrm{M}+\mathrm{H}\rceil^{+}\right)$.

$\mathrm{N}$-MeZ-protected cyclohexadepsipeptide $\mathbf{1 0 3} \mathbf{b}\left(\mathrm{Cyclo}-\mathrm{F}_{2} 6-\mathrm{MeZ}\right)$ : The hexadepsipeptide $101 \mathbf{b}$

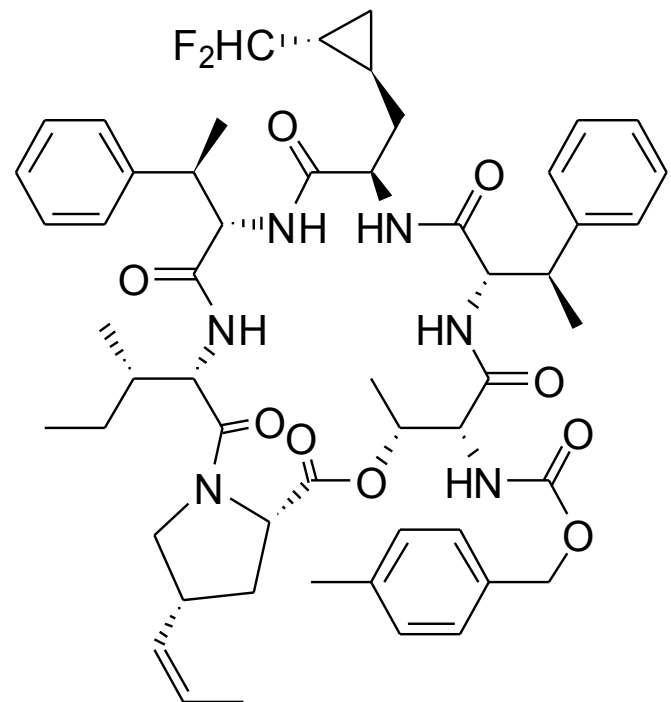
(136 mg, $122 \mu \mathrm{mol})$ was ends-deprotected by treating with $2 \mathrm{M} \mathrm{HCl}$ solution in ethyl acetate $(5 \mathrm{~mL})$. The reaction mixture was stirred for $20 \mathrm{~min}$ in dark place (Al foil jacket) at ambient temperature and all volatiles were removed under reduced pressure in vacuo without any heating. The residue was triturated with anhydrous diethyl ether to give the hydrochloride of the deprotected material $\mathbf{1 0 2} \mathbf{b}$ as a colorless solid. HRMS for $\left.(\mathrm{M}+\mathrm{H}\rceil^{+}\right)$: calculated 1001.51943, measured 1001.51859. The ends-deprotected hexadepsipeptide

$102 \mathrm{~b}, \mathrm{HATU}(54 \mathrm{mg}, 142 \mu \mathrm{mol})$ and HOAt $(16 \mathrm{mg}, 121 \mu \mathrm{mol})$ were dissolved in cold $\left(4{ }^{\circ} \mathrm{C}\right.$, internal temperature) anhydrous $\mathrm{CH}_{2} \mathrm{Cl}_{2}(1,5 \mathrm{~L})$, and the solution of DIEA (46 mg, $\left.354 \mu \mathrm{mol}\right)$ in $\mathrm{CH}_{2} \mathrm{Cl}_{2}(100 \mathrm{~mL})$ was added dropwise within $1 \mathrm{~h}$, the cooling bath was removed and the mixture was stirred for $2 \mathrm{~h}$ at ambient temperature. Then the mixture was cooled again to $4{ }^{\circ} \mathrm{C}$ (internal temperature), the second portions of HATU $(54 \mathrm{mg}, 142 \mu \mathrm{mol})$ and HOAt $(16 \mathrm{mg}, 121 \mu \mathrm{mol})$ were added, followed with dropwise addition of the solution of DIEA (46 mg, $354 \mu \mathrm{mol})$ in $\mathrm{CH}_{2} \mathrm{Cl}_{2}(100 \mathrm{~mL})$ within $1 \mathrm{~h}$. The cooling bath was removed and the mixture was stirred for $18 \mathrm{~h}$ at ambient temperature. The mixture was concentrated under reduced pressure, subjected to aqueous work-up according to GP 2 to give the crude protected cyclohexadepsipeptide (118 mg, $120 \mu \mathrm{mol}, 98 \%$ ) which was finally purified with the HPLC to give pure product $\mathbf{1 0 3} \mathbf{~ b}$ (72 $\mathrm{mg}$, $73 \mu \mathrm{mol}, 60 \%$ ). Preparative HPLC: isocratic, $60 \%$ B for $8 \mathrm{~min}$, then gradient $60 \% \rightarrow 100 \%$ B for $6 \mathrm{~min}$, then isocratic $100 \% \mathrm{~B}$ for $11 \mathrm{~min}, \mathrm{t}_{\mathrm{R}}=17.2 \mathrm{~min}$, purity $>98 \%$; analytical HPLC: isocratic $60 \%$ B for $10 \mathrm{~min}$, then gradient $60 \% \rightarrow 100 \%$ B for $20 \mathrm{~min}$, then isocratic $100 \%$ B for $15 \min$ $\mathrm{t}_{\mathrm{R}}=27.8 \mathrm{~min}$, purity $>98 \% ;[\alpha]_{\mathrm{D}}{ }^{20}-20.0(\mathrm{c}=0.15$, THF $) ;{ }^{1} \mathrm{H}$ NMR $(600 \mathrm{MHz},[\mathrm{D} 8] \mathrm{THF}): \delta=-$ 0.05-0.02 (m, 1 H), 0.12-0.18 (m, 1 H), $0.27-0.34(\mathrm{~m}, 1 \mathrm{H}), \quad 0.34-0.39(\mathrm{~m}, 1 \mathrm{H}), \quad 0.74(\mathrm{~d}$, $J=6.6 \mathrm{~Hz}, 3 \mathrm{H}), 0.79(\mathrm{t}, J=7.5 \mathrm{~Hz}, 3 \mathrm{H}), 1.10-1.24(\mathrm{~m}, 2 \mathrm{H}), 1.20(\mathrm{~d}, J=7.2,3 \mathrm{H}) 1.28(\mathrm{~d}$, $J=7.2 \mathrm{~Hz}, 3 \mathrm{H}), 1.36-1.47$ (m, $1 \mathrm{H}), 1.49$ (d, $J=6.9 \mathrm{~Hz}, 3 \mathrm{H}), 1.60-1.70$ (m, $1 \mathrm{H}), 1.66$ (dd, $J=1.7 \mathrm{~Hz}, 6.9 \mathrm{~Hz}, 3 \mathrm{H}), 1.95-2.04(\mathrm{~m}, 1 \mathrm{H}), 2.12-2.18(\mathrm{~m}, 1 \mathrm{H}), 2.30$ (s, $3 \mathrm{H}), 2.88-2.96(\mathrm{~m}$, $1 \mathrm{H}), 3.12-3.17(\mathrm{~m}, 1 \mathrm{H}), 3.22-3.31(\mathrm{~m}, 1 \mathrm{H}), 3.45-3.51(\mathrm{~m}, 1 \mathrm{H}), 3.70-3.76(\mathrm{~m}, 1 \mathrm{H}), 3.83-$ $3.88(\mathrm{~m}, 1 \mathrm{H}), 4.17-4.23(\mathrm{~m}, 1 \mathrm{H}), 4.40-4.46(\mathrm{~m}, 1 \mathrm{H}), 4.52-4.70(\mathrm{~m}, 4 \mathrm{H}), 4.93-4.99(\mathrm{~m}, 1 \mathrm{H})$, 
4.99-5.02, 5.07-5.14 (2×m, 2 H), 5.27-5.33 (m, $1 \mathrm{H}), 5.36-5.41(\mathrm{~m}, 1 \mathrm{H}), 5.51-5.58(\mathrm{~m}, 1 \mathrm{H})$, 6.74-6.82 (m, 1 H), 7.07-7.22 (m, 9 H), 7.23-7.29 (m, 5 H), 7.30-7.40 (m, 1 H), $7.64(\mathrm{~d}$, $J=8.5 \mathrm{~Hz}, 1 \mathrm{H}), \quad 7.69(\mathrm{~d}, J=7.8 \mathrm{~Hz}, 1 \mathrm{H}), \quad 7.80-7.90, \quad 8.18-8.22(2 \times \mathrm{m}, 1 \mathrm{H}) ; \quad{ }^{13} \mathrm{C} \mathrm{NMR}$ (125.7 MHz, [D8]THF): $\delta=7.89(-), 7.94(-), 10.40(+), 11.56,11.61(+), 13.15(+), 13.20(+)$, $15.32(+), 18.45(+), 18.60(+), 21.07(+), 20.45,21.43(+, \mathrm{t}, J=27.5 \mathrm{~Hz}), 30.42(-), 31.12(-)$, $34.85(-), 35.95(-), 36.07(+), 37.29(+), 39.19(+), 46.26(+), 52.52(-), 54.81(+), 55.01(+)$, $58.88(+), 60.25(+), 60.66(+), 61.70(+), 73.01(+), 118.17,118.70(+, \mathrm{t}, J=236.3 \mathrm{~Hz})$, $126.81(+), 126.90(+), 126.96(+), 127.55(+), 128.07(+), 128.47(+), 128.50(+), 128.59(+)$, $128.65(+), 128.72(+), 128.94(+), 129.46(+), 129.71(+), 130.70(+), 135.35(-), 137.67(-)$, $143.81(-), 144.84(-), 156.58(-), 168.96(-), 170.94(-), 171.04(-), 171.35(-), 172.90(-)$, $172.90(-)$; MS-ESI: positive $\left.-1005.6(100 \%, \mathrm{M}+\mathrm{Na}\rceil^{+}\right)$; negative $\left.-981.4(100 \%, \mathrm{M}-\mathrm{H}\rceil^{-}\right)$.

$\mathrm{N}$-Teoc-(2S, 1'S, 2'R)-3-(2'-Difluoromethylcyclopropyl)alanine (Teoc-(S)dFmcpA-OH, 105 b): A

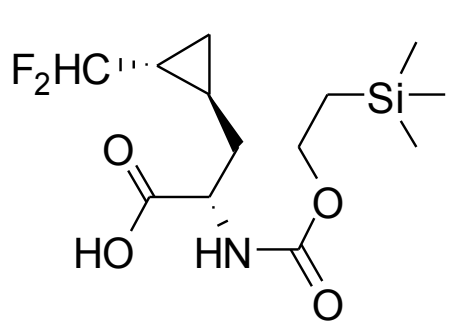
solution of TeocOSu $(59 \mathrm{mg}, 228 \mu \mathrm{mol})$ in acetone $(1 \mathrm{~mL})$ was added to a vigorously stirred solution of $\left(2 S, 1^{\prime} S, 2^{\prime} R\right)-3-\left(2^{\prime}-\right.$ difluoromethylcyclopropyl)alanine $\boldsymbol{S - 9 6}$ b $(34 \mathrm{mg}, 190 \mu \mathrm{mol})$ and $\mathrm{NaHCO}_{3}(34 \mathrm{mg}, 396 \mu \mathrm{mol})$ in water $(1 \mathrm{~mL})$ (if an emulsion formed, acetone and/or water was added to obtain a homogeneous solution), and stirring was continued for another $2 \mathrm{~h}$. $N, N$-dimethylaminopropylamine $(10 \mu \mathrm{L}$, $7.5 \mathrm{mg}, 73 \mu \mathrm{mol}$ ) was then added. After an additional $10 \mathrm{~min}$ acetone was removed under reduced pressure and the $\mathrm{pH}$ of the residual water solution was adjusted to 2-3 with aq. $1 \mathrm{M} \mathrm{KHSO}_{4}$. The resulting emulsion was extracted with diethyl ether $(50 \mathrm{~mL})$, and the ethereal layer was washed with aq. $1 \mathrm{M} \mathrm{KHSO}_{4}(2 \times 10 \mathrm{~mL})$, water $(3 \times 10 \mathrm{~mL})$, brine $(2 \times 5 \mathrm{~mL})$, dried over $\mathrm{MgSO}_{4}$, filtered and concentrated under reduced pressure. The residual oil was dried overnight in vacuo to give glass-like product $105 \mathbf{b}$ (41 mg, $127 \mu \mathrm{mol}, 67 \%)$. $R_{\mathrm{f}}=0.24$ $($ EtOAc/hexane $1: 3+2 \% \mathrm{AcOH}) ;[\alpha]_{\mathrm{D}}{ }^{20}=22.80\left(\mathrm{c}=0.46, \mathrm{CHCl}_{3}\right) ;{ }^{1} \mathrm{H} \mathrm{NMR}\left(250 \mathrm{MHz}, \mathrm{CDCl}_{3}\right.$, as cyclohexylammonium salt): $\delta=0.01(\mathrm{~s}, 9 \mathrm{H}), 0.38-0.57(\mathrm{~m}, 1 \mathrm{H}), 0.65-0.80(\mathrm{~m}, 1 \mathrm{H}), 0.85-$ 1.49 (m, 9 H), 1.50-1.85 (m, $5 \mathrm{H}), 1.85-2.03(\mathrm{~m}, 2 \mathrm{H}), 2.72-3.00$ (m, $1 \mathrm{H}), 3.89-4.22(\mathrm{~m}, 3 \mathrm{H})$, $5.50(\mathrm{td}, J=57.6 \mathrm{~Hz}, 4.1 \mathrm{~Hz}, 1 \mathrm{H}), 5.71(\mathrm{~d}, J=7.3 \mathrm{~Hz}, 1 \mathrm{H}), 7.35(\mathrm{bs}, 3 \mathrm{H}) ;{ }^{13} \mathrm{C} \mathrm{NMR}$ $\left(62.9 \mathrm{MHz}, \mathrm{CDCl}_{3}\right.$, as cyclohexylammonium salt): $\delta=-1.36,7.24,11.23,17.39,19.58(\mathrm{t}$, $J=24.0 \mathrm{~Hz}), 24.04,24.74,30.67,35.36,50.21,55.84,62.78,117.02$ (t, $J=237.5 \mathrm{~Hz}), 156.25$, 177.41 . 
Teoc-(S)dFmcpA-Cyclo-F $F_{2} 6$ (106 b): $N$-MeZ-protected cyclohexadepsipeptide $\mathbf{1 0 3}$ b (62 mg,

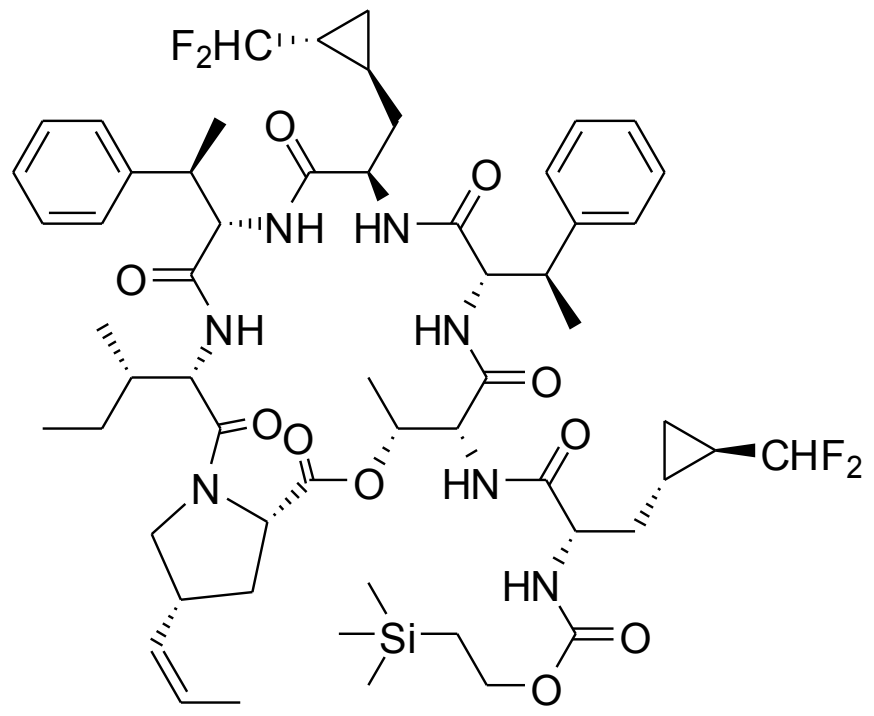
$63 \mu \mathrm{mol})$ was deprotected with $10 \%$ anisole in TFA $(4 \mathrm{~mL})$ in the dark at ambient temperature for $2 \mathrm{~h}$, the residue was treated with toluene $(5 \mathrm{~mL})$, concentrated under reduced pressure and the residue was dried in vacuo at ambient temperature for 2 hours. The solution of Teoc- $(S)$ dFmcpA-OH $\mathbf{1 0 5} \mathbf{b} \quad(31 \mathrm{mg}$, $96 \mu \mathrm{mol})$, HATU (72 $\mathrm{mg}, 189 \mu \mathrm{mol})$ and HOAt $(26 \mathrm{mg}, 190 \mu \mathrm{mol})$ in $\mathrm{CH}_{2} \mathrm{Cl}_{2}$

( $3 \mathrm{~mL}$ ) was added at $4{ }^{\circ} \mathrm{C}$, followed with DIEA (8.4 mg, $\left.65 \mu \mathrm{mol}\right)$ and TMP (69 $\left.\mathrm{mg}, 568 \mu \mathrm{mol}\right)$ solution in $\mathrm{CH}_{2} \mathrm{Cl}_{2}(5.5 \mathrm{~mL})$ and the mixture was stirred at ambient temperature for $15 \mathrm{~h}$. The reaction mixture was then diluted with diethyl ether $(50 \mathrm{~mL})$ and the crude product obtained after the usual aqueous work-up (GP 2) was purified by column chromatography (silica gel, eluted

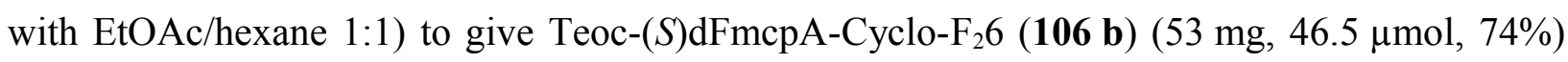
as a colorless solid. $R_{\mathrm{f}}=0.34$, EtOAc/hexane $1: 1 ;[\alpha]_{\mathrm{D}}{ }^{20}=-7.0\left(\mathrm{c}=0.3, \mathrm{CHCl}_{3}\right) ;{ }^{1} \mathrm{H} \mathrm{NMR}$ $\left(500 \mathrm{MHz}, \mathrm{CDCl}_{3}\right): \delta=-0.15--0.02(\mathrm{~m}, 2 \mathrm{H}), 0.01-0.02(\mathrm{~m}, 1 \mathrm{H}), 0.05(\mathrm{~s}, 9 \mathrm{H}), 0.06-0.10(\mathrm{~m}$, $1 \mathrm{H}), 0.28-0.42(\mathrm{~m}, 2 \mathrm{H}), 0.43-0.58(\mathrm{~m}, 2 \mathrm{H}), 0.78-0.88(\mathrm{~m}, 2 \mathrm{H}), 0.90(\mathrm{t}, J=7.3 \mathrm{~Hz}, 3 \mathrm{H})$, $0.96(\mathrm{~d}, J=6.8 \mathrm{~Hz}, 3 \mathrm{H}), 1.02(\mathrm{t}, J=8.5 \mathrm{~Hz}, 2 \mathrm{H}), 1.07-1.20(\mathrm{~m}, 3 \mathrm{H}), 1.26(\mathrm{~d}, J=7.1 \mathrm{~Hz}, 3 \mathrm{H})$, $1.31(\mathrm{~d}, J=7.2 \mathrm{~Hz}, 3 \mathrm{H}), 1.54(\mathrm{~d}, J=6.8 \mathrm{~Hz}, 3 \mathrm{H}), 1.63(\mathrm{~d}, J=6.8 \mathrm{~Hz}, 3 \mathrm{H}), 1.71(\mathrm{dd}$, $J=11.8 \mathrm{~Hz}, 23.4 \mathrm{~Hz}, 1 \mathrm{H}), 1.86-1.95(\mathrm{~m}, 1 \mathrm{H}), 2.15-2.35(\mathrm{~m}, 3 \mathrm{H}), 3.11-3.27(\mathrm{~m}, 1 \mathrm{H}), 3.23(\mathrm{t}$, $J=9.6 \mathrm{~Hz}, 1 \mathrm{H}), 3.62-3.69(\mathrm{~m}, 1 \mathrm{H}), 3.71-3.82(\mathrm{~m}, 1 \mathrm{H}), 4.02-4.18(\mathrm{~m}, 3 \mathrm{H}), 4.20-4.30$ (m, $1 \mathrm{H}), 4.34-4.41(\mathrm{~m}, 1 \mathrm{H}), 4.48-4.57(\mathrm{~m}, 1 \mathrm{H}), 4.58-4.70(\mathrm{~m}, 2 \mathrm{H}), 4.74(\mathrm{~d}, J=8.9 \mathrm{~Hz}, 1 \mathrm{H})$, $5.17(\mathrm{td}, J=60 \mathrm{~Hz}, 5.0 \mathrm{~Hz}, 1 \mathrm{H}), 5.19-5.25(\mathrm{~m}, 1 \mathrm{H}), 5.27-5.34(\mathrm{~m}, 1 \mathrm{H}), 5.47(\mathrm{td}, J=55 \mathrm{~Hz}$, $4.6 \mathrm{~Hz}, 1 \mathrm{H}), 5.53-5.61(\mathrm{~d}, J=8.9 \mathrm{~Hz}, 1 \mathrm{H}), 6.64(\mathrm{~d}, J=8.5 \mathrm{~Hz}, 1 \mathrm{H}), 7.00-7.40(\mathrm{~m}, 14 \mathrm{H})$, $8.16(\mathrm{~s}, 1 \mathrm{H}) ;{ }^{13} \mathrm{C} \mathrm{NMR}\left(125.7 \mathrm{MHz}, \mathrm{CDCl}_{3}\right): \delta=-1.47(+), 7.00(-, \mathrm{t}, J=2.6 \mathrm{~Hz}), 7.44(-, \mathrm{t}$, $J=4.2 \mathrm{~Hz}), 10.12(+), 10.40(+), 10.60(+), 13.27(+), 13.54(+), 15.27(+), 17.58(-), 17.93(+)$, $18.33(+), 20.35(+), 20.57(+), 20.79\left(^{+}\right), 24.82(-), 34.70(-), 35.50(-), 36.51(+), 37.07(+)$, $39.16(+), 43.65(+), 52.61(-), 53.38(+), 53.96(+), 54.70(+), 55.90(+), 59.05(+), 59.68(+)$, $61.02(+), 63.41(-), 71.45(+), 117.01(+, \mathrm{t}, J=237.3 \mathrm{~Hz}), 117.24(+, \mathrm{t}, J=237.7 \mathrm{~Hz})$, $126.83(+), 126.90(+), 127.20(+), 127.61(+), 127.75(+), 127.99(+), 128.44(+), 128.58(+)$, $128.63(+), 141.79(-), 156.34(-), 168.38(-), 170.09(-), 170.75(-), 171.17(-), 171.27(-)$, 
171.90 (-); MS-ESI: (positive) m/z (\%) $\left.1163(100, \mathrm{M}+\mathrm{Na}\rceil^{+}\right)$, (negative) m/z (\%) $1139(100, \mathrm{M}-$ $\left.\mathrm{H}\rceil^{-}\right)$.

MOM-O-protected difluoromethylcyclopropylalanyl Hormaomycin (MOM-O-F $F_{2} H o r m, 108$ b):

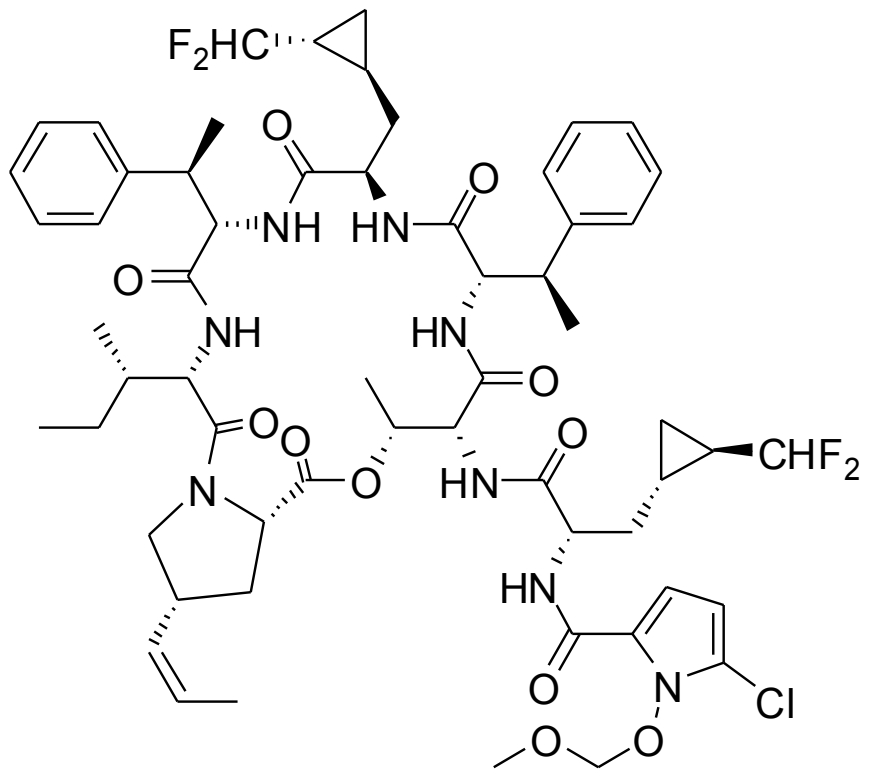

Teoc group was cleaved from the compound $106 \mathbf{b}(8.0 \mathrm{mg}, 7.08 \mu \mathrm{mol})$ with TFA $(0.6 \mathrm{~mL})$ for $1 \mathrm{~h}$. The mixture was concentrated under reduced pressure at $20{ }^{\circ} \mathrm{C}$ and then taken up with toluene $(3 \times 15 \mathrm{~mL})$ which was distilled off to remove the last traces of TFA. The resulting deprotected depsipeptide $\mathbf{1 0 7} \mathbf{b}$ was coupled with $O$-MOM protected acid 81 (2.9 mg, $14.10 \mu \mathrm{mol})$ using HATU (5.4 $\mathrm{mg}, 14.20 \mu \mathrm{mol})$, DIEA $(0.92 \mathrm{mg}, 7.12$

$\mu \mathrm{mol})$ and TMP $(5.14 \mathrm{mg}, 42.42 \mu \mathrm{mol})$ in $\mathrm{CH}_{2} \mathrm{Cl}_{2}(1 \mathrm{~mL})$ according to $\mathrm{GP} 6$ for $2.5 \mathrm{~h}$. The mixture was then taken up with $\mathrm{Et}_{2} \mathrm{O}(20 \mathrm{~mL})$ and the crude product obtained after usual aqueous work-up (GP 2) was crystallized from $\mathrm{CH}_{2} \mathrm{Cl}_{2} /$ pentane to give $O$-MOM protected difluoromethylcyclopropylalanyl Hormaomycin $\mathbf{1 0 8} \mathbf{b}\left(8.0 \mathrm{mg}, 90 \%, R_{\mathrm{f}}=0.36\right.$ acetone/hexanes $\left.1: 2\right)$ as a colorless glass. ${ }^{1} \mathrm{H} \mathrm{NMR}\left(500 \mathrm{MHz}, \mathrm{CDCl}_{3}\right): \delta=-0.47--0.37(\mathrm{~m}, 2 \mathrm{H}), 0.15-0.26(\mathrm{~m}, 4 \mathrm{H})$, 0.45-0.55 (m, 2 H), 0.80-0.92 (m, $3 \mathrm{H}), 0.87(\mathrm{t}, J=7.3 \mathrm{~Hz}, 3 \mathrm{H}), 1.01(\mathrm{~d}, J=6.8 \mathrm{~Hz}, 3 \mathrm{H})$, $1.05-1.15(\mathrm{~m}, 1 \mathrm{H}), 1.20-1.28(\mathrm{~m}, 2 \mathrm{H}), 1.26(\mathrm{~d}, J=7.1 \mathrm{~Hz}, 3 \mathrm{H}), 1.37(\mathrm{~d}, J=7.2 \mathrm{~Hz}, 3 \mathrm{H})$, $1.56(\mathrm{~d}, J=6.9 \mathrm{~Hz}, 3 \mathrm{H}), 1.66(\mathrm{~d}, J=6.9 \mathrm{~Hz}, 3 \mathrm{H}), 1.70-1.82(\mathrm{~m}, 3 \mathrm{H}), 1.85-1.95(\mathrm{~m}, 1 \mathrm{H}), 2.31-$ 2.38 (m, $1 \mathrm{H}), 2.80-2.89(\mathrm{~m}, 1 \mathrm{H}), 3.20-3.30$ (m, $2 \mathrm{H}), 3.65-3.70(\mathrm{~m}, 1 \mathrm{H}), 3.73(\mathrm{~s}, 3 \mathrm{H}), 3.82-$ $3.88(\mathrm{~m}, 1 \mathrm{H}), 3.91-3.99(\mathrm{~m}, 1 \mathrm{H}), 4.21-4.29$ (m, $2 \mathrm{H}), 4.62-4.73(\mathrm{~m}, 3 \mathrm{H}), 5.05$ (td, $J=55.0 \mathrm{~Hz}$, $5.0 \mathrm{~Hz}, 1 \mathrm{H}), 5.08-5.14(\mathrm{~m}, 1 \mathrm{H}), 5.22-5.27$ (m, $1 \mathrm{H}), 5.30-5.37$ (m, $1 \mathrm{H}), 5.43-5.48(\mathrm{~m}, 1 \mathrm{H})$, $5.52-5.59(\mathrm{~m}, 2 \mathrm{H}), 5.60-5.67(\mathrm{~m}, 1 \mathrm{H}), 6.12(\mathrm{~d}, J=4.7 \mathrm{~Hz}, 1 \mathrm{H}), 6.77-6.87(\mathrm{~m}, 2 \mathrm{H}), 7.02-$ $7.10(\mathrm{~m}, \quad 3 \mathrm{H}), \quad 7.11-7.17(\mathrm{~m}, \quad 3 \mathrm{H}), \quad 7.20-7.28(\mathrm{~m}, \quad 3 \mathrm{H}), \quad 7.24-7.43(\mathrm{~m}, \quad 2 \mathrm{H}), \quad 7.55(\mathrm{~d}$, $J=9.5 \mathrm{~Hz}, 1 \mathrm{H}), \quad 8.86(\mathrm{~d}, \quad J=9.5 \mathrm{~Hz}, 1 \mathrm{H}) ;{ }^{13} \mathrm{C} \mathrm{NMR} \quad\left(125.7 \mathrm{MHz}, \mathrm{CDCl}_{3}\right): \delta=6.82(-, \mathrm{t}$, $J=3.4 \mathrm{~Hz}), 8.18(-, \mathrm{t}, J=4.2 \mathrm{~Hz}), 10.01(+, \mathrm{t}, J=4.4 \mathrm{~Hz}), 10.72(+), 10.94(+, \mathrm{t}, J=4.7 \mathrm{~Hz})$, $13.31(+), 13.50(+), 14.09(+), 15.07(+), 17.45(+), 18.55(+), 19.69(+, \mathrm{t}, J=26.9 \mathrm{~Hz})$, $20.24(+, \mathrm{t}, J=27.8 \mathrm{~Hz}), 24.94(-), 26.90(-), 29.68(-), 35.51(-), 36.00(-), 36.72(+)$, $37.07(-), 38.62(+), 39.54(+), 43.14(+), 50.96(+), 52.65(+), 52.72(-), 54.70(+), 54.90(+)$, $59.26(+), \quad 59.41(+), \quad 59.97(+), \quad 61.44(+), \quad 70.11(+), \quad 104.24(+), \quad 106.05(-), \quad 111.15(+)$, 
$116.70(+, \mathrm{t}, J=237.5 \mathrm{~Hz}), 117.51(+, \mathrm{t}, J=237.8 \mathrm{~Hz}), 119.36(-), 121.83(-), 126.72(+)$, $126.96(+), 127.31(+), 127.51(+), 127.53(+), 128.25(+), 128.37(+), 128.64(+), 142.14(-)$, $142.54(-), 158.11(-), 168.84(-), 169.99(-), 170.17(-), 170.92(-), 171.32(-), 171.43(-)$, 171.90 (-); MS-ESI: (positive) m/z (\%) $\left.1205(100, \mathrm{M}+\mathrm{Na}\rceil^{+}\right)$, (negative) m/z (\%) 1181 (100, M$\left.\mathrm{H}\rceil^{-}\right)$.

Difluoromethylcyclopropylalanyl Hormaomycin $\left(F_{2} H o r m, 109\right.$ b): O-MOM protected difluoro-

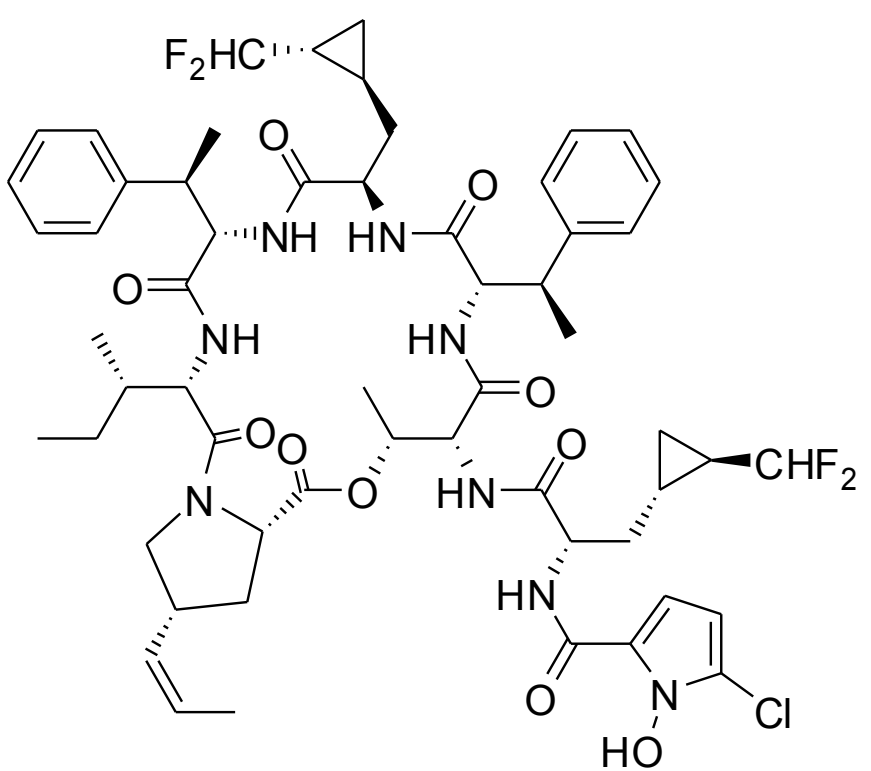
methylcyclopropylalanyl Hormaomycin 108 b (35 mg, $29.5 \mu \mathrm{mol}$ ) was deprotected using $\mathrm{MgBr}_{2} \times \mathrm{Et}_{2} \mathrm{O}$ (204 mg, $\left.788 \mu \mathrm{mol}\right)$ and EtSH $(50 \mu \mathrm{L}, 0.7 \mathrm{mmol})$ in $\mathrm{CH}_{2} \mathrm{Cl}_{2}$ $(17 \mathrm{~mL})$ according to GP 7 for $3 \mathrm{~h}$. The mixture was taken up with EtOAc and the crude product obtained after usual aqueous work-up was crystallized from $\mathrm{CH}_{2} \mathrm{Cl}_{2}$ / pentane to give $\mathbf{1 0 9} \mathbf{b}(33 \mathrm{mg})$ as a white solid, which was finally purified with preparative HPLC. $\mathrm{R}_{\mathrm{f}}=0.24$ acetone/ hexanes 3:7; preparative HPLC: isocratic, $82 \% \mathrm{~B}$ for $25 \mathrm{~min}, \mathrm{t}_{\mathrm{R}}=15.4 \mathrm{~min}$, purity $>98 \%$; analytical HPLC: isocratic $60 \%$ B for $10 \mathrm{~min}$, then gradient $60 \% \rightarrow 100 \%$ B for $20 \mathrm{~min}$, then isocratic $100 \% \mathrm{~B}$ for $15 \mathrm{~min} \mathrm{t}_{\mathrm{R}}=28.8 \mathrm{~min}$, purity $>98 \%$; $[\alpha]_{\mathrm{D}}{ }^{20} 20.0(\mathrm{c}=0.1, \mathrm{MeOH}) ;{ }^{1} \mathrm{H} \mathrm{NMR}$ $\left(250 \mathrm{Mhz}, \mathrm{CDCl}_{3}\right): \delta=-0.62--0.50(\mathrm{~m}, 2 \mathrm{H}),-0.18--0.08(\mathrm{~m}, 1 \mathrm{H}),-0.07-0.01(\mathrm{~m}, 1 \mathrm{H})$, $0.31-0.50(\mathrm{~m}, 2 \mathrm{H}), 0.86(\mathrm{~d}, J=6.3 \mathrm{~Hz}, 3 \mathrm{H}), 0.92(\mathrm{t}, J=7.3 \mathrm{~Hz}, 3 \mathrm{H}), 1.03(\mathrm{~d}, J=6.5 \mathrm{~Hz}, 3 \mathrm{H})$, 1.11-1.19 (m, $2 \mathrm{H}), 1.21-1.40(\mathrm{~m}, 5 \mathrm{H}), 1.47(\mathrm{~d}, J=7.1 \mathrm{~Hz}, 3 \mathrm{H}), 1.54(\mathrm{~d}, J=6.8 \mathrm{~Hz}, 3 \mathrm{H})$, $1.69(\mathrm{~m}, J=6.7 \mathrm{~Hz}, 3 \mathrm{H}), 1.78-1.85$ (m, $2 \mathrm{H}), 1.91-2.05$ (m, $2 \mathrm{H}), 2.34$ (dd, $J=7.1 \mathrm{~Hz}, 7.4 \mathrm{~Hz}$, $2 \mathrm{H}), 2.95-3.17(\mathrm{~m}, 1 \mathrm{H}), 3.18-3.32(\mathrm{~m}, 1 \mathrm{H}), 3.33-3.59(\mathrm{~m}, 3 \mathrm{H}), 3.70(\mathrm{dd}, J=4.7 \mathrm{~Hz}, 6.7 \mathrm{~Hz}$, $1 \mathrm{H}), 3.91-4.47(\mathrm{~m}, 3 \mathrm{H}), 4.51-4.75(\mathrm{~m}, 3 \mathrm{H}), 5.02-5.18(\mathrm{~m}, 1 \mathrm{H}), 5.22-5.42(\mathrm{~m}, 2 \mathrm{H}), 5.56-$ $5.73(\mathrm{~m}, 1 \mathrm{H}), 6.14(\mathrm{~d}, J=4.6 \mathrm{~Hz}, 1 \mathrm{H}), 6.57-6.78(\mathrm{~m}, 2 \mathrm{H}), 6.82(\mathrm{~d}, J=4.7 \mathrm{~Hz}, 1 \mathrm{H}), 6.98(\mathrm{~d}$, $J=9.8 \mathrm{~Hz}, 1 \mathrm{H}), 7.10-7.37(\mathrm{~m}, 10 \mathrm{H}), 7.90(\mathrm{~d}, J=9.3 \mathrm{~Hz}, 1 \mathrm{H}), 9.17$ (d, $J=8.9 \mathrm{~Hz}, 1 \mathrm{H})$; ${ }^{13} \mathrm{C}$ NMR $\left(62.9 \mathrm{MHz}, \mathrm{CDCl}_{3}\right): \delta=6.82(-), 8.18(-), 10.43(+), 13.33(+), 14.92(+), 17.18(+)$, $17.94(+), 18.72(+), 19.65(+), 20.02(+), 22.68(+), 24.98(+), 29.11(+), 29.35(-), 29.68(-)$, $31.42(-), 31.91(-), 33.66(-), 35.53(+), 36.64(-), 37.62(+), 39.14(+), 41.63(+), 47.03(+)$, $51.87(+), 52.65(-), 52.88(+), 54.67(+), 54.85(+), 59.43(+), 61.03(+), 61.33(+), 69.11(+)$, $103.62(+, \mathrm{t}, J=235.7 \mathrm{~Hz}), 109.46(+, \mathrm{t}, J=237.3 \mathrm{~Hz}), 111.15(+), 119.13(-), 121.67(-)$, 
$126.78(+), 127.23(+), 127.45(+), 127.64(+), 127.94(+), 128.20(+), 128.49(+), 128.87(+)$, $141.70(-), 141.88(-), 168.56(-), 169.03(-), 170.10(-), 170.45(-), 171.39(-), 171.63(-)$, $171.90(-), 172.62(-)$; MS-ESI: (positive) m/z (\%) $\left.1161(100, \mathrm{M}+\mathrm{Na}\rceil^{+}\right)$, (negative) $\mathrm{m} / \mathrm{z}(\%)$ $\left.1137(100, \mathrm{M}-\mathrm{H}\rceil^{-}\right)$.

\section{3. (Monofluoromethylcyclopropyl)alanyl-Hormaomycin}

N-Fmoc-(2R, 1'R, 2'R)-3-(2'-Monofluoromethylcyclopropyl)alanine

(Fmoc-(R)mFmcpA-OH,

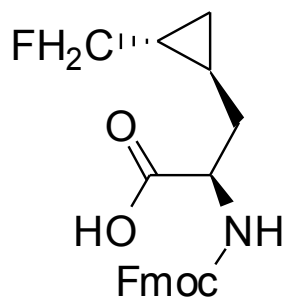

$97 \mathrm{a})$ : A solution of Fmoc-OSu (459 mg, $1.36 \mathrm{mmol})$ in acetone $(7 \mathrm{~mL})$ was added to a vigorously stirred solution of $\left(2 R, 1^{\prime} R, 2^{\prime} R\right)-3-\left(2^{\prime}-\right.$-monofluoromethyl cyclopropyl) alanine $\boldsymbol{R}-96$ a $(225 \mathrm{mg}, 1.14 \mathrm{mmol})$ and $\mathrm{NaHCO}_{3}(0.202 \mathrm{~g}$, $2.40 \mathrm{mmol}$ ) in water $(5 \mathrm{~mL})$ (if a precipitate formed, acetone and/or water was added to obtain a homogeneous solution) and stirring continued for an

additional $3 \mathrm{~h}$. Acetone was then removed under reduced pressure, and the $\mathrm{pH}$ of the residual water solution was adjusted to 1 with aq. $1 \mathrm{M} \mathrm{KHSO}_{4}$. The resulting emulsion was extracted with diethyl ether $(30 \mathrm{~mL})$ and the ethereal layer was back-extracted with aq. $3 \% \mathrm{NaHCO}_{3}$ $(5 \times 10 \mathrm{~mL})$. The combined aqueous fractions were washed with diethyl ether $(2 \times 10 \mathrm{~mL})$, acidified to $\mathrm{pH} 2$ with aq. $1 \mathrm{M} \mathrm{KHSO}_{4}$, and the resulting emulsion was extracted with diethyl ether $(4 \times 10 \mathrm{~mL})$. The organic phase was washed with aq. $1 \mathrm{M} \mathrm{KHSO}_{4}(2 \times 10 \mathrm{~mL})$, water $(3 \times 10 \mathrm{~mL})$, brine $(2 \times 5 \mathrm{~mL})$, dried, filtered and concentrated under reduced pressure. The residue was triturated with cold pentane and filtered. The resulting extremely viscous oil was dried at 0.02 Torr for prolonged time to give the target protected amino acid $97 \mathbf{a}(390 \mathrm{mg}$, $0.930 \mathrm{mmol}, 73 \%$ ) as a colorless foam. $R_{\mathrm{f}}=0.08$ (EtOAc/hexane 1:1); m.p. (softening) $50-57^{\circ} \mathrm{C}$; $[\alpha]_{\mathrm{D}}{ }^{20}-56.7\left(\mathrm{c}=0.36, \mathrm{CHCl}_{3}\right) ;{ }^{1} \mathrm{H} \mathrm{NMR}\left(600 \mathrm{MHz}, \mathrm{CDCl}_{3}\right): 0.38-0.44+0.57-0.64+0.79-0.86$ $(3 \times \mathrm{m}, 1 \mathrm{H}), 0.91-0.97+1.00-1.09(2 \times \mathrm{m}, 1 \mathrm{H}), 1.14-1.22+1.26-1.34(2 \times \mathrm{m}, 1 \mathrm{H}), 1.35-$ $1.53+1.85-1.88(2 \times \mathrm{m}, 1 \mathrm{H}), 1.80-1.85(\mathrm{~m}, 1 \mathrm{H}), 3.75-3.79+4.53-4.67(2 \times \mathrm{m}, 1 \mathrm{H}), 3.95-$ $4.01+4.47-4.52(2 \times \mathrm{m}, 1 \mathrm{H}), 4.16-4.23(\mathrm{~m}, 1 \mathrm{H}), 4.36-4.47(\mathrm{~m}, 1 \mathrm{H}), 5.52(\mathrm{~d}, J=7.9 \mathrm{~Hz}$, $0.7 \mathrm{H}), 6.76(\mathrm{~d}, J=5.9 \mathrm{~Hz}, 0.3 \mathrm{H}), 7.27-7.31(\mathrm{~m}, 2 \mathrm{H}), 7.35-7.41(\mathrm{~m}, 2 \mathrm{H}), 7.51(\mathrm{t}, \mathrm{J}=8.1 \mathrm{~Hz}$, $0.6 \mathrm{H}), 7.58$ (t, J = 8.4 Hz, $1.4 \mathrm{H}), 7.74(\mathrm{~d}, J=7.4 \mathrm{~Hz}, 2 \mathrm{H}), 7.85-8.65$ (bs, $1 \mathrm{H}) ;{ }^{13} \mathrm{C}$ NMR $\left(125.7 \mathrm{MHz}, \mathrm{CDCl}_{3}\right): \delta=7.94(-), 11.46(+), 19.73(+, \mathrm{d}, J=37.0 \mathrm{~Hz}), 34.65(-), 47.10(+)$, $53.48+53.82(+), 67.14+67.87(-), 120.00(+), 124.97(+), 125.96(-, \mathrm{d}, J=272.4 \mathrm{~Hz}), 127.07$ $(+), \quad 127.76(+), \quad 141.32 \quad\left(\mathrm{C}_{\text {quat }}\right), \quad 143.51 \quad\left(\mathrm{C}_{\text {quat }}\right), 143.76 \quad\left(\mathrm{C}_{\text {quat }}\right), 155.79+156.71 \quad\left(\mathrm{C}_{\text {quat }}\right)$, $174.96+175.79\left(\mathrm{C}_{\text {quat }}\right)$; MS-ESI: (positive) $\mathrm{m} / \mathrm{z}(\%)$ : $\left.1302(35,3 \mathrm{M}-\mathrm{H}+2 \mathrm{Na}\rceil^{+}\right), 861 \quad(100$, 
$\left.\left.2 \mathrm{M}+\mathrm{Na}\rceil^{+}\right), 442(\mathrm{M}+\mathrm{Na}\rceil^{+}\right),\left(\right.$negative) $\left.\left.\mathrm{m} / \mathrm{z}(\%): 837(100,2 \mathrm{M}-\mathrm{H}\rceil^{-}\right), 418(16, \mathrm{M}-\mathrm{H}\rceil^{-}\right), 222(14$, $\left.\mathrm{M}-\mathrm{FmOH}-\mathrm{H}^{-}\right), 196\left(15, \mathrm{FmOH}^{-}\right)$.

Fmoc-(R)mFmcpA-MeF-Ile-ODCPM (98 a): Dipeptide 60 (434 mg, $834 \mu \mathrm{mol})$ was taken up

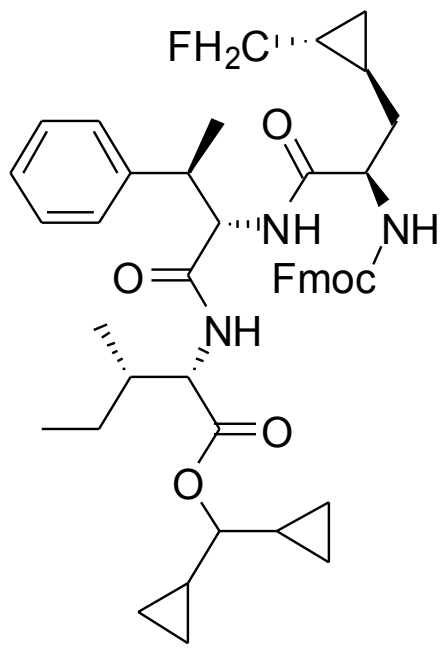
with EtOAc $(20 \mathrm{~mL})$ and hydrogenated over 10\% $\mathrm{Pd} / \mathrm{C}(250 \mathrm{mg})$ under ambient pressure of hydrogen for $2 \mathrm{~h}$. The reaction mixture was filtered through a pad of Celite ${ }^{\circledR}$ and concentrated under reduced pressure to give deprotected dipeptide 62, which was directly used for the coupling with Fmoc- $(R)$ mFmcpA-OH 97 a (360 mg, $860 \mu \mathrm{mol})$, using EDC (172 mg, $896 \mu \mathrm{mol})$, HOAt (120 mg, $883 \mu \mathrm{mol})$ and TMP $(310 \mu \mathrm{L}, 2.5 \mathrm{mmol})$ according to GP 2. The mixture was diluted with chloroform $(50 \mathrm{~mL})$ and subjected usual aqueous work-up according to GP 2 to give the pure tripeptide 98 a as white solid (535 mg, $679 \mu \mathrm{mol}, 72 \%) . R_{\mathrm{f}}=0.52$;

EtOAc/hexane 2:3; m.p. $151-155^{\circ} \mathrm{C} ;[\alpha]_{\mathrm{D}}{ }^{20}-3,8\left(c=0.26, \mathrm{CHCl}_{3}\right) ;{ }^{1} \mathrm{H}$ NMR $\left(500 \mathrm{MHz}, \mathrm{CDCl}_{3}\right)$ : $\delta=0.28-0.40(\mathrm{~m}, 4 \mathrm{H}), 0.43-0.53(\mathrm{~m}, 2 \mathrm{H}), 0.57-0.66(\mathrm{~m}, 3 \mathrm{H}), 0.88(\mathrm{~d}, J=6.7 \mathrm{~Hz}, 3 \mathrm{H}), 0.90$ (t, $J=7.4 \mathrm{~Hz}, 3 \mathrm{H}), 0.94-1.00$ (m, $1 \mathrm{H}), 1.03-1.13$ (m, $2 \mathrm{H}), 1.13-1.22$ (m, $2 \mathrm{H}), 1.30-1.50$ (m, $2 \mathrm{H}), 1.41(\mathrm{~d}, J=6.8 \mathrm{~Hz}, 3 \mathrm{H}), 1.58-1.70(\mathrm{~m}, 1 \mathrm{H}), 1.81-1.97(\mathrm{~m}, 2 \mathrm{H}), 3.28-3.38(\mathrm{~m}, 1 \mathrm{H}), 3.86$ $(\mathrm{t}, J=8.4 \mathrm{~Hz}, 1 \mathrm{H}), 4.28(\mathrm{t}, J=6.9 \mathrm{~Hz}, 1 \mathrm{H}), 4.39-4.48(\mathrm{~m}, 3 \mathrm{H}), 4.48-4.57(\mathrm{~m}, 1 \mathrm{H}), 4.76(\mathrm{t}, J=$ $8.0 \mathrm{~Hz}, 1 \mathrm{H}), 5.76(\mathrm{~d}, J=7.7 \mathrm{~Hz}, 1 \mathrm{H}), 6.40(\mathrm{~d}, J=7.5 \mathrm{~Hz}, 1 \mathrm{H}), 7.11(\mathrm{~d}, J=8.1 \mathrm{~Hz}, 1 \mathrm{H}), 7.20$ $7.34(\mathrm{~m}, 5 \mathrm{H}), 7.35$ (t, $J=7.4 \mathrm{~Hz}, 2 \mathrm{H}), 7.46$ (t, $J=7.4 \mathrm{~Hz}, 2 \mathrm{H}), 7.60$ (t, $J=8.0 \mathrm{~Hz}, 2 \mathrm{H}), 7.79$ $(\mathrm{d}, J=7.5 \mathrm{~Hz}, 2 \mathrm{H}) ;{ }^{13} \mathrm{C}$ NMR $\left(125.7 \mathrm{MHz}, \mathrm{CDCl}_{3}\right) \delta=2.57 ; 2.90,8.18,11.62,14.39$, 14.66, 15.07, 15.07, 16.86, 19.70 (q, $J=37.1 \mathrm{~Hz}$ ), 25.14, 35.08, 38.13, 42.05, 47.07, 54.69,56.46, 58.79, $67.18,83.44,119.94,124.99,127.06,127.55,127.71,128.54,141.23,141.28,141.70,143.57$, $143.78,155.95,169.64,170.55,170.94$.

Fmoc-MeF-(R)mFmcpA-MeF-Ile-ODCPM (100 a): The tripeptide 98 a (394 mg, $500 \mu \mathrm{mol})$ was

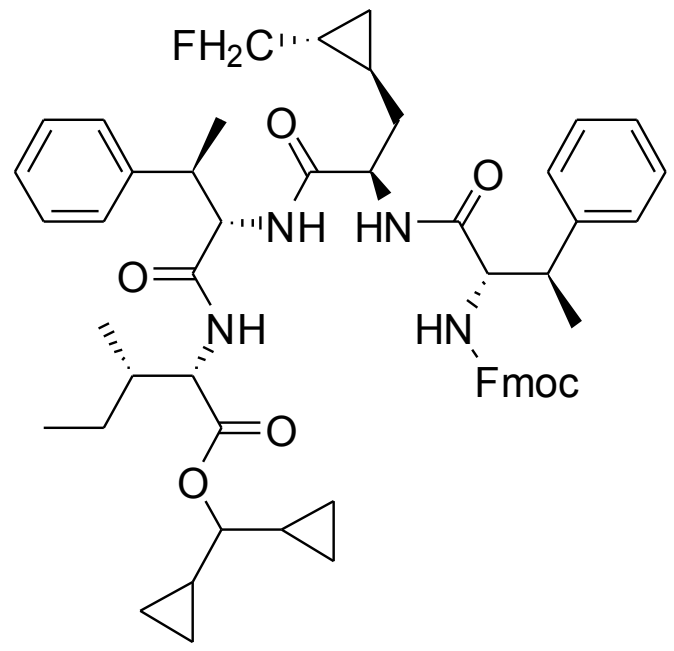
deprotected according to GP 1 and the resulting $C$-protected tripeptide 99 a was then directly coupled with Fmoc-MeF-OH 64 (211 mg, 525 mol) according to GP 2 using EDC (99 mg, $518 \mu \mathrm{mol})$, HOAt (70 mg, $512 \mu \mathrm{mol})$ and TMP $(175 \mathrm{mg}, 1440 \mu \mathrm{mol})$ in $\mathrm{CH}_{2} \mathrm{Cl}_{2}$ $(3 \mathrm{~mL})$. After $16 \mathrm{~h}$ the reaction mixture was diluted with chloroform $(50 \mathrm{~mL})$ and subjected usual aqueous work-up according to GP 2 to give the crude 
tetrapeptide, which was twice re-crystallized from THF/hexane, giving the pure target tetrapeptide 100 a as off-white solid (440 mg, $463 \mu \mathrm{mol}, 88 \%$ ). $R_{\mathrm{f}}=0.29 ; \mathrm{CHCl}_{3} / \mathrm{MeOH} 70: 1$; m.p. $210-215^{\circ} \mathrm{C}$ (decomp.); $[\alpha]_{\mathrm{D}}{ }^{20}-26,3\left(c=0.32\right.$, THF); ${ }^{1} \mathrm{H}$ NMR $\left(250 \mathrm{MHz}, \mathrm{CDCl}_{3}\right): \delta=$ $0.22-0.36(\mathrm{~m}, 4 \mathrm{H}), 0.41(\mathrm{t}, J=8.9 \mathrm{~Hz}, 3 \mathrm{H}), 0.48-0.61(\mathrm{~m}, 2 \mathrm{H}), 0.62-0.71(\mathrm{~m}, 1 \mathrm{H}), 0.76(\mathrm{~d}, J$ $=6.9 \mathrm{~Hz}, 3 \mathrm{H}), 0.82(\mathrm{t}, J=7.3 \mathrm{~Hz}, 3 \mathrm{H}), 0.92-1.09(\mathrm{~m}, 4 \mathrm{H}), 1.09-1.18(\mathrm{~m}, 1 \mathrm{H}), 1.22-1.46(\mathrm{~m}$, $2 \mathrm{H}) 1.26(\mathrm{~d}, J=6.9 \mathrm{~Hz}, 3 \mathrm{H}), 1.39(\mathrm{~d}, J=6.6 \mathrm{~Hz}, 3 \mathrm{H}), 1.71-1.89$ (m, $1 \mathrm{H}), 3.09-3.36(\mathrm{~m}, 3 \mathrm{H})$, $3.81(\mathrm{t}, J=8.4 \mathrm{~Hz}, 1 \mathrm{H}), 4.15-4.33(\mathrm{~m}, 3 \mathrm{H}), 4.35-4.64(\mathrm{~m}, 3 \mathrm{H}), 4.62(\mathrm{t}, J=8.3 \mathrm{~Hz}, 1 \mathrm{H}), 5.95$ (d, $J=7.1 \mathrm{~Hz}, 1 \mathrm{H}), 6.40(\mathrm{~d}, J=7.4 \mathrm{~Hz}, 1 \mathrm{H}), 7.08-7.45(\mathrm{~m}, 16 \mathrm{H}), 7.57(\mathrm{t}, J=8.9 \mathrm{~Hz}, 2 \mathrm{H})$, $7.76(\mathrm{~d}, J=7.3 \mathrm{~Hz}, 2 \mathrm{H}) ;{ }^{13} \mathrm{C} \mathrm{NMR}\left(62.9 \mathrm{MHz}, \mathrm{CDCl}_{3}\right): \delta=3.3(-), 3.5(-), 3.6(-), 3.7(-), 12.2$ $(+), 15.6(+), 15.9(+), 16.1(+), 17.7(+), 18.7(+), 18.9(-), 23.2(+), 26.2(-), 35.0(-), 39.1\left(^{+}\right)$, $42.8(+), 44.1(+), 48.5(+), 52.8(+), 57.4(+), 59.3(+), 60.2(+), 62.1(+), 67.8(-), 83.4(+)$, $115,1(+, \mathrm{q}, J=291.4 \mathrm{~Hz}), 126.4(+), 127.5(+), 127.7(+), 128.1(+), 128.6(+), 129.1(+), 129.1$ $(+), 129.3(+), 129.4(+), 142.5\left(\mathrm{C}_{\text {quat }}\right), 144.1\left(\mathrm{C}_{\text {quat }}\right), 144.4\left(\mathrm{C}_{\text {quat }}\right), 145.4\left(\mathrm{C}_{\text {quat }}\right), 145.6\left(\mathrm{C}_{\text {quat }}\right)$, $157.8\left(\mathrm{C}_{\text {quat }}\right), 171.1\left(\mathrm{C}_{\text {quat }}\right), 171.5\left(\mathrm{C}_{\text {quat }}\right), 171.9\left(\mathrm{C}_{\text {quat }}\right), 172.1\left(\mathrm{C}_{\text {quat }}\right)$.

Boc-(4-Pe)Pro-[MeZ-a-Thr]-MeF-(S)mFmcpA-MeF-Ile-ODCPM (101 a): The tetrapeptide

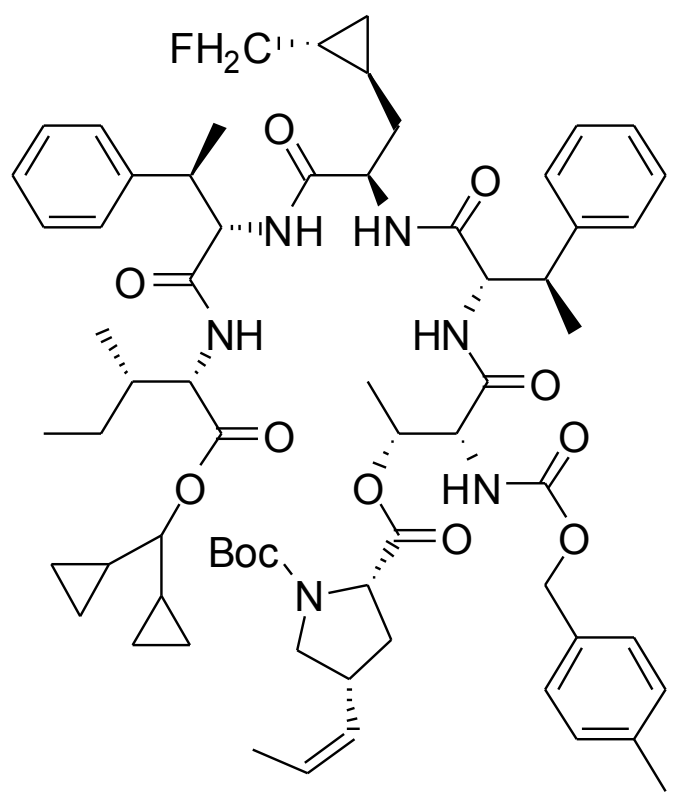

100 a $\quad(332 \mathrm{mg}, \quad 350 \mu \mathrm{mol}) \quad$ was $\quad N$-deprotected according to GP 1 , taken up with anhydrous $\mathrm{CH}_{2} \mathrm{Cl}_{2}$ $(5 \mathrm{~mL})$, the solution of the ester acid 71 (194 mg, $385 \mu \mathrm{mol})$, HATU $(160 \mathrm{mg}, 420 \mu \mathrm{mol})$ and HOAt (53 mg, $385 \mu \mathrm{mol})$ in $\mathrm{CH}_{2} \mathrm{Cl}_{2}(3 \mathrm{~mL})$ was added, and the reaction mixture was cooled to $4{ }^{\circ} \mathrm{C}$. After this, a solution of DIEA ( $65 \mu \mathrm{L}, 48 \mathrm{mg}, 368 \mu \mathrm{mol})$ and TMP $(140 \mu \mathrm{L}, 127 \mathrm{mg}, 1050 \mu \mathrm{mol})$ in $\mathrm{CH}_{2} \mathrm{Cl}_{2}(2 \mathrm{~mL})$ was added at the same temperature within $5 \mathrm{~min}$. The temperature was allowed to reach $20^{\circ} \mathrm{C}$, and stirring was continued for an additional 15 hours. After aqueous work-up according to GP 2 and two recrystallizations from EtOAc/hexane (1:2), the target hexadepsipeptide 101 a (390 mg, $321 \mu \mathrm{mol}, 86 \%)$ was obtained as a colorless solid. $R_{\mathrm{f}}=0.46$ (EtOAc/hexane 1:1); m.p. 125$127{ }^{\circ} \mathrm{C} ;[\alpha]_{\mathrm{D}}{ }^{20}-29.0(\mathrm{c}=0.2, \mathrm{THF}) ;{ }^{1} \mathrm{H} \mathrm{NMR}\left(250 \mathrm{MHz}, \mathrm{CDCl}_{3}\right): \delta=0.24-0.68(\mathrm{~m}, 12 \mathrm{H}), 0.75$ $(\mathrm{d}, J=6.9 \mathrm{~Hz}, 3 \mathrm{H}), 0.88(\mathrm{t}, J=7.1 \mathrm{~Hz}, 3 \mathrm{H}), 0.98-1.17(\mathrm{~m}, 5 \mathrm{H}), 1.18-1.46(\mathrm{~m}, 1 \mathrm{H}), 1.24(\mathrm{~d}$, $J=6.6 \mathrm{~Hz}, 3 \mathrm{H}), 1.27(\mathrm{~d}, J=6.7 \mathrm{~Hz}, 3 \mathrm{H}), 1.36(\mathrm{~s}, 9 \mathrm{H}), 1.40(\mathrm{~d}, J=6.7 \mathrm{~Hz}, 3 \mathrm{H}), 1.68(\mathrm{~d}, J=$ $7.0 \mathrm{~Hz}, 3 \mathrm{H}), 1.75-1.94(\mathrm{~m}, 2 \mathrm{H}), 2.29-2.46$ (m, $1 \mathrm{H}), 2.32$ (s, $3 \mathrm{H}), 3.07-3.33(\mathrm{~m}, 4 \mathrm{H}), 3.68$ (t, $J=8.6 \mathrm{~Hz}, 1 \mathrm{H}), 3.93(\mathrm{t}, J=8.3 \mathrm{~Hz}, 1 \mathrm{H}), 4.18-4.27(\mathrm{~m}, 1 \mathrm{H}), 4.32-4.54(\mathrm{~m}, 4 \mathrm{H}), 4.64(\mathrm{t}$, 
$J=9.6 \mathrm{~Hz}, 1 \mathrm{H}), 4.95-5.13(\mathrm{~m}, 2 \mathrm{H}), 5.20-5.34(\mathrm{~m}, 1 \mathrm{H}), 5.44-5.63(\mathrm{~m}, 2 \mathrm{H}), 6.60(\mathrm{~d}, J=$ $7.7 \mathrm{~Hz}, 1 \mathrm{H}), 6.70$ (d, $J=8.8 \mathrm{~Hz}, 1 \mathrm{H}), 6.82(\mathrm{~d}, J=8.4 \mathrm{~Hz}, 1 \mathrm{H}), 7.05-7.37$ (m, $14 \mathrm{H}), 7.45$ (d, $J=10.1 \mathrm{~Hz}, 1 \mathrm{H}), 7.76(\mathrm{~d}, J=8.1 \mathrm{~Hz}, 1 \mathrm{H}) ;{ }^{13} \mathrm{C} \mathrm{NMR}\left(62.9 \mathrm{MHz}, \mathrm{CDCl}_{3}\right): \delta=2.48(-), 2.82(-)$, $2.90(-), 3.01(-), 11.59(+), 13.20(+), 14.16(+), 14.64(+), 15.57(+), 17.77(+), 18.41(-)$, $18.86(+), 19.43(+), 21.11(+), 21.77(+), 25.23(-), 26.85(-), 28.23(+), 31.46(-), 31.53(-)$, $36.28(-), 36.32(+), 37.30(+), 40.45(+), 42.00(+), 50.62(+), 52.08(+), 56.43(+), 59.30(+)$, $59.49(+), 61.01(+), 61.62(+), 61.99(+), 66.89(-), 70.53(+), 80.93\left(\mathrm{C}_{\text {quat }}\right), 83.24(+), 116,2(+$, q, $J=287.3 \mathrm{~Hz}), 127.00(+), 127.06(+), 127.10(+), 127.60(+), 127.69(+), 128.47(+), 128.63$ $(+), \quad 128.68(+), \quad 128.82(+), \quad 128.93(+), \quad 133.21\left(\mathrm{C}_{\text {quat }}\right), \quad 137.83\left(\mathrm{C}_{\text {quat }}\right), 141.73\left(\mathrm{C}_{\text {quat }}\right)$, $141.90\left(\mathrm{C}_{\text {quat }}\right), \quad 154.76\left(\mathrm{C}_{\text {quat }}\right), \quad 155.75\left(\mathrm{C}_{\text {quat }}\right), \quad 170.38\left(\mathrm{C}_{\text {quat }}\right), \quad 170.43\left(\mathrm{C}_{\text {quat }}\right), \quad 170.79\left(\mathrm{C}_{\text {quat }}\right)$, $171.37\left(\mathrm{C}_{\text {quat }}\right), 173.41\left(\mathrm{C}_{\text {quat }}\right), 174.06\left(\mathrm{C}_{\text {quat }}\right)$.

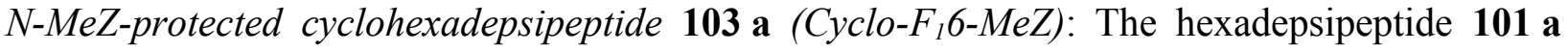

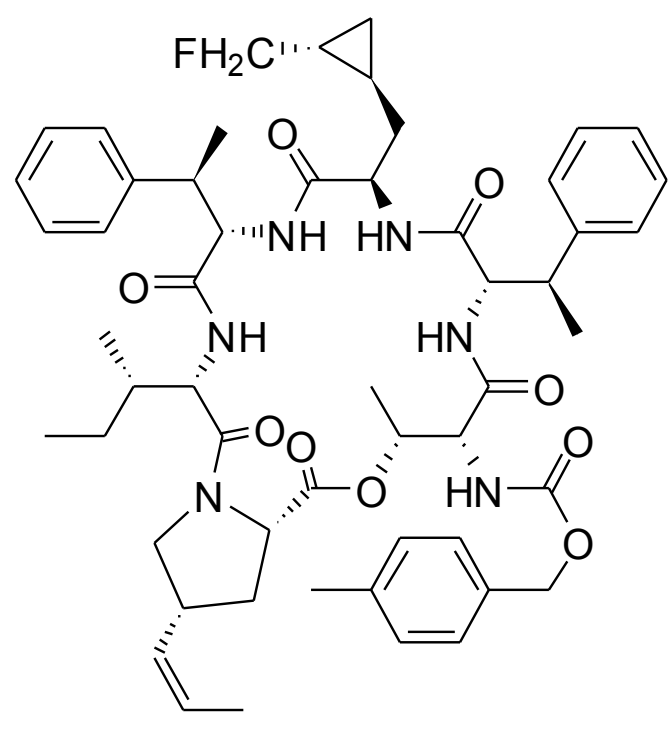
(300 mg, $247 \mu \mathrm{mol})$ was ends-deprotected by treating with $2 \mathrm{M} \mathrm{HCl}$ solution in ethyl acetate $(5 \mathrm{~mL})$. The reaction mixture was stirred for $20 \mathrm{~min}$ in dark place (Al foil jacket) at ambient temperature and all volatiles were removed in vacuo without any heating. The residue was triturated with anhydrous diethyl ether to give the hydrochloride of the deprotected material $102 \mathrm{a}$ as a colorless solid (232 mg, $220 \mu \mathrm{mol}, 89 \%)$. The ends-deprotected hexadepsipeptide 102 a, HATU (110 mg, $288 \mu \mathrm{mol})$ and HOAt $(33 \mathrm{mg}, 244 \mu \mathrm{mol})$ were dissolved in cold $\left(4{ }^{\circ} \mathrm{C}\right.$, internal temperature $)$ anhydrous $\mathrm{CH}_{2} \mathrm{Cl}_{2}(2,5 \mathrm{~L})$, and the solution of DIEA $(120 \mu \mathrm{L}, 93 \mathrm{mg}, 720 \mu \mathrm{mol})$ in $\mathrm{CH}_{2} \mathrm{Cl}_{2}$ $(100 \mathrm{~mL})$ was added dropwise within 1 hour, the cooling bath was removed and the mixture was stirred for 2 hours at ambient temperature. Then the mixture was cooled again to $4{ }^{\circ} \mathrm{C}$ (internal temperature), the second portions of HATU $(110 \mathrm{mg}, 288 \mu \mathrm{mol})$ and HOAt $(33 \mathrm{mg}, 244 \mu \mathrm{mol})$ were added, followed with dropwise addition of the solution of DIEA $(120 \mu \mathrm{L}, 93 \mathrm{mg}$, $720 \mu \mathrm{mol})$ in $\mathrm{CH}_{2} \mathrm{Cl}_{2}(100 \mathrm{~mL})$ within 1 hour. The cooling bath was removed and the mixture was stirred for 18 hours at ambient temperature. The mixture was concentrated under reduced pressure, subjected to aqueous work-up according to GP 2 to give the crude protected cyclohexadepsipeptide (180 mg, $180 \mu \mathrm{mol}, 73 \%$ ) which was finally purified with the HPLC to give pure product 103 a $(132 \mathrm{mg}, 132 \mu \mathrm{mol}, 49 \%)$. 
N-Teoc-(2S, 1'S, 2'R)-3-(2'-Monofluoromethylcyclopropyl)alanine (Teoc-(S)mFmcA-OH, 105 a):

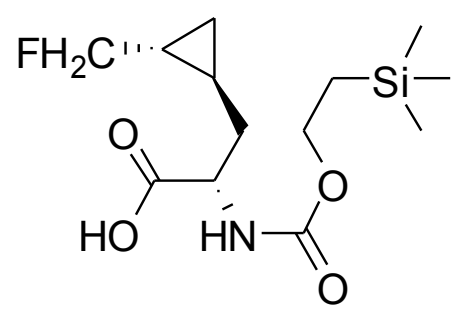

A solution of TeocOSu $(43 \mathrm{mg}, 164 \mu \mathrm{mol})$ in acetone $(1 \mathrm{~mL})$ was added to a vigorously stirred solution of $\left(2 S, 1^{\prime} S, 2^{\prime} R\right)-3-\left(2^{\prime}-\right.$ monofluoromethylcyclopropyl)alanine $\mathbf{S - 9 6}$ a $(27 \mathrm{mg}, 137 \mu \mathrm{mol})$ and $\mathrm{NaHCO}_{3}(24 \mathrm{mg}, 286 \mu \mathrm{mol})$ in water $(1 \mathrm{~mL})$ (if an emulsion formed, acetone and/or water was added to obtain a homogeneous solution), and stirring was continued for another $2 \mathrm{~h}$. $N, N$-dimethylaminopropylamine ( $8 \mu \mathrm{L}$, $6,4 \mathrm{mg}, 52 \mu \mathrm{mol})$ was then added. After an additional $10 \mathrm{~min}$ acetone was removed under reduced pressure and the $\mathrm{pH}$ of the residual water solution was adjusted to 2-3 with aq. $1 \mathrm{M} \mathrm{KHSO}_{4}$. The resulting emulsion was extracted with diethyl ether $(50 \mathrm{~mL})$, and the ethereal layer was washed with aq. $1 \mathrm{MKHSO}_{4}(2 \times 10 \mathrm{~mL})$, water $(3 \times 10 \mathrm{~mL})$, brine $(2 \times 5 \mathrm{~mL})$, dried over $\mathrm{MgSO}_{4}$, filtered and concentrated under reduced pressure. The residual oil was dried overnight in vacuo to give glass-like product 105 a (38 mg, $111 \mu \mathrm{mol}, 71 \%) . R_{\mathrm{f}}=0.24$ [EtOAc/hexane 1:3 (2\% AcOH)]; $[\alpha]_{\mathrm{D}}{ }^{20}=22.80\left(\mathrm{c}=0.46, \mathrm{CHCl}_{3}\right) ;{ }^{1} \mathrm{H} \mathrm{NMR}\left(250 \mathrm{MHz}, \mathrm{CDCl}_{3}\right)$ : $\delta=0.04(\mathrm{~s}, 9 \mathrm{H}), 1.00(\mathrm{dd}, J=9.5 \mathrm{~Hz}, 7.3 \mathrm{~Hz}, 2 \mathrm{H}), 1.11-1.18(\mathrm{~m}, 1 \mathrm{H}), 1.60-1.95(\mathrm{~m}, 2 \mathrm{H})$, 1.98-2.19 (m, 2 H), 4.14-4.23 (m, 3 H), 4.33-4.59 (m, 1 H), 5.33-5.46 (m, 1 H), 7.08-7.25 (bs, $1 \mathrm{H}) ;{ }^{13} \mathrm{C}$ NMR (62.9 MHz, $\left.\mathrm{CDCl}_{3}\right): \delta=-1.9(+), 10.2(-), 17.3(-), 22.0(+), 33.1+33.3(-)$, $52.7(+), 53.2(+), 59.0(+), 63.7(+), 64.8(-), 115.6(\mathrm{~d}, J=271.4 \mathrm{~Hz}), 157.4\left(\mathrm{C}_{\text {quat }}\right)$, $174.5+174.8\left(\mathrm{C}_{\text {quat }}\right)$.

Teoc-(S)mFmcpA-Cyclo-F 6 (106 a): N-MeZ-protected cyclohexadepsipeptide 103 a (25 mg,

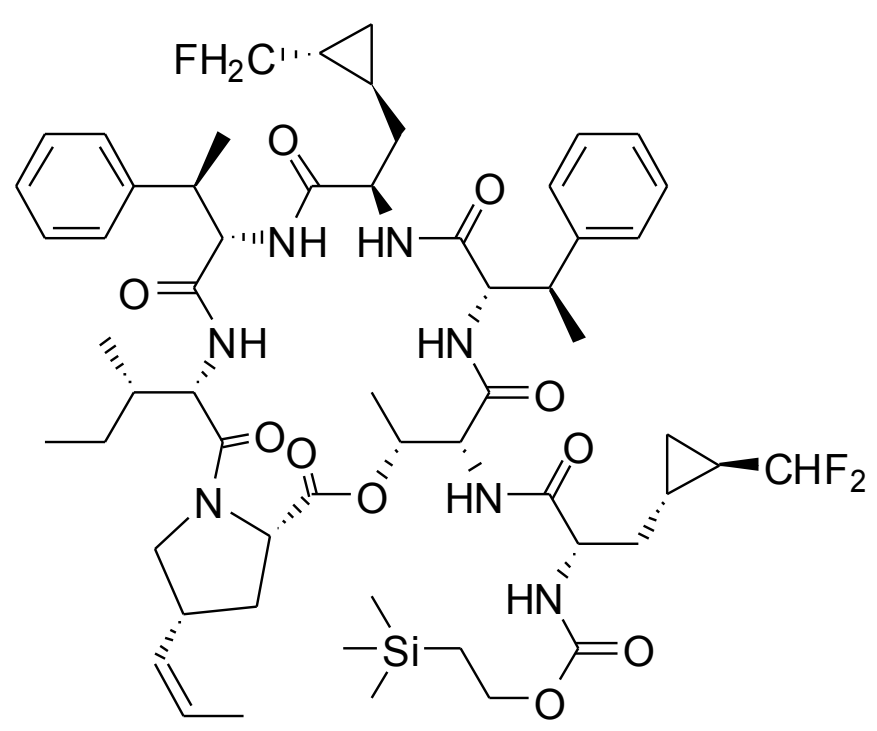
$25 \mu \mathrm{mol})$ was deprotected with $10 \%$ anisole in TFA $(1.1 \mathrm{~mL})$ in the dark at ambient temperature for $2 \mathrm{~h}$, the residue was treated with toluene $(5 \mathrm{~mL})$, concentrated under reduced pressure and the residue was dried in vacuo at ambient temperature for 2 hours. The solution of Teoc- $(S) m F m c p A-O H \quad 105$ a, HATU (29 $\mathrm{mg}, 75 \mu \mathrm{mol})$ and HOAt $(10 \mathrm{mg}$, $75 \mu \mathrm{mol})$ in $\mathrm{CH}_{2} \mathrm{Cl}_{2}(1.5 \mathrm{~mL})$ was added at $4{ }^{\circ} \mathrm{C}$, followed with DIEA (3 $5 \mathrm{mg}$, $27 \mu \mathrm{mol})$ and TMP $(27 \mathrm{mg}, 225 \mu \mathrm{mol})$ solution in $\mathrm{CH}_{2} \mathrm{Cl}_{2}(1.5 \mathrm{~mL})$ and the mixture was stirred at ambient temperature for $15 \mathrm{~h}$. The reaction mixture was then diluted with diethyl ether $(50 \mathrm{~mL})$ and the crude product obtained after the usual aqueous work-up (GP 2) was purified by 
crystallization from $\mathrm{CH}_{2} \mathrm{Cl}_{2} /$ pentane to give Teoc- $(S)$ mFmcpA-Cyclo- $\mathrm{F}_{2} 6$ (106 a) (29 mg, $24,7 \mu \mathrm{mol}, 89 \%)$ as a colorless solid $\left(R_{\mathrm{f}}=0.43\right.$, acetone/hexane $\left.1: 2\right)$ which was used for the next step without any characterization.

MOM-O-protected monofluoromethylcyclopropylalanyl Hormaomycin (MOM-O-F $F_{1} H o r m$,

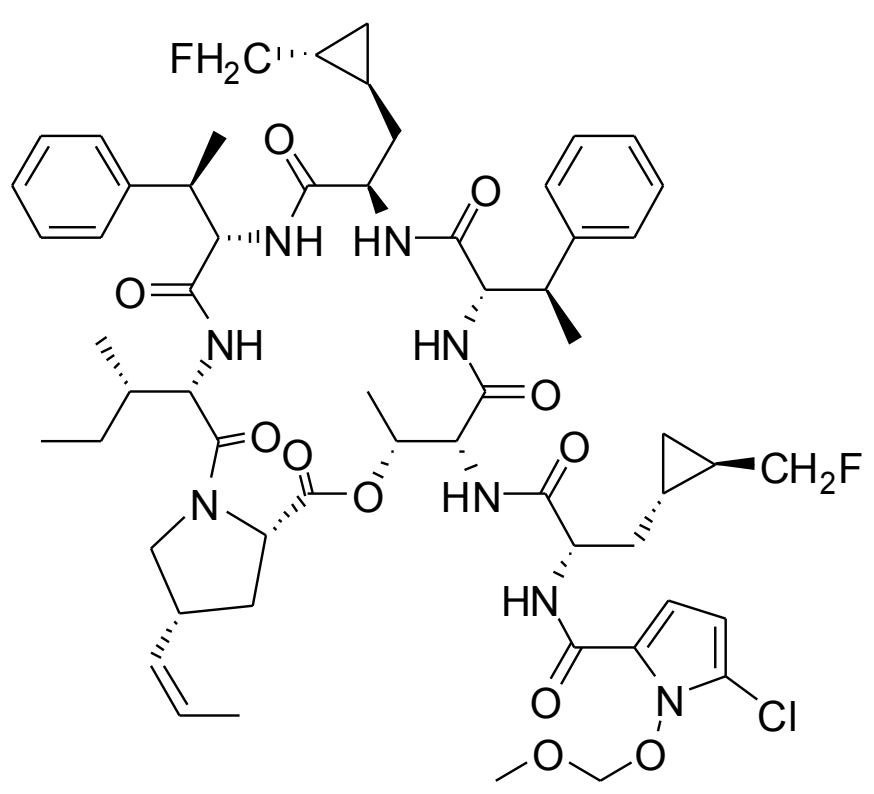

108 a): Teoc group was cleaved from the compound 106 a $(8.0 \mathrm{mg}, 7.08 \mu \mathrm{mol})$ with TFA $(0.6 \mathrm{~mL})$ for $1 \mathrm{~h}$. The mixture was concentrated under reduced pressure at $20{ }^{\circ} \mathrm{C}$ and then taken up with toluene $(3 \times 15 \mathrm{~mL})$ which was distilled off to remove the last traces of TFA. The resulting deprotected depsipeptide 107 a was coupled with $O$-MOM protected acid 81 (2.9 mg, $14.10 \mu \mathrm{mol})$ using HATU (5.4 mg, $14.20 \mu \mathrm{mol})$, DIEA (0.92 mg, 7.12 $\mu \mathrm{mol})$ and TMP (5.14 mg, $42.42 \mu \mathrm{mol})$ in

$\mathrm{CH}_{2} \mathrm{Cl}_{2}(1 \mathrm{~mL})$ according to $\mathrm{GP} 6$ for $2.5 \mathrm{~h}$. The mixture was then taken up with $\mathrm{Et}_{2} \mathrm{O}(20 \mathrm{~mL})$ and the crude product obtained after usual aqueous work-up (GP 2) was crystallized from $\mathrm{CH}_{2} \mathrm{Cl}_{2}$ / pentane to give $O$-MOM protected monofluoromethylcyclopropylalanyl Hormaomycin 108 a $\left(8.0 \mathrm{mg}, 93 \%, R_{\mathrm{f}}=0.36\right.$ acetone/hexanes $\left.1: 2\right)$ as a colorless glass which was used for the next step without any characterization.

Monofluoromethylcyclopropylalanyl Hormaomycin ( $F_{1} H o r m, 109$ b): O-MOM protected mono-

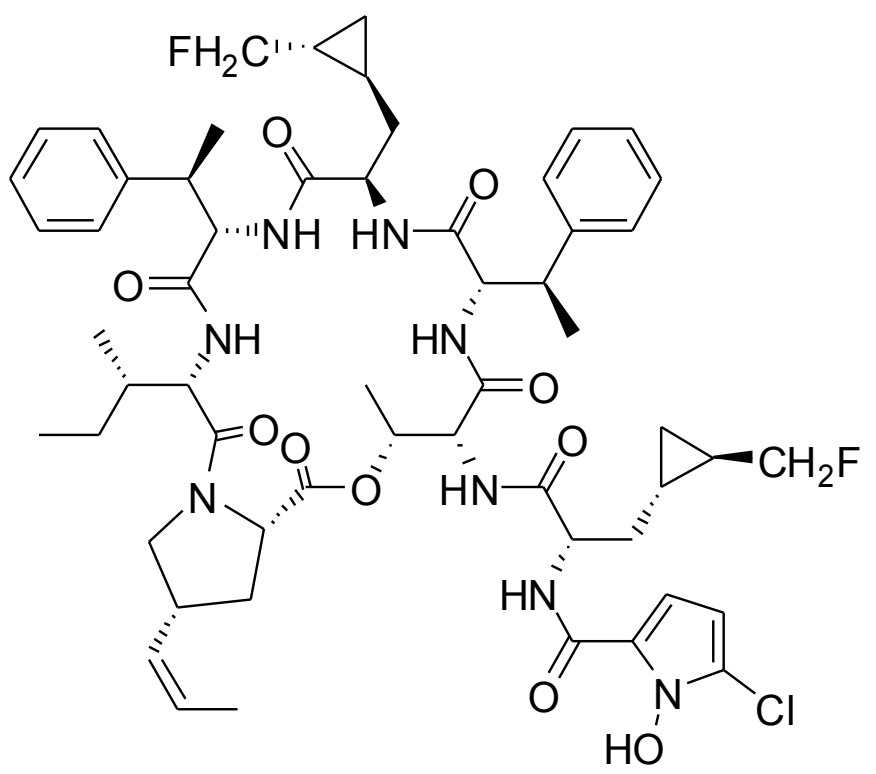
fluoromethylcyclopropylalanyl Hormaomycin 108 a $(8.0 \mathrm{mg}, 6.82 \mu \mathrm{mol})$ was deprotected using $\mathrm{MgBr}_{2} \times \mathrm{Et}_{2} \mathrm{O}(52 \mathrm{mg}$, $201.36 \mu \mathrm{mol})$ and EtSH $(0.10 \mu \mathrm{mL}$, $1.9 \mathrm{mmol})$ in $\mathrm{CH}_{2} \mathrm{Cl}_{2}(10 \mathrm{~mL})$ according to GP 7 for $3 \mathrm{~h}$. The mixture was taken up with EtOAc and the crude product obtained after usual aqueous work-up was crystallized from $\mathrm{CH}_{2} \mathrm{Cl}_{2}$ / pentane to give 109 a $(5.5 \mathrm{mg}, 78 \%, 50 \%$ on 5 steps from 103 a) as a white solid, which was finally 
purified with preparative HPLC. $\mathrm{R}_{\mathrm{f}}=0.24$ acetone/hexanes 3:7; analytical HPLC: column B, isocratic, $65 \% \mathrm{MeCN}$ in $\mathrm{H}_{2} \mathrm{O}$ for $15 \mathrm{~min}$, then gradient $65 \rightarrow 99 \% \mathrm{MeCN}$ in $\mathrm{H}_{2} \mathrm{O}$ for $5 \mathrm{~min}$, then isocratic, $99 \% \mathrm{MeCN}$, flow rate $=0.5 \mathrm{~mL} / \mathrm{min}, \mathrm{t}_{\mathrm{R}}=14.54 \mathrm{~min}$, purity $>92 \%$; preparative HPLC: isocratic, $62 \% \mathrm{MeCN}$ in $\mathrm{H}_{2} \mathrm{O}\left(+0.1 \%\right.$ TFA) for 7 min, then gradient $65 \rightarrow 99 \% \mathrm{MeCN}$ in $\mathrm{H}_{2} \mathrm{O}$ $(+0.1 \% \mathrm{TFA})$ for $10 \mathrm{~min}$, then isocratic, $62 \% \mathrm{MeCN}$ in $\mathrm{H}_{2} \mathrm{O}(+0.1 \% \mathrm{TFA})$, flow rate $=18 \mathrm{~mL} /$ $\min , \mathrm{t}_{\mathrm{R}}=12.54 \mathrm{~min} ;[\alpha]_{\mathrm{D}}{ }^{20} 20.0(\mathrm{c}=0.1, \mathrm{MeOH}) ;{ }^{1} \mathrm{H} \mathrm{NMR}(600 \mathrm{MHz}, \mathrm{CDCl}): \delta=-0.71--$ $0.63(\mathrm{~m}, 1 \mathrm{H}),-0.20-0.10(\mathrm{~m}, 1 \mathrm{H}), 0.23-0.32(\mathrm{~m}, 1 \mathrm{H}), 0.49-0.56(\mathrm{~m}, 1 \mathrm{H}), 0.88(\mathrm{t}, J=7.4 \mathrm{~Hz}$, $3 \mathrm{H}), 0.95-1.01(\mathrm{~m}, 1 \mathrm{H}), 1.02(\mathrm{~d}, J=6.9 \mathrm{~Hz}, 3 \mathrm{H}), 1.04-1.14(\mathrm{~m}, 1 \mathrm{H}), 1.17-1.35(\mathrm{~m}, 1 \mathrm{H}), 1.30$ $(\mathrm{d}, J=7.0 \mathrm{~Hz}, 3 \mathrm{H}), 1.39$ (d, $J=7.3 \mathrm{~Hz}, 3 \mathrm{H}), 1.47-1.54(\mathrm{~m}, 1 \mathrm{H}), 1.53$ (d, $J=6.9 \mathrm{~Hz}, 3 \mathrm{H})$, 1.55-1.62 (m, $1 \mathrm{H}), 1.67$ (dd, $J=6.9 \mathrm{~Hz}, 1.6 \mathrm{~Hz}, 3 \mathrm{H}), 1.75-1.84$ (m, $2 \mathrm{H}), 1.85-1.94$ (m, $3 \mathrm{H})$, 2.30-2.40 (m, $1 \mathrm{H}), 2.88-2.91(\mathrm{~m}, 1 \mathrm{H}), 2.96-3.02(\mathrm{~m}, 1 \mathrm{H}), 3.22-3.31(\mathrm{~m}, 2 \mathrm{H}), 3.43-3.50(\mathrm{~m}$, $1 \mathrm{H}), 3.62-3.70(\mathrm{~m}, 1 \mathrm{H}), 3.93-4.00(\mathrm{~m}, 1 \mathrm{H}), 4.03$ (ddd, $J=6.8 \mathrm{~Hz}, 3.4 \mathrm{~Hz}, 3.4 \mathrm{~Hz}, 1 \mathrm{H}), 4.26$ (dd, $J=11.5 \mathrm{~Hz}, 6.0 \mathrm{~Hz}, 1 \mathrm{H}), 4.38(\mathrm{dd}, J=10.6 \mathrm{~Hz}, 10.6 \mathrm{~Hz}, 1 \mathrm{H}), 4.46(\mathrm{dd}, J=9.4 \mathrm{~Hz}, 4.5 \mathrm{~Hz}$, $1 \mathrm{H}), 4.57(\mathrm{dd}, J=9.3 \mathrm{~Hz}, 2.3 \mathrm{~Hz}, 1 \mathrm{H}), 4.66(\mathrm{dd}, J=9.0,9.0 \mathrm{~Hz}, 1 \mathrm{H}), 5.08-5.14(\mathrm{~m}, 1 \mathrm{H})$, 5.22-5.28 (m, $1 \mathrm{H}), 5.40(\mathrm{qd}, J=6.9 \mathrm{~Hz}, 2.4 \mathrm{~Hz}, 1 \mathrm{H}), 5.58-5.65(\mathrm{~m}, 1 \mathrm{H}), 6.13(\mathrm{~d}, J=4.7 \mathrm{~Hz}$, $1 \mathrm{H}), 6.56(\mathrm{~d}, J=6.5 \mathrm{~Hz}, 1 \mathrm{H}), 6.80(\mathrm{~d}, J=9.3 \mathrm{~Hz}, 1 \mathrm{H}), 6.82(\mathrm{~d}, J=4.7 \mathrm{~Hz}, 1 \mathrm{H}), 6.98-7.03(\mathrm{~m}$, $1 \mathrm{H}), 7.09-7.18$ (m, 5 H), 7.20-7.27 (m, $7 \mathrm{H}), 8.05$ (d, $J=9.1 \mathrm{~Hz}, 1 \mathrm{H}), 9.06$ (d, $J=9.3 \mathrm{~Hz}$, $1 \mathrm{H}) ;{ }^{13} \mathrm{C}$ NMR $\left(150.8 \mathrm{MHz}, \mathrm{CDCl}_{3}\right): \delta=10.50(+), 13.24(+), 13.33(+), 14.94(+), 16.99(+)$, $17.41(-), 17.74(+), 20.00(+), 21.66(+), 24.90(-), 26.88(-), 33.02(-), 35.03(-), 35.51(-)$, $36.66(-), 37.97(+), 39.24(+) 41.75(+), 50.99(+), 51.79(+), 52.78(-), 54.61(+), 54.93(+)$, $58.11(+), 59.12(+), 59.86(+), 60.04(+), 61.37(+), 69.07(+), 103.59(+), 109.85(+), 119.86$ $\left(\mathrm{C}_{\text {quat }}\right), 121.55\left(\mathrm{C}_{\text {quat }}\right), 126.98(+), 127.17(+), 127.44(+), 127.47(+), 127.67(+), 128.33(+)$, $128.49(+), 128.64(+), 141.55\left(\mathrm{C}_{\text {quat }}\right), 142.11\left(\mathrm{C}_{\text {quat }}\right), 159.27\left(\mathrm{C}_{\text {quat }}\right), 168.54\left(\mathrm{C}_{\text {quat }}\right), 168.73\left(\mathrm{C}_{\text {quat }}\right)$, 169.75 ( $\left.\mathrm{C}_{\text {quat }}\right), 170.74$ ( $\left.\mathrm{C}_{\text {quat }}\right), 171.26$ (Cquat), 171.55 ( $\left.\mathrm{C}_{\text {quat }}\right), 172.86$ ( $\left.\mathrm{C}_{\text {quat }}\right) ;$ MS-ESI: positive, $\mathrm{m} / \mathrm{z}$ $=292(100), 1151\left(80, \mathrm{M}+\mathrm{Na}^{\dagger}\right)$; negative, $\mathrm{m} / \mathrm{z}=1127\left(100, \mathrm{M}-\mathrm{H}^{\dagger}\right)$. 


\section{Other new non-proteinogenic amino acids}

\section{1. $\quad \beta$-Methylphenylalanine}

Racemic 1-phenylethanol (118): Acetophenone $(12.0 \mathrm{~g}, 100 \mathrm{mmol})$ was reduced with $\mathrm{LiAlH}_{4}$

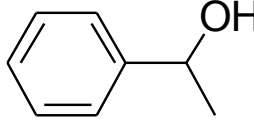
solution according to GP 8, giving the target racemic alcohol as colorless liquid $(10.8 \mathrm{~g}, 88 \mathrm{mmol}, 88 \%) ;{ }^{1} \mathrm{H} \mathrm{NMR}\left(250 \mathrm{MHz}, \mathrm{CDCl}_{3}\right): \delta=1.49(\mathrm{~d}$, $J=6.5 \mathrm{~Hz}, 3 \mathrm{H}), 2.20(\mathrm{~d}, J=3.1,1 \mathrm{H}), 4.82-4.92(\mathrm{~m}, 1 \mathrm{H}), 7.23-7.44(\mathrm{~m}$, $5 \mathrm{H}) ;{ }^{13} \mathrm{C} \mathrm{NMR}\left(62.9 \mathrm{MHz}, \mathrm{CDCl}_{3}\right): \delta=25.12,70.33,125.35,127.41,128.45,145.77$.

Racemic 1-iod-1-phenylethane (119): Racemic 1-phenyl ethanol 118 (10.8 g, 88 mmol) was

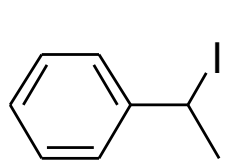
iodinated according to GP 9 using triphenylphosphine (40.0 g, $153 \mathrm{mmol})$, imidazole $(10.9 \mathrm{~g}, 160 \mathrm{mmol})$ and iodine $(43.1 \mathrm{~g}, 170 \mathrm{mmol})$ in diethyl ether/acetonitrile mixture $(260+175 \mathrm{ml})$, giving the target iodide 119 as yellowish liquid (18.9 g, $81.7 \mathrm{mmol}, 92,8 \%) ;{ }^{1} \mathrm{H} \mathrm{NMR} \quad\left(250 \mathrm{MHz}, \mathrm{CDCl}_{3}\right): \delta=2.25(\mathrm{~d}$, $J=7.1 \mathrm{~Hz}, 3 \mathrm{H}), 5.44(\mathrm{q}, J=7.1,1 \mathrm{H}), 7.24-7.38(\mathrm{~m}, 3 \mathrm{H}), 7.44-7.55(\mathrm{~m}, 2 \mathrm{H}) ;{ }^{13} \mathrm{C} \mathrm{NMR}$ $\left(62.9 \mathrm{MHz}, \mathrm{CDCl}_{3}\right): \delta=26.27,28.99,126.56,127.95,128.71,145.32$.

(S)-Belokon' (2S,3R)- $\beta$-methylphenylalanine complex [(S)-BFC, (2S,3R)-120]: (S)-BGC (10.0 g,

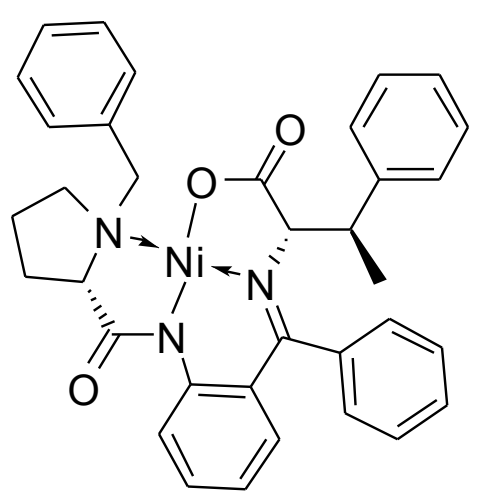
$20 \mathrm{mmol}$ ) was was alkylated with the racemic 1-iod-1phenylethane 119 (4.9 g, $21 \mathrm{mmol}$ ) according to GP 10 using $\mathrm{NaH}$ $(60 \%$ in oil, $1.0 \mathrm{~g}, 25 \mathrm{mmol})$ in $\mathrm{DMF} / \mathrm{MeCN}$ mixture $(10+20 \mathrm{~mL})$, giving $(2 S, 3 R)$ component $(3.78 \mathrm{~g}, 6.3 \mathrm{mmol}, 63 \%$ on $(S)$-BGC), $(2 S, 3 S)$ component $(3.13 \mathrm{~g}, 5.2 \mathrm{mmol}, 52 \%$ on $(S)$-BGC) and mixed fractions $(3.87 \mathrm{~g}, 6.4 \mathrm{mmol}, 64 \%$ on $(S)$-BGC) as well as products of the anion oxidation $(0.94 \mathrm{~g})$; $(2 S, 3 R)$ component: ${ }^{1} \mathrm{H}$ NMR $\left(500 \mathrm{MHz}, \mathrm{CDCl}_{3}\right): \delta=1,13(\mathrm{~d}$, $J=7.34 \mathrm{~Hz}, 3 \mathrm{H}), 1.34-1.51(\mathrm{~m}, 2 \mathrm{H}), 1.69-2.01(\mathrm{~m}, 2 \mathrm{H}), 2.22(\mathrm{q}, J=7.90 \mathrm{~Hz}, 2 \mathrm{H}), 2.71-$ $2.95(\mathrm{~m}, 2 \mathrm{H}), 3.25(\mathrm{t}, J=8.72 \mathrm{~Hz}, 1 \mathrm{H}), 3.39(\mathrm{~d}, J=12.62 \mathrm{~Hz}, 1 \mathrm{H}), 3.49(\mathrm{~d}, J=5.49 \mathrm{~Hz}, 3 \mathrm{H})$, $4.12(\mathrm{~d}, J=3.17 \mathrm{~Hz}, 1 \mathrm{H}), 4.23(\mathrm{~d}, J=12.56 \mathrm{~Hz}, 1 \mathrm{H}), 6.62-6.78(\mathrm{~m}, 2 \mathrm{H}), 6.99-7.07(\mathrm{~m}, 1 \mathrm{H})$, $7.08-7.70(\mathrm{~m}, \quad 13 \mathrm{H}), \quad 7.98(\mathrm{~d}, \quad J=8.28 \mathrm{~Hz}, \quad 2 \mathrm{H}), \quad 8.26(\mathrm{~d}, \quad J=8.56 \mathrm{~Hz}, \quad 1 \mathrm{H}) ;{ }^{13} \mathrm{C} \mathrm{NMR}$ $\left(125.7 \mathrm{MHz}, \mathrm{CDCl}_{3}\right): \delta=18.27(+), 22.92(-), 30.67(-), 44.84(+), 50.56(+, \mathrm{MeOH}), 57.19(-)$, $63.45(-), 70.28(+), 75.48(+), 120.39(+), 123.07(+), 125.90(-), 127.09(+), 127.53(+)$, $127.89(+), 128.37(+), 128.46(+), 128.57(+), 128.61(+), 128.75(+), 128.79+129.08(+)$, $129.38(+), \quad 129.60(+), \quad 131.47(+), \quad 131.85+131.87(+), \quad 131.95+132.03(+), \quad 132.28(+)$, 
$132.78(-), 133.17(-), 133.48(+), 134.30(-), 141.04(-), 142.90(-), 171.02(-), 177.37(-)$, $180.36(-) ;[\alpha]_{\mathrm{D}}{ }^{20}=+2190.0^{\circ}\left(\mathrm{c}=0.2\right.$ in $\left.\mathrm{CHCl}_{3}\right)$; MS-ESI $\left.(\mathrm{MeOH}): 1827.6(85 \%, 3 \mathrm{M}+\mathrm{Na}\rceil^{+}\right)$, $\left.\left.\left.1225.1(70 \%, \quad 2 \mathrm{M}+\mathrm{Na}\rceil^{+}\right), \quad 1205.0(20 \%, \quad 2 \mathrm{M}+\mathrm{H}\rceil^{+}\right), \quad 624.3(100 \%, \quad \mathrm{M}+\mathrm{Na}\rceil^{+}\right), \quad 602.3(48 \%$, $\left.\mathrm{M}+\mathrm{H}\rceil^{+}\right)$.

(2S,3R)- $\beta$-methylphenylalanine (MeF, 55): $(S)$-BFC (3.78 g, $6.3 \mathrm{mmol})$ was decomposed and the

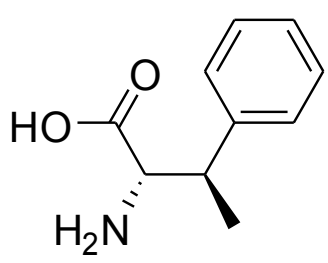
amino acid was separated and purified according to GP 11 to give pure target amino acid $(0.67 \mathrm{~g}, 3.7 \mathrm{mmol}, 59 \%)$; ${ }^{1} \mathrm{H} \mathrm{NMR}\left(300 \mathrm{MHz}, \mathrm{D}_{2} \mathrm{O}\right)$ : $\delta=1.43(\mathrm{~d}, J=7.3 \mathrm{~Hz}, 3 \mathrm{H}), 3.57(\mathrm{qd}, J=7.3 \mathrm{~Hz}, 5.0 \mathrm{~Hz}, 1 \mathrm{H}), 3.98(\mathrm{~d}$, $J=5.0 \mathrm{~Hz}, 1 \mathrm{H}), 4.72(\mathrm{bs}, 3 \mathrm{H}), 7.36-7.53(\mathrm{~m}, 5 \mathrm{H}) ;{ }^{13} \mathrm{C} \mathrm{NMR}(125.7 \mathrm{MHz}$, $\left.\mathrm{D}_{2} \mathrm{O}\right): \quad \delta=13.81(+), \quad 39.46(+), \quad 60.75(+), \quad 127.81(+), \quad 127.86(+), \quad 129.17(+), \quad 140.38(-)$, $173.24(-)$; $[\alpha]_{\mathrm{D}}{ }^{20}-7.4\left(\mathrm{c}=0.5\right.$ in $\left.\mathrm{H}_{2} \mathrm{O}\right)$; MS-ESI $(\mathrm{MeOH})$ : (positive) $\left.\mathrm{m} / \mathrm{z}(\%) 180(100, \mathrm{M}+\mathrm{H}\rceil^{+}\right)$, (negative) $\left.\mathrm{m} / \mathrm{z}(\%) 178(100, \mathrm{M}-\mathrm{H}\rceil^{-}\right)$.

\subsection{Substituted $\beta$-methylphenylalanines}

Racemic 1-(p-chlorophenyl)ethanol (121): p-Chloroacetophenone (3.10 g, $20 \mathrm{mmol})$ was

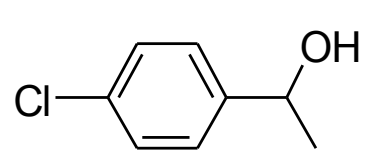
reduced with $\mathrm{LiAlH}_{4}$ solution according to GP 8, giving the target racemic alcohol 121 as colorless liquid $(3.07 \mathrm{~g}, 19.6 \mathrm{mmol}, 98 \%)$; ${ }^{1} \mathrm{H} \mathrm{NMR}\left(250 \mathrm{MHz}, \mathrm{CDCl}_{3}\right): \delta=1.42(\mathrm{~d}, J=6.5 \mathrm{~Hz}, 3 \mathrm{H}), 2.65$ (bs, $\left.1 \mathrm{H}\right)$, $5.36(\mathrm{dq}, J=3.0 \mathrm{~Hz}, 6.5 \mathrm{~Hz}, 1 \mathrm{H}), 7.20-7.35(\mathrm{~m}, 4 \mathrm{H}),{ }^{13} \mathrm{C} \mathrm{NMR}\left(62.9 \mathrm{MHz}, \mathrm{CDCl}_{3}\right): \delta=25.12$, $69.50,126.70,128.43,132.85,144.14$.

Racemic 1-iod-1-(p-chlorophenyl)ethane (122): Racemic 1-(p-chlorophenyl)ethanol 121 (3.0 g,<smiles>CC(I)c1ccc(Cl)cc1</smiles>
$19.2 \mathrm{mmol}$ ) was iodinated according to GP 9 using triphenylphosphine $(6.7 \mathrm{~g}, 25.5 \mathrm{mmol})$, imidazole $(2.0 \mathrm{~g}, 30 \mathrm{mmol})$ and iodine $(9.4 \mathrm{~g}$, $37.0 \mathrm{mmol})$ in toluene/acetonitrile mixture $(100+20 \mathrm{ml})$. The mixture was heated to reflux for $30 \mathrm{~min}$ before work-up, diluted with tert-buthyl methyl ether $(50 \mathrm{~mL})$, washed with $10 \% \mathrm{w} / \mathrm{w}$ aqueous $\mathrm{NaHSO}_{3}(3 \times 50 \mathrm{~mL}), \mathrm{H}_{2} \mathrm{O}(50 \mathrm{~mL})$ and brine $(50 \mathrm{~mL})$, dried over $\mathrm{MgSO}_{4}$, and concentrated under reduced pressure. Crude iodide was purified with column chromatography (silica gel, eluted with pentane), giving the target iodide $\mathbf{1 2 2}$ as yellowish liquid (5.01 g, $18.8 \mathrm{mmol}, 98 \%)$; TLC: pentane, $R_{\mathrm{f}}=0.28$; ${ }^{1} \mathrm{H}$ NMR $\left(250 \mathrm{MHz}, \mathrm{CDCl}_{3}\right): \delta=2.19(\mathrm{~d}$, $J=7.1 \mathrm{~Hz}, 3 \mathrm{H}), 5.36(\mathrm{q}, J=7.1 \mathrm{~Hz}, 1 \mathrm{H}), 7.23-7.41(\mathrm{~m}, 4 \mathrm{H}),{ }^{13} \mathrm{C} \mathrm{NMR}\left(62.9 \mathrm{MHz}, \mathrm{CDCl}_{3}\right)$ : $\delta=24.35,28.81,127.85,128.82,133.38,143.88$; MS-EI $\left.(70 \mathrm{eV}): \mathrm{m} / \mathrm{z}(\%) 141\left(30, \mathrm{M}\left({ }^{37} \mathrm{Cl}\right)-\mathrm{I}\right\rceil^{+}\right)$, $\left.\left.139\left(100, \mathrm{M}\left({ }^{35} \mathrm{Cl}\right)-\mathrm{I}\right\rceil^{+}\right), 103\left(50, \mathrm{C}_{8} \mathrm{H}_{7}{ }^{+}\right), 77\left(18, \mathrm{C}_{6} \mathrm{H}_{5}\right\rceil^{+}\right)$. 
(S)-Belokon' (2S,3R)-beta-methyl(p-chlorophenyl)alanine complex [(S)-BpCFC (2S,3R)-123]:

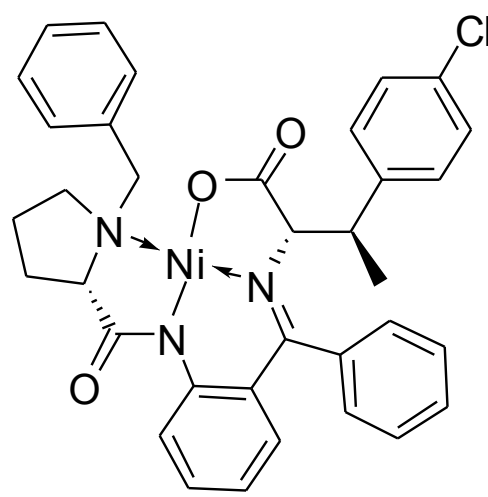

$(S)$-BGC $(3.45 \mathrm{~g}, 6.9 \mathrm{mmol})$ was alkylated with the racemic 1iod-1-( $p$-chlorophenyl)ethane 122 (1.95 g,7.3 mmol) according to GP 10 using $\mathrm{NaH}$ (60\% in oil, $0.33 \mathrm{~g}, 8.3 \mathrm{mmol})$ in $\mathrm{DMF} / \mathrm{MeCN}$ mixture $(3.5+7.0 \mathrm{~mL})$, giving after chromatogarphy $(2 S, 3 R)$ component $(1.77 \mathrm{~g}, 2.8 \mathrm{mmol}, 40.3 \%$ on $(S)$-BGC, d.e. $\geq 98 \%)$, $(2 S, 3 S)$ component $(1.83 \mathrm{~g}, 2.9 \mathrm{mmol}, 41.6 \%$ on $(S)$-BGC, d.e. $\geq 98 \%)$ and mixed fractions $(0.67 \mathrm{~g}, 1.1 \mathrm{mmol}, 15,2 \%$ on $(S)$-BGC) as well as products of the anion oxidation $(0.11 \mathrm{~g})$; $(2 S, 3 R)$ component: TLC: $R_{\mathrm{f}}=0.17$ (EtOAc); ${ }^{1} \mathrm{H}$ NMR $\left(250 \mathrm{MHz}, \mathrm{CDCl}_{3}\right): \delta=1.07(\mathrm{~d}, 7.3 \mathrm{~Hz}), 1.45-1.65(\mathrm{~m}, 1 \mathrm{H})$, 1.80-2.06 (m, 2 H), 2.14-2.36 (m, 3 H), 2.70-2.90 (m, 2 H), 3.29 (t, J=8.6 Hz, 1 H), 3.40 (d, $J=12.5 \mathrm{~Hz}, 1 \mathrm{H}), 4.10(\mathrm{~d}, J=3.1 \mathrm{~Hz}, 1 \mathrm{H}), 4.24(\mathrm{~d}, J=12.5 \mathrm{~Hz}, 1 \mathrm{H}), 6.62-6.74(\mathrm{~m}, 2 \mathrm{H}), 7.00-$ $7.06(\mathrm{~m}, 1 \mathrm{H}), 7.08-7.19(\mathrm{~m}, 2 \mathrm{H}), 7.22-7.37$ (m, $5 \mathrm{H}), 7.42-7.63$ (m, $5 \mathrm{H}), 7.94-8.03$ (m, $2 \mathrm{H})$, 8.22-8.30 (m, $1 \mathrm{H}) ;{ }^{13} \mathrm{C} \mathrm{NMR}\left(62.9 \mathrm{MHz}, \mathrm{CDCl}_{3}\right)$ : 18.02, 22.69, 30.67, 44.18, 50.38, 57.40, $63.53,70.18,75.05,106.97,113.70,120.39,122.98,125.66,126.94,127.64,128.52,128.76$, $129.09,129.62,130.61,131.32$, 132.32, 133.12, 133.41, 133.51, 134.04, 139.37, 142.69, 171.12, $177.14,180.32$.

(2S,3R)- $\beta$-methyl(p-chlorophenyl)alanine $\quad[(2 S, 3 R)-51]: \quad(S)-\mathrm{BpCFC} \quad(2 S, 3 R)-\mathbf{1 2 2} \quad(1.70 \mathrm{~g}$,

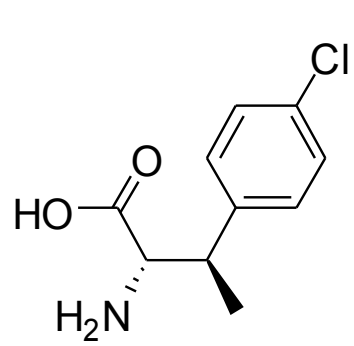
$2.7 \mathrm{mmol}$ ) was decomposed and the amino acid was separated and purified according to GP 11 to give pure target amino acid $(512 \mathrm{mg}$, $2.4 \mathrm{mmol}, 89 \%) ;{ }^{1} \mathrm{H}$ NMR $\left(300 \mathrm{MHz}, \mathrm{D}_{2} \mathrm{O}\right): \delta=1.43(\mathrm{~d}, J=7.3 \mathrm{~Hz}, 3 \mathrm{H})$, 3.57 (qd, $J=7.3 \mathrm{~Hz}, 5.0 \mathrm{~Hz}, 1 \mathrm{H}), 3.98$ (d, $J=5.0 \mathrm{~Hz}, 1 \mathrm{H}), 4.72$ (bs, $3 \mathrm{H}), 7.36-7.53(\mathrm{~m}, 5 \mathrm{H}) ;{ }^{13} \mathrm{C} \mathrm{NMR}\left(125.7 \mathrm{MHz}, \mathrm{D}_{2} \mathrm{O}\right): \delta=13.81(+)$, $39.46(+), \quad 60.75(+), \quad 127.81(+), \quad 127.86(+), \quad 129.17(+), \quad 140.38(-), \quad 173.24(-) ;[\alpha]_{\mathrm{D}}{ }^{20}-$ 7.4 (c=0.5 in $\left.\mathrm{H}_{2} \mathrm{O}\right)$; MS-ESI $(\mathrm{MeOH})$ : positive 180.0 (100\%), negative $178.2(100 \%)$.

Racemic 1-(m-chlorophenyl)ethanol (124): m-Chloroacetophenone (3.10 g, $20 \mathrm{mmol})$ was

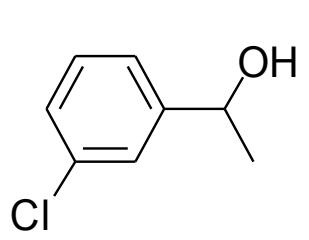
reduced with $\mathrm{LiAlH}_{4}$ solution according to GP 8, giving the target racemic alcohol $\mathbf{1 2 4}$ as colorless liquid $(3.09 \mathrm{~g}, 19.7 \mathrm{mmol}$, 98\%); ${ }^{1} \mathrm{H}$ NMR $\left(250 \mathrm{MHz}, \mathrm{CDCl}_{3}\right): \delta=1.44(\mathrm{~d}, J=6.4 \mathrm{~Hz}, 3 \mathrm{H}), 2.48(\mathrm{bs}, 1 \mathrm{H})$, $4.81(\mathrm{dq}, J=6.4 \mathrm{~Hz}, 3.3 \mathrm{~Hz}, 1 \mathrm{H}), 7.16-7.30(\mathrm{~m}, 3 \mathrm{H}), 7.32-7.36(\mathrm{~m}, 1 \mathrm{H})$; ${ }^{13} \mathrm{C}$ NMR (62.9 MHz, $\left.\mathrm{CDCl}_{3}\right): \delta=25.12,59.59,123.48,125.54,127.41,129.70,134.22,147.77$; MS-EI $\left.\left.(70 \mathrm{eV}): \mathrm{m} / \mathrm{z}(\%) 158\left(10 \%, \mathrm{M}\left({ }^{37} \mathrm{Cl}\right)\right\rceil^{+}\right), 156\left(30 \%, \mathrm{M}\left({ }^{35} \mathrm{Cl}\right)\right\rceil^{+}\right), 143\left(25 \%, \mathrm{M}\left({ }^{37} \mathrm{Cl}\right)-\right.$ 
$\left.\left.\left.\left.\mathrm{O}+\mathrm{H}\rceil^{+}\right), 141\left(100 \%, \mathrm{M}\left({ }^{35} \mathrm{Cl}\right)-\mathrm{O}+\mathrm{H}\right\rceil^{+}, \mathrm{M}\left({ }^{37} \mathrm{Cl}\right)-\mathrm{OH}\right\rceil^{+}\right), 139.1\left(45 \%, \mathrm{M}\left({ }^{35} \mathrm{Cl}\right)-\mathrm{OH}\right\rceil^{+}\right), 121.1(15 \%$, $\left.\left.\mathrm{M}-\mathrm{Cl}\rceil^{+}\right), 115.1\left(13 \%, \mathrm{C}_{6} \mathrm{H}_{6}{ }^{37} \mathrm{Cl}^{+}\right), 113.1\left(40 \%, \mathrm{C}_{6} \mathrm{H}_{6}{ }^{35} \mathrm{Cl}\right\rceil^{+}\right), 77.1\left(75 \%, \mathrm{C}_{6} \mathrm{H}_{5} 7^{+}\right)$.

Racemic 1-iod-1-(m-chlorophenyl)ethane (125): Racemic 1-(m-chlorophenyl)ethanol 124 (3.0 g,

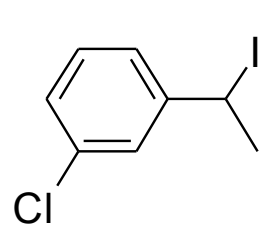
$19.2 \mathrm{mmol}$ ) was iodinated according to GP 9 using triphenylphosphine $(6.7 \mathrm{~g}$, $25.5 \mathrm{mmol})$, imidazole $(2.0 \mathrm{~g}, 30 \mathrm{mmol})$ and iodine $(9.4 \mathrm{~g}, 37.0 \mathrm{mmol})$ in toluene/acetonitrile mixture $(100+20 \mathrm{ml})$. The mixture was heated to reflux for $30 \mathrm{~min}$ before work-up, diluted with tert-buthyl methyl ether $(50 \mathrm{~mL})$, washed with $10 \% \mathrm{w} / \mathrm{w}$ aqueous $\mathrm{NaHSO}_{3}(3 \times 50 \mathrm{~mL}), \mathrm{H}_{2} \mathrm{O}(50 \mathrm{~mL})$ and brine $(50 \mathrm{~mL})$, dried over $\mathrm{MgSO}_{4}$, and concentrated under reduced pressure. Crude iodide was purified with column chromatography (silica, eluted with pentane), giving the target iodide $\mathbf{1 2 5}$ as yellowish liquid (5.04 g, $18.9 \mathrm{mmol}, 98 \%)$; TLC: $R_{\mathrm{f}}=0.28$, pentane; ${ }^{1} \mathrm{H}$ NMR $\left(250 \mathrm{MHz}, \mathrm{CDCl}_{3}\right): \delta=2.19(\mathrm{~d}$, $J=7.1 \mathrm{~Hz}, 3 \mathrm{H}), 5.31(\mathrm{q}, J=7.1 \mathrm{~Hz}, 1 \mathrm{H}), 7.20-7.35(\mathrm{~m}, 3 \mathrm{H}), 7.41-7.44(\mathrm{~m}, 1 \mathrm{H}) ;{ }^{13} \mathrm{C} \mathrm{NMR}$ $\left(62.9 \mathrm{MHz}, \mathrm{CDCl}_{3}\right): \delta=23.72,28,67,124.80,126.67,128.01,129.92,134.28,147.21$.

(S)-Belokon' beta-methyl(m-chlorophenyl)alanine complex $\quad[(S)-B m C F C, \quad(2 S, 3 R)-\mathbf{1 2 6}]$ :

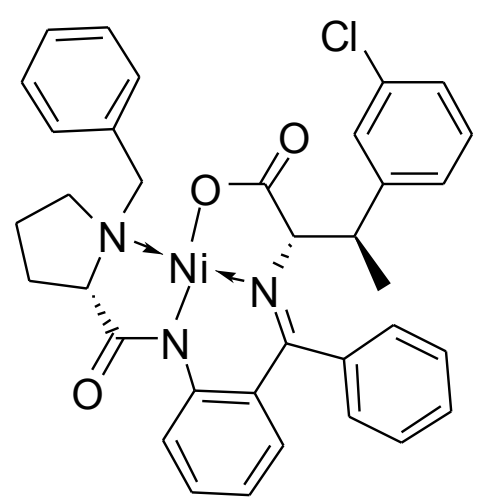

$(S)$-BGC (3.45 g, $6.9 \mathrm{mmol})$ was alkylated with racemic 1-iod-1( $m$-chlorophenyl)ethane $(1.95 \mathrm{~g}, 7.3 \mathrm{mmol})$ according to GP 10 using $\mathrm{NaH}(60 \%$ in oil, $0.33 \mathrm{~g}, 8.3 \mathrm{mmol})$ in $\mathrm{DMF} / \mathrm{MeCN}$ mixture $(3.5+7.0 \mathrm{~mL})$, giving $(2 S, 3 R)$ component $(1.83 \mathrm{~g}, 2.9 \mathrm{mmol}$, $41.6 \%$ on $(S)$-BGC, d.e. $\geq 98 \%),(2 S, 3 S)$ component $(1.62 \mathrm{~g}$, $2.5 \mathrm{mmol}, 36.9 \%$ on $(S)$-BGC, d.e. $\geq 98 \%$ ) and mixed fractions $(0.87 \mathrm{~g}, 1.4 \mathrm{mmol}, 19.8 \%$ on $(S)$-BGC) as well as products of the anion oxidation $(0.18 \mathrm{~g}) . \quad(2 S, 3 R)$-Component: ${ }^{1} \mathrm{H} \mathrm{NMR}\left(250 \mathrm{MHz}, \mathrm{CDCl}_{3}\right): \delta=1.11(\mathrm{~d}$, $J=7.3 \mathrm{~Hz}, 3 \mathrm{H}), 1.43-1.64(\mathrm{~m}, 1 \mathrm{H}), 1.73-2.02(\mathrm{~m}, 2 \mathrm{H}), 2.27(\mathrm{q}, J=7.6 \mathrm{~Hz}, 2 \mathrm{H}), 2.75-$ $2.94(\mathrm{~m}, 2 \mathrm{H}), 3.27$ (t, $J=8.6 \mathrm{~Hz}, 1 \mathrm{H}), 3.42(\mathrm{~d}, J=12.6 \mathrm{~Hz}, 1 \mathrm{H}), 4.05-4.16(\mathrm{~m}, 1 \mathrm{H}), 4.25(\mathrm{~d}$, $J=12.6 \mathrm{~Hz}, 1 \mathrm{H}), 6.62-6.74(\mathrm{~m}, 2 \mathrm{H}), 6.98-7.06$ (m, $1 \mathrm{H}), 7.08-7.19$ (m, $2 \mathrm{H}), 7.19-7.35$ (m, $5 \mathrm{H}), \quad 7.37-7.46(\mathrm{~m}, 3 \mathrm{H}), \quad 7.46-7.66(\mathrm{~m}, 3 \mathrm{H}), \quad 7.93-8.00(\mathrm{~m}, 2 \mathrm{H}), \quad 8.26-8.32(\mathrm{~m}, 1 \mathrm{H})$; ${ }^{13} \mathrm{C} \mathrm{NMR}\left(62.9 \mathrm{MHz}, \mathrm{CDCl}_{3}\right): 30.84,44.62,57.25,63.45,70.30,75.18,120.46,123.17,125.81$, $127.86,128.68,128.91,129.25,131.52,143.05,143.34,177.12,180.39,209,51$.

$(2 S, 3 R)-\beta$-methyl(p-chlorophenyl)alanine $\quad[(2 S, 3 R)-\mathbf{5 0}]: \quad(S)$-BmCFC $\quad(2 S, 3 R)-\mathbf{1 2 6} \quad(1.80 \mathrm{~g}$,

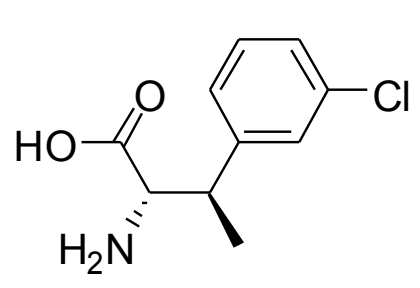

$2.8 \mathrm{mmol})$ was decomposed and the amino acid was separated and purified according to GP 11 to give pure target amino acid $(573 \mathrm{mg}$, 2,7 mmol, 96\%); ${ }^{1} \mathrm{H}$ NMR (300 MHz, $\left.\mathrm{D}_{2} \mathrm{O}\right): \delta=1.43(\mathrm{~d}, J=7.3 \mathrm{~Hz}$, $3 \mathrm{H}), 3.57(\mathrm{qd}, J=7.3 \mathrm{~Hz}, 5.0 \mathrm{~Hz}, 1 \mathrm{H}), 3.98(\mathrm{~d}, J=5.0 \mathrm{~Hz}, 1 \mathrm{H})$, 
$4.72(\mathrm{bs}, 3 \mathrm{H}), \quad 7.36-7.53(\mathrm{~m}, 5 \mathrm{H}) ;{ }^{13} \mathrm{C} \mathrm{NMR}\left(125.7 \mathrm{MHz}, \mathrm{D}_{2} \mathrm{O}\right): \delta=13.81(+), 39.46(+)$, $60.75(+), 127.81(+), 127.86(+), 129.17(+), 140.38(-), 173.24(-) ;[\alpha]_{\mathrm{D}}^{20}-7.4\left(\mathrm{c}=0.5\right.$ in $\left.\mathrm{H}_{2} \mathrm{O}\right)$; MS-ESI (MeOH): positive 180.0 (100\%), negative $178.2(100 \%)$.

Racemic 1-(o-chlorophenyl) ethanol (127): o-Chloroacetophenone (3.10 g, $20 \mathrm{mmol}$ ) was

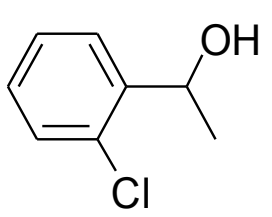
reduced with $\mathrm{LiAlH}_{4}$ solution according to GP 8, giving the target racemic alcohol 127 as colorless liquid (2.98 g, $19.0 \mathrm{mmol}, 95 \%) ;{ }^{1} \mathrm{H} \mathrm{NMR}(250 \mathrm{MHz}$, $\left.\mathrm{CDCl}_{3}\right): \delta=1.46(\mathrm{~d}, J=6.3 \mathrm{~Hz}, 3 \mathrm{H}), 2.50$ (bs, $\left.1 \mathrm{H}\right), 5.26$ (q, $\left.J=6.3 \mathrm{~Hz}, 1 \mathrm{H}\right)$, 7.12-7.34 (m, $3 \mathrm{H}), \quad 7.53-7.60(\mathrm{~m}, \quad 1 \mathrm{H}) ; \quad{ }^{13} \mathrm{C} \mathrm{NMR}\left(62.9 \mathrm{MHz}, \mathrm{CDCl}_{3}\right)$ : $\delta=23.43,66.81,126.33,127.12,128.29,129.28,131.48,142.99 ;$ MS-EI $(70 \mathrm{eV}): \mathrm{m} / \mathrm{z}(\%)$ $\left.158.2\left(4 \%, \quad \mathrm{M}\left({ }^{37} \mathrm{Cl}\right) 7^{+}\right), \quad 156.2\left(12 \%, \quad \mathrm{M}\left({ }^{35} \mathrm{Cl}\right) 7^{+}\right), \quad 143.1\left(18 \%, \quad \mathrm{M}\left({ }^{37} \mathrm{Cl}\right)-\mathrm{O}+\mathrm{H}\right\rceil^{+}\right), \quad 141.1(55 \%$, $\left.\left.\left.\left.\left.\mathrm{M}\left({ }^{35} \mathrm{Cl}\right)-\mathrm{O}+\mathrm{H}\right\rceil^{+}\right), 115.1\left(10 \%, \mathrm{C}_{6} \mathrm{H}_{6}{ }^{37} \mathrm{Cl}\right\rceil^{+}\right), 113.1\left(30 \%, \mathrm{C}_{6} \mathrm{H}_{6}{ }^{35} \mathrm{Cl}\right\rceil^{+}\right), 77.1\left(100 \%, \mathrm{C}_{6} \mathrm{H}_{5}\right\rceil^{+}\right)$.

Racemic 1-iod-1-(o-chlorophenyl) ethane (128): Racemic 1-(o-chlorophenyl)ethanol 127 (2.9 g,

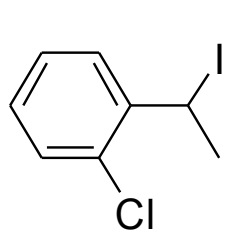
$18.5 \mathrm{mmol})$ was iodinated according to GP 7 using triphenylphosphine $(6.7 \mathrm{~g}$, $25.5 \mathrm{mmol})$, imidazole $(2.0 \mathrm{~g}, 30.0 \mathrm{mmol})$ and iodine $(9.4 \mathrm{~g}, 37.0 \mathrm{mmol})$ in toluene/acetonitrile mixture $(100+20 \mathrm{ml})$. The mixture was heated to reflux for 30 min before work-up, diluted with tert-buthyl methyl ether $(50 \mathrm{~mL})$, washed with $10 \% \mathrm{w} / \mathrm{w}$ aqueous $\mathrm{NaHSO}_{3}(3 \times 50 \mathrm{~mL}), \mathrm{H}_{2} \mathrm{O}(50 \mathrm{~mL})$ and brine $(50 \mathrm{~mL})$, dried over $\mathrm{MgSO}_{4}$, and concentrated under reduced pressure. Crude iodide was purified with column chromatography (silica gel, eluted with pentane), giving the target iodide $\mathbf{1 2 8}$ as yellowish liquid (4.82 g, $18.1 \mathrm{mmol}, 98 \%)$;LC: pentane, $R_{\mathrm{f}}=0.28 ;{ }^{1} \mathrm{H} \mathrm{NMR}\left(250 \mathrm{MHz}, \mathrm{CDCl}_{3}\right): \delta=2.25(\mathrm{~d}$, $J=7.1 \mathrm{~Hz}, \quad 3 \mathrm{H}), \quad 5.72(\mathrm{q}, \quad J=7.1 \mathrm{~Hz}, \quad 1 \mathrm{H}), \quad 7.15-7.35(\mathrm{~m}, \quad 3 \mathrm{H}), \quad 7.60-7.67(\mathrm{~m}, 1 \mathrm{H})$; ${ }^{13} \mathrm{C}$ NMR (62.9 MHz, $\mathrm{CDCl}_{3}$ ): $\delta=20.54,27.75,127.50,127.57,128.93,129.89,132.05,142.11$; MS-EI (70 eV): m/z (\%) $\left.\left.141.1\left(33 \%, \mathrm{M}\left({ }^{37} \mathrm{Cl}\right)-\mathrm{I}\right\rceil^{+}\right), 139.1\left(100 \%, \mathrm{M}\left({ }^{35} \mathrm{Cl}\right)-\mathrm{I}\right\rceil^{+}\right), 103.1(100 \%$, M$\left.(\mathrm{H}+\mathrm{Cl}+\mathrm{I})\rceil^{+}\right), 77.1\left(75 \%, \mathrm{C}_{6} \mathrm{H}_{5} 7^{+}\right)$.

(S)-Belokon' $\beta$-methyl(o-chlorophenyl)alanine complex [(S)-BoCFC, (2S,3R)-129]: (S)-BGC

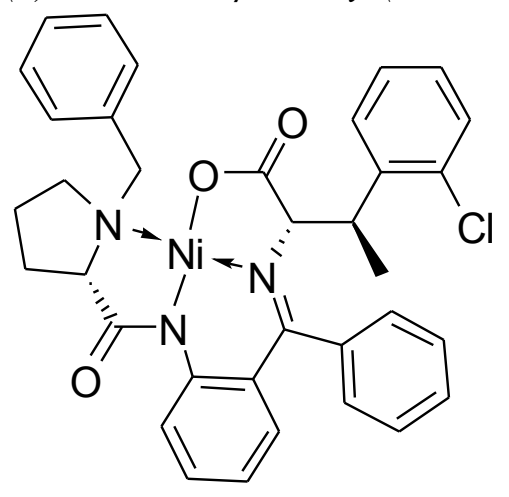
$(3.08 \mathrm{~g}, 6.2 \mathrm{mmol})$ was alkylated with racemic 1-iod-1-(ochlorophenyl)ethane $\mathbf{1 2 8}(1.74 \mathrm{~g}, 6.5 \mathrm{mmol})$ according to GP 10 using $\mathrm{NaH}(60 \%$ in oil, $0.30 \mathrm{~g}, 7.4 \mathrm{mmol})$ in $\mathrm{DMF} / \mathrm{MeCN}$ mixture $(3.0+6.0 \mathrm{~mL})$, giving $(2 S, 3 R)$ component $[1.64 \mathrm{~g}$, $2.6 \mathrm{mmol}, 41.6 \%$ on $(S)$-BGC, d.e. $\geq 98 \%$ ], $(2 S, 3 S)$ component [1.49 g, $2.3 \mathrm{mmol}, 37.7 \%$ on $(S)$-BGC, d.e. $\geq 98 \%$ ] and mixed fractions $[0.76 \mathrm{~g}, 1.2 \mathrm{mmol}, 19.2 \%$ on $(S)$-BGC] as well as products of the anion oxidation $(0.12 \mathrm{~g})$. 
(2S,3R)-B-Methyl(o-chlorophenyl)alanine $\quad[(\mathbf{2 S}, 3 \mathbf{R})-\mathbf{4 9}]: \quad(\mathrm{S})-\mathrm{BoCFC} \quad(2 \mathrm{~S}, 3 \mathrm{R})-\mathbf{1 2 9} \quad$ was

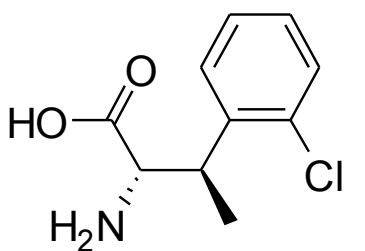

decomposed and the amino acid was separated and purified according to GP 9 to give pure target amino acid (513 $\mathrm{mg}, 2,4 \mathrm{mmol}, 96 \%)$.

Racemic 1-(p-fluorophenyl)ethanol (130): p-Fluoroacetophenone (2.76 g, $20.0 \mathrm{mmol})$ was

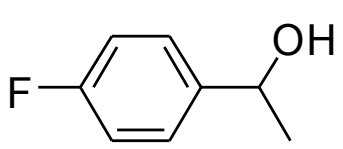
reduced with $\mathrm{LiAlH}_{4}$ solution according to GP 8, giving the target racemic alcohol 130 as colorless liquid (2.72 g, $19.4 \mathrm{mmol}, 97 \%)$; TLC: $\mathrm{R}_{f}=0.27$, pentane; $\quad{ }^{1} \mathrm{H}$ NMR $\left(250 \mathrm{MHz}, \mathrm{CDCl}_{3}\right): \quad \delta=1.45(\mathrm{~d}, \quad J=6.5 \mathrm{~Hz}, \quad 3 \mathrm{H})$, $2.30(\mathrm{bs}, \quad 1 \mathrm{H}), \quad 4.85(\mathrm{q}, \quad J=6.5 \mathrm{~Hz}, \quad 1 \mathrm{H}), \quad 6.96-7.08(\mathrm{~m}, \quad 2 \mathrm{H}), \quad 7.25-7.37(\mathrm{~m}, \quad 2 \mathrm{H})$; ${ }^{13} \mathrm{C} \mathrm{NMR}\left(62.9 \mathrm{MHz}, \mathrm{CDCl}_{3}\right): \delta=25.20(+), 69.65(+), 115.16(+, \mathrm{d}, J=21.3 \mathrm{~Hz}), 126.98(+, \mathrm{d}$, $J=8.1 \mathrm{~Hz}), 141.45\left(\mathrm{C}_{\text {quat }}, \mathrm{d}, J=3.1 \mathrm{~Hz}\right), 162.00\left(\mathrm{C}_{\text {quat, }}, \mathrm{d}, J=245.1 \mathrm{~Hz}\right) ; \mathrm{MS}-\mathrm{EI}(70 \mathrm{eV}): \mathrm{m} / \mathrm{z}(\%)$ $\left.\left.\left.\left.140.1(22 \%, M\rceil^{+}\right), \quad 125.1\left(100 \%, \quad \mathrm{C}_{8} \mathrm{H}_{10} \mathrm{~F}\right\rceil^{+}\right), \quad 97.1\left(60 \%, \mathrm{C}_{6} \mathrm{H}_{6} \mathrm{~F}\right\rceil^{+}\right), \quad 77.1\left(20 \%, \mathrm{C}_{6} \mathrm{H}_{5}\right\rceil^{+}\right)$, $43.2\left(24 \%, \mathrm{C}_{2} \mathrm{H}_{3} \mathrm{O}^{+}\right)$.

Racemic 1-iod-1-(p-fluorophenyl)ethane (131): Racemic 1-(p-fluorophenyl)ethanol 130 (2.5 g,<smiles>CC(I)c1ccc(F)cc1</smiles>
$17.8 \mathrm{mmol}$ ) was iodinated according to GP 9 using triphenylphosphine $(8.1 \mathrm{~g}, 30.7 \mathrm{mmol})$, imidazole $(2.2 \mathrm{~g}, 32.6 \mathrm{mmol})$ and iodine $(9.0 \mathrm{~g}$, $35.6 \mathrm{mmol})$ in diethyl ether/acetonitrile mixture $(50+35 \mathrm{ml})$, giving the target iodide 131 as yellowish liquid $(4.31 \mathrm{~g}, 17.2 \mathrm{mmol}, 96,8 \%)$; TLC: $\mathrm{R}_{f}=0.37$ pentane; ${ }^{1} \mathrm{H}$ NMR $\left(250 \mathrm{MHz}, \mathrm{CDCl}_{3}\right): \delta=2.20(\mathrm{~d}, J=7.1,3 \mathrm{H}), 5.40(\mathrm{q}, J=7.1 \mathrm{~Hz}, 1 \mathrm{H}), 6.92-7.06(\mathrm{~m}$, $2 \mathrm{H}), 7.36-7.48(\mathrm{~m}, 2 \mathrm{H}) ;{ }^{13} \mathrm{C} \mathrm{NMR}\left(62.9 \mathrm{MHz}, \mathrm{CDCl}_{3}\right): \delta=24.85(+), 29.13(+), 115.54(+, \mathrm{d}$, $J=21.6 \mathrm{~Hz}), 128.20(+, \mathrm{d}, J=8.3 \mathrm{~Hz}), 141.24\left(\mathrm{C}_{\text {quat }}\right), 161.86\left(\mathrm{C}_{\text {quat }}, \mathrm{d}, J=247.2 \mathrm{~Hz}\right)$.

(S)-Belokon' $\beta$-methyl(p-fluorophenyl)alanine complex [(S)-BpFFC, (2S,3R)-132]: (S)-BGC

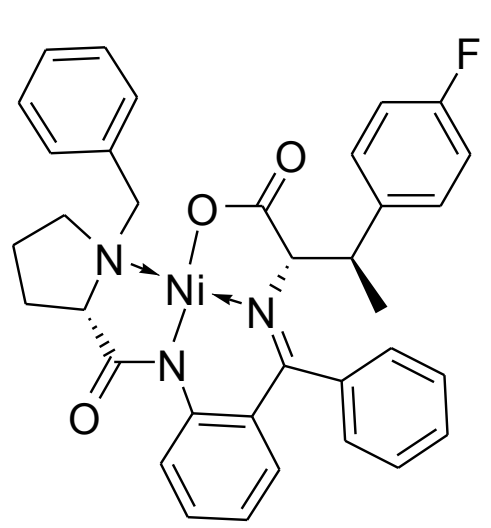
$(2.84 \mathrm{~g}, 5.7 \mathrm{mmol})$ was alkylated with racemic 1 -iod-1- $(p-$ fluorophenyl)ethane $131(1.50 \mathrm{~g}, 6.0 \mathrm{mmol})$ according to GP 10 using $\mathrm{NaH}$ (60\% in oil, $0.28 \mathrm{~g}, 6.8 \mathrm{mmol})$ in $\mathrm{DMF} / \mathrm{MeCN}$ mixture $(3.0+6.0 \mathrm{~mL})$, giving $(2 S, 3 R)$ component $[1.53 \mathrm{~g}, 2.5 \mathrm{mmol}$, $43.2 \%$ on $(S)$-BGC, d.e. $\geq 98 \%],(2 S, 3 S)$ component $[1.63 \mathrm{~g}$, $2.6 \mathrm{mmol}, 46.1 \%$ on $(S)$-BGC, d.e. $\geq 98 \%$ ] and mixed fractions $[0.33 \mathrm{~g}, 0.52 \mathrm{mmol}, 9.2 \%$ on $(S)-\mathrm{BGC}]$ as well as products of the anion oxidation $(0.13 \mathrm{~g})$ after chromatography (silica gel, eluted with ethyl acetate). ${ }^{1} \mathrm{H}$ NMR $\left(250 \mathrm{MHz}, \mathrm{CDCl}_{3}\right): \delta=1.85-2.02(\mathrm{~m}, 1 \mathrm{H}), 2.03-2.21(\mathrm{~m}, 1 \mathrm{H})$, $2.06(\mathrm{~d}, J=7.1 \mathrm{~Hz}, 3 \mathrm{H}) 2.50-2.75(\mathrm{~m}, 1 \mathrm{H}), 2.80-2.98(\mathrm{~m}, 1 \mathrm{H}), 3.31-3.52(\mathrm{~m}, 4 \mathrm{H}), 3.59(\mathrm{~d}$, 
$J=12.7 \mathrm{~Hz}, 1 \mathrm{H}), 4.09(\mathrm{~d}, J=5.0 \mathrm{~Hz}, 1 \mathrm{H}), 4.39(\mathrm{~d}, J=2.6 \mathrm{~Hz}, 1 \mathrm{H}), 6.50-6.71(\mathrm{~m}, 5 \mathrm{H})$, $6.75(\mathrm{t}, J=8.6 \mathrm{~Hz}, 2 \mathrm{H}), 7.01-7.17(\mathrm{~m}, 2 \mathrm{H}), 7.22-7.34(\mathrm{~m}, 4 \mathrm{H}), 7.47-7.58(\mathrm{~m}, 2 \mathrm{H}), 8.04(\mathrm{~d}$, $J=7.3 \mathrm{~Hz}, 2 \mathrm{H}), 8.20(\mathrm{~d}, J=8.6 \mathrm{~Hz}, 1 \mathrm{H}) ;{ }^{13} \mathrm{C} \mathrm{NMR}\left(62.9 \mathrm{MHz}, \mathrm{CDCl}_{3}\right): \delta=16.12,23.27$, 30.71, 43.94, 46.16, 56.71, 63.19, 70.39, 75.66, 114.83 (d, $J=21.0 \mathrm{~Hz}), 120.63,123.13,126.31$, $127.46,128.20,128.75,128.88,128.94,129.09$ (d, $J=7.9 \mathrm{~Hz}), 129.58,131.43,133.04,133.52$, $133.75,134.48(\mathrm{~d}, J=269.7 \mathrm{~Hz}), 142.39,170.75,176.73,180.14$.

(2S,3R)- $\beta$-Methyl-(p-fluorophenyl)alanine $\quad$ [(2S,3R)-52]: $\quad(\mathrm{S})-\mathrm{BpFFC} \quad(2 \mathrm{~S}, 3 \mathrm{R})-\mathbf{1 3 2} \quad$ was

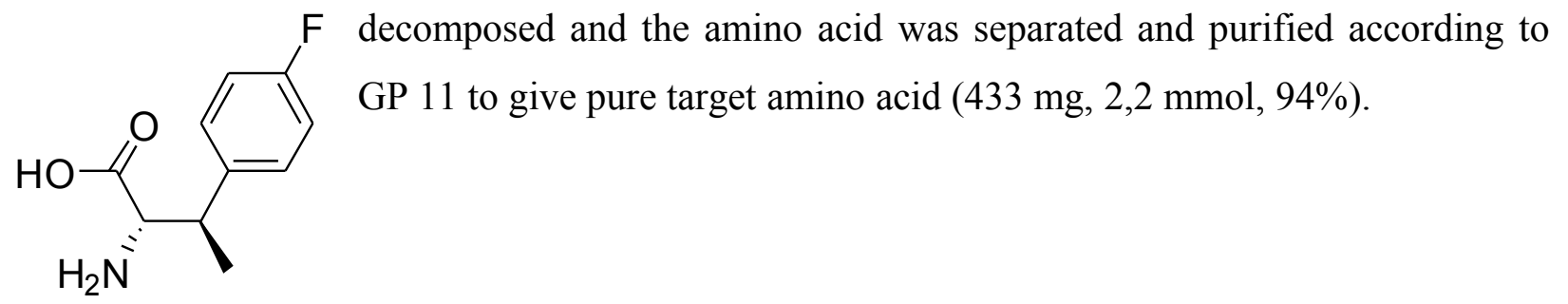

11.3. (R)-allo-Threonine

(R)-Belokon' (R)-allo-Threonine complex [(R)-BTC, 133]: (R)-BGC (21.4 g, $43.0 \mathrm{mmol})$ was

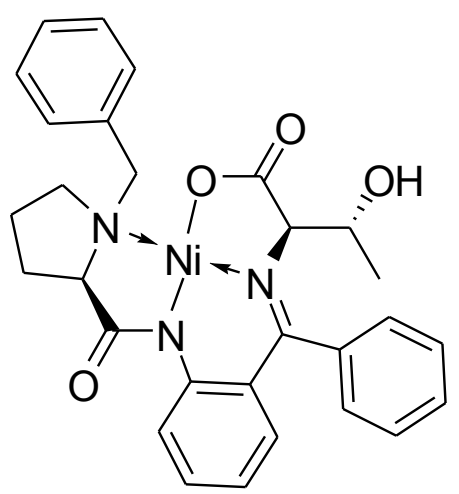
suspended in $\mathrm{DMF} / \mathrm{MeCN}$ mixture $(20+40 \mathrm{~mL})$ and degassed with two freeze-pump-thaw cycles (dry ice/acetone bath) under stirring, then $\mathrm{NaH}(60 \%$ in oil, $2.0 \mathrm{~g}, 50 \mathrm{mmol})$ was added to cold mixture and the system was thawed to $0{ }^{\circ} \mathrm{C}$ under stirring till $\mathrm{H}_{2}$ gas evolution ceased. The mixture was frozen, acetaldehyde $(2.0 \mathrm{~g}$, $45 \mathrm{mmol}$ ) was added under stirring and system was left to warm to $0{ }^{\circ} \mathrm{C}$ on air under stirring. When ice cover on flask started to thaw, $60 \%$ aqueous acetic acid $(4.5 \mathrm{~mL})$ was added. After additional 10 min stirring the mixture was concentrated under reduced pressure (bath temp $\sim 50{ }^{\circ} \mathrm{C}$ ) and liquid residue was poured into a vigorously stirred mixture of $\mathrm{H}_{2} \mathrm{O}(0.5 \mathrm{~L})$ and $\mathrm{CHCl}_{3}(100 \mathrm{~mL})$. Organic layer was separated and washed with $\mathrm{H}_{2} \mathrm{O}(2 \times 100 \mathrm{~mL})$, combined water phases were extracted with $\mathrm{CHCl}_{3}(3 \times 50 \mathrm{~mL})$, combined organic phases were concentrated under reduced pressure to $\sim 20 \mathrm{ml}$ and left overnight at ambient temperature to crystallize. Crystals were filtered out, washed with cold $\mathrm{CHCl}_{3}(\sim 10 \mathrm{ml})$ and recrystallized from $\mathrm{CHCl}_{3}$, giving orange crystals, uniform by TLC (1.76 g, $3.2 \mathrm{mmol}, 7.5 \%) ;{ }^{1} \mathrm{H} \mathrm{NMR}\left(250 \mathrm{MHz}, \mathrm{CDCl}_{3}\right): \delta=1.08(\mathrm{~d}, J=6.5 \mathrm{~Hz}$, $3 \mathrm{H}), 1.94-2.12(\mathrm{~m}, 3 \mathrm{H}), 2.25-2.59(\mathrm{~m}, 2 \mathrm{H}), 2.67-2.84(\mathrm{~m}, 1 \mathrm{H}), 3.38-3.61(\mathrm{~m}, 1 \mathrm{H}), 3.45$ (dd, $J=11.1 \mathrm{~Hz}, 5.8 \mathrm{~Hz}, 1 \mathrm{H}), 3.55(\mathrm{~d}, 12.6 \mathrm{~Hz}, 1 \mathrm{H}), 3.75-3.97(\mathrm{~m}, 3 \mathrm{H}), 4.42(\mathrm{~d}, J=12.6 \mathrm{~Hz}, 1 \mathrm{H})$, $6.65(\mathrm{~d}, J=3.87 \mathrm{~Hz}, 2 \mathrm{H}), 6.95(\mathrm{~m}, 1 \mathrm{H}), 7.09-7.23(\mathrm{~m}, 2 \mathrm{H}), 7.35(\mathrm{t}, J=7.52 \mathrm{~Hz}, 2 \mathrm{H}), 7.41-$ 
$7.56(\mathrm{~m}, 3 \mathrm{H}), 8.06(\mathrm{~d}, J=7.02 \mathrm{~Hz}, 2 \mathrm{H}), 8.14(\mathrm{~d}, J=8.61 \mathrm{~Hz}, 1 \mathrm{H}) ;{ }^{1} \mathrm{H} \mathrm{NMR}(250 \mathrm{MHz}$, [D6]DMSO): $\delta=0.88(\mathrm{~d}, J=6.3 \mathrm{~Hz}, 3 \mathrm{H}), 1.81-2.18(\mathrm{~m}, 2 \mathrm{H}), 2.29-2.40(\mathrm{~m}, 2 \mathrm{H}), 3.45-$ $3.58(\mathrm{~m}, 3 \mathrm{H}), 3.61-3.87(\mathrm{~m}, 2 \mathrm{H}), 4.04(\mathrm{~d}, J=12.2 \mathrm{~Hz}, 1 \mathrm{H}), 6.12(\mathrm{~d}, J=5.1 \mathrm{~Hz}, 1 \mathrm{H}), 6.54(\mathrm{~d}$, $J=7.8 \mathrm{~Hz}, 1 \mathrm{H}), 6.64(\mathrm{t}, J=7.1 \mathrm{~Hz}, 1 \mathrm{H}), 7.02-7.19(\mathrm{~m}, 3 \mathrm{H}), 7.30-7.62(\mathrm{~m}, 6 \mathrm{H}), 7.99(\mathrm{~d}$, $J=8.6 \mathrm{~Hz}, 1 \mathrm{H}), 8.35(\mathrm{~d}, J=7.4 \mathrm{~Hz}, 2 \mathrm{H})$, hydroxyl sygnal is masked with $\mathrm{H}_{2} \mathrm{O}$ absorption; ${ }^{13} \mathrm{C}$ NMR (62.9 MHz, [D6]DMSO): $\delta=20.19,22.61,30.24,57.43,62.38,69.27,69.71,75.82$, $119.78,123.20,126.05,127.24,127.75,128.20,128.31,128.46,128.86,128.96,129.38,130.96$, $131.45,131.55,132.48,134.01,134.76,142.61,169.08,174.67,180.18$.

(2R,3R)-2-Amino-3-hydroxybutyric acid [(R)-allo-Threonine, 67]: (R)-Belokon' (R)-alloThreonine complex $133(1.76 \mathrm{~g}, 3.2 \mathrm{mmol})$ was decomposed and the amino
$2.9 \mathrm{mmol}, 92 \%)$.

11.4. 1-Hydroxy-5-chloropyrrole-2-carboxylic acid

2,6-Dichloropyridine-1-oxide (134): ${ }^{[123]}$ 2,6-dichloropyridine (11.10 g; $\left.75.5 \mathrm{mmol}\right)$ was dissolved<smiles></smiles>
in trifluoroacetic acid $(88 \mathrm{~mL})$ at ambient temperature under stirring and aqueous $\mathrm{H}_{2} \mathrm{O}_{2}\left(30 \%\right.$ in $\left.\mathrm{H}_{2} \mathrm{O}, 16 \mathrm{~mL}\right)$ was added dropwise during $10 \mathrm{~min}$. and the mixture was carefully heated to reflux. Additional portions of $\mathrm{H}_{2} \mathrm{O}_{2}(2 \mathrm{~mL}$ each) were added twice after $1 \mathrm{~h}$ and $2 \mathrm{~h}$. The mixture was refluxed for $3 \mathrm{~h}$ under stirring, then the heating bath was removed and after short ( $\sim 15 \mathrm{~min})$ air-cooling the reflux condenser was changed to distillation head. The solvent mixture was distilled out under reduced pressure (bath temperature $\leq 50{ }^{\circ} \mathrm{C}$ ) and liquid dark red-brown residue was poured to ice/water mixture $(200 \mathrm{~mL})$ under stirring. Solid $\mathrm{K}_{2} \mathrm{CO}_{3}$ was added portionswise to vigorously stirring mixture (carefully-foam!) till $\mathrm{CO}_{2}$ gas evolution ceased, then solid $\mathrm{NaCl}$ (about $60 \mathrm{~g}$ ) was added to saturation. The precipitate formed was filtered off, wet precipitate was dissolved in $\mathrm{CH}_{2} \mathrm{Cl}_{2}$ $(50 \mathrm{~mL})$ and last drops of water phase were separated in separation funnel. Combined water phases were extracted with $\mathrm{CH}_{2} \mathrm{Cl}_{2}(5 \times 50 \mathrm{ml})$. Extracts and the solution of precipitate were combined, dried over $\mathrm{MgSO}_{4}$, filtered and concentrated under reduced pressure at ambient temperature till first crystals appeared. The solution was homogenized with $\mathrm{CH}_{2} \mathrm{Cl}_{2}(\sim 1 \mathrm{~mL})$ and diluted with hexane $(150 \mathrm{~mL})$. The precipitate formed was filtered off, washed on filter with pentane $(2 \times 50 \mathrm{~mL})$ and dried in vacuo overnight, giving target product $134(9.11 \mathrm{~g}, 55.5 \mathrm{mmol}$, $73.5 \%)$ as white solid. Starting 2,6-dichloropyridine (2.09 g, $14.1 \mathrm{mmol}, 18.7 \%)$ was recovered from filtrate; TLC: $R_{\mathrm{f}}=0.12$ EtOAc:hexane $1: 1$. 
2-Amino-6-chloropyridine-1-oxide(135):[28] 2,6-dichloropyridine-1-oxide $134 \quad(4.50$ g; 27.4 mmol) was placed to thick-wall ampoule $(250 \mathrm{~mL})$, covered with $75 \mathrm{~mL}$ of<smiles></smiles>
methanolic ammonia solution $(25 \% \mathrm{w} / \mathrm{w}$, obtained by anhydrous gaseous ammonia condensation in absolute methanol), sealed and heated at $105{ }^{\circ} \mathrm{C}$ (bath temperature) under stirring. After $24 \mathrm{~h}$ the mixture was cooled in ice/ water bath, the ampoule was open and solvents were removed at the reduced pressure. The darkbrown crystalline residue was treated with methanol/chloroform mixture (1:4, $10 \mathrm{~mL}), \mathrm{NH}_{4} \mathrm{Cl}$ precipitate was filtered out, washed with chloroform $(3 \times 5 \mathrm{~mL})$ and discarded, the filtrate was concentrated under reduced pressure and residing oil was purified with column chromatography (methanol/chloroform mixture, 1:10), giving target product $\mathbf{1 3 5}$ as off-white solid (2.32 $\mathrm{g}$, 16.0 mmol, 58.6\%); TLC: $\mathrm{MeOH}: \mathrm{CHCl}_{3}=1: 10, R_{\mathrm{f}}=0.3$; m.p. $=133-134{ }^{\circ} \mathrm{C}$.

2-Azido-6-chloropyridine-1-oxide (136): ${ }^{[28]}$ 2-amino-6-chloropyridine-1-oxide 135 (4.33 g, 30.0<smiles></smiles>
mmol) was dissolved in $10 \%$ aqueous $\mathrm{HCl}(96 \mathrm{~mL})$ at $+5^{\circ} \mathrm{C}$ (inner temperature, during the reaction time reagents addition rates were selected not to overheat the reaction mixture higher than $+5^{\circ} \mathrm{C}$ ) under vigorous stirring, the mixture was stirred for additional $15 \mathrm{~min}$ and aq. $\mathrm{NaNO}_{2}(2.5 \mathrm{M}, 13 \mathrm{~mL}$, $32.5 \mathrm{mmol})$ was added dropwise. The mixture was stirred for $15 \mathrm{~min}$ and aq. $\mathrm{NaN}_{3}(2.5 \mathrm{M}$, $13 \mathrm{ml}, 32.5 \mathrm{mmol}$ ) was added dropwise, cooling bath was removed and the mixture was left to stir and warm to ambient temperature. The reaction mixture was extracted with $\mathrm{CH}_{2} \mathrm{Cl}_{2}$ $(8 \times 50 \mathrm{~mL})$, combined extracts were dried over $\mathrm{MgSO}_{4}$, filtered and concentrated under reduced pressure to $\sim 100 \mathrm{~mL}$, diluted with hexane $(300 \mathrm{~mL})$ and concentrated under reduced pressure to $\sim 50 \mathrm{~mL}$. The precipitate was filtered off, washed with hexane $(3 \times 10 \mathrm{~mL})$ and dried in vacuo, giving target product 136 as a pale-yellow crystalline solid (4.42g, 25.9 mmol, 86.4\%); m.p. 82$83{ }^{\circ} \mathrm{C}$ (decomp.); ${ }^{1} \mathrm{H}$ NMR $\left(250 \mathrm{MHz}, \mathrm{CDCl}_{3}\right): \delta=6.85$ (dd, $\left.J=8.3 \mathrm{~Hz}, 1.8 \mathrm{~Hz}, 1 \mathrm{H}\right), 7.12$ (dd, $J=8.3 \mathrm{~Hz}, 1 \mathrm{H}), 7.25(\mathrm{dd}, J=8.3 \mathrm{~Hz}, 1.8 \mathrm{~Hz}, 1 \mathrm{H})$.

5-Chloro-1-hydroxy-1H-pyrrole-2-carbonitrile (137): 2-Azido-6-chloropyridine-1-oxide 136<smiles>N#Cc1ccc(Cl)n1O</smiles>
(3.91 g; $22.9 \mathrm{mmol})$ was dissolved in toluene $(150 \mathrm{~mL})$, the system was degassed, filled with $\mathrm{N}_{2}$ and the solution was refluxed and stirred under $\mathrm{N}_{2}$ flow for $30 \mathrm{~min}$. The reaction mixture was filtered through silica gel $(50 \mathrm{~mL})$ to remove tar products; the silica gel on filter was washed with toluene $(200 \mathrm{~mL})$, filtrates were combined and the toluene was removed under reduced pressure. Light-brown crystal residue was dissolved in minimum chloroform volume and filtered through silica gel $(50 \mathrm{~mL})$. Traces of starting material and side products were washed out with chloroform (TLC control), the product 
was eluted with ethyl acetate/hexane mixture (1:3). Eluate concentration under reduced pressure gives target product 137 as off-white crystals $(2.47 \mathrm{~g}, 17.3 \mathrm{mmol}, 76 \%)$ uniform by TLC; m.p. $100-101{ }^{\circ} \mathrm{C} ;{ }^{1} \mathrm{H}$ NMR $\left(250 \mathrm{MHz}, \mathrm{CDCl}_{3}\right): \delta=6.03(\mathrm{~d}, J=5.0 \mathrm{~Hz}, 1 \mathrm{H}), 6.66(\mathrm{~d}, J=5.0 \mathrm{~Hz}$, $1 \mathrm{H})$,

$8.94(\mathrm{~s}, 1 \mathrm{H})$.

5-Chloro-1-methoxymethoxy-1H-pyrrole-2-carbonitrile (138): ${ }^{[28]} \quad$ 5-Chloro-1-hydroxy-1H-<smiles>COCOn1c(Cl)ccc1C#N</smilespyrrole-2-carbonitrile 137 (1.0g, 7.0mmol), and TEBAC (110 mg, $0.5 \mathrm{mmol})$ were dissolved in $\mathrm{CH}_{2} \mathrm{Cl}_{2}(15 \mathrm{~mL})$ and aqueous $\mathrm{NaOH}$ solution $(24 \% \mathrm{w} / \mathrm{w}, 2.2 \mathrm{~mL})$ was added dropwise under vigorous stirring at ambient temperature. Chloro-methoxy-methane (MOM-Cl, 1.6g, $14 \mathrm{mmol}$ ) was added as one portion and stirring was continued for $30 \mathrm{~min}$. The mixture was diluted with $\mathrm{CH}_{2} \mathrm{Cl}_{2}(20 \mathrm{ml})$ and organic layer was separated. Water layer was diluted with $\mathrm{H}_{2} \mathrm{O}(10 \mathrm{~mL})$ and extracted with $\mathrm{CH}_{2} \mathrm{Cl}_{2}(3 \times 10 \mathrm{~mL})$. Combined organic layers were concentrated under reduced pressure giving $1.29 \mathrm{~g}$ crude product, which was purified with the column chromatography (silica gel, eluted with EtOAc:hexane 1:7), giving the target product 138 as colorless liquid (1.15 g, $6.3 \mathrm{mmol}, 88.0 \%) ;{ }^{1} \mathrm{H} \mathrm{NMR}\left(250 \mathrm{MHz}, \mathrm{CDCl}_{3}\right): \delta=3.73(\mathrm{~s}, 3 \mathrm{H}), 5.17(\mathrm{~s}, 2 \mathrm{H}), 6.05(\mathrm{~d}$, $J=4.8 \mathrm{~Hz}, 1 \mathrm{H}), 6.64(\mathrm{~d}, J=4.8 \mathrm{~Hz}, 1 \mathrm{H})$.

5-Chloro-1-methoxymethoxy-1H-pyrrole-2-carboxamide

$$
\text { (139): }{ }^{[28]}
$$

5-Chloro-1-<smiles>COCOn1c(Cl)ccc1C(N)=O</smilesmethoxymethoxy-1H-pyrrole-2-carbonitrile 138 (3.53g, $18.9 \mathrm{mmol})$, and tetrabutylammonium hydrosulfate $(1.35 \mathrm{~g}, 3.9 \mathrm{mmol})$ were dissolved in $\mathrm{CH}_{2} \mathrm{Cl}_{2}(40 \mathrm{~mL})$ in $250-\mathrm{ml}$ round-bottomed flask under vigorous stirring, the solution of $2.0 \mathrm{~g} \mathrm{NaOH}$ in $8.0 \mathrm{~mL} \mathrm{H}_{2} \mathrm{O}$ was added, followed with aqueous $\mathrm{H}_{2} \mathrm{O}_{2}$ solution $(30 \%, 14 \mathrm{~mL}$; carefully-foam!). The mixture was vigorously stirred for $30 \mathrm{~min}$, organic layer was separated, water layer was washed with $\mathrm{CH}_{2} \mathrm{Cl}_{2}$ $(10 \mathrm{~mL})$, saturated with solid $\mathrm{NaCl}$ and extracted with $\mathrm{CH}_{2} \mathrm{Cl}_{2}(2 \times 20 \mathrm{~mL})$. Combined organic layers were concentrated under reduced pressure. The residue $(4.88 \mathrm{~g})$ was purified with column chromatography (silica gel, eluted with EtOAc: hexane 1:1), giving the target product as lightyellow extremely viscose oil $(3.65 \mathrm{~g})$. The crystalline product was obtained after dissolving the product in diethyl ether $(10 \mathrm{~mL})$ and precipitation with hexane $(100 \mathrm{~mL})$. The crystalline precipitate was filtered out, rinsed with pentane $(3 \times 10 \mathrm{~mL})$ and dried in vacuo, giving the target product 139 as white solid (3.18 g, $15.5 \mathrm{mmol}, 82.2 \%)$; m.p.: $60-61{ }^{\circ} \mathrm{C}$. 
Di-tert-butyl [5-Chloro-1-(methoxymethoxy)pyrrol-2-yl]carbonylimidodicarbamate (140): ${ }^{[28]}$<smiles>C=C(C)OC(=O)N(C(=O)OC(C)(C)C)C(=O)c1ccc(Cl)n1OCOC</smiles>
The solution of 4-pyrrolidino pyridine $(0.22 \mathrm{~g}, 1.5 \mathrm{mmol})$ in anhydrous acetonitrile $(20 \mathrm{~mL})$ was added dropwise to vigorously stirred solution of 5-chloro-1-methoxymethoxy-1H-pyrrole-2-carboxamide 139 (3.45 g, $16.9 \mathrm{mmol})$ and di-tert-butyl pyrocarbonate $(22.1 \mathrm{~g}, 101.2 \mathrm{mmol})$ in anhydrous acetonitrile $(65 \mathrm{~mL})$ and the stirring was continued for 1.5 hour. Solvents were evaporated under reduced pressure, oily yellowish residue was purified with column chromatography (silica gel, eluted with ethyl acetate/hexane 1:8), giving the target product 140 as colorless oil, solidified while drying in vacuo overnight to white solid (5.46 g, $13.5 \mathrm{mmol}, 79.8 \%)$; m.p.: 62$69{ }^{\circ} \mathrm{C}$.

5-Chloro-1-methoxymethoxy-1H-pyrrole-2-carboxylic acid (81): ${ }^{[28]}$ Di-tert-butyl [5-Chloro-1-<smiles>COCOn1c(Cl)ccc1C(=O)O</smiles(methoxymethoxy)pyrrol-2-yl]carbonylimidodicarbamate $\mathbf{1 4 0} \quad(5.42 \mathrm{~g}$, $13.4 \mathrm{mmol})$ was dissolved in dioxane $(65 \mathrm{~mL})$ under stirring at $55^{\circ} \mathrm{C}$ (bath temperature) and aq. $1 \mathrm{M} \mathrm{NaOH}(18 \mathrm{~mL})$ was added as one portion. The mixture was stirred overnight, and then all volatiles were removed under reduced pressure. Paste-like residue was dissolved in $\mathrm{H}_{2} \mathrm{O}(150 \mathrm{~mL})$ and the solution was washed with $\mathrm{CH}_{2} \mathrm{Cl}_{2}(3 \times 50 \mathrm{~mL})$. Water layer was separated, acidified with aq. $1 \mathrm{M} \mathrm{KHSO}_{4}$ to $\mathrm{pH} \sim 2$ and extracted with $\mathrm{CH}_{2} \mathrm{Cl}_{2}(3 \times 50 \mathrm{~mL})$. Combined organic layers were concentrated under reduced pressure, giving $2.08 \mathrm{~g}$ crude product as pink solid, which was purified with flash-chromatography (silica gel, eluted with diethyl ether) to give target product 81 as colorless solid (2.05 g, $10.0 \mathrm{mmol}, 74.6 \%)$; m.p.: $123-124{ }^{\circ} \mathrm{C}$; ${ }^{1} \mathrm{H}$ NMR $(250 \mathrm{MHz}$, $\left.\mathrm{CDCl}_{3}\right): \delta=3.68(\mathrm{~s}, 3 \mathrm{H}), 5.25(\mathrm{~s}, 2 \mathrm{H}), 6.06(\mathrm{~d}, J=5.0 \mathrm{~Hz}, 1 \mathrm{H}), 6.95(\mathrm{~d}, J=5.0 \mathrm{~Hz}, 1 \mathrm{H})$, 11.75 (bs, $1 \mathrm{H})$.

\section{5. (2S,4R)-N-Boc-4-(Z)-Propenylproline}

(2S,4R)-N-Boc-4-Hydroxyproline (2): $:^{[124]}$ (2S,4R)-4-Hydroxyproline (32.8 g, $\left.250 \mathrm{mmol}\right)$ was

$\mathrm{HO}$,<smiles>CC(=O)OC(=O)N1CCC[C@H]1C(=O)O</smiles>

aqueous layer was extracted with ethyl acetate $(3 \times 100 \mathrm{~mL})$, combined extracts were washed $\mathrm{NaOH}(11.0 \mathrm{~g}, 275 \mathrm{mmol})$ in $\mathrm{H}_{2} \mathrm{O}(100 \mathrm{~mL})$ were successively added with vigorous stirring, followed with di-tert-butyl dicarbonate $(82.6 \mathrm{~g}$, $367 \mathrm{mmol}$ ) and the mixture was left to stir overnight. The mixture was acidified with aq. $1 \mathrm{M} \mathrm{KHSO}_{4}$ to $\mathrm{pH} \sim 2$, organic layer was separated, dissolved in $\mathrm{H}_{2} \mathrm{O}(170 \mathrm{~mL})$ with stirring, THF $(340 \mathrm{~mL})$ and the solution of 
with brine $(2 \times 20 \mathrm{~mL})$, dried over $\mathrm{MgSO}_{4}$, filtered and concentrated under reduced pressure to give crude $N$-protected amino acid as colorless sypup. The syrup was dissolved in EtOAc $(300 \mathrm{~mL})$ and back-extracted with aq. $10 \% \mathrm{NaOH}(3 \times 100 \mathrm{~mL})$. Organic layer was discarded, combined aqueous phases were acidified with aq. $1 \mathrm{M} \mathrm{KHSO}_{4}$ to $\mathrm{pH} \sim 2$ and extracted with diethyl ether $(3 \times 100 \mathrm{~mL})$. Combined extracts were washed with aq. $1 \mathrm{M} \mathrm{KHSO}_{4}(3 \times 100 \mathrm{~mL})$ and brine $(2 \times 100 \mathrm{~mL})$, dried over $\mathrm{MgSO}_{4}$, filtered and concentrated under reduced pressure giving target $N$-protected amino acid 2 as glass-like solid $(50.2 \mathrm{~g}, 217 \mathrm{mmol}, 87 \%)$; TLC: $R_{\mathrm{f}}=0.18$ (ethyl acetate/ hexane 1:1+5\% acetic acid).

(2S,4R)-N-Boc-4-Hydroxyprolinol (3):[28] To a solution of (2S,4R)-N-Boc-4-Hydroxyproline 2

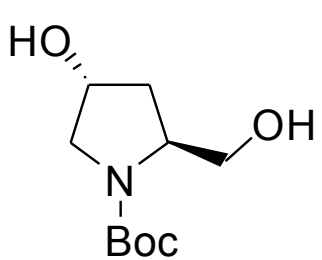
$(50.2 \mathrm{~g}, \quad 217 \mathrm{mmol})$ and triethylamine $(33.6 \mathrm{~mL}, \quad 239 \mathrm{mmol})$ in $\mathrm{CH}_{2} \mathrm{Cl}_{2}(600 \mathrm{~mL})$ was added at $-30{ }^{\circ} \mathrm{C}$ ethyl chloroformate $(21.9 \mathrm{~mL}$, $229 \mathrm{mmol}$ ), and the mixture was stirred for $40 \mathrm{~min}$. To this mixture tetrabutylammonium bromide $(7.44 \mathrm{~g}, 23.1 \mathrm{mmol})$ was added and then carefully, by small portions a suspension of $\mathrm{NaBH}_{4}(35.0 \mathrm{~g}, 925 \mathrm{mmol})$ in ice-cold water $(44 \mathrm{~mL})$. The reaction mixture was allowed to warm to $-10^{\circ} \mathrm{C}$ and stirred for $1 \mathrm{~h}$. The temperature of the mixture was further increased to $0{ }^{\circ} \mathrm{C}$, and stirring was continued at this temperature for $1 \mathrm{~h}$. The $\mathrm{pH}$ value of the aqueous layer was then carefully adjusted to 5-6 with $50 \%$ acetic acid, the mixture was stirred till $\mathrm{H}_{2}$ gas evolution ceased and filtered through Celite ${ }^{\circledR}$ pad. The organic layer was separated, and the aqueous layer was extracted with $\mathrm{CH}_{2} \mathrm{Cl}_{2}$ $(3 \times 20 \mathrm{~mL})$. The aqueous layer was discarded, and the combined organic fractions were dried over $\mathrm{MgSO}_{4}$, filtered, concentrated under reduced pressure, and the residue was purified by column chromatography (silica gel, eluted with EtOAc/hexane 2:1, $R_{\mathrm{f}}=0.13$ ) to give the target diol 3 (21.44 g, $98.7 \mathrm{mmol}, 45 \%)$ as a colorless syrup. $[\alpha]_{\mathrm{D}}{ }^{20}-58.8\left(\mathrm{c}=1.0\right.$, EtOH); ${ }^{1} \mathrm{H}$ NMR $\left(250 \mathrm{MHz}, \mathrm{CDCl}_{3}\right): \delta=1.47$ (s, $\left.9 \mathrm{H}\right), 1.57-1.80(\mathrm{~m}, 1 \mathrm{H}), 1.89-1.98$ (bs, $\left.1 \mathrm{H}\right), 1.98-2.11(\mathrm{~m}$, $1 \mathrm{H}), 3.35-3.63(\mathrm{~m}, 3 \mathrm{H}), 3.70$ (t, $J=9.4 \mathrm{~Hz}, 1 \mathrm{H}), 4.04-4.25(\mathrm{~m}, 1 \mathrm{H}), 4.29-4.45(\mathrm{~m}, 1 \mathrm{H})$, 4.95-5.09 (bs, $1 \mathrm{H}) ;{ }^{13} \mathrm{C}$ NMR $\left(62.9 \mathrm{MHz}, \mathrm{CDCl}_{3}\right): \delta=28.3(+), 37.3(-), 54.9+55.6(-)$, $57.7+58.6(+), 63.8+66.4(-), 68.8(+), 80.4\left(\mathrm{C}_{\text {quat }}\right), 155.0+156.9\left(\mathrm{C}_{\text {quat }}\right)$.

$(2 S, 4 R)-N$-Boc-O-TBDMS-4-Hydroxyprolinol (4): ${ }^{[125]}(2 S, 4 R)-N$-Boc-4-Hydroxyprolinol 3
$(3.28 \mathrm{~g}, 15 \mathrm{mmol})$ was dissolved under $\mathrm{N}_{2}$-flow in ice-cold
vigorously stirred mixture of triethyl amine $(2.5 \mathrm{~mL}, 1.8 \mathrm{~g}, 18 \mathrm{mmol})$
and $\mathrm{CH}_{2} \mathrm{Cl}_{2}(100 \mathrm{~mL})$, DMAP $(0.1 \mathrm{~g})$ was added, followed with
TBDMS-Cl toluene solution $(55.2 \% \mathrm{w} / \mathrm{w}, 4.55 \mathrm{~g}, 16 \mathrm{mmol})$ and the mixture was left to stir at ambient temperature overnight. The mixture was diluted with diethyl 
ether $(300 \mathrm{~mL})$ to form precipitate, which was filtered out; filter cake was washed with diethyl ether $(3 \times 20 \mathrm{~mL})$ and discarded. Combined filtrates were concentrated under reduced pressure to give oily residue, which was purified with the column chromatography (silica gel, eluted with ethyl acetate/hexane 1:3) to give target product 4 as colorless oil (2.2 g, $6.6 \mathrm{mmol}, 44 \%)$.

(2S,4R)-N-Boc-O-TBDMS-4-Mesyloxyprolinol (5): ${ }^{[28]}$ To a solution of $(2 S, 4 R)-N$-Boc- $O$ -

$\mathrm{MsO}$,

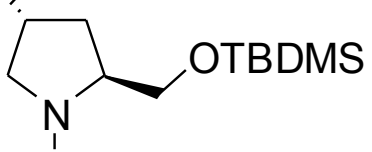

Boc TBDMS-4-Hydroxyprolinol 4 (2.26 g, $6.8 \mathrm{mmol})$ and triethylamine (1.4 mL, $10.2 \mathrm{mmol})$ in $\mathrm{CH}_{2} \mathrm{Cl}_{2}(6 \mathrm{~mL})$ at $-78^{\circ} \mathrm{C}$ was added mesyl chloride $(0.7 \mathrm{~mL}, 1.05 \mathrm{~g}, 9.2 \mathrm{mmol})$ within $5 \mathrm{~min}$. The mixture was allowed to warm to $0{ }^{\circ} \mathrm{C}$ and stirred for an additional $3 \mathrm{~h}$, before aq. sat. $\mathrm{NaHCO}_{3}(5 \mathrm{~mL})$ was added. The reaction mixture was extracted with diethyl ether $(3 \times 20 \mathrm{~mL})$, combined extracts were washed with $\mathrm{H}_{2} \mathrm{O}(3 \times 20 \mathrm{~mL})$, aq. $1 \mathrm{M} \mathrm{KHSO}_{4}$ $(3 \times 20 \mathrm{~mL}), \mathrm{H}_{2} \mathrm{O}(3 \times 20 \mathrm{~mL})$, brine $(2 \times 20 \mathrm{~mL})$ and dried over $\mathrm{MgSO}_{4}$. Concentration under reduced pressure gave the target product $5(2.53 \mathrm{~g}, 6.2 \mathrm{mmol}, 94 \%)$ as a light yellow oil. $R_{\mathrm{f}}=0.53\left(\right.$ EtOAc/hexane, 2:5); $[\alpha]_{\mathrm{D}}{ }^{20}-38.5 \quad\left(\mathrm{c}=0.55, \mathrm{CHCl}_{3}\right) ;{ }^{1} \mathrm{H}$ NMR $(250 \mathrm{MHz}$, $\left.\mathrm{CDCl}_{3}\right): \delta=-0.01(\mathrm{~s}, 6 \mathrm{H}), 0.85(\mathrm{~s}, 9 \mathrm{H}), 1.44(\mathrm{~s}, 9 \mathrm{H}), 2.30-2.49(\mathrm{~m}, 2 \mathrm{H}), 3.02(\mathrm{~s}, 3 \mathrm{H}), 3.44-$ $3.63(\mathrm{~m}, 2 \mathrm{H}), 3.63-4.15(\mathrm{~m}, 3 \mathrm{H}), 5.25-5.33(\mathrm{~m}, 1 \mathrm{H}) ;{ }^{13} \mathrm{C} \mathrm{NMR}\left(75.5 \mathrm{MHz}, \mathrm{C}_{2} \mathrm{D}_{2} \mathrm{Cl}_{4}, 353 \mathrm{~K}\right)$ : $\delta=-5.7(+), 17.8\left(\mathrm{C}_{\text {quat }}\right), 25.6(+), 28.3(+), 34.8(-), 38.4(+), 52.4(-), 57.0(+), 63.4(-)$, $78.9(+), 79.6\left(\mathrm{C}_{\text {quat }}\right), 153.6\left(\mathrm{C}_{\text {quat }}\right)$.

$(2 S, 4 S)-N-B o c-O-T B D M S-4-C y a n o p r o l i n o l(6):{ }^{[28]}$ A sealed round-bottomed flask, containing a

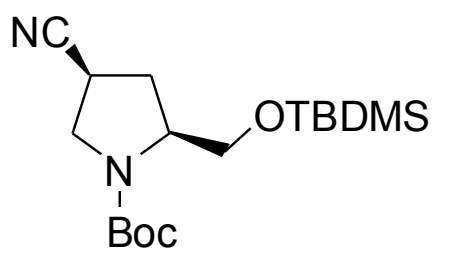
solution of the mesyl ester $5(2.53 \mathrm{~g}, 6.2 \mathrm{mmol})$ and tetrabutylammonium cyanide $(3.44 \mathrm{~g}, 12.8 \mathrm{mmol})$ in anhydrous $\mathrm{MeCN}(3 \mathrm{~mL}$ ) was immersed to an oil-bath which was preheated to $65-68{ }^{\circ} \mathrm{C}$. The mixture was stirred for $6 \mathrm{~h}$, diluted with ethyl acetate/ hexane mixture $(1: 4,25 \mathrm{~mL})$, washed with water $(5 \times 5 \mathrm{~mL})$ and brine $(2 \times 5 \mathrm{~mL})$, dried over $\mathrm{MgSO}_{4}$ and filtered through a pad of silica gel $(1 \mathrm{~cm})$. The solvents were removed under reduced pressure, and the residue was purified by column chromatography (silica gel, eluted with EtOAc/ hexane $\left.1: 3, R_{\mathrm{f}}=0.50\right)$ to give target nitrile $6(1.31 \mathrm{~g}, 62 \%)$ as a yellowish oil which solidified to a colorless solid upon seeding. M.p. $55-58^{\circ} \mathrm{C} ;[\alpha]_{\mathrm{D}}{ }^{20}-25.9\left(\mathrm{c}=0.9, \mathrm{CHCl}_{3}\right) ;{ }^{1} \mathrm{H} \mathrm{NMR}$ $\left(300 \mathrm{MHz}, \mathrm{C}_{2} \mathrm{D}_{2} \mathrm{Cl}_{4}, 358 \mathrm{~K}\right): \delta=0.10(\mathrm{~s}, 6 \mathrm{H}), 0.94(\mathrm{~s}, 9 \mathrm{H}), 1.48(\mathrm{~s}, 9 \mathrm{H}), 2.29-2.46(\mathrm{~m}, 2 \mathrm{H})$, 2.90-3.01 (m, $1 \mathrm{H}), 3.42(\mathrm{dd}, J=8.2 \mathrm{~Hz}, 10.6 \mathrm{~Hz}, 1 \mathrm{H}), 3.74(\mathrm{dd}, J=3.0 \mathrm{~Hz}, 9.8 \mathrm{~Hz}, 1 \mathrm{H})$, 3.79-3.95 (m, $2 \mathrm{H}), 3.96(\mathrm{dd}, J=8.2 \mathrm{~Hz}, 10.6 \mathrm{~Hz}, 1 \mathrm{H}) ;{ }^{13} \mathrm{C} \mathrm{NMR}\left(75.5 \mathrm{MHz}, \mathrm{C}_{2} \mathrm{D}_{2} \mathrm{Cl}_{4}, 358 \mathrm{~K}\right)$ : $\delta=-5.6(+), 17.9\left(\mathrm{C}_{\text {quat }}\right), 25.7(+), 26.3(+), 28.2(+), 31.9(-), 49.8(-), 57.8(+), 62.9(-)$, $80.0\left(\mathrm{C}_{\text {quat }}\right), 119.8\left(\mathrm{C}_{\text {quat }}\right), 153.2\left(\mathrm{C}_{\text {quat }}\right)$. 
(2S,4S)-N-Boc-O-TBDMS-4-Formylprolinol (7): ${ }^{[28]}$ A $1 \mathrm{M}$ solution of DIBAH in hexane

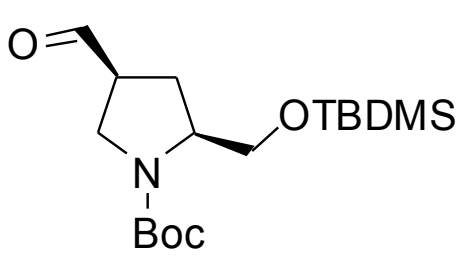

(36.3 $\mathrm{mL}, 36.3 \mathrm{mmol}$ ) was added dropwise at $-30{ }^{\circ} \mathrm{C}$ over $10 \mathrm{~min}$ to a stirred solution of the $(2 S, 4 S)-N$-Boc- $O$-TBDMS-4cyanoprolinol $6(9.15 \mathrm{~g}, 26.9 \mathrm{mmol})$ in anhydrous $\mathrm{CH}_{2} \mathrm{Cl}_{2}(90 \mathrm{~mL})$. The reaction mixture was stirred at -30 to $-20{ }^{\circ} \mathrm{C}$ for $2 \mathrm{~h}$, then

methanol $(2.5 \mathrm{~mL})$ was added dropwise at $0{ }^{\circ} \mathrm{C}$ within $3 \mathrm{~min}$, and stirring was continued at the same temperature for $15 \mathrm{~min}$. A saturated aqueous $\mathrm{NH}_{4} \mathrm{Cl}$ solution $(8.5 \mathrm{~mL})$ was added, and the mixture was allowed to warm to $20^{\circ} \mathrm{C}$. After $45 \mathrm{~min}$, the reaction mixture was diluted with diethyl ether $(80 \mathrm{~mL})$, saturated aqueous potassium sodium tartrate $(14 \mathrm{~mL})$ was added and vigorous stirring was continued for an additional $1 \mathrm{~h}$. The phases were separated, and the organic fraction was washed twice with a solution of citric acid $(5.14 \mathrm{~g}, 26.72 \mathrm{mmol})$ in water $(120 \mathrm{~mL})$, with water $(5 \times 50 \mathrm{~mL})$, brine $(2 \times 20 \mathrm{~mL})$, dried over $\mathrm{MgSO}_{4}$, filtered and concentrated under reduced pressure. The residue was taken up with hexane $(30 \mathrm{~mL})$, filtered through a pad of Celite $^{\circledR}$ and concentrated under reduced pressure to give the target aldehyde 7 (8.76 g, 95\% crude) as a colorless oil, which was used for the next step without further purification. $R_{\mathrm{f}}=0.37$ (EtOAc/hexane, 1:4); ${ }^{1} \mathrm{H}$ NMR $\left(250 \mathrm{MHz}, \mathrm{CDCl}_{3}\right): \delta=0.03$ (s, $\left.6 \mathrm{H}\right), 0.86(\mathrm{~s}, 9 \mathrm{H}), 1.43$ (s, $\left.9 \mathrm{H}\right)$, 1.78-2.20 (m, $1 \mathrm{H}), 2.22-2.41(\mathrm{~m}, 1 \mathrm{H}), 2.78-3.12(\mathrm{~m}, 1 \mathrm{H}), 3.45-4.02(\mathrm{~m}, 5 \mathrm{H}), 9.63$ (s, $1 \mathrm{H})$. $(2 S, 4 R)-N-B o c-4-(Z)-P r o p e n y l p r o l i n o l ~(9):{ }^{[28]}$ A freshly prepared solution of $t \mathrm{BuOK}(10.0 \mathrm{~g}$,

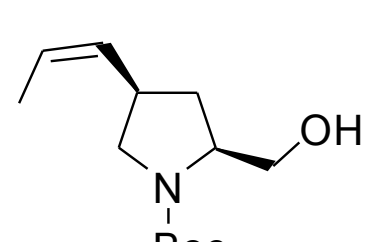

Boc $89.3 \mathrm{mmol})$ in THF $(100 \mathrm{~mL})$ was added to a suspension of ethyltriphenylphosponium bromide $(40.0 \mathrm{~g}, 107.6 \mathrm{mmol})$ in THF $(50 \mathrm{~mL})$ at $0{ }^{\circ} \mathrm{C}$. The cooling bath was removed, and stirring continued for an additional $2 \mathrm{~h}$. The mixture was cooled to $-78^{\circ} \mathrm{C}$ (dry ice/acetone bath), and a solution of $(2 S, 4 S)$ - $N$-Boc-O-TBDMS-4-formylprolinol 7 (8.8 g, $25.5 \mathrm{mmol})$ in THF $(30 \mathrm{~mL})$ was added dropwise within $2 \mathrm{~h}$. Stirring was continued at the same temperature for an additional $24 \mathrm{~h}$, and then the mixture was allowed to warm to $20^{\circ} \mathrm{C}$ for $24 \mathrm{~h}$. After $48 \mathrm{~h}$, the reaction flask was immersed into an ice/water bath, and aq. sat. $\mathrm{Na}_{2} \mathrm{SO}_{4}(50 \mathrm{~mL})$ was added. Organic layer was separated, water layer was washed with THF $(2 \times 50 \mathrm{~mL})$, combined organic phases were concentrated under reduced pressure, yellow oily residue was taken up with $\mathrm{CH}_{2} \mathrm{Cl}_{2} /$ diethyl ether mixture $(1: 4,200 \mathrm{~mL})$, precipitate was filtered out through the pad of silica gel $(5 \mathrm{~cm})$ and filter cake was washed with $\mathrm{CH}_{2} \mathrm{Cl}_{2} /$ diethyl ether mixture $(1: 4,100 \mathrm{~mL})$, filtrate was concentrated under reduced pressure, the residue was dissolved in diethyl ether $(20 \mathrm{~mL})$, the solution was filtered, concentrated under reduced pressure, the residue was dissolved in diethyl ether /hexane mixture $(1: 1,20 \mathrm{~mL})$, the solution was filtered, concentrated under reduced pressure, the residue was dissolved in hexane $(20 \mathrm{~mL})$, the solution was filtered, concentrated 
under reduced pressure, the residue was dissolved in pentane $(20 \mathrm{~mL})$, the solution was filtered, concentrated, and the residue was finally purified by column chromatography (silica gel, eluted with EtOAc/ hexane 1:8, $\left.R_{\mathrm{f}}=0.51\right)$ to give pure $(2 S, 4 R)-N$-Boc- $O$-TBDMS-4- $(Z)$ propenylprolinol 8 (5.3 g), which was dissolved in THF $(10 \mathrm{~mL})$ and deprotected by treating with the tetra- $n$-butylammonium fluoride trihydrate $(6.25 \mathrm{~g}, 19.8 \mathrm{mmol})$ with stirring at ambient temperature. After $2 \mathrm{~h}$, the mixture was diluted with diethyl ether $(100 \mathrm{~mL})$, washed with water $(5 \times 20 \mathrm{~mL})$, brine $(2 \times 20 \mathrm{~mL})$, dried over $\mathrm{MgSO}_{4}$, concentrated under reduced pressure, and the residue was purified by column chromatography (silica gel, eluted with EtOAc/hexane 2:5, $\left.R_{\mathrm{f}}=0.32\right)$ to give target unsaturated alcohol $9(1.70 \mathrm{~g}, 7.0 \mathrm{mmol}, 28 \%$ over 2 steps from aldehyde) as a colorless oil which solidified into a colorless solid upon seeding. M.p. $41-43{ }^{\circ} \mathrm{C}$; $[\alpha]_{\mathrm{D}}{ }^{20}-47.9\left(\mathrm{c}=0.97, \mathrm{CHCl}_{3}\right) ;{ }^{1} \mathrm{H} \mathrm{NMR}\left(250 \mathrm{MHz}, \mathrm{CDCl}_{3}\right): \delta=1.16-1.27(\mathrm{~m}, 1 \mathrm{H}), 1.46(\mathrm{~s}$, $9 \mathrm{H}), 1.65(\mathrm{dd}, J=6.9 \mathrm{~Hz}, 0.8 \mathrm{~Hz}, 3 \mathrm{H}), 2.06-2.19$ (m, $1 \mathrm{H}), 2.83-2.96$ (m, $1 \mathrm{H}), 2.85-3.10$ (m, $1 \mathrm{H}), 3.52-3.77(\mathrm{~m}, 3 \mathrm{H}), 3.96(\mathrm{dd}, J=14.9 \mathrm{~Hz}, 7.6 \mathrm{~Hz}, 1 \mathrm{H}), 5.11-2.24(\mathrm{~m}, 1 \mathrm{H}), 5.30(\mathrm{dd}$, $J=8.9 \mathrm{~Hz}, 1.8 \mathrm{~Hz}, 1 \mathrm{H}), 5.52(\mathrm{dq}, J=9.8 \mathrm{~Hz}, 6.9 \mathrm{~Hz}, 1 \mathrm{H}) ;{ }^{13} \mathrm{C} \mathrm{NMR}\left(62.9 \mathrm{MHz}, \mathrm{CDCl}_{3}\right)$ : $\delta=13.2(+), 28.4(+), 35.2(+), 35.8(-), 52.7(-), 61.1(+), 67.6(-), 80.4\left(\mathrm{C}_{\text {quat }}\right), 126.3(+)$, $129.8(+), 156.8\left(\mathrm{C}_{\text {quat }}\right)$.

$(2 S, 4 R)-N$-Boc-4-(Z)-Propenylproline (10): ${ }^{[28]}$ A $2.67 \mathrm{M}$ solution of Jones reagent ${ }^{[126]}(30.5 \mathrm{~mL}$,

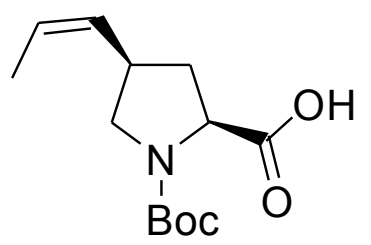
$81.4 \mathrm{mmol})$ was added to a solution of unsaturated alcohol 9 (1.96 g, $8.14 \mathrm{mmol}$ ) in freshly distilled acetone $\left(670 \mathrm{~mL}\right.$ ) at $4{ }^{\circ} \mathrm{C}$ (inner temperature) within $1 \mathrm{~h}$, and the mixture was stirred at the same temperature for an additional $4 \mathrm{~h}$. Isopropyl alcohol $(10 \mathrm{~mL})$ was then added dropwise within $10 \mathrm{~min}$, and the mixture was allowed to warm to $20^{\circ} \mathrm{C}$. Organic solution was decanted out of inorganic solid, latter was washed with diethyl ether $(100 \mathrm{ml})$ and combined organics was concentrated under reduced pressure at ambient temperature to ca. $20 \mathrm{~mL}$. Inorganic solid precipitate was dissolved in water $(100 \mathrm{~mL})$ and extracted with diethyl ether $(2 \times 50 \mathrm{~mL})$. Extracts were combined with the organic concentrate, washed with water $(2 \times 50 \mathrm{~mL})$, dried over $\mathrm{MgSO}_{4}$, filtered, concentrated under reduced pressure, giving the crude product $(2.15 \mathrm{~g})$ as colorless oil. This was dissolved in diethyl ether $(100 \mathrm{~mL})$ and extracted with saturated aqueous $\mathrm{NaHCO}_{3}$ solution $(5 \times 40 \mathrm{~mL})$. The combined aqueous fractions were washed with diethyl ether $(2 \times 50 \mathrm{~mL})$, the $\mathrm{pH}$ of the aqueous fractions was carefully adjusted to $2.5-3$ with solid $\mathrm{KHSO}_{4}$, the formed emulsion was extracted with diethyl ether $(2 \times 100 \mathrm{~mL})$ and the organic fraction was washed with aq. $1 \mathrm{MKHSO}_{4}(3 \times 50 \mathrm{~mL})$, water $(3 \times 50 \mathrm{~mL})$, brine $(2 \times 20 \mathrm{~mL})$, dried over $\mathrm{MgSO}_{4}$, filtered and concentrated under reduced pressure. The residue was finally purified by column chromatography (silica gel, eluted with EtOAc/hexane 1:3+2\% 
$\left.\mathrm{AcOH}, R_{\mathrm{f}}=0.27\right)$ to give the target unsaturated amino acid $\mathbf{1 0}(1.44 \mathrm{~g}, 69 \%)$ as a colorless solid. M.p. $84-85{ }^{\circ} \mathrm{C} ;[\alpha]_{\mathrm{D}}{ }^{20}-84.4\left(\mathrm{c}=0.86, \mathrm{CHCl}_{3}\right) ;{ }^{1} \mathrm{H} \mathrm{NMR}\left(250 \mathrm{MHz}, \mathrm{CDCl}_{3}\right): \delta=1.42,1.48(2 \mathrm{~s}$, $9 \mathrm{H}), 1.66(\mathrm{~d}, J=6.8 \mathrm{~Hz}, 3 \mathrm{H}), 1.72-1.84,1.93-2.12$ (m, $1 \mathrm{H}), 2.27-2.54$ (m, $1 \mathrm{H}), 2.87-3.24$ $(\mathrm{m}, 2 \mathrm{H}), 3.64-3.86(\mathrm{~m}, 1 \mathrm{H}), 4.25+4.35(2 \times \mathrm{dd}, J=8.3 \mathrm{~Hz}, 8.3 \mathrm{~Hz}, 1 \mathrm{H}), 5.26$ (ddq, $J=$ $9.6 \mathrm{~Hz}, 8.5 \mathrm{~Hz}, 1.8 \mathrm{~Hz}, 1 \mathrm{H}), 5.52$ (dq, $J=8.5 \mathrm{~Hz}, 6.8 \mathrm{~Hz}, 1 \mathrm{H}), 10.30-11.40$ (bs, $1 \mathrm{H}) .{ }^{13} \mathrm{C}$ NMR $\left(62.9 \mathrm{MHz}, \mathrm{CDCl}_{3}\right): \delta=13.1(+), 28.1+28.3(+), 35.7+36.1(+), 36.2+37.3(-), 51.4+51.9$ $(-), 58.9+59.1(+), 80.6\left(\mathrm{C}_{\text {quat }}\right), 126.6+126.9(+), 129.0+129.2(+), 153.6+154.8\left(\mathrm{C}_{\text {quat }}\right)$, $177.3+178.4\left(\mathrm{C}_{\text {quat }}\right)$. 


\section{REFERENCES}

[1] A. Fleming, Br. J. Exp. Pathol. 1929, 10, 226-236.

[2] Benzylpenicillin, commonly known as penicillin G, 6-(2-phenylacetamido)-penicillan acid

[3] M. F. Vincente, A. Basilio, A. Cabello, F. Peläez, Clin. Microbiol. Infect. 2003, 9, 15-32.

[4] K. Stephens, CRC Crit. Rev. Microbiol. 1986, 13, 309-334.

[5] Quorum sensing coordinates certain behavior or actions between bacteria, based on the local density of the bacterial population. Quorum sensing can occur within a single bacterial species as well as between diverse species, and can regulate a host of different processes, essentially serving as a simple communication network.

[6] Bacillus regulates DNA uptake (competence) and sporulation using a small peptide called the Competence and $\underline{\text { Sporulation }}$ Factor (CSF). The CSF peptide is chromosomally encoded and secreted as a long precursor, which is cleaved outside the cell into the active peptide sequence.

[7] J. Engebrecht, K. Nealson, M. Silverman, Cell 1983, 32, 773-781.

[8] M. Perego, J. A. Hoch, Proc. Natl. Acad. Sci. USA 1996, 93, 1549-1553.

[9] H. B. Kaplan, L. Plamam, FEMS Microbiol. Lett. 1996, 139, 89-95.

[10] A. S. Khokhlow in Frontiers of bioorganic chemistry and molecular biology (Ed.: S. N. Ananchenko), Pergamon Press, Oxford 1980, pp. 201-210.

[11] K. Shigeru, K. Hiroshi, N. Takuya, Y. Yasuhiro, J. Bacteriol. 1999, 181, 5081-5084.

[12] A. L. Demain, Internatl. Microbiol. 1998, 1, 259-264.

[13] N. Andres, H. Wolf, H. Zähner, Z. Naturforsch. 1990, 45, 851-855.

[14] N. Andres, H. Wolf, H. Zähner, E. Rössner, A. Zeeck, W. A. König, V. Sinnwell, Helv. Chim. Acta 1989, 426-437.

[15] There are few groups of bacteria of which the typical representatives are easier to recognize and the aberrant types more numerous and more difficult to separate than those which originally constituted the genus Corynebacterium, and which may be cautiously call the group of coryneform bacteria. H. L. Jensen, Annu. Rev. Microbiol., 1952, 6, 77-90.

[16] E. Rössner, Dissertation, Universität Göttingen, 1989. 
[17] E. Rössner, A. Zeeck, W. A. König, Angew. Chem. 1990, 102, 84-85; Angew. Chem. Int. Ed. Engl. 1990, 29, 64-65.

[18] J. Zindel, A. de Meijere, J. Org. Chem. 1995, 60, 2968-2973.

[19] J. Zindel, A. Zeeck, W. A. König, A. de Meijere, Tetrahedron Lett. 1993, 34, 1917-1920.

[20] K. Loscha, Diplomarbeit, Universität Göttingen, 2001.

[21] P. Alvermann, Dissertation, Universität Göttingen, 2001.

[22] B. D. Zlatopolskiy, K. Loscha, P. Alvermann, S. I. Kozhushkov, S. V. Nikolaev, A. Zeeck, A. de Meijere, Chem. Eur. J. 2004, 10, 4708-4717.

[23] J. Zindel, Dissertation, Universität Göttingen, 1993.

[24] M. Brandl, S. I. Kozhushkov, K. Loscha, O. V. Kokoreva, D. S. Yufit, J. A. K. Howard, A. de Meijere, Synlett 2000, 12, 1741-1744.

[25] O. V. Larionov, T. F. Savel'eva, K. A. Kochetkov， N S. Ikonnokov， S. I. Kozhushkov, D. S. Yufit, J. A. K. Howard, V. N. Khrustalev, Yu. N. Belokon, A. De Meijere Eur. J. Org. Chem. 2003, 869-877.

[26] K. Osugi, Yakugaki Zasshi 1958, 78, 1338-1342; C. A. 53, 8110e.

[27] E. Melotto, Dissertation, Universität Göttingen, 1999.

[28] B. D. Zlatopolskiy, H.-P. Kroll, E. Melotto, A. de Meijere, Eur. J. Org. Chem. 2004, 44924502 .

[29] X.-1. Qiu and F.-L. Qing, J. Org. Chem. 2002, 67, 7162-7164.

[30] Yu. Aoyagi, R. M. Williams, Tetrahedron 1998, 54, 13045-13058.

[31] M. Ritzau, Dissertation, Universität Göttingen, 1992.

[32] M. Brandl, Dissertation, Universität Göttingen, 2000.

[33] K. Wagner-Gillen, Diplomarbeit, Universität Göttingen, 1998.

[34] K. Ohba, H. Nakayama, K. Furihata, A. Shimazu, T. Endo, U. Seto, N. Otake, J. Antibiot. 1987, 40, 709-713.

[35] S. Hanessian, M. Bayrakdarian, X. Luo, J. Am. Chem. Soc. 2002, 124, 4716-4721.

[36] A. Pojitkov, E. Efremenko, S. Varfolomeyev, J. Mol. Catal. B: Enzym. 2000, 10, 47. 
[37] R. Larsson, S. Dhar, H. Ehrsson, P. Nygren, R. Lewensohn, Br. J. Cancer 1998, 78, 328335.

[38] S. D. Varfolomeyev, T. K. Aliev, E. N. Efremenko, Pure Appl. Chem. 2004, 76, 1781.

[39] Y. Tang, G. Ghirlanda, N. Vaidehi, J. Kua, D. T. Mainz, W. A. Goddard III, W. F. De Grado, D. A. Tirrell, Biochemistry 2001, 40 2790-2796.

[40] N. C. Yoder, K. Kumar, Chem. Soc. Rev. 2002, 31 335-341.

[41] K. Fukuda, M. Watanabe, K. Asano, K. Ouchi, S. Takasawa, Curr. Genetics 1991, 20, 449

[42] P. Gueguen, M. Padron, B. Perbal, G. Herve, Biochim. Biophys. Acta 1980, 615, 59.

[43] J. Parsons, G. Xiao, G. Gilliland, R. Armstrong, Biochemistry 1998, 37, 6286.

[44] J. T. Welch, S. Eswarakrishan, Fluorine in bioorganic chemistry 1991 Wiley, New York

[45] R. Filler, Y. Kobayashi, L. M. Yagupolskii, Organofluorine compounds in medicinal chemistry and biomedicinal applications 1993, Elsevier, Amsterdam

[46] M. Dominguez, K. Thornton, M. Melendez, C. Dupureur, Proteins 2001, 45, 55

[47] G. Xiao, J. F. Parsons, K. Tesh, R. N. Armstrong, G. L. Gilliland, J. Mol. Biol. 1998, 281323

[48] J. A. K. Howard, V. J. Hoy, D. O’Hagan, G. T. Smith, Tetrahedron 1996, 52 12613-12622

[49] B. Zlatopolski Dissertation, Universität Göttingen, 2003

[50] G. A. Olah, J. T. Welch, Y. D. Vankar, M. Nojima, I. Kerekes, J. A. Olah, J. Org. Chem. 1979, 44, 3872-3881

[51] T. Furuya, T. Fukuhara, S. Hara, J. Fluorine Chem. 2005, 126, 721-725.

[52] A. M. Sipyagin, V. S. Enshov, S. A. Kashtanov, V. A. Potemkin, J. S. Thrasher, A. Waterfeld, Russian Chemical Bulletin, Int. Ed. 2004, 53, 420-434.

[53] F. Mathey, J. Bensoam, Tetrahedron, 1975, 31, 391-401

[54] W. C. Smith, C. W. Tullock, E. L. Muetterties, W. R. Hasek, F. S. Fawsett, V. A. Engelhardt, D. D. Coffman, J. Am. Chem. Soc. 1959, 81, 3165-3166.

[55] W. R. Hasek, W. C. Smith, V. A. Engelhardt, J. Am. Chem. Soc. 1960, 82, 543-551.

[56] C. W. Tullock, F. C. Fawcett, W. C. Smith, D. D. Coffman, J. Am. Chem. Soc. 1960, 82, $539-542$. 
[57] G. C. Demitras, R. A. Kent, A. G. MacDiarmid, Chemistry \& Industry (London, UK), 1964, 41, 1712-1714.

[58] L. N. Markovskii, V. E. Pashinnik, A. V. Kirsanov, Synthesis 1973, 12, 787-789.

[59] R. P. Singh, J. M. Shreeve, Synthesis 2002, 17, 2561-2578.

[60] I am grateful to Dr. Sergei Kozhushkov, Göttingen, for a gift of monomethyl cyclopropane-trans-1,2-dicarboxylate and to Dr. Heiko Schill, Brisbane, Australia, for a gift of dimethyl cyclopropane-trans-1,2-dicarboxylate.

[61] D. Kaufmann, A. de Meijere, B. Hingerty, W. Saenger, Angew. Chem. 1975, 87, 842-843; Angew. Chem. Int. Ed. Engl. 1975, 14, 816-817.

[62] Y. Hara, M. Matsuda, J. Org. Chem. 1975, 40, 2786-2791.

[63] P. Brown, J. Burdon, T. J. Smith, J. C. Tatlow Tetrahedron, 1960, 10, 164-170.

[64] M. Ratier, M. Pereyre, A. G. Davies, R. Sutcliffe J. Chem. Soc., Perkin Trans. 2 1984, $1907-1915$.

[65] J. T. Koh, L. Delaude, R. Breslow J. Am. Chem. Soc. 1994, 116, 11234-11240.

[66] T. Kimura, V. P. Vassilev, Gwo-Jenn Shen, Chi-Huey Wong, J. Am. Chem. Soc. 1997, 119, 11734-11742.

[67] B. T. Lotz, C. M. Gasparski, K. Peterson, M. J. Miller, J. Chem. Soc., Chem. Commun. 1990, 1107-1109.

[68] J. Steinreiber, K. Fesko, C. Mayer, C. Reisinger, M. Schürmann, H. Griengl, Tetrahedron 2007, 63, 8088-8093.

[69] M. E. Jung, Y. H. Jung, Tetrahedron Lett. 1989, 30, 6637-6640.

[70] Y. Ito, M. Sawamura, M. Kobayashi, T. Hayashi, Tetrahedron Lett. 1988, 29, 6321-6324.

[71] P. G. Andersson, D. Guijarro, D. Tanner, J. Org. Chem. 1997, 62, 7364-7375.

[72] L. Addadi, E. Gati, M. Lahav, J. Am. Chem. Soc. 1981, 103, 1251-1252.

[73] E. Gil-Av, A. Tishbee, P. E. Hare, J. Am. Chem. Soc. 1980, 102, 5115-5117.

[74] H. Kiniwa, Y. Baba, T. Ishida, H. Katoh, J. Chromatography 1989, 461, 397-405.

[75] T. Shiraiwa, H. Miyazaki, H. Kurokawa, Chirality 1994, 6, 654-657.

[76] M. Sato, K.Okawa, S. Akabori, Bull. Chem. Soc. Jpn. 1957, 30, 937-938. 
[77] T. Wieland, Angew. Chem. 1960, 72, 892-895.

[78] Y. N. Belokon, V. I. Bakhmutov, N. I. Chernoglazova, K. A. Kochetkov， S. V. Vitt, N. S. Garbalinskaya, V. M. Belikov, J. Chem. Soc., Perkin Trans. 1 1988, 305-312.

[79] Y. N. Belokon', A. G. Bulychev, V. A. Pavlov, E. B. Fedorova, V. A. Tsyryapkin, V. A. Bakhmutov, V. M. Belikov, J. Chem. Soc., Perkin Trans. 1 1988, 2075-2083.

[80] Y. N. Belokon'， 1. E. Zel'tzer， M. G. Ryzhov， M. B. Saporovskaya, V. I. Bakhmutov, V. M. Belikov, J. Chem. Soc., Chem. Commun. 1982, 181-182.

[81] Y. N.Belokon, V. I. Maleyev, S. V. Vitt, M. G. Ryzhov, Y. D. Kondrashov, S. N. Golubev, Y. P. Vauchskii, A. I. Kazika, M. I. Novikova, P. A. Krasutskii, A. G. Yurchenko, I. L. Dubchak, V. E. Shklover， Y. T. Struchkov， V. I. Bakhmutov， V. M. Belikov, J. Chem. Soc., Dalton Trans. 1985, 17-26.

[82] Y. N. Belokon', A. N. Popkov, N. I. Chernoglazova, M. B. Saporovskaya, V. I. Bakhmutov V. M. Belikov, J. Chem. Soc., Chem. Commun. 1988, 1336-1338.

[83] Y. N. Belokon', A. G. Bulychev, S. V. Vitt, Y. T. Struchkov, A. S. Batsanov, T. V. Timofeeva, V. A. Tsyryapkin, M. C. Ryzhov， L. A. Lysova， V. I. Bakhmutov, V. M. Belikov, J. Am. Chem. Soc. 1985, 107, 4252-4259.

[84] V. A. Soloshonok, D. V. Avilov, V. P.Kukhar, V. I. Tararov, T. F. Saver'eva, T. D. Churkina, N. S. Ikonnikov, K. A. Kochetkov, S. A. Orlova, A. P. Pysarevsky, Y. T. Struchkov, N. I. Raevsky, Y. N. Belokon', Tetrahedron: Asymmetry 1995, 6, 17411756 .

[85] Y. N. Belokon', A. S. Sagyan, S. A. Djamgaryan, V. 1. Bakhmutov, S. V. Vitt, A. S. Batsanov, Y. T. Struchkov, V. M. Belikov, J. Chem. Soc. Perkin Trans. 1 1990, 2301-2310.

[86] P. Balaram, Curr. Opin. Struct. Biol. 1992, 2, 845-851.

[87] V. J. Hruby, F. A. Al-Obeidi, W. Kazmierski, Biochem. J. 1990, 268, 249-262.

[88] V. J. Hruby, Acc. Chem. Res. 2001, 34, 389-397.

[89] G. G. Bonner, P. Davis, D. Stropova, E. Sidney, H. I. Yamamura, F. Porreca, V. J. Hruby, J. Med. Chem. 2000, 43, 569-580.

[90] S. E. Gibson, N. Guillo, M. J. Tozer, Tetrahedron 1999, 55, 585-615. 
[91] Z. Huang, Y.-B. He, K. Raynor, M. Tallent, T. Reisine, M. Goodman, J. Am. Chem. Soc. 1992, 114, 9390-9401.

[92] D. Tourwé, E. Mannekeuse, T. Nguyen Thi Diem, P. Verheyden, H. Jaspers, G. Tóth, A. Péter, I. Kertész, G. Török, N. N. Chung, P. W. Schiller, J. Med. Chem. 1998, 41, 5167-5176.

[93] H. I. Mosberg, J. R. Omnaas, A. Lonize, D. L. Heyl, I. Nordan, C. Mousigian, P. Davis, F. Porreca, J. Med. Chem. 1994, 37, 4384-4391.

[94] Y. Kataoka, Y. Seto, M. Yamamoto, T. Yamada, S. Kuwata, H. Watanabe, Bull. Chem. Soc. Jpn. 1976, 49, 1081-1084.

[95] M. Alías, M. P. López, C. Cativiela, Tetrahedron 2004, 60, 885-891.

[96] M. Pastó, A. Moyano, M. A. Pericàs, A. Riera, J. Org. Chem. 1997, 62, 8425-8431.

[97] F. A. Davis, C. H. Liang, H. Liu, J. Org. Chem. 1997, 62, 3796-3797.

[98] F. Effenberger, T. Weber, Angew. Chem. Int. Ed. Engl. 1987, 26, 142-143.

[99] R. Dharanipragada, E. Nicolas, G. Toth, V. J. Hruby, Tetrahedron Lett. 1989, 30, 68416844.

[100] G. Li, M. A. Jarosinski, V. J. Hruby, Tetrahedron Lett. 1993, 34, 2561-2564.

[101] W. Oppolzer, O. Tamura, J. Deerberg, Helv. Chim. Acta 1992, 75, 1965-1978.

[102] G. Shapiro, D. Buecheler, M. Marzi, K. Schmidt, B. Gomez-Lor, J. Org. Chem. 1995, 60, 4978-4979.

[103] M. J. Burk, M. F. Gross, J. P. Martinez, J. Am. Chem. Soc. 1995, 117, 9375-9376.

[104] M. J. Burk, K. M. Bedingfield, W. F. Kiesman, J. G. Allen, Tetrahedron Lett. 1999, 40, 3093-3096.

[105] O. Baron, P. Knochel, Angew. Chem. 2005, 117, 3193-3195, Angew. Chem. Int. Ed. 2005, 44, 3133-3135.

[106] P. Knochel, M. C. P. Yeh, S. C. Berk, J. Talbert, J. Org. Chem. 1988, 53, 2390.

[107] R. Dharanipragada, K. VanHulle, A. Bannister, S. Bear, L. Kennedy, V. J. Hruby, Tetrahedron, 1992, 48, 4733-4748.

[108] E. Nicolas, K. C. Russell, V. J. Hruby, J. Org. Chem. 1993, 58, 766-770.

[109] B. D. Zlatopolskiy, A. de Meijere, Chem. Eur. J. 2004, 10, 4718-4727. 
[110] U. M. Reinscheid, B. D. Zlatopolskiy, C. Griesinger, A. Zeeck, A. de Meijere, Chem. Eur. J. 2005, 11, 2929-2945.

[111] All in vitro and in vivo tests were performed by the group of Dr. M. Kaiser (Swiss Tropical Institute, Basel).

[112] For a review on peptide coupling agents see: F. Albericio, R. Chinchilla, D. J. Dodsworth, C. Najera, Org. Prep. Proc. Int. 2001, 33, 203-303 or annual issues of Amino Acids, Peptides And Proteins, The Royal Society of Chemistry; vol. 36 (issued 2007) covers literature published during 2003.

[113] L. A. Carpino, A. El-Faham, Tetrahedron 1999, 55, 6813-6830.

[114] W. R. Kobertz, J. M. Essigmann, J. Am. Chem. Soc. 1996, 118, 7101-7107.

[115] W.-R. Li, W. R. Ewing, B. D. Harris, M. M. Joulie, J. Am. Chem. Soc. 1990, 112, 76597672.

[116] S. F. Brady, S. L. Varga, R. M. Freidinger, D. A. Schwenk, M. Mendlowski, F. W. Holly, D. F. Veber, J. Org. Chem. 1979, 44, 3101-3105.

[117] V. V. Sokolov, S. I. Kozhushkov, S. Nikolskaya, V. N. Belov, M. Es-Sayed, A. de Meijere, Eur. J. Org. Chem. 1998, 777-783.

[118] A. F. Abdel-Magid, J. H. Cohen, C. A. Maryanoff, R. D. Shah, F. J. Villani, F. Zhang, Tetrahedron Lett. 1998, 39, 3391-3394.

[119] The $\mathbf{I C}_{\mathbf{5 0}}$ is a measure of drug effectiveness. It indicates how much of a particular drug or other substance (inhibitor) is needed to inhibit a given biological process (or component of a process, i.e. an enzyme, cell, cell receptor or microorganism) by half. In other words, it is the half maximal $(50 \%)$ inhibitory concentration (IC) of a substance $\left(50 \% \mathrm{IC}\right.$, or $\left.\mathrm{IC}_{50}\right)$. It is commonly used as a measure of antagonist drug potency in pharmacological research. Sometimes, it is also converted to the $\mathbf{p I C}_{\mathbf{5 0}}$ scale $\left(-\log \mathrm{IC}_{50}\right)$, in which higher values indicate exponentially greater potency. According to the FDA, $\mathrm{IC}_{50}$ represents the concentration of a drug that is required for $50 \%$ inhibition in vitro. It is comparable to an $\mathrm{EC}_{50}$ for agonist drugs. $\mathrm{EC}_{50}$ also represents the plasma concentration required for obtaining 50\% of a maximum effect in vivo (http://en.wikipedia.org/wiki/IC50).

[120] M. Hanack, H. Meyer, Justus Liebigs Ann. Chem. 1968, 720, 81-97. 
[121] V. Bahutski, Dissertation, Universität Göttingen, 2003.

[122] J. E. Baldwin, G. E. C. Chang, Tetrahedron 1982, 36, 6, 625-835.

[123] S. V. D'Andrea， D. Bonner， J. J. Bronson， J. Clark， K. Denbleyker， J. Fung-Tomc, S. E. Hoeft， T. W. Hudyma， J. D. Matiskella， R. F. Miller， P. F. Misco， M. Pucci, R. Sterzycki, Y. Tsai, Y. Ueda, J. A. Wichtowski, J. Singh, T. P. Kissick, J. T. North, A. Pullockaran, M. Humora, B. Boyhan, T. Vu, A. Fritz, J. Heikes, R. Fox, J. D. Godfrey, R. Perrone, M. Kaplan, D. Kronenthal, R. H. Mueller, Tetrahedron 2000, 56, 5687-5698.

[124] X.-L. Qiu, F.-L. Qing, J. Org. Chem. 2002, 67, 7162-7164.

[125] Y. Aoyagi, R. M. Williams, Tetrahedron 1998, 54, 13045-13058.

[126] L. F. Fieser, M. Fieser, Reagents for Organic Synthesis, 1967, 1, 142. 


\section{SUMMARY}

Hormaomycin $\mathbf{1}$ and its all-peptide aza-analogue $\mathbf{5 3}$ were synthesized in quantities of 39 and $34 \mathrm{mg}$ respectively, using the protocol developed by B. Zlatopolskiy, to have enough material for in vitro and in vivo biological tests.

$(R)$-allo-Threonine 67 was prepared on a multigram scale employing a modified Belokon' protocol providing kinetically controlled conditions for an aldol reaction between the glycine complex enolate and acetaldehyde.

A new synthesis of $\beta$-methyl(haloaryl)alanines $\mathbf{4 8 - 5 2}$ was developed on the basis of the Belokon' protocol using commercially available haloacetophenones as starting materials for the preparation of 1-(haloaryl)ethyl iodides to alkylate the (S)-configured Belokon' glycine complex

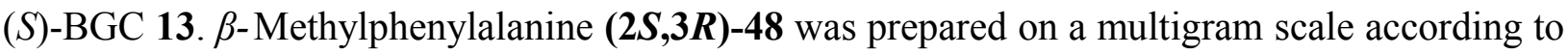
this protocol.

(2R)- and (2S)-3-(1'S, 2'R)-(2'-Fluoromethylcyclopropyl)alanines [mono- $(\boldsymbol{R}-\mathbf{9 6}$ a and $\boldsymbol{S}-\mathbf{9 6}$ a), di- $(\boldsymbol{R}-96 \mathrm{~b}$ and $\boldsymbol{S}-96 \mathrm{~b})$ and trifluoromethyl $(\boldsymbol{R}-96 \mathrm{c}$ and $\boldsymbol{S}-96 \mathrm{c})$ derivatives] were prepared employing the protocol developed by O. Larionov for the preparation of 3-(2-trans-nitrocyclopropyl)alanines. ${ }^{[25]}$

New Hormaomycin analogues 109 a-c, containing $(2 R)$ - and $(2 S)-3-\left(1^{\prime} S, 2^{\prime} R\right)-\left(2^{\prime}-\right.$ fluoromethylcyclopropyl)alanine moieties instead of $(2 R)$ - and $(2 S)-3-\left(1^{\prime} R, 2^{\prime} R\right)-\left(2^{\prime}-\right.$ nitrocyclopropyl)alanine were synthesized, and their in vitro antimalarial activities were tested. The activities turned out to be comparable to the one for native Hormaomycin and in the case of the MeZ-protected cyclic peptidolactone core with $(2 R)-3-\left(1^{\prime} S, 2^{\prime} R\right)$-(2'-trifluoromethylcyclopropyl)alanine $103 \mathbf{c}$ twice better than that of the reference drug Chloroquine against the malaria parasite Plasmodium falciparum strain K1. 


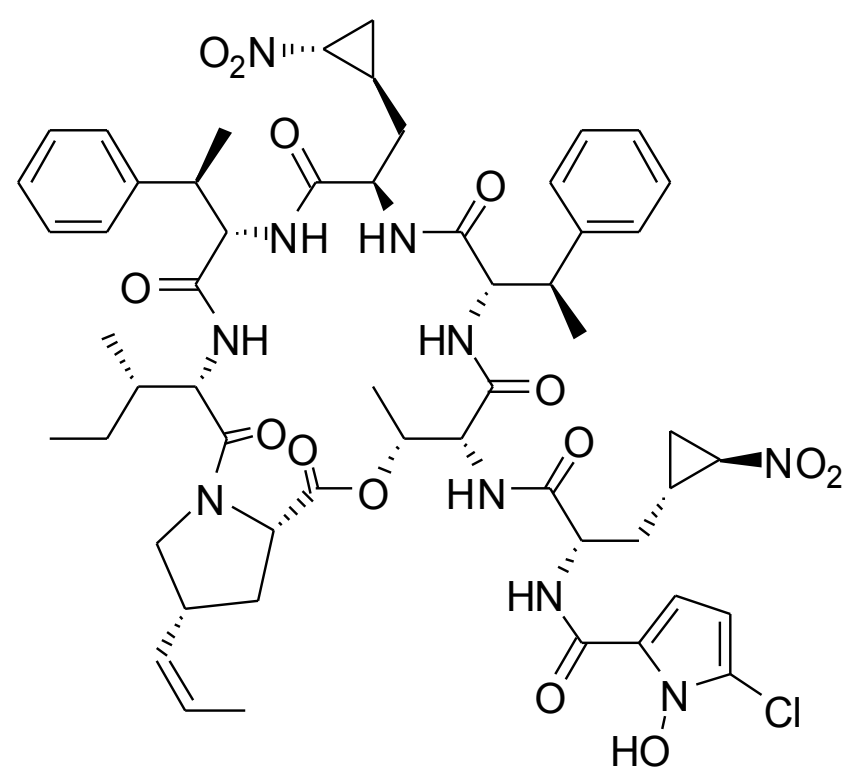

Hormaomycin $\mathbf{1}$

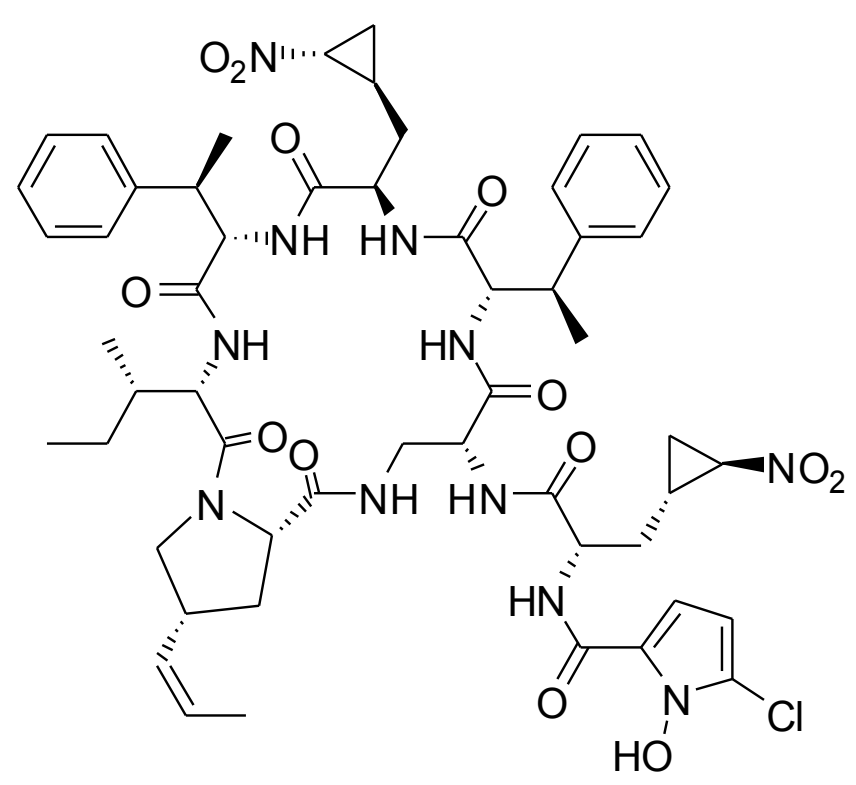

Hormaomycin all-peptide aza-analogue $\mathbf{5 3}$<smiles>CC(O)C(N)C(=O)O</smiles>

(R)-allo-Threonine 67 
$\beta$-Methylphenylalanines

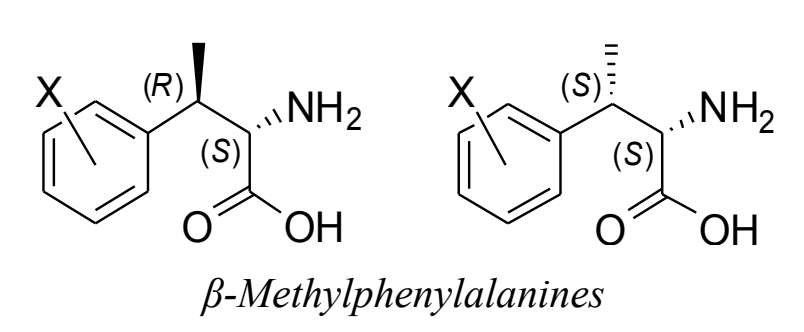

(
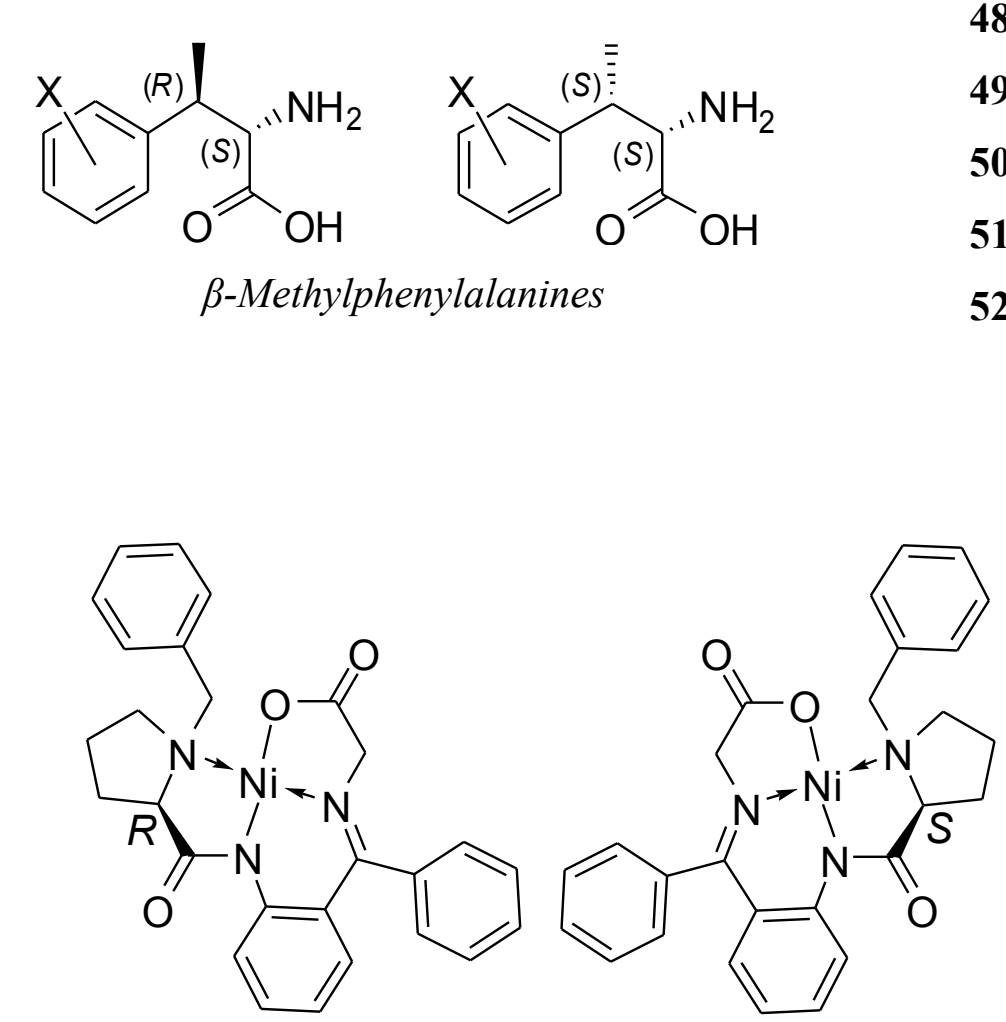

Belokon' glycine complexes $\mathbf{R - 1 3}$ and $\mathbf{S - 1 3}$

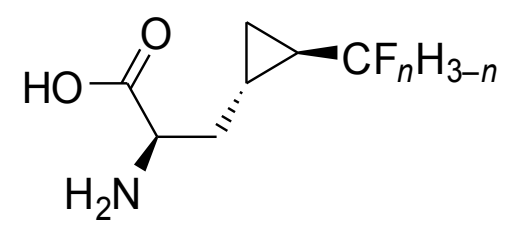

(2R, 1'S, 2'R)-3-(fluoromethylcyclopropyl)alanines

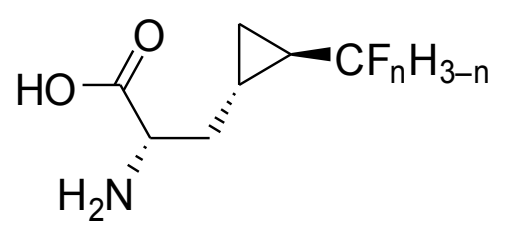

(2S, 1'S, 2'R)-3-(fluoromethylcyclopropyl)alanines
$48 \quad \mathrm{X}=\mathrm{H}$

$49 \mathrm{X}=o-\mathrm{Cl}$

$50 \quad \mathrm{X}=m-\mathrm{Cl}$

$51 \mathrm{X}=p-\mathrm{Cl}$

$52 \quad \mathrm{X}=p-\mathrm{F}$
R-96 a $n=1$

$\boldsymbol{R}-96$ b $n=2$

R-96 с $n=3$
$S-96$ a $(n=1)$

$S-96$ b $(n=2)$

$S-96$ c $(n=3)$ 


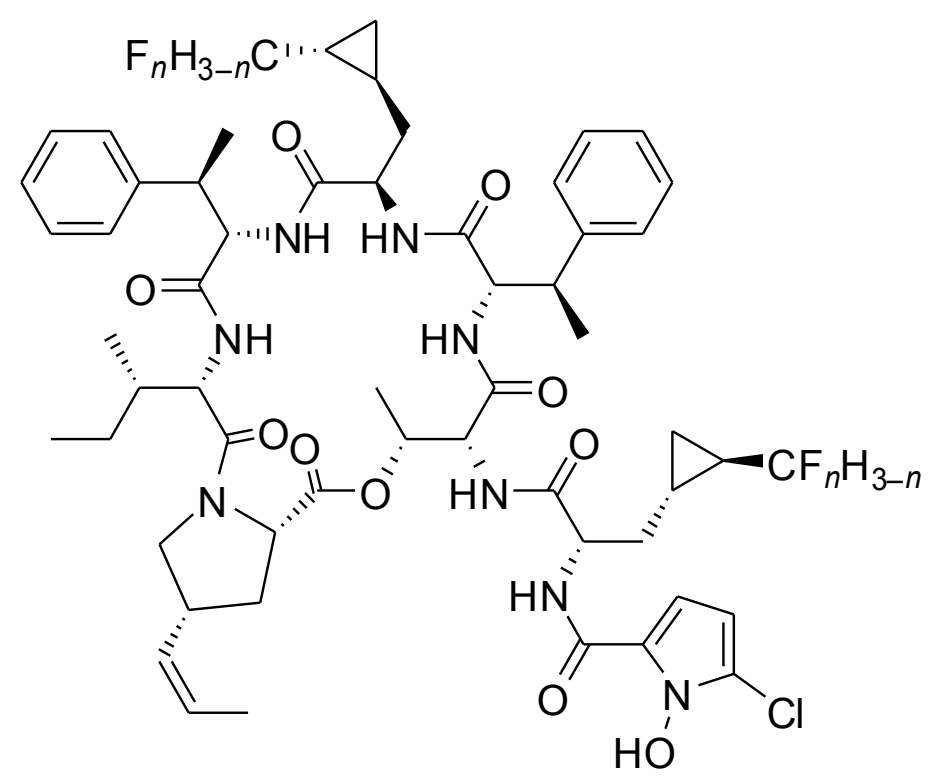

(Fluoromethylcyclopropyl)alanyl

Hormaomycin analogues

$$
\begin{aligned}
& 109 \text { a }(n=1) \\
& 109 \text { b }(n=2) \\
& 109 \text { c }(n=3)
\end{aligned}
$$

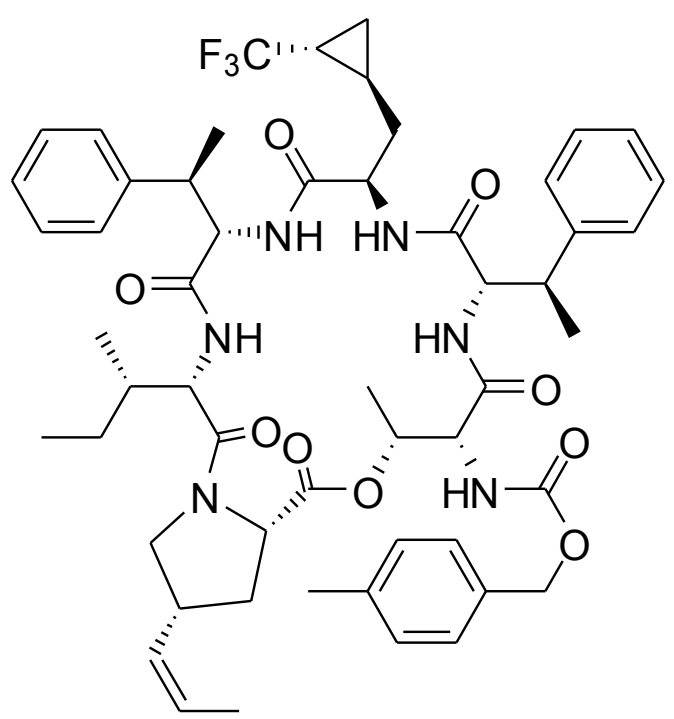

MeZ-protected cyclohexadepsipeptide 
SPECTRAL DATA

1. NMR 
Hormaomycin 1

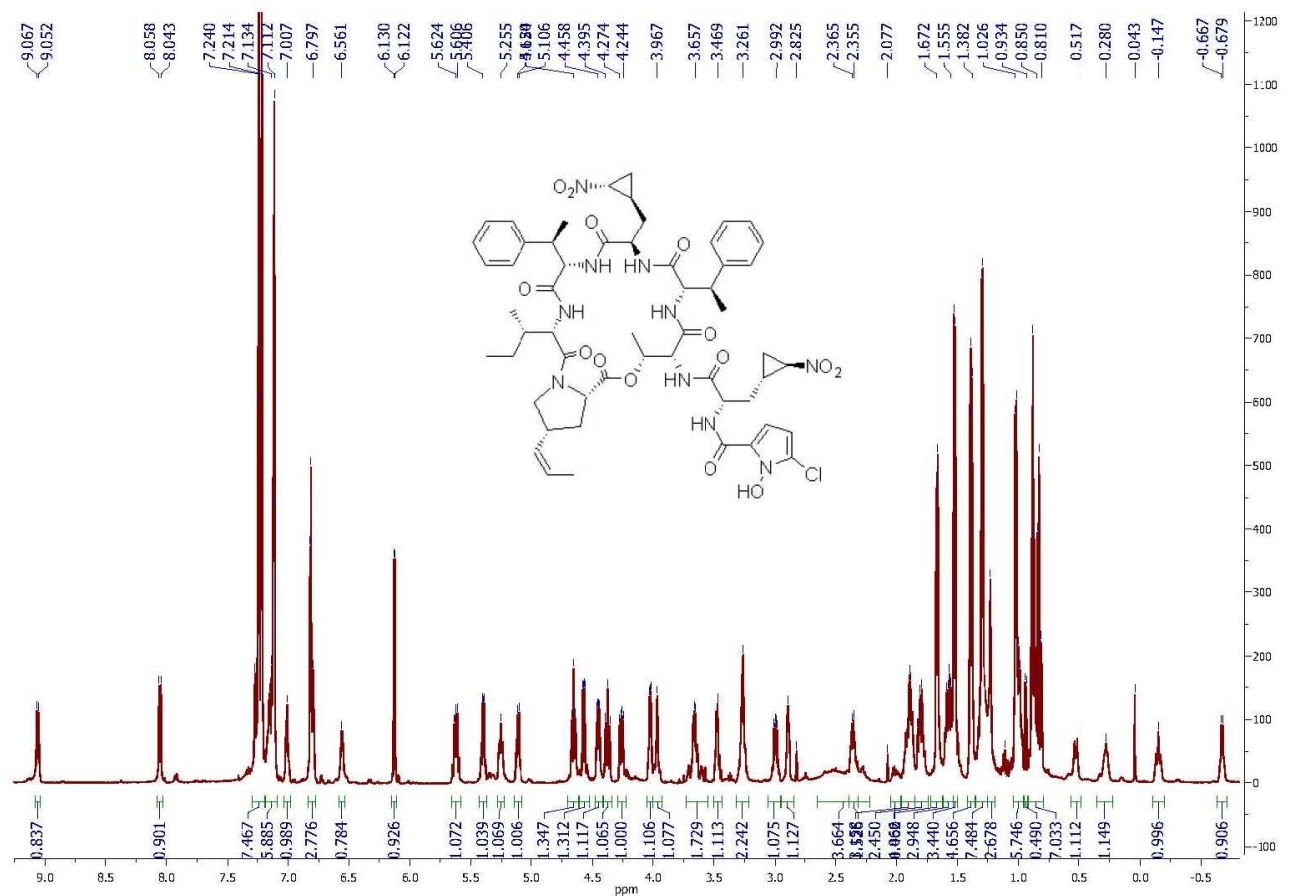

${ }^{1} \mathrm{H}$ NMR $\left(600 \mathrm{MHz}, \mathrm{CDCl}_{3}\right)$

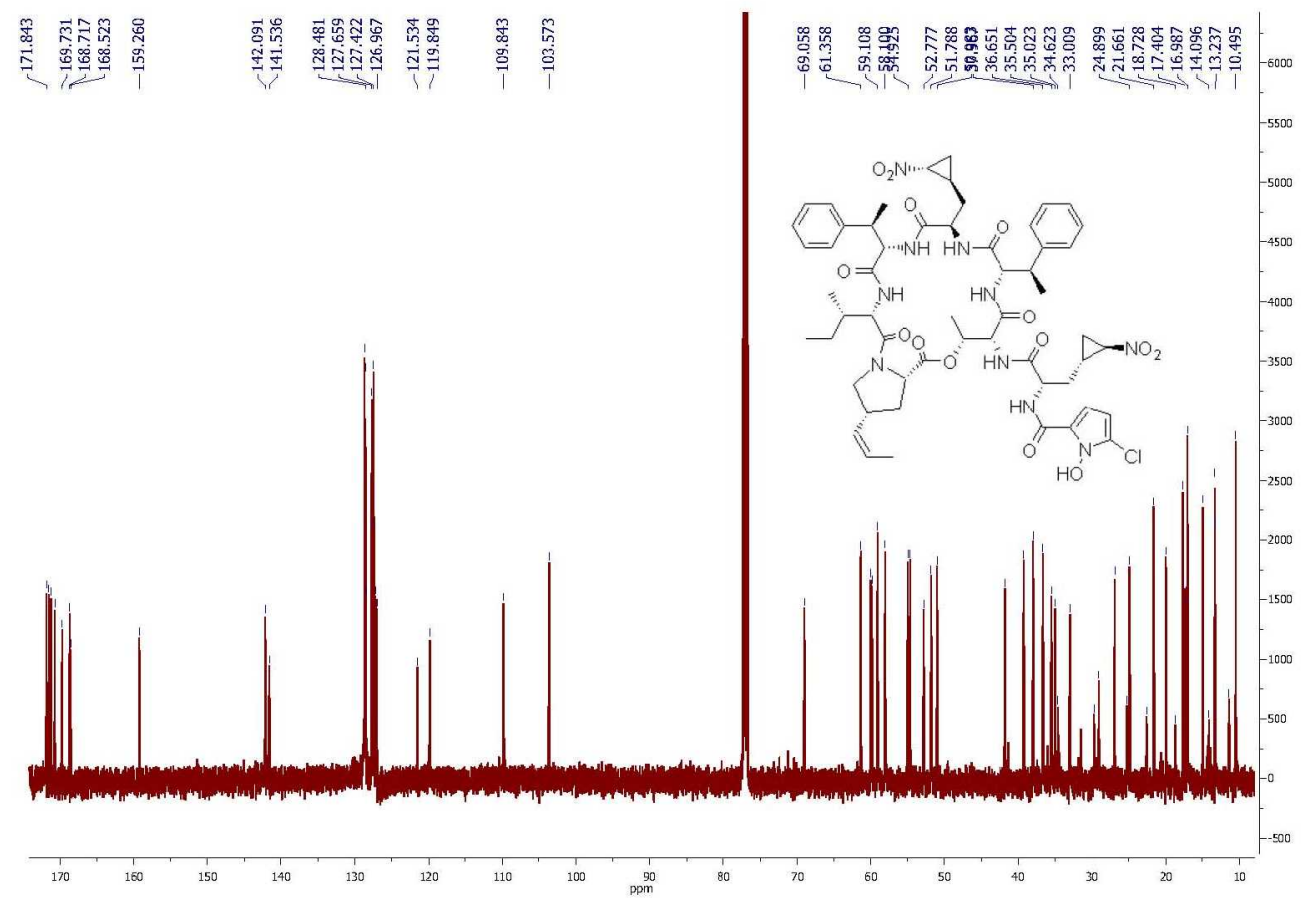

${ }^{13} \mathrm{C}$ NMR (150.8 MHz, $\left.\mathrm{CDCl}_{3}\right)$ 
Hormaomycin all-peptide aza-analogue 53

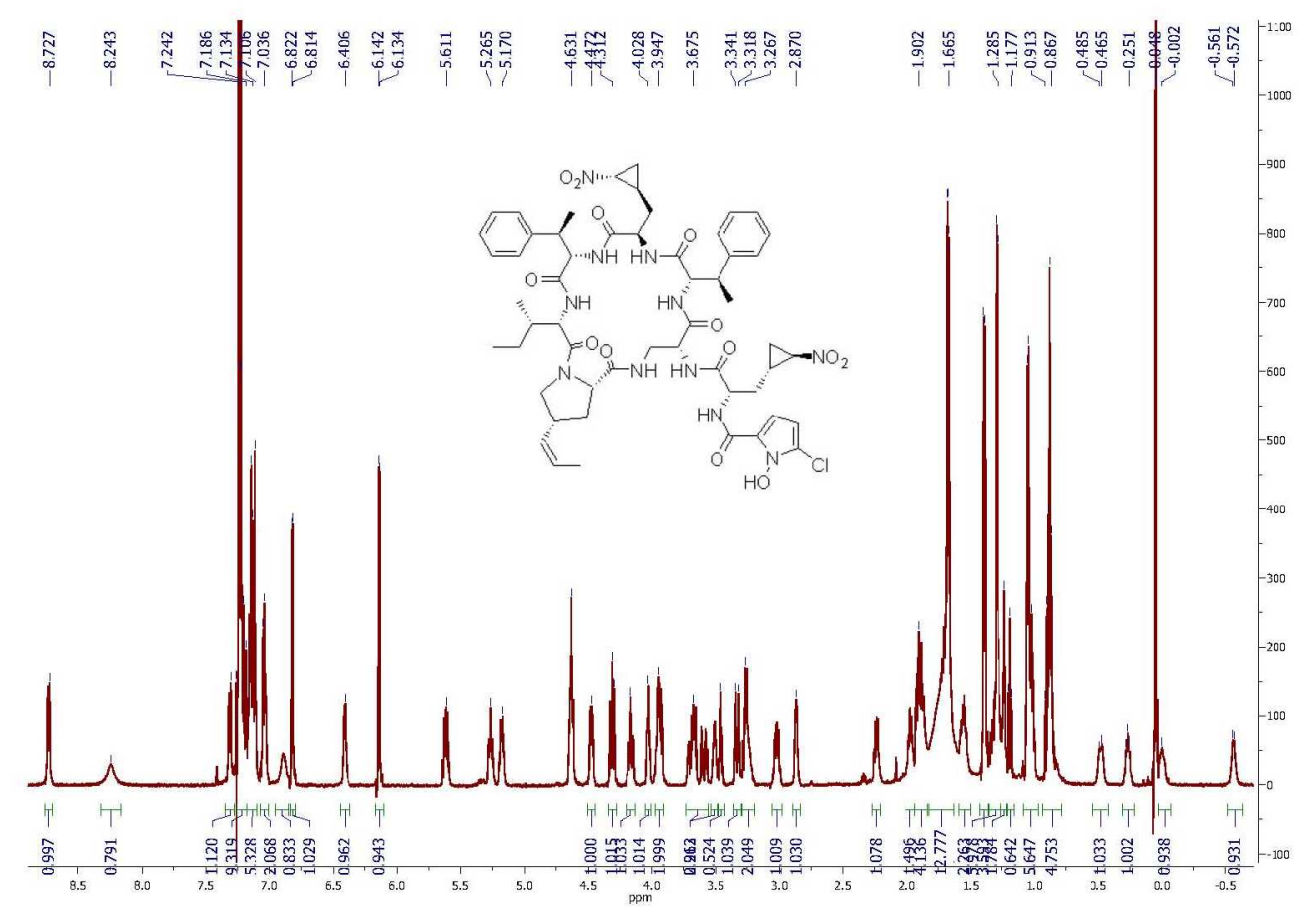

${ }^{1} \mathrm{H}$ NMR $\left(600 \mathrm{MHz}, \mathrm{CDCl}_{3}\right)$

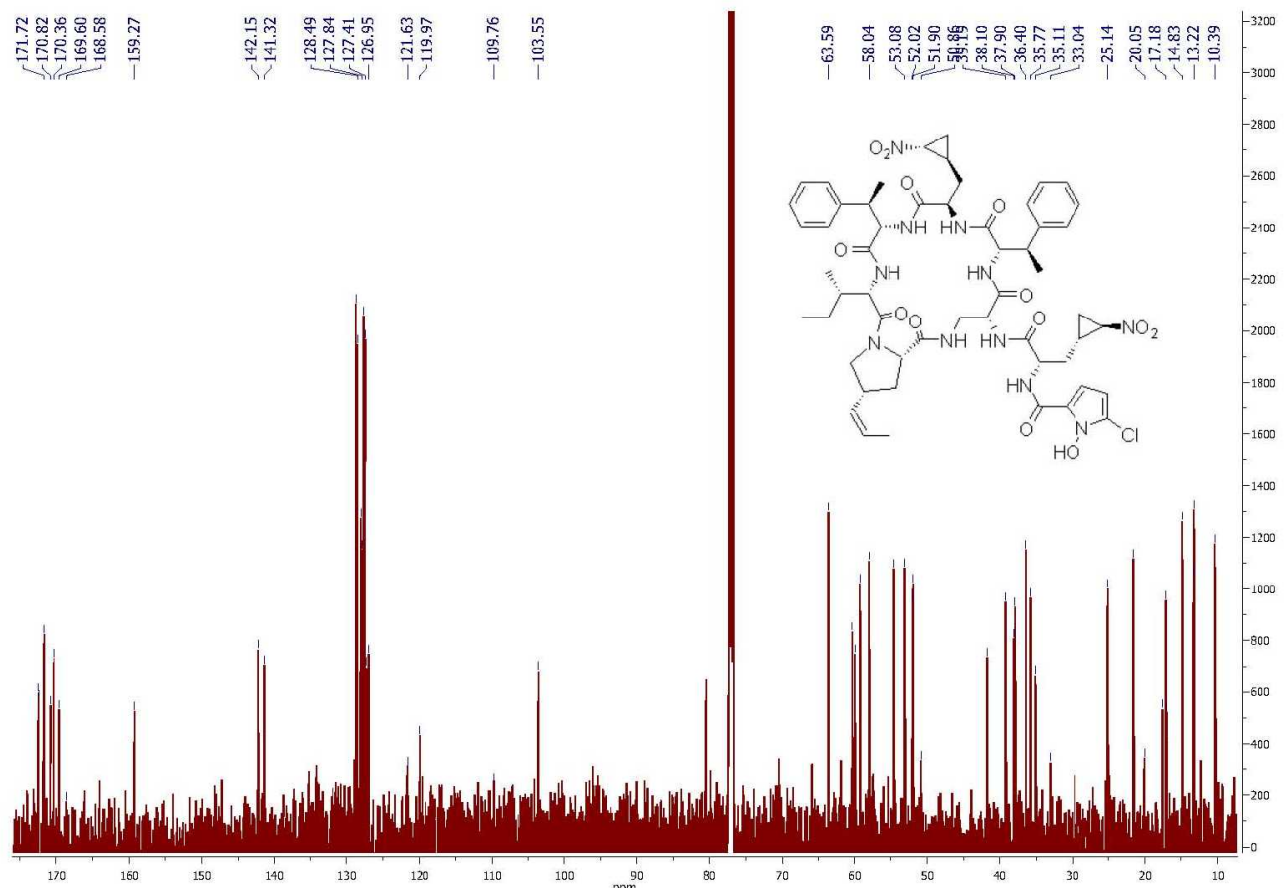

${ }^{13} \mathrm{C}$ NMR (150.8 MHz, $\left.\mathrm{CDCl}_{3}\right)$ 
(2R,1'S,2'R)-3-(2'-trifluoromethylcyclopropyl)alanine

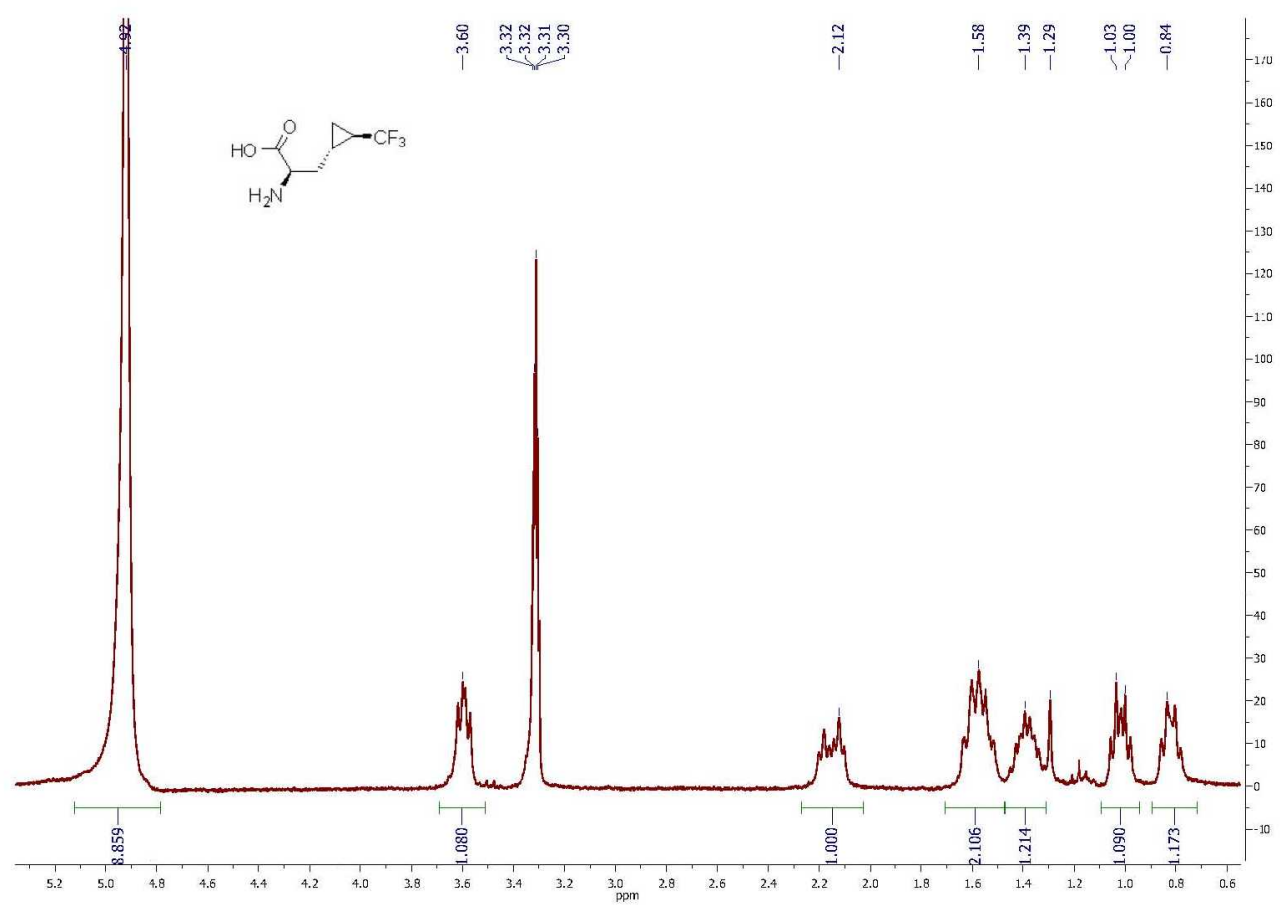

${ }^{1} \mathrm{H}$ NMR $\left(600 \mathrm{MHz}, \mathrm{CD}_{3} \mathrm{OD}\right)$

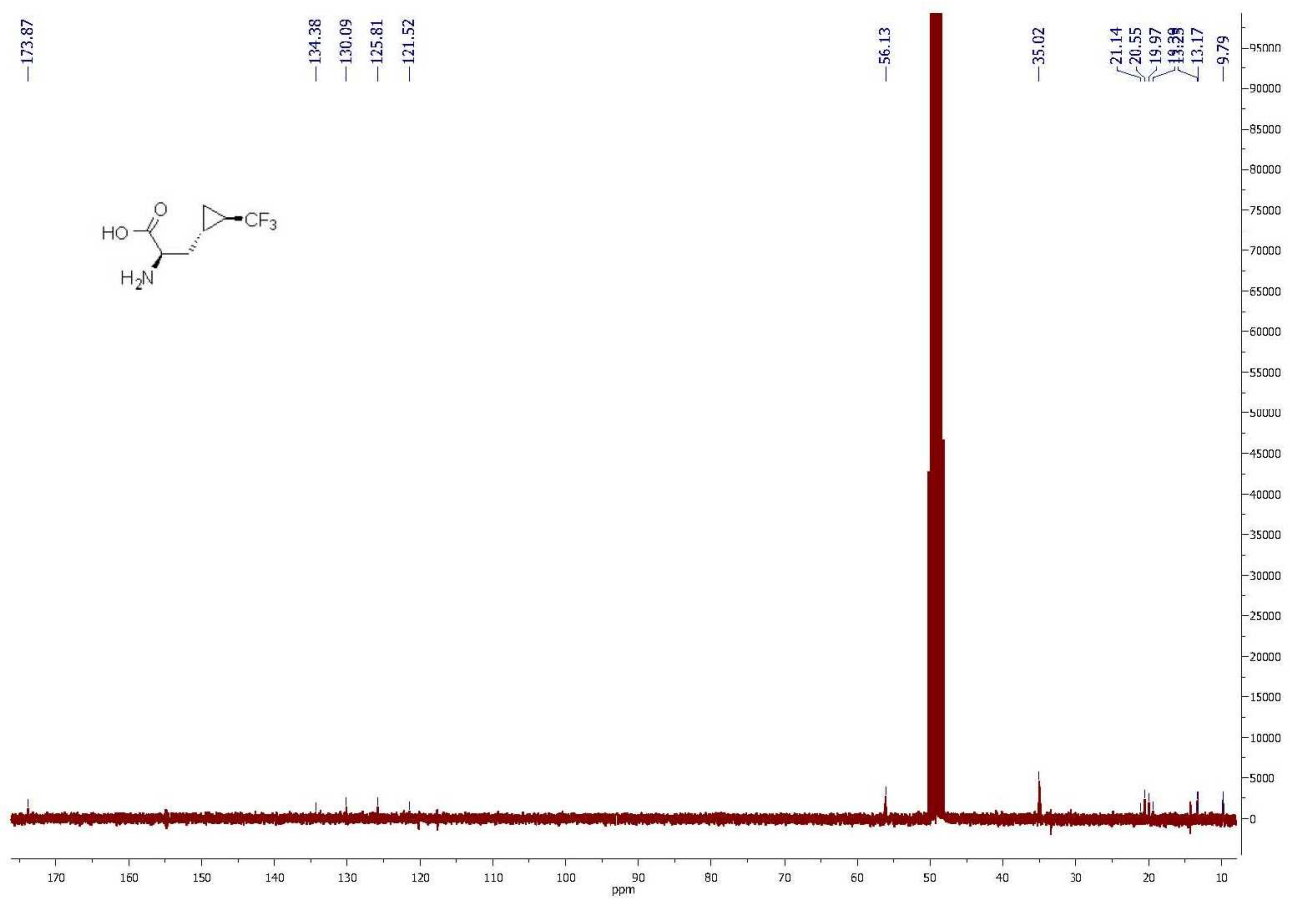

${ }^{13} \mathrm{C}$ NMR (125.7 MHz, $\left.\mathrm{CD}_{3} \mathrm{OD}\right)$ 
(2S,1'S,2'R)-3-(2'-difluoromethylcyclopropyl)alanine

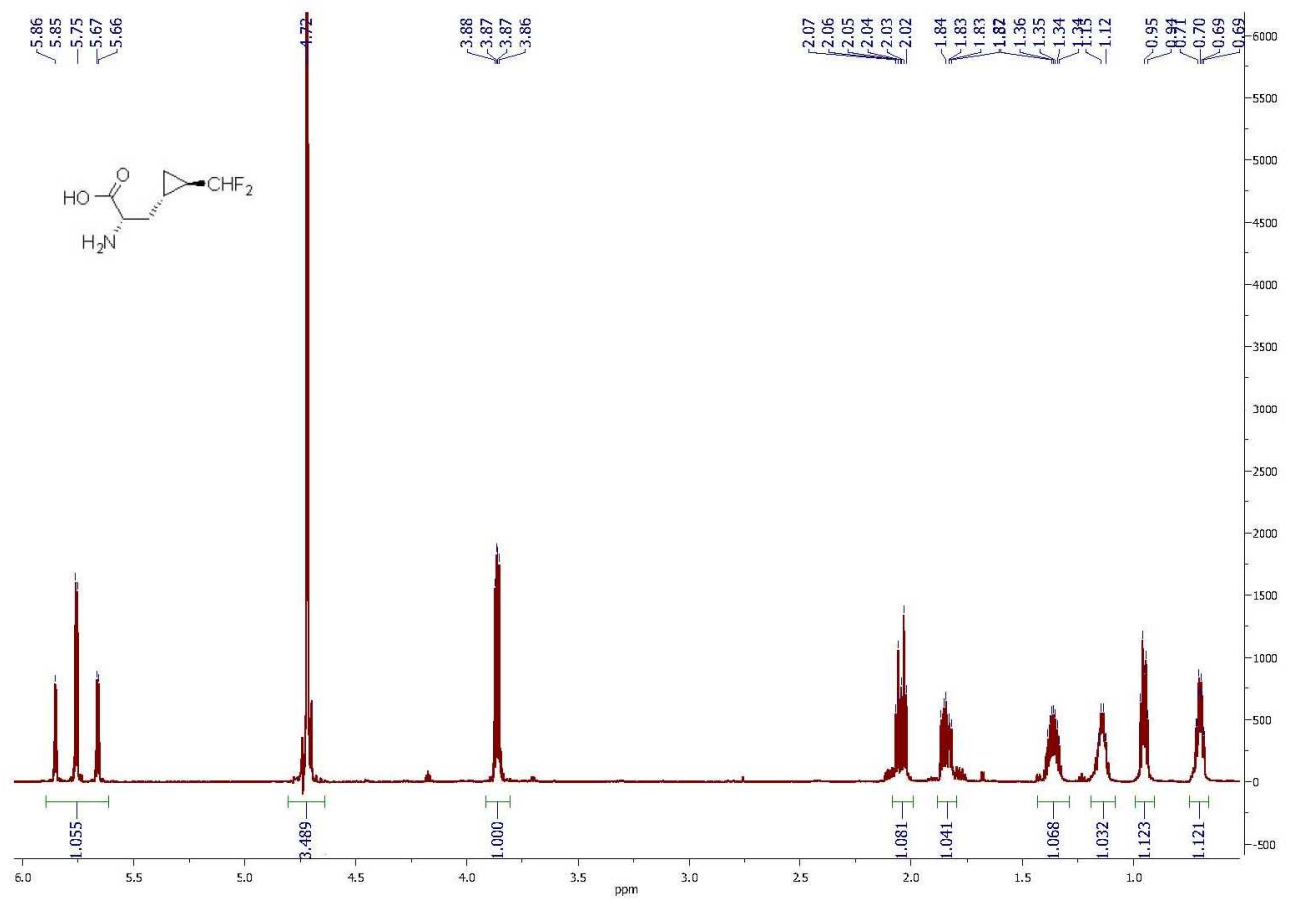

${ }^{1} \mathrm{H}$ NMR $\left(600 \mathrm{MHz}, \mathrm{D}_{2} \mathrm{O}\right)$

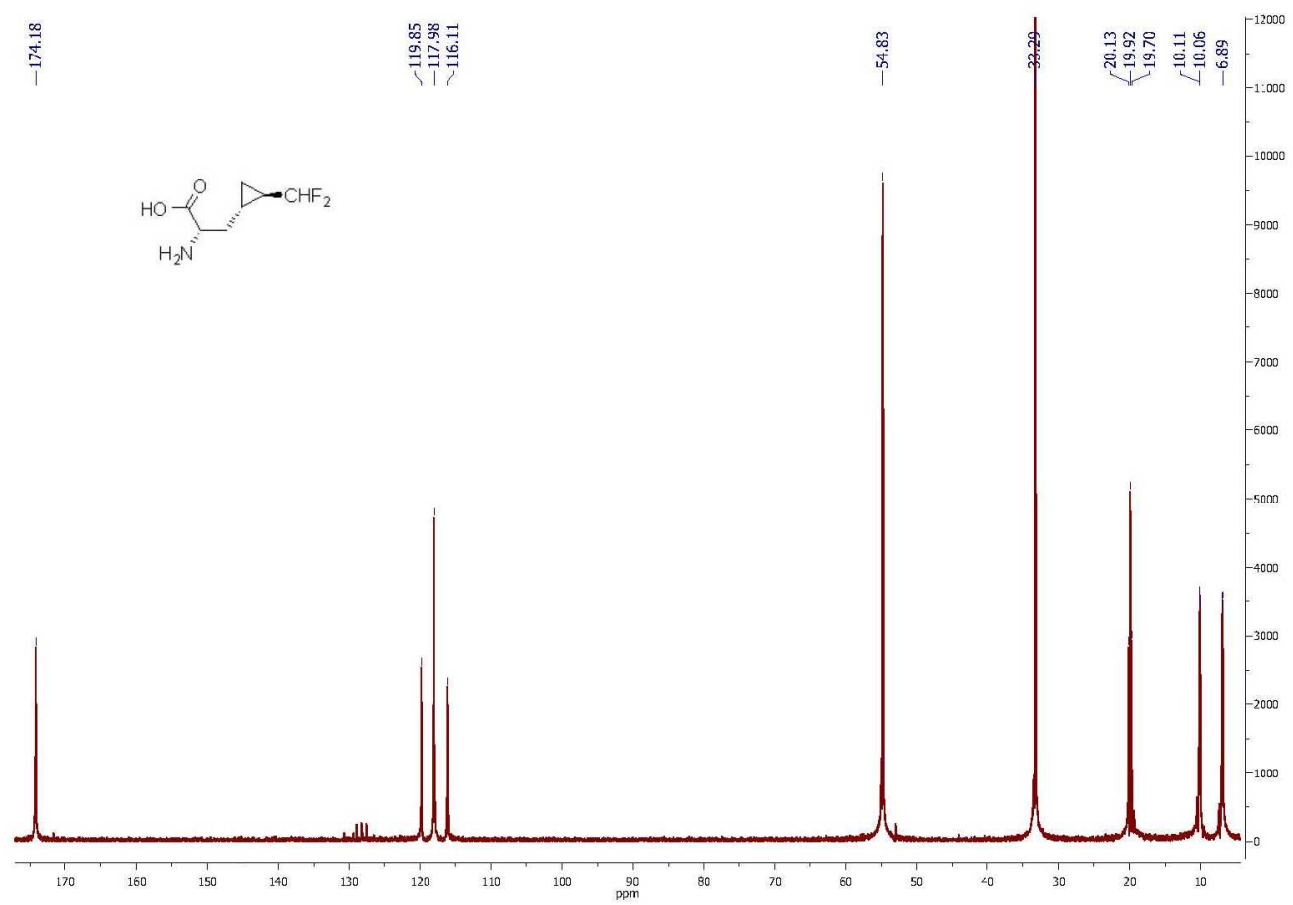

${ }^{13} \mathrm{C}$ NMR (125.7 MHz, $\left.\mathrm{D}_{2} \mathrm{O}\right)$ 
(Trifluoromethylcyclopropyl)alanyl Hormaomycin

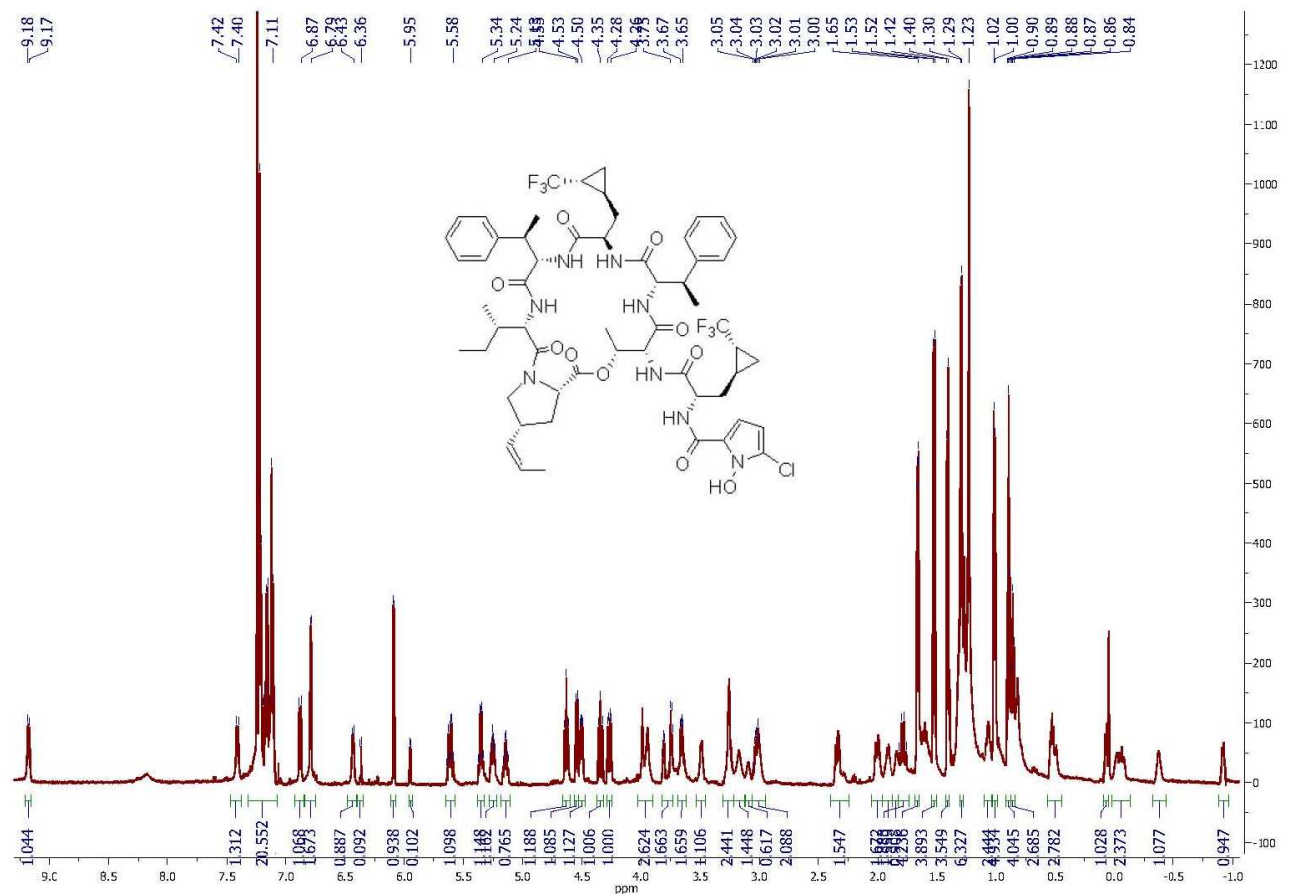

${ }^{1} \mathrm{H}$ NMR $\left(600 \mathrm{MHz}, \mathrm{CDCl}_{3}\right)$

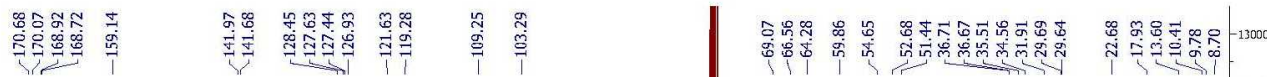
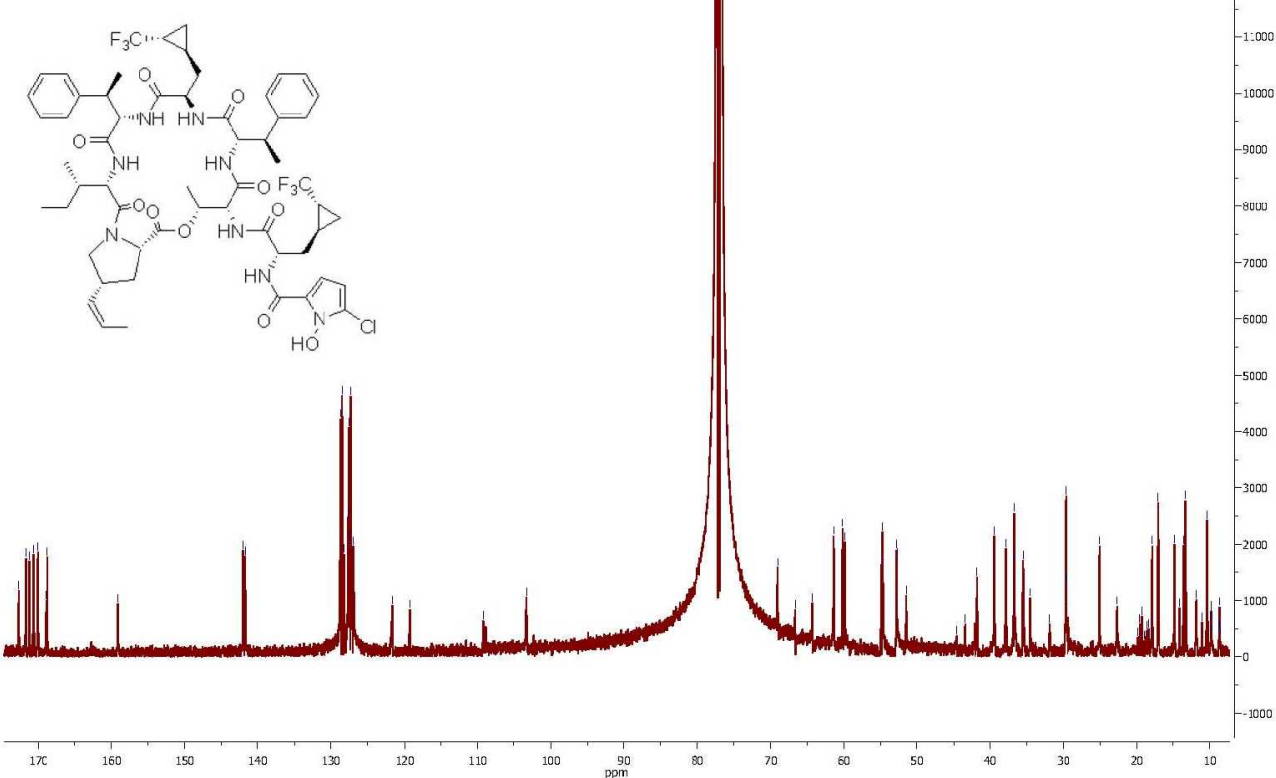

${ }^{13} \mathrm{C}$ NMR $\left(125.7 \mathrm{MHz}, \mathrm{CDCl}_{3}\right)$ 


\section{X-Ray}

Belokon' (2S, 1'S, 2'R)-3-(2'-trifluoromethylcycloprpopyl)alanine complex

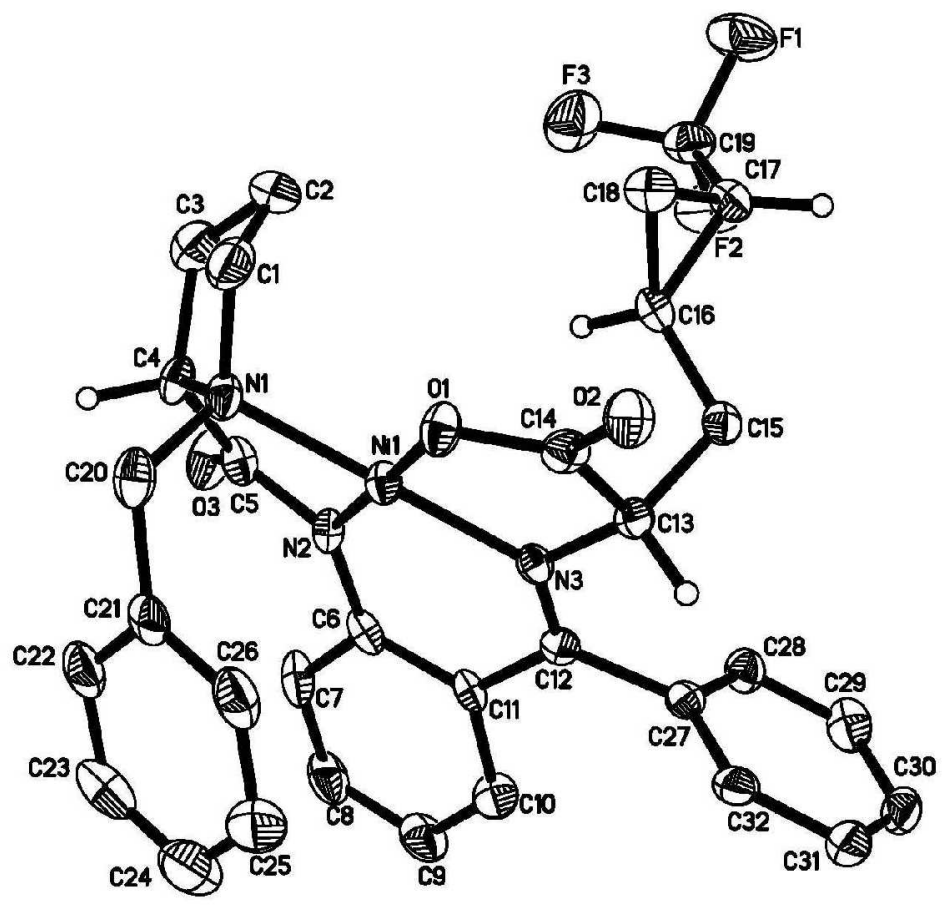


Table 1. Crystal data and structure refinement for $\mathrm{dk} 25$.

Identification code

Empirical formula

Formula weight

Temperature

Wavelength

Crystal system

Space group

Unit cell dimensions

Volume

Z

Density (calculated)

Absorption coefficient

$\mathrm{F}(000)$

Crystal size

Theta range for data collection

Index ranges

Reflections collected

Independent reflections

Completeness to theta $=25.49^{\circ}$

Absorption correction

Max. and min. transmission

Refinement method

Data / restraints / parameters

Goodness-of-fit on $\mathrm{F}^{2}$

Final R indices [I $>2 \operatorname{sigma}(\mathrm{I})]$

$\mathrm{R}$ indices (all data)

Absolute structure parameter

Largest diff. peak and hole $\mathrm{dk} 25$

$\mathrm{C}_{32} \mathrm{H}_{30} \mathrm{~F}_{3} \mathrm{~N}_{3} \mathrm{NiO}_{3}$

620.30

120(2) K

$0.71073 \AA$

Tetragonal

$\mathrm{P} 4_{3} 2_{1} 2$

$\mathrm{a}=9.9745(2) \AA$

$\alpha=90^{\circ}$.

$\mathrm{b}=9.9745(2)$

(2) $\AA$

$\beta=90^{\circ}$.

$\mathrm{c}=57.255$

(2) $\AA$

$\gamma=90^{\circ}$.

5696.4(2) $\AA^{3}$

8

$1.447 \mathrm{Mg} / \mathrm{m}^{3}$

$0.740 \mathrm{~mm}^{-1}$

2576

$0.52 \times 0.14 \times 0.10 \mathrm{~mm}^{3}$

2.49 to $25.49^{\circ}$.

$-11<=\mathrm{h}<=11,-12<=\mathrm{k}<=11,-68<=1<=69$

35391

$5151[\mathrm{R}(\mathrm{int})=0.0644]$

$98.3 \%$

Semi-empirical from equivalents

0.9297 and 0.6996

Full-matrix least-squares on $\mathrm{F}^{2}$

$5151 / 0 / 379$

1.144

$\mathrm{R}_{1}=0.0480, \mathrm{wR}_{2}=0.0945$

$\mathrm{R}_{1}=0.0571, \mathrm{wR}_{2}=0.0968$

$0.03(2)$

0.466 and -0.706 e. $\AA^{-3}$ 
Table 2. Atomic coordinates $\left(\times 10^{4}\right)$ and equivalent isotropic displacement parameters $\left(\AA^{2} \times 10^{3}\right)$ for $\mathrm{dk} 25$. $\mathrm{U}(\mathrm{eq})$ is defined as one third of the trace of the orthogonalized $\mathrm{U}^{\mathrm{ij}}$ tensor.

\begin{tabular}{rrrrr}
\hline Atom & $\mathrm{x}$ & $\mathrm{y}$ & $\mathrm{z}$ & $\mathrm{U}(\mathrm{eq})$ \\
\hline $\mathrm{Ni}(1)$ & $650(1)$ & $5721(1)$ & $9554(1)$ & $18(1)$ \\
$\mathrm{O}(1)$ & $1058(3)$ & $6987(3)$ & $9783(1)$ & $23(1)$ \\
$\mathrm{O}(2)$ & $1591(3)$ & $9151(3)$ & $9834(1)$ & $27(1)$ \\
$\mathrm{O}(3)$ & $-1249(3)$ & $2638(3)$ & $9289(1)$ & $35(1)$ \\
$\mathrm{N}(1)$ & $93(3)$ & $4427(3)$ & $9789(1)$ & $22(1)$ \\
$\mathrm{N}(2)$ & $250(3)$ & $4424(3)$ & $9332(1)$ & $20(1)$ \\
$\mathrm{N}(3)$ & $1218(3)$ & $6989(3)$ & $9339(1)$ & $17(1)$ \\
$\mathrm{C}(1)$ & $-754(5)$ & $4990(4)$ & $9980(1)$ & $35(1)$ \\
$\mathrm{C}(2)$ & $-2129(5)$ & $5080(5)$ & $9871(1)$ & $40(1)$ \\
$\mathrm{C}(3)$ & $-2244(4)$ & $3720(5)$ & $9748(1)$ & $32(1)$ \\
$\mathrm{C}(4)$ & $-794(4)$ & $3408(4)$ & $9670(1)$ & $23(1)$ \\
$\mathrm{C}(5)$ & $-621(4)$ & $3446(4)$ & $9410(1)$ & $25(1)$ \\
$\mathrm{C}(6)$ & $872(4)$ & $4348(4)$ & $9114(1)$ & $22(1)$ \\
$\mathrm{C}(7)$ & $886(5)$ & $3121(4)$ & $8984(1)$ & $29(1)$ \\
$\mathrm{C}(8)$ & $1604(5)$ & $3014(4)$ & $8779(1)$ & $34(1)$ \\
$\mathrm{C}(9)$ & $2324(5)$ & $4076(5)$ & $8691(1)$ & $35(1)$ \\
$\mathrm{C}(10)$ & $2283(4)$ & $5285(4)$ & $8807(1)$ & $26(1)$ \\
$\mathrm{C}(11)$ & $1550(4)$ & $5446(4)$ & $9017(1)$ & $20(1)$ \\
$\mathrm{C}(12)$ & $1543(4)$ & $6797(4)$ & $9124(1)$ & $18(1)$ \\
$\mathrm{C}(13)$ & $1267(4)$ & $8361(4)$ & $9443(1)$ & $21(1)$ \\
$\mathrm{C}(14)$ & $1327(4)$ & $8193(4)$ & $9709(1)$ & $22(1)$ \\
$\mathrm{C}(15)$ & $22(4)$ & $9203(4)$ & $9377(1)$ & $20(1)$ \\
$\mathrm{C}(16)$ & $-1261(4)$ & $8621(4)$ & $9468(1)$ & $21(1)$ \\
$\mathrm{C}(17)$ & $-2486(4)$ & $9515(4)$ & $9476(1)$ & $25(1)$ \\
$\mathrm{C}(18)$ & $-1814(4)$ & $9061(4)$ & $9700(1)$ & $26(1)$ \\
$\mathrm{C}(19)$ & $-3768(4)$ & $8888(5)$ & $9408(1)$ & $30(1)$ \\
$\mathrm{C}(20)$ & $1351(4)$ & $3822(4)$ & $9893(1)$ & $27(1)$ \\
$\mathrm{C}(21)$ & $2408(4)$ & $3540(4)$ & $9709(1)$ & $25(1)$ \\
$\mathrm{C}(22)$ & $2419(4)$ & $2358(4)$ & $9583(1)$ & $33(1)$ \\
$\mathrm{C}(23)$ & $3324(5)$ & $2169(5)$ & $9403(1)$ & $43(1)$ \\
$\mathrm{C}(24)$ & $4265(6)$ & $3142(6)$ & $9352(1)$ & $53(1)$ \\
$\mathrm{C}(25)$ & $4304(5)$ & $4315(6)$ & $9483(1)$ & $49(1)$ \\
$\mathrm{C}(26)$ & $3367(4)$ & $4514(5)$ & $9661(1)$ & $34(1)$ \\
$\mathrm{C}(27)$ & $1940(4)$ & $7937(4)$ & $8962(1)$ & $19(1)$ \\
$\mathrm{C}(28)$ & $1088(4)$ & $8283(4)$ & $8779(1)$ & $25(1)$ \\
$\mathrm{C}(29)$ & $1483(5)$ & $9227(5)$ & $8616(1)$ & $31(1)$ \\
$\mathrm{C}(30)$ & $2731(5)$ & $9841(4)$ & $8638(1)$ & $33(1)$ \\
$\mathrm{C}(31)$ & $3571(4)$ & $9531(4)$ & $8822(1)$ & $31(1)$ \\
$\mathrm{C}(32)$ & $3168(4)$ & $8568(4)$ & $8986(1)$ & $24(1)$ \\
$\mathrm{F}(1)$ & $-4847(3)$ & $9639(3)$ & $9465(1)$ & $47(1)$ \\
$\mathrm{F}(2)$ & $-3841(3)$ & $8699(3)$ & $9176(1)$ & $47(1)$ \\
$\mathrm{F}(3)$ & $-3995(3)$ & $7678(3)$ & $9506(1)$ & $46(1)$ \\
\hline & & & & \\
& & & \\
& & &
\end{tabular}


Table 3. Selected bond lengths $[\AA]$ and angles $\left[{ }^{\circ}\right]$ for $\mathrm{dk} 25$.

$\begin{aligned} \mathrm{Ni}(1)-\mathrm{N}(3) & 1.855(3) \\ \mathrm{Ni}(1)-\mathrm{N}(2) & 1.860(3) \\ \mathrm{Ni}(1)-\mathrm{O}(1) & 1.864(3) \\ \mathrm{Ni}(1)-\mathrm{N}(1) & 1.942(3) \\ \mathrm{O}(1)-\mathrm{C}(14) & 1.303(5) \\ \mathrm{O}(2)-\mathrm{C}(14) & 1.220(5) \\ \mathrm{O}(3)-\mathrm{C}(5) & 1.237(5) \\ \mathrm{N}(1)-\mathrm{C}(1) & 1.495(5) \\ \mathrm{N}(1)-\mathrm{C}(4) & 1.510(5) \\ \mathrm{N}(1)-\mathrm{C}(20) & 1.515(5) \\ \mathrm{N}(2)-\mathrm{C}(5) & 1.382(5) \\ \mathrm{N}(2)-\mathrm{C}(6) & 1.396(4) \\ \mathrm{N}(3)-\mathrm{C}(12) & 1.290(4) \\ \mathrm{N}(3)-\mathrm{C}(13) & 1.494(5) \\ \mathrm{C}(1)-\mathrm{C}(2) & 1.509(7) \\ \mathrm{C}(2)-\mathrm{C}(3) & 1.534(6) \\ \mathrm{C}(3)-\mathrm{C}(4) & 1.545(6)\end{aligned}$

$\begin{aligned} \mathrm{C}(4)-\mathrm{C}(5) & 1.496(5) \\ \mathrm{C}(6)-\mathrm{C}(11) & 1.403(5) \\ \mathrm{C}(6)-\mathrm{C}(7) & 1.431(5) \\ \mathrm{C}(7)-\mathrm{C}(8) & 1.379(6) \\ \mathrm{C}(8)-\mathrm{C}(9) & 1.377(7) \\ \mathrm{C}(9)-\mathrm{C}(10) & 1.378(6) \\ \mathrm{C}(10)-\mathrm{C}(11) & 1.415(5) \\ \mathrm{C}(11)-\mathrm{C}(12) & 1.480(5) \\ \mathrm{C}(12)-\mathrm{C}(27) & 1.517(5) \\ \mathrm{C}(13)-\mathrm{C}(14) & 1.532(5) \\ \mathrm{C}(13)-\mathrm{C}(15) & 1.547(5) \\ \mathrm{C}(15)-\mathrm{C}(16) & 1.499(5) \\ \mathrm{C}(16)-\mathrm{C}(18) & 1.506(5) \\ \mathrm{C}(16)-\mathrm{C}(17) & 1.513(5) \\ \mathrm{C}(17)-\mathrm{C}(19) & 1.477(6) \\ \mathrm{C}(17)-\mathrm{C}(18) & 1.516(5)\end{aligned}$

$\mathrm{C}(19)-\mathrm{F}(2)$
$\mathrm{C}(19)-\mathrm{F}(3)$
$\mathrm{C}(19)-\mathrm{F}(1)$
$\mathrm{C}(20)-\mathrm{C}(21)$
$\mathrm{C}(21)-\mathrm{C}(22)$
$\mathrm{C}(21)-\mathrm{C}(26)$
$\mathrm{C}(22)-\mathrm{C}(23)$
$\mathrm{C}(23)-\mathrm{C}(24)$
$\mathrm{C}(24)-\mathrm{C}(25)$
$\mathrm{C}(25)-\mathrm{C}(26)$
$\mathrm{C}(27)-\mathrm{C}(32)$
$\mathrm{C}(27)-\mathrm{C}(28)$
$\mathrm{C}(28)-\mathrm{C}(29)$
$\mathrm{C}(29)-\mathrm{C}(30)$
$\mathrm{C}(30)-\mathrm{C}(31)$
$\mathrm{C}(31)-\mathrm{C}(32)$

$1.345(4)$

$1.348(5)$

$1.351(5)$

$1.517(6)$

$1.382(6)$

$1.391(6)$

$1.381(6)$

$1.382(8)$

$1.389(8)$

$1.398(7)$

$1.384(6)$

$1.392(5)$

$1.384(6)$

$1.393(6)$

1.381(6)

$1.402(6)$

$\begin{array}{rrr}94.78(13) & \mathrm{N}(3)-\mathrm{C}(12)-\mathrm{C}(27) & 122.4(3) \\ 86.47(12) & \mathrm{C}(11)-\mathrm{C}(12)-\mathrm{C}(27) & 115.4(3) \\ 178.60(12) & \mathrm{N}(3)-\mathrm{C}(13)-\mathrm{C}(14) & 107.4(3) \\ 177.93(14) & \mathrm{N}(3)-\mathrm{C}(13)-\mathrm{C}(15) & 111.8(3) \\ 87.14(13) & \mathrm{C}(14)-\mathrm{C}(13)-\mathrm{C}(15) & 109.7(3) \\ 91.60(12) & \mathrm{O}(2)-\mathrm{C}(14)-\mathrm{O}(1) & 125.3(4) \\ 116.2(2) & \mathrm{O}(2)-\mathrm{C}(14)-\mathrm{C}(13) & 120.2(4) \\ 104.6(3) & \mathrm{O}(1)-\mathrm{C}(14)-\mathrm{C}(13) & 114.6(3) \\ 109.2(3) & \mathrm{C}(16)-\mathrm{C}(15)-\mathrm{C}(13) & 112.9(3) \\ 113.3(3) & \mathrm{C}(15)-\mathrm{C}(16)-\mathrm{C}(18) & 120.5(3) \\ 114.8(2) & \mathrm{C}(15)-\mathrm{C}(16)-\mathrm{C}(17) & 118.2(3) \\ 107.7(2) & \mathrm{C}(18)-\mathrm{C}(16)-\mathrm{C}(17) & 60.3(3) \\ 107.5(2) & \mathrm{C}(19)-\mathrm{C}(17)-\mathrm{C}(16) & 116.2(4) \\ 122.2(3) & \mathrm{C}(19)-\mathrm{C}(17)-\mathrm{C}(18) & 118.7(4) \\ 113.7(2) & \mathrm{C}(16)-\mathrm{C}(17)-\mathrm{C}(18) & 59.6(3) \\ 123.8(3) & \mathrm{C}(16)-\mathrm{C}(18)-\mathrm{C}(17) & 60.1(2) \\ 120.7(3) & \mathrm{F}(2)-\mathrm{C}(19)-\mathrm{F}(3) & 106.0(4) \\ 127.7(3) & \mathrm{F}(2)-\mathrm{C}(19)-\mathrm{F}(1) & 105.8(3) \\ 111.6(2) & \mathrm{F}(3)-\mathrm{C}(19)-\mathrm{F}(1) & 105.2(3) \\ 103.5(3) & \mathrm{F}(2)-\mathrm{C}(19)-\mathrm{C}(17) & 111.5(3) \\ 101.9(4) & \mathrm{F}(3)-\mathrm{C}(19)-\mathrm{C}(17) & 114.5(3) \\ 104.0(3) & \mathrm{F}(1)-\mathrm{C}(19)-\mathrm{C}(17) & 113.0(4) \\ 111.2(3) & \mathrm{N}(1)-\mathrm{C}(20)-\mathrm{C}(21) & 112.1(3) \\ 112.9(3) & \mathrm{C}(22)-\mathrm{C}(21)-\mathrm{C}(26) & 119.2(4) \\ 106.4(3) & \mathrm{C}(22)-\mathrm{C}(21)-\mathrm{C}(20) & 121.8(4) \\ 126.5(3) & \mathrm{C}(26)-\mathrm{C}(21)-\mathrm{C}(20) & 119.0(4) \\ 119.0(4) & \mathrm{C}(23)-\mathrm{C}(22)-\mathrm{C}(21) & 120.7(5) \\ 114.5(3) & \mathrm{C}(22)-\mathrm{C}(23)-\mathrm{C}(24) & 120.4(5) \\ 121.7(3) & \mathrm{C}(23)-\mathrm{C}(24)-\mathrm{C}(25) & 119.7(5) \\ 121.0(4) & \mathrm{C}(24)-\mathrm{C}(25)-\mathrm{C}(26) & 119.7(5)\end{array}$




$\begin{array}{rlll}\mathrm{C}(11)-\mathrm{C}(6)-\mathrm{C}(7) & 117.3(3) & \mathrm{C}(21)-\mathrm{C}(26)-\mathrm{C}(25) & 120.2(5) \\ \mathrm{C}(8)-\mathrm{C}(7)-\mathrm{C}(6) & 120.8(4) & \mathrm{C}(32)-\mathrm{C}(27)-\mathrm{C}(28) & 120.1(4) \\ \mathrm{C}(9)-\mathrm{C}(8)-\mathrm{C}(7) & 121.7(4) & \mathrm{C}(32)-\mathrm{C}(27)-\mathrm{C}(12) & 120.8(3) \\ \mathrm{C}(8)-\mathrm{C}(9)-\mathrm{C}(10) & 118.7(4) & \mathrm{C}(28)-\mathrm{C}(27)-\mathrm{C}(12) & 119.0(3) \\ \mathrm{C}(9)-\mathrm{C}(10)-\mathrm{C}(11) & 121.6(4) & \mathrm{C}(29)-\mathrm{C}(28)-\mathrm{C}(27) & 120.2(4) \\ \mathrm{C}(6)-\mathrm{C}(11)-\mathrm{C}(10) & 119.8(4) & \mathrm{C}(28)-\mathrm{C}(29)-\mathrm{C}(30) & 119.5(4) \\ \mathrm{C}(6)-\mathrm{C}(11)-\mathrm{C}(12) & 123.0(3) & \mathrm{C}(31)-\mathrm{C}(30)-\mathrm{C}(29) & 120.9(4) \\ \mathrm{C}(10)-\mathrm{C}(11)-\mathrm{C}(12) & 117.2(3) & \mathrm{C}(30)-\mathrm{C}(31)-\mathrm{C}(32) & 119.4(4) \\ \mathrm{N}(3)-\mathrm{C}(12)-\mathrm{C}(11) & 122.1(3) & \mathrm{C}(27)-\mathrm{C}(32)-\mathrm{C}(31) & 119.9(4)\end{array}$

Table 4. Anisotropic displacement parameters $\left(\AA^{2} \times 10^{3}\right)$ for $\mathrm{dk} 25$. The anisotropic displacement factor exponent takes the form: $-2 \pi^{2}\left[h^{2} a^{* 2} U^{11}+\ldots+2 h k a^{*} b^{*} U^{12}\right]$

\begin{tabular}{|c|c|c|c|c|c|c|}
\hline Atom & $\mathrm{U}^{11}$ & $\mathrm{U}^{22}$ & $\mathrm{U}^{33}$ & $\mathrm{U}^{23}$ & $\mathrm{U}^{13}$ & $\mathrm{U}^{12}$ \\
\hline $\mathrm{Ni}(1)$ & $23(1)$ & $16(1)$ & $17(1)$ & $0(1)$ & $-2(1)$ & $0(1)$ \\
\hline $\mathrm{O}(1)$ & $32(2)$ & $17(1)$ & 21(1) & $1(1)$ & $-2(1)$ & $-5(1)$ \\
\hline $\mathrm{O}(2)$ & $31(2)$ & $21(2)$ & $30(2)$ & $-4(1)$ & $-3(1)$ & $-6(1)$ \\
\hline $\mathrm{O}(3)$ & $51(2)$ & $26(2)$ & $27(2)$ & $-1(1)$ & $-8(1)$ & $-15(2)$ \\
\hline $\mathrm{N}(1)$ & $29(2)$ & $19(2)$ & $17(2)$ & $3(1)$ & $0(1)$ & $1(2)$ \\
\hline $\mathrm{N}(2)$ & $24(2)$ & $15(2)$ & $21(2)$ & $4(1)$ & $-3(1)$ & $-1(2)$ \\
\hline $\mathrm{N}(3)$ & $17(2)$ & $16(2)$ & $17(2)$ & $0(1)$ & $-3(1)$ & $2(1)$ \\
\hline $\mathrm{C}(1)$ & $50(3)$ & $23(2)$ & $31(2)$ & $0(2)$ & $14(2)$ & $-3(2)$ \\
\hline$C(2)$ & $40(3)$ & $31(3)$ & $48(3)$ & $3(2)$ & $20(2)$ & $11(2)$ \\
\hline$C(3)$ & $28(3)$ & $37(3)$ & $30(2)$ & $6(2)$ & $-2(2)$ & $-4(2)$ \\
\hline$C(4)$ & $26(2)$ & $17(2)$ & $25(2)$ & $5(2)$ & $-1(2)$ & $-8(2)$ \\
\hline$C(5)$ & $29(2)$ & $20(2)$ & $26(2)$ & $3(2)$ & $-6(2)$ & $4(2)$ \\
\hline$C(6)$ & $25(2)$ & $16(2)$ & $24(2)$ & $-3(2)$ & $-8(2)$ & $6(2)$ \\
\hline$C(7)$ & $49(3)$ & $15(2)$ & $24(2)$ & $2(2)$ & $-6(2)$ & $3(2)$ \\
\hline $\mathrm{C}(8)$ & $51(3)$ & 21(2) & $30(2)$ & $-5(2)$ & $1(2)$ & $10(2)$ \\
\hline $\mathrm{C}(9)$ & $47(3)$ & $33(3)$ & $24(2)$ & $-5(2)$ & $6(2)$ & $14(2)$ \\
\hline$C(10)$ & $35(3)$ & $29(3)$ & $15(2)$ & $-2(2)$ & $1(2)$ & $2(2)$ \\
\hline $\mathrm{C}(11)$ & $23(2)$ & $15(2)$ & $22(2)$ & $1(2)$ & $-4(2)$ & $6(2)$ \\
\hline$C(12)$ & $11(2)$ & $22(2)$ & $21(2)$ & $2(2)$ & $-5(2)$ & $0(2)$ \\
\hline$C(13)$ & $22(2)$ & $16(2)$ & $24(2)$ & $-1(2)$ & $4(2)$ & $-1(2)$ \\
\hline$C(14)$ & $17(2)$ & $24(2)$ & $26(2)$ & $-2(2)$ & $0(2)$ & $-3(2)$ \\
\hline$C(15)$ & $27(2)$ & $17(2)$ & $16(2)$ & $-2(2)$ & $1(2)$ & $1(2)$ \\
\hline$C(16)$ & $23(2)$ & $16(2)$ & $24(2)$ & $-2(2)$ & $-3(2)$ & $4(2)$ \\
\hline$C(17)$ & $23(2)$ & $22(2)$ & $30(2)$ & $0(2)$ & $2(2)$ & $5(2)$ \\
\hline $\mathrm{C}(18)$ & $23(2)$ & $33(3)$ & $22(2)$ & $-2(2)$ & $-2(2)$ & $2(2)$ \\
\hline$C(19)$ & $27(2)$ & $40(3)$ & $24(2)$ & $2(2)$ & $2(2)$ & $6(2)$ \\
\hline $\mathrm{C}(20)$ & $33(3)$ & $24(2)$ & $23(2)$ & $5(2)$ & $-10(2)$ & $-3(2)$ \\
\hline $\mathrm{C}(21)$ & $22(2)$ & $28(2)$ & $24(2)$ & $7(2)$ & $-10(2)$ & $6(2)$ \\
\hline$C(22)$ & $28(3)$ & $27(3)$ & $43(2)$ & $9(2)$ & $-10(2)$ & $7(2)$ \\
\hline $\mathrm{C}(23)$ & $39(3)$ & 41(3) & $50(3)$ & $-6(2)$ & $-9(2)$ & $20(3)$ \\
\hline $\mathrm{C}(24)$ & $46(3)$ & $59(4)$ & $54(3)$ & $8(3)$ & $10(3)$ & $23(3)$ \\
\hline$C(25)$ & $29(3)$ & $45(3)$ & $74(4)$ & $14(3)$ & $8(3)$ & $2(3)$ \\
\hline$C(26)$ & $31(3)$ & $26(3)$ & $44(3)$ & $6(2)$ & $-11(2)$ & $5(2)$ \\
\hline$C(27)$ & $21(2)$ & $16(2)$ & $18(2)$ & $-5(2)$ & 2(2) & $0(2)$ \\
\hline
\end{tabular}




\begin{tabular}{cccrrrr}
\hline Atom & $\mathrm{U}^{11}$ & $\mathrm{U}^{22}$ & $\mathrm{U}^{33}$ & $\mathrm{U}^{23}$ & $\mathrm{U}^{13}$ & $\mathrm{U}^{12}$ \\
\hline $\mathrm{C}(28)$ & $28(2)$ & $24(2)$ & $23(2)$ & $4(2)$ & $-1(2)$ & $2(2)$ \\
$\mathrm{C}(29)$ & $46(3)$ & $28(2)$ & $20(2)$ & $0(2)$ & $-1(2)$ & $4(2)$ \\
$\mathrm{C}(30)$ & $45(3)$ & $21(2)$ & $31(2)$ & $2(2)$ & $17(2)$ & $1(2)$ \\
$\mathrm{C}(31)$ & $30(2)$ & $24(2)$ & $40(2)$ & $-1(2)$ & $10(2)$ & $-2(2)$ \\
$\mathrm{C}(32)$ & $21(2)$ & $24(2)$ & $28(2)$ & $-3(2)$ & $5(2)$ & $2(2)$ \\
$\mathrm{F}(1)$ & $27(1)$ & $67(2)$ & $46(2)$ & $0(1)$ & $0(1)$ & $19(1)$ \\
$\mathrm{F}(2)$ & $30(2)$ & $80(2)$ & $31(1)$ & $-11(1)$ & $-6(1)$ & $-4(1)$ \\
$\mathrm{F}(3)$ & $31(2)$ & $48(2)$ & $59(2)$ & $10(1)$ & $-3(1)$ & $-10(1)$ \\
\hline
\end{tabular}

Table 5. Hydrogen coordinates $\left(\times 10^{4}\right)$ and isotropic displacement parameters $\left(\AA^{2} \times 10^{3}\right)$ for dk25.

\begin{tabular}{rrrrr}
\hline Atom & \multicolumn{1}{c}{$\mathrm{x}$} & $\mathrm{y}$ & $\mathrm{z}$ & $\mathrm{U}(\mathrm{eq})$ \\
\hline $\mathrm{H}(1 \mathrm{~A})$ & -430 & 5886 & 10029 & 42 \\
$\mathrm{H}(1 \mathrm{~B})$ & -760 & 4389 & 10118 & 42 \\
$\mathrm{H}(2 \mathrm{~A})$ & -2184 & 5831 & 9759 & 48 \\
$\mathrm{H}(2 \mathrm{~B})$ & -2834 & 5189 & 9992 & 48 \\
$\mathrm{H}(3 \mathrm{~A})$ & -2852 & 3778 & 9611 & 38 \\
$\mathrm{H}(3 \mathrm{~B})$ & -2582 & 3023 & 9856 & 38 \\
$\mathrm{H}(4 \mathrm{~A})$ & -546 & 2494 & 9727 & 27 \\
$\mathrm{H}(7 \mathrm{~A})$ & 394 & 2371 & 9040 & 35 \\
$\mathrm{H}(8 \mathrm{~A})$ & 1603 & 2186 & 8697 & 41 \\
$\mathrm{H}(9 \mathrm{~A})$ & 2838 & 3978 & 8552 & 41 \\
$\mathrm{H}(10 \mathrm{~A})$ & 2759 & 6029 & 8745 & 32 \\
$\mathrm{H}(13 \mathrm{~A})$ & 2095 & 8831 & 9389 & 25 \\
$\mathrm{H}(15 \mathrm{~A})$ & 131 & 10122 & 9439 & 24 \\
$\mathrm{H}(15 \mathrm{~B})$ & -31 & 9268 & 9204 & 24 \\
$\mathrm{H}(16 \mathrm{~A})$ & -1433 & 7664 & 9425 & 25 \\
$\mathrm{H}(17 \mathrm{~A})$ & -2354 & 10473 & 9430 & 30 \\
$\mathrm{H}(18 \mathrm{~A})$ & -1297 & 9735 & 9790 & 31 \\
$\mathrm{H}(18 \mathrm{~B})$ & -2283 & 8384 & 9796 & 31 \\
$\mathrm{H}(20 \mathrm{~A})$ & 1118 & 2976 & 9974 & 32 \\
$\mathrm{H}(20 \mathrm{~B})$ & 1723 & 4447 & 10011 & 32 \\
$\mathrm{H}(22 \mathrm{~A})$ & 1797 & 1669 & 9620 & 39 \\
$\mathrm{H}(23 \mathrm{~A})$ & 3300 & 1364 & 9315 & 52 \\
$\mathrm{H}(24 \mathrm{~A})$ & 4884 & 3009 & 9228 & 63 \\
$\mathrm{H}(25 \mathrm{~A})$ & 4962 & 4978 & 9451 & 59 \\
$\mathrm{H}(26 \mathrm{~A})$ & 3386 & 5319 & 9750 & 41 \\
$\mathrm{H}(28 \mathrm{~A})$ & 233 & 7871 & 8767 & 30 \\
$\mathrm{H}(29 \mathrm{~A})$ & 908 & 9453 & 8490 & 37 \\
$\mathrm{H}(30 \mathrm{~A})$ & 3007 & 10482 & 8525 & 39 \\
$\mathrm{H}(31 \mathrm{~A})$ & 4413 & 9966 & 8837 & 38 \\
$\mathrm{H}(32 \mathrm{~A})$ & 3738 & 8349 & 9113 & 29 \\
\hline & & & &
\end{tabular}


Belokon' (2S, 1'R, 2'S)-3-(2'-difluoromethylcycloprpopyl)alanine complex

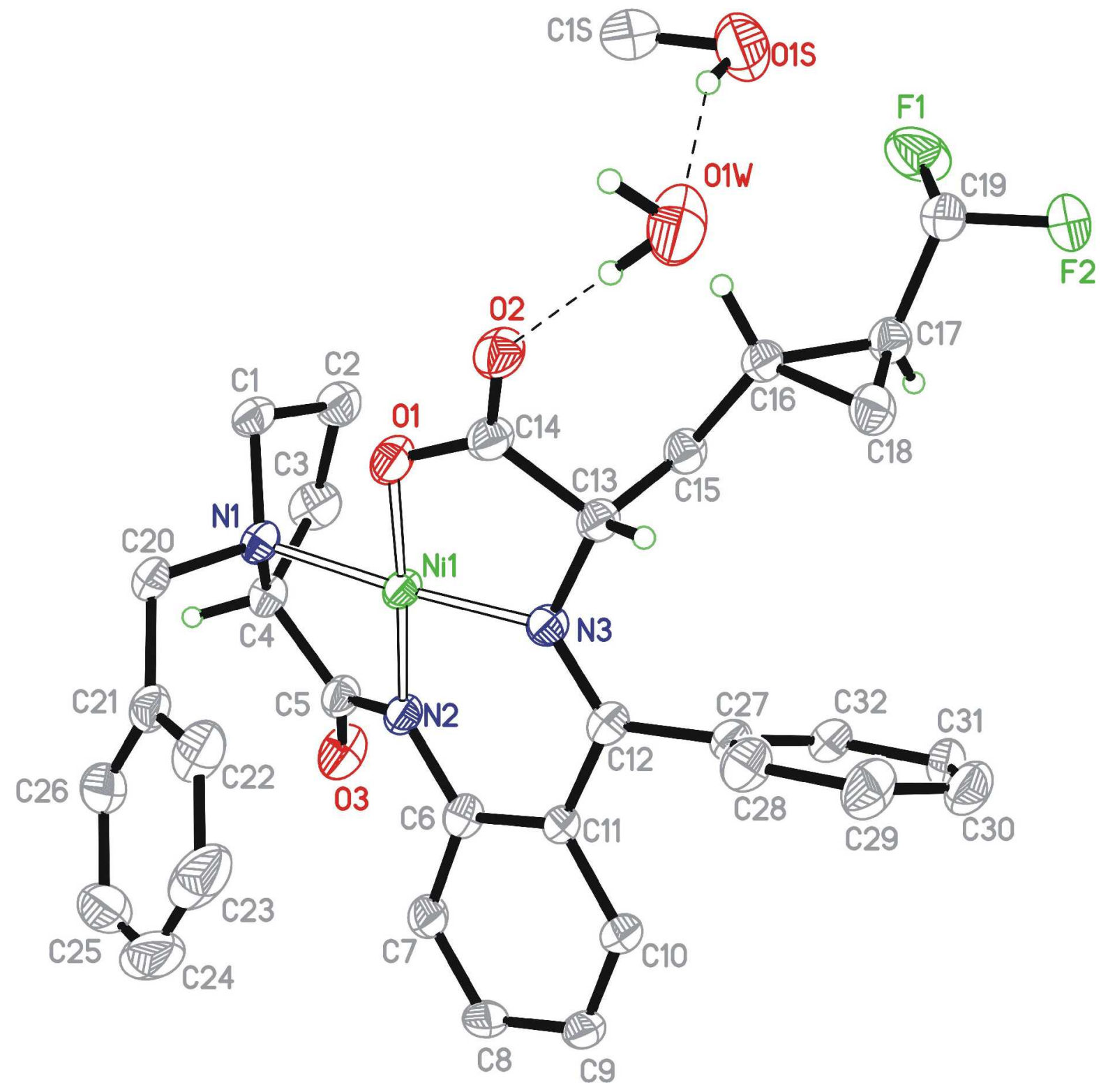

Table 1. Crystal data and structure refinement for DK33.

Identification code

Empirical formula

Formula weight

Temperature

Wavelength

Crystal system

Space group dk33

$\mathrm{C}_{32} \mathrm{H}_{31} \mathrm{~F}_{2} \mathrm{~N}_{3} \mathrm{NiO}_{3} \times \mathrm{CH}_{3} \mathrm{OH} \times \mathrm{H}_{2} \mathrm{O}$

651.36

120(2) K

$0.71073 \AA$

Monoclinic

P 21 
Unit cell dimensions

Volume

Z

Density (calculated)

Absorption coefficient

$\mathrm{F}(000)$

Crystal size

Theta range for data collection

Index ranges

Reflections collected

Independent reflections

Completeness to theta $=29.50^{\circ}$

Absorption correction

Max. and min. transmission

Refinement method

Data / restraints / parameters

Goodness-of-fit on $\mathrm{F}^{2}$

Final $\mathrm{R}$ indices [I $>2 \operatorname{sigma}(\mathrm{I})]$

$\mathrm{R}$ indices (all data)

Absolute structure parameter

Largest diff. peak and hole

$$
\begin{array}{ll}
\mathrm{a}=9.2565(6) \AA & \alpha=90^{\circ} . \\
\mathrm{b}=11.8858(6) \AA & \beta=90.67(3)^{\circ} . \\
\mathrm{c}=14.020(1) \AA & \gamma=90^{\circ} .
\end{array}
$$

1542.4(5) $\AA^{3}$

2

$1.403 \mathrm{Mg} / \mathrm{m}^{3}$

$0.687 \mathrm{~mm}^{-1}$

682

$0.33 \times 0.22 \times 0.08 \mathrm{~mm}^{3}$

1.45 to $29.50^{\circ}$.

$-12<=\mathrm{h}<=12,-16<=\mathrm{k}<=16,-19<=\mathrm{l}<=19$

16960

$8238[\mathrm{R}($ int $)=0.0657]$

$99.6 \%$

Numerical

0.9101 and 0.7030

Full-matrix least-squares on $\mathrm{F}^{2}$

$8238 / 1 / 532$

1.009

$\mathrm{R}_{1}=0.0487, \mathrm{wR}_{2}=0.1122$

$\mathrm{R}_{1}=0.0542, \mathrm{wR}_{2}=0.1203$

$0.008(11)$

0.733 and -0.498 e. $\AA^{-3}$

Table 2. Atomic coordinates $\left(\times 10^{4}\right)$ and equivalent isotropic displacement parameters $\left(\AA^{2} \times 10^{3}\right)$ for DK33. U(eq) is defined as one third of the trace of the orthogonalized $\mathrm{U}^{\mathrm{ij}}$ tensor.

\begin{tabular}{rrrrr}
\hline Atom & $\mathrm{x}$ & $\mathrm{y}$ & $\mathrm{z}$ & $\mathrm{U}(\mathrm{eq})$ \\
\hline $\mathrm{Ni}(1)$ & $6637(1)$ & $6446(1)$ & $6486(1)$ & $22(1)$ \\
$\mathrm{O}(1)$ & $6124(3)$ & $4947(2)$ & $6282(2)$ & $30(1)$ \\
$\mathrm{O}(2)$ & $6366(3)$ & $3228(2)$ & $6888(2)$ & $33(1)$ \\
$\mathrm{O}(3)$ & $6212(3)$ & $9717(2)$ & $6378(2)$ & $37(1)$ \\
$\mathrm{F}(1)$ & $3635(2)$ & $3038(2)$ & $10665(2)$ & $52(1)$ \\
$\mathrm{F}(2)$ & $5499(3)$ & $2441(2)$ & $11481(1)$ & $45(1)$ \\
$\mathrm{N}(1)$ & $5336(3)$ & $6946(2)$ & $5473(2)$ & $23(1)$ \\
$\mathrm{N}(2)$ & $7204(3)$ & $7937(2)$ & $6611(2)$ & $23(1)$ \\
$\mathrm{N}(3)$ & $7775(3)$ & $5924(2)$ & $7494(2)$ & $22(1)$
\end{tabular}




\begin{tabular}{|c|c|c|c|c|}
\hline Atom & $\mathrm{x}$ & $\mathrm{y}$ & $\mathrm{z}$ & $\mathrm{U}(\mathrm{eq})$ \\
\hline $\mathrm{C}(1)$ & $3886(3)$ & 6393(3) & $5495(2)$ & $27(1)$ \\
\hline$C(2)$ & $3156(4)$ & $6952(3)$ & $6339(2)$ & $34(1)$ \\
\hline $\mathrm{C}(3)$ & $3586(4)$ & $8180(3)$ & $6219(2)$ & $37(1)$ \\
\hline $\mathrm{C}(4)$ & $5026(3)$ & $8166(2)$ & $5674(2)$ & $26(1)$ \\
\hline $\mathrm{C}(5)$ & $6227(3)$ & $8684(2)$ & $6253(2)$ & $26(1)$ \\
\hline$C(6)$ & $8506(3)$ & $8276(2)$ & $7030(2)$ & $24(1)$ \\
\hline$C(7)$ & $9127(4)$ & $9322(2)$ & $6794(2)$ & $29(1)$ \\
\hline $\mathrm{C}(8)$ & $10420(4)$ & $9675(2)$ & $7193(2)$ & $30(1)$ \\
\hline $\mathrm{C}(9)$ & $11153(3)$ & $9014(3)$ & $7854(2)$ & $27(1)$ \\
\hline$C(10)$ & $10608(4)$ & $7967(3)$ & $8075(2)$ & $26(1)$ \\
\hline$C(11)$ & $9284(3)$ & $7582(2)$ & $7674(2)$ & $22(1)$ \\
\hline$C(12)$ & $8817(3)$ & $6436(3)$ & $7944(2)$ & $22(1)$ \\
\hline$C(13)$ & $7327(3)$ & $4784(2)$ & $7793(2)$ & $25(1)$ \\
\hline$C(14)$ & $6564(4)$ & $4254(2)$ & $6932(2)$ & $27(1)$ \\
\hline$C(15)$ & $6288(3)$ & $4840(2)$ & $8651(2)$ & $25(1)$ \\
\hline$C(16)$ & $6127(3)$ & $3697(2)$ & $9111(2)$ & $25(1)$ \\
\hline$C(17)$ & $5959(3)$ & $3622(2)$ & $10178(2)$ & $27(1)$ \\
\hline$C(18)$ & $7360(4)$ & $3275(3)$ & $9732(2)$ & $29(1)$ \\
\hline$C(19)$ & $5052(4)$ & $2705(3)$ & $10571(2)$ & $33(1)$ \\
\hline$C(20)$ & 5991(3) & $6763(2)$ & $4511(2)$ & $24(1)$ \\
\hline $\mathrm{C}(21)$ & $7481(3)$ & $7251(3)$ & $4425(2)$ & $29(1)$ \\
\hline$C(22)$ & $8695(4)$ & $6612(4)$ & $4676(2)$ & $41(1)$ \\
\hline$C(23)$ & $10067(4)$ & $7065(5)$ & $4615(3)$ & $61(1)$ \\
\hline $\mathrm{C}(24)$ & $10253(6)$ & $8161(7)$ & $4305(3)$ & $81(2)$ \\
\hline$C(25)$ & $9038(6)$ & $8823(4)$ & $4048(3)$ & $63(1)$ \\
\hline$C(26)$ & $7681(5)$ & $8347(3)$ & $4101(2)$ & $39(1)$ \\
\hline$C(27)$ & $9624(3)$ & $5890(2)$ & $8759(2)$ & $24(1)$ \\
\hline $\mathrm{C}(28)$ & $10658(4)$ & $5072(3)$ & $8583(2)$ & $30(1)$ \\
\hline$C(29)$ & $11461(4)$ & $4622(3)$ & $9341(3)$ & $37(1)$ \\
\hline$C(30)$ & $11249(4)$ & $4989(3)$ & $10260(2)$ & $36(1)$ \\
\hline $\mathrm{C}(31)$ & $10219(4)$ & $5811(3)$ & $10439(2)$ & $31(1)$ \\
\hline$C(32)$ & 9412(3) & $6263(2)$ & $9690(2)$ & $27(1)$ \\
\hline $\mathrm{O}(1 \mathrm{~W})$ & $6817(4)$ & $1184(2)$ & $7878(2)$ & $55(1)$ \\
\hline $\mathrm{O}(1 \mathrm{~S})$ & $4185(5)$ & $1132(2)$ & $8736(2)$ & $62(1)$ \\
\hline$C(1 S)$ & $3302(6)$ & $1648(4)$ & $8054(3)$ & $62(1)$ \\
\hline
\end{tabular}

Table 3. Selected bond lengths $[\AA]$ and angles $\left[{ }^{\circ}\right]$ for DK33.

$\begin{array}{rrrrrr}\mathrm{Ni}(1)-\mathrm{N}(2) & 1.856(2) & \mathrm{C}(2)-\mathrm{C}(3) & 1.523(5) & \mathrm{C}(17)-\mathrm{C}(19) & 1.486(4) \\ \mathrm{Ni}(1)-\mathrm{N}(3) & 1.859(2) & \mathrm{C}(3)-\mathrm{C}(4) & 1.545(5) & \mathrm{C}(17)-\mathrm{C}(18) & 1.504(5) \\ \mathrm{Ni}(1)-\mathrm{O}(1) & 1.865(2) & \mathrm{C}(4)-\mathrm{C}(5) & 1.501(4) & \mathrm{C}(20)-\mathrm{C}(21) & 1.503(4) \\ \mathrm{Ni}(1)-\mathrm{N}(1) & 1.944(2) & \mathrm{C}(6)-\mathrm{C}(7) & 1.410(4) & \mathrm{C}(21)-\mathrm{C}(26) & 1.392(5) \\ \mathrm{O}(1)-\mathrm{C}(14) & 1.290(4) & \mathrm{C}(6)-\mathrm{C}(11) & 1.414(4) & \mathrm{C}(21)-\mathrm{C}(22) & 1.398(5) \\ \mathrm{O}(2)-\mathrm{C}(14) & 1.235(4) & \mathrm{C}(7)-\mathrm{C}(8) & 1.381(4) & \mathrm{C}(22)-\mathrm{C}(23) & 1.383(6) \\ \mathrm{O}(3)-\mathrm{C}(5) & 1.240(4) & \mathrm{C}(8)-\mathrm{C}(9) & 1.386(4) & \mathrm{C}(23)-\mathrm{C}(24) & 1.385(9) \\ \mathrm{F}(1)-\mathrm{C}(19) & 1.378(4) & \mathrm{C}(9)-\mathrm{C}(10) & 1.379(4) & \mathrm{C}(24)-\mathrm{C}(25) & 1.415(9)\end{array}$




$\begin{array}{rlllll}\mathrm{F}(2)-\mathrm{C}(19) & 1.372(3) & \mathrm{C}(10)-\mathrm{C}(11) & 1.418(4) & \mathrm{C}(25)-\mathrm{C}(26) & 1.380(6) \\ \mathrm{N}(1)-\mathrm{C}(1) & 1.495(4) & \mathrm{C}(11)-\mathrm{C}(12) & 1.480(4) & \mathrm{C}(27)-\mathrm{C}(28) & 1.389(4) \\ \mathrm{N}(1)-\mathrm{C}(20) & 1.501(4) & \mathrm{C}(12)-\mathrm{C}(27) & 1.504(4) & \mathrm{C}(27)-\mathrm{C}(32) & 1.395(4) \\ \mathrm{N}(1)-\mathrm{C}(4) & 1.506(4) & \mathrm{C}(13)-\mathrm{C}(14) & 1.527(4) & \mathrm{C}(28)-\mathrm{C}(29) & 1.396(4) \\ \mathrm{N}(2)-\mathrm{C}(5) & 1.359(4) & \mathrm{C}(13)-\mathrm{C}(15) & 1.551(4) & \mathrm{C}(29)-\mathrm{C}(30) & 1.376(5) \\ \mathrm{N}(2)-\mathrm{C}(6) & 1.395(4) & \mathrm{C}(15)-\mathrm{C}(16) & 1.512(4) & \mathrm{C}(30)-\mathrm{C}(31) & 1.390(5) \\ \mathrm{N}(3)-\mathrm{C}(12) & 1.299(3) & \mathrm{C}(16)-\mathrm{C}(17) & 1.509(4) & \mathrm{C}(31)-\mathrm{C}(32) & 1.390(4) \\ \mathrm{N}(3)-\mathrm{C}(13) & 1.478(3) & \mathrm{C}(16)-\mathrm{C}(18) & 1.513(4) & \mathrm{O}(1 \mathrm{~S})-\mathrm{C}(1 \mathrm{~S}) & 1.393(5) \\ \mathrm{C}(1)-\mathrm{C}(2) & 1.523(4) & & & & \end{array}$

$$
\begin{array}{r}
\mathrm{N}(2)-\mathrm{Ni}(1)-\mathrm{N}(3) \\
\mathrm{N}(2)-\mathrm{Ni}(1)-\mathrm{O}(1) \\
\mathrm{N}(3)-\mathrm{Ni}(1)-\mathrm{O}(1) \\
\mathrm{N}(2)-\mathrm{Ni}(1)-\mathrm{N}(1) \\
\mathrm{N}(3)-\mathrm{Ni}(1)-\mathrm{N}(1) \\
\mathrm{O}(1)-\mathrm{Ni}(1)-\mathrm{N}(1) \\
\mathrm{C}(14)-\mathrm{O}(1)-\mathrm{Ni}(1) \\
\mathrm{C}(1)-\mathrm{N}(1)-\mathrm{C}(20) \\
\mathrm{C}(1)-\mathrm{N}(1)-\mathrm{C}(4) \\
\mathrm{C}(20)-\mathrm{N}(1)-\mathrm{C}(4) \\
\mathrm{C}(1)-\mathrm{N}(1)-\mathrm{Ni}(1) \\
\mathrm{C}(20)-\mathrm{N}(1)-\mathrm{Ni}(1) \\
\mathrm{C}(4)-\mathrm{N}(1)-\mathrm{Ni}(1) \\
\mathrm{C}(5)-\mathrm{N}(2)-\mathrm{C}(6) \\
\mathrm{C}(5)-\mathrm{N}(2)-\mathrm{Ni}(1) \\
\mathrm{C}(6)-\mathrm{N}(2)-\mathrm{Ni}(1) \\
\mathrm{C}(12)-\mathrm{N}(3)-\mathrm{C}(13) \\
\mathrm{C}(12)-\mathrm{N}(3)-\mathrm{Ni}(1) \\
\mathrm{C}(13)-\mathrm{N}(3)-\mathrm{Ni}(1) \\
\mathrm{N}(1)-\mathrm{C}(1)-\mathrm{C}(2) \\
\mathrm{C}(1)-\mathrm{C}(2)-\mathrm{C}(3) \\
\mathrm{C}(2)-\mathrm{C}(3)-\mathrm{C}(4) \\
\mathrm{C}(5)-\mathrm{C}(4)-\mathrm{N}(1) \\
\mathrm{C}(5)-\mathrm{C}(4)-\mathrm{C}(3) \\
\mathrm{N}(1)-\mathrm{C}(4)-\mathrm{C}(3) \\
\mathrm{O}(3)-\mathrm{C}(5)-\mathrm{N}(2) \\
\mathrm{O}(3)-\mathrm{C}(5)-\mathrm{C}(4) \\
\mathrm{N}(2)-\mathrm{C}(5)-\mathrm{C}(4) \\
\mathrm{N}(2)-\mathrm{C}(6)-\mathrm{C}(7) \\
\mathrm{N}(2)-\mathrm{C}(6)-\mathrm{C}(11) \\
\mathrm{C}(7)-\mathrm{C}(6)-\mathrm{C}(11) \\
\mathrm{C}(8)-\mathrm{C}(7)-\mathrm{C}(6) \\
\mathrm{C}(7)-\mathrm{C}(8)-\mathrm{C}(9) \\
\mathrm{C}(10)-\mathrm{C}(9)-\mathrm{C}(8) \\
\mathrm{C}(9)-\mathrm{C}(10)-\mathrm{C}(11) \\
\mathrm{C}(6)-\mathrm{C}(11)-\mathrm{C}(10) \\
\mathrm{C}(6)-\mathrm{C}(11)-\mathrm{C}(12) \\
\mathrm{C}(10)-\mathrm{C}(11)-\mathrm{C}(12) \\
\end{array}
$$

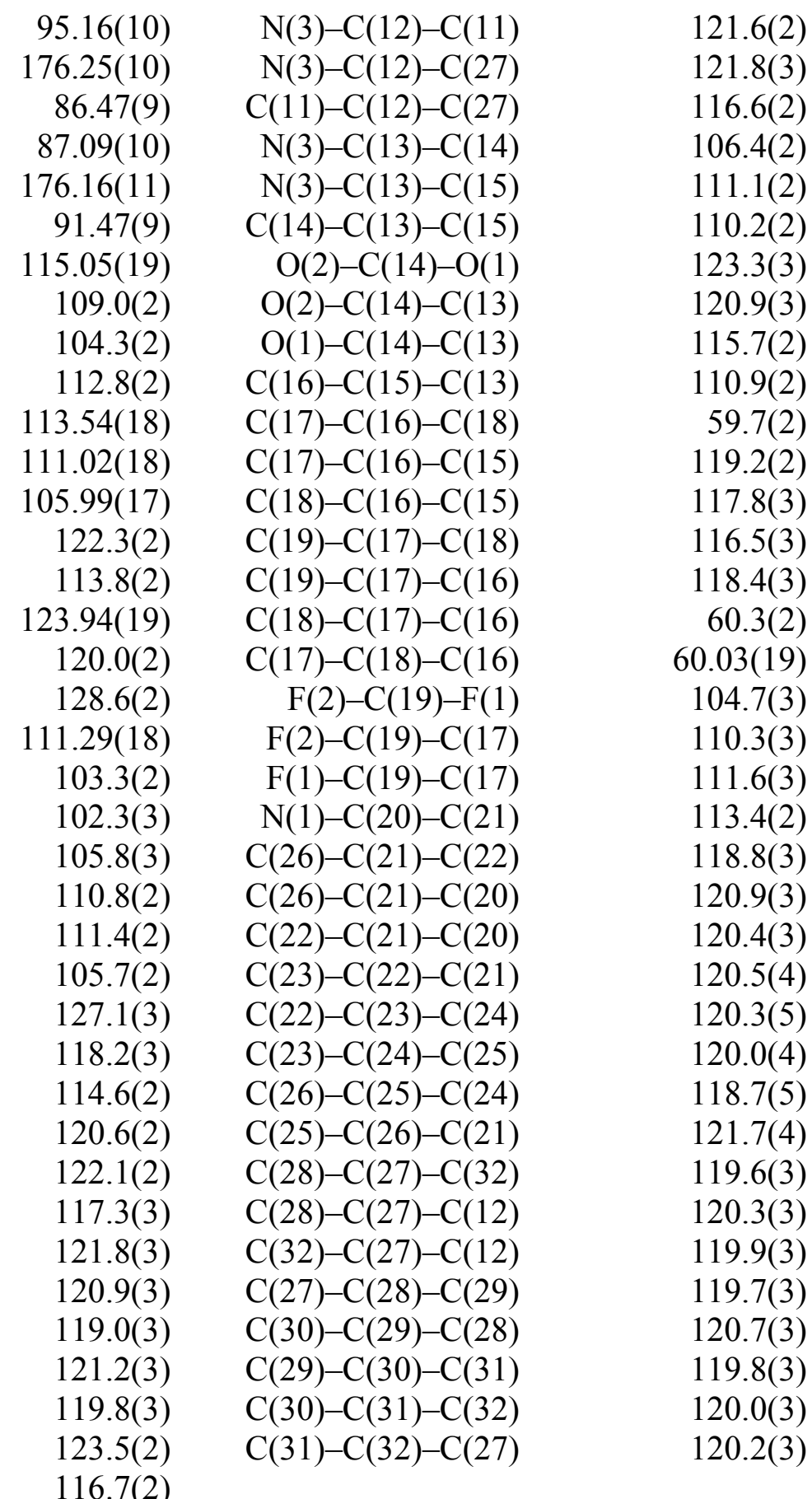

$21.6(2)$

$21.8(3)$

$16.6(2)$

06.4(2)

11.1(2)

$10.2(2)$

120.9(3)

$15.7(2)$

$59.7(2)$

19.2(2)

17.8(3)

$16.5(3)$

$18.4(3)$

$0.03(19)$

104.7(3)

$11.6(3)$

113.4(2)

(18.8(3)

20.9(3)

$0.4(3)$

$20.5(4)$

$20.3(5)$

20.0(4)

$118.7(5)$

$119.6(3)$

20.3(3)

119.7(3)

20.7(3)

$19.8(3)$

120.2(3) 
Table 4. Hydrogen bonds for DK33 [ and $\left.^{\circ}\right]$.

\begin{tabular}{ccccc}
\hline $\mathrm{D}-\mathrm{H} \ldots \mathrm{A}$ & $\mathrm{d}(\mathrm{D}-\mathrm{H})$ & $\mathrm{d}(\mathrm{H} \ldots \mathrm{A})$ & $\mathrm{d}(\mathrm{D} \ldots \mathrm{A})$ & $<(\mathrm{DHA})$ \\
\hline $\mathrm{O}(1 \mathrm{~S})-\mathrm{H}(1 \mathrm{OS}) \ldots \mathrm{O}(1 \mathrm{~W})$ & $1.04(4)$ & $1.79(4)$ & $2.731(6)$ & $148(3)$ \\
$\mathrm{O}(1 \mathrm{~W})-\mathrm{H}(1 \mathrm{OW}) \ldots \mathrm{O}(2)$ & $0.95(7)$ & $1.88(7)$ & $2.827(3)$ & $171(7)$ \\
$\mathrm{O}(1 \mathrm{~W})-\mathrm{H}(2 \mathrm{OW}) \ldots \mathrm{O}(3) \# 1$ & $0.91(8)$ & $1.88(8)$ & $2.784(4)$ & $169(7)$ \\
\hline
\end{tabular}

Symmetry transformations used to generate equivalent atoms:

$\# 1 \mathrm{x}, \mathrm{y}-1, \mathrm{z}$

Table 5. Anisotropic displacement parameters $\left(\AA^{2} \times 10^{3}\right)$ for DK33. The anisotropic displacement factor exponent takes the form: $-2 \pi^{2}\left[\mathrm{~h}^{2} \mathrm{a}^{* 2} \mathrm{U}^{11}+\ldots+2 \mathrm{~h} \mathrm{k} \mathrm{a}^{*} \mathrm{~b}^{*} \mathrm{U}^{12}\right]$

\begin{tabular}{|c|c|c|c|c|c|c|}
\hline Atom & $\mathrm{U}^{11}$ & $\mathrm{U}^{22}$ & $U^{33}$ & $\mathrm{U}^{23}$ & $\mathrm{U}^{13}$ & $\mathrm{U}^{12}$ \\
\hline $\mathrm{Ni}(1)$ & $23(1)$ & $20(1)$ & $22(1)$ & 1(1) & $-6(1)$ & $-2(1)$ \\
\hline $\mathrm{O}(1)$ & $37(1)$ & $24(1)$ & $27(1)$ & $0(1)$ & $-11(1)$ & $-2(1)$ \\
\hline $\mathrm{O}(2)$ & $43(1)$ & $22(1)$ & $32(1)$ & $-2(1)$ & $-8(1)$ & $-4(1)$ \\
\hline $\mathrm{O}(3)$ & $45(1)$ & $24(1)$ & $41(1)$ & $-2(1)$ & $-14(1)$ & $8(1)$ \\
\hline $\mathrm{F}(1)$ & $28(1)$ & $59(1)$ & $71(2)$ & $23(1)$ & $4(1)$ & $-2(1)$ \\
\hline$F(2)$ & $52(1)$ & $51(1)$ & $32(1)$ & $15(1)$ & $-9(1)$ & $-10(1)$ \\
\hline $\mathrm{N}(1)$ & $22(1)$ & $23(1)$ & $23(1)$ & $-2(1)$ & $-5(1)$ & $3(1)$ \\
\hline $\mathrm{N}(2)$ & $26(1)$ & $22(1)$ & $22(1)$ & 1(1) & $-6(1)$ & $-2(1)$ \\
\hline $\mathrm{N}(3)$ & $24(1)$ & $18(1)$ & $24(1)$ & $1(1)$ & $-3(1)$ & $0(1)$ \\
\hline$C(1)$ & $20(1)$ & $35(1)$ & $27(1)$ & $-2(1)$ & $-4(1)$ & $-5(2)$ \\
\hline$C(2)$ & $29(2)$ & $45(2)$ & $29(1)$ & $-3(1)$ & $0(1)$ & $-5(2)$ \\
\hline$C(3)$ & $32(2)$ & $39(2)$ & $41(2)$ & $-8(1)$ & $2(1)$ & $2(2)$ \\
\hline$C(4)$ & $26(2)$ & $27(1)$ & $24(1)$ & $-1(1)$ & $-4(1)$ & $1(1)$ \\
\hline$C(5)$ & $30(2)$ & $23(1)$ & $24(1)$ & $2(1)$ & $-7(1)$ & $-1(1)$ \\
\hline$C(6)$ & $24(1)$ & $23(1)$ & $24(1)$ & $-1(1)$ & $-2(1)$ & 4(1) \\
\hline$C(7)$ & $34(2)$ & $25(1)$ & $26(1)$ & $7(1)$ & $-6(1)$ & $-5(1)$ \\
\hline$C(8)$ & $32(2)$ & $28(1)$ & $30(1)$ & $0(1)$ & $0(1)$ & $-13(1)$ \\
\hline $\mathrm{C}(9)$ & $24(1)$ & $34(1)$ & $24(1)$ & $-4(1)$ & $0(1)$ & $-8(1)$ \\
\hline$C(10)$ & $25(2)$ & $27(1)$ & $25(1)$ & $-1(1)$ & $-4(1)$ & $-5(1)$ \\
\hline $\mathrm{C}(11)$ & $23(1)$ & $23(1)$ & 19(1) & $0(1)$ & $-2(1)$ & $-4(1)$ \\
\hline$C(12)$ & $22(1)$ & $22(1)$ & $23(1)$ & $-4(1)$ & $-3(1)$ & $3(1)$ \\
\hline $\mathrm{C}(13)$ & $27(2)$ & $20(1)$ & $27(1)$ & $3(1)$ & $-7(1)$ & $-2(1)$ \\
\hline$C(14)$ & $28(2)$ & $24(1)$ & $30(1)$ & $0(1)$ & $-5(1)$ & $-3(1)$ \\
\hline$C(15)$ & $26(2)$ & 23(1) & $26(1)$ & $2(1)$ & $-3(1)$ & $2(1)$ \\
\hline$C(16)$ & $26(2)$ & 23(1) & $26(1)$ & $2(1)$ & $-5(1)$ & $-3(1)$ \\
\hline$C(17)$ & $28(2)$ & $25(1)$ & $27(1)$ & $3(1)$ & $-5(1)$ & 1(1) \\
\hline $\mathrm{C}(18)$ & $27(2)$ & $30(1)$ & $31(1)$ & $7(1)$ & $-4(1)$ & $4(1)$ \\
\hline$C(19)$ & $32(2)$ & $33(2)$ & $33(2)$ & $7(1)$ & $-7(1)$ & $-4(1)$ \\
\hline $\mathrm{C}(20)$ & $23(1)$ & $28(1)$ & $21(1)$ & $-2(1)$ & $-5(1)$ & $0(1)$ \\
\hline
\end{tabular}




\begin{tabular}{crrrrrr}
\hline Atom & $\mathrm{U}^{11}$ & $\mathrm{U}^{22}$ & $\mathrm{U}^{33}$ & $\mathrm{U}^{23}$ & $\mathrm{U}^{13}$ & $\mathrm{U}^{12}$ \\
\hline $\mathrm{C}(21)$ & $27(2)$ & $39(2)$ & $21(1)$ & $-6(1)$ & $0(1)$ & $-1(1)$ \\
$\mathrm{C}(22)$ & $29(2)$ & $59(3)$ & $33(2)$ & $-12(2)$ & $-2(1)$ & $9(2)$ \\
$\mathrm{C}(23)$ & $25(2)$ & $115(4)$ & $44(2)$ & $-28(2)$ & $-1(2)$ & $1(2)$ \\
$\mathrm{C}(24)$ & $41(3)$ & $157(6)$ & $44(2)$ & $-32(3)$ & $16(2)$ & $-48(4)$ \\
$\mathrm{C}(25)$ & $71(3)$ & $83(3)$ & $35(2)$ & $-12(2)$ & $13(2)$ & $-46(3)$ \\
$\mathrm{C}(26)$ & $45(2)$ & $44(2)$ & $28(2)$ & $-4(1)$ & $3(1)$ & $-11(2)$ \\
$\mathrm{C}(27)$ & $22(1)$ & $25(1)$ & $26(1)$ & $3(1)$ & $-5(1)$ & $-2(1)$ \\
$\mathrm{C}(28)$ & $27(2)$ & $32(2)$ & $33(2)$ & $-2(1)$ & $-3(1)$ & $6(1)$ \\
$\mathrm{C}(29)$ & $32(2)$ & $35(2)$ & $43(2)$ & $3(1)$ & $-11(1)$ & $9(1)$ \\
$\mathrm{C}(30)$ & $34(2)$ & $36(2)$ & $39(2)$ & $7(1)$ & $-16(1)$ & $-2(1)$ \\
$\mathrm{C}(31)$ & $35(2)$ & $35(2)$ & $22(1)$ & $4(1)$ & $-8(1)$ & $-4(1)$ \\
$\mathrm{C}(32)$ & $24(1)$ & $31(2)$ & $26(1)$ & $0(1)$ & $-6(1)$ & $-3(1)$ \\
$\mathrm{O}(1 \mathrm{~W})$ & $89(2)$ & $32(1)$ & $43(1)$ & $-2(1)$ & $-20(2)$ & $6(1)$ \\
$\mathrm{O}(1 \mathrm{~S})$ & $102(3)$ & $50(2)$ & $36(1)$ & $8(1)$ & $-7(2)$ & $-22(2)$ \\
$\mathrm{C}(1 \mathrm{~S})$ & $73(3)$ & $68(3)$ & $45(2)$ & $9(2)$ & $-12(2)$ & $-40(3)$ \\
\hline
\end{tabular}

Table 6. Hydrogen coordinates $\left(\times 10^{4}\right)$ and isotropic displacement parameters $\left(\AA^{2} \times 10^{3}\right)$ for DK33.

\begin{tabular}{rrrrr}
\hline Atom & \multicolumn{1}{c}{$\mathrm{x}$} & $\mathrm{y}$ & $\mathrm{U}(\mathrm{eq})$ \\
\hline $\mathrm{H}(1 \mathrm{~S})$ & 2334 & 1757 & 8315 & 75 \\
$\mathrm{H}(2 \mathrm{~S})$ & 3238 & 1171 & 7485 & 75 \\
$\mathrm{H}(3 \mathrm{~S})$ & 3712 & 2380 & 7882 & 75 \\
$\mathrm{H}(101)$ & $3390(40)$ & $6510(30)$ & $4920(20)$ & $23(7)$ \\
$\mathrm{H}(102)$ & $4100(50)$ & $5470(30)$ & $5500(30)$ & $39(11)$ \\
$\mathrm{H}(201)$ & $3540(40)$ & $6630(30)$ & $6940(20)$ & $30(9)$ \\
$\mathrm{H}(202)$ & $2050(50)$ & $6820(30)$ & $6260(30)$ & $43(11)$ \\
$\mathrm{H}(301)$ & $2800(50)$ & $8590(30)$ & $5870(30)$ & $35(10)$ \\
$\mathrm{H}(302)$ & $3730(40)$ & $8560(30)$ & $6840(20)$ & $17(7)$ \\
$\mathrm{H}(4)$ & $4940(40)$ & $8560(20)$ & $5060(20)$ & $17(7)$ \\
$\mathrm{H}(7)$ & $8770(40)$ & $9700(30)$ & $6390(20)$ & $18(8)$ \\
$\mathrm{H}(8)$ & $10870(40)$ & $10430(30)$ & $6960(20)$ & $23(8)$ \\
$\mathrm{H}(9)$ & $12170(50)$ & $9280(30)$ & $8140(30)$ & $36(10)$ \\
$\mathrm{H}(10)$ & $11130(30)$ & $7540(20)$ & $8496(19)$ & $4(6)$ \\
$\mathrm{H}(13)$ & $8100(40)$ & $4270(30)$ & $7980(20)$ & $18(7)$ \\
$\mathrm{H}(151)$ & $5360(40)$ & $5130(30)$ & $8470(20)$ & $25(9)$ \\
$\mathrm{H}(152)$ & $6730(30)$ & $5390(30)$ & $9090(20)$ & $14(7)$ \\
$\mathrm{H}(16)$ & $5480(40)$ & $3200(30)$ & $8770(20)$ & $23(8)$ \\
$\mathrm{H}(17)$ & $5950(40)$ & $4260(30)$ & $10520(20)$ & $25(8)$ \\
$\mathrm{H}(181)$ & $8190(50)$ & $3740(30)$ & $9790(30)$ & $37(10)$ \\
$\mathrm{H}(182)$ & $7630(40)$ & $2350(30)$ & $9760(30)$ & $31(9)$ \\
$\mathrm{H}(19)$ & $4910(50)$ & $2070(30)$ & $10190(30)$ & $34(10)$ \\
$\mathrm{H}(20 \mathrm{~A})$ & $5270(40)$ & $7110(30)$ & $4050(20)$ & $26(8)$ \\
$\mathrm{H}(20 \mathrm{~B})$ & $6080(50)$ & $5990(30)$ & $4360(30)$ & $41(11)$ \\
$\mathrm{H}(22)$ & $8530(50)$ & $5900(40)$ & $4850(30)$ & $43(12)$
\end{tabular}


146

\begin{tabular}{rrrrr}
\hline Atom & $\mathrm{x}$ & $\mathrm{y}$ & $\mathrm{z}$ & $\mathrm{U}(\mathrm{eq})$ \\
\hline $\mathrm{H}(23)$ & $10960(50)$ & $6320(40)$ & $4850(30)$ & $60(13)$ \\
$\mathrm{H}(24)$ & $11300(60)$ & $8520(50)$ & $4280(40)$ & $77(16)$ \\
$\mathrm{H}(25)$ & $9180(7)$ & $9650(5)$ & $3830(4)$ & $80(17)$ \\
$\mathrm{H}(26)$ & $6880(40)$ & $8820(30)$ & $3900(20)$ & $23(9)$ \\
$\mathrm{H}(28)$ & $10740(40)$ & $4840(30)$ & $7910(30)$ & $30(10)$ \\
$\mathrm{H}(29)$ & $12180(40)$ & $4070(30)$ & $9200(30)$ & $31(9)$ \\
$\mathrm{H}(30)$ & $11920(50)$ & $4710(30)$ & $10790(30)$ & $39(11)$ \\
$\mathrm{H}(31)$ & $10010(40)$ & $5960(20)$ & $11070(20)$ & $16(7)$ \\
$\mathrm{H}(32)$ & $8610(40)$ & $6830(30)$ & $9810(30)$ & $31(9)$ \\
$\mathrm{H}(1 \mathrm{OW})$ & $6740(90)$ & $1840(60)$ & $7500(50)$ & $108(16)$ \\
$\mathrm{H}(2 \mathrm{OW})$ & $6620(80)$ & $780(60)$ & $7340(50)$ & $108(16)$ \\
$\mathrm{H}(1 \mathrm{OS})$ & $5040(40)$ & $890(30)$ & $8310(30)$ & $28(9)$ \\
\hline
\end{tabular}


$(R)$-Belokon' $(R)$-allo-Threonine complex $[(R)$-BTC, 133]
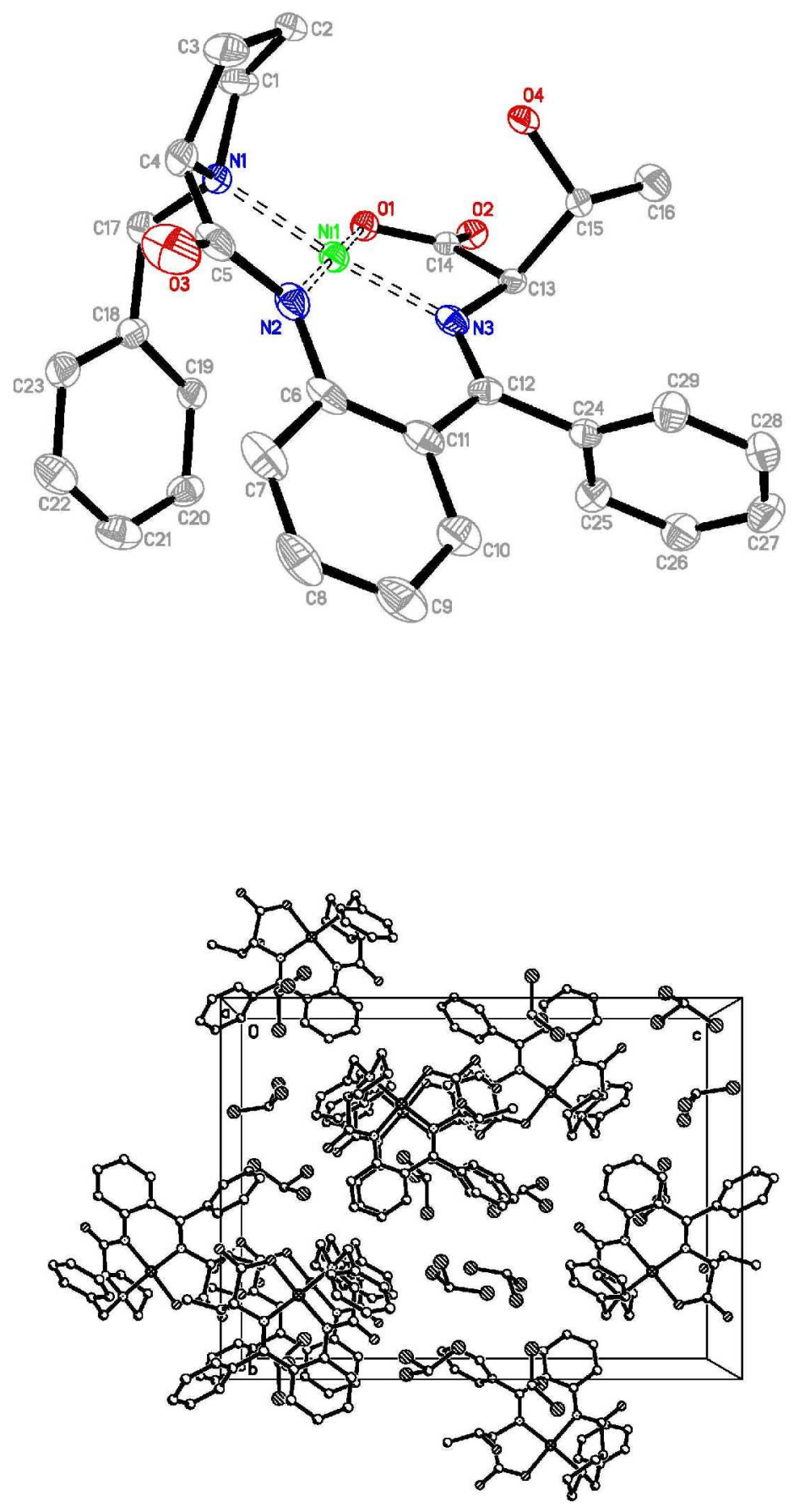
Table 1. Crystal data and structure refinement for $\mathrm{dk} 14$.

Identification code

Empirical formula

Formula weight

Temperature

Wavelength

Crystal system

Space group

Unit cell dimensions

Volume

Z

Density (calculated)

Absorption coefficient

$\mathrm{F}(000)$

Crystal size

Theta range for data collection

Index ranges

Reflections collected

Independent reflections

Completeness to theta $=27.50^{\circ}$

Absorption correction

Max. and min. transmission

Refinement method

Data / restraints / parameters

Goodness-of-fit on $\mathrm{F}^{2}$

Final $\mathrm{R}$ indices [I $>2 \operatorname{sigma}(\mathrm{I})]$

$\mathrm{R}$ indices (all data)

Absolute structure parameter

Largest diff. peak and hole dk14

$\mathrm{C}_{29} \mathrm{H}_{29} \mathrm{~N}_{3} \mathrm{NiO}_{4} \times 3 \mathrm{CHCl}_{3}$

900.37

120(2) K

$0.71073 \AA$

Orthorhombic

$\mathrm{P} 2{ }_{1} 2_{1}$

$\begin{array}{ll}\mathrm{a}=10.3057(3) \AA & \alpha=90^{\circ} . \\ \mathrm{b}=16.3778(5) \AA & \beta=90^{\circ} . \\ \mathrm{c}=22.3882(7) \AA & \gamma=90^{\circ} .\end{array}$

$3778.8(2) \AA^{3}$

4

$1.583 \mathrm{Mg} / \mathrm{m}^{3}$

$1.191 \mathrm{~mm}^{-1}$

1832

$0.46 \times 0.04 \times 0.02 \mathrm{~mm}^{3}$

1.54 to $27.50^{\circ}$.

$-13<=\mathrm{h}<=13,-21<=\mathrm{k}<=21,-29<=1<=29$

39822

$8679[\mathrm{R}($ int $)=0.0734]$

$100.0 \%$

Multi-scan

0.9766 and 0.6104

Full-matrix least-squares on $\mathrm{F}^{2}$

8679 / 0 / 442

1.056

$\mathrm{R}_{1}=0.0523, \mathrm{wR}_{2}=0.1207$

$\mathrm{R}_{1}=0.0763, \mathrm{wR}_{2}=0.1319$

$-0.01(2)$

0.943 and -0.977 e. $\AA^{-3}$ 
Table 2. Atomic coordinates $\left(\times 10^{4}\right)$ and equivalent isotropic displacement parameters $\left(\AA^{2} \times 10^{3}\right)$ for $\mathrm{dk} 14$. $\mathrm{U}(\mathrm{eq})$ is defined as one third of the trace of the orthogonalized $\mathrm{U}^{\mathrm{ij}}$ tensor.

\begin{tabular}{rrrrr}
\hline Atom & $\mathrm{x}$ & $\mathrm{y}$ & $\mathrm{z}$ & $\mathrm{U}(\mathrm{eq})$ \\
\hline $\mathrm{Ni}(1)$ & $1621(1)$ & $7738(1)$ & $1538(1)$ & $18(1)$ \\
$\mathrm{O}(1)$ & $1901(3)$ & $6843(2)$ & $1026(1)$ & $20(1)$ \\
$\mathrm{O}(2)$ & $2393(3)$ & $6539(2)$ & $84(1)$ & $21(1)$ \\
$\mathrm{O}(3)$ & $384(4)$ & $8930(3)$ & $2948(2)$ & $44(1)$ \\
$\mathrm{O}(4)$ & $-288(3)$ & $7772(2)$ & $382(1)$ & $23(1)$ \\
$\mathrm{N}(1)$ & $906(4)$ & $7036(2)$ & $2154(2)$ & $24(1)$ \\
$\mathrm{N}(2)$ & $1504(4)$ & $8592(2)$ & $2075(2)$ & $23(1)$ \\
$\mathrm{N}(3)$ & $2163(3)$ & $8384(2)$ & $907(2)$ & $19(1)$ \\
$\mathrm{C}(1)$ & $-103(5)$ & $6470(4)$ & $1913(2)$ & $36(1)$ \\
$\mathrm{C}(2)$ & $-1229(5)$ & $7041(4)$ & $1784(2)$ & $39(1)$ \\
$\mathrm{C}(3)$ & $-1254(5)$ & $7569(4)$ & $2344(2)$ & $38(1)$ \\
$\mathrm{C}(4)$ & $175(5)$ & $7590(3)$ & $2559(2)$ & $29(1)$ \\
$\mathrm{C}(5)$ & $705(4)$ & $8448(3)$ & $2551(2)$ & $27(1)$ \\
$\mathrm{C}(6)$ & $2226(4)$ & $9319(3)$ & $2030(2)$ & $23(1)$ \\
$\mathrm{C}(7)$ & $2512(5)$ & $9803(3)$ & $2537(2)$ & $31(1)$ \\
$\mathrm{C}(8)$ & $3249(5)$ & $10506(3)$ & $2491(2)$ & $32(1)$ \\
$\mathrm{C}(9)$ & $3713(5)$ & $10775(3)$ & $1944(2)$ & $34(1)$ \\
$\mathrm{C}(10)$ & $3449(5)$ & $10311(3)$ & $1446(2)$ & $28(1)$ \\
$\mathrm{C}(11)$ & $2725(4)$ & $9589(3)$ & $1476(2)$ & $23(1)$ \\
$\mathrm{C}(12)$ & $2582(4)$ & $9127(3)$ & $913(2)$ & $18(1)$ \\
$\mathrm{C}(13)$ & $2014(4)$ & $7946(2)$ & $333(2)$ & $16(1)$ \\
$\mathrm{C}(14)$ & $2107(4)$ & $7037(3)$ & $475(2)$ & $18(1)$ \\
$\mathrm{C}(15)$ & $708(4)$ & $8148(3)$ & $40(2)$ & $19(1)$ \\
$\mathrm{C}(16)$ & $668(5)$ & $7890(3)$ & $-609(2)$ & $28(1)$ \\
$\mathrm{C}(17)$ & $1948(4)$ & $6563(3)$ & $2472(2)$ & $25(1)$ \\
$\mathrm{C}(18)$ & $3116(4)$ & $7075(3)$ & $2621(2)$ & $20(1)$ \\
$\mathrm{C}(19)$ & $4111(4)$ & $7139(3)$ & $2199(2)$ & $22(1)$ \\
$\mathrm{C}(20)$ & $5197(4)$ & $7616(3)$ & $2317(2)$ & $25(1)$ \\
$\mathrm{C}(21)$ & $5293(5)$ & $8037(3)$ & $2853(2)$ & $32(1)$ \\
$\mathrm{C}(22)$ & $4322(5)$ & $7973(3)$ & $3268(2)$ & $30(1)$ \\
$\mathrm{C}(23)$ & $3227(4)$ & $7486(3)$ & $3154(2)$ & $25(1)$ \\
$\mathrm{C}(24)$ & $2990(4)$ & $9539(3)$ & $347(2)$ & $20(1)$ \\
$\mathrm{C}(25)$ & $4193(5)$ & $9347(3)$ & $99(2)$ & $23(1)$ \\
$\mathrm{C}(26)$ & $4582(5)$ & $9712(3)$ & $-427(2)$ & $30(1)$ \\
$\mathrm{C}(27)$ & $3791(5)$ & $10279(3)$ & $-705(2)$ & $37(1)$ \\
$\mathrm{C}(28)$ & $2598(5)$ & $10470(3)$ & $-452(2)$ & $36(1)$ \\
$\mathrm{C}(29)$ & $2207(5)$ & $10109(3)$ & $72(2)$ & $30(1)$ \\
$\mathrm{C}(1 \mathrm{~S})$ & $-2507(5)$ & $9689(3)$ & $1036(3)$ & $37(1)$ \\
$\mathrm{Cl}(1)$ & $-3508(2)$ & $9117(1)$ & $1511(1)$ & $53(1)$ \\
$\mathrm{Cl}(2)$ & $-878(2)$ & $9638(1)$ & $1264(1)$ & $52(1)$ \\
$\mathrm{Cl})$ & $-3050(1)$ & $10702(1)$ & $1021(1)$ & $45(1)$ \\
$\mathrm{Cl})$ & $3213(5)$ & $4962(3)$ & $1011(2)$ & $32(1)$ \\
$\mathrm{C}(5)(1)$ & $4515(1)$ & $1453(1)$ & $31(1)$ \\
$\mathrm{C}(3)(1)$ & $5146(1)$ & $1447(1)$ & $43(1)$ \\
$\mathrm{C}(3)$ & $4345(1)$ & $401(1)$ & $53(1)$ \\
& & & $613(3)$ & $56(2)$
\end{tabular}




\begin{tabular}{crrrr}
\hline Atom & $\mathrm{x}$ & $\mathrm{y}$ & $\mathrm{z}$ & $\mathrm{U}(\mathrm{eq})$ \\
\hline $\mathrm{Cl}(7)$ & $2278(3)$ & $2266(2)$ & $926(1)$ & $97(1)$ \\
$\mathrm{Cl}(8)$ & $-368(3)$ & $1910(2)$ & $776(1)$ & $126(1)$ \\
$\mathrm{Cl}(9)$ & $948(4)$ & $2770(2)$ & $-127(1)$ & $158(2)$ \\
\hline
\end{tabular}

Table 3. Selected bond lengths $[\AA]$ and angles $\left[{ }^{\circ}\right]$ for $\mathrm{dk} 14$.

\begin{tabular}{|c|c|c|c|c|}
\hline $\mathrm{Ni}(1)-\mathrm{N}(2)$ & $1.848(4)$ & $C(4)-C(5)$ & $1.508(7)$ & $C(21)-C(22)$ \\
\hline $\mathrm{Ni}(1)-\mathrm{N}(3)$ & $1.853(4)$ & $C(6)-C(7)$ & $1.414(6)$ & $\mathrm{C}(22)-\mathrm{C}(23)$ \\
\hline $\mathrm{Ni}(1)-\mathrm{O}(1)$ & $1.883(3)$ & $C(6)-C(11)$ & $1.415(7)$ & $C(24)-C(29)$ \\
\hline $\mathrm{Ni}(1)-\mathrm{N}(1)$ & $1.940(4)$ & $\mathrm{C}(7)-\mathrm{C}(8)$ & $1.384(7)$ & $C(24)-C(25)$ \\
\hline $\mathrm{O}(1)-\mathrm{C}(14)$ & $1.291(5)$ & $\mathrm{C}(8)-\mathrm{C}(9)$ & $1.386(8)$ & $C(25)-C(26)$ \\
\hline $\mathrm{O}(2)-\mathrm{C}(14)$ & $1.231(5)$ & $C(9)-C(10)$ & $1.377(6)$ & $C(26)-C(27)$ \\
\hline $\mathrm{O}(3)-\mathrm{C}(5)$ & $1.235(6)$ & $C(10)-C(11)$ & $1.399(6)$ & $\mathrm{C}(27)-\mathrm{C}(28)$ \\
\hline $\mathrm{O}(4)-\mathrm{C}(15)$ & $1.421(5)$ & $C(11)-C(12)$ & $1.478(6)$ & $\mathrm{C}(28)-\mathrm{C}(29)$ \\
\hline $\mathrm{N}(1)-\mathrm{C}(4)$ & $1.487(6)$ & $C(12)-C(24)$ & $1.496(6)$ & $\mathrm{C}(1 \mathrm{~S})-\mathrm{Cl}(3)$ \\
\hline $\mathrm{N}(1)-\mathrm{C}(1)$ & $1.495(6)$ & $C(13)-C(14)$ & $1.526(6)$ & $\mathrm{C}(1 \mathrm{~S})-\mathrm{Cl}(1)$ \\
\hline $\mathrm{N}(1)-\mathrm{C}(17)$ & $1.503(6)$ & $C(13)-C(15)$ & $1.533(6)$ & $\mathrm{C}(1 \mathrm{~S})-\mathrm{Cl}(2)$ \\
\hline $\mathrm{N}(2)-\mathrm{C}(5)$ & $1.367(6)$ & $C(15)-C(16)$ & $1.513(6)$ & $\mathrm{C}(2 \mathrm{~S})-\mathrm{Cl}(6)$ \\
\hline $\mathrm{N}(2)-\mathrm{C}(6)$ & $1.407(6)$ & $\mathrm{C}(17)-\mathrm{C}(18)$ & $1.506(6)$ & $\mathrm{C}(2 \mathrm{~S})-\mathrm{Cl}(5)$ \\
\hline $\mathrm{N}(3)-\mathrm{C}(12)$ & $1.290(6)$ & $C(18)-C(23)$ & $1.375(6)$ & $\mathrm{C}(2 \mathrm{~S})-\mathrm{Cl}(4)$ \\
\hline$N(3)-C(13)$ & $1.480(5)$ & $\mathrm{C}(18)-\mathrm{C}(19)$ & $1.397(6)$ & $\mathrm{C}(3 \mathrm{~S})-\mathrm{Cl}(9)$ \\
\hline $\mathrm{C}(1)-\mathrm{C}(2)$ & $1.519(8)$ & $C(19)-C(20)$ & $1.391(6)$ & $\mathrm{C}(3 \mathrm{~S})-\mathrm{Cl}(7)$ \\
\hline$C(2)-C(3)$ & $1.522(8)$ & $\mathrm{C}(20)-\mathrm{C}(21)$ & $1.388(7)$ & $\mathrm{C}(3 \mathrm{~S})-\mathrm{Cl}(8)$ \\
\hline$C(3)-C(4)$ & $1.550(7)$ & & & \\
\hline \multicolumn{2}{|c|}{$\mathrm{N}(2)-\mathrm{Ni}(1)-\mathrm{N}(3)$} & $94.71(16)$ & $\mathrm{N}(3)-\mathrm{C}(12)-\mathrm{C}(11)$ & $121.6(4$ \\
\hline \multicolumn{2}{|c|}{$\mathrm{N}(2)-\mathrm{Ni}(1)-\mathrm{O}(1)$} & $174.34(15)$ & $\mathrm{N}(3)-\mathrm{C}(12)-\mathrm{C}(24)$ & $120.8(4$ \\
\hline \multicolumn{2}{|c|}{$\mathrm{N}(3)-\mathrm{Ni}(1)-\mathrm{O}(1)$} & $86.21(14)$ & $\mathrm{C}(11)-\mathrm{C}(12)-\mathrm{C}(24)$ & $117.6(4)$ \\
\hline \multicolumn{2}{|c|}{$\mathrm{N}(2)-\mathrm{Ni}(1)-\mathrm{N}(1)$} & $87.83(17)$ & $\mathrm{N}(3)-\mathrm{C}(13)-\mathrm{C}(14)$ & $106.6(3)$ \\
\hline \multicolumn{2}{|c|}{$\mathrm{N}(3)-\mathrm{Ni}(1)-\mathrm{N}(1)$} & $174.48(16)$ & $\mathrm{N}(3)-\mathrm{C}(13)-\mathrm{C}(15)$ & $111.0(3)$ \\
\hline \multicolumn{2}{|c|}{$\mathrm{O}(1)-\mathrm{Ni}(1)-\mathrm{N}(1)$} & $91.75(15)$ & $\mathrm{C}(14)-\mathrm{C}(13)-\mathrm{C}(15)$ & $110.8(3)$ \\
\hline \multicolumn{2}{|c|}{$\mathrm{C}(14)-\mathrm{O}(1)-\mathrm{Ni}(1)$} & $114.6(3)$ & $\mathrm{O}(2)-\mathrm{C}(14)-\mathrm{O}(1)$ & $123.7(4)$ \\
\hline \multicolumn{2}{|c|}{$\mathrm{C}(15)-\mathrm{O}(4)-\mathrm{H}(4 \mathrm{O})$} & 109.5 & $\mathrm{O}(2)-\mathrm{C}(14)-\mathrm{C}(13)$ & $120.9(4)$ \\
\hline \multicolumn{2}{|c|}{$\mathrm{C}(4)-\mathrm{N}(1)-\mathrm{C}(1)$} & $104.3(4)$ & $\mathrm{O}(1)-\mathrm{C}(14)-\mathrm{C}(13)$ & $115.4(4)$ \\
\hline \multicolumn{2}{|c|}{$\mathrm{C}(4)-\mathrm{N}(1)-\mathrm{C}(17)$} & $112.9(3)$ & $\mathrm{O}(4)-\mathrm{C}(15)-\mathrm{C}(16)$ & $112.1(4)$ \\
\hline \multicolumn{2}{|c|}{$\mathrm{C}(1)-\mathrm{N}(1)-\mathrm{C}(17)$} & $110.3(4)$ & $\mathrm{O}(4)-\mathrm{C}(15)-\mathrm{C}(13)$ & $108.0(3)$ \\
\hline \multicolumn{2}{|c|}{$\mathrm{C}(4)-\mathrm{N}(1)-\mathrm{Ni}(1)$} & $105.3(3)$ & $C(16)-C(15)-C(13)$ & $112.0(4$ \\
\hline \multicolumn{2}{|c|}{$\mathrm{C}(1)-\mathrm{N}(1)-\mathrm{Ni}(1)$} & $112.0(3)$ & $\mathrm{C}(18)-\mathrm{C}(17)-\mathrm{N}(1)$ & $112.9(4$ \\
\hline \multicolumn{2}{|c|}{$\mathrm{C}(17)-\mathrm{N}(1)-\mathrm{Ni}(1)$} & $111.7(3)$ & $\mathrm{C}(23)-\mathrm{C}(18)-\mathrm{C}(19)$ & $119.3(4)$ \\
\hline \multicolumn{2}{|c|}{$\mathrm{C}(5)-\mathrm{N}(2)-\mathrm{C}(6)$} & $121.3(4)$ & $\mathrm{C}(23)-\mathrm{C}(18)-\mathrm{C}(17)$ & $122.1(4)$ \\
\hline \multicolumn{2}{|c|}{$\mathrm{C}(5)-\mathrm{N}(2)-\mathrm{Ni}(1)$} & $114.5(3)$ & $\mathrm{C}(19)-\mathrm{C}(18)-\mathrm{C}(17)$ & $118.6(4$ \\
\hline \multicolumn{2}{|c|}{$\mathrm{C}(6)-\mathrm{N}(2)-\mathrm{Ni}(1)$} & $124.1(3)$ & $\mathrm{C}(20)-\mathrm{C}(19)-\mathrm{C}(18)$ & $120.3(4$ \\
\hline \multicolumn{2}{|c|}{$\mathrm{C}(12)-\mathrm{N}(3)-\mathrm{C}(13)$} & $120.0(4)$ & $C(21)-C(20)-C(19)$ & $120.0(4$ \\
\hline \multicolumn{2}{|c|}{$\mathrm{C}(12)-\mathrm{N}(3)-\mathrm{Ni}(1)$} & $129.2(3)$ & $\mathrm{C}(22)-\mathrm{C}(21)-\mathrm{C}(20)$ & $119.8(5$ \\
\hline \multicolumn{2}{|c|}{$\mathrm{C}(13)-\mathrm{N}(3)-\mathrm{Ni}(1)$} & $110.7(3)$ & $C(21)-C(22)-C(23)$ & $120.5(4$ \\
\hline \multicolumn{2}{|c|}{$\mathrm{N}(1)-\mathrm{C}(1)-\mathrm{C}(2)$} & $102.6(5)$ & $\mathrm{C}(18)-\mathrm{C}(23)-\mathrm{C}(22)$ & $120.1(4$ \\
\hline \multicolumn{2}{|c|}{$\mathrm{C}(1)-\mathrm{C}(2)-\mathrm{C}(3)$} & $101.9(4)$ & $C(29)-C(24)-C(25)$ & $119.7(4$ \\
\hline
\end{tabular}

$1.370(7)$ $1.405(7)$ $1.378(6)$ $1.395(6)$ 1.380(6) $1.384(7)$ $1.390(8)$ $1.374(7)$ $1.750(5)$ 1.754(6) $1.757(6)$ $1.746(5)$ $1.759(5)$ $1.764(5)$ $1.680(8)$ $1.720(7)$ 1.744(7) 


$\begin{array}{rrrr}\mathrm{C}(2)-\mathrm{C}(3)-\mathrm{C}(4) & 104.7(4) & \mathrm{C}(29)-\mathrm{C}(24)-\mathrm{C}(12) & 121.3(4) \\ \mathrm{N}(1)-\mathrm{C}(4)-\mathrm{C}(5) & 112.1(4) & \mathrm{C}(25)-\mathrm{C}(24)-\mathrm{C}(12) & 119.0(4) \\ \mathrm{N}(1)-\mathrm{C}(4)-\mathrm{C}(3) & 106.1(4) & \mathrm{C}(26)-\mathrm{C}(25)-\mathrm{C}(24) & 120.0(5) \\ \mathrm{C}(5)-\mathrm{C}(4)-\mathrm{C}(3) & 111.2(4) & \mathrm{C}(25)-\mathrm{C}(26)-\mathrm{C}(27) & 120.3(5) \\ \mathrm{O}(3)-\mathrm{C}(5)-\mathrm{N}(2) & 127.8(5) & \mathrm{C}(26)-\mathrm{C}(27)-\mathrm{C}(28) & 119.2(5) \\ \mathrm{O}(3)-\mathrm{C}(5)-\mathrm{C}(4) & 119.3(4) & \mathrm{C}(29)-\mathrm{C}(28)-\mathrm{C}(27) & 120.8(5) \\ \mathrm{N}(2)-\mathrm{C}(5)-\mathrm{C}(4) & 112.9(4) & \mathrm{C}(28)-\mathrm{C}(29)-\mathrm{C}(24) & 120.0(5) \\ \mathrm{N}(2)-\mathrm{C}(6)-\mathrm{C}(7) & 121.8(4) & \mathrm{Cl}(3)-\mathrm{C}(1 \mathrm{~S})-\mathrm{Cl}(1) & 109.3(3) \\ \mathrm{N}(2)-\mathrm{C}(6)-\mathrm{C}(11) & 121.2(4) & \mathrm{Cl}(3)-\mathrm{C}(1 \mathrm{~S})-\mathrm{Cl}(2) & 110.9(3) \\ \mathrm{C}(7)-\mathrm{C}(6)-\mathrm{C}(11) & 116.9(4) & \mathrm{Cl}(1)-\mathrm{C}(1 \mathrm{~S})-\mathrm{Cl}(2) & 111.1(3) \\ \mathrm{C}(8)-\mathrm{C}(7)-\mathrm{C}(6) & 121.4(5) & \mathrm{Cl}(6)-\mathrm{C}(2 \mathrm{~S})-\mathrm{Cl}(5) & 110.3(3) \\ \mathrm{C}(7)-\mathrm{C}(8)-\mathrm{C}(9) & 121.4(4) & \mathrm{Cl}(6)-\mathrm{C}(2 \mathrm{~S})-\mathrm{Cl}(4) & 111.2(3) \\ \mathrm{C}(10)-\mathrm{C}(9)-\mathrm{C}(8) & 118.1(5) & \mathrm{Cl}(5)-\mathrm{C}(2 \mathrm{~S})-\mathrm{Cl}(4) & 110.1(3) \\ \mathrm{C}(9)-\mathrm{C}(10)-\mathrm{C}(11) & 122.2(5) & \mathrm{Cl}(9)-\mathrm{C}(3 \mathrm{~S})-\mathrm{Cl}(7) & 113.9(5) \\ \mathrm{C}(10)-\mathrm{C}(11)-\mathrm{C}(6) & 120.0(4) & \mathrm{Cl}(9)-\mathrm{C}(3 \mathrm{~S})-\mathrm{Cl}(8) & 110.5(4) \\ \mathrm{C}(10)-\mathrm{C}(11)-\mathrm{C}(12) & 116.5(4) & \mathrm{Cl}(7)-\mathrm{C}(3 \mathrm{~S})-\mathrm{Cl}(8) & 108.3(4) \\ \mathrm{C}(6)-\mathrm{C}(11)-\mathrm{C}(12) & 123.5(4) & & \end{array}$

Table 4. Anisotropic displacement parameters $\left(\AA^{2} \times 10^{3}\right)$ for $\mathrm{dk} 14$. The anisotropic displacement factor exponent takes the form: $-2 \pi^{2}\left[h^{2} a^{* 2} U^{11}+\ldots+2 h k a^{*} b^{*} U^{12}\right]$

\begin{tabular}{rrrrrrr}
\hline Atom & $\mathrm{U}^{11}$ & $\mathrm{U}^{22}$ & $\mathrm{U}^{33}$ & $\mathrm{U}^{23}$ & $\mathrm{U}^{13}$ & $\mathrm{U}^{12}$ \\
\hline $\mathrm{Ni}(1)$ & $16(1)$ & $25(1)$ & $14(1)$ & $-1(1)$ & $1(1)$ & $0(1)$ \\
$\mathrm{O}(1)$ & $19(2)$ & $25(2)$ & $16(1)$ & $1(1)$ & $1(1)$ & $-1(1)$ \\
$\mathrm{O}(2)$ & $22(2)$ & $19(2)$ & $21(2)$ & $-3(1)$ & $2(1)$ & $-1(1)$ \\
$\mathrm{O}(3)$ & $28(2)$ & $72(3)$ & $32(2)$ & $-27(2)$ & $9(2)$ & $3(2)$ \\
$\mathrm{O}(4)$ & $14(1)$ & $33(2)$ & $21(1)$ & $4(1)$ & $-2(1)$ & $-1(1)$ \\
$\mathrm{N}(1)$ & $17(2)$ & $40(2)$ & $15(2)$ & $4(2)$ & $0(2)$ & $-6(2)$ \\
$\mathrm{N}(2)$ & $19(2)$ & $32(2)$ & $18(2)$ & $-1(2)$ & $-1(2)$ & $4(2)$ \\
$\mathrm{N}(3)$ & $14(2)$ & $24(2)$ & $20(2)$ & $-4(2)$ & $-1(2)$ & $3(2)$ \\
$\mathrm{C}(1)$ & $20(2)$ & $60(4)$ & $28(3)$ & $-2(2)$ & $-1(2)$ & $-16(2)$ \\
$\mathrm{C}(2)$ & $18(2)$ & $75(4)$ & $25(2)$ & $17(3)$ & $-7(2)$ & $-16(2)$ \\
$\mathrm{C}(3)$ & $15(2)$ & $57(4)$ & $44(3)$ & $14(3)$ & $0(2)$ & $0(2)$ \\
$\mathrm{C}(4)$ & $22(2)$ & $46(3)$ & $18(2)$ & $4(2)$ & $3(2)$ & $-3(2)$ \\
$\mathrm{C}(5)$ & $17(2)$ & $43(3)$ & $20(2)$ & $-8(2)$ & $-2(2)$ & $5(2)$ \\
$\mathrm{C}(6)$ & $16(2)$ & $30(2)$ & $23(2)$ & $-11(2)$ & $-6(2)$ & $7(2)$ \\
$\mathrm{C}(7)$ & $28(3)$ & $37(3)$ & $27(3)$ & $-11(2)$ & $-8(2)$ & $11(2)$ \\
$\mathrm{C}(8)$ & $39(3)$ & $29(2)$ & $29(2)$ & $-13(2)$ & $-15(2)$ & $13(2)$ \\
$\mathrm{C}(9)$ & $36(3)$ & $26(2)$ & $38(3)$ & $-12(2)$ & $-14(2)$ & $4(2)$ \\
$\mathrm{C}(10)$ & $27(2)$ & $23(2)$ & $32(2)$ & $-4(2)$ & $-5(2)$ & $3(2)$ \\
$\mathrm{C}(11)$ & $17(2)$ & $25(2)$ & $25(2)$ & $-8(2)$ & $-4(2)$ & $4(2)$ \\
$\mathrm{C}(12)$ & $12(2)$ & $19(2)$ & $24(2)$ & $-1(2)$ & $0(2)$ & $1(2)$ \\
$\mathrm{C}(13)$ & $15(2)$ & $19(2)$ & $15(2)$ & $-1(2)$ & $0(2)$ & $-3(2)$ \\
$\mathrm{C}(14)$ & $14(2)$ & $22(2)$ & $17(2)$ & $2(2)$ & $-1(2)$ & $-5(2)$ \\
$\mathrm{C}(15)$ & $16(2)$ & $22(2)$ & $19(2)$ & $5(2)$ & $-2(2)$ & $-2(2)$ \\
$\mathrm{C}(16)$ & $25(2)$ & $37(3)$ & $22(2)$ & $-3(2)$ & $1(2)$ & $4(2)$ \\
$\mathrm{C}(17)$ & $22(2)$ & $30(2)$ & $23(2)$ & $1(2)$ & $0(2)$ & $-2(2)$ \\
$\mathrm{C}(18)$ & $16(2)$ & $20(2)$ & $25(2)$ & $6(2)$ & $-2(2)$ & $2(2)$
\end{tabular}




\begin{tabular}{rrrrrrr}
\hline Atom & $\mathrm{U}^{11}$ & $\mathrm{U}^{22}$ & $\mathrm{U}^{33}$ & $\mathrm{U}^{23}$ & $\mathrm{U}^{13}$ & $\mathrm{U}^{12}$ \\
\hline $\mathrm{C}(19)$ & $17(2)$ & $30(2)$ & $19(2)$ & $4(2)$ & $0(2)$ & $1(2)$ \\
$\mathrm{C}(20)$ & $18(2)$ & $24(2)$ & $32(2)$ & $4(2)$ & $1(2)$ & $1(2)$ \\
$\mathrm{C}(21)$ & $22(2)$ & $30(3)$ & $44(3)$ & $-2(2)$ & $-7(2)$ & $0(2)$ \\
$\mathrm{C}(22)$ & $31(3)$ & $27(3)$ & $32(3)$ & $-8(2)$ & $-6(2)$ & $2(2)$ \\
$\mathrm{C}(23)$ & $21(2)$ & $31(2)$ & $23(2)$ & $3(2)$ & $2(2)$ & $1(2)$ \\
$\mathrm{C}(24)$ & $21(2)$ & $18(2)$ & $19(2)$ & $-1(2)$ & $-4(2)$ & $-4(2)$ \\
$\mathrm{C}(25)$ & $26(2)$ & $21(2)$ & $23(2)$ & $-2(2)$ & $2(2)$ & $-1(2)$ \\
$\mathrm{C}(26)$ & $26(3)$ & $39(3)$ & $25(2)$ & $-2(2)$ & $4(2)$ & $-1(2)$ \\
$\mathrm{C}(27)$ & $42(3)$ & $42(3)$ & $28(3)$ & $8(2)$ & $0(2)$ & $-14(2)$ \\
$\mathrm{C}(28)$ & $31(3)$ & $35(3)$ & $41(3)$ & $16(2)$ & $-13(2)$ & $-2(2)$ \\
$\mathrm{C}(29)$ & $31(3)$ & $25(2)$ & $33(3)$ & $5(2)$ & $-4(2)$ & $1(2)$ \\
$\mathrm{C}(1 \mathrm{~S})$ & $41(3)$ & $30(3)$ & $40(3)$ & $-7(2)$ & $-2(3)$ & $4(2)$ \\
$\mathrm{Cl}(1)$ & $52(1)$ & $45(1)$ & $64(1)$ & $21(1)$ & $2(1)$ & $-5(1)$ \\
$\mathrm{Cl}(2)$ & $33(1)$ & $62(1)$ & $62(1)$ & $4(1)$ & $0(1)$ & $12(1)$ \\
$\mathrm{Cl}(3)$ & $41(1)$ & $27(1)$ & $67(1)$ & $8(1)$ & $7(1)$ & $3(1)$ \\
$\mathrm{C}(2 \mathrm{~S})$ & $29(3)$ & $24(2)$ & $43(3)$ & $7(2)$ & $-8(2)$ & $1(2)$ \\
$\mathrm{Cl}(4)$ & $27(1)$ & $31(1)$ & $33(1)$ & $6(1)$ & $1(1)$ & $1(1)$ \\
$\mathrm{Cl}(5)$ & $29(1)$ & $35(1)$ & $65(1)$ & $-5(1)$ & $-10(1)$ & $-4(1)$ \\
$\mathrm{Cl}(6)$ & $56(1)$ & $66(1)$ & $37(1)$ & $-10(1)$ & $13(1)$ & $-11(1)$ \\
$\mathrm{C}(3 \mathrm{~S})$ & $50(4)$ & $39(3)$ & $80(5)$ & $8(3)$ & $-1(4)$ & $-8(3)$ \\
$\mathrm{Cl}(7)$ & $103(2)$ & $75(1)$ & $112(2)$ & $-16(1)$ & $-50(2)$ & $35(1)$ \\
$\mathrm{Cl}(8)$ & $132(2)$ & $161(3)$ & $85(2)$ & $-77(2)$ & $71(2)$ & $-107(2)$ \\
$\mathrm{Cl}(9)$ & $175(3)$ & $186(3)$ & $114(2)$ & $104(2)$ & $-52(2)$ & $-100(3)$ \\
\hline
\end{tabular}

Table 5. Hydrogen coordinates $\left(\times 10^{4}\right)$ and isotropic displacement parameters $\left(\AA^{2} \times 10^{3}\right)$ for dk14.

\begin{tabular}{rrrrr}
\hline Atom & \multicolumn{1}{c}{$\mathrm{x}$} & \multicolumn{1}{c}{$\mathrm{y}$} & \multicolumn{1}{c}{$\mathrm{z}$} & $\mathrm{U}(\mathrm{eq})$ \\
\hline $\mathrm{H}(4 \mathrm{O})$ & -1015 & 7901 & 241 & 27 \\
$\mathrm{H}(1 \mathrm{~A})$ & -347 & 6050 & 2211 & 43 \\
$\mathrm{H}(1 \mathrm{~B})$ & 201 & 6196 & 1544 & 43 \\
$\mathrm{H}(2 \mathrm{~A})$ & -2052 & 6736 & 1734 & 47 \\
$\mathrm{H}(2 \mathrm{~B})$ & -1067 & 7373 & 1422 & 47 \\
$\mathrm{H}(3 \mathrm{~A})$ & -1824 & 7325 & 2652 & 46 \\
$\mathrm{H}(3 \mathrm{~B})$ & -1568 & 8125 & 2251 & 46 \\
$\mathrm{H}(4 \mathrm{~A})$ & 220 & 7372 & 2976 & 34 \\
$\mathrm{H}(7 \mathrm{~A})$ & 2191 & 9641 & 2917 & 37 \\
$\mathrm{H}(8 \mathrm{~A})$ & 3440 & 10811 & 2841 & 39 \\
$\mathrm{H}(9 \mathrm{~A})$ & 4201 & 11266 & 1914 & 40 \\
$\mathrm{H}(10 \mathrm{~A})$ & 3769 & 10487 & 1069 & 33 \\
$\mathrm{H}(13 \mathrm{~A})$ & 2736 & 8103 & 58 & 19 \\
$\mathrm{H}(15 \mathrm{~A})$ & 580 & 8753 & 58 & 23 \\
$\mathrm{H}(16 \mathrm{~A})$ & -178 & 8032 & -780 & 42 \\
$\mathrm{H}(16 \mathrm{~B})$ & 801 & 7299 & -637 & 42 \\
$\mathrm{H}(16 \mathrm{C})$ & 1355 & 8173 & -830 & 42 \\
$\mathrm{H}(17 \mathrm{~A})$ & 1585 & 6333 & 2845 & 30
\end{tabular}




\begin{tabular}{crrrr}
\hline Atom & $x$ & $y$ & \multicolumn{1}{c}{ z } & $\mathrm{U}(\mathrm{eq})$ \\
\hline $\mathrm{H}(17 \mathrm{~B})$ & 2222 & 6101 & 2216 & 30 \\
$\mathrm{H}(19 \mathrm{~A})$ & 4044 & 6855 & 1831 & 26 \\
$\mathrm{H}(20 \mathrm{~A})$ & 5873 & 7654 & 2030 & 30 \\
$\mathrm{H}(21 \mathrm{~A})$ & 6029 & 8369 & 2932 & 38 \\
$\mathrm{H}(22 \mathrm{~A})$ & 4390 & 8259 & 3636 & 36 \\
$\mathrm{H}(23 \mathrm{~A})$ & 2561 & 7441 & 3446 & 30 \\
$\mathrm{H}(25 \mathrm{~A})$ & 4745 & 8964 & 292 & 28 \\
$\mathrm{H}(26 \mathrm{~A})$ & 5396 & 9574 & -598 & 36 \\
$\mathrm{H}(27 \mathrm{~A})$ & 4060 & 10535 & -1065 & 45 \\
$\mathrm{H}(28 \mathrm{~A})$ & 2047 & 10854 & -643 & 43 \\
$\mathrm{H}(29 \mathrm{~A})$ & 1396 & 10252 & 245 & 36 \\
$\mathrm{H}(1 \mathrm{SA})$ & -2575 & 9459 & 623 & 44 \\
$\mathrm{H}(2 \mathrm{SA})$ & 2886 & 5497 & 858 & 38 \\
$\mathrm{H}(3 \mathrm{SA})$ & 627 & 3141 & 808 & 67 \\
\hline
\end{tabular}

Table 6. Hydrogen bonds for $\mathrm{dk} 14$ [A and $\left.{ }^{\circ}\right]$.

\begin{tabular}{rrrrr}
\hline D-H...A & d(D-H) & d(H...A $)$ & d(D...A $)$ & $<$ (DHA) \\
\hline $\mathrm{O}(4)-\mathrm{H}(4 \mathrm{O}) \ldots \mathrm{O}(2) \# 1$ & 0.84 & 2.02 & $2.842(4)$ & 167.4 \\
\hline
\end{tabular}

Symmetry transformations used to generate equivalent atoms:

$\# 1 x-1 / 2,-y+3 / 2,-z$ 
Belokon' (2S,3S)- $\beta$-methylphenylalanine complex
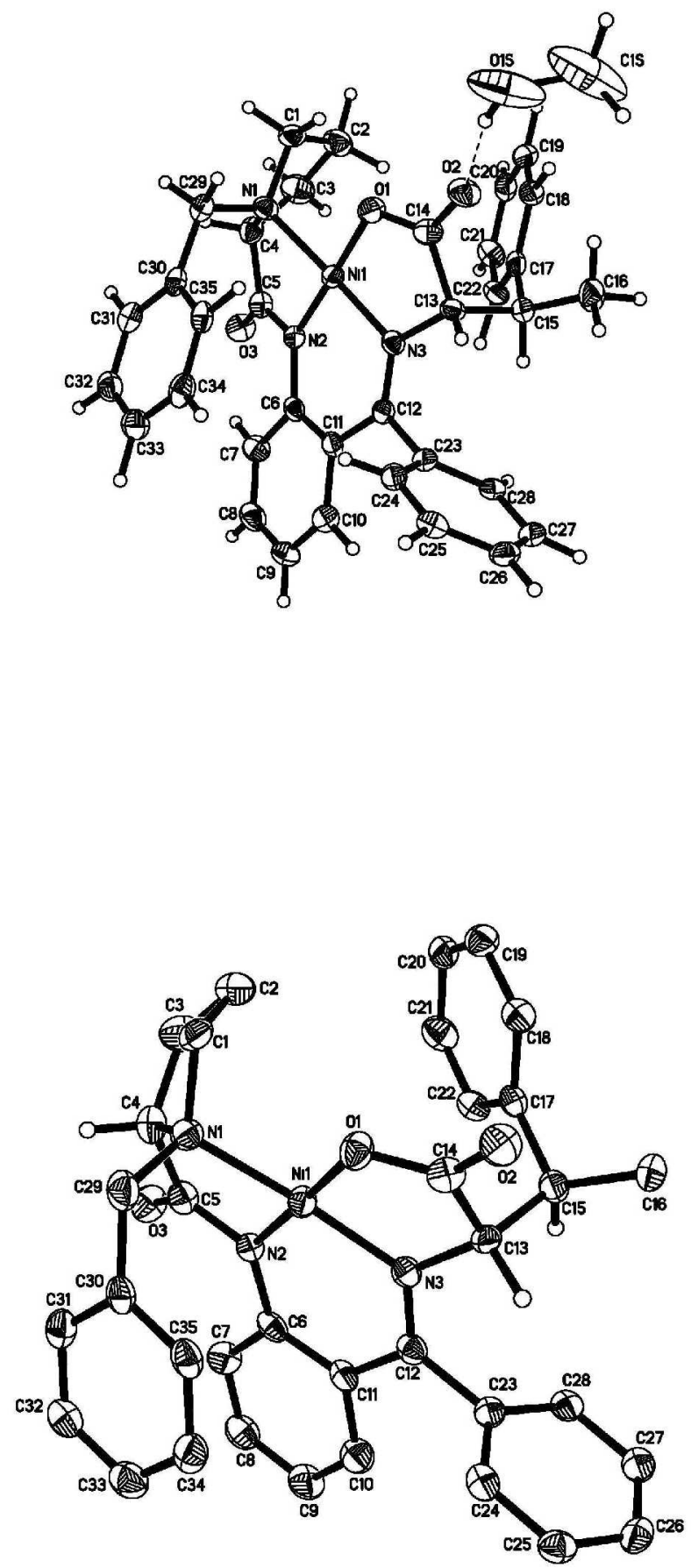
Table 1. Crystal data and structure refinement for dk24.

Identification code

dk24

Empirical formula

$\mathrm{C}_{35} \mathrm{H}_{33} \mathrm{~N}_{3} \mathrm{NiO}_{3} \times \mathrm{CH}_{3} \mathrm{OH}$

Formula weight

634.40

Temperature

$120(2) \mathrm{K}$

Wavelength

$0.71073 \AA$

Crystal system

Orthorhombic

Space group

Unit cell dimensions

P $22_{1} 2_{1}$

Volume

$\mathrm{a}=8.5832(5) \AA$

$\alpha=90^{\circ}$.

$\mathrm{b}=15.6673(9) \AA$

$\beta=90^{\circ}$.

$\mathrm{c}=23.0536(14) \AA$ $\gamma=90^{\circ}$.

Z

3100.1(3) $\AA^{3}$

Density (calculated)

4

Absorption coefficient

$1.359 \mathrm{Mg} / \mathrm{m}^{3}$

$0.670 \mathrm{~mm}^{-1}$

$\mathrm{F}(000)$

1336

Crystal size

$0.44 \times 0.05 \times 0.03 \mathrm{~mm}^{3}$

Theta range for data collection

1.57 to $27.50^{\circ}$.

Index ranges

$-11<=\mathrm{h}<=11,-20<=\mathrm{k}<=20,-29<=1<=29$

Reflections collected

30404

Independent reflections

$7118[\mathrm{R}(\mathrm{int})=0.1278]$

Completeness to theta $=27.50^{\circ}$

Absorption correction

$100.0 \%$

Max. and min. transmission

Refinement method

None

0.9802 and 0.7569

Full-matrix least-squares on $\mathrm{F}^{2}$

Data / restraints / parameters

$7118 / 0 / 530$

Goodness-of-fit on $\mathrm{F}^{2}$

0.880

Final $\mathrm{R}$ indices [I $>2 \operatorname{sigma}(\mathrm{I})]$

$\mathrm{R}_{1}=0.0412, \mathrm{wR}_{2}=0.0691$

$\mathrm{R}$ indices (all data)

$\mathrm{R}_{1}=0.0805, \mathrm{wR}_{2}=0.0785$

Absolute structure parameter

$-0.003(13)$

Largest diff. peak and hole

0.432 and -0.449 e. $\AA^{-3}$ 
Table 2. Atomic coordinates $\left(\times 10^{4}\right)$ and equivalent isotropic displacement parameters $\left(\AA^{2} \times 10^{3}\right)$ for $\mathrm{dk} 24$. $\mathrm{U}(\mathrm{eq})$ is defined as one third of the trace of the orthogonalized $\mathrm{U}^{\mathrm{ij}}$ tensor.

$\begin{array}{rrrrr}\text { Atom } & \mathrm{X} & \mathrm{y} & \mathrm{z} & \mathrm{U}(\mathrm{eq}) \\ \mathrm{Ni}(1) & 2054(1) & 9205(1) & 8575(1) & 20(1) \\ \mathrm{O}(1) & 988(3) & 8645(2) & 7982(1) & 26(1) \\ \mathrm{O}(2) & -724(3) & 8848(2) & 7268(1) & 31(1) \\ \mathrm{O}(3) & 5523(3) & 9569(2) & 9650(1) & 32(1) \\ \mathrm{N}(1) & 3170(3) & 8165(2) & 8772(1) & 23(1) \\ \mathrm{N}(2) & 3123(3) & 9736(2) & 9178(1) & 20(1) \\ \mathrm{N}(3) & 791(3) & 10126(2) & 8417(1) & 20(1) \\ \mathrm{C}(1) & 3683(4) & 7635(2) & 8268(2) & 28(1) \\ \mathrm{C}(2) & 4977(5) & 8140(3) & 8009(2) & 34(1) \\ \mathrm{C}(3) & 5862(4) & 8458(3) & 8549(2) & 40(1) \\ \mathrm{C}(4) & 4656(4) & 8460(2) & 9041(2) & 27(1) \\ \mathrm{C}(5) & 4469(4) & 9320(2) & 9327(1) & 25(1) \\ \mathrm{C}(6) & 2561(4) & 10441(2) & 9496(1) & 22(1) \\ \mathrm{C}(7) & 3320(4) & 10734(2) & 10007(1) & 28(1) \\ \mathrm{C}(8) & 2701(5) & 11377(2) & 10333(2) & 31(1) \\ \mathrm{C}(9) & 1339(5) & 11774(3) & 10182(2) & 34(1) \\ \mathrm{C}(10) & 600(4) & 11522(2) & 9682(2) & 30(1) \\ \mathrm{C}(11) & 1206(4) & 10870(2) & 9321(1) & 23(1) \\ \mathrm{C}(12) & 352(3) & 10717(2) & 8779(1) & 21(1) \\ \mathrm{C}(13) & -2(4) & 10052(2) & 7849(1) & 21(1) \\ \mathrm{C}(14) & 58(4) & 9111(2) & 7679(1) & 25(1) \\ \mathrm{C}(15) & 811(4) & 10641(2) & 7391(1) & 23(1) \\ \mathrm{C}(16) & -223(4) & 10760(3) & 6856(2) & 34(1) \\ \mathrm{C}(17) & 2459(4) & 10362(2) & 7250(1) & 22(1) \\ \mathrm{C}(18) & 2775(4) & 9750(2) & 6827(1) & 25(1) \\ \mathrm{C}(19) & 4306(4) & 9511(2) & 6703(2) & 27(1) \\ \mathrm{C}(20) & 5523(4) & 9877(2) & 6990(2) & 28(1) \\ \mathrm{C}(21) & 5231(4) & 10488(3) & 7409(2) & 32(1) \\ \mathrm{C}(22) & 3717(4) & 10736(3) & 7538(1) & 26(1) \\ \mathrm{C}(23) & -1059(4) & 11243(2) & 8656(1) & 21(1) \\ \mathrm{C}(24) & -2483(4) & 10956(2) & 8866(1) & 25(1) \\ \mathrm{C}(25) & -3839(4) & 11411(2) & 8761(1) & 27(1) \\ \mathrm{C}(26) & -3779(4) & 12155(2) & 8434(2) & 27(1) \\ \mathrm{C}(27) & -2367(4) & 12443(2) & 8228(2) & 27(1) \\ \mathrm{C}(28) & -1009(4) & 11995(2) & 8338(1) & 23(1) \\ \mathrm{C}(29) & 2144(5) & 7639(2) & 9163(2) & 29(1) \\ \mathrm{C}(30) & 1379(4) & 8146(2) & 9640(2) & 24(1) \\ \mathrm{C}(31) & 2088(5) & 8289(2) & 10171(1) & 28(1) \\ \mathrm{C}(32) & 1397(4) & 8795(2) & 10586(2) & 30(1) \\ \mathrm{C}(33) & -35(4) & 9173(3) & 10477(2) & 32(1) \\ \mathrm{C}(34) & -790(4) & 9025(2) & 9954(2) & 30(1) \\ \mathrm{C}(35) & -74(4) & 8512(2) & 9538(2) & 26(1) \\ \mathrm{O}(1 \mathrm{~S}) & -2649(3) & 7889(2) & 6589(2) & 87(1) \\ \mathrm{C}(1 \mathrm{~S}) & -2335(6) & 8264(5) & 6031(3) & 125(3)\end{array}$


Table 3. Selected bond lengths $[\AA]$ and angles $\left[{ }^{\circ}\right]$ for $\mathrm{dk} 24$.

$\begin{array}{rrrrrr}\mathrm{Ni}(1)-\mathrm{N}(3) & 1.841(3) & \mathrm{C}(4)-\mathrm{C}(5) & 1.508(5) & \mathrm{C}(20)-\mathrm{C}(21) & 1.383(5) \\ \mathrm{Ni}(1)-\mathrm{N}(2) & 1.861(3) & \mathrm{C}(6)-\mathrm{C}(11) & 1.402(4) & \mathrm{C}(21)-\mathrm{C}(22) & 1.388(5) \\ \mathrm{Ni}(1)-\mathrm{O}(1) & 1.865(2) & \mathrm{C}(6)-\mathrm{C}(7) & 1.423(4) & \mathrm{C}(23)-\mathrm{C}(28) & 1.389(4) \\ \mathrm{Ni}(1)-\mathrm{N}(1) & 1.944(3) & \mathrm{C}(7)-\mathrm{C}(8) & 1.364(5) & \mathrm{C}(23)-\mathrm{C}(24) & 1.390(4) \\ \mathrm{O}(1)-\mathrm{C}(14) & 1.287(4) & \mathrm{C}(8)-\mathrm{C}(9) & 1.370(5) & \mathrm{C}(24)-\mathrm{C}(25) & 1.386(4) \\ \mathrm{O}(2)-\mathrm{C}(14) & 1.232(4) & \mathrm{C}(9)-\mathrm{C}(10) & 1.373(5) & \mathrm{C}(25)-\mathrm{C}(26) & 1.390(5) \\ \mathrm{O}(3)-\mathrm{C}(5) & 1.235(4) & \mathrm{C}(10)-\mathrm{C}(11) & 1.416(5) & \mathrm{C}(26)-\mathrm{C}(27) & 1.377(5) \\ \mathrm{N}(1)-\mathrm{C}(4) & 1.492(4) & \mathrm{C}(11)-\mathrm{C}(12) & 1.469(4) & \mathrm{C}(27)-\mathrm{C}(28) & 1.384(5) \\ \mathrm{N}(1)-\mathrm{C}(1) & 1.495(4) & \mathrm{C}(12)-\mathrm{C}(23) & 1.492(4) & \mathrm{C}(29)-\mathrm{C}(30) & 1.507(5) \\ \mathrm{N}(1)-\mathrm{C}(29) & 1.507(4) & \mathrm{C}(13)-\mathrm{C}(14) & 1.525(5) & \mathrm{C}(30)-\mathrm{C}(31) & 1.384(5) \\ \mathrm{N}(2)-\mathrm{C}(5) & 1.370(4) & \mathrm{C}(13)-\mathrm{C}(15) & 1.566(5) & \mathrm{C}(30)-\mathrm{C}(35) & 1.393(5) \\ \mathrm{N}(2)-\mathrm{C}(6) & 1.411(4) & \mathrm{C}(15)-\mathrm{C}(17) & 1.516(4) & \mathrm{C}(31)-\mathrm{C}(32) & 1.378(5) \\ \mathrm{N}(3)-\mathrm{C}(12) & 1.302(4) & \mathrm{C}(15)-\mathrm{C}(16) & 1.531(5) & \mathrm{C}(32)-\mathrm{C}(33) & 1.387(5) \\ \mathrm{N}(3)-\mathrm{C}(13) & 1.481(4) & \mathrm{C}(17)-\mathrm{C}(18) & 1.395(4) & \mathrm{C}(33)-\mathrm{C}(34) & 1.389(5) \\ \mathrm{C}(1)-\mathrm{C}(2) & 1.489(5) & \mathrm{C}(17)-\mathrm{C}(22) & 1.395(4) & \mathrm{C}(34)-\mathrm{C}(35) & 1.394(5) \\ \mathrm{C}(2)-\mathrm{C}(3) & 1.543(6) & \mathrm{C}(18)-\mathrm{C}(19) & 1.396(5) & \mathrm{O}(1 \mathrm{~S})-\mathrm{C}(1 \mathrm{~S}) & 1.439(7) \\ \mathrm{C}(3)-\mathrm{C}(4) & 1.535(5) & \mathrm{C}(19)-\mathrm{C}(20) & 1.362(5) & & \end{array}$

$\begin{array}{rrrr}\mathrm{N}(3)-\mathrm{Ni}(1)-\mathrm{N}(2) & 95.05(12) & \mathrm{N}(3)-\mathrm{C}(12)-\mathrm{C}(11) & 121.1(3) \\ \mathrm{N}(3)-\mathrm{Ni}(1)-\mathrm{O}(1) & 86.25(11) & \mathrm{N}(3)-\mathrm{C}(12)-\mathrm{C}(23) & 120.4(3) \\ \mathrm{N}(2)-\mathrm{Ni}(1)-\mathrm{O}(1) & 178.45(11) & \mathrm{C}(11)-\mathrm{C}(12)-\mathrm{C}(23) & 118.5(3) \\ \mathrm{N}(3)-\mathrm{Ni}(1)-\mathrm{N}(1) & 173.33(12) & \mathrm{N}(3)-\mathrm{C}(13)-\mathrm{C}(14) & 106.7(3) \\ \mathrm{N}(2)-\mathrm{Ni}(1)-\mathrm{N}(1) & 87.57(11) & \mathrm{N}(3)-\mathrm{C}(13)-\mathrm{C}(15) & 110.2(3) \\ \mathrm{O}(1)-\mathrm{Ni}(1)-\mathrm{N}(1) & 91.06(11) & \mathrm{C}(14)-\mathrm{C}(13)-\mathrm{C}(15) & 112.4(3) \\ \mathrm{C}(14)-\mathrm{O}(1)-\mathrm{Ni}(1) & 115.8(2) & \mathrm{O}(2)-\mathrm{C}(14)-\mathrm{O}(1) & 124.4(3) \\ \mathrm{C}(4)-\mathrm{N}(1)-\mathrm{C}(1) & 104.1(3) & \mathrm{O}(2)-\mathrm{C}(14)-\mathrm{C}(13) & 120.1(3) \\ \mathrm{C}(4)-\mathrm{N}(1)-\mathrm{C}(29) & 114.8(3) & \mathrm{O}(1)-\mathrm{C}(14)-\mathrm{C}(13) & 115.4(3) \\ \mathrm{C}(1)-\mathrm{N}(1)-\mathrm{C}(29) & 109.5(3) & \mathrm{C}(17)-\mathrm{C}(15)-\mathrm{C}(16) & 113.8(3) \\ \mathrm{C}(4)-\mathrm{N}(1)-\mathrm{Ni}(1) & 105.0(2) & \mathrm{C}(17)-\mathrm{C}(15)-\mathrm{C}(13) & 113.0(3) \\ \mathrm{C}(1)-\mathrm{N}(1)-\mathrm{Ni}(1) & 115.5(2) & \mathrm{C}(16)-\mathrm{C}(15)-\mathrm{C}(13) & 110.9(3) \\ \mathrm{C}(29)-\mathrm{N}(1)-\mathrm{Ni}(1) & 108.1(2) & \mathrm{C}(18)-\mathrm{C}(17)-\mathrm{C}(22) & 118.0(3) \\ \mathrm{C}(5)-\mathrm{N}(2)-\mathrm{C}(6) & 122.0(3) & \mathrm{C}(18)-\mathrm{C}(17)-\mathrm{C}(15) & 122.0(3) \\ \mathrm{C}(5)-\mathrm{N}(2)-\mathrm{Ni}(1) & 113.0(2) & \mathrm{C}(22)-\mathrm{C}(17)-\mathrm{C}(15) & 120.0(3) \\ \mathrm{C}(6)-\mathrm{N}(2)-\mathrm{Ni}(1) & 124.7(2) & \mathrm{C}(17)-\mathrm{C}(18)-\mathrm{C}(19) & 120.7(3) \\ \mathrm{C}(12)-\mathrm{N}(3)-\mathrm{C}(13) & 119.3(3) & \mathrm{C}(20)-\mathrm{C}(19)-\mathrm{C}(18) & 120.7(3) \\ \mathrm{C}(12)-\mathrm{N}(3)-\mathrm{Ni}(1) & 127.0(2) & \mathrm{C}(19)-\mathrm{C}(20)-\mathrm{C}(21) & 119.4(3) \\ \mathrm{C}(13)-\mathrm{N}(3)-\mathrm{Ni}(1) & 112.6(2) & \mathrm{C}(20)-\mathrm{C}(21)-\mathrm{C}(22) & 120.9(4) \\ \mathrm{C}(2)-\mathrm{C}(1)-\mathrm{N}(1) & 103.6(3) & \mathrm{C}(21)-\mathrm{C}(22)-\mathrm{C}(17) & 120.4(3) \\ \mathrm{C}(1)-\mathrm{C}(2)-\mathrm{C}(3) & 102.4(3) & \mathrm{C}(28)-\mathrm{C}(23)-\mathrm{C}(24) & 119.0(3) \\ \mathrm{C}(4)-\mathrm{C}(3)-\mathrm{C}(2) & 105.4(3) & \mathrm{C}(28)-\mathrm{C}(23)-\mathrm{C}(12) & 122.9(3) \\ \mathrm{N}(1)-\mathrm{C}(4)-\mathrm{C}(5) & 111.6(3) & \mathrm{C}(24)-\mathrm{C}(23)-\mathrm{C}(12) & 118.0(3) \\ \mathrm{N}(1)-\mathrm{C}(4)-\mathrm{C}(3) & 105.6(3) & \mathrm{C}(25)-\mathrm{C}(24)-\mathrm{C}(23) & 120.8(3) \\ \mathrm{C}(5)-\mathrm{C}(4)-\mathrm{C}(3) & 113.3(3) & \mathrm{C}(24)-\mathrm{C}(25)-\mathrm{C}(26) & 119.7(3) \\ \mathrm{O}(3)-\mathrm{C}(5)-\mathrm{N}(2) & 128.2(3) & \mathrm{C}(27)-\mathrm{C}(26)-\mathrm{C}(25) & 119.6(3) \\ \mathrm{O}(3)-\mathrm{C}(5)-\mathrm{C}(4) & 117.9(3) & \mathrm{C}(26)-\mathrm{C}(27)-\mathrm{C}(28) & 120.8(3) \\ \mathrm{N}(2)-\mathrm{C}(5)-\mathrm{C}(4) & 113.9(3) & \mathrm{C}(27)-\mathrm{C}(28)-\mathrm{C}(23) & 120.0(3) \\ & & & \end{array}$




$\begin{array}{rrrr}\mathrm{C}(11)-\mathrm{C}(6)-\mathrm{N}(2) & 120.6(3) & \mathrm{C}(30)-\mathrm{C}(29)-\mathrm{N}(1) & 113.8(3) \\ \mathrm{C}(11)-\mathrm{C}(6)-\mathrm{C}(7) & 117.6(3) & \mathrm{C}(31)-\mathrm{C}(30)-\mathrm{C}(35) & 118.4(3) \\ \mathrm{N}(2)-\mathrm{C}(6)-\mathrm{C}(7) & 121.7(3) & \mathrm{C}(31)-\mathrm{C}(30)-\mathrm{C}(29) & 122.6(3) \\ \mathrm{C}(8)-\mathrm{C}(7)-\mathrm{C}(6) & 121.0(3) & \mathrm{C}(35)-\mathrm{C}(30)-\mathrm{C}(29) & 118.9(3) \\ \mathrm{C}(7)-\mathrm{C}(8)-\mathrm{C}(9) & 121.9(4) & \mathrm{C}(32)-\mathrm{C}(31)-\mathrm{C}(30) & 121.3(4) \\ \mathrm{C}(8)-\mathrm{C}(9)-\mathrm{C}(10) & 118.5(4) & \mathrm{C}(31)-\mathrm{C}(32)-\mathrm{C}(33) & 120.0(4) \\ \mathrm{C}(9)-\mathrm{C}(10)-\mathrm{C}(11) & 122.1(4) & \mathrm{C}(32)-\mathrm{C}(33)-\mathrm{C}(34) & 120.0(4) \\ \mathrm{C}(6)-\mathrm{C}(11)-\mathrm{C}(10) & 118.8(3) & \mathrm{C}(33)-\mathrm{C}(34)-\mathrm{C}(35) & 119.2(3) \\ \mathrm{C}(6)-\mathrm{C}(11)-\mathrm{C}(12) & 125.5(3) & \mathrm{C}(30)-\mathrm{C}(35)-\mathrm{C}(34) & 121.0(3) \\ \mathrm{C}(10)-\mathrm{C}(11)-\mathrm{C}(12) & 115.7(3) & & \end{array}$

Table 4. Anisotropic displacement parameters $\left(\AA^{2} \times 10^{3}\right)$ for $\mathrm{dk} 24$. The anisotropic displacement factor exponent takes the form: $-2 \pi^{2}\left[h^{2} a^{* 2} U^{11}+\ldots+2 h k a^{*} b^{*} U^{12}\right]$

\begin{tabular}{rrrrrrr}
\hline Atom & $\mathrm{U}^{11}$ & $\mathrm{U}^{22}$ & $\mathrm{U}^{33}$ & $\mathrm{U}^{23}$ & $\mathrm{U}^{13}$ & $\mathrm{U}^{12}$ \\
\hline $\mathrm{Ni}(1)$ & $24(1)$ & $18(1)$ & $20(1)$ & $0(1)$ & $-3(1)$ & $1(1)$ \\
$\mathrm{O}(1)$ & $27(1)$ & $23(1)$ & $27(1)$ & $-2(1)$ & $-7(1)$ & $2(1)$ \\
$\mathrm{O}(2)$ & $32(1)$ & $33(1)$ & $29(2)$ & $-8(1)$ & $-8(1)$ & $-1(1)$ \\
$\mathrm{O}(3)$ & $26(1)$ & $33(1)$ & $37(2)$ & $-2(1)$ & $-7(1)$ & $0(1)$ \\
$\mathrm{N}(1)$ & $23(2)$ & $25(1)$ & $20(2)$ & $-3(1)$ & $-3(1)$ & $3(1)$ \\
$\mathrm{N}(2)$ & $21(2)$ & $20(1)$ & $19(1)$ & $1(1)$ & $0(1)$ & $1(1)$ \\
$\mathrm{N}(3)$ & $21(1)$ & $21(2)$ & $18(2)$ & $-1(1)$ & $-2(1)$ & $-2(1)$ \\
$\mathrm{C}(1)$ & $33(2)$ & $25(2)$ & $26(2)$ & $-5(2)$ & $-3(2)$ & $12(2)$ \\
$\mathrm{C}(2)$ & $34(2)$ & $39(2)$ & $29(2)$ & $-4(2)$ & $4(2)$ & $8(2)$ \\
$\mathrm{C}(3)$ & $33(2)$ & $45(2)$ & $42(2)$ & $-7(2)$ & $6(2)$ & $0(2)$ \\
$\mathrm{C}(4)$ & $29(2)$ & $26(2)$ & $26(2)$ & $3(2)$ & $-5(2)$ & $-1(2)$ \\
$\mathrm{C}(5)$ & $25(2)$ & $27(2)$ & $24(2)$ & $2(2)$ & $-1(1)$ & $-2(2)$ \\
$\mathrm{C}(6)$ & $28(2)$ & $20(2)$ & $17(2)$ & $1(1)$ & $3(1)$ & $-8(1)$ \\
$\mathrm{C}(7)$ & $27(2)$ & $31(2)$ & $28(2)$ & $-3(2)$ & $-1(1)$ & $-3(2)$ \\
$\mathrm{C}(8)$ & $36(2)$ & $34(2)$ & $22(2)$ & $-4(2)$ & $-3(2)$ & $-6(2)$ \\
$\mathrm{C}(9)$ & $50(3)$ & $29(2)$ & $23(2)$ & $-8(2)$ & $-1(2)$ & $2(2)$ \\
$\mathrm{C}(10)$ & $36(2)$ & $26(2)$ & $28(2)$ & $0(2)$ & $1(2)$ & $0(2)$ \\
$\mathrm{C}(11)$ & $30(2)$ & $23(2)$ & $16(2)$ & $2(2)$ & $0(1)$ & $-2(2)$ \\
$\mathrm{C}(12)$ & $26(2)$ & $18(2)$ & $20(2)$ & $2(2)$ & $3(1)$ & $-2(2)$ \\
$\mathrm{C}(13)$ & $21(2)$ & $24(2)$ & $18(2)$ & $-4(1)$ & $-2(1)$ & $7(2)$ \\
$\mathrm{C}(14)$ & $22(2)$ & $24(2)$ & $28(2)$ & $-4(2)$ & $0(1)$ & $-2(2)$ \\
$\mathrm{C}(15)$ & $28(2)$ & $22(2)$ & $19(2)$ & $1(2)$ & $-1(1)$ & $1(2)$ \\
$\mathrm{C}(16)$ & $30(2)$ & $44(2)$ & $27(2)$ & $6(2)$ & $-3(2)$ & $14(2)$ \\
$\mathrm{C}(17)$ & $25(2)$ & $22(2)$ & $18(2)$ & $5(1)$ & $2(1)$ & $-1(1)$ \\
$\mathrm{C}(18)$ & $28(2)$ & $23(2)$ & $23(2)$ & $0(1)$ & $0(2)$ & $-2(2)$ \\
$\mathrm{C}(19)$ & $32(2)$ & $21(2)$ & $27(2)$ & $0(2)$ & $3(2)$ & $2(2)$ \\
$\mathrm{C}(20)$ & $27(2)$ & $32(2)$ & $25(2)$ & $7(2)$ & $4(2)$ & $4(2)$ \\
$\mathrm{C}(21)$ & $31(2)$ & $38(2)$ & $26(2)$ & $-3(2)$ & $-1(2)$ & $-4(2)$ \\
$\mathrm{C}(22)$ & $31(2)$ & $30(2)$ & $19(2)$ & $-4(2)$ & $0(1)$ & $1(2)$ \\
$\mathrm{C}(23)$ & $26(2)$ & $17(2)$ & $20(2)$ & $-2(1)$ & $-1(1)$ & $1(1)$ \\
$\mathrm{C}(24)$ & $32(2)$ & $20(2)$ & $21(2)$ & $0(1)$ & $-2(1)$ & $-3(2)$ \\
$\mathrm{C}(25)$ & $28(2)$ & $29(2)$ & $24(2)$ & $-6(2)$ & $2(2)$ & $0(2)$
\end{tabular}




\begin{tabular}{lrrrrrr}
\hline Atom & $\mathrm{U}^{11}$ & $\mathrm{U}^{22}$ & $\mathrm{U}^{33}$ & $\mathrm{U}^{23}$ & $\mathrm{U}^{13}$ & $\mathrm{U}^{12}$ \\
\hline $\mathrm{C}(26)$ & $30(2)$ & $24(2)$ & $26(2)$ & $-8(2)$ & $-7(2)$ & $8(2)$ \\
$\mathrm{C}(27)$ & $37(2)$ & $19(2)$ & $24(2)$ & $-3(2)$ & $-1(2)$ & $4(2)$ \\
$\mathrm{C}(28)$ & $31(2)$ & $18(2)$ & $21(2)$ & $-5(1)$ & $1(2)$ & $1(2)$ \\
$\mathrm{C}(29)$ & $39(2)$ & $21(2)$ & $26(2)$ & $4(1)$ & $-1(2)$ & $0(2)$ \\
$\mathrm{C}(30)$ & $26(2)$ & $21(2)$ & $24(2)$ & $7(1)$ & $0(2)$ & $-4(2)$ \\
$\mathrm{C}(31)$ & $26(2)$ & $25(2)$ & $31(2)$ & $8(1)$ & $-2(2)$ & $-1(2)$ \\
$\mathrm{C}(32)$ & $34(2)$ & $30(2)$ & $25(2)$ & $3(2)$ & $1(2)$ & $-6(2)$ \\
$\mathrm{C}(33)$ & $34(2)$ & $31(2)$ & $31(2)$ & $3(2)$ & $8(2)$ & $-5(2)$ \\
$\mathrm{C}(34)$ & $23(2)$ & $29(2)$ & $36(2)$ & $11(2)$ & $2(2)$ & $-2(2)$ \\
$\mathrm{C}(35)$ & $26(2)$ & $26(2)$ & $25(2)$ & $9(2)$ & $1(2)$ & $-5(2)$ \\
$\mathrm{O}(1 \mathrm{~S})$ & $40(2)$ & $114(3)$ & $107(3)$ & $-78(3)$ & $1(2)$ & $-3(2)$ \\
$\mathrm{C}(1 \mathrm{~S})$ & $68(4)$ & $219(8)$ & $88(5)$ & $-93(5)$ & $-24(3)$ & $49(5)$ \\
\hline
\end{tabular}

Table 5. Hydrogen coordinates $\left(\times 10^{4}\right)$ and isotropic displacement parameters $\left(\AA^{2} \times 10^{3}\right)$ for dk24.

\begin{tabular}{rrrrr}
\hline Atom & $\mathrm{x}$ & $\mathrm{y}$ & $\mathrm{z}$ & $\mathrm{U}(\mathrm{eq})$ \\
\hline $\mathrm{H}(1 \mathrm{OS})$ & -2088 & 8124 & 6841 & 104 \\
$\mathrm{H}(1 \mathrm{~S})$ & -1229 & 8415 & 6006 & 150 \\
$\mathrm{H}(2 \mathrm{~S})$ & -2970 & 8779 & 5983 & 150 \\
$\mathrm{H}(3 \mathrm{~S})$ & -2592 & 7853 & 5725 & 150 \\
$\mathrm{H}(1 \mathrm{~A})$ & $2800(40)$ & $7562(19)$ & $7978(13)$ & $20(8)$ \\
$\mathrm{H}(1 \mathrm{~B})$ & $4130(40)$ & $7080(20)$ & $8455(15)$ & $32(9)$ \\
$\mathrm{H}(2 \mathrm{~A})$ & $5730(50)$ & $7770(30)$ & $7699(17)$ & $56(12)$ \\
$\mathrm{H}(2 \mathrm{~B})$ & $4560(40)$ & $8670(20)$ & $7786(15)$ & $34(10)$ \\
$\mathrm{H}(3 \mathrm{~A})$ & $6930(40)$ & $8070(20)$ & $8677(15)$ & $52(11)$ \\
$\mathrm{H}(3 \mathrm{~B})$ & $6360(40)$ & $8940(20)$ & $8469(16)$ & $38(12)$ \\
$\mathrm{H}(4)$ & $4900(40)$ & $8070(30)$ & $9346(17)$ & $48(12)$ \\
$\mathrm{H}(7)$ & $4370(40)$ & $10450(20)$ & $10095(13)$ & $25(9)$ \\
$\mathrm{H}(8)$ & $3310(40)$ & $11550(20)$ & $10627(14)$ & $24(9)$ \\
$\mathrm{H}(9)$ & $920(40)$ & $12210(20)$ & $10396(15)$ & $28(10)$ \\
$\mathrm{H}(10)$ & $-380(30)$ & $11841(19)$ & $9577(12)$ & $14(8)$ \\
$\mathrm{H}(13)$ & $-1240(40)$ & $10206(19)$ & $7887(13)$ & $21(8)$ \\
$\mathrm{H}(15)$ & $1010(30)$ & $11220(20)$ & $7618(13)$ & $20(9)$ \\
$\mathrm{H}(161)$ & $-430(40)$ & $10200(30)$ & $6623(16)$ & $49(12)$ \\
$\mathrm{H}(162)$ & $280(40)$ & $11200(20)$ & $6548(16)$ & $42(11)$ \\
$\mathrm{H}(163)$ & $-1230(30)$ & $10991(18)$ & $6955(11)$ & $9(7)$ \\
$\mathrm{H}(18)$ & $1920(30)$ & $9494(17)$ & $6604(12)$ & $16(8)$ \\
$\mathrm{H}(19)$ & $4420(30)$ & $9070(20)$ & $6402(14)$ & $24(8)$ \\
$\mathrm{H}(20)$ & $6610(40)$ & $9770(20)$ & $6916(14)$ & $29(10)$ \\
$\mathrm{H}(21)$ & $6150(40)$ & $10730(30)$ & $7590(16)$ & $57(12)$ \\
$\mathrm{H}(22)$ & $3480(30)$ & $11154(19)$ & $7886(13)$ & $16(8)$ \\
$\mathrm{H}(24)$ & $-2450(30)$ & $10482(19)$ & $9103(13)$ & $17(8)$ \\
$\mathrm{H}(25)$ & $-4830(40)$ & $11220(20)$ & $8952(15)$ & $38(11)$ \\
$\mathrm{H}(26)$ & $-4820(30)$ & $12457(18)$ & $8357(11)$ & $9(7)$ \\
& & & &
\end{tabular}


160

\begin{tabular}{rrrrr}
\hline Atom & $\mathrm{x}$ & $\mathrm{y}$ & $\mathrm{z}$ & $\mathrm{U}(\mathrm{eq})$ \\
\hline $\mathrm{H}(27)$ & $-2460(40)$ & $12950(20)$ & $8013(14)$ & $29(10)$ \\
$\mathrm{H}(28)$ & $-10(40)$ & $12220(20)$ & $8236(15)$ & $34(11)$ \\
$\mathrm{H}(291)$ & $2830(40)$ & $7190(20)$ & $9332(14)$ & $34(10)$ \\
$\mathrm{H}(292)$ & $1190(40)$ & $7360(20)$ & $8881(15)$ & $48(11)$ \\
$\mathrm{H}(31)$ & $3080(50)$ & $8000(30)$ & $10253(17)$ & $67(14)$ \\
$\mathrm{H}(32)$ & $1950(40)$ & $8878(19)$ & $10950(14)$ & $32(9)$ \\
$\mathrm{H}(33)$ & $-710(40)$ & $9550(20)$ & $10782(15)$ & $32(10)$ \\
$\mathrm{H}(34)$ & $-1890(40)$ & $9240(20)$ & $9864(14)$ & $48(10)$ \\
$\mathrm{H}(35)$ & $-680(40)$ & $8380(20)$ & $9135(15)$ & $31(10)$ \\
\hline
\end{tabular}

Table 6. Hydrogen bonds for $\mathrm{dk} 24$ [ $\AA$ and $\left.^{\circ}\right]$.

\begin{tabular}{ccccc}
\hline $\mathrm{D}-\mathrm{H} \ldots \mathrm{A}$ & $\mathrm{d}(\mathrm{D}-\mathrm{H})$ & $\mathrm{d}(\mathrm{H} \ldots \mathrm{A})$ & $\mathrm{d}(\mathrm{D} \ldots \mathrm{A})$ & $<(\mathrm{DHA})$ \\
\hline $\mathrm{O}(1 \mathrm{~S})-\mathrm{H}(1 \mathrm{OS}) \ldots \mathrm{O}(2)$ & 0.84 & 1.90 & $2.727(4)$ & 166.1 \\
\hline
\end{tabular}




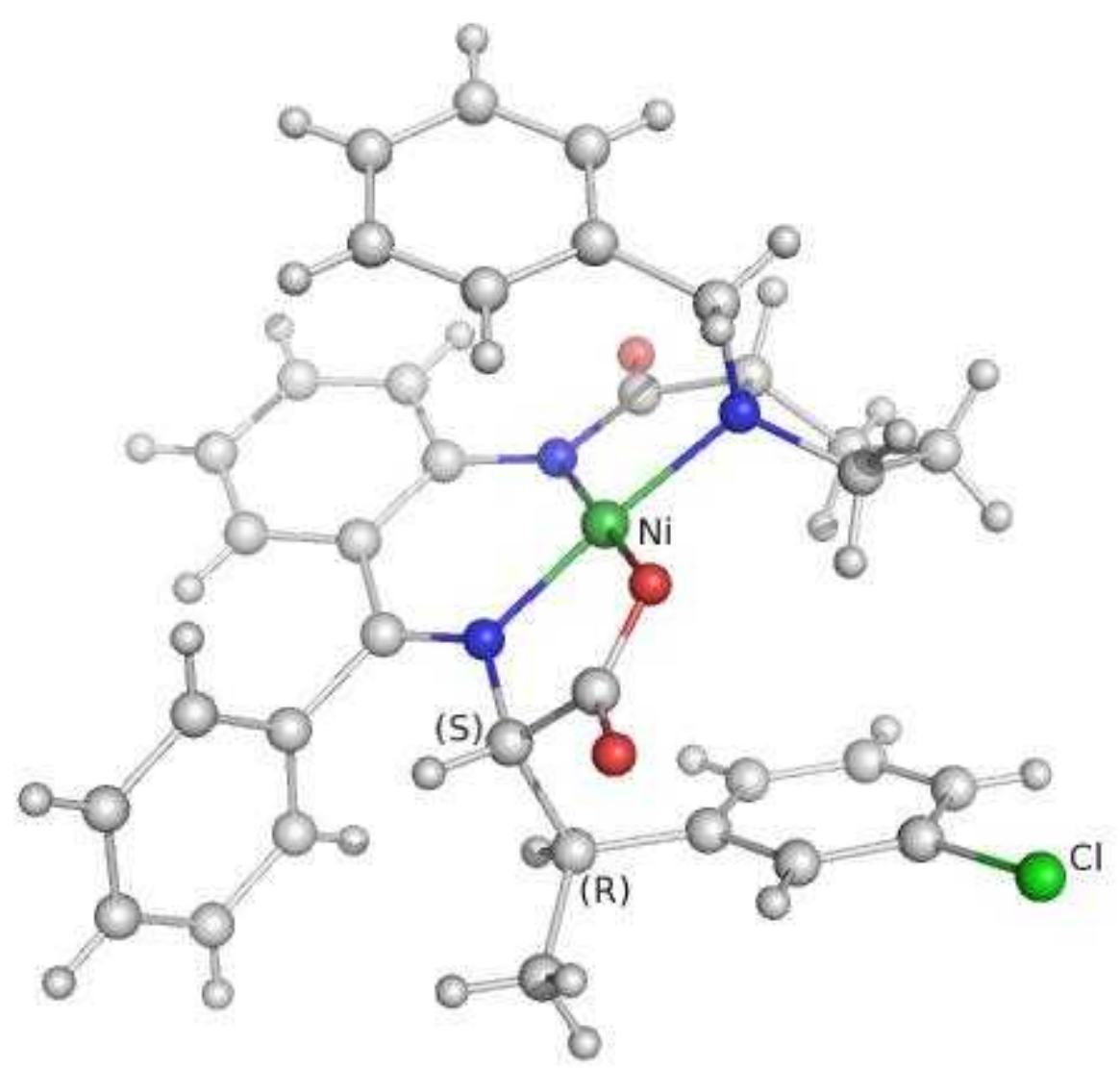

Table 1. Crystal data and structure refinement for sw005.

Identification code

Empirical formula

Formula weight

Temperature

Wavelength

Crystal system

Space group

Unit cell dimensions

Volume

Z sw005

$\mathrm{C}_{35} \mathrm{H}_{32} \mathrm{ClN}_{3} \mathrm{NiO}_{3}$

636.80

100(2) K

$1.54178 \AA$

orthorhombic

P $22_{1} 2_{1}$

$$
\begin{array}{ll}
\mathrm{a}=8.0379(16) \AA & \alpha=90^{\circ} . \\
\mathrm{b}=15.338(3) \AA & \beta=90^{\circ} . \\
\mathrm{c}=24.070(5) \AA & \gamma=90^{\circ} .
\end{array}
$$

2967.4(10) $\AA^{3}$

4 
Density (calculated)

Absorption coefficient

$\mathrm{F}(000)$

Crystal size

Theta range for data collection

Index ranges

Reflections collected

Independent reflections

Completeness to theta $=61.04^{\circ}$

Max. and min. transmission

Refinement method

Data / restraints / parameters

Goodness-of-fit on $\mathrm{F}^{2}$

Final $\mathrm{R}$ indices [I $>2 \operatorname{sigma}(\mathrm{I})]$

$\mathrm{R}$ indices (all data)

Absolute structure parameter

Largest diff. peak and hole
$1.425 \mathrm{Mg} / \mathrm{m}^{3}$

$2.103 \mathrm{~mm}^{-1}$

1328

$0.40 \times 0.25 \times 0.03 \mathrm{~mm}^{3}$

3.42 to $61.04^{\circ}$.

$-8<=\mathrm{h}<=8,-13<=\mathrm{k}<=17,-26<=\mathrm{l}<=27$

21464

$4364[\mathrm{R}($ int $)=0.0350]$

$96.9 \%$

0.9396 and 0.4868

Full-matrix least-squares on $\mathrm{F}^{2}$

4364 / 0 / 389

1.040

$\mathrm{R}_{1}=0.0215, \mathrm{wR}_{2}=0.0515$

$\mathrm{R}_{1}=0.0230, \mathrm{wR}_{2}=0.0525$

$-0.008(12)$

0.135 and -0.204 e. $\AA^{-3}$

Table 2. Atomic coordinates $\left(\times 10^{4}\right)$ and equivalent isotropic displacement parameters $\left(\AA^{2} \times 10^{3}\right)$ for sw005. U(eq) is defined as one third of the trace of the orthogonalized $\mathrm{U}^{\mathrm{ij}}$ tensor.

\begin{tabular}{rrrrr}
\hline & $\mathrm{x}$ & $\mathrm{y}$ & $\mathrm{z}$ & $\mathrm{U}(\mathrm{eq})$ \\
\hline $\mathrm{C}(1)$ & $10555(3)$ & $10303(1)$ & $2012(1)$ & $27(1)$ \\
$\mathrm{C}(2)$ & $11820(3)$ & $10222(1)$ & $2401(1)$ & $37(1)$ \\
$\mathrm{C}(3)$ & $11444(3)$ & $10002(1)$ & $2947(1)$ & $40(1)$ \\
$\mathrm{C}(4)$ & $9809(3)$ & $9884(1)$ & $3104(1)$ & $35(1)$ \\
$\mathrm{C}(5)$ & $8540(3)$ & $9973(1)$ & $2721(1)$ & $28(1)$ \\
$\mathrm{C}(6)$ & $8910(2)$ & $10166(1)$ & $2170(1)$ & $21(1)$ \\
$\mathrm{C}(7)$ & $7555(2)$ & $10205(1)$ & $1743(1)$ & $21(1)$ \\
$\mathrm{N}(8)$ & $7104(2)$ & $9491(1)$ & $1492(1)$ & $18(1)$ \\
$\mathrm{C}(9)$ & $6799(3)$ & $11047(1)$ & $1615(1)$ & $22(1)$ \\
$\mathrm{C}(10)$ & $7575(3)$ & $11794(1)$ & $1834(1)$ & $29(1)$ \\
$\mathrm{C}(11)$ & $7010(3)$ & $12621(1)$ & $1731(1)$ & $34(1)$ \\
$\mathrm{C}(12)$ & $5570(3)$ & $12719(1)$ & $1416(1)$ & $33(1)$ \\
$\mathrm{C}(13)$ & $4747(3)$ & $12009(1)$ & $1204(1)$ & $29(1)$
\end{tabular}


163

\begin{tabular}{|c|c|c|c|c|}
\hline & $\mathrm{x}$ & $\mathrm{y}$ & $\mathrm{z}$ & $\mathrm{U}(\mathrm{eq})$ \\
\hline$C(14)$ & $5344(3)$ & 11151(1) & $1282(1)$ & $22(1)$ \\
\hline $\mathrm{N}(15)$ & $4598(2)$ & $10432(1)$ & $1015(1)$ & $20(1)$ \\
\hline$C(16)$ & $3098(2)$ & $10501(1)$ & $752(1)$ & $21(1)$ \\
\hline $\mathrm{O}(17)$ & $2038(2)$ & $11077(1)$ & 801(1) & $28(1)$ \\
\hline $\mathrm{C}(18)$ & $2705(2)$ & $9740(1)$ & $376(1)$ & $21(1)$ \\
\hline$C(19)$ & $1383(2)$ & $9135(1)$ & $614(1)$ & $25(1)$ \\
\hline $\mathrm{C}(20)$ & $1661(2)$ & $8304(1)$ & 281(1) & $25(1)$ \\
\hline $\mathrm{C}(21)$ & $3542(2)$ & $8232(1)$ & $268(1)$ & $22(1)$ \\
\hline $\mathrm{N}(22)$ & $4190(2)$ & $9169(1)$ & 281(1) & $18(1)$ \\
\hline $\mathrm{C}(23)$ & $5042(2)$ & $9375(1)$ & $-261(1)$ & $21(1)$ \\
\hline$C(24)$ & $5769(3)$ & $10280(1)$ & $-267(1)$ & $23(1)$ \\
\hline$C(25)$ & $4908(3)$ & $10992(1)$ & $-484(1)$ & $28(1)$ \\
\hline$C(26)$ & $5606(3)$ & $11826(1)$ & $-450(1)$ & $37(1)$ \\
\hline$C(27)$ & $7119(3)$ & $11953(2)$ & $-191(1)$ & $39(1)$ \\
\hline $\mathrm{C}(28)$ & 7997(3) & $11250(1)$ & $14(1)$ & $35(1)$ \\
\hline C(29) & $7323(3)$ & $10421(1)$ & $-30(1)$ & $26(1)$ \\
\hline $\mathrm{O}(30)$ & $6896(2)$ & 8369(1) & $720(1)$ & $20(1)$ \\
\hline $\mathrm{C}(31)$ & $7904(2)$ & $8102(1)$ & $1104(1)$ & $19(1)$ \\
\hline $\mathrm{O}(32)$ & $8789(2)$ & $7453(1)$ & $1075(1)$ & $22(1)$ \\
\hline C(33) & $7893(2)$ & $8649(1)$ & $1633(1)$ & $19(1)$ \\
\hline$C(34)$ & $6928(2)$ & $8161(1)$ & $2108(1)$ & $21(1)$ \\
\hline$C(35)$ & $8142(3)$ & $7582(1)$ & $2436(1)$ & $26(1)$ \\
\hline$C(36)$ & $5420(3)$ & $7673(1)$ & 1894(1) & $21(1)$ \\
\hline$C(37)$ & $3845(2)$ & $8048(1)$ & 1913(1) & $27(1)$ \\
\hline $\mathrm{C}(38)$ & 2473(3) & $7605(2)$ & $1712(1)$ & $34(1)$ \\
\hline C(39) & $2621(3)$ & $6789(2)$ & $1481(1)$ & $32(1)$ \\
\hline $\mathrm{C}(40)$ & 4193(3) & $6420(1)$ & $1459(1)$ & $27(1)$ \\
\hline $\mathrm{Cl}(41)$ & $4435(1)$ & $5391(1)$ & $1153(1)$ & $39(1)$ \\
\hline$C(42)$ & $5580(3)$ & $6838(1)$ & $1666(1)$ & $23(1)$ \\
\hline Ni(43) & $5684(1)$ & $9377(1)$ & 894(1) & $18(1)$ \\
\hline
\end{tabular}

Table 3. Bond lengths $[\AA]$ and angles $\left[^{\circ}\right]$ for sw005.

$\begin{array}{lrrrrr}\mathrm{C}(1)-\mathrm{C}(2) & 1.387(3) & \mathrm{N}(15)-\mathrm{Ni}(43) & 1.8617(15) & \mathrm{C}(28)-\mathrm{C}(29) & 1.388(3) \\ \mathrm{C}(1)-\mathrm{C}(6) & 1.392(3) & \mathrm{C}(16)-\mathrm{O}(17) & 1.233(2) & \mathrm{C}(28)-\mathrm{H}(28) & 0.9500 \\ \mathrm{C}(1)-\mathrm{H}(1) & 0.9500 & \mathrm{C}(16)-\mathrm{C}(18) & 1.509(3) & \mathrm{C}(29)-\mathrm{H}(29) & 0.9500 \\ \mathrm{C}(2)-\mathrm{C}(3) & 1.390(4) & \mathrm{C}(18)-\mathrm{N}(22) & 1.498(2) & \mathrm{O}(30)-\mathrm{C}(31) & 1.296(2) \\ \mathrm{C}(2)-\mathrm{H}(2) & 0.9500 & \mathrm{C}(18)-\mathrm{C}(19) & 1.523(3) & \mathrm{O}(30)-\mathrm{Ni}(43) & 1.8749(13) \\ \mathrm{C}(3)-\mathrm{C}(4) & 1.380(4) & \mathrm{C}(18)-\mathrm{H}(18) & 1.0000 & \mathrm{C}(31)-\mathrm{O}(32) & 1.225(2) \\ \mathrm{C}(3)-\mathrm{H}(3) & 0.9500 & \mathrm{C}(19)-\mathrm{C}(20) & 1.522(3) & \mathrm{C}(31)-\mathrm{C}(33) & 1.525(3) \\ \mathrm{C}(4)-\mathrm{C}(5) & 1.383(3) & \mathrm{C}(19)-\mathrm{H}(19 \mathrm{~A}) & 0.9900 & \mathrm{C}(33)-\mathrm{C}(34) & 1.571(3) \\ \mathrm{C}(4)-\mathrm{H}(4) & 0.9500 & \mathrm{C}(19)-\mathrm{H}(19 \mathrm{~B}) & 0.9900 & \mathrm{C}(33)-\mathrm{H}(33) & 1.0000 \\ \mathrm{C}(5)-\mathrm{C}(6) & 1.390(3) & \mathrm{C}(20)-\mathrm{C}(21) & 1.517(3) & \mathrm{C}(34)-\mathrm{C}(36) & 1.514(3) \\ \mathrm{C}(5)-\mathrm{H}(5) & 0.9500 & \mathrm{C}(20)-\mathrm{H}(20 \mathrm{~A}) & 0.9900 & \mathrm{C}(34)-\mathrm{C}(35) & 1.538(3) \\ \mathrm{C}(6)-\mathrm{C}(7) & 1.499(3) & \mathrm{C}(20)-\mathrm{H}(20 \mathrm{~B}) & 0.9900 & \mathrm{C}(34)-\mathrm{H}(34) & 1.0000 \\ \mathrm{C}(7)-\mathrm{N}(8) & 1.302(2) & \mathrm{C}(21)-\mathrm{N}(22) & 1.529(2) & \mathrm{C}(35)-\mathrm{H}(35 \mathrm{~A}) & 0.9800\end{array}$




$\begin{array}{rrrrrr}\mathrm{C}(7)-\mathrm{C}(9) & 1.460(3) & \mathrm{C}(21)-\mathrm{H}(21 \mathrm{~A}) & 0.9900 & \mathrm{C}(35)-\mathrm{H}(35 \mathrm{~B}) & 0.9800 \\ \mathrm{~N}(8)-\mathrm{C}(33) & 1.479(2) & \mathrm{C}(21)-\mathrm{H}(21 \mathrm{~B}) & 0.9900 & \mathrm{C}(35)-\mathrm{H}(35 \mathrm{C}) & 0.9800 \\ \mathrm{~N}(8)-\mathrm{Ni}(43) & 1.8441(15) & \mathrm{N}(22)-\mathrm{C}(23) & 1.506(2) & \mathrm{C}(36)-\mathrm{C}(37) & 1.391(3) \\ \mathrm{C}(9)-\mathrm{C}(10) & 1.406(3) & \mathrm{N}(22)-\mathrm{Ni}(43) & 1.9298(16) & \mathrm{C}(36)-\mathrm{C}(42) & 1.399(3) \\ \mathrm{C}(9)-\mathrm{C}(14) & 1.427(3) & \mathrm{C}(23)-\mathrm{C}(24) & 1.507(3) & \mathrm{C}(37)-\mathrm{C}(38) & 1.382(3) \\ \mathrm{C}(10)-\mathrm{C}(11) & 1.370(3) & \mathrm{C}(23)-\mathrm{H}(23 \mathrm{~A}) & 0.9900 & \mathrm{C}(37)-\mathrm{H}(37) & 0.9500 \\ \mathrm{C}(10)-\mathrm{H}(10) & 0.9500 & \mathrm{C}(23)-\mathrm{H}(23 \mathrm{~B}) & 0.9900 & \mathrm{C}(38)-\mathrm{C}(39) & 1.375(3) \\ \mathrm{C}(11)-\mathrm{C}(12) & 1.392(3) & \mathrm{C}(24)-\mathrm{C}(29) & 1.391(3) & \mathrm{C}(38)-\mathrm{H}(38) & 0.9500 \\ \mathrm{C}(11)-\mathrm{H}(11) & 0.9500 & \mathrm{C}(24)-\mathrm{C}(25) & 1.393(3) & \mathrm{C}(39)-\mathrm{C}(40) & 1.385(3) \\ \mathrm{C}(12)-\mathrm{C}(13) & 1.373(3) & \mathrm{C}(25)-\mathrm{C}(26) & 1.400(3) & \mathrm{C}(39)-\mathrm{H}(39) & 0.9500 \\ \mathrm{C}(12)-\mathrm{H}(12) & 0.9500 & \mathrm{C}(25)-\mathrm{H}(25) & 0.9500 & \mathrm{C}(40)-\mathrm{C}(42) & 1.379(3) \\ \mathrm{C}(13)-\mathrm{C}(14) & 1.414(3) & \mathrm{C}(26)-\mathrm{C}(27) & 1.380(3) & \mathrm{C}(40)-\mathrm{Cl}(41) & 1.753(2) \\ \mathrm{C}(13)-\mathrm{H}(13) & 0.9500 & \mathrm{C}(26)-\mathrm{H}(26) & 0.9500 & \mathrm{C}(42)-\mathrm{H}(42) & 0.9500 \\ \mathrm{C}(14)-\mathrm{N}(15) & 1.409(2) & \mathrm{C}(27)-\mathrm{C}(28) & 1.380(3) & & \\ \mathrm{N}(15)-\mathrm{C}(16) & 1.367(2) & \mathrm{C}(27)-\mathrm{H}(27) & 0.9500 & & \end{array}$

\begin{tabular}{|c|c|c|c|}
\hline$C(2)-C(1)-C(6)$ & $119.9(2)$ & $\mathrm{C}(23)-\mathrm{N}(22)-\mathrm{C}(21)$ & $109.53(14)$ \\
\hline $\mathrm{C}(2)-\mathrm{C}(1)-\mathrm{H}(1)$ & 120.1 & $\mathrm{C}(18)-\mathrm{N}(22)-\mathrm{Ni}(43)$ & $106.36(11)$ \\
\hline $\mathrm{C}(6)-\mathrm{C}(1)-\mathrm{H}(1)$ & 120.1 & $\mathrm{C}(23)-\mathrm{N}(22)-\mathrm{Ni}(43)$ & $110.17(11)$ \\
\hline $\mathrm{C}(1)-\mathrm{C}(2)-\mathrm{C}(3)$ & $120.0(2)$ & $\mathrm{C}(21)-\mathrm{N}(22)-\mathrm{Ni}(43)$ & $112.49(11)$ \\
\hline $\mathrm{C}(1)-\mathrm{C}(2)-\mathrm{H}(2)$ & 120.0 & $\mathrm{~N}(22)-\mathrm{C}(23)-\mathrm{C}(24)$ & $112.18(14$ \\
\hline $\mathrm{C}(3)-\mathrm{C}(2)-\mathrm{H}(2)$ & 120.0 & $\mathrm{~N}(22)-\mathrm{C}(23)-\mathrm{H}(23 \mathrm{~A})$ & \\
\hline $\mathrm{C}(4)-\mathrm{C}(3)-\mathrm{C}(2)$ & $119.9(2)$ & $\mathrm{C}(24)-\mathrm{C}(23)-\mathrm{H}(23 \mathrm{~A})$ & \\
\hline $\mathrm{C}(4)-\mathrm{C}(3)-\mathrm{H}(3)$ & 120.0 & $\mathrm{~N}(22)-\mathrm{C}(23)-\mathrm{H}(23 \mathrm{~B})$ & 109. \\
\hline $\mathrm{C}(2)-\mathrm{C}(3)-\mathrm{H}(3)$ & 120.0 & $\mathrm{C}(24)-\mathrm{C}(23)-\mathrm{H}(23 \mathrm{~B})$ & 109 \\
\hline$C(3)-C(4)-C(5)$ & $120.4(2)$ & $\mathrm{H}(23 \mathrm{~A})-\mathrm{C}(23)-\mathrm{H}(23 \mathrm{~B})$ & 107 \\
\hline $\mathrm{C}(3)-\mathrm{C}(4)-\mathrm{H}(4)$ & 119.8 & $C(29)-C(24)-C(25)$ & $118.62(18$ \\
\hline $\mathrm{C}(5)-\mathrm{C}(4)-\mathrm{H}(4)$ & 119.8 & $C(29)-C(24)-C(23)$ & $119.11(17$ \\
\hline$C(4)-C(5)-C(6)$ & $120.0(2)$ & $C(25)-C(24)-C(23)$ & $122.22(18$ \\
\hline $\mathrm{C}(4)-\mathrm{C}(5)-\mathrm{H}(5)$ & 120.0 & $C(24)-C(25)-C(26)$ & $119.7(2$ \\
\hline $\mathrm{C}(6)-\mathrm{C}(5)-\mathrm{H}(5)$ & 120.0 & $\mathrm{C}(24)-\mathrm{C}(25)-\mathrm{H}(25)$ & 120 \\
\hline$C(5)-C(6)-C(1)$ & 119.73(19) & $\mathrm{C}(26)-\mathrm{C}(25)-\mathrm{H}(25)$ & 120. \\
\hline$C(5)-C(6)-C(7)$ & $120.50(18)$ & $C(27)-C(26)-C(25)$ & $120.6(2$ \\
\hline$C(1)-C(6)-C(7)$ & $119.76(17)$ & $\mathrm{C}(27)-\mathrm{C}(26)-\mathrm{H}(26)$ & 119 \\
\hline $\mathrm{N}(8)-\mathrm{C}(7)-\mathrm{C}(9)$ & $122.03(17)$ & $\mathrm{C}(25)-\mathrm{C}(26)-\mathrm{H}(26)$ & 119 \\
\hline $\mathrm{N}(8)-\mathrm{C}(7)-\mathrm{C}(6)$ & $119.17(16)$ & $C(28)-C(27)-C(26)$ & $120.2(2$ \\
\hline $\mathrm{C}(9)-\mathrm{C}(7)-\mathrm{C}(6)$ & $118.79(16)$ & $\mathrm{C}(28)-\mathrm{C}(27)-\mathrm{H}(27)$ & 119 \\
\hline $\mathrm{C}(7)-\mathrm{N}(8)-\mathrm{C}(33)$ & $120.52(15)$ & $\mathrm{C}(26)-\mathrm{C}(27)-\mathrm{H}(27)$ & 119. \\
\hline $\mathrm{C}(7)-\mathrm{N}(8)-\mathrm{Ni}(43)$ & $127.87(13)$ & $\mathrm{C}(27)-\mathrm{C}(28)-\mathrm{C}(29)$ & $119.3(2$ \\
\hline $\mathrm{C}(33)-\mathrm{N}(8)-\mathrm{Ni}(43)$ & $111.25(11)$ & $\mathrm{C}(27)-\mathrm{C}(28)-\mathrm{H}(28)$ & 120. \\
\hline $\mathrm{C}(10)-\mathrm{C}(9)-\mathrm{C}(14)$ & $118.91(17)$ & $\mathrm{C}(29)-\mathrm{C}(28)-\mathrm{H}(28)$ & 120 \\
\hline $\mathrm{C}(10)-\mathrm{C}(9)-\mathrm{C}(7)$ & $117.19(17)$ & $\mathrm{C}(28)-\mathrm{C}(29)-\mathrm{C}(24)$ & $121.6(2$ \\
\hline $\mathrm{C}(14)-\mathrm{C}(9)-\mathrm{C}(7)$ & $123.90(16)$ & $\mathrm{C}(28)-\mathrm{C}(29)-\mathrm{H}(29)$ & 119 \\
\hline $\mathrm{C}(11)-\mathrm{C}(10)-\mathrm{C}(9)$ & $122.6(2)$ & $\mathrm{C}(24)-\mathrm{C}(29)-\mathrm{H}(29)$ & 119 \\
\hline $\mathrm{C}(11)-\mathrm{C}(10)-\mathrm{H}(10)$ & 118.7 & $\mathrm{C}(31)-\mathrm{O}(30)-\mathrm{Ni}(43)$ & $115.20(11$ \\
\hline $\mathrm{C}(9)-\mathrm{C}(10)-\mathrm{H}(10)$ & 118.7 & $\mathrm{O}(32)-\mathrm{C}(31)-\mathrm{O}(30)$ & $125.45(17$ \\
\hline $\mathrm{C}(10)-\mathrm{C}(11)-\mathrm{C}(12)$ & $118.34(19)$ & $\mathrm{O}(32)-\mathrm{C}(31)-\mathrm{C}(33)$ & $119.77(16$ \\
\hline $\mathrm{C}(10)-\mathrm{C}(11)-\mathrm{H}(11)$ & 120.8 & $\mathrm{O}(30)-\mathrm{C}(31)-\mathrm{C}(33)$ & $114.75(16$ \\
\hline $\mathrm{C}(12)-\mathrm{C}(11)-\mathrm{H}(11)$ & 120.8 & $\mathrm{~N}(8)-\mathrm{C}(33)-\mathrm{C}(31)$ & $106.87(14$ \\
\hline $\mathrm{C}(13)-\mathrm{C}(12)-\mathrm{C}(11)$ & $121.16(19)$ & $\mathrm{N}(8)-\mathrm{C}(33)-\mathrm{C}(34)$ & $111.82(15$ \\
\hline
\end{tabular}




\begin{tabular}{|c|c|c|c|}
\hline $\mathrm{C}(13)-\mathrm{C}(12)-\mathrm{H}(12)$ & 119.4 & $\mathrm{C}(31)-\mathrm{C}(33)-\mathrm{C}(34)$ & $110.39(15)$ \\
\hline $\mathrm{C}(11)-\mathrm{C}(12)-\mathrm{H}(12)$ & 119.4 & $\mathrm{~N}(8)-\mathrm{C}(33)-\mathrm{H}(33)$ & 109.2 \\
\hline$C(12)-C(13)-C(14)$ & $121.7(2)$ & $\mathrm{C}(31)-\mathrm{C}(33)-\mathrm{H}(33)$ & 109.2 \\
\hline $\mathrm{C}(12)-\mathrm{C}(13)-\mathrm{H}(13)$ & 119.1 & $\mathrm{C}(34)-\mathrm{C}(33)-\mathrm{H}(33)$ & 109.2 \\
\hline $\mathrm{C}(14)-\mathrm{C}(13)-\mathrm{H}(13)$ & 119.1 & $C(36)-C(34)-C(35)$ & $113.43(16)$ \\
\hline $\mathrm{N}(15)-\mathrm{C}(14)-\mathrm{C}(13)$ & $121.57(17)$ & $C(36)-C(34)-C(33)$ & $112.53(15)$ \\
\hline $\mathrm{N}(15)-\mathrm{C}(14)-\mathrm{C}(9)$ & $121.17(16)$ & $C(35)-C(34)-C(33)$ & $109.60(16)$ \\
\hline $\mathrm{C}(13)-\mathrm{C}(14)-\mathrm{C}(9)$ & $117.15(17)$ & $\mathrm{C}(36)-\mathrm{C}(34)-\mathrm{H}(34)$ & 107.0 \\
\hline $\mathrm{C}(16)-\mathrm{N}(15)-\mathrm{C}(14)$ & $121.79(15)$ & $\mathrm{C}(35)-\mathrm{C}(34)-\mathrm{H}(34)$ & 107.0 \\
\hline $\mathrm{C}(16)-\mathrm{N}(15)-\mathrm{Ni}(43)$ & $114.06(12)$ & $\mathrm{C}(33)-\mathrm{C}(34)-\mathrm{H}(34)$ & 107.0 \\
\hline $\mathrm{C}(14)-\mathrm{N}(15)-\mathrm{Ni}(43)$ & $123.48(13)$ & $\mathrm{C}(34)-\mathrm{C}(35)-\mathrm{H}(35 \mathrm{~A})$ & 109.5 \\
\hline $\mathrm{O}(17)-\mathrm{C}(16)-\mathrm{N}(15)$ & $128.29(17)$ & $\mathrm{C}(34)-\mathrm{C}(35)-\mathrm{H}(35 \mathrm{~B})$ & 109.5 \\
\hline $\mathrm{O}(17)-\mathrm{C}(16)-\mathrm{C}(1$ & 117.87( & $\mathrm{H}(35 \mathrm{~A})$ & 109.5 \\
\hline $\mathrm{N}(15)-\mathrm{C}(16)-\mathrm{C}(18)$ & $113.77(16)$ & $\mathrm{C}(34)-\mathrm{C}$ & 109.5 \\
\hline $\mathrm{N}(22)-\mathrm{C}(18)-\mathrm{C}(16)$ & $112.17(15)$ & $\mathrm{H}(35 \mathrm{~A})-\mathrm{C}(35)-\mathrm{H}(35 \mathrm{C})$ & 109.5 \\
\hline $\mathrm{N}(22)-\mathrm{C}(18)-\mathrm{C}(19)$ & $104.95(14)$ & $\mathrm{H}(35 \mathrm{~B})-\mathrm{C}(35$ & 109.5 \\
\hline $\mathrm{C}(16$ & 113.10( & $-C(42)$ & $118.31(18)$ \\
\hline $\mathrm{N}(22)-\mathrm{C}(1$ & 108.8 & $\mathrm{C}(37)-\mathrm{C}($ & $120.88(17)$ \\
\hline $\mathrm{C}(16)-\mathrm{C}(18)-\mathrm{H}(18)$ & 108.8 & $\mathrm{C}(42)-\mathrm{C}($ & $120.80(17)$ \\
\hline $\mathrm{C}(19)-\mathrm{C}(18)-\mathrm{H}(18)$ & 10 & & $120.8(2)$ \\
\hline $\mathrm{C}(2 \mathrm{C}$ & $102.15(16)$ & $-\mathrm{H}(37)$ & 119.6 \\
\hline$C(20)-C(19)-1$ & 111.3 & $-\mathrm{H}(37)$ & 119.6 \\
\hline & 111.3 & $\mathrm{C}(39)-\mathrm{C}($ & $121.3(2)$ \\
\hline & & & 119.4 \\
\hline & 111.3 & $\mathrm{H}(38)$ & 119.4 \\
\hline $\mathrm{H}(19 \mathrm{~A})-\mathrm{C}(19)-\mathrm{H}(19 \mathrm{~B})$ & 109.2 & $\mathrm{C}(38)-\mathrm{C}(39)-\mathrm{C}(40)$ & $117.8(2)$ \\
\hline $\mathrm{C}(21)-\mathrm{C}(20)-\mathrm{C}(19)$ & $102.59(16)$ & $\mathrm{C}(38)-\mathrm{C}(39)-\mathrm{H}(39)$ & 121.1 \\
\hline $\mathrm{C}(2)$ & & & \\
\hline $\mathrm{C}(19)-\mathrm{C}(20)-\mathrm{H}(20 \mathrm{~A})$ & 111.2 & $\mathrm{C}(42)-\mathrm{C}(40)-\mathrm{C}(39)$ & $122.29(19)$ \\
\hline $\mathrm{C}(21)-\mathrm{C}(20)-\mathrm{H}(20 \mathrm{~B})$ & 111.2 & $\mathrm{C}(42)-\mathrm{C}(40)-\mathrm{Cl}(41)$ & $118.69(17)$ \\
\hline $\mathrm{C}(19)-\mathrm{C}(20)-\mathrm{H}$ & & $\mathrm{C}(39)-\mathrm{C}(40)-\mathrm{Cl}(41)$ & $119.02(16)$ \\
\hline $\mathrm{H}(20 \mathrm{~A})-\mathrm{C}(20)-\mathrm{H}(20 \mathrm{~B})$ & 109.2 & $\mathrm{C}(40)-\mathrm{C}(42)-\mathrm{C}(36)$ & $119.51(19)$ \\
\hline $\mathrm{C}(20)-\mathrm{C}(21)-\mathrm{N}(22)$ & $105.74(15)$ & $\mathrm{C}(40)-\mathrm{C}(42)-\mathrm{H}(42)$ & 120.2 \\
\hline $\mathrm{C}(20)-\mathrm{C}(21)-\mathrm{H}(21 \mathrm{~A})$ & 110.6 & $\mathrm{C}(36)-\mathrm{C}(42)-\mathrm{H}(42)$ & 120.2 \\
\hline $\mathrm{N}(22)-\mathrm{C}(21)-\mathrm{H}(21 \mathrm{~A})$ & & $\mathrm{N}(8)-\mathrm{Ni}(43)-\mathrm{N}(15)$ & $94.91(7)$ \\
\hline $\mathrm{C}(20)-\mathrm{C}(21)-\mathrm{H}(21 \mathrm{~B})$ & 110.6 & $\mathrm{~N}(8)-\mathrm{Ni}(43)-\mathrm{O}(30)$ & $86.07(6)$ \\
\hline $\mathrm{N}(22)-\mathrm{C}(21)-\mathrm{H}(21 \mathrm{~B})$ & 110.6 & $\mathrm{~N}(15)-\mathrm{Ni}(43)-\mathrm{O}(30)$ & $174.53(6)$ \\
\hline $\mathrm{I}(21 \mathrm{~A})-\mathrm{C}(21)-\mathrm{H}(21 \mathrm{~B})$ & 108.7 & $\mathrm{~N}(8)-\mathrm{Ni}(43)-\mathrm{N}(22)$ & $175.85(6)$ \\
\hline $\mathrm{C}(18)-\mathrm{N}(22)-\mathrm{C}(23)$ & $111.90(14)$ & $\mathrm{N}(15)-\mathrm{Ni}(43)-\mathrm{N}(22)$ & $88.38(6)$ \\
\hline $\mathrm{C}(18)-\mathrm{N}(22)-\mathrm{C}(21)$ & $106.33(14)$ & $\mathrm{O}(30)-\mathrm{Ni}(43)-\mathrm{N}(22)$ & $90.89(6)$ \\
\hline
\end{tabular}


Table 4. Anisotropic displacement parameters $\left(\AA^{2} \times 10^{3}\right)$ for sw005. The anisotropic displacement factor exponent takes the form: $-2 \pi^{2}\left[h^{2} a^{* 2} U^{11}+\ldots+2 h k a^{*} b^{*} U^{12}\right]$

\begin{tabular}{|c|c|c|c|c|c|c|}
\hline & $\mathrm{U}^{11}$ & $\mathrm{U}^{22}$ & $\mathrm{U}^{33}$ & $\mathrm{U}^{23}$ & $\mathrm{U}^{13}$ & $\mathrm{U}^{12}$ \\
\hline$C(1)$ & $30(1)$ & $18(1)$ & $32(1)$ & $-6(1)$ & $-2(1)$ & $-2(1)$ \\
\hline$C(2)$ & $31(1)$ & $22(1)$ & $58(2)$ & $-14(1)$ & $-11(1)$ & 1(1) \\
\hline$C(3)$ & $55(2)$ & $20(1)$ & $46(2)$ & $-8(1)$ & $-29(1)$ & $7(1)$ \\
\hline$C(4)$ & $57(2)$ & $22(1)$ & $26(1)$ & $-2(1)$ & $-13(1)$ & $2(1)$ \\
\hline$C(5)$ & $38(1)$ & $20(1)$ & $25(1)$ & $-3(1)$ & $-4(1)$ & 1(1) \\
\hline$C(6)$ & $29(1)$ & $12(1)$ & $22(1)$ & $-4(1)$ & $-3(1)$ & $0(1)$ \\
\hline$C(7)$ & $23(1)$ & $19(1)$ & $20(1)$ & 1(1) & $3(1)$ & $-1(1)$ \\
\hline $\mathrm{N}(8)$ & $21(1)$ & $13(1)$ & 21(1) & $0(1)$ & $2(1)$ & 1(1) \\
\hline$C(9)$ & $31(1)$ & $16(1)$ & $18(1)$ & $0(1)$ & $-1(1)$ & $0(1)$ \\
\hline$C(10)$ & $40(1)$ & $20(1)$ & $26(1)$ & $-2(1)$ & $-8(1)$ & $-1(1)$ \\
\hline$C(11)$ & $49(2)$ & $16(1)$ & $36(1)$ & $-3(1)$ & $-9(1)$ & $-4(1)$ \\
\hline$C(12)$ & $49(1)$ & $17(1)$ & $34(1)$ & $-1(1)$ & $-5(1)$ & $7(1)$ \\
\hline$C(13)$ & $38(1)$ & $19(1)$ & $29(1)$ & $-1(1)$ & $-6(1)$ & $4(1)$ \\
\hline $\mathrm{C}(14)$ & $31(1)$ & $16(1)$ & $18(1)$ & $0(1)$ & $0(1)$ & 2(1) \\
\hline $\mathrm{N}(15)$ & $24(1)$ & $16(1)$ & $20(1)$ & $0(1)$ & $0(1)$ & $0(1)$ \\
\hline$C(16)$ & $22(1)$ & $18(1)$ & $23(1)$ & $5(1)$ & $3(1)$ & $0(1)$ \\
\hline $\mathrm{O}(17)$ & $25(1)$ & $20(1)$ & $39(1)$ & $0(1)$ & 1(1) & $6(1)$ \\
\hline$C(18)$ & $22(1)$ & $20(1)$ & $22(1)$ & $2(1)$ & $-2(1)$ & $5(1)$ \\
\hline$C(19)$ & $20(1)$ & $24(1)$ & $32(1)$ & $0(1)$ & $0(1)$ & $0(1)$ \\
\hline $\mathrm{C}(20)$ & $21(1)$ & $23(1)$ & $31(1)$ & $0(1)$ & $-2(1)$ & $-2(1)$ \\
\hline $\mathrm{C}(21)$ & $23(1)$ & $16(1)$ & $26(1)$ & $-3(1)$ & $-2(1)$ & $-3(1)$ \\
\hline $\mathrm{N}(22)$ & $18(1)$ & $15(1)$ & $22(1)$ & 1(1) & $2(1)$ & $-1(1)$ \\
\hline$C(23)$ & $24(1)$ & $22(1)$ & $18(1)$ & $-1(1)$ & $1(1)$ & 1(1) \\
\hline$C(24)$ & $27(1)$ & $23(1)$ & $18(1)$ & $0(1)$ & $7(1)$ & 1(1) \\
\hline$C(25)$ & $36(1)$ & $28(1)$ & 21(1) & $3(1)$ & $8(1)$ & $6(1)$ \\
\hline$C(26)$ & $60(2)$ & $20(1)$ & 31(1) & $5(1)$ & $16(1)$ & $8(1)$ \\
\hline $\mathrm{C}(27)$ & $56(2)$ & $27(1)$ & $34(1)$ & $-3(1)$ & $15(1)$ & $-12(1)$ \\
\hline $\mathrm{C}(28)$ & $39(1)$ & $34(1)$ & $33(1)$ & $-3(1)$ & $9(1)$ & $-14(1)$ \\
\hline C(29) & $29(1)$ & $26(1)$ & $24(1)$ & $1(1)$ & $6(1)$ & $-5(1)$ \\
\hline $\mathrm{O}(30)$ & $21(1)$ & $17(1)$ & $22(1)$ & $-2(1)$ & $-2(1)$ & $0(1)$ \\
\hline $\mathrm{C}(31)$ & $18(1)$ & $17(1)$ & $22(1)$ & 1(1) & 1(1) & $-5(1)$ \\
\hline $\mathrm{O}(32)$ & $19(1)$ & $18(1)$ & $28(1)$ & $-2(1)$ & 1(1) & $4(1)$ \\
\hline$C(33)$ & $17(1)$ & $15(1)$ & $25(1)$ & $0(1)$ & $-4(1)$ & 1(1) \\
\hline$C(34)$ & $25(1)$ & $17(1)$ & $21(1)$ & $0(1)$ & $0(1)$ & $2(1)$ \\
\hline$C(35)$ & $27(1)$ & $22(1)$ & $27(1)$ & $4(1)$ & $-6(1)$ & $-2(1)$ \\
\hline$C(36)$ & $22(1)$ & $21(1)$ & 21(1) & $7(1)$ & $3(1)$ & 1(1) \\
\hline$C(37)$ & $26(1)$ & $25(1)$ & $30(1)$ & $10(1)$ & $3(1)$ & $3(1)$ \\
\hline $\mathrm{C}(38)$ & $20(1)$ & $42(1)$ & $41(1)$ & $22(1)$ & $2(1)$ & $2(1)$ \\
\hline C(39) & $24(1)$ & $38(1)$ & $33(1)$ & $17(1)$ & $-5(1)$ & $-8(1)$ \\
\hline $\mathrm{C}(40)$ & $30(1)$ & $27(1)$ & $24(1)$ & $6(1)$ & 1(1) & $-9(1)$ \\
\hline $\mathrm{Cl}(41)$ & $43(1)$ & $36(1)$ & $40(1)$ & $-8(1)$ & $-2(1)$ & $-17(1)$ \\
\hline $\mathrm{C}(42)$ & $23(1)$ & $23(1)$ & $24(1)$ & $5(1)$ & $0(1)$ & $-1(1)$ \\
\hline Ni(43) & $20(1)$ & $15(1)$ & $20(1)$ & $-1(1)$ & $-2(1)$ & $1(1)$ \\
\hline
\end{tabular}


Table 5. Hydrogen coordinates $\left(\times 10^{4}\right)$ and isotropic displacement parameters $\left(\AA^{2} \times 10^{3}\right)$ for sw005.

\begin{tabular}{|c|c|c|c|c|}
\hline & $\mathrm{X}$ & $\mathrm{y}$ & $\mathrm{Z}$ & $\mathrm{U}(\mathrm{eq})$ \\
\hline $\mathrm{H}(1)$ & 10811 & 10451 & 1638 & 32 \\
\hline $\mathrm{H}(2)$ & 12943 & 10318 & 2294 & 44 \\
\hline $\mathrm{H}(3)$ & 12312 & 9932 & 3211 & 48 \\
\hline $\mathrm{H}(4)$ & 9554 & 9742 & 3479 & 42 \\
\hline $\mathrm{H}(5)$ & 7416 & 9903 & 2833 & 33 \\
\hline $\mathrm{H}(10)$ & 8530 & 11722 & 2062 & 34 \\
\hline $\mathrm{H}(11)$ & 7585 & 13115 & 1872 & 40 \\
\hline $\mathrm{H}(12)$ & 5151 & 13288 & 1347 & 40 \\
\hline $\mathrm{H}(13)$ & 3749 & 12097 & 1000 & 35 \\
\hline $\mathrm{H}(18)$ & 2310 & 9968 & 10 & 26 \\
\hline $\mathrm{H}(19 \mathrm{~A})$ & 1555 & 9035 & 1016 & 30 \\
\hline $\mathrm{H}(19 \mathrm{~B})$ & 251 & 9371 & 553 & 30 \\
\hline $\mathrm{H}(20 \mathrm{~A})$ & 1155 & 7794 & 468 & 30 \\
\hline $\mathrm{H}(20 \mathrm{~B})$ & 1196 & 8355 & -98 & 30 \\
\hline $\mathrm{H}(21 \mathrm{~A})$ & 3913 & 7931 & -74 & 26 \\
\hline $\mathrm{H}(21 \mathrm{~B})$ & 3952 & 7902 & 594 & 26 \\
\hline $\mathrm{H}(23 \mathrm{~A})$ & 4230 & 9318 & -568 & 26 \\
\hline $\mathrm{H}(23 \mathrm{~B})$ & 5941 & 8945 & -326 & 26 \\
\hline $\mathrm{H}(25)$ & 3852 & 10911 & -654 & 34 \\
\hline $\mathrm{H}(26)$ & 5034 & 12309 & -607 & 44 \\
\hline $\mathrm{H}(27)$ & 7557 & 12525 & -155 & 46 \\
\hline $\mathrm{H}(28)$ & 9052 & 11334 & 184 & 42 \\
\hline $\mathrm{H}(29)$ & 7937 & 9936 & 106 & 31 \\
\hline $\mathrm{H}(33)$ & 9065 & 8755 & 1755 & 23 \\
\hline $\mathrm{H}(34)$ & 6511 & 8616 & 2370 & 26 \\
\hline $\mathrm{H}(35 \mathrm{~A})$ & 7531 & 7265 & 2725 & 38 \\
\hline $\mathrm{H}(35 \mathrm{~B})$ & 8996 & 7947 & 2610 & 38 \\
\hline $\mathrm{H}(35 \mathrm{C})$ & 8671 & 7165 & 2184 & 38 \\
\hline $\mathrm{H}(37)$ & 3711 & 8614 & 2066 & 32 \\
\hline $\mathrm{H}(38)$ & 1407 & 7871 & 1733 & 41 \\
\hline $\mathrm{H}(39)$ & 1677 & 6488 & 1341 & 38 \\
\hline $\mathrm{H}(42)$ & 6636 & 6560 & 1654 & 28 \\
\hline
\end{tabular}


Table 6. Torsion angles $\left[{ }^{\circ}\right]$ for sw005.

$$
\begin{aligned}
& \mathrm{C}(6)-\mathrm{C}(1)-\mathrm{C}(2)-\mathrm{C}(3) \\
& \mathrm{C}(1)-\mathrm{C}(2)-\mathrm{C}(3)-\mathrm{C}(4) \\
& \mathrm{C}(2)-\mathrm{C}(3)-\mathrm{C}(4)-\mathrm{C}(5) \\
& \mathrm{C}(3)-\mathrm{C}(4)-\mathrm{C}(5)-\mathrm{C}(6) \\
& \mathrm{C}(4)-\mathrm{C}(5)-\mathrm{C}(6)-\mathrm{C}(1) \\
& \mathrm{C}(4)-\mathrm{C}(5)-\mathrm{C}(6)-\mathrm{C}(7) \\
& \mathrm{C}(2)-\mathrm{C}(1)-\mathrm{C}(6)-\mathrm{C}(5) \\
& \mathrm{C}(2)-\mathrm{C}(1)-\mathrm{C}(6)-\mathrm{C}(7) \\
& \mathrm{C}(5)-\mathrm{C}(6)-\mathrm{C}(7)-\mathrm{N}(8) \\
& \mathrm{C}(1)-\mathrm{C}(6)-\mathrm{C}(7)-\mathrm{N}(8) \\
& \mathrm{C}(5)-\mathrm{C}(6)-\mathrm{C}(7)-\mathrm{C}(9) \\
& \mathrm{C}(1)-\mathrm{C}(6)-\mathrm{C}(7)-\mathrm{C}(9) \\
& \mathrm{C}(9)-\mathrm{C}(7)-\mathrm{N}(8)-\mathrm{C}(33) \\
& \mathrm{C}(6)-\mathrm{C}(7)-\mathrm{N}(8)-\mathrm{C}(33) \\
& \text { C(9)-C(7)-N(8)-Ni(43) } \\
& \mathrm{C}(6)-\mathrm{C}(7)-\mathrm{N}(8)-\mathrm{Ni}(43) \\
& \mathrm{N}(8)-\mathrm{C}(7)-\mathrm{C}(9)-\mathrm{C}(10) \\
& \mathrm{C}(6)-\mathrm{C}(7)-\mathrm{C}(9)-\mathrm{C}(10) \\
& \mathrm{N}(8)-\mathrm{C}(7)-\mathrm{C}(9)-\mathrm{C}(14) \\
& \mathrm{C}(6)-\mathrm{C}(7)-\mathrm{C}(9)-\mathrm{C}(14) \\
& \mathrm{C}(14)-\mathrm{C}(9)-\mathrm{C}(10)-\mathrm{C}(11) \\
& \mathrm{C}(7)-\mathrm{C}(9)-\mathrm{C}(10)-\mathrm{C}(11) \\
& \text { C(9)-C(10)-C(11)-C(12) } \\
& \mathrm{C}(10)-\mathrm{C}(11)-\mathrm{C}(12)-\mathrm{C}(13) \\
& \mathrm{C}(11)-\mathrm{C}(12)-\mathrm{C}(13)-\mathrm{C}(14) \\
& \mathrm{C}(12)-\mathrm{C}(13)-\mathrm{C}(14)-\mathrm{N}(15) \\
& \mathrm{C}(12)-\mathrm{C}(13)-\mathrm{C}(14)-\mathrm{C}(9) \\
& \mathrm{C}(10)-\mathrm{C}(9)-\mathrm{C}(14)-\mathrm{N}(15) \\
& \mathrm{C}(7)-\mathrm{C}(9)-\mathrm{C}(14)-\mathrm{N}(15) \\
& \mathrm{C}(10)-\mathrm{C}(9)-\mathrm{C}(14)-\mathrm{C}(13) \\
& \mathrm{C}(7)-\mathrm{C}(9)-\mathrm{C}(14)-\mathrm{C}(13) \\
& \mathrm{C}(13)-\mathrm{C}(14)-\mathrm{N}(15)-\mathrm{C}(16) \\
& \mathrm{C}(9)-\mathrm{C}(14)-\mathrm{N}(15)-\mathrm{C}(16) \\
& \text { C(13)-C(14)-N(15)-Ni(43) } \\
& \mathrm{C}(9)-\mathrm{C}(14)-\mathrm{N}(15)-\mathrm{Ni}(43) \\
& \mathrm{C}(14)-\mathrm{N}(15)-\mathrm{C}(16)-\mathrm{O}(17) \\
& \mathrm{Ni}(43)-\mathrm{N}(15)-\mathrm{C}(16)-\mathrm{O}(17) \\
& \text { C(14)-N(15)-C(16)-C(18) } \\
& \mathrm{Ni}(43)-\mathrm{N}(15)-\mathrm{C}(16)-\mathrm{C}(18) \\
& \mathrm{O}(17)-\mathrm{C}(16)-\mathrm{C}(18)-\mathrm{N}(22) \\
& \mathrm{N}(15)-\mathrm{C}(16)-\mathrm{C}(18)-\mathrm{N}(22) \\
& \mathrm{O}(17)-\mathrm{C}(16)-\mathrm{C}(18)-\mathrm{C}(19) \\
& \mathrm{N}(15)-\mathrm{C}(16)-\mathrm{C}(18)-\mathrm{C}(19) \\
& \mathrm{N}(22)-\mathrm{C}(18)-\mathrm{C}(19)-\mathrm{C}(20) \\
& \mathrm{C}(16)-\mathrm{C}(18)-\mathrm{C}(19)-\mathrm{C}(20) \\
& \mathrm{C}(18)-\mathrm{C}(19)-\mathrm{C}(20)-\mathrm{C}(21) \\
& \mathrm{C}(19)-\mathrm{C}(20)-\mathrm{C}(21)-\mathrm{N}(22) \\
& \mathrm{C}(16)-\mathrm{C}(18)-\mathrm{N}(22)-\mathrm{C}(23) \\
& 0.3(3) \quad \mathrm{C}(29)-\mathrm{C}(24)-\mathrm{C}(25)-\mathrm{C}(26) \quad-1.0(3) \\
& -1.6(3) \quad \mathrm{C}(23)-\mathrm{C}(24)-\mathrm{C}(25)-\mathrm{C}(26) \quad 176.43(17) \\
& 0.9(3) \quad \mathrm{C}(24)-\mathrm{C}(25)-\mathrm{C}(26)-\mathrm{C}(27) \quad-1.7(3) \\
& \text { 1.2(3) } \mathrm{C}(25)-\mathrm{C}(26)-\mathrm{C}(27)-\mathrm{C}(28) \quad 3.1(3) \\
& -2.6(3) \quad \mathrm{C}(26)-\mathrm{C}(27)-\mathrm{C}(28)-\mathrm{C}(29) \quad-1.7(3) \\
& 175.87(18) \quad \mathrm{C}(27)-\mathrm{C}(28)-\mathrm{C}(29)-\mathrm{C}(24) \quad-1.1(3) \\
& 1.8(3) \quad \mathrm{C}(25)-\mathrm{C}(24)-\mathrm{C}(29)-\mathrm{C}(28) \quad 2.4(3) \\
& -176.63(17) \quad \mathrm{C}(23)-\mathrm{C}(24)-\mathrm{C}(29)-\mathrm{C}(28) \quad-175.07(18) \\
& -86.7(2) \quad \mathrm{Ni}(43)-\mathrm{O}(30)-\mathrm{C}(31)-\mathrm{O}(32) \quad 179.03(14) \\
& \text { 91.7(2) } \mathrm{Ni}(43)-\mathrm{O}(30)-\mathrm{C}(31)-\mathrm{C}(33) \quad 1.12(19) \\
& \text { 94.2(2) } \quad \mathrm{C}(7)-\mathrm{N}(8)-\mathrm{C}(33)-\mathrm{C}(31) \quad-148.24(16) \\
& -87.4(2) \quad \mathrm{Ni}(43)-\mathrm{N}(8)-\mathrm{C}(33)-\mathrm{C}(31) \quad 25.46(17) \\
& \begin{array}{rrr}
-179.76(17) & \mathrm{C}(7)-\mathrm{N}(8)-\mathrm{C}(33)-\mathrm{C}(34) & 90.9(2) \\
1.1(2) & \mathrm{Ni}(43)-\mathrm{N}(8)-\mathrm{C}(33)-\mathrm{C}(34) & -95.44(15)
\end{array} \\
& \text { 7.7(3) } \quad \mathrm{O}(32)-\mathrm{C}(31)-\mathrm{C}(33)-\mathrm{N}(8) \quad 164.80(16) \\
& -171.41(13) \quad \mathrm{O}(30)-\mathrm{C}(31)-\mathrm{C}(33)-\mathrm{N}(8) \quad-17.2(2) \\
& -168.78(18) \quad \mathrm{O}(32)-\mathrm{C}(31)-\mathrm{C}(33)-\mathrm{C}(34) \quad-73.4(2) \\
& 10.3(3) \quad \mathrm{O}(30)-\mathrm{C}(31)-\mathrm{C}(33)-\mathrm{C}(34) \quad 104.65(18) \\
& 10.7(3) \quad \mathrm{N}(8)-\mathrm{C}(33)-\mathrm{C}(34)-\mathrm{C}(36) \quad 81.08(19) \\
& -170.22(17) \quad \mathrm{C}(31)-\mathrm{C}(33)-\mathrm{C}(34)-\mathrm{C}(36) \quad-37.8(2) \\
& -1.3(3) \quad \mathrm{N}(8)-\mathrm{C}(33)-\mathrm{C}(34)-\mathrm{C}(35) \quad-151.71(16) \\
& 178.2(2) \quad \mathrm{C}(31)-\mathrm{C}(33)-\mathrm{C}(34)-\mathrm{C}(35) \quad 89.45(18) \\
& \text { 2.6(3) } \quad \mathrm{C}(35)-\mathrm{C}(34)-\mathrm{C}(36)-\mathrm{C}(37) \quad 139.08(18) \\
& -1.1(3) \quad \mathrm{C}(33)-\mathrm{C}(34)-\mathrm{C}(36)-\mathrm{C}(37) \quad-95.8(2) \\
& -1.8(3) \quad \mathrm{C}(35)-\mathrm{C}(34)-\mathrm{C}(36)-\mathrm{C}(42) \quad-41.7(2) \\
& -173.16(19) \quad \mathrm{C}(33)-\mathrm{C}(34)-\mathrm{C}(36)-\mathrm{C}(42) \quad 83.5(2) \\
& \text { 3.1(3) } \quad \mathrm{C}(42)-\mathrm{C}(36)-\mathrm{C}(37)-\mathrm{C}(38) \quad 0.0(3) \\
& 174.72(17) \quad \mathrm{C}(34)-\mathrm{C}(36)-\mathrm{C}(37)-\mathrm{C}(38) \quad 179.25(18) \\
& -4.7(3) \quad \mathrm{C}(36)-\mathrm{C}(37)-\mathrm{C}(38)-\mathrm{C}(39) \quad-0.8(3) \\
& -1.6(3) \quad \mathrm{C}(37)-\mathrm{C}(38)-\mathrm{C}(39)-\mathrm{C}(40) \quad 0.4(3) \\
& 178.96(18) \quad \mathrm{C}(38)-\mathrm{C}(39)-\mathrm{C}(40)-\mathrm{C}(42) \quad 0.9(3) \\
& -12.0(3) \quad \mathrm{C}(38)-\mathrm{C}(39)-\mathrm{C}(40)-\mathrm{Cl}(41) \quad-178.58(15) \\
& 171.86(16) \quad \mathrm{C}(39)-\mathrm{C}(40)-\mathrm{C}(42)-\mathrm{C}(36) \quad-1.7(3) \\
& 158.05(15) \quad \mathrm{Cl}(41)-\mathrm{C}(40)-\mathrm{C}(42)-\mathrm{C}(36) \quad 177.77(14) \\
& -18.1(2) \quad \mathrm{C}(37)-\mathrm{C}(36)-\mathrm{C}(42)-\mathrm{C}(40) \quad 1.2(3) \\
& -17.3(3) \quad \mathrm{C}(34)-\mathrm{C}(36)-\mathrm{C}(42)-\mathrm{C}(40) \quad-178.03(17) \\
& 171.77(15) \quad \mathrm{C}(7)-\mathrm{N}(8)-\mathrm{Ni}(43)-\mathrm{N}(15) \quad-22.72(16) \\
& 165.75(16) \quad \mathrm{C}(33)-\mathrm{N}(8)-\mathrm{Ni}(43)-\mathrm{N}(15) \quad 164.15(12) \\
& -5.17(19) \quad \mathrm{C}(7)-\mathrm{N}(8)-\mathrm{Ni}(43)-\mathrm{O}(30) \quad 151.88(16) \\
& 170.58(15) \quad \mathrm{C}(33)-\mathrm{N}(8)-\mathrm{Ni}(43)-\mathrm{O}(30) \quad-21.24(12) \\
& -12.1(2) \quad \mathrm{C}(7)-\mathrm{N}(8)-\mathrm{Ni}(43)-\mathrm{N}(22) \quad-165.1(9) \\
& -71.0(2) \quad \mathrm{C}(33)-\mathrm{N}(8)-\mathrm{Ni}(43)-\mathrm{N}(22) \quad 21.7(10) \\
& 106.34(18) \quad \mathrm{C}(16)-\mathrm{N}(15)-\mathrm{Ni}(43)-\mathrm{N}(8) \quad-162.12(12) \\
& -38.25(18) \quad \mathrm{C}(14)-\mathrm{N}(15)-\mathrm{Ni}(43)-\mathrm{N}(8) \quad 27.13(14) \\
& -160.83(16) \quad \mathrm{C}(16)-\mathrm{N}(15)-\mathrm{Ni}(43)-\mathrm{O}(30) \quad 97.8(7) \\
& \text { 42.26(19) } \quad \mathrm{C}(14)-\mathrm{N}(15)-\mathrm{Ni}(43)-\mathrm{O}(30) \quad-73.0(7) \\
& -30.77(19) \quad \mathrm{C}(16)-\mathrm{N}(15)-\mathrm{Ni}(43)-\mathrm{N}(22) \quad 15.35(12) \\
& -98.07(17) \quad \mathrm{C}(14)-\mathrm{N}(15)-\mathrm{Ni}(43)-\mathrm{N}(22) \quad-155.40(14)
\end{aligned}
$$




$\begin{array}{rrrr}\mathrm{C}(19)-\mathrm{C}(18)-\mathrm{N}(22)-\mathrm{C}(23) & 138.74(15) & \mathrm{C}(31)-\mathrm{O}(30)-\mathrm{Ni}(43)-\mathrm{N}(8) & 11.66(12) \\ \mathrm{C}(16)-\mathrm{C}(18)-\mathrm{N}(22)-\mathrm{C}(21) & 142.37(15) & \mathrm{C}(31)-\mathrm{O}(30)-\mathrm{Ni}(43)-\mathrm{N}(15) & 112.2(6) \\ \mathrm{C}(19)-\mathrm{C}(18)-\mathrm{N}(22)-\mathrm{C}(21) & 19.19(18) & \mathrm{C}(31)-\mathrm{O}(30)-\mathrm{Ni}(43)-\mathrm{N}(22) & -165.51(12) \\ \mathrm{C}(16)-\mathrm{C}(18)-\mathrm{N}(22)-\mathrm{Ni}(43) & 22.28(16) & \mathrm{C}(18)-\mathrm{N}(22)-\mathrm{Ni}(43)-\mathrm{N}(8) & 122.0(9) \\ \mathrm{C}(19)-\mathrm{C}(18)-\mathrm{N}(22)-\mathrm{Ni}(43) & -100.90(13) & \mathrm{C}(23)-\mathrm{N}(22)-\mathrm{Ni}(43)-\mathrm{N}(8) & -116.6(9) \\ \mathrm{C}(20)-\mathrm{C}(21)-\mathrm{N}(22)-\mathrm{C}(18) & 7.30(19) & \mathrm{C}(21)-\mathrm{N}(22)-\mathrm{Ni}(43)-\mathrm{N}(8) & 5.9(10) \\ \mathrm{C}(20)-\mathrm{C}(21)-\mathrm{N}(22)-\mathrm{C}(23) & -113.78(17) & \mathrm{C}(18)-\mathrm{N}(22)-\mathrm{Ni}(43)-\mathrm{N}(15) & -20.61(11) \\ \mathrm{C}(20)-\mathrm{C}(21)-\mathrm{N}(22)-\mathrm{Ni}(43) & 123.34(14) & \mathrm{C}(23)-\mathrm{N}(22)-\mathrm{Ni}(43)-\mathrm{N}(15) & 100.86(12) \\ \mathrm{C}(18)-\mathrm{N}(22)-\mathrm{C}(23)-\mathrm{C}(24) & 64.54(19) & \mathrm{C}(21)-\mathrm{N}(22)-\mathrm{Ni}(43)-\mathrm{N}(15) & -136.63(12) \\ \mathrm{C}(21)-\mathrm{N}(22)-\mathrm{C}(23)-\mathrm{C}(24) & -177.81(15) & \mathrm{C}(18)-\mathrm{N}(22)-\mathrm{Ni}(43)-\mathrm{O}(30) & 164.81(11) \\ \mathrm{Ni}(43)-\mathrm{N}(22)-\mathrm{C}(23)-\mathrm{C}(24) & -53.56(17) & \mathrm{C}(23)-\mathrm{N}(22)-\mathrm{Ni}(43)-\mathrm{O}(30) & -73.72(12) \\ \mathrm{N}(22)-\mathrm{C}(23)-\mathrm{C}(24)-\mathrm{C}(29) & 83.0(2) & \mathrm{C}(21)-\mathrm{N}(22)-\mathrm{Ni}(43)-\mathrm{O}(30) & 48.80(12) \\ \mathrm{N}(22)-\mathrm{C}(23)-\mathrm{C}(24)-\mathrm{C}(25) & -94.5(2) & & \end{array}$




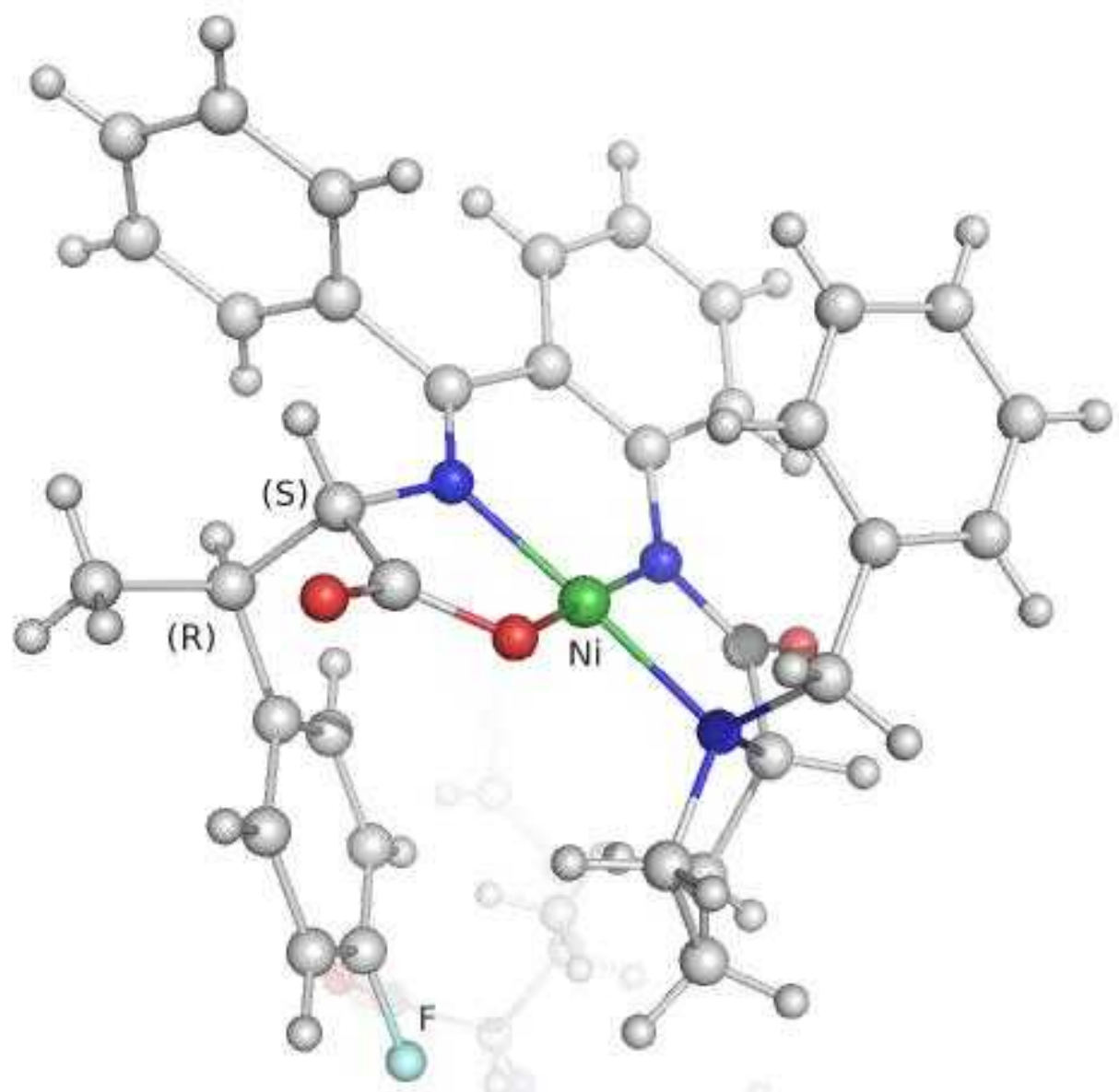

Table 1. Crystal data and structure refinement for sw007.

Identification code

Empirical formula

Formula weight

Temperature

Wavelength

Crystal system

Space group

Unit cell dimensions

Volume

Z sw007 1

$\mathrm{C}_{35} \mathrm{H}_{32} \mathrm{FN}_{3} \mathrm{NiO}_{3}$

620.35

100(2) K

$1.54178 \AA$

orthorhombic

P $22_{1} 2_{1}$

$$
\begin{array}{ll}
\mathrm{a}=11.146(2) \AA & \alpha=90^{\circ} . \\
\mathrm{b}=19.625(4) \AA & \beta=90^{\circ} . \\
\mathrm{c}=26.276(5) \AA & \gamma=90^{\circ} .
\end{array}
$$

$5748(2) \AA^{3}$ 
Density (calculated)

Absorption coefficient

$\mathrm{F}(000)$

Crystal size

Theta range for data collection

Index ranges

Reflections collected

Independent reflections

Completeness to theta $=60.02^{\circ}$

Max. and min. transmission

Refinement method

Data / restraints / parameters

Goodness-of-fit on $\mathrm{F}^{2}$

Final $\mathrm{R}$ indices [I $>2 \operatorname{sigma}(\mathrm{I})]$

$\mathrm{R}$ indices (all data)

Absolute structure parameter

Largest diff. peak and hole
$1.434 \mathrm{Mg} / \mathrm{m}^{3}$

$1.372 \mathrm{~mm}^{-1}$

2592

$0.25 \times 0.15 \times 0.10 \mathrm{~mm}^{3}$

2.81 to $60.02^{\circ}$.

$-11<=\mathrm{h}<=12,-20<=\mathrm{k}<=14,-29<=\mathrm{l}<=26$

40395

$7958[\mathrm{R}($ int $)=0.0404]$

$95.4 \%$

0.8750 and 0.7255

Full-matrix least-squares on $\mathrm{F}^{2}$

7958 / 0 / 777

1.024

$\mathrm{R}_{1}=0.0253, \mathrm{wR}_{2}=0.0615$

$\mathrm{R}_{1}=0.0275, \mathrm{wR}_{2}=0.0628$

$-0.032(14)$

0.371 and -0.244 e. $\AA^{-3}$

Table 2. Atomic coordinates $\left(\times 10^{4}\right)$ and equivalent isotropic displacement parameters $\left(\AA^{2} \times 10^{3}\right)$ for sw007. $U(e q)$ is defined as one third of the trace of the orthogonalized $U^{i j}$ tensor.

\begin{tabular}{rrrrr}
\hline & $\mathrm{x}$ & $\mathrm{y}$ & $\mathrm{z}$ & $\mathrm{U}(\mathrm{eq})$ \\
\hline $\mathrm{Ni}(01)$ & $8667(1)$ & $909(1)$ & $9527(1)$ & $24(1)$ \\
$\mathrm{Ni}(02)$ & $2579(1)$ & $7972(1)$ & $8358(1)$ & $26(1)$ \\
$\mathrm{O}(003)$ & $2613(2)$ & $5999(1)$ & $8385(1)$ & $34(1)$ \\
$\mathrm{O}(004)$ & $2427(2)$ & $9973(1)$ & $8393(1)$ & $32(1)$ \\
$\mathrm{F}(005)$ & $2685(1)$ & $7881(1)$ & $10638(1)$ & $44(1)$ \\
$\mathrm{O}(006)$ & $8806(1)$ & $85(1)$ & $9178(1)$ & $27(1)$ \\
$\mathrm{N}(007)$ & $8671(2)$ & $1707(1)$ & $9909(1)$ & $24(1)$ \\
$\mathrm{N}(008)$ & $10048(2)$ & $1254(1)$ & $9169(1)$ & $25(1)$ \\
$\mathrm{F}(009)$ & $7167(1)$ & $2101(1)$ & $7733(1)$ & $50(1)$ \\
$\mathrm{O}(010)$ & $7830(1)$ & $-889(1)$ & $9017(1)$ & $31(1)$ \\
$\mathrm{C}(011)$ & $2819(2)$ & $6611(1)$ & $8394(1)$ & $28(1)$ \\
$\mathrm{O}(012)$ & $9513(1)$ & $2802(1)$ & $9872(1)$ & $31(1)$ \\
$\mathrm{C}(013)$ & $4499(2)$ & $7733(1)$ & $9504(1)$ & $30(1)$
\end{tabular}




\begin{tabular}{|c|c|c|c|c|}
\hline & $\mathrm{x}$ & $\mathrm{y}$ & $\mathrm{z}$ & $\mathrm{U}(\mathrm{eq})$ \\
\hline $\mathrm{O}(014)$ & $2013(1)$ & $7085(1)$ & $8442(1)$ & $30(1)$ \\
\hline$C(015)$ & $6853(2)$ & 704(1) & $10273(1)$ & $23(1)$ \\
\hline$C(016)$ & $4801(2)$ & $6788(1)$ & 8868(1) & $29(1)$ \\
\hline $\mathrm{N}(017)$ & $7345(2)$ & $528(1)$ & $9842(1)$ & $24(1)$ \\
\hline $\mathrm{C}(018)$ & $2310(2)$ & $9351(1)$ & $8416(1)$ & $27(1)$ \\
\hline $\mathrm{C}(019)$ & $4643(2)$ & 479(1) & $10409(1)$ & $26(1)$ \\
\hline $\mathrm{C}(020)$ & $1337(2)$ & $9059(1)$ & $8752(1)$ & $32(1)$ \\
\hline $\mathrm{C}(021)$ & $10064(2)$ & $2007(1)$ & $9242(1)$ & $27(1)$ \\
\hline $\mathrm{C}(022)$ & $3733(2)$ & $57(1)$ & $10574(1)$ & $30(1)$ \\
\hline $\mathrm{C}(023)$ & $8168(2)$ & 1761(1) & 10401(1) & $25(1)$ \\
\hline $\mathrm{C}(024)$ & $3939(2)$ & $9025(1)$ & $7856(1)$ & $26(1)$ \\
\hline $\mathrm{C}(025)$ & $4900(2)$ & $8566(1)$ & $7757(1)$ & $25(1)$ \\
\hline $\mathrm{C}(026)$ & $6146(2)$ & 681(1) & $8805(1)$ & $25(1)$ \\
\hline $\mathrm{C}(027)$ & $2812(2)$ & 6991(1) & $10044(1)$ & $33(1)$ \\
\hline $\mathrm{C}(028)$ & $5836(2)$ & 286(1) & $10483(1)$ & $24(1)$ \\
\hline $\mathrm{C}(029)$ & $11224(2)$ & 992(1) & $9956(1)$ & $25(1)$ \\
\hline $\mathrm{C}(030)$ & $4104(2)$ & $6871(1)$ & $8358(1)$ & $27(1)$ \\
\hline $\mathrm{C}(031)$ & $5843(2)$ & $9398(1)$ & $7196(1)$ & $34(1)$ \\
\hline $\mathrm{C}(032)$ & $7249(2)$ & $1827(1)$ & $11406(1)$ & $30(1)$ \\
\hline $\mathrm{C}(033)$ & $3985(2)$ & $-552(1)$ & $10818(1)$ & $32(1)$ \\
\hline $\mathrm{C}(034)$ & $3175(2)$ & $7623(1)$ & $10200(1)$ & $33(1)$ \\
\hline $\mathrm{N}(035)$ & $3017(2)$ & $8857(1)$ & 8197(1) & $26(1)$ \\
\hline$C(036)$ & $4872(2)$ & $9826(1)$ & $7269(1)$ & $33(1)$ \\
\hline $\mathrm{C}(037)$ & $8098(2)$ & $2289(1)$ & $11238(1)$ & $31(1)$ \\
\hline $\mathrm{C}(038)$ & $9991(2)$ & $1125(1)$ & 8594(1) & $29(1)$ \\
\hline $\mathrm{C}(039)$ & $6582(2)$ & $478(1)$ & $8332(1)$ & $30(1)$ \\
\hline $\mathrm{C}(040)$ & $4952(2)$ & $7868(1)$ & 7951(1) & $26(1)$ \\
\hline $\mathrm{C}(041)$ & $6093(2)$ & $-312(1)$ & $10744(1)$ & $27(1)$ \\
\hline $\mathrm{N}(042)$ & $1087(2)$ & $8334(1)$ & $8618(1)$ & $33(1)$ \\
\hline$C(043)$ & $6046(2)$ & $7437(1)$ & $7852(1)$ & $27(1)$ \\
\hline $\mathrm{C}(044)$ & $8548(2)$ & $2258(1)$ & $10750(1)$ & $30(1)$ \\
\hline $\mathrm{C}(045)$ & $6035(2)$ & $6970(1)$ & 7451(1) & $28(1)$ \\
\hline $\mathrm{C}(046)$ & $5841(2)$ & $8770(1)$ & $7434(1)$ & $31(1)$ \\
\hline $\mathrm{C}(047)$ & $6927(2)$ & $951(2)$ & $7966(1)$ & $35(1)$ \\
\hline $\mathrm{C}(048)$ & $4006(2)$ & $8005(1)$ & 9944(1) & $33(1)$ \\
\hline $\mathrm{C}(049)$ & $7306(2)$ & $1275(1)$ & $10573(1)$ & $24(1)$ \\
\hline $\mathrm{C}(050)$ & $9391(2)$ & $2215(1)$ & $9716(1)$ & $26(1)$ \\
\hline $\mathrm{C}(051)$ & $10558(2)$ & $567(1)$ & $10279(1)$ & $28(1)$ \\
\hline $\mathrm{C}(052)$ & 3942(2) & $9648(1)$ & 7591(1) & $31(1)$ \\
\hline $\mathrm{C}(053)$ & $5163(3)$ & $6049(1)$ & $8948(1)$ & $39(1)$ \\
\hline $\mathrm{C}(054)$ & $155(2)$ & $8712(2)$ & $7786(1)$ & $36(1)$ \\
\hline $\mathrm{C}(055)$ & $9525(2)$ & $2290(1)$ & $8748(1)$ & $30(1)$ \\
\hline$C(056)$ & $11226(2)$ & $1196(1)$ & $11009(1)$ & $34(1)$ \\
\hline $\mathrm{C}(057)$ & $6366(2)$ & $1856(1)$ & $8540(1)$ & $35(1)$ \\
\hline $\mathrm{C}(058)$ & 764(3) & $7986(2)$ & $9120(1)$ & $52(1)$ \\
\hline $\mathrm{C}(059)$ & $6863(2)$ & 1331(1) & $11074(1)$ & $28(1)$ \\
\hline $\mathrm{C}(060)$ & $11147(2)$ & 919(1) & $9386(1)$ & $29(1)$ \\
\hline $\mathrm{C}(061)$ & $7899(2)$ & $-326(1)$ & 9221(1) & $26(1)$ \\
\hline $\mathrm{C}(062)$ & $11921(2)$ & 1499(1) & $10175(1)$ & $30(1)$ \\
\hline
\end{tabular}




\begin{tabular}{rrrrr}
\hline \multicolumn{1}{c}{$\mathrm{x}$} & $\mathrm{y}$ & $\mathrm{z}$ & $\mathrm{U}(\mathrm{eq})$ \\
\hline $\mathrm{C}(063)$ & $-523(2)$ & $9303(2)$ & $7759(1)$ & $46(1)$ \\
$\mathrm{N}(064)$ & $4048(2)$ & $7591(1)$ & $8191(1)$ & $25(1)$ \\
$\mathrm{C}(065)$ & $5785(2)$ & $175(1)$ & $9211(1)$ & $26(1)$ \\
$\mathrm{C}(066)$ & $4174(2)$ & $7092(1)$ & $9329(1)$ & $26(1)$ \\
$\mathrm{C}(067)$ & $11931(2)$ & $1595(1)$ & $10697(1)$ & $32(1)$ \\
$\mathrm{C}(068)$ & $3316(2)$ & $6727(1)$ & $9604(1)$ & $32(1)$ \\
$\mathrm{C}(069)$ & $6037(2)$ & $1374(1)$ & $8902(1)$ & $33(1)$ \\
$\mathrm{C}(070)$ & $905(2)$ & $8541(2)$ & $7382(1)$ & $37(1)$ \\
$\mathrm{C}(071)$ & $10073(2)$ & $1823(1)$ & $8350(1)$ & $32(1)$ \\
$\mathrm{C}(072)$ & $6871(2)$ & $-53(1)$ & $9545(1)$ & $25(1)$ \\
$\mathrm{C}(073)$ & $314(3)$ & $9560(2)$ & $6943(1)$ & $53(1)$ \\
$\mathrm{C}(074)$ & $6825(2)$ & $1624(2)$ & $8087(1)$ & $37(1)$ \\
$\mathrm{C}(075)$ & $10547(2)$ & $672(1)$ & $10800(1)$ & $30(1)$ \\
$\mathrm{C}(076)$ & $5171(2)$ & $-733(1)$ & $10911(1)$ & $30(1)$ \\
$\mathrm{C}(077)$ & $7915(2)$ & $6503(1)$ & $7725(1)$ & $35(1)$ \\
$\mathrm{C}(078)$ & $7035(2)$ & $7449(1)$ & $8175(1)$ & $31(1)$ \\
$\mathrm{C}(079)$ & $989(2)$ & $8968(2)$ & $6967(1)$ & $47(1)$ \\
$\mathrm{C}(080)$ & $876(3)$ & $8514(2)$ & $9525(1)$ & $60(1)$ \\
$\mathrm{C}(081)$ & $-451(3)$ & $9725(2)$ & $7337(1)$ & $54(1)$ \\
$\mathrm{C}(082)$ & $7948(2)$ & $6977(2)$ & $8117(1)$ & $37(1)$ \\
$\mathrm{C}(083)$ & $5109(2)$ & $-446(1)$ & $9007(1)$ & $34(1)$ \\
$\mathrm{C}(084)$ & $6959(2)$ & $6511(1)$ & $7386(1)$ & $33(1)$ \\
$\mathrm{C}(085)$ & $1702(3)$ & $9029(1)$ & $9316(1)$ & $41(1)$ \\
$\mathrm{C}(086)$ & $68(2)$ & $8257(1)$ & $8250(1)$ & $42(1)$ \\
\hline & & & &
\end{tabular}

Table 3. Bond lengths $[\AA]$ and angles $\left[^{\circ}\right]$ for sw007.

$\begin{array}{lrlllr}\mathrm{Ni}(01)-\mathrm{N}(017) & 1.8484(18) & \mathrm{C}(026)-\mathrm{C}(065) & 1.511(3) & \mathrm{C}(055)-\mathrm{H}(05 \mathrm{G}) & 0.9900 \\ \mathrm{Ni}(01)-\mathrm{N}(007) & 1.8618(19) & \mathrm{C}(027)-\mathrm{C}(034) & 1.369(4) & \mathrm{C}(056)-\mathrm{C}(067) & 1.379(3) \\ \mathrm{Ni}(01)-\mathrm{O}(006) & 1.8651(16) & \mathrm{C}(027)-\mathrm{C}(068) & 1.386(3) & \mathrm{C}(056)-\mathrm{C}(075) & 1.389(3) \\ \mathrm{Ni}(01)-\mathrm{N}(008) & 1.9273(19) & \mathrm{C}(027)-\mathrm{H}(02 \mathrm{D}) & 0.9500 & \mathrm{C}(056)-\mathrm{H}(05 \mathrm{H}) & 0.9500 \\ \mathrm{Ni}(02)-\mathrm{N}(035) & 1.853(2) & \mathrm{C}(028)-\mathrm{C}(041) & 1.388(3) & \mathrm{C}(057)-\mathrm{C}(074) & 1.373(4) \\ \mathrm{Ni}(02)-\mathrm{N}(064) & 1.853(2) & \mathrm{C}(029)-\mathrm{C}(062) & 1.387(3) & \mathrm{C}(057)-\mathrm{C}(069) & 1.392(3) \\ \mathrm{Ni}(02)-\mathrm{O}(014) & 1.8640(17) & \mathrm{C}(029)-\mathrm{C}(051) & 1.403(3) & \mathrm{C}(057)-\mathrm{H}(05 \mathrm{I}) & 0.9500 \\ \mathrm{Ni}(02)-\mathrm{N}(042) & 1.934(2) & \mathrm{C}(029)-\mathrm{C}(060) & 1.507(3) & \mathrm{C}(058)-\mathrm{C}(080) & 1.492(4) \\ \mathrm{O}(003)-\mathrm{C}(011) & 1.224(3) & \mathrm{C}(030)-\mathrm{N}(064) & 1.480(3) & \mathrm{C}(058)-\mathrm{H}(05 \mathrm{~J}) & 0.9900 \\ \mathrm{O}(004)-\mathrm{C}(018) & 1.230(3) & \mathrm{C}(030)-\mathrm{H}(03 \mathrm{~A}) & 1.0000 & \mathrm{C}(058)-\mathrm{H}(05 \mathrm{~K}) & 0.9900 \\ \mathrm{~F}(005)-\mathrm{C}(034) & 1.372(3) & \mathrm{C}(031)-\mathrm{C}(046) & 1.381(3) & \mathrm{C}(059)-\mathrm{H}(05 \mathrm{~L}) & 0.9500\end{array}$




\begin{tabular}{|c|c|c|c|c|c|}
\hline $\mathrm{O}(006)-\mathrm{C}(061)$ & $1.298(3)$ & $\mathrm{C}(031)-\mathrm{C}(036)$ & $1.384(4)$ & $\mathrm{C}(060)-\mathrm{H}(06 \mathrm{~A})$ & 0.9900 \\
\hline $\mathrm{N}(007)-\mathrm{C}(050)$ & $1.376(3)$ & $\mathrm{C}(031)-\mathrm{H}(03 \mathrm{~B})$ & 0.9500 & $\mathrm{C}(060)-\mathrm{H}(06 \mathrm{~B})$ & 0.9900 \\
\hline $\mathrm{N}(007)-\mathrm{C}(023)$ & $1.412(3)$ & $\mathrm{C}(032)-\mathrm{C}(059)$ & $1.376(3)$ & $\mathrm{C}(061)-\mathrm{C}(072)$ & $1.524(3)$ \\
\hline $\mathrm{N}(008)-\mathrm{C}(021)$ & $1.490(3)$ & $\mathrm{C}(032)-\mathrm{C}(037)$ & $1.382(3)$ & $\mathrm{C}(062)-\mathrm{C}(067)$ & $1.385(3)$ \\
\hline $\mathrm{N}(008)-\mathrm{C}(060)$ & $1.504(3)$ & $\mathrm{C}(032)-\mathrm{H}(03 \mathrm{C})$ & 0.9500 & $\mathrm{C}(062)-\mathrm{H}(06 \mathrm{C})$ & 0.9500 \\
\hline $\mathrm{N}(008)-\mathrm{C}(038)$ & $1.533(3)$ & $\mathrm{C}(033)-\mathrm{C}(076)$ & $1.391(3)$ & $\mathrm{C}(063)-\mathrm{C}(081)$ & $1.387(4)$ \\
\hline F(009)-C(074) & $1.373(3)$ & $\mathrm{C}(033)-\mathrm{H}(03 \mathrm{D})$ & 0.9500 & $\mathrm{C}(063)-\mathrm{H}(06 \mathrm{D})$ & 0.9500 \\
\hline $\mathrm{O}(010)-\mathrm{C}(061)$ & $1.230(3)$ & $C(034)-C(048)$ & $1.368(4)$ & $\mathrm{C}(065)-\mathrm{C}(083)$ & $1.530(3)$ \\
\hline $\mathrm{C}(011)-\mathrm{O}(014)$ & $1.299(3)$ & $C(036)-C(052)$ & $1.383(3)$ & $\mathrm{C}(065)-\mathrm{C}(072)$ & $1.560(3)$ \\
\hline $\mathrm{C}(011)-\mathrm{C}(030)$ & $1.524(3)$ & $\mathrm{C}(036)-\mathrm{H}(03 \mathrm{E})$ & 0.9500 & $\mathrm{C}(065)-\mathrm{H}(06 \mathrm{E})$ & 1.0000 \\
\hline $\mathrm{O}(012)-\mathrm{C}(050)$ & $1.231(3)$ & $\mathrm{C}(037)-\mathrm{C}(044)$ & $1.379(3)$ & $\mathrm{C}(066)-\mathrm{C}(068)$ & $1.396(3)$ \\
\hline $\mathrm{C}(013)-\mathrm{C}(048)$ & $1.388(3)$ & $\mathrm{C}(037)-\mathrm{H}(03 \mathrm{~F})$ & 0.9500 & $\mathrm{C}(067)-\mathrm{H}(06 \mathrm{~F})$ & 0.9500 \\
\hline$C(013)-C(066)$ & $1.387(3)$ & $\mathrm{C}(038)-\mathrm{C}(071)$ & $1.516(3)$ & $\mathrm{C}(068)-\mathrm{H}(06 \mathrm{G})$ & 0.9500 \\
\hline $\mathrm{C}(013)-\mathrm{H}(01 \mathrm{~A})$ & 0.9500 & $\mathrm{C}(038)-\mathrm{H}(03 \mathrm{G})$ & 0.9900 & $\mathrm{C}(069)-\mathrm{H}(06 \mathrm{H})$ & 0.9500 \\
\hline $\mathrm{C}(015)-\mathrm{N}(017)$ & $1.304(3)$ & $\mathrm{C}(038)-\mathrm{H}(03 \mathrm{H})$ & 0.9900 & $\mathrm{C}(070)-\mathrm{C}(079)$ & $1.377(4)$ \\
\hline $\mathrm{C}(015)-\mathrm{C}(049)$ & $1.460(3)$ & $\mathrm{C}(039)-\mathrm{C}(047)$ & $1.392(3)$ & $\mathrm{C}(070)-\mathrm{H}(07 \mathrm{~A})$ & 0.9500 \\
\hline $\mathrm{C}(015)-\mathrm{C}(028)$ & $1.504(3)$ & $\mathrm{C}(039)-\mathrm{H}(03 \mathrm{I})$ & 0.9500 & $\mathrm{C}(071)-\mathrm{H}(07 \mathrm{~B})$ & 0.9900 \\
\hline $\mathrm{C}(016)-\mathrm{C}(053)$ & $1.520(3)$ & $\mathrm{C}(040)-\mathrm{N}(064)$ & $1.306(3)$ & $\mathrm{C}(071)-\mathrm{H}(07 \mathrm{C})$ & 0.9900 \\
\hline $\mathrm{C}(016)-\mathrm{C}(066)$ & $1.521(3)$ & $C(040)-C(043)$ & $1.506(3)$ & $\mathrm{C}(072)-\mathrm{H}(07 \mathrm{D})$ & 1.0000 \\
\hline $\mathrm{C}(016)-\mathrm{C}(030)$ & $1.558(3)$ & $\mathrm{C}(041)-\mathrm{C}(076)$ & $1.390(3)$ & $\mathrm{C}(073)-\mathrm{C}(081)$ & $1.378(4)$ \\
\hline $\mathrm{C}(016)-\mathrm{H}(016)$ & 1.0000 & $\mathrm{C}(041)-\mathrm{H}(04 \mathrm{~A})$ & 0.9500 & $\mathrm{C}(073)-\mathrm{C}(079)$ & $1.385(4)$ \\
\hline $\mathrm{N}(017)-\mathrm{C}(072)$ & $1.480(3)$ & $\mathrm{N}(042)-\mathrm{C}(086)$ & $1.500(3)$ & $\mathrm{C}(073)-\mathrm{H}(07 \mathrm{E})$ & 0.9500 \\
\hline $\mathrm{C}(018)-\mathrm{N}(035)$ & $1.376(3)$ & $\mathrm{N}(042)-\mathrm{C}(058)$ & $1.527(3)$ & $\mathrm{C}(075)-\mathrm{H}(07 \mathrm{~F})$ & 0.9500 \\
\hline $\mathrm{C}(018)-\mathrm{C}(020)$ & $1.510(3)$ & $\mathrm{C}(043)-\mathrm{C}(078)$ & $1.392(3)$ & $\mathrm{C}(076)-\mathrm{H}(07 \mathrm{G})$ & 0.9500 \\
\hline $\mathrm{C}(019)-\mathrm{C}(022)$ & $1.380(3)$ & $C(043)-C(045)$ & $1.396(3)$ & $\mathrm{C}(077)-\mathrm{C}(084)$ & $1.387(4)$ \\
\hline $\mathrm{C}(019)-\mathrm{C}(028)$ & $1.397(3)$ & $\mathrm{C}(044)-\mathrm{H}(04 \mathrm{~B})$ & 0.9500 & $\mathrm{C}(077)-\mathrm{C}(082)$ & $1.388(4)$ \\
\hline $\mathrm{C}(019)-\mathrm{H}(01 \mathrm{C})$ & 0.9500 & $\mathrm{C}(045)-\mathrm{C}(084)$ & $1.379(3)$ & $\mathrm{C}(077)-\mathrm{H}(07 \mathrm{H})$ & 0.9500 \\
\hline $\mathrm{C}(020)-\mathrm{N}(042)$ & $1.491(3)$ & $\mathrm{C}(045)-\mathrm{H}(04 \mathrm{C})$ & 0.9500 & $\mathrm{C}(078)-\mathrm{C}(082)$ & $1.384(4$ \\
\hline $\mathrm{C}(020)-\mathrm{C}(085)$ & $1.539(3)$ & $\mathrm{C}(046)-\mathrm{H}(04 \mathrm{D})$ & 0.9500 & $\mathrm{C}(078)-\mathrm{H}(07 \mathrm{I})$ & 0.950 \\
\hline
\end{tabular}




$\begin{array}{lrlrlr}\mathrm{C}(020)-\mathrm{H}(02 \mathrm{~A}) & 1.0000 & \mathrm{C}(047)-\mathrm{C}(074) & 1.362(4) & \mathrm{C}(079)-\mathrm{H}(07 \mathrm{~J}) & 0.9500 \\ \mathrm{C}(021)-\mathrm{C}(050) & 1.510(3) & \mathrm{C}(047)-\mathrm{H}(04 \mathrm{E}) & 0.9500 & \mathrm{C}(080)-\mathrm{C}(085) & 1.474(4) \\ \mathrm{C}(021)-\mathrm{C}(055) & 1.535(3) & \mathrm{C}(048)-\mathrm{H}(04 \mathrm{~F}) & 0.9500 & \mathrm{C}(080)-\mathrm{H}(08 \mathrm{~A}) & 0.9900 \\ \mathrm{C}(021)-\mathrm{H}(02 \mathrm{~B}) & 1.0000 & \mathrm{C}(049)-\mathrm{C}(059) & 1.409(3) & \mathrm{C}(080)-\mathrm{H}(08 \mathrm{~B}) & 0.9900 \\ \mathrm{C}(022)-\mathrm{C}(033) & 1.384(3) & \mathrm{C}(051)-\mathrm{C}(075) & 1.385(3) & \mathrm{C}(081)-\mathrm{H}(08 \mathrm{C}) & 0.9500 \\ \mathrm{C}(022)-\mathrm{H}(02 \mathrm{C}) & 0.9500 & \mathrm{C}(051)-\mathrm{H}(05 \mathrm{~A}) & 0.9500 & \mathrm{C}(082)-\mathrm{H}(08 \mathrm{D}) & 0.9500 \\ \mathrm{C}(023)-\mathrm{C}(044) & 1.404(3) & \mathrm{C}(052)-\mathrm{H}(05 \mathrm{~B}) & 0.9500 & \mathrm{C}(083)-\mathrm{H}(08 \mathrm{E}) & 0.9800 \\ \mathrm{C}(023)-\mathrm{C}(049) & 1.427(3) & \mathrm{C}(053)-\mathrm{H}(05 \mathrm{C}) & 0.9800 & \mathrm{C}(083)-\mathrm{H}(08 \mathrm{~F}) & 0.9800 \\ \mathrm{C}(024)-\mathrm{N}(035) & 1.403(3) & \mathrm{C}(053)-\mathrm{H}(05 \mathrm{D}) & 0.9800 & \mathrm{C}(083)-\mathrm{H}(08 \mathrm{G}) & 0.9800 \\ \mathrm{C}(024)-\mathrm{C}(052) & 1.408(3) & \mathrm{C}(053)-\mathrm{H}(05 \mathrm{E}) & 0.9800 & \mathrm{C}(084)-\mathrm{H}(08 \mathrm{H}) & 0.9500 \\ \mathrm{C}(024)-\mathrm{C}(025) & 1.423(3) & \mathrm{C}(054)-\mathrm{C}(063) & 1.387(4) & \mathrm{C}(085)-\mathrm{H}(08 \mathrm{I}) & 0.9900 \\ \mathrm{C}(025)-\mathrm{C}(046) & 1.409(3) & \mathrm{C}(054)-\mathrm{C}(070) & 1.394(4) & \mathrm{C}(085)-\mathrm{H}(08 \mathrm{~J}) & 0.9900 \\ \mathrm{C}(025)-\mathrm{C}(040) & 1.464(3) & \mathrm{C}(054)-\mathrm{C}(086) & 1.513(4) & \mathrm{C}(086)-\mathrm{H}(08 \mathrm{~K}) & 0.9900 \\ \mathrm{C}(026)-\mathrm{C}(069) & 1.388(3) & \mathrm{C}(055)-\mathrm{C}(071) & 1.519(3) & \mathrm{C}(086)-\mathrm{H}(08 \mathrm{~L}) & 0.9900 \\ \mathrm{C}(026)-\mathrm{C}(039) & 1.393(3) & \mathrm{C}(055)-\mathrm{H}(05 \mathrm{~F}) & 0.9900 & & \end{array}$

$\mathrm{N}(017)-\mathrm{Ni}(01)-\mathrm{N}(007) \quad 95.73(8) \quad \mathrm{C}(048)-\mathrm{C}(034)-\mathrm{F}(005) \quad 118.7(2) \quad \mathrm{O}(010)-\mathrm{C}(061)-\mathrm{C}(072) \quad 120.9(2)$ $\mathrm{N}(017)-\mathrm{Ni}(01)-\mathrm{O}(006) \quad 86.36(8) \quad \mathrm{C}(027)-\mathrm{C}(034)-\mathrm{F}(005) \quad 117.9(2) \quad \mathrm{O}(006)-\mathrm{C}(061)-\mathrm{C}(072) \quad 114.6(2)$ $\mathrm{N}(007)-\mathrm{Ni}(01)-\mathrm{O}(006) \quad 174.24(8) \quad \mathrm{C}(018)-\mathrm{N}(035)-\mathrm{C}(024) \quad 121.5(2) \quad \mathrm{C}(067)-\mathrm{C}(062)-\mathrm{C}(029) 120.8(2)$ $\mathrm{N}(017)-\mathrm{Ni}(01)-\mathrm{N}(008) \quad 176.23(8) \quad \mathrm{C}(018)-\mathrm{N}(035)-\mathrm{Ni}(02) \quad 114.40(15) \mathrm{C}(067)-\mathrm{C}(062)-\mathrm{H}(06 \mathrm{C}) 119.6$ $\mathrm{N}(007)-\mathrm{Ni}(01)-\mathrm{N}(008) \quad 88.03(8) \quad \mathrm{C}(024)-\mathrm{N}(035)-\mathrm{Ni}(02) \quad 124.01(16) \mathrm{C}(029)-\mathrm{C}(062)-\mathrm{H}(06 \mathrm{C}) 119.6$ $\mathrm{O}(006)-\mathrm{Ni}(01)-\mathrm{N}(008) \quad 89.91(7) \quad \mathrm{C}(031)-\mathrm{C}(036)-\mathrm{C}(052) \quad 121.1(2) \quad \mathrm{C}(054)-\mathrm{C}(063)-\mathrm{C}(081)$ 120.6(3) $\mathrm{N}(035)-\mathrm{Ni}(02)-\mathrm{N}(064) \quad 95.23(9) \quad \mathrm{C}(031)-\mathrm{C}(036)-\mathrm{H}(03 \mathrm{E}) 119.4$ $\mathrm{N}(035)-\mathrm{Ni}(02)-\mathrm{O}(014) \quad 172.41(7) \quad \mathrm{C}(052)-\mathrm{C}(036)-\mathrm{H}(03 \mathrm{E}) 119.4$ $\mathrm{N}(064)-\mathrm{Ni}(02)-\mathrm{O}(014) \quad 87.16(8) \quad \mathrm{C}(044)-\mathrm{C}(037)-\mathrm{C}(032) \quad 121.1(2)$ $\mathrm{N}(035)-\mathrm{Ni}(02)-\mathrm{N}(042) \quad 87.88(8) \quad \mathrm{C}(044)-\mathrm{C}(037)-\mathrm{H}(03 \mathrm{~F}) 119.4$ $\mathrm{N}(064)-\mathrm{Ni}(02)-\mathrm{N}(042) \quad 172.78(8) \quad \mathrm{C}(032)-\mathrm{C}(037)-\mathrm{H}(03 \mathrm{~F}) 119.4$ (014)-Ni(02)-N(042) 90.60(8) $\quad \mathrm{C}(071)-\mathrm{C}(038)-\mathrm{N}(008)-105.37(19) \mathrm{C}(026)-\mathrm{C}(065)-\mathrm{C}(083)$ 114.08(18) $\mathrm{C}(061)-\mathrm{O}(006)-\mathrm{Ni}(01) \quad 115.52(14) \mathrm{C}(071)-\mathrm{C}(038)-\mathrm{H}(03 \mathrm{G}) 110.7$ $\mathrm{C}(050)-\mathrm{N}(007)-\mathrm{C}(023) 121.07(19) \mathrm{N}(008)-\mathrm{C}(038)-\mathrm{H}(03 \mathrm{G}) 110.7$ $\mathrm{C}(050)-\mathrm{N}(007)-\mathrm{Ni}(01) \quad 114.28(14) \mathrm{C}(071)-\mathrm{C}(038)-\mathrm{H}(03 \mathrm{H}) 110.7$ $\mathrm{C}(023)-\mathrm{N}(007)-\mathrm{Ni}(01) \quad 123.70(16) \mathrm{N}(008)-\mathrm{C}(038)-\mathrm{H}(03 \mathrm{H}) 110.7$ $\mathrm{C}(021)-\mathrm{N}(008)-\mathrm{C}(060) 112.03(18) \mathrm{H}(03 \mathrm{G})-\mathrm{C}(038)-\mathrm{H}(03 \mathrm{H}) 108.8$ $\mathrm{C}(021)-\mathrm{N}(008)-\mathrm{C}(038) 106.97(17) \mathrm{C}(047)-\mathrm{C}(039)-\mathrm{C}(026) \quad 121.4(2)$ $\mathrm{C}(060)-\mathrm{N}(008)-\mathrm{C}(038) \quad 109.60(17) \mathrm{C}(047)-\mathrm{C}(039)-\mathrm{H}(03 \mathrm{I}) \quad 119.3$ $\mathrm{C}(021)-\mathrm{N}(008)-\mathrm{Ni}(01) \quad 107.17(13) \mathrm{C}(026)-\mathrm{C}(039)-\mathrm{H}(03 \mathrm{I}) \quad 119.3$ $\mathrm{C}(060)-\mathrm{N}(008)-\mathrm{Ni}(01) \quad 108.11(13) \mathrm{N}(064)-\mathrm{C}(040)-\mathrm{C}(025) \quad 121.8(2)$ $\mathrm{C}(038)-\mathrm{N}(008)-\mathrm{Ni}(01) \quad 113.00(14) \mathrm{N}(064)-\mathrm{C}(040)-\mathrm{C}(043) \quad 118.4(2)$ $\mathrm{O}(003)-\mathrm{C}(011)-\mathrm{O}(014) 125.1(2) \quad \mathrm{C}(025)-\mathrm{C}(040)-\mathrm{C}(043) \quad 119.8(2)$ $\mathrm{O}(003)-\mathrm{C}(011)-\mathrm{C}(030)$ 120.3(2) $\quad \mathrm{C}(028)-\mathrm{C}(041)-\mathrm{C}(076) \quad 120.4(2)$ $\mathrm{O}(014)-\mathrm{C}(011)-\mathrm{C}(030)$ 114.6(2) $\quad \mathrm{C}(028)-\mathrm{C}(041)-\mathrm{H}(04 \mathrm{~A}) 119.8$ $\mathrm{C}(054)-\mathrm{C}(063)-\mathrm{H}(06 \mathrm{D}) 119.7$ $\mathrm{C}(081)-\mathrm{C}(063)-\mathrm{H}(06 \mathrm{D}) 119.7$ $\mathrm{C}(040)-\mathrm{N}(064)-\mathrm{C}(030) \quad 120.4(2)$ $\mathrm{C}(040)-\mathrm{N}(064)-\mathrm{Ni}(02) \quad 128.90(17)$ $\mathrm{C}(030)-\mathrm{N}(064)-\mathrm{Ni}(02) \quad 110.66(14)$ C(026)-C(065)-C(072) $112.26(18)$ $\mathrm{C}(083)-\mathrm{C}(065)-\mathrm{C}(072) \quad 110.5(2)$ $\mathrm{C}(026)-\mathrm{C}(065)-\mathrm{H}(06 \mathrm{E}) 106.5$ $\mathrm{C}(083)-\mathrm{C}(065)-\mathrm{H}(06 \mathrm{E}) 106.5$ $\mathrm{C}(072)-\mathrm{C}(065)-\mathrm{H}(06 \mathrm{E}) 106.5$ $\mathrm{C}(013)-\mathrm{C}(066)-\mathrm{C}(068) \quad 118.2(2)$ $\mathrm{C}(013)-\mathrm{C}(066)-\mathrm{C}(016) \quad 119.9(2)$ $\mathrm{C}(068)-\mathrm{C}(066)-\mathrm{C}(016) \quad 121.7(2)$ $\mathrm{C}(056)-\mathrm{C}(067)-\mathrm{C}(062) \quad 120.4(2)$ $\mathrm{C}(056)-\mathrm{C}(067)-\mathrm{H}(06 \mathrm{~F}) 119.8$ $\mathrm{C}(062)-\mathrm{C}(067)-\mathrm{H}(06 \mathrm{~F}) 119.8$ $\mathrm{C}(027)-\mathrm{C}(068)-\mathrm{C}(066) \quad 121.2(2)$ C(027)-C(068)-H(06G) 119.4 
$\mathrm{C}(048)-\mathrm{C}(013)-\mathrm{C}(066) 121.4(2)$ $\mathrm{C}(048)-\mathrm{C}(013)-\mathrm{H}(01 \mathrm{~A}) 119.3$ $\mathrm{C}(066)-\mathrm{C}(013)-\mathrm{H}(01 \mathrm{~A}) 119.3$ $\mathrm{N}(017)-\mathrm{C}(015)-\mathrm{C}(049) \quad 121.8(2) \quad \mathrm{C}(020)-\mathrm{N}(042)-\mathrm{Ni}(02)$ $\mathrm{N}(017)-\mathrm{C}(015)-\mathrm{C}(028) 119.4(2) \quad \mathrm{C}(086)-\mathrm{N}(042)-\mathrm{Ni}(02)$ $\mathrm{C}(049)-\mathrm{C}(015)-\mathrm{C}(028)$ 118.74(18) C(058)-N(042)-Ni(02) $\mathrm{C}(053)-\mathrm{C}(016)-\mathrm{C}(066) 112.68(19) \mathrm{C}(078)-\mathrm{C}(043)-\mathrm{C}(045)$ $\mathrm{C}(053)-\mathrm{C}(016)-\mathrm{C}(030) 110.61(19) \mathrm{C}(078)-\mathrm{C}(043)-\mathrm{C}(040) 121.7(2)$ $\mathrm{C}(066)-\mathrm{C}(016)-\mathrm{C}(030) 114.53(19) \mathrm{C}(045)-\mathrm{C}(043)-\mathrm{C}(040) \quad 119.5(2)$ $\mathrm{C}(053)-\mathrm{C}(016)-\mathrm{H}(016) 106.1$ $\mathrm{C}(066)-\mathrm{C}(016)-\mathrm{H}(016) 106.1$ $\mathrm{C}(030)-\mathrm{C}(016)-\mathrm{H}(016) 106.1$ $\mathrm{C}(015)-\mathrm{N}(017)-\mathrm{C}(072) 120.85(19) \mathrm{C}(084)-\mathrm{C}(045)-\mathrm{C}(043) \quad 121.0(2)$ $\mathrm{C}(015)-\mathrm{N}(017)-\mathrm{Ni}(01) \quad 128.11(16) \mathrm{C}(084)-\mathrm{C}(045)-\mathrm{H}(04 \mathrm{C}) 119.5$ $\mathrm{C}(072)-\mathrm{N}(017)-\mathrm{Ni}(01) \quad 111.02(13) \mathrm{C}(043)-\mathrm{C}(045)-\mathrm{H}(04 \mathrm{C}) 119.5$ $\mathrm{O}(004)-\mathrm{C}(018)-\mathrm{N}(035) 128.1(2) \quad \mathrm{C}(031)-\mathrm{C}(046)-\mathrm{C}(025) \quad 121.9(2)$ $\mathrm{O}(004)-\mathrm{C}(018)-\mathrm{C}(020) 118.8(2)$ $\mathrm{N}(035)-\mathrm{C}(018)-\mathrm{C}(020) 112.9(2)$ $\mathrm{C}(022)-\mathrm{C}(019)-\mathrm{C}(028) 119.5(2)$ $\mathrm{C}(022)-\mathrm{C}(019)-\mathrm{H}(01 \mathrm{C}) 120.2$ $\mathrm{C}(028)-\mathrm{C}(019)-\mathrm{H}(01 \mathrm{C}) 120.2$ $\mathrm{N}(042)-\mathrm{C}(020)-\mathrm{C}(018) 110.96(18) \mathrm{C}(034)-\mathrm{C}(048)-\mathrm{C}(013) \quad 117.8(2)$ $\mathrm{N}(042)-\mathrm{C}(020)-\mathrm{C}(085)$ 103.89(19) C(034)-C(048)-H(04F) 121.1 $\mathrm{C}(018)-\mathrm{C}(020)-\mathrm{C}(085) 112.8(2)$ $\mathrm{N}(042)-\mathrm{C}(020)-\mathrm{H}(02 \mathrm{~A}) 109.7$ $\mathrm{C}(018)-\mathrm{C}(020)-\mathrm{H}(02 \mathrm{~A}) 109.7$ $\mathrm{C}(085)-\mathrm{C}(020)-\mathrm{H}(02 \mathrm{~A}) 109.7$ $\mathrm{N}(008)-\mathrm{C}(021)-\mathrm{C}(050)$ 111.64(19) $\mathrm{O}(012)-\mathrm{C}(050)-\mathrm{N}(007)$ 128.2(2) $\mathrm{N}(008)-\mathrm{C}(021)-\mathrm{C}(055) 104.18(18) \mathrm{O}(012)-\mathrm{C}(050)-\mathrm{C}(021) \quad 118.3(2)$ $\mathrm{C}(050)-\mathrm{C}(021)-\mathrm{C}(055)$ 113.91(19) $\mathrm{N}(007)-\mathrm{C}(050)-\mathrm{C}(021) \quad 113.5(2)$ $\mathrm{N}(008)-\mathrm{C}(021)-\mathrm{H}(02 \mathrm{~B}) 109.0$ $\mathrm{C}(050)-\mathrm{C}(021)-\mathrm{H}(02 \mathrm{~B}) 109.0$ $\mathrm{C}(055)-\mathrm{C}(021)-\mathrm{H}(02 \mathrm{~B}) 109.0$ $\mathrm{C}(019)-\mathrm{C}(022)-\mathrm{C}(033) 121.0(2)$ $\mathrm{C}(019)-\mathrm{C}(022)-\mathrm{H}(02 \mathrm{C}) 119.5$ $\mathrm{C}(033)-\mathrm{C}(022)-\mathrm{H}(02 \mathrm{C}) 119.5$ $\mathrm{C}(044)-\mathrm{C}(023)-\mathrm{N}(007) 121.9(2)$ $\mathrm{C}(044)-\mathrm{C}(023)-\mathrm{C}(049)$ 117.36(19) C(016)-C(053)-H(05D) 109.5 $\mathrm{N}(007)-\mathrm{C}(023)-\mathrm{C}(049) 120.59(19) \mathrm{H}(05 \mathrm{C})-\mathrm{C}(053)-\mathrm{H}(05 \mathrm{D}) 109.5$ $\mathrm{N}(035)-\mathrm{C}(024)-\mathrm{C}(052) 121.5(2)$ $\mathrm{N}(035)-\mathrm{C}(024)-\mathrm{C}(025) 121.2(2)$ $\mathrm{C}(052)-\mathrm{C}(024)-\mathrm{C}(025) 117.3(2)$ $\mathrm{C}(046)-\mathrm{C}(025)-\mathrm{C}(024) 119.3(2)$ $\mathrm{C}(046)-\mathrm{C}(025)-\mathrm{C}(040) 116.6(2)$ $\mathrm{C}(024)-\mathrm{C}(025)-\mathrm{C}(040) 124.0(2)$ $\mathrm{C}(069)-\mathrm{C}(026)-\mathrm{C}(039) 118.4(2)$ $\mathrm{C}(069)-\mathrm{C}(026)-\mathrm{C}(065) 119.4(2)$ $\mathrm{C}(039)-\mathrm{C}(026)-\mathrm{C}(065) 122.2(2)$ $\mathrm{C}(034)-\mathrm{C}(027)-\mathrm{C}(068) 117.9(2)$ $\mathrm{C}(034)-\mathrm{C}(027)-\mathrm{H}(02 \mathrm{D}) 121.0$ $\mathrm{C}(068)-\mathrm{C}(027)-\mathrm{H}(02 \mathrm{D}) 121.0$ $\mathrm{C}(041)-\mathrm{C}(028)-\mathrm{C}(019) 119.6(2) \quad \mathrm{C}(067)-\mathrm{C}(056)-\mathrm{C}(075) \quad 119.7(2)$ $\mathrm{C}(041)-\mathrm{C}(028)-\mathrm{C}(015) 119.13(19) \mathrm{C}(067)-\mathrm{C}(056)-\mathrm{H}(05 \mathrm{H}) 120.1$ $\mathrm{C}(019)-\mathrm{C}(028)-\mathrm{C}(015) \quad 121.2(2) \quad \mathrm{C}(075)-\mathrm{C}(056)-\mathrm{H}(05 \mathrm{H}) 120.1$ $\mathrm{C}(037)-\mathrm{C}(044)-\mathrm{C}(023) \quad 121.9(2)$ $\mathrm{C}(037)-\mathrm{C}(044)-\mathrm{H}(04 \mathrm{~B}) 119.1$ $\mathrm{C}(023)-\mathrm{C}(044)-\mathrm{H}(04 \mathrm{~B}) 119.1$ $\mathrm{C}(031)-\mathrm{C}(046)-\mathrm{H}(04 \mathrm{D}) 119.1$ $\mathrm{C}(025)-\mathrm{C}(046)-\mathrm{H}(04 \mathrm{D}) 119.1$ $\mathrm{C}(074)-\mathrm{C}(047)-\mathrm{C}(039) \quad 117.6(2)$ $\mathrm{C}(074)-\mathrm{C}(047)-\mathrm{H}(04 \mathrm{E}) \quad 121.2$ $\mathrm{C}(039)-\mathrm{C}(047)-\mathrm{H}(04 \mathrm{E}) \quad 121.2$ $\mathrm{C}(013)-\mathrm{C}(048)-\mathrm{H}(04 \mathrm{~F}) 121.1$ $\mathrm{C}(059)-\mathrm{C}(049)-\mathrm{C}(023) \quad 118.8(2)$ $\mathrm{C}(059)-\mathrm{C}(049)-\mathrm{C}(015) \quad 116.3(2)$ $\mathrm{C}(023)-\mathrm{C}(049)-\mathrm{C}(015) \quad 124.97(18) \mathrm{C}(056)-\mathrm{C}(075)-\mathrm{H}(07 \mathrm{~F}) 120.1$ $\mathrm{C}(075)-\mathrm{C}(051)-\mathrm{C}(029) \quad 120.9(2)$ $\mathrm{C}(075)-\mathrm{C}(051)-\mathrm{H}(05 \mathrm{~A}) 119.5$ $\mathrm{C}(029)-\mathrm{C}(051)-\mathrm{H}(05 \mathrm{~A}) 119.5$ $\mathrm{C}(036)-\mathrm{C}(052)-\mathrm{C}(024) \quad 121.6(2)$ $\mathrm{C}(036)-\mathrm{C}(052)-\mathrm{H}(05 \mathrm{~B}) 119.2$ $\mathrm{C}(024)-\mathrm{C}(052)-\mathrm{H}(05 \mathrm{~B}) 119.2$

$\mathrm{C}(066)-\mathrm{C}(068)-\mathrm{H}(06 \mathrm{G}) 119.4$ $\mathrm{C}(026)-\mathrm{C}(069)-\mathrm{C}(057) \quad 121.2(2)$ $\mathrm{C}(026)-\mathrm{C}(069)-\mathrm{H}(06 \mathrm{H}) 119.4$ $\mathrm{C}(057)-\mathrm{C}(069)-\mathrm{H}(06 \mathrm{H}) 119.4$ $\mathrm{C}(079)-\mathrm{C}(070)-\mathrm{C}(054) \quad 119.9(3)$ (054)-C(070)-H(07A) 120.0 $\mathrm{C}(038)-\mathrm{C}(071)-\mathrm{C}(055) \quad 103.28(18)$ $\mathrm{C}(038)-\mathrm{C}(071)-\mathrm{H}(07 \mathrm{~B}) 111.1$ $\mathrm{C}(055)-\mathrm{C}(071)-\mathrm{H}(07 \mathrm{~B}) 111.1$ $\mathrm{C}(038)-\mathrm{C}(071)-\mathrm{H}(07 \mathrm{C}) 111.1$ $\mathrm{C}(055)-\mathrm{C}(071)-\mathrm{H}(07 \mathrm{C}) 111.1$ $\mathrm{H}(07 \mathrm{~B})-\mathrm{C}(071)-\mathrm{H}(07 \mathrm{C}) 109.1$ $\mathrm{N}(017)-\mathrm{C}(072)-\mathrm{C}(061) \quad 107.32(18)$ $\mathrm{N}(017)-\mathrm{C}(072)-\mathrm{C}(065) 110.66(18)$ $\mathrm{C}(061)-\mathrm{C}(072)-\mathrm{C}(065)$ 111.72(17) $\mathrm{N}(017)-\mathrm{C}(072)-\mathrm{H}(07 \mathrm{D}) 109.0$ $\mathrm{C}(061)-\mathrm{C}(072)-\mathrm{H}(07 \mathrm{D}) 109.0$ $\mathrm{C}(065)-\mathrm{C}(072)-\mathrm{H}(07 \mathrm{D}) 109.0$ $\mathrm{C}(081)-\mathrm{C}(073)-\mathrm{C}(079) \quad 119.9(3)$ C(081)-C(073)-H(07E) 120.0 $\mathrm{C}(079)-\mathrm{C}(073)-\mathrm{H}(07 \mathrm{E}) 120.0$ $\mathrm{C}(047)-\mathrm{C}(074)-\mathrm{C}(057) \quad 123.6(2)$ $\mathrm{C}(047)-\mathrm{C}(074)-\mathrm{F}(009) \quad 118.7(2)$ $\mathrm{C}(057)-\mathrm{C}(074)-\mathrm{F}(009) \quad 117.6(3)$ $C(051)-C(075)-C(056) 119.7(2)$ $\mathrm{C}(051)-\mathrm{C}(075)-\mathrm{H}(07 \mathrm{~F}) 120.1$ $\mathrm{C}(033)-\mathrm{C}(076)-\mathrm{C}(041) \quad 119.7(2)$ $\mathrm{C}(033)-\mathrm{C}(076)-\mathrm{H}(07 \mathrm{G}) 120.1$ C(041)-C(076)-H(07G) 120.1 $\mathrm{C}(084)-\mathrm{C}(077)-\mathrm{C}(082) \quad 119.2(2)$ $\mathrm{C}(084)-\mathrm{C}(077)-\mathrm{H}(07 \mathrm{H}) 120.4$ $\mathrm{C}(082)-\mathrm{C}(077)-\mathrm{H}(07 \mathrm{H}) 120.4$ $\mathrm{C}(082)-\mathrm{C}(078)-\mathrm{C}(043) \quad 120.2(2)$ $\mathrm{C}(082)-\mathrm{C}(078)-\mathrm{H}(07 \mathrm{I}) \quad 119.9$ $\mathrm{C}(043)-\mathrm{C}(078)-\mathrm{H}(07 \mathrm{I}) \quad 119.9$ $\mathrm{C}(070)-\mathrm{C}(079)-\mathrm{C}(073) 120.6(3)$ $\mathrm{C}(070)-\mathrm{C}(079)-\mathrm{H}(07 \mathrm{~J}) \quad 119.7$ $\mathrm{C}(073)-\mathrm{C}(079)-\mathrm{H}(07 \mathrm{~J}) \quad 119.7$ $\mathrm{C}(085)-\mathrm{C}(080)-\mathrm{C}(058) \quad 105.2(2)$ $\mathrm{C}(085)-\mathrm{C}(080)-\mathrm{H}(08 \mathrm{~A}) 110.7$ $\mathrm{C}(058)-\mathrm{C}(080)-\mathrm{H}(08 \mathrm{~A}) 110.7$ $\mathrm{C}(085)-\mathrm{C}(080)-\mathrm{H}(08 \mathrm{~B}) 110.7$ $\mathrm{C}(058)-\mathrm{C}(080)-\mathrm{H}(08 \mathrm{~B}) 110.7$ $\mathrm{H}(08 \mathrm{~A})-\mathrm{C}(080)-\mathrm{H}(08 \mathrm{~B}) 108.8$ $\mathrm{C}(070)-\mathrm{C}(054)-\mathrm{C}(086) \quad 120.8(2)$ $\mathrm{C}(071)-\mathrm{C}(055)-\mathrm{C}(021) \quad 101.93(18) \mathrm{C}(073)-\mathrm{C}(081)-\mathrm{C}(063)$ 119.7(3) $\mathrm{C}(071)-\mathrm{C}(055)-\mathrm{H}(05 \mathrm{~F}) \quad 111.4$ $\mathrm{C}(021)-\mathrm{C}(055)-\mathrm{H}(05 \mathrm{~F}) \quad 111.4$ $\mathrm{C}(071)-\mathrm{C}(055)-\mathrm{H}(05 \mathrm{G}) 111.4$ $\mathrm{C}(021)-\mathrm{C}(055)-\mathrm{H}(05 \mathrm{G}) 111.4$ $\mathrm{H}(05 \mathrm{~F})-\mathrm{C}(055)-\mathrm{H}(05 \mathrm{G}) 109.2$ $\mathrm{C}(073)-\mathrm{C}(081)-\mathrm{H}(08 \mathrm{C}) 120.1$ $\mathrm{C}(063)-\mathrm{C}(081)-\mathrm{H}(08 \mathrm{C}) 120.1$ $\mathrm{C}(078)-\mathrm{C}(082)-\mathrm{C}(077) 120.7(2)$ $\mathrm{C}(078)-\mathrm{C}(082)-\mathrm{H}(08 \mathrm{D}) 119.6$ $\mathrm{C}(077)-\mathrm{C}(082)-\mathrm{H}(08 \mathrm{D}) 119.6$ $\mathrm{C}(065)-\mathrm{C}(083)-\mathrm{H}(08 \mathrm{E}) 109.5$ $\mathrm{C}(065)-\mathrm{C}(083)-\mathrm{H}(08 \mathrm{~F}) 109.5$ $\mathrm{H}(08 \mathrm{E})-\mathrm{C}(083)-\mathrm{H}(08 \mathrm{~F}) 109.5$ $\mathrm{C}(079)-\mathrm{C}(070)-\mathrm{H}(07 \mathrm{~A}) 120.0$ 
$\mathrm{C}(062)-\mathrm{C}(029)-\mathrm{C}(051) \quad 118.2(2) \quad \mathrm{C}(074)-\mathrm{C}(057)-\mathrm{C}(069) \quad 117.7(3)$ $\mathrm{C}(062)-\mathrm{C}(029)-\mathrm{C}(060) \quad 120.9(2) \quad \mathrm{C}(074)-\mathrm{C}(057)-\mathrm{H}(05 \mathrm{I}) \quad 121.1$ $\mathrm{C}(051)-\mathrm{C}(029)-\mathrm{C}(060) \quad 120.9(2) \quad \mathrm{C}(069)-\mathrm{C}(057)-\mathrm{H}(05 \mathrm{I}) \quad 121.1$ $\mathrm{N}(064)-\mathrm{C}(030)-\mathrm{C}(011)$ 107.36(19) $\mathrm{C}(080)-\mathrm{C}(058)-\mathrm{N}(042) \quad 106.6(2)$ $\mathrm{N}(064)-\mathrm{C}(030)-\mathrm{C}(016)$ 112.04(18) C(080)-C(058)-H(05J) 110.4 $\mathrm{C}(011)-\mathrm{C}(030)-\mathrm{C}(016) \quad 112.32(18) \mathrm{N}(042)-\mathrm{C}(058)-\mathrm{H}(05 \mathrm{~J}) \quad 110.4$ $\mathrm{N}(064)-\mathrm{C}(030)-\mathrm{H}(03 \mathrm{~A}) 108.3$ $\mathrm{C}(011)-\mathrm{C}(030)-\mathrm{H}(03 \mathrm{~A}) 108.3$ $\mathrm{C}(016)-\mathrm{C}(030)-\mathrm{H}(03 \mathrm{~A}) 108.3$ $\mathrm{C}(046)-\mathrm{C}(031)-\mathrm{C}(036) 118.6(2)$ $\mathrm{C}(046)-\mathrm{C}(031)-\mathrm{H}(03 \mathrm{~B}) 120.7$ $\mathrm{C}(036)-\mathrm{C}(031)-\mathrm{H}(03 \mathrm{~B}) 120.7$ $\mathrm{C}(059)-\mathrm{C}(032)-\mathrm{C}(037) 118.4(2)$ $\mathrm{C}(059)-\mathrm{C}(032)-\mathrm{H}(03 \mathrm{C}) 120.8$ $\mathrm{C}(037)-\mathrm{C}(032)-\mathrm{H}(03 \mathrm{C}) 120.8$ $\mathrm{C}(022)-\mathrm{C}(033)-\mathrm{C}(076) 119.7(2)$ $\mathrm{C}(022)-\mathrm{C}(033)-\mathrm{H}(03 \mathrm{D}) 120.2$ $\mathrm{C}(076)-\mathrm{C}(033)-\mathrm{H}(03 \mathrm{D}) 120.2$ $\mathrm{C}(048)-\mathrm{C}(034)-\mathrm{C}(027) 123.4(2)$ $\mathrm{C}(080)-\mathrm{C}(058)-\mathrm{H}(05 \mathrm{~K}) 110.4$ $\mathrm{N}(042)-\mathrm{C}(058)-\mathrm{H}(05 \mathrm{~K}) 110.4$ $\mathrm{H}(05 \mathrm{~J})-\mathrm{C}(058)-\mathrm{H}(05 \mathrm{~K}) 108.6$ $\mathrm{C}(032)-\mathrm{C}(059)-\mathrm{C}(049) \quad 122.4(2)$ $\mathrm{C}(032)-\mathrm{C}(059)-\mathrm{H}(05 \mathrm{~L}) \quad 118.8$ $\mathrm{C}(049)-\mathrm{C}(059)-\mathrm{H}(05 \mathrm{~L}) \quad 118.8$ $\mathrm{N}(008)-\mathrm{C}(060)-\mathrm{C}(029) \quad 112.42(18) \mathrm{N}(042)-\mathrm{C}(086)-\mathrm{C}(054) 114.3(2)$ $\mathrm{N}(008)-\mathrm{C}(060)-\mathrm{H}(06 \mathrm{~A}) 109.1$ $\mathrm{C}(029)-\mathrm{C}(060)-\mathrm{H}(06 \mathrm{~A}) 109.1$ $\mathrm{N}(008)-\mathrm{C}(060)-\mathrm{H}(06 \mathrm{~B}) 109.1$ C(029)-C(060)-H(06B) 109.1 $\mathrm{H}(06 \mathrm{~A})-\mathrm{C}(060)-\mathrm{H}(06 \mathrm{~B}) 107.9$ $\mathrm{O}(010)-\mathrm{C}(061)-\mathrm{O}(006) \quad 124.6(2)$
$\mathrm{C}(065)-\mathrm{C}(083)-\mathrm{H}(08 \mathrm{G}) 109.5$ $\mathrm{H}(08 \mathrm{E})-\mathrm{C}(083)-\mathrm{H}(08 \mathrm{G}) 109.5$ $\mathrm{H}(08 \mathrm{~F})-\mathrm{C}(083)-\mathrm{H}(08 \mathrm{G}) 109.5$ $\mathrm{C}(045)-\mathrm{C}(084)-\mathrm{C}(077) \quad 120.2(2)$ $\mathrm{C}(045)-\mathrm{C}(084)-\mathrm{H}(08 \mathrm{H}) 119.9$ $\mathrm{C}(077)-\mathrm{C}(084)-\mathrm{H}(08 \mathrm{H}) 119.9$ $\mathrm{C}(080)-\mathrm{C}(085)-\mathrm{C}(020) \quad 102.7(2)$ C(080)-C(085)-H(08I) 111.2 $\mathrm{C}(020)-\mathrm{C}(085)-\mathrm{H}(08 \mathrm{I}) \quad 111.2$ $\mathrm{C}(080)-\mathrm{C}(085)-\mathrm{H}(08 \mathrm{~J}) \quad 111.2$ $\mathrm{C}(020)-\mathrm{C}(085)-\mathrm{H}(08 \mathrm{~J}) \quad 111.2$ $\mathrm{H}(08 \mathrm{I})-\mathrm{C}(085)-\mathrm{H}(08 \mathrm{~J}) \quad 109.1$ $\mathrm{N}(042)-\mathrm{C}(086)-\mathrm{H}(08 \mathrm{~K}) 108.7$ $\mathrm{C}(054)-\mathrm{C}(086)-\mathrm{H}(08 \mathrm{~K}) 108.7$ $\mathrm{N}(042)-\mathrm{C}(086)-\mathrm{H}(08 \mathrm{~L}) 108.7$ $\mathrm{C}(054)-\mathrm{C}(086)-\mathrm{H}(08 \mathrm{~L}) 108.7$ $\mathrm{H}(08 \mathrm{~K})-\mathrm{C}(086)-\mathrm{H}(08 \mathrm{~L}) 107.6$

Table 4. Anisotropic displacement parameters $\left(\AA^{2} \times 10^{3}\right)$ for sw007. The anisotropic displacement factor exponent takes the form: $-2 \pi^{2}\left[h^{2} a^{* 2} U^{11}+\ldots+2 h k a^{*} b^{*} U^{12}\right]$

\begin{tabular}{rrrrrrr}
\hline & $\mathrm{U}^{11}$ & $\mathrm{U}^{22}$ & $\mathrm{U}^{33}$ & $\mathrm{U}^{23}$ & $\mathrm{U}^{13}$ & $\mathrm{U}^{12}$ \\
\hline $\mathrm{Ni}(01)$ & $23(1)$ & $26(1)$ & $25(1)$ & $-1(1)$ & $2(1)$ & $0(1)$ \\
$\mathrm{Ni}(02)$ & $30(1)$ & $25(1)$ & $24(1)$ & $0(1)$ & $3(1)$ & $-2(1)$ \\
$\mathrm{O}(003)$ & $44(1)$ & $23(1)$ & $36(1)$ & $-1(1)$ & $7(1)$ & $-7(1)$ \\
$\mathrm{O}(004)$ & $35(1)$ & $27(1)$ & $36(1)$ & $0(1)$ & $0(1)$ & $2(1)$ \\
$\mathrm{F}(005)$ & $56(1)$ & $45(1)$ & $30(1)$ & $-6(1)$ & $14(1)$ & $3(1)$ \\
$\mathrm{O}(006)$ & $26(1)$ & $26(1)$ & $29(1)$ & $-1(1)$ & $3(1)$ & $0(1)$ \\
$\mathrm{N}(007)$ & $22(1)$ & $26(1)$ & $25(1)$ & $0(1)$ & $0(1)$ & $1(1)$ \\
$\mathrm{N}(008)$ & $27(1)$ & $23(1)$ & $25(1)$ & $0(1)$ & $-1(1)$ & $3(1)$ \\
$\mathrm{F}(009)$ & $54(1)$ & $52(1)$ & $44(1)$ & $24(1)$ & $-12(1)$ & $-14(1)$ \\
$\mathrm{O}(010)$ & $37(1)$ & $26(1)$ & $29(1)$ & $-2(1)$ & $-1(1)$ & $0(1)$ \\
$\mathrm{C}(011)$ & $38(1)$ & $27(2)$ & $20(1)$ & $-2(1)$ & $4(1)$ & $-4(1)$ \\
$\mathrm{O}(012)$ & $31(1)$ & $23(1)$ & $38(1)$ & $-3(1)$ & $2(1)$ & $1(1)$ \\
$\mathrm{C}(013)$ & $33(1)$ & $31(2)$ & $26(1)$ & $5(1)$ & $1(1)$ & $-1(1)$ \\
$\mathrm{O}(014)$ & $32(1)$ & $29(1)$ & $30(1)$ & $-2(1)$ & $4(1)$ & $-3(1)$ \\
$\mathrm{C}(015)$ & $21(1)$ & $25(2)$ & $23(1)$ & $3(1)$ & $-3(1)$ & $3(1)$ \\
$\mathrm{C}(016)$ & $33(1)$ & $27(2)$ & $27(1)$ & $2(1)$ & $0(1)$ & $2(1)$ \\
$\mathrm{N}(017)$ & $24(1)$ & $25(1)$ & $21(1)$ & $1(1)$ & $-3(1)$ & $3(1)$ \\
$\mathrm{C}(018)$ & $30(1)$ & $25(2)$ & $25(1)$ & $1(1)$ & $-5(1)$ & $1(1)$ \\
$\mathrm{C}(019)$ & $27(1)$ & $28(2)$ & $25(1)$ & $3(1)$ & $1(1)$ & $3(1)$ \\
$\mathrm{C}(020)$ & $40(1)$ & $26(2)$ & $30(1)$ & $1(1)$ & $6(1)$ & $4(1)$ \\
$\mathrm{C}(021)$ & $22(1)$ & $26(2)$ & $33(1)$ & $0(1)$ & $2(1)$ & $2(1)$ \\
$\mathrm{C}(022)$ & $21(1)$ & $35(2)$ & $33(1)$ & $-1(1)$ & $-1(1)$ & $3(1)$ \\
$\mathrm{C}(023)$ & $22(1)$ & $27(2)$ & $25(1)$ & $-2(1)$ & $-4(1)$ & $7(1)$ \\
$\mathrm{C}(024)$ & $33(1)$ & $27(2)$ & $19(1)$ & $-2(1)$ & $-2(1)$ & $-7(1)$
\end{tabular}




\begin{tabular}{|c|c|c|c|c|c|c|}
\hline & $\mathrm{U}^{11}$ & $\mathrm{U}^{22}$ & $\mathrm{U}^{33}$ & $\mathrm{U}^{23}$ & $\mathrm{U}^{13}$ & $\mathrm{U}^{12}$ \\
\hline $\mathrm{C}(025)$ & $31(1)$ & $22(2)$ & $22(1)$ & $-1(1)$ & $2(1)$ & $-2(1)$ \\
\hline $\mathrm{C}(026)$ & $22(1)$ & $27(2)$ & $27(1)$ & $-1(1)$ & $-4(1)$ & 1(1) \\
\hline $\mathrm{C}(027)$ & $37(1)$ & $34(2)$ & $27(1)$ & $4(1)$ & $4(1)$ & $0(1)$ \\
\hline $\mathrm{C}(028)$ & $25(1)$ & $28(2)$ & $20(1)$ & $-1(1)$ & $2(1)$ & $-1(1)$ \\
\hline $\mathrm{C}(029)$ & $21(1)$ & $23(2)$ & $32(1)$ & $0(1)$ & $-2(1)$ & $5(1)$ \\
\hline $\mathrm{C}(030)$ & $34(1)$ & $22(2)$ & $25(1)$ & $0(1)$ & $6(1)$ & $1(1)$ \\
\hline $\mathrm{C}(031)$ & $41(1)$ & $33(2)$ & $28(1)$ & $2(1)$ & $5(1)$ & $-9(1)$ \\
\hline $\mathrm{C}(032)$ & $32(1)$ & $35(2)$ & $22(1)$ & $-1(1)$ & $-2(1)$ & $6(1)$ \\
\hline $\mathrm{C}(033)$ & $32(1)$ & $30(2)$ & $33(1)$ & $-3(1)$ & $6(1)$ & $-5(1)$ \\
\hline $\mathrm{C}(034)$ & $38(1)$ & $39(2)$ & $23(1)$ & $0(1)$ & $3(1)$ & $10(1)$ \\
\hline $\mathrm{N}(035)$ & $31(1)$ & $26(1)$ & $22(1)$ & $0(1)$ & $0(1)$ & $-1(1)$ \\
\hline $\mathrm{C}(036)$ & $46(2)$ & $26(2)$ & $27(1)$ & $4(1)$ & $-1(1)$ & $-5(1)$ \\
\hline $\mathrm{C}(037)$ & $34(1)$ & $31(2)$ & $27(1)$ & $-8(1)$ & $-6(1)$ & $4(1)$ \\
\hline $\mathrm{C}(038)$ & $30(1)$ & $34(2)$ & $24(1)$ & $-1(1)$ & $3(1)$ & $-2(1)$ \\
\hline $\mathrm{C}(039)$ & $34(1)$ & $28(2)$ & $29(1)$ & $2(1)$ & $-4(1)$ & 1(1) \\
\hline $\mathrm{C}(040)$ & $32(1)$ & $29(2)$ & $19(1)$ & $-1(1)$ & $-2(1)$ & $-3(1)$ \\
\hline $\mathrm{C}(041)$ & $26(1)$ & $31(2)$ & $24(1)$ & $-1(1)$ & $2(1)$ & $6(1)$ \\
\hline $\mathrm{N}(042)$ & $39(1)$ & $27(1)$ & $33(1)$ & 1(1) & $10(1)$ & $-4(1)$ \\
\hline $\mathrm{C}(043)$ & $29(1)$ & $27(2)$ & $24(1)$ & $4(1)$ & $4(1)$ & $-3(1)$ \\
\hline $\mathrm{C}(044)$ & $26(1)$ & $31(2)$ & $32(1)$ & $-1(1)$ & $-3(1)$ & $3(1)$ \\
\hline $\mathrm{C}(045)$ & $28(1)$ & $31(2)$ & $26(1)$ & $4(1)$ & $2(1)$ & $-2(1)$ \\
\hline $\mathrm{C}(046)$ & $35(1)$ & $30(2)$ & $26(1)$ & 1(1) & $2(1)$ & $-4(1)$ \\
\hline $\mathrm{C}(047)$ & $33(1)$ & $45(2)$ & $26(1)$ & $4(1)$ & $-2(1)$ & $2(1)$ \\
\hline $\mathrm{C}(048)$ & $41(1)$ & $29(2)$ & $30(1)$ & $-3(1)$ & $-1(1)$ & (1) \\
\hline $\mathrm{C}(049)$ & 21(1) & $27(1)$ & $24(1)$ & 1(1) & $0(1)$ & $3(1)$ \\
\hline $\mathrm{C}(050)$ & $22(1)$ & $24(2)$ & $33(1)$ & $3(1)$ & $-4(1)$ & $2(1)$ \\
\hline $\mathrm{C}(051)$ & $25(1)$ & $21(2)$ & $38(1)$ & 1(1) & $-4(1)$ & 1(1) \\
\hline $\mathrm{C}(052)$ & $41(2)$ & $27(2)$ & $23(1)$ & 1(1) & $-1(1)$ & $0(1)$ \\
\hline $\mathrm{C}(053)$ & $53(2)$ & $31(2)$ & $33(1)$ & 1(1) & $3(1)$ & $8(1)$ \\
\hline $\mathrm{C}(054)$ & $24(1)$ & $39(2)$ & $45(1)$ & $-4(1)$ & $-6(1)$ & $-1(1)$ \\
\hline $\mathrm{C}(055)$ & $29(1)$ & $28(2)$ & $33(1)$ & $6(1)$ & 1(1) & 1(1) \\
\hline $\mathrm{C}(056)$ & $32(1)$ & $35(2)$ & $34(1)$ & $-2(1)$ & $-2(1)$ & $2(1)$ \\
\hline $\mathrm{C}(057)$ & $41(1)$ & $26(2)$ & $38(1)$ & $4(1)$ & $-17(1)$ & $-3(1)$ \\
\hline $\mathrm{C}(058)$ & $70(2)$ & $40(2)$ & $47(2)$ & $10(1)$ & $34(1)$ & $9(2)$ \\
\hline $\mathrm{C}(059)$ & $27(1)$ & $32(2)$ & $25(1)$ & 1(1) & $-1(1)$ & $3(1)$ \\
\hline $\mathrm{C}(060)$ & $26(1)$ & $27(2)$ & $34(1)$ & $3(1)$ & 1(1) & $5(1)$ \\
\hline $\mathrm{C}(061)$ & $27(1)$ & $31(2)$ & $22(1)$ & $3(1)$ & $-4(1)$ & $2(1)$ \\
\hline $\mathrm{C}(062)$ & $25(1)$ & $28(2)$ & $36(1)$ & $4(1)$ & 1(1) & $3(1)$ \\
\hline $\mathrm{C}(063)$ & $37(2)$ & $54(2)$ & $48(2)$ & $-6(1)$ & $-3(1)$ & $11(2)$ \\
\hline $\mathrm{N}(064)$ & $34(1)$ & $22(1)$ & $20(1)$ & $-2(1)$ & 1(1) & $-4(1)$ \\
\hline $\mathrm{C}(065)$ & $23(1)$ & $32(2)$ & $25(1)$ & $-2(1)$ & $-2(1)$ & $0(1)$ \\
\hline $\mathrm{C}(066)$ & $30(1)$ & $26(2)$ & $23(1)$ & $4(1)$ & $-3(1)$ & $2(1)$ \\
\hline $\mathrm{C}(067)$ & $29(1)$ & $29(2)$ & $39(1)$ & $-6(1)$ & $-2(1)$ & $-3(1)$ \\
\hline $\mathrm{C}(068)$ & $39(1)$ & 29(2) & $27(1)$ & $1(1)$ & $1(1)$ & $-4(1)$ \\
\hline $\mathrm{C}(069)$ & $31(1)$ & $37(2)$ & $30(1)$ & $-2(1)$ & $-9(1)$ & $3(1)$ \\
\hline $\mathrm{C}(070)$ & $32(1)$ & $42(2)$ & $38(1)$ & $-11(1)$ & $-5(1)$ & $3(1)$ \\
\hline $\mathrm{C}(071)$ & $30(1)$ & $35(2)$ & $31(1)$ & 4(1) & $4(1)$ & (1) \\
\hline $\mathrm{C}(072)$ & $28(1)$ & $24(1)$ & $23(1)$ & $-1(1)$ & $0(1)$ & $-2(1)$ \\
\hline $\mathrm{C}(073)$ & $54(2)$ & $63(2)$ & $43(2)$ & $9(2)$ & $-18(1)$ & $-3(2)$ \\
\hline
\end{tabular}




\begin{tabular}{rrrrrrr}
\hline & $\mathrm{U}^{11}$ & $\mathrm{U}^{22}$ & $\mathrm{U}^{33}$ & $\mathrm{U}^{23}$ & $\mathrm{U}^{13}$ & \multicolumn{1}{c}{$\mathrm{U}^{12}$} \\
\hline $\mathrm{C}(074)$ & $32(1)$ & $41(2)$ & $37(1)$ & $16(1)$ & $-9(1)$ & $-8(1)$ \\
$\mathrm{C}(075)$ & $28(1)$ & $27(2)$ & $35(1)$ & $7(1)$ & $2(1)$ & $2(1)$ \\
$\mathrm{C}(076)$ & $36(1)$ & $25(2)$ & $30(1)$ & $4(1)$ & $5(1)$ & $2(1)$ \\
$\mathrm{C}(077)$ & $33(1)$ & $31(2)$ & $42(1)$ & $11(1)$ & $10(1)$ & $2(1)$ \\
$\mathrm{C}(078)$ & $31(1)$ & $32(2)$ & $30(1)$ & $4(1)$ & $1(1)$ & $-5(1)$ \\
$\mathrm{C}(079)$ & $40(2)$ & $68(2)$ & $32(1)$ & $-8(1)$ & $-6(1)$ & $-1(2)$ \\
$\mathrm{C}(080)$ & $58(2)$ & $80(3)$ & $41(2)$ & $19(2)$ & $-8(1)$ & $-12(2)$ \\
$\mathrm{C}(081)$ & $56(2)$ & $53(2)$ & $53(2)$ & $-2(2)$ & $-14(2)$ & $17(2)$ \\
$\mathrm{C}(082)$ & $31(1)$ & $41(2)$ & $38(1)$ & $10(1)$ & $-2(1)$ & $-5(1)$ \\
$\mathrm{C}(083)$ & $33(1)$ & $34(2)$ & $34(1)$ & $4(1)$ & $-6(1)$ & $-9(1)$ \\
$\mathrm{C}(084)$ & $37(1)$ & $30(2)$ & $32(1)$ & $2(1)$ & $9(1)$ & $-2(1)$ \\
$\mathrm{C}(085)$ & $68(2)$ & $31(2)$ & $24(1)$ & $3(1)$ & $6(1)$ & $9(2)$ \\
$\mathrm{C}(086)$ & $28(1)$ & $39(2)$ & $57(2)$ & $-1(1)$ & $10(1)$ & $-5(1)$ \\
\hline
\end{tabular}

Table 5. Hydrogen coordinates $\left(\times 10^{4}\right)$ and isotropic displacement parameters $\left(\AA^{2} \times 10^{3}\right)$ for sw007.

\begin{tabular}{rrrrr}
\hline & $x$ & $y$ & \multicolumn{1}{c}{$z$} & $\mathrm{U}(\mathrm{eq})$ \\
\hline $\mathrm{H}(01 \mathrm{~A})$ & 5072 & 7991 & 9318 & 36 \\
$\mathrm{H}(016)$ & 5566 & 7049 & 8826 & 35 \\
$\mathrm{H}(01 \mathrm{C})$ & 4458 & 898 & 10245 & 31 \\
$\mathrm{H}(02 \mathrm{~A})$ & 588 & 9335 & 8714 & 38 \\
$\mathrm{H}(02 \mathrm{~B})$ & 10915 & 2163 & 9271 & 32 \\
$\mathrm{H}(02 \mathrm{C})$ & 2921 & 185 & 10519 & 36 \\
$\mathrm{H}(02 \mathrm{D})$ & 2232 & 6740 & 10232 & 39 \\
$\mathrm{H}(03 \mathrm{~A})$ & 4531 & 6603 & 8089 & 32 \\
$\mathrm{H}(03 \mathrm{~B})$ & 6498 & 9533 & 6988 & 40 \\
$\mathrm{H}(03 \mathrm{C})$ & 6938 & 1852 & 11742 & 36 \\
$\mathrm{H}(03 \mathrm{D})$ & 3350 & -844 & 10922 & 38 \\
$\mathrm{H}(03 \mathrm{E})$ & 4844 & 10250 & 7095 & 40 \\
$\mathrm{H}(03 \mathrm{~F})$ & 8376 & 2633 & 11463 & 37 \\
$\mathrm{H}(03 \mathrm{G})$ & 10668 & 833 & 8483 & 35 \\
$\mathrm{H}(03 \mathrm{H})$ & 9229 & 898 & 8501 & 35 \\
$\mathrm{H}(03 \mathrm{I})$ & 6645 & 5 & 8258 & 36 \\
$\mathrm{H}(04 \mathrm{~A})$ & 6904 & -433 & 10810 & 33 \\
$\mathrm{H}(04 \mathrm{~B})$ & 9132 & 2582 & 10646 & 35 \\
$\mathrm{H}(04 \mathrm{C})$ & 5382 & 6970 & 7218 & 34 \\
$\mathrm{H}(04 \mathrm{D})$ & 6492 & 8468 & 7377 & 37 \\
$\mathrm{H}(04 \mathrm{E})$ & 7224 & 811 & 7644 & 42 \\
$\mathrm{H}(04 \mathrm{~F})$ & 4239 & 8442 & 10064 & 40 \\
$\mathrm{H}(05 \mathrm{~A})$ & 10110 & 201 & 10138 & 33 \\
$\mathrm{H}(05 \mathrm{~B})$ & 3289 & 9954 & 7634 & 37 \\
$\mathrm{H}(05 \mathrm{C})$ & 5557 & 6001 & 9279 & 58 \\
$\mathrm{H}(05 \mathrm{D})$ & 5719 & 5910 & 8678 & 58 \\
$\mathrm{H}(05 \mathrm{E})$ & 4447 & 5759 & 8938 & 58 \\
$\mathrm{H}(05 \mathrm{~F})$ & 8639 & 2258 & 8749 & 36 \\
$\mathrm{H}(05 \mathrm{G})$ & 9764 & 2770 & 8692 & 36 \\
$\mathrm{H}(05 \mathrm{H})$ & 11204 & 1278 & 11365 & 40 \\
& & & &
\end{tabular}




\begin{tabular}{rrrrr}
\hline & $\mathrm{x}$ & $\mathrm{y}$ & $\mathrm{z}$ & $\mathrm{U}(\mathrm{eq})$ \\
\hline $\mathrm{H}(05 \mathrm{I})$ & 6277 & 2330 & 8604 & 42 \\
$\mathrm{H}(05 \mathrm{~J})$ & -67 & 7808 & 9106 & 63 \\
$\mathrm{H}(05 \mathrm{~K})$ & 1317 & 7602 & 9187 & 63 \\
$\mathrm{H}(05 \mathrm{~L})$ & 6277 & 1013 & 11187 & 34 \\
$\mathrm{H}(06 \mathrm{~A})$ & 11870 & 1123 & 9230 & 35 \\
$\mathrm{H}(06 \mathrm{~B})$ & 11137 & 428 & 9298 & 35 \\
$\mathrm{H}(06 \mathrm{C})$ & 12398 & 1784 & 9964 & 35 \\
$\mathrm{H}(06 \mathrm{D})$ & -1043 & 9420 & 8032 & 56 \\
$\mathrm{H}(06 \mathrm{E})$ & 5219 & 419 & 9443 & 32 \\
$\mathrm{H}(06 \mathrm{~F})$ & 12426 & 1938 & 10842 & 39 \\
$\mathrm{H}(06 \mathrm{G})$ & 3073 & 6290 & 9487 & 38 \\
$\mathrm{H}(06 \mathrm{H})$ & 5732 & 1521 & 9221 & 39 \\
$\mathrm{H}(07 \mathrm{~A})$ & 1358 & 8131 & 7391 & 45 \\
$\mathrm{H}(07 \mathrm{~B})$ & 9612 & 1840 & 8028 & 39 \\
$\mathrm{H}(07 \mathrm{C})$ & 10918 & 1949 & 8280 & 39 \\
$\mathrm{H}(07 \mathrm{D})$ & 6601 & -418 & 9785 & 30 \\
$\mathrm{H}(07 \mathrm{E})$ & 379 & 9852 & 6656 & 64 \\
$\mathrm{H}(07 \mathrm{~F})$ & 10076 & 387 & 11014 & 36 \\
$\mathrm{H}(07 \mathrm{G})$ & 5350 & -1143 & 11087 & 36 \\
$\mathrm{H}(07 \mathrm{H})$ & 8539 & 6177 & 7688 & 42 \\
$\mathrm{H}(07 \mathrm{I})$ & 7084 & 7782 & 8436 & 37 \\
$\mathrm{H}(07 \mathrm{~J})$ & 1513 & 8855 & 6695 & 56 \\
$\mathrm{H}(08 \mathrm{~A})$ & 85 & 8719 & 9602 & 71 \\
$\mathrm{H}(08 \mathrm{~B})$ & 1206 & 8312 & 9841 & 71 \\
$\mathrm{H}(08 \mathrm{C})$ & -926 & 10126 & 7319 & 65 \\
$\mathrm{H}(08 \mathrm{D})$ & 8604 & 6977 & 8347 & 44 \\
$\mathrm{H}(08 \mathrm{E})$ & 4427 & -294 & 8801 & 51 \\
$\mathrm{H}(08 \mathrm{~F})$ & 4819 & -721 & 9293 & 51 \\
$\mathrm{H}(08 \mathrm{G})$ & 5650 & -721 & 8796 & 51 \\
$\mathrm{H}(08 \mathrm{H})$ & 6941 & 6199 & 7110 & 40 \\
$\mathrm{H}(08 \mathrm{I})$ & 2549 & 8886 & 9355 & 49 \\
$\mathrm{H}(08 \mathrm{~J})$ & 1588 & 9476 & 9484 & 49 \\
$\mathrm{H}(08 \mathrm{~K})$ & -692 & 8358 & 8430 & 50 \\
$\mathrm{H}(08 \mathrm{~L})$ & 34 & 7776 & 8136 & 50 \\
\hline & & & &
\end{tabular}

Table 6. Torsion angles $\left[^{\circ}\right]$ for sw007.

$\begin{array}{rrrr}\mathrm{N}(017)-\mathrm{Ni}(01)-\mathrm{O}(006)-\mathrm{C}(061) & 11.08(15) & \mathrm{C}(024)-\mathrm{C}(025)-\mathrm{C}(046)-\mathrm{C}(031) & -1.9(3) \\ \mathrm{N}(007)-\mathrm{Ni}(01)-\mathrm{O}(006)-\mathrm{C}(061) & 122.6(7) & \mathrm{C}(040)-\mathrm{C}(025)-\mathrm{C}(046)-\mathrm{C}(031) & 175.0(2) \\ \mathrm{N}(008)-\mathrm{Ni}(01)-\mathrm{O}(006)-\mathrm{C}(061) & -168.31(15) & \mathrm{C}(026)-\mathrm{C}(039)-\mathrm{C}(047)-\mathrm{C}(074) & 0.1(3) \\ \mathrm{N}(017)-\mathrm{Ni}(01)-\mathrm{N}(007)-\mathrm{C}(050) & -166.44(15) & \mathrm{C}(027)-\mathrm{C}(034)-\mathrm{C}(048)-\mathrm{C}(013) & 0.2(4) \\ \mathrm{O}(006)-\mathrm{Ni}(01)-\mathrm{N}(007)-\mathrm{C}(050) & 82.5(8) & \mathrm{F}(005)-\mathrm{C}(034)-\mathrm{C}(048)-\mathrm{C}(013) & 179.5(2) \\ \mathrm{N}(008)-\mathrm{Ni}(01)-\mathrm{N}(007)-\mathrm{C}(050) & 13.31(15) & \mathrm{C}(066)-\mathrm{C}(013)-\mathrm{C}(048)-\mathrm{C}(034) & -0.7(3) \\ \mathrm{N}(017)-\mathrm{Ni}(01)-\mathrm{N}(007)-\mathrm{C}(023) & 24.62(17) & \mathrm{C}(044)-\mathrm{C}(023)-\mathrm{C}(049)-\mathrm{C}(059) & 1.6(3) \\ \mathrm{O}(006)-\mathrm{Ni}(01)-\mathrm{N}(007)-\mathrm{C}(023) & -86.5(8) & \mathrm{N}(007)-\mathrm{C}(023)-\mathrm{C}(049)-\mathrm{C}(059) & 177.35(19) \\ \mathrm{N}(008)-\mathrm{Ni}(01)-\mathrm{N}(007)-\mathrm{C}(023) & -155.63(17) & \mathrm{C}(044)-\mathrm{C}(023)-\mathrm{C}(049)-\mathrm{C}(015) & -176.8(2) \\ \mathrm{N}(017)-\mathrm{Ni}(01)-\mathrm{N}(008)-\mathrm{C}(021) & 155.7(12) & \mathrm{N}(007)-\mathrm{C}(023)-\mathrm{C}(049)-\mathrm{C}(015) & -1.1(3)\end{array}$


$\mathrm{N}(007)-\mathrm{Ni}(01)-\mathrm{N}(008)-\mathrm{C}(021)$ $\mathrm{O}(006)-\mathrm{Ni}(01)-\mathrm{N}(008)-\mathrm{C}(021)$ $\mathrm{N}(017)-\mathrm{Ni}(01)-\mathrm{N}(008)-\mathrm{C}(060)$ $\mathrm{N}(007)-\mathrm{Ni}(01)-\mathrm{N}(008)-\mathrm{C}(060)$ $\mathrm{O}(006)-\mathrm{Ni}(01)-\mathrm{N}(008)-\mathrm{C}(060)$ $\mathrm{N}(017)-\mathrm{Ni}(01)-\mathrm{N}(008)-\mathrm{C}(038)$ $\mathrm{N}(007)-\mathrm{Ni}(01)-\mathrm{N}(008)-\mathrm{C}(038)$ $\mathrm{O}(006)-\mathrm{Ni}(01)-\mathrm{N}(008)-\mathrm{C}(038)$ $\mathrm{O}(003)-\mathrm{C}(011)-\mathrm{O}(014)-\mathrm{Ni}(02)$ $\mathrm{C}(030)-\mathrm{C}(011)-\mathrm{O}(014)-\mathrm{Ni}(02)$ $\mathrm{N}(035)-\mathrm{Ni}(02)-\mathrm{O}(014)-\mathrm{C}(011)$ $\mathrm{N}(064)-\mathrm{Ni}(02)-\mathrm{O}(014)-\mathrm{C}(011)$ $\mathrm{N}(042)-\mathrm{Ni}(02)-\mathrm{O}(014)-\mathrm{C}(011)$ $\mathrm{C}(049)-\mathrm{C}(015)-\mathrm{N}(017)-\mathrm{C}(072)$ $\mathrm{C}(028)-\mathrm{C}(015)-\mathrm{N}(017)-\mathrm{C}(072)$ $\mathrm{C}(049)-\mathrm{C}(015)-\mathrm{N}(017)-\mathrm{Ni}(01)$ $\mathrm{C}(028)-\mathrm{C}(015)-\mathrm{N}(017)-\mathrm{Ni}(01)$ $\mathrm{N}(007)-\mathrm{Ni}(01)-\mathrm{N}(017)-\mathrm{C}(015)$ $\mathrm{O}(006)-\mathrm{Ni}(01)-\mathrm{N}(017)-\mathrm{C}(015)$ $\mathrm{N}(008)-\mathrm{Ni}(01)-\mathrm{N}(017)-\mathrm{C}(015)$ $\mathrm{N}(007)-\mathrm{Ni}(01)-\mathrm{N}(017)-\mathrm{C}(072)$ $\mathrm{O}(006)-\mathrm{Ni}(01)-\mathrm{N}(017)-\mathrm{C}(072)$ $\mathrm{N}(008)-\mathrm{Ni}(01)-\mathrm{N}(017)-\mathrm{C}(072)$ $\mathrm{O}(004)-\mathrm{C}(018)-\mathrm{C}(020)-\mathrm{N}(042)$ $\mathrm{N}(035)-\mathrm{C}(018)-\mathrm{C}(020)-\mathrm{N}(042)$ $\mathrm{O}(004)-\mathrm{C}(018)-\mathrm{C}(020)-\mathrm{C}(085)$ $\mathrm{N}(035)-\mathrm{C}(018)-\mathrm{C}(020)-\mathrm{C}(085)$ $\mathrm{C}(060)-\mathrm{N}(008)-\mathrm{C}(021)-\mathrm{C}(050)$ $\mathrm{C}(038)-\mathrm{N}(008)-\mathrm{C}(021)-\mathrm{C}(050)$ $\mathrm{Ni}(01)-\mathrm{N}(008)-\mathrm{C}(021)-\mathrm{C}(050)$ $\mathrm{C}(060)-\mathrm{N}(008)-\mathrm{C}(021)-\mathrm{C}(055)$ $\mathrm{C}(038)-\mathrm{N}(008)-\mathrm{C}(021)-\mathrm{C}(055)$ $\mathrm{Ni}(01)-\mathrm{N}(008)-\mathrm{C}(021)-\mathrm{C}(055)$ $\mathrm{C}(028)-\mathrm{C}(019)-\mathrm{C}(022)-\mathrm{C}(033)$ $\mathrm{C}(050)-\mathrm{N}(007)-\mathrm{C}(023)-\mathrm{C}(044)$ $\mathrm{Ni}(01)-\mathrm{N}(007)-\mathrm{C}(023)-\mathrm{C}(044)$ $\mathrm{C}(050)-\mathrm{N}(007)-\mathrm{C}(023)-\mathrm{C}(049)$ $\mathrm{Ni}(01)-\mathrm{N}(007)-\mathrm{C}(023)-\mathrm{C}(049)$ $\mathrm{N}(035)-\mathrm{C}(024)-\mathrm{C}(025)-\mathrm{C}(046)$ $\mathrm{C}(052)-\mathrm{C}(024)-\mathrm{C}(025)-\mathrm{C}(046)$ $\mathrm{N}(035)-\mathrm{C}(024)-\mathrm{C}(025)-\mathrm{C}(040)$ $\mathrm{C}(052)-\mathrm{C}(024)-\mathrm{C}(025)-\mathrm{C}(040)$ $\mathrm{C}(022)-\mathrm{C}(019)-\mathrm{C}(028)-\mathrm{C}(041)$ $\mathrm{C}(022)-\mathrm{C}(019)-\mathrm{C}(028)-\mathrm{C}(015)$ $\mathrm{N}(017)-\mathrm{C}(015)-\mathrm{C}(028)-\mathrm{C}(041)$ $\mathrm{C}(049)-\mathrm{C}(015)-\mathrm{C}(028)-\mathrm{C}(041)$ $\mathrm{N}(017)-\mathrm{C}(015)-\mathrm{C}(028)-\mathrm{C}(019)$ $\mathrm{C}(049)-\mathrm{C}(015)-\mathrm{C}(028)-\mathrm{C}(019)$ $\mathrm{O}(003)-\mathrm{C}(011)-\mathrm{C}(030)-\mathrm{N}(064)$ $\mathrm{O}(014)-\mathrm{C}(011)-\mathrm{C}(030)-\mathrm{N}(064)$ $\mathrm{O}(003)-\mathrm{C}(011)-\mathrm{C}(030)-\mathrm{C}(016)$
$-20.47(13)$

164.91(13)

$-83.4(12)$

$100.47(15)$

$-74.15(14)$

38.1(13)

$-138.05(16)$

47.33(16)

$-172.36(17)$

$8.8(2)$

113.2(6)

4.65(14)

$-168.48(14)$

179.95(19)

$3.4(3)$

$1.7(3)$

$-174.86(15)$

$-16.2(2)$

158.43(19)

$167.7(11)$

165.40(14)

$-19.99(14)$

$-10.7(13)$

163.1(2)

$-21.1(3)$

$-80.8(3)$

95.0(2)

$-94.8(2)$

145.12(18)

23.7(2)

141.87(17)

21.8(2)

$-99.70(16)$

$0.8(3)$

$-12.3(3)$

155.89(17)

172.2(2)

$-19.6(3)$

$-176.5(2)$

$4.6(3)$

$6.8(3)$

$-172.1(2)$

$-3.1(3)$

175.2(2)

80.9(3)

$-95.7(3)$

$-97.4(3)$

86.0(3)

$159.75(18)$

$-21.4(2)$

$-76.6(3)$
$\mathrm{N}(017)-\mathrm{C}(015)-\mathrm{C}(049)-\mathrm{C}(059)$ $\mathrm{C}(028)-\mathrm{C}(015)-\mathrm{C}(049)-\mathrm{C}(059)$ $\mathrm{N}(017)-\mathrm{C}(015)-\mathrm{C}(049)-\mathrm{C}(023)$ $\mathrm{C}(028)-\mathrm{C}(015)-\mathrm{C}(049)-\mathrm{C}(023)$ $\mathrm{C}(023)-\mathrm{N}(007)-\mathrm{C}(050)-\mathrm{O}(012)$ $\mathrm{Ni}(01)-\mathrm{N}(007)-\mathrm{C}(050)-\mathrm{O}(012)$ $\mathrm{C}(023)-\mathrm{N}(007)-\mathrm{C}(050)-\mathrm{C}(021)$ $\mathrm{Ni}(01)-\mathrm{N}(007)-\mathrm{C}(050)-\mathrm{C}(021)$ $\mathrm{N}(008)-\mathrm{C}(021)-\mathrm{C}(050)-\mathrm{O}(012)$ $\mathrm{C}(055)-\mathrm{C}(021)-\mathrm{C}(050)-\mathrm{O}(012)$ $\mathrm{N}(008)-\mathrm{C}(021)-\mathrm{C}(050)-\mathrm{N}(007)$ $\mathrm{C}(055)-\mathrm{C}(021)-\mathrm{C}(050)-\mathrm{N}(007)$ $\mathrm{C}(062)-\mathrm{C}(029)-\mathrm{C}(051)-\mathrm{C}(075)$ $\mathrm{C}(060)-\mathrm{C}(029)-\mathrm{C}(051)-\mathrm{C}(075)$ $\mathrm{C}(031)-\mathrm{C}(036)-\mathrm{C}(052)-\mathrm{C}(024)$ $\mathrm{N}(035)-\mathrm{C}(024)-\mathrm{C}(052)-\mathrm{C}(036)$ $\mathrm{C}(025)-\mathrm{C}(024)-\mathrm{C}(052)-\mathrm{C}(036)$ $\mathrm{N}(008)-\mathrm{C}(021)-\mathrm{C}(055)-\mathrm{C}(071)$ $\mathrm{C}(050)-\mathrm{C}(021)-\mathrm{C}(055)-\mathrm{C}(071)$ $\mathrm{C}(020)-\mathrm{N}(042)-\mathrm{C}(058)-\mathrm{C}(080)$ $\mathrm{C}(086)-\mathrm{N}(042)-\mathrm{C}(058)-\mathrm{C}(080)$ $\mathrm{Ni}(02)-\mathrm{N}(042)-\mathrm{C}(058)-\mathrm{C}(080)$ $\mathrm{C}(037)-\mathrm{C}(032)-\mathrm{C}(059)-\mathrm{C}(049)$ $\mathrm{C}(023)-\mathrm{C}(049)-\mathrm{C}(059)-\mathrm{C}(032)$ $\mathrm{C}(015)-\mathrm{C}(049)-\mathrm{C}(059)-\mathrm{C}(032)$ $\mathrm{C}(021)-\mathrm{N}(008)-\mathrm{C}(060)-\mathrm{C}(029)$ $\mathrm{C}(038)-\mathrm{N}(008)-\mathrm{C}(060)-\mathrm{C}(029)$ $\mathrm{Ni}(01)-\mathrm{N}(008)-\mathrm{C}(060)-\mathrm{C}(029)$ $\mathrm{C}(062)-\mathrm{C}(029)-\mathrm{C}(060)-\mathrm{N}(008)$ $\mathrm{C}(051)-\mathrm{C}(029)-\mathrm{C}(060)-\mathrm{N}(008)$ $\mathrm{Ni}(01)-\mathrm{O}(006)-\mathrm{C}(061)-\mathrm{O}(010)$ $\mathrm{Ni}(01)-\mathrm{O}(006)-\mathrm{C}(061)-\mathrm{C}(072)$ $\mathrm{C}(051)-\mathrm{C}(029)-\mathrm{C}(062)-\mathrm{C}(067)$ $\mathrm{C}(060)-\mathrm{C}(029)-\mathrm{C}(062)-\mathrm{C}(067)$ $\mathrm{C}(070)-\mathrm{C}(054)-\mathrm{C}(063)-\mathrm{C}(081)$ $\mathrm{C}(086)-\mathrm{C}(054)-\mathrm{C}(063)-\mathrm{C}(081)$ $\mathrm{C}(025)-\mathrm{C}(040)-\mathrm{N}(064)-\mathrm{C}(030)$ $\mathrm{C}(043)-\mathrm{C}(040)-\mathrm{N}(064)-\mathrm{C}(030)$ $\mathrm{C}(025)-\mathrm{C}(040)-\mathrm{N}(064)-\mathrm{Ni}(02)$ $\mathrm{C}(043)-\mathrm{C}(040)-\mathrm{N}(064)-\mathrm{Ni}(02)$ $\mathrm{C}(011)-\mathrm{C}(030)-\mathrm{N}(064)-\mathrm{C}(040)$ $\mathrm{C}(016)-\mathrm{C}(030)-\mathrm{N}(064)-\mathrm{C}(040)$ $\mathrm{C}(011)-\mathrm{C}(030)-\mathrm{N}(064)-\mathrm{Ni}(02)$ $\mathrm{C}(016)-\mathrm{C}(030)-\mathrm{N}(064)-\mathrm{Ni}(02)$ $\mathrm{N}(035)-\mathrm{Ni}(02)-\mathrm{N}(064)-\mathrm{C}(040)$ $\mathrm{O}(014)-\mathrm{Ni}(02)-\mathrm{N}(064)-\mathrm{C}(040)$ $\mathrm{N}(042)-\mathrm{Ni}(02)-\mathrm{N}(064)-\mathrm{C}(040)$ $\mathrm{N}(035)-\mathrm{Ni}(02)-\mathrm{N}(064)-\mathrm{C}(030)$ $\mathrm{O}(014)-\mathrm{Ni}(02)-\mathrm{N}(064)-\mathrm{C}(030)$ $\mathrm{N}(042)-\mathrm{Ni}(02)-\mathrm{N}(064)-\mathrm{C}(030)$ $\mathrm{C}(069)-\mathrm{C}(026)-\mathrm{C}(065)-\mathrm{C}(083)$
$-167.7(2)$

$8.9(3)$

$10.8(3)$

$-172.6(2)$

$-15.1(3)$

175.60(18)

167.21(18)

$-2.0(2)$

167.16(19)

$-75.2(3)$

$-14.9(3)$

102.7(2)

3.4(3)

$-174.3(2)$

$-0.3(4)$

177.6(2)

$-3.5(3)$

$-39.4(2)$

$-161.3(2)$

$-1.8(3)$

$-123.6(3)$

112.1(2)

$0.3(4)$

$-1.3(3)$

177.3(2)

64.6(2)

$-176.8(2)$

$-53.3(2)$

$-97.6(3)$

80.1(3)

179.72(17)

$0.9(2)$

$-2.0(3)$

$175.8(2)$

$-0.7(4)$

179.9(3)

176.55(18)

$-0.3(3)$

$-1.6(3)$

$-178.44(14)$

$-154.51(18)$

81.7(2)

23.95(19)

$-99.82(18)$

$-11.33(19)$

161.44(19)

$-126.5(7)$

$170.38(14)$

$-16.85(14)$

55.2(8)

139.1(2) 


\begin{tabular}{|c|c|c|c|}
\hline $\mathrm{O}(014)-\mathrm{C}(011)-\mathrm{C}(030)-\mathrm{C}(016)$ & $102.2(2)$ & $\mathrm{C}(039)-\mathrm{C}(026)-\mathrm{C}(065)-\mathrm{C}(083)$ & $-40.6(3)$ \\
\hline $\mathrm{C}(053)-\mathrm{C}(016)-\mathrm{C}(030)-\mathrm{N}(064)$ & $-163.25(19)$ & $\mathrm{C}(069)-\mathrm{C}(026)-\mathrm{C}(065)-\mathrm{C}(072)$ & $-94.2(2)$ \\
\hline $\mathrm{C}(066)-\mathrm{C}(016)-\mathrm{C}(030)-\mathrm{N}(064)$ & $68.1(3)$ & $\mathrm{C}(039)-\mathrm{C}(026)-\mathrm{C}(065)-\mathrm{C}(072)$ & $86.1(3)$ \\
\hline $\mathrm{C}(053)-\mathrm{C}(016)-\mathrm{C}(030)-\mathrm{C}(011)$ & $75.8(3)$ & $\mathrm{C}(048)-\mathrm{C}(013)-\mathrm{C}(066)-\mathrm{C}(068)$ & $1.0(3)$ \\
\hline $\mathrm{C}(066)-\mathrm{C}(016)-\mathrm{C}(030)-\mathrm{C}(011)$ & $-52.8(3)$ & $\mathrm{C}(048)-\mathrm{C}(013)-\mathrm{C}(066)-\mathrm{C}(016)$ & $-175.4(2)$ \\
\hline $\mathrm{C}(019)-\mathrm{C}(022)-\mathrm{C}(033)-\mathrm{C}(076)$ & $1.7(3)$ & $\mathrm{C}(053)-\mathrm{C}(016)-\mathrm{C}(066)-\mathrm{C}(013)$ & $134.2(2)$ \\
\hline $\mathrm{C}(068)-\mathrm{C}(027)-\mathrm{C}(034)-\mathrm{C}(048)$ & $-0.1(4)$ & $\mathrm{C}(030)-\mathrm{C}(016)-\mathrm{C}(066)-\mathrm{C}(013)$ & $-98.2(3)$ \\
\hline $\mathrm{C}(068)-\mathrm{C}(027)-\mathrm{C}(034)-\mathrm{F}(005)$ & $-179.4(2)$ & $\mathrm{C}(053)-\mathrm{C}(016)-\mathrm{C}(066)-\mathrm{C}(068)$ & $-42.1(3)$ \\
\hline $\mathrm{O}(004)-\mathrm{C}(018)-\mathrm{N}(035)-\mathrm{C}(024)$ & $-7.2(3)$ & $\mathrm{C}(030)-\mathrm{C}(016)-\mathrm{C}(066)-\mathrm{C}(068)$ & $85.5(3)$ \\
\hline $\mathrm{C}(020)-\mathrm{C}(018)-\mathrm{N}(035)-\mathrm{C}(024)$ & $177.40(18)$ & $\mathrm{C}(075)-\mathrm{C}(056)-\mathrm{C}(067)-\mathrm{C}(062)$ & $3.2(4)$ \\
\hline $\mathrm{O}(004)-\mathrm{C}(018)-\mathrm{N}(035)-\mathrm{Ni}(02)$ & $176.56(18)$ & $\mathrm{C}(029)-\mathrm{C}(062)-\mathrm{C}(067)-\mathrm{C}(056)$ & $-1.3(4)$ \\
\hline $\mathrm{C}(020)-\mathrm{C}(018)-\mathrm{N}(035)-\mathrm{Ni}(02)$ & $1.2(2)$ & $\mathrm{C}(034)-\mathrm{C}(027)-\mathrm{C}(068)-\mathrm{C}(066)$ & $0.5(4)$ \\
\hline $\mathrm{C}(052)-\mathrm{C}(024)-\mathrm{N}(035)-\mathrm{C}(018)$ & $-21.7(3)$ & $\mathrm{C}(013)-\mathrm{C}(066)-\mathrm{C}(068)-\mathrm{C}(027)$ & $-0.9(3)$ \\
\hline $\mathrm{C}(025)-\mathrm{C}(024)-\mathrm{N}(035)-\mathrm{C}(018)$ & $159.4(2)$ & $\mathrm{C}(016)-\mathrm{C}(066)-\mathrm{C}(068)-\mathrm{C}(027)$ & $175.4(2)$ \\
\hline $\mathrm{C}(052)-\mathrm{C}(024)-\mathrm{N}(035)-\mathrm{Ni}(02)$ & $154.10(17)$ & $\mathrm{C}(039)-\mathrm{C}(026)-\mathrm{C}(069)-\mathrm{C}(057)$ & $-0.8(3)$ \\
\hline $\mathrm{C}(025)-\mathrm{C}(024)-\mathrm{N}(035)-\mathrm{Ni}(02)$ & $-24.8(3)$ & $C(065)-C(026)-C(069)-C(057)$ & $179.6(2)$ \\
\hline $\mathrm{N}(064)-\mathrm{Ni}(02)-\mathrm{N}(035)-\mathrm{C}(018)$ & $-159.97(15)$ & $\mathrm{C}(074)-\mathrm{C}(057)-\mathrm{C}(069)-\mathrm{C}(026)$ & $-1.2(4)$ \\
\hline $\mathrm{O}(014)-\mathrm{Ni}(02)-\mathrm{N}(035)-\mathrm{C}(018)$ & $92.0(6)$ & $\mathrm{C}(063)-\mathrm{C}(054)-\mathrm{C}(070)-\mathrm{C}(079)$ & $1.7(4)$ \\
\hline $\mathrm{N}(042)-\mathrm{Ni}(02)-\mathrm{N}(035)-\mathrm{C}(018)$ & $13.50(15)$ & $\mathrm{C}(086)-\mathrm{C}(054)-\mathrm{C}(070)-\mathrm{C}(079)$ & $-178.8(2)$ \\
\hline $\mathrm{N}(064)-\mathrm{Ni}(02)-\mathrm{N}(035)-\mathrm{C}(024)$ & 23.93(17) & $\mathrm{N}(008)-\mathrm{C}(038)-\mathrm{C}(071)-\mathrm{C}(055)$ & $-28.7(2)$ \\
\hline $\mathrm{O}(014)-\mathrm{Ni}(02)-\mathrm{N}(035)-\mathrm{C}(024)$ & $-84.1(6)$ & $\mathrm{C}(021)-\mathrm{C}(055)-\mathrm{C}(071)-\mathrm{C}(038)$ & $41.8(2)$ \\
\hline $\mathrm{N}(042)-\mathrm{Ni}(02)-\mathrm{N}(035)-\mathrm{C}(024)$ & $-162.60(17)$ & $\mathrm{C}(015)-\mathrm{N}(017)-\mathrm{C}(072)-\mathrm{C}(061)$ & $-154.60(19)$ \\
\hline $\mathrm{C}(046)-\mathrm{C}(031)-\mathrm{C}(036)-\mathrm{C}(052)$ & $3.1(4)$ & $\mathrm{Ni}(01)-\mathrm{N}(017)-\mathrm{C}(072)-\mathrm{C}(061)$ & $23.9(2)$ \\
\hline $\mathrm{C}(059)-\mathrm{C}(032)-\mathrm{C}(037)-\mathrm{C}(044)$ & $0.3(4)$ & $\mathrm{C}(015)-\mathrm{N}(017)-\mathrm{C}(072)-\mathrm{C}(065)$ & $83.3(2)$ \\
\hline $\mathrm{C}(021)-\mathrm{N}(008)-\mathrm{C}(038)-\mathrm{C}(071)$ & & & $-98.19(17)$ \\
\hline $\mathrm{C}(060)-\mathrm{N}(008)-\mathrm{C}(038)-\mathrm{C}(071)$ & $-117.5(2)$ & $\mathrm{O}(010)-\mathrm{C}(061)-\mathrm{C}(072)-\mathrm{N}(017)$ & $165.01(19)$ \\
\hline $\mathrm{Ni}(01)-\mathrm{N}(008)-\mathrm{C}(038)-\mathrm{C}(071)$ & 121.91(16) & $\mathrm{O}(006)-\mathrm{C}(061)-\mathrm{C}(072)-\mathrm{N}(017)$ & $-16.2(2)$ \\
\hline $\mathrm{C}(069)-\mathrm{C}(026)-\mathrm{C}(039)-\mathrm{C}(047)$ & $1.4(3)$ & $\mathrm{O}(010)-\mathrm{C}(061)-\mathrm{C}(072)-\mathrm{C}(065)$ & $-73.5(3)$ \\
\hline $\mathrm{C}(065)-\mathrm{C}(026)-\mathrm{C}(039)-\mathrm{C}(047)$ & $-179.0(2)$ & $\mathrm{O}(006)-\mathrm{C}(061)-\mathrm{C}(0$ & $105.3(2)$ \\
\hline $\mathrm{C}(046)-\mathrm{C}(025)-\mathrm{C}(040)-\mathrm{N}(064)$ & $-169.8(2)$ & $\mathrm{C}(026)-\mathrm{C}(065)-\mathrm{C}(072)-\mathrm{N}(017)$ & $65.2(2)$ \\
\hline $\mathrm{C}(024)-\mathrm{C}(025)-\mathrm{C}(040)-\mathrm{N}(064)$ & $7.0(3)$ & $\mathrm{C}(083)-\mathrm{C}(065)-\mathrm{C}(072)-\mathrm{N}(017)$ & $-166.16(18)$ \\
\hline $\mathrm{C}(046)-\mathrm{C}(025)-\mathrm{C}(040)-\mathrm{C}(043)$ & $7.0(3)$ & $\mathrm{C}(026)-\mathrm{C}(065)-\mathrm{C}(072)-\mathrm{C}(061)$ & $-54.3(3)$ \\
\hline $\mathrm{C}(024)-\mathrm{C}(025)-\mathrm{C}(040)-\mathrm{C}(043)$ & $-176.2(2)$ & $\mathrm{C}(083)-\mathrm{C}(065)-\mathrm{C}(072)-\mathrm{C}(061)$ & $74.3(2)$ \\
\hline $\mathrm{C}(019)-\mathrm{C}(028)-\mathrm{C}(041)-\mathrm{C}(076)$ & $3.0(3)$ & $\mathrm{C}(039)-\mathrm{C}(047)-\mathrm{C}(074)-\mathrm{C}(057)$ & $-2.2(4)$ \\
\hline $\mathrm{C}(015)-\mathrm{C}(028)-\mathrm{C}(041)-\mathrm{C}(076)$ & $-175.4(2)$ & $\mathrm{C}(039)-\mathrm{C}(047)-\mathrm{C}(074)-\mathrm{F}(009)$ & $179.6(2)$ \\
\hline $\mathrm{C}(018)-\mathrm{C}(020)-\mathrm{N}(042)-\mathrm{C}(086)$ & $-94.4(2)$ & $\mathrm{C}(069)-\mathrm{C}(057)-\mathrm{C}(074)-\mathrm{C}(04$ & $2.8(4)$ \\
\hline $\mathrm{C}(085)-\mathrm{C}(020)-\mathrm{N}(042)-\mathrm{C}(086)$ & $144.1(2)$ & $\mathrm{C}(069)-\mathrm{C}(057)-\mathrm{C}(074)-\mathrm{F}(009)$ & $-179.0(2)$ \\
\hline $\mathrm{C}(018)-\mathrm{C}(020)-\mathrm{N}(042)-\mathrm{C}(058)$ & $146.1(2)$ & $\mathrm{C}(029)-\mathrm{C}(051)-\mathrm{C}(075)-\mathrm{C}(056)$ & $-1.6(4)$ \\
\hline $\mathrm{C}(085)-\mathrm{C}(020)-\mathrm{N}(042)-\mathrm{C}(058)$ & $24.6(2)$ & $\mathrm{C}(067)-\mathrm{C}(056)-\mathrm{C}(075)-\mathrm{C}(051)$ & $-1.7(4)$ \\
\hline $\mathrm{C}(018)-\mathrm{C}(020)-\mathrm{N}(042)-\mathrm{Ni}(02)$ & $29.4(2)$ & $\mathrm{C}(022)-\mathrm{C}(033)-\mathrm{C}(076)-\mathrm{C}(041)$ & $-1.9(3)$ \\
\hline $\mathrm{C}(085)-\mathrm{C}(020)-\mathrm{N}(042)-\mathrm{Ni}(02)$ & $-92.11(18)$ & $\mathrm{C}(028)-\mathrm{C}(041)-\mathrm{C}(076)-\mathrm{C}(033)$ & $-0.5(3)$ \\
\hline $\mathrm{N}(035)-\mathrm{Ni}(02)-\mathrm{N}(042)-\mathrm{C}(020)$ & $-23.84(14)$ & $\mathrm{C}(045)-\mathrm{C}(043)-\mathrm{C}(078)-\mathrm{C}(082)$ & $-4.1(3)$ \\
\hline $\mathrm{N}(064)-\mathrm{Ni}(02)-\mathrm{N}(042)-\mathrm{C}(020)$ & 91.8(7) & $\mathrm{C}(040)-\mathrm{C}(043)-\mathrm{C}(078)-\mathrm{C}(082)$ & $170.4(2)$ \\
\hline $\mathrm{O}(014)-\mathrm{Ni}(02)-\mathrm{N}(042)-\mathrm{C}(020)$ & $163.60(13)$ & $\mathrm{C}(054)-\mathrm{C}(070)-\mathrm{C}(079)-\mathrm{C}(073)$ & $-1.3(4)$ \\
\hline $\mathrm{N}(035)-\mathrm{Ni}(02)-\mathrm{N}(042)-\mathrm{C}(086)$ & $100.09(17)$ & $\mathrm{C}(081)-\mathrm{C}(073)-\mathrm{C}(079)-\mathrm{C}(070)$ & $-0.2(4)$ \\
\hline $\mathrm{N}(064)-\mathrm{Ni}(02)-\mathrm{N}(042)-\mathrm{C}(086)$ & $-144.3(7)$ & $\mathrm{N}(042)-\mathrm{C}(058)-\mathrm{C}(080)-\mathrm{C}(085)$ & $-23.0(3)$ \\
\hline $\mathrm{O}(014)-\mathrm{Ni}(02)-\mathrm{N}(042)-\mathrm{C}(086)$ & $-72.47(17)$ & $\mathrm{C}(079)-\mathrm{C}(073)-\mathrm{C}(081)-\mathrm{C}(063)$ & $1.3(4)$ \\
\hline $\mathrm{N}(035)-\mathrm{Ni}(02)-\mathrm{N}(042)-\mathrm{C}(058)$ & $-137.47(18)$ & $\mathrm{C}(054)-\mathrm{C}(063)-\mathrm{C}(081)-\mathrm{C}(073)$ & $-0.8(4)$ \\
\hline $\mathrm{N}(064)-\mathrm{Ni}(02)-\mathrm{N}(042)-\mathrm{C}(058)$ & $-21.8(8)$ & $\mathrm{C}(043)-\mathrm{C}(078)-\mathrm{C}(082)-\mathrm{C}(077)$ & $2.5(4)$ \\
\hline $\mathrm{O}(014)-\mathrm{Ni}(02)-\mathrm{N}(042)-\mathrm{C}(058)$ & 49.97(18) & $\mathrm{C}(084)-\mathrm{C}(077)-\mathrm{C}(082)-\mathrm{C}(078)$ & $0.6(4)$ \\
\hline $\mathrm{N}(064)-\mathrm{C}(040)-\mathrm{C}(043)-\mathrm{C}(078)$ & $-95.6(3)$ & $\mathrm{C}(043)-\mathrm{C}(045)-\mathrm{C}(084)-\mathrm{C}(077)$ & $0.4(3)$ \\
\hline
\end{tabular}


183

$\begin{array}{lrlr}\mathrm{C}(025)-\mathrm{C}(040)-\mathrm{C}(043)-\mathrm{C}(078) & 87.5(3) & \mathrm{C}(082)-\mathrm{C}(077)-\mathrm{C}(084)-\mathrm{C}(045) & -2.1(4) \\ \mathrm{N}(064)-\mathrm{C}(040)-\mathrm{C}(043)-\mathrm{C}(045) & 78.9(3) & \mathrm{C}(058)-\mathrm{C}(080)-\mathrm{C}(085)-\mathrm{C}(020) & 37.9(3) \\ \mathrm{C}(025)-\mathrm{C}(040)-\mathrm{C}(043)-\mathrm{C}(045) & -98.0(3) & \mathrm{N}(042)-\mathrm{C}(020)-\mathrm{C}(085)-\mathrm{C}(080) & -38.9(3) \\ \mathrm{C}(032)-\mathrm{C}(037)-\mathrm{C}(044)-\mathrm{C}(023) & 0.1(4) & \mathrm{C}(018)-\mathrm{C}(020)-\mathrm{C}(085)-\mathrm{C}(080) & -159.2(2) \\ \mathrm{N}(007)-\mathrm{C}(023)-\mathrm{C}(044)-\mathrm{C}(037) & -176.8(2) & \mathrm{C}(020)-\mathrm{N}(042)-\mathrm{C}(086)-\mathrm{C}(054) & 49.5(3) \\ \mathrm{C}(049)-\mathrm{C}(023)-\mathrm{C}(044)-\mathrm{C}(037) & -1.1(3) & \mathrm{C}(058)-\mathrm{N}(042)-\mathrm{C}(086)-\mathrm{C}(054) & 166.8(2) \\ \mathrm{C}(078)-\mathrm{C}(043)-\mathrm{C}(045)-\mathrm{C}(084) & 2.7(3) & \mathrm{Ni}(02)-\mathrm{N}(042)-\mathrm{C}(086)-\mathrm{C}(054) & -70.4(3) \\ \mathrm{C}(040)-\mathrm{C}(043)-\mathrm{C}(045)-\mathrm{C}(084) & -172.0(2) & \mathrm{C}(063)-\mathrm{C}(054)-\mathrm{C}(086)-\mathrm{N}(042) & -101.4(3) \\ \mathrm{C}(036)-\mathrm{C}(031)-\mathrm{C}(046)-\mathrm{C}(025) & -2.0(3) & \mathrm{C}(070)-\mathrm{C}(054)-\mathrm{C}(086)-\mathrm{N}(042) & 79.1(3)\end{array}$




\section{LIST OF PUBLICATIONS}

1. V. Raev, N. Vasilieva, S. Kruglov, A. Kurinov Migration of Radionuclides from Chernobyl's losses by vertical soil profile of meadows. Theses of reports of the third All-Union conference on agricultural radiology. Obninsk, 1990, Vol. IV, 16.

2. S. Kruglov, R. Alexakhin, V. Raev, N. Sanzharova, S. Fesenko, V. Anisimov, S. Spiridonov, V. Osipov Radionuclides Migration in Natural and Semi-natural ecosystems. In: The behaviour of radionuclide in natural and semi-natural environments. Report for the Period 1 October 1991 - 30 November 1992. CEC coordinated ECP-5 Project. Contract Number COSU-CT-91-0005. Doc. ENEADISP/ARA-MET 1992, 6, 49-108.

3. S. Kruglov, V. Raev Parameters of cerium, cesium, ruthenium and strontium radionuclide migration in the basic soil types of $30-\mathrm{km}$ Chernobyl NPP zone. Radiobiological congress. Kiev 20-25 sept. 1993. Theses of reports. Part II. Puschino 1993, 528-529.

4. V. V. Razin, N. V. Ulin, V. A. Raev, N. Yu. Zadonskaya, D. S. Zuev Synthesis and dehydrbromination of nitrile and methyl ester of 3-bromomethyl- and 3methoxymethyl-3-bromocyclobutanecarboxylic acids. Russian J. Org. Chem., 2003, 39, 1, 40-48; Zh. Org. Khim. (russ.), 2003, 39, 1, 51-59.

5. V. A. Raev, E. M. Kharicheva Microwave irradiation using for synthesis of pyrazolines and cyclopropanes from aliphatic diazo compounds. Theses of report of the fourth youth conference on Organic Chemistry, St.Petersburg, 2004, 128

6. D. Kuznetsov, V. Raev, G. Kuranov, O. Arapov, R. Kostikov, Microwave Activation in Organic Synthesis (Review), Russian J. Org. Chem., 2005, 41, 12, 1719-1749; Zh. Org. Khim. (russ.), 2005, 41, 12, 1757-1787. 


\section{LEBENSLAUF}

Vitaly Raev

Hermann-Rein Str. 11,

D-37075 Göttingen

Email: vraev@gwdg.de

phone: 0551 / 2726844

Ich wurde am 21. September 1965 als erster von zwei Söhnen des Schlossers Alexander Raev und seiner Ehefrau, der Hausfrau Valentina Raeva geb. Kiseleva, in Murmansk (UdSSR) geboren.

Von September 1972 bis Juni 1982 besuchte ich die Grund- und Mittelschule No. 45 in Murmansk, an der ich im Juni 1982 das Abitur ablegte. Während der Schulzeit nahm ich im Jahre 1981 an der Physikalischen Regional-Schülerolympiade (3. Platz) und im Jahre 1982 an der Chemischen Regional-Schülerolympiade (2. Platz) teil.

Im Herbstsemester 1982 begann ich das Studium der Chemie an der Chemischen Fakultät der Staatlichen Universität Leningrad, UdSSR. Meine Freizeit verbrachte ich als freiwilliger wissenschaftlicher Mitarbeiter in der Gruppe von Prof. Dr. V. I. Ivanskij.

Im Herbstsemester 1982 begann ich das Studium der Organischen Chemie unter der wissenschaftlichen Anleitung von Dr. V. V. Razin und zum Juni 1987 fertigte ich meine Diplomarbeit zu dem Thema „Zum Problem der Synthese des Bicyclo[1.1.1]pentans“ an. Am 22. Juni 1987 bestand ich meine Diplomprüfung vor der Staatlichen Prüfungskommission, wobei mir die Qualifizierung des Diplom-Chemikers zuerkannt wurde.

Nach dem Universitätsstudium war ich als Diplom-Ingenieur in Chemie in dem AllunionsForschungs-Institut für Landwirtschaftliche Radiologie in Obninsk, Kaluga Gebiet, UdSSR, tätig. Im April und im August 1988, im Juni 1989 und im Juli 1990 habe ich je zweiwöchige Dienstreisen in das Gebiet des Chernobyl-Kernunfalls als offizieller Reise-Ingenieur durchgeführt.

Im Mai 1993 habe ich einen zusätzlichen Kurs zur Ausbildung in ökonomischen Fragen und von Juni 1993 bis Oktober 1995 arbeitete ich als Manager in der Bescheinigung von medizinischen Präparaten in der Firma „Jablochko SO“ in St. Peterburg, Russland. 
Im Oktober 1995 habe ich ein gemeinsames Finanzprojekt mit der Bank „Inkombank“ durchgeführt und von Oktober 1995 bis April 1997 arbeitete ich als Vorstandsvorsitzender der Firma „Investitionsfond „Slavutych“ in Simferopol, Krim, Ukraine.

Im Mai 1997 wurde dieses Projekt Teil eines anderen Projektes bei der Firma „Geschäftsbank „Kreditprombank“, und von Mai 1997 bis März 2002 arbeitete ich als Hauptwirtschaftswissenschaftler an der Aktienabteilung der Firma „Geschäftsbank „Kreditprombank“ in Kiew, Ukraine.

Ab Juli 2002 setzte ich das Studium der Chemie an der Chemischen Fakultät der Staatlichen Universität Sankt-Petersburg, Russland, unter der wissenschaftlichen Anleitung von Prof. Dr. R. R. Kostikov fort und zum Juni 2004 fertigte ich meine Magisterarbeit zu dem Thema „Einfluss von Mikrowellenbestrahlung des Ethyldiazoacetats, Reaktionsweisen mit und ohne StickstoffAbspaltung“"an.

Am 22. Juni 2004 bestand ich meine Magisterprüfung vor der Staatlichen Prüfungskommission, wobei mir die Qualifizierung eines Magisters im Fachgebiet Chemie zuerkannt wurde.

Seit Januar 2005 arbeite ich an meiner Dissertation unter der wissenschaftlichen Anleitung von Prof. Dr. Armin de Meijere im Institut für Organische und Biomolekulare Chemie der GeorgAugust-Universität Göttingen.

Meine Sprachkenntnisse sind: Englisch - gut, Deutsch - Grundkenntnisse. 


\section{ACKNOLEDGEMENT}

I would like to thank Professor Dr. A. de Meijere for giving me an opportunity to work in his excellent group, for his help and support during the period of the work on my $\mathrm{PhD}$ thesis. I am grateful also for opening me new perspectives in my life and carrier.

I am extremely grateful to Prof. Dr. Rafael Kostikov, who helped me to come back to chemistry after a too long period of "business".

My thanks are also due to Dr. V. Belov for his valuable support at the beginning and help in and outside the laboratory. I would like to thank Dr. S. Kozhushkov for his advice, support, and scientific and non-scientific discussions.

I am also very grateful to my nice colleagues for the wonderful working atmosphere: Alessandra Zanobini, Alexander Lygin, Andrey Savchenko, Daniel Frank, Farina Brackmann, Hans-Wolf Sünemann, Heiko Schill, Irina Martynova, Karsten Rauch, Ligang Zhao, Marco Marradi, Oleg Larionov, Sergey Kozhushkov, Shamil Nizamov, Stefan Beußhausen, Vadim Korotkov and Viktar Bahutski.

I am indebted to Prof. Dr. A. de Meijere and Deutsche Forschungsgemeinschaft (SFB 416 project) for the financial support of my research.

I am very thankful to Prof. Dr. A. Zeck for co-refereeing this thesis.

I am very grateful to Mr. Machinek and his entire group for the fast and precise NMR measurements and discussions of NMR problems; to Dr. H. Frauendorf - for MS and LC-MS measurements; to Mr. F. Hambloch - for the elemental analysis; to Mrs. Pfeil - for the measurements of optical rotatory power; to Stefan and Heiko - for their help with computers; Alessandra, Hans and Heiko - for their help with the NMR instrument, Heiko - for his help with GC chromatograph, Vadim - for his help with FT-IR instrument.

I am very thankful to Dr. Dmitrii Yufit (Durham University, UK) and Mr. Christian Grosse for determining of the X-ray structures of my compounds.

I appreciate the assistance and help of Mrs. Gabriele Keil-Knepel in most of the organizational issues.

I am very grateful to Alexander Lygin and Vadim Korotkov for their careful proofreading of this manuscript. 
I am very grateful to Farina Brackmann for the corrections of my German in important documents.

I am grateful to Andrey Savchenko for mushrooms.

Thank you all! 